\title{
1979 Bibliography of Atomic and Molecular Processes
}

August 1980

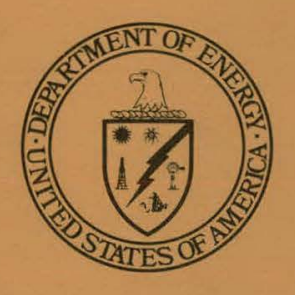

U.S. Department of Energy

Office of Basic Energy Sciences

Division of Chemical Sciences 


\section{DISCLAIMER}

This report was prepared as an account of work sponsored by an agency of the United States Government. Neither the United States Government nor any agency Thereof, nor any of their employees, makes any warranty, express or implied, or assumes any legal liability or responsibility for the accuracy, completeness, or usefulness of any information, apparatus, product, or process disclosed, or represents that its use would not infringe privately owned rights. Reference herein to any specific commercial product, process, or service by trade name, trademark, manufacturer, or otherwise does not necessarily constitute or imply its endorsement, recommendation, or favoring by the United States Government or any agency thereof. The views and opinions of authors expressed herein do not necessarily state or reflect those of the United States Government or any agency thereof. 


\section{DISCLAIMER}

Portions of this document may be illegible in electronic image products. Images are produced from the best available original document. 
Printed in the United States of America Available from

National Technical Information Service U.S. Department of Commerce 5285 Port Royal Road

Springfield, VA 22161

NTIS price codes

Printed Copy: $\$ 18.00$

Microfiche Copy: $\$ 3.50$ 


\title{
1979 Bibliography of Atomic and Molecular Processes
}

\author{
August 1980
}

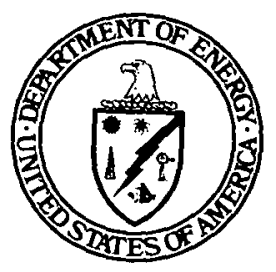

\section{U.S. Department of Energy \\ Office of Basic Energy Sciences Division of Chemical Sciences \\ Washington, D.C. 20545}


THIS WORK IS PUBLISHED UNDER THE AUSPICES OF THE OFFICE OF BASIC ENERGY SCIENCES WITHIN THE U. S. DEPARTMENT OF ENERGY. ITS PUBLICATION IS DESIGNED TO HELP INCREASE THE UTILITY OF EXISTING SOURCES OF ENERGY AND TO ASSIST THE DEVELOPMENT OF NEW ONES. IN ADDITION, THE BIBLIOGRAPHY WILL ASSIST THE IMPROVED UNDERSTANDING OF THE FUNDAMENTAL PKUCESSES INVOT.VFT TN STIITIES RELATED TO THIE DEEIGN. THE DEPARTMENT OF ENERGY ACKNOWLEDGES THE CONTRIBUTIONS BY SCIENTISTS ASSOCIATED WITH THE OAR RIDGE NATIONAL LABORATORY THAT MADE THE APPEARANCE OF THIS PUBLICATION POSSIBI,E.

J. V. MARTINEZ ATOMIC PHYSICS PROGRAM AUGUST 1980 
BIBLIOGRAPHY OF ATOMIC AND MOLECULAR PROCESSES

1979

compiled by.

C. F. Barnett

D. H. Crandall

B. J. Farmer

H. B. Gilbody
P. M. Hafford

M. I. Kirkpattick

E. W. McDaniel

R. H. McKnight
F. W. Meyer

T. G. Morgan

R. A. Phaneuf

E. W. Thomas

Physics Division

Oak Ridge National Laboratory

Oak Ridge, Tennessee 37830 


\section{CONTENTS}

Abstract ............................ . v v

Introduction . . . . . . . . . . . . . . . . . . . . . 1

Categorization List. . . . . . . . . . . . . . . .... . 4

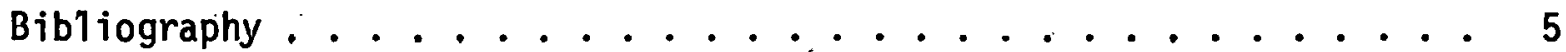

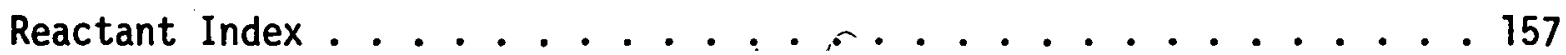

Author Index . . . . . . . . . . . . . . . . . . . 245 
THIS PAGE

WAS INTENTIONALLY

LEFT BLANK 
ABSTRACT

This annotated bibliography lists 2146 works on atomic and molecular processes reported in publications dated 1979. Sources include scientific journals, conference proceedings, and books. Each entry is designated by one or more of the 114 categories of atomic and molecuar processes used by the Controlled Fusion Atomic Data Center, Oak Ridge National Laboratory, to classify data. Also indicated is whether the work was experimental or theoretical, what energy range was covered, what reactants were investigated, and the country of origin of the first author. Following the bibliographical listing are indexes of reactants and authors.

v 


\section{INTRODUCTION}

This annotated bibliography lists 2146 works on atomic and molecular processes reported in publications dated 1979. It is the first such bibliography to be formatted under an open-field computer code developed for the Controlled Fusion Atomic Data Center, Oak Ridge National Laboratory. Unlike previous bibliographies which listed entries alphabetically by first author, there is no such ordering of entries in this publication. An alphabetical arrangement by author can be found in the author index. Also unlike bibliographies formatted using an earlier computer code, entries in this bibliography are not grouped according to a particular atomic or molecular process (i.e., there is no independent section on electron transfer, ionization, etc.). Instead, each entry is labeled by one or more of the 114 categories of atomic and molecular processes used by the Controlled Fusion Atomic Data Center to classify data. (A list of these categories is found on page 4.) Grouping according to specific categories is found in the reactants index. The author index is likewise arranged by assigned category. The absence of an author and reactants index for any one of these 114 categories indicates that there were no entries designated to that category in 1979. Each entry indicated whether the work was experimental (E) or theoretical $(T)$, what energy range was covered, and what reactants were investigated. 
The following remarks are offered to facilitate the use of the bibliography:

1. Entries were edited for incorrect categorization. Misplaced reactants were removed from their original reactants index category and added to the correct index. Authors listed for these entries were similarly removed to the appropriate index. The addition of re= actant to the reactants index is indicated by an asterisk beside the entry's alphabetically correct position; the reactant is given at the end of the appropriate column. The same method is used to add authors to the author index. When necessary, double asterisks, triple asterisks, and daggers are used to show second, third, or fourth additions to a column.

2. Due to the computer manipulation, any differences in symbolic representation or formatting resulted in different entries in the index. For example, a superscript +t is handled differently from a superscript 2+. All attempts have been made to minimize these discrepancies. However, the user should keep these possible differences in mind when performing searches.

3. Sequencing of reactants in the index follows the order $N, N^{*}$ (excited state), $\mathrm{N}+, \mathrm{N}_{2}, \mathrm{~N}^{-}, \mathrm{NO}, \mathrm{Na}, \mathrm{Ne}$, etc.

4. Many papers do not refer to a particular collision system. Reactants in these cases are listed as undefined, denoted as Undef. Review papers are labeled Review. rather than listing all reactants 
discussed in the paper. The abbreviation Seq, preceded by an atom, indicates all members of the isoelectronic sequence for that particular atom. PERT symbolizes "periodic table"; this notation is used when reactions involving a large number of the elements are covered by a publication. All of these codes are used in a general sense to avoid handling thousands of additional reactants at every stage in the production of these bibliographies.

5. The country listed at the end of each bibliographic entry is derived from the address of the first author given in the original publication. 
A. HEAVY PARTICLE - HEAYY PARTICLI INTERAITIONS
1. General
. Dissociation
Chemiluminescence, Fi:sorescence, and LumI nescence
(photon emission by nispecified proces se
Electron Captor
Recombination or Mutieal, Nestralization Leadfig to Neutra
10. Electronic, Vibrat10r.a.1, and Rotationai Imeryy Iransfer
(process unknown)
13. Heavy Particie Interctange
ingement, asd Association (one or more ionic reactants) Heavy Particle Interctanges
(only neutral reactants)
16. Spin Exchange
16.ectron Detachment from Negative Lons into Continuum
17. Interaction Potentials
19. Angular Scattering (specified process or dherw/se)
19. Inecific categories:
20. Attenuation (unspecified process)

B. INTERACTIONS WITH STATIC OR TIME-VARYIMGi ELE:T VIC ANG MAGNETIC

1. General

. Ionization

5. Quenching

6. Excitation in Presence of Intense Eectronegnatic Fields

c. PARTICle penetPation IN MacrosCo:IC Matter (10NS, revtrals, ayo ELECTRONS)
. General Los
Eergy to Create an Io.r Pa
Particle Range
Charge State Popula
: Excited State Population

D. PARTICLE INTERACTIONS WITH SOLIDS
1. General
3. Sputtered Particle Charce and Quantin (ExciLed State Distritution Secondary Electron Ejection oy Heavy Particle and Electrons

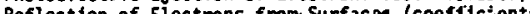 Reflection of Heavy Particle: from Sirfaces
(total reflection coefficleñs)
8. Charge and Quantum State O1s:ribut lors of Feflected Heavy Particles
9. De-Excitation, Neutralizatior, Jonizasion, or [-1ssoclation of Particles Interacting with Surfaces

\section{"aATEGORIZATION LIST}

10. Interaction Potentials Between Surfaces and Free Particles

. Located Externar to the Surface (electrons and heavy particies)

12. Electranagnetic Radiation Induced by Electron or Heavy Particle

3. Desorpt on of coses from Surfaces
4. Blistering, Volds, end Surface Strain in Metals

16. Partion tamage in Me surs

17. Electron-, Ion-, and Photon-Induced Chemical Changes to Surfaces

E. electrun-particle interaction
1. General Collistons
4. Excitation
6. Recombination (electron-ion)
Collis ional De-Excitation
Collisional Line Braddening
9. Negat ive Ion Formation
Ons (Bremsstrahlung),

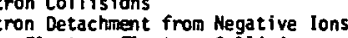
4. Binary Electron-Electron
16. Fluorescence and Luminescence
17. Angular Scattering (specified process)

G. TRANSPORT PHENOMENA AND AYERAGE PROPERTIES IN LASES

1. General 01 ffusion of Neutrals

Diffusion of Electrons

Diffusion of lons
Drift Velocity of Electrons
Orift Velocity of Iors

8. Meutrals, and Ions in Gases
Energy Distributicn (energy distribution of ions and

9. Momentum Transfer

10. First and Second Tounsend Coeffictents
11. Electron Attachment Coefficients

A. PHOTON COLLISIONS WITH hEAYY PARTICLES, ELECTROAS, AND
1. General
2. Total Absorpt ion
3. Elastic Scottering
. Excitation
. Dissociation
7. Photodetachment
. Fluorescence and Luminescence Yields
. Free-Free Absorption or Inverse Bremsstrahlung
Photon-Electron Scattering
. Photon-Photon Collitsions
. Photo-De-Excitation, Quenching, and Stinul azed

J. DATA COMPILATION

1. Heavy Particle

3. Photons

4. Particles on Surfaces

5. Ind Sols ids
6. Transort
Structure

K. REVIENS AND BOOKS

1. Heavy Particle

3. Photons

4. Particles on Surfaces

5. Transport

BIBLIOGRAPHI

1. Heavy Particle

2. Electrons

4. Particles on Surfaces

5. Thansport

6. Structure

ABBFEVIATIONS:

L1 Seq - sequence (Li)
PERT - pertodic table
Undef - undefined 
BIBLIOGRAPHY OF ATOMIC AND MOLECULAR PROCESSES

1979 


\section{BIBLIOGRAPHY}




\begin{tabular}{|c|c|c|c|c|}
\hline $\begin{array}{l}\text { QEF. } \\
\text { No. }\end{array}$ & REACTANTS & $\begin{array}{l}\text { EXP } \\
\text { NR: } \\
\text { THEOR. }\end{array}$ & $\begin{array}{l}\text { ENERGY } \\
\text { RANGE }\end{array}$ & REFERENCE \\
\hline 1 & $e^{E}+c_{12} ; e+\mathrm{Cl}_{2}+\mathrm{Ar}$ & $\mathbf{E}$ & $\operatorname{sen} x$ & 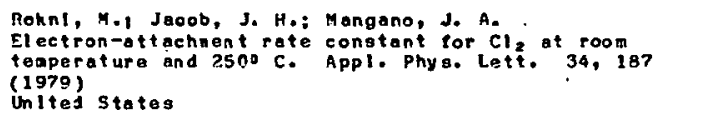 \\
\hline 2 & $\mathrm{Hr}_{\mathrm{H}}^{\mathrm{H}}+\mathrm{N}_{2}$ & E. & $9440=n-1$ & $\begin{array}{l}\text { Baravtan, G.j Godart, J.: Sultan, G. Experimental } \\
\text { reaults on ihe multiphoton lonizat ton of molecular } \\
\text { nltrogen. Appl. Phys. Lett. } 34,190 \text { (1979) } \\
\text { France }\end{array}$ \\
\hline 3 & $\begin{array}{l}\mathrm{Al} 4 \\
\mathrm{KrF}\end{array}+\mathrm{Ar}+\mathrm{Ar}: \mathrm{KrF}+\mathrm{Kr}+\mathrm{Kr}$ & $T$ & $30 \mathrm{c}-830 \mathrm{~K}$ & 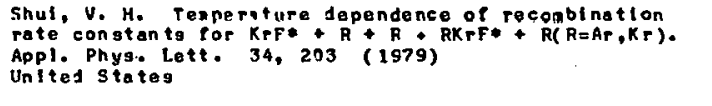 \\
\hline 4. & 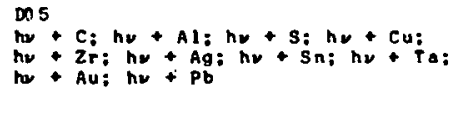 & $\mathbf{E}$ & $5-130 \mathrm{keV}$ & 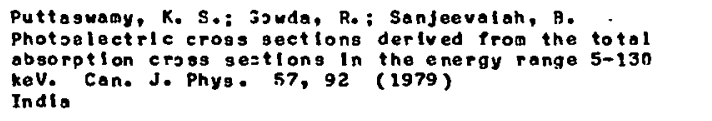 \\
\hline 5 & $\operatorname{Mr}_{\text {ho }}+\mathrm{KI}$ & $\mathbf{E}$ & $265-335 \mathrm{~nm}$ & 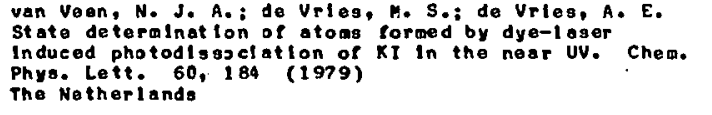 \\
\hline 6 & $\begin{array}{l}\mathrm{HO}_{2} \\
\mathrm{hom}_{4}+\mathrm{H}_{2} \mathrm{O} \\
\mathrm{mon}_{2}+\mathrm{H}_{2} \mathrm{O} \\
\mathrm{HO}_{6} \\
\mathrm{Ho}+\mathrm{H}_{2} \mathrm{O}\end{array}$ & $T$ & $4-60$ eV & 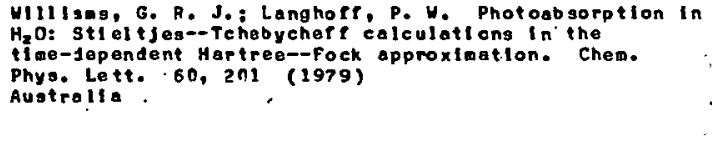 \\
\hline$?$ & $\begin{array}{l}\mathrm{AlO} \\
\mathrm{C}^{*}+\mathrm{Co}\end{array}$ & $T$ & $3 n n k$ & 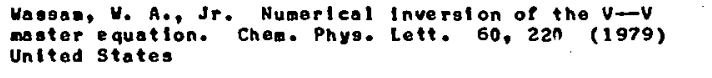 \\
\hline 8 & 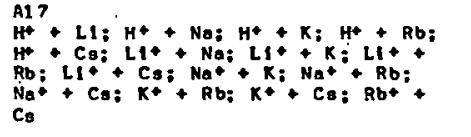 & $T$ & Under & $\begin{array}{l}\text { Kolke, F. Core--coro Interaction potentlals of alkall } \\
\text { diatodic aolecular long. Heteronuclear case. Chem. } \\
\text { Phys. Lett. } 60,271 \text { (1979) } \\
\text { Japan }\end{array}$ \\
\hline 9 & 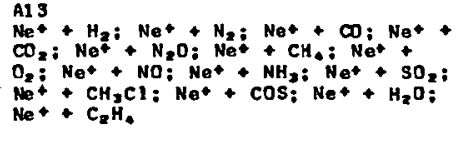 & $\mathbf{E}$ & $300 \mathrm{~K}$ & 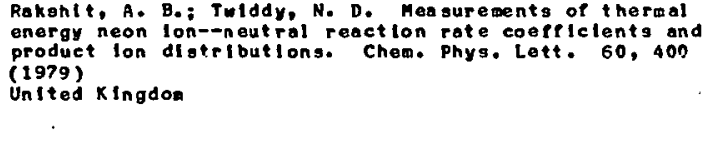 \\
\hline 13 & $\begin{array}{l}\mathrm{Al}^{2} \text { + } \mathrm{LI}: \mathrm{Nat}+\mathrm{Na}: \mathrm{Rb}+\mathrm{Rb}: \mathrm{Cs}++ \\
\mathrm{Cs}_{3}: \mathrm{K}+\mathrm{K}\end{array}$ & $\mathbf{T}$ & Under & 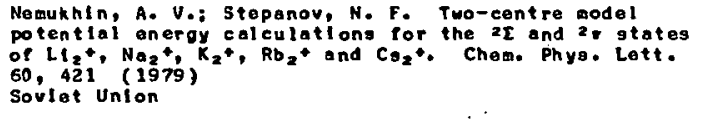 \\
\hline 11 & $\begin{array}{l}\mathrm{HOO}^{\mathrm{O}}+\mathrm{SO}_{2} \\
\mathrm{HLO} \\
\mathrm{HL}+\mathrm{SO}_{2}\end{array}$ & E & $4 \mu$ & 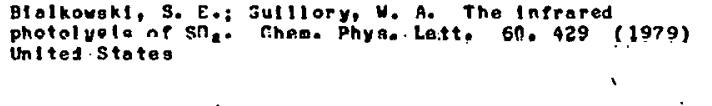 \\
\hline 12 & $\begin{array}{l}10404 \\
2 h v+I_{2}\end{array}$ & E & $\begin{array}{l}4250-3950 \\
A O\end{array}$ & $\begin{array}{l}\text { Wlllinson, A. D: Stepulge two-photon exctitatlon of } \\
\text { molecular iodine: the E tate vindicated. Chem. Phys. } \\
\text { Lett. } 60,451 \text { (1979) } \\
\text { United States }\end{array}$ \\
\hline 13 & $\mathrm{PO}_{\mathrm{H}}^{\mathrm{PO}}+\mathrm{BO}_{2}$ & E & 579 ח & 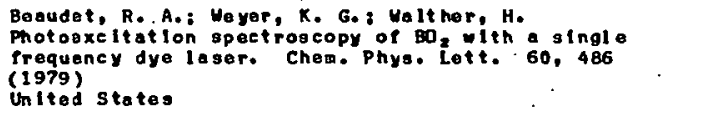 \\
\hline 14 & $\begin{array}{l}\mathrm{AO}_{2} \\
\mathrm{LI}+\mathrm{H}_{2}: \mathrm{H}++\mathrm{H}_{2} \\
\mathrm{AOS} \\
\mathrm{LI}+\mathrm{H}_{2}: \mathrm{H}+\mathrm{H}_{2}\end{array}$ & $\mathbf{T}$ & $0.6-3.7 \mathrm{eV}$ & 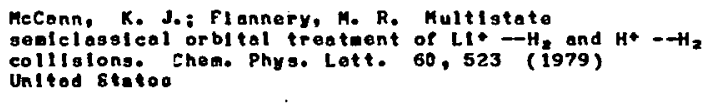 \\
\hline 15 & $\mathrm{HI}_{\mathrm{h}}^{\mathrm{H}}+\mathrm{HF}$ & $\mathbf{E}$ & $0.28 \mathrm{BV}$ & $\begin{array}{l}\text { Hinehen, J. J.: Hobbs, R. H. Rotattonal population } \\
\text { tranofar in HF. J. APpl. Phys. 50, 628 (1979) } \\
\text { United States }\end{array}$ \\
\hline
\end{tabular}




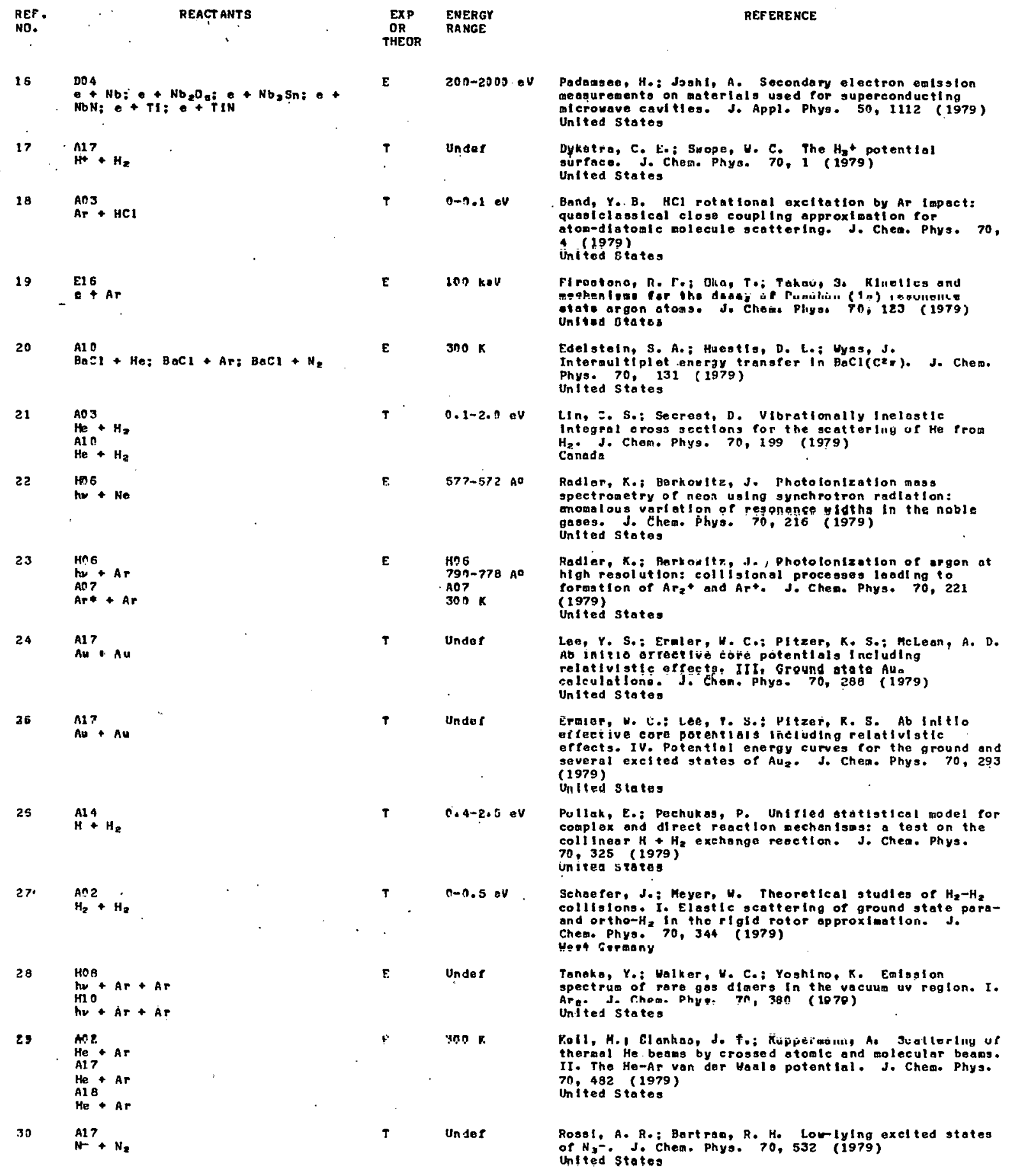




\begin{tabular}{|c|c|c|c|c|}
\hline $\begin{array}{l}\text { REF. } \\
\text { ND. }\end{array}$ & REACT ANTS & $\begin{array}{l}\text { EXP } \\
\text { OR } \\
\text { THEOR }\end{array}$ & $\begin{array}{l}\text { ENERGY } \\
\text { RANGE }\end{array}$ & REF ERENCE. \\
\hline 31 & $\begin{array}{l}\mathrm{AO2} \\
\mathrm{He}+\mathrm{N}_{2}: \mathrm{He}+\mathrm{O}_{2}: \mathrm{He}+\mathrm{CO}: \mathrm{He}+\mathrm{NO} \\
\mathrm{Al} \text { ? } \\
\mathrm{He}+\mathrm{N}_{2}: \mathrm{He}+\mathrm{O}_{2}: \mathrm{He}+\mathrm{CO} \mathrm{He}+\mathrm{NO} \\
\mathrm{Al} 1 \mathrm{~B}+\mathrm{N}_{2}: \mathrm{He}+\mathrm{O}_{2}: \mathrm{He}+\mathrm{CO} \mathrm{He}+\mathrm{NO}\end{array}$ & E & $300 \mathrm{~K}$ & 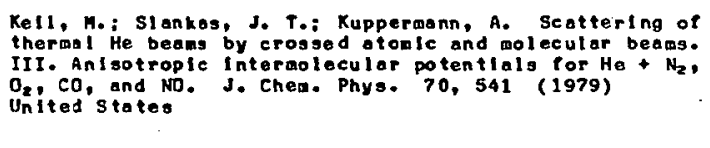 \\
\hline 32 & $\begin{array}{l}\mathrm{Al} 1 \\
\mathrm{BaCl}+\mathrm{He}: \mathrm{BaCl}+\mathrm{Ar}: \mathrm{BaCl}+\mathrm{N}_{2}\end{array}$ & $\varepsilon$ & $300 \cdot K$ & 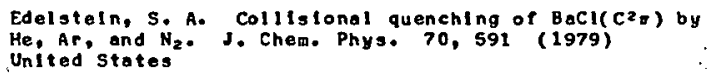 \\
\hline 33 & 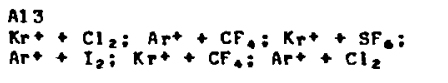 & E & $300 \mathrm{~K}$ & $\begin{array}{l}\text { Kuen, I.: Howorks, F. Noble gns hnilde lons: KrCl+, } \\
\text { KrFt, ArC1+, Arlt. J. Chem. Phys. 7C, S95 (1979) } \\
\text { Austrla }\end{array}$ \\
\hline 34. & ${ }_{\text {hN }}^{\mathrm{Hn}}+\mathrm{H}_{2} \mathrm{O}$ & $\mathbf{E}$ & $90(-4)) \cdot A^{\circ}$ & 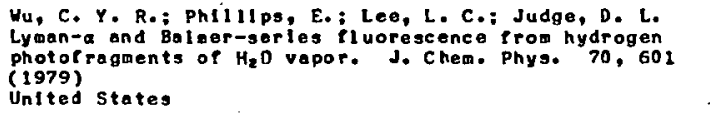 \\
\hline 35 & $\stackrel{\mathrm{Al3}}{\mathrm{N}^{+}+\mathrm{H}_{2}: \mathrm{N}+}+\mathrm{CH}_{*}$ & E & $1.6-150 \mathrm{eV}$ & 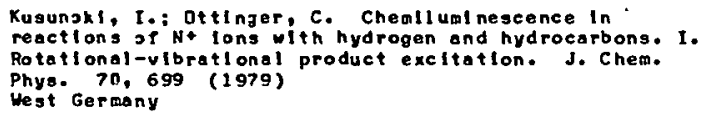 \\
\hline 36 & $\mathrm{~N}^{\mathrm{A1} 3}+\mathrm{H}_{2}: \mathrm{N}++\mathrm{CH}_{*}$ & $E$ & $1.6-453 \mathrm{eV}$ & 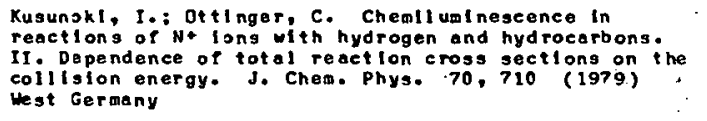 \\
\hline 37 & $\mathrm{Al}_{\mathrm{Ne}}+\mathrm{He}_{2}+$ & $T$ & Unde f & 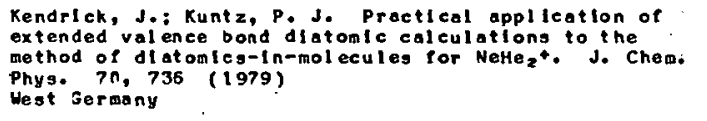 \\
\hline 33 & $\mathrm{H}_{\mathrm{Hg} *}+\mathrm{CO} ; \mathrm{Hg} *+\mathrm{NO}$ & E & $300 \mathrm{~K}$ & 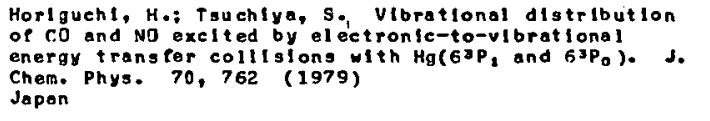 \\
\hline 39 & $\mathrm{He}_{\mathrm{He}}+\mathrm{NH}_{3}: \mathrm{He}+\mathrm{H}_{2} \mathrm{CO}$ & 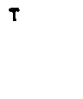 & $\begin{array}{l}103-234 \\
\mathrm{~cm}^{-1}\end{array}$ & 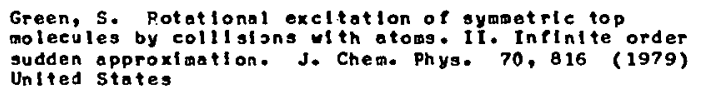 \\
\hline 40 & $\mathrm{Fl}^{\mathrm{Fe}}+\mathrm{Fe} ; \mathrm{NI}_{1}+\mathrm{N}_{1} ; \mathrm{TI}+\mathrm{Tl} ; \mathrm{K}+\mathrm{K}$ & $T$ & Undef & $\begin{array}{l}\text { Harrls, J.: Jones, R. O. Density functional theory and } \\
\text { molecular, bonding, III. Iron-series dimers. J. Chem. } \\
\text { Phys, } 70,830 \text { (ig79) } \\
\text { West Germany }\end{array}$ \\
\hline 41 & $\begin{array}{l}\text { A1 } 7 \\
\text { si }+0\end{array}$ & $\top$ & Unde? & 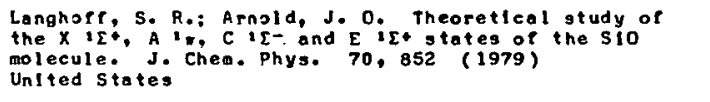 \\
\hline 42 & ${ }_{\mathrm{Ar}}^{\mathrm{Al}}+\mathrm{C}+$ & $\top$ & Unisef & 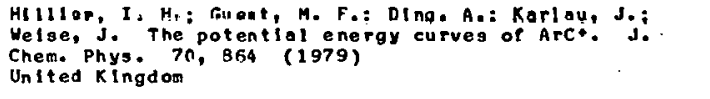 \\
\hline 43 & $\mathrm{HnS}_{\mathrm{HL}}+\mathrm{NO}_{2}$ & $E$ & $455 \mathrm{nn}$ & $\begin{array}{l}\text { Imasoka, T.: Ogava, T.: Ishibashl, N. Tlme-resolved } \\
\text { gpectroscopy on the excited electronic states of } \mathrm{NO}_{2} \text { In } \\
\text { the nelghoorhood of } 454.6 \mathrm{~nm} \text {. J. Chem. Phys. } 70,881 \\
\text { (1979) } \\
\text { Japon }\end{array}$ \\
\hline 44 & A1 + C & 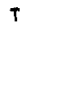 & Undet & $\begin{array}{l}\text { Kirby, K.: Liu, B. The valence states of } C_{2} \text { : } \\
\text { onflguration interaction study. J. Chen. Phys. } 70 \text {, } \\
893 \text { (1979) } \\
\text { Unlled Stutes }\end{array}$ \\
\hline 45 & $\stackrel{A}{s}+s$ & $\top$ & Under & 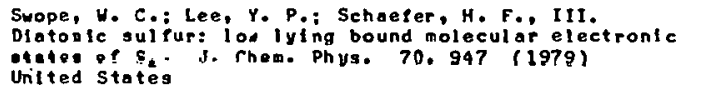 \\
\hline 46 & $\begin{array}{l}\mathrm{Al}^{2} 4 \\
\mathrm{OH}\end{array}$ & $E$ & $238-357 \times$ & 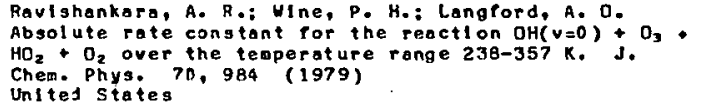 \\
\hline
\end{tabular}




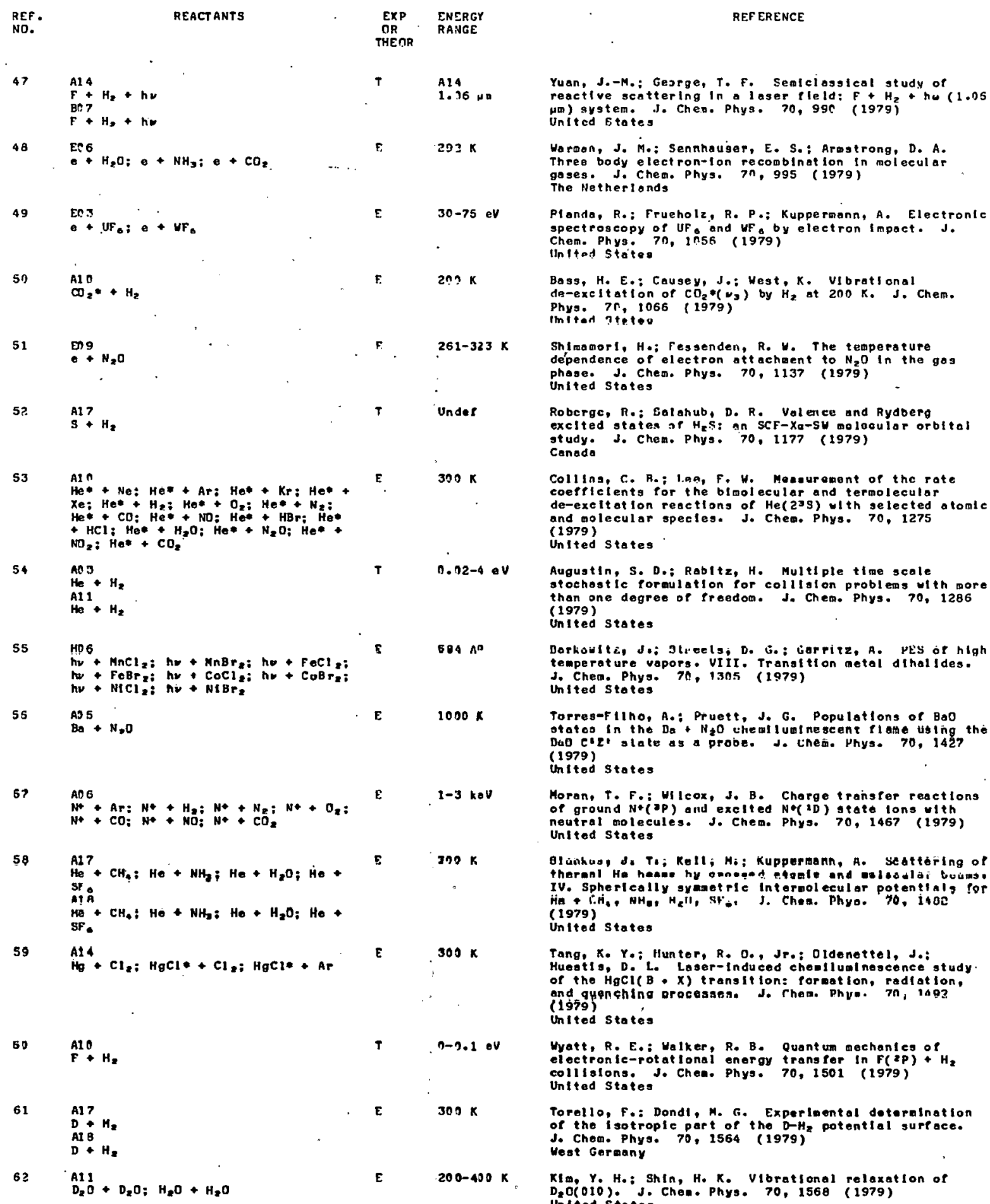

$261-323 x$

Inder

$300 \mathrm{~K}$

.12-4 ev

$\cos A^{\circ}$

$1000 \times$

$-3 k \circ v$

E Ino $\mathrm{K}$

$300 x$

$300 \times$

$200-400 K$

Yuan, J.-M.: George, T. F. Senlclasgleal study or reactlve senttering In a laser fleld: $F+H_{2}+$ hu (1.0S HIJ) system. J. Chex. Phys. 70, 99C (1979)

Warman, J. Mo: Sennhauser, E. S.: Arostrong, D. A. Three body electron-ion recombination in molecular gases. J. Chen. Phys. 7n, 995 (1979) The Netherlands

Planda, R.: Frueholz, R. P.: Kupperaann, A, Electronlc spectroscopy of UF and UF, by electron impact. J. Chem. Phys 70,1 ins $(1979)$

Bass, H. E.: Causey, J.: West, K. VIbrational da-excltation of $\mathrm{CO}_{2} *\left(v_{3}\right)$ by $H_{2}$ at $200 \mathrm{~K}$. J. Chem. Phys. 7r, 1066 (1979)

imiter itoto Shlmamorl, Ho; ressenden, R. W. The temperature
dependence of electron attachaent to $\mathrm{N}_{2} \mathrm{O}$ In the go phase. Jo Ches. Phys. 70, 1137 (1979) United states

Roberge, R.: Ealahub, D. R. Val ence and Rydberg exclted states of $H_{2} S$ : An SCF-Xa-SW molaeulor orbltal study. J. Chem. Phys. 70, 1177 (1979) Canada

Colling, C. R.: l.an, f. W. Meaurement of the rote coefficients for the bimolecular and termolecular de-excitation reactlons of $\mathrm{He}\left(2^{3} 3\right)$ w th selected atoml and molecular spectes. J. Chem. Phys. 70, 1275 (1979)

UnIted States

Augugtin, S. D.: Rabltz, H. Multiple tlme scale stochastic formulation for collision problems ulth more than one degree of Preedom. J. Chem. Phys. 70, 1286 (1979)

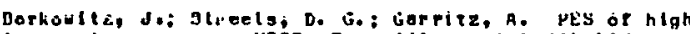
temperature vapors. VIII. Transftion metal dithalldes. J. Chen. Phys. 70, 1305 (1979)

Torrestfllno, A.: Pruett, J. G. Populations of BaO

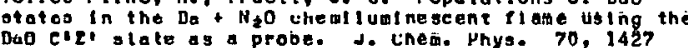
United stotes

Moran, T. F.: Wslcox, J. B. Charge transter reactlons of ground $N^{+}(J P)$ and exclted $N^{\circ}($ ND) state lons with neutrat molecules. J. Chem. Phys. 70, 1467 (1979) Olainkua, do Ta: Kell; Ma: Kupperwanh, A. Seattoring of

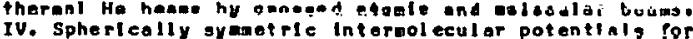
IV. Spherteally symetrle Internol ecular potentlal, (1979) Unted states

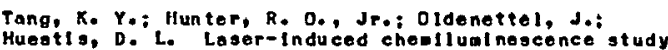
Hueatls, D. L. Laser- Induced ehenll unt nescence study. of the HgCl(B - X) transition: pormation, radiation and quenching oracessean Jo rhan. phyo $7 \mathrm{n}_{1} 1003$

United states

Wyatt, R. E.; Walker, R. B. Quant um mechantes of electronic-rotat lonal energy trans $\left(a r\right.$ in $F(2 P)+\mathrm{H}_{2}$ colliglons. J. Chea. Phys. 70, 1501 (1979) United States

Torello, F.: Dondl, M. G. Experlental deteralnation of the isotrople part of the $\mathrm{D}-\mathrm{H}_{z}$ potentlal surface. J. Chem. Phys. 70, 1564 (1979) West Gormany

Y. H.: Shin, H. K. Vibratlional relaxatlon of $D_{2} O(010)$. J. Chen. Phys. 70, 1568 (1979) 


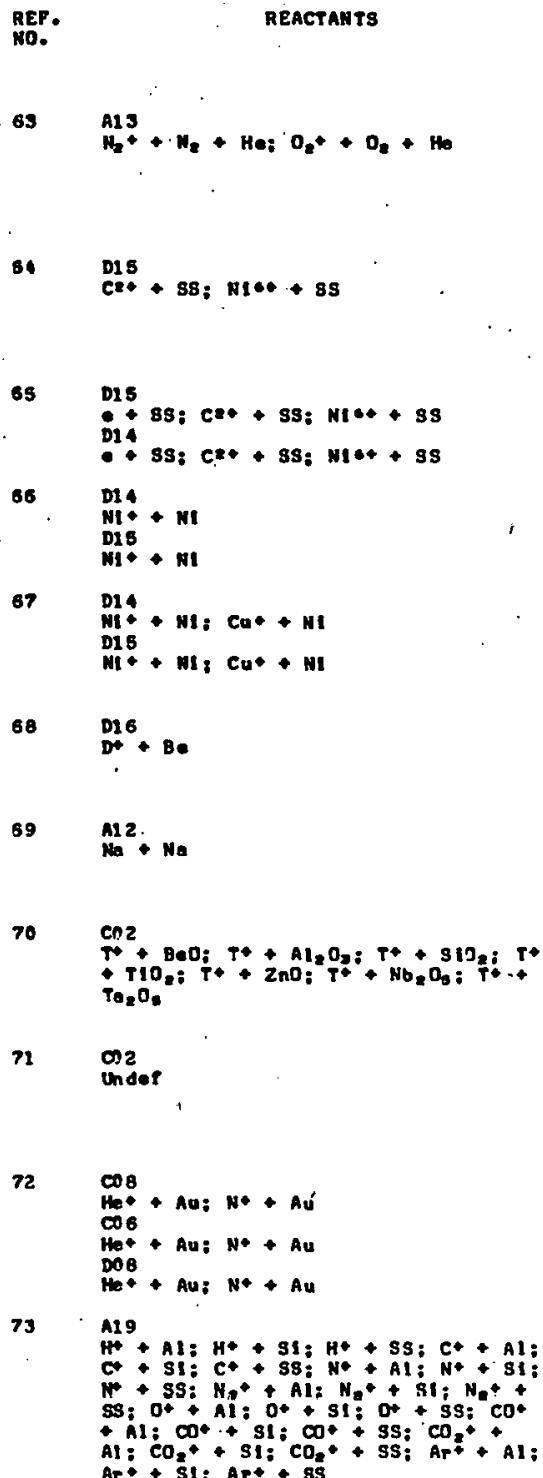

$\begin{array}{lll}\text { EXP } & \text { ENERGY } & \text { REF EREMCE } \\ \text { OR } & \text { PHEOR } & \end{array}$

E $80 x$

o...

Foheenteld, F. C.: Farguson, E. E. Comente on Dservation of eeturation in thrci-body lon-neut rel aseoclat lon reactl ons by D. K. Bohes, D. B. Dunk In, F. 1579 (1979) united stateo

E 22-46 M:V

villtams, T. H. Intoratitlal loop nucleat lon and growth in solotlon-treated type s16 stuinl oes oteol irradiated to 100 doses ul th 22 mav cat and 46.5 Mav wiot lons. Jo Nuel. Nat or. 79, 28 (1979) United KIngdo:

Forouan, A: J. E. i Makin, Mo J. The effoct of veenneg loops on the ewiling of 1 rradiated materlal a. J. thel. Natar. 79, 43 (1979) United Kingdom

Yoo, H. H. A generallzed wodel of vold swollling-the depth dependent swolling by zolf-i on irradiotion. J. Nucl. Mater. 79, 155 (1979)

United states

E $\quad 14 \mathrm{KeV}$

Inttley, J. B.: Kuletnekl, G. t.: Hlikes, P.; Salth, H. V., Jp. the depth dependent daege profile in nickel irrodiat od uith nlekel or copper lone. Jo Nue 1. Moter. 79,189 (1979)

E $10 \mathrm{kev}$

LIu, M. B.: Sheft, I.: Gruen, D. N. Deutert us trapping In ion-boebarded beryilfun. J. Muel. Mater. 79, 28 ? (1979)

United stotes

E $\quad 620-770 \mathrm{~K}$

Werdanen. J. P. Sel f-brooden ing of the Ne 3S-5s ond 3s-40 two-phot on transitione. Dgpt. Comun. 28,69 (1979) two-photo The Metherlands:

Ianlt, Ko: Blondlaux, G.: Volledon, Mo: Debrun, J. L. the study of etoppling powere by the method of the everage otoppling power. Nucl. Ino trun. Mothods 158, 199 (1979) Danle1, He: Leon, M. The effect of aultiple Coulonb scattering on the stoppl ing. Patlos of onarged partleles, ${ }_{233}^{\text {In }}$ (1979) 233 (1979)

E $\quad 1.7-2.3 \mathrm{MaV}$

KIdo, Y.: Yado, T.; Fukuzava, F. meseureaents of

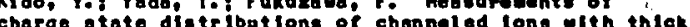
charge olate dictributhes of channoged 158,525 (1979)

Japan

50-120 heV

Cal rns, J. A.: Holloway, D. F. An exeatnatl on of the orfgln of molecular erbital X-rays erom atoale and molecular tepact on sertes of olld targots. . Mucl. Inetrum. Mothede 160, 00 (1978)

United Kingdom

Jean-Morlo, B.: Lepeltler, V.: LiHote, D. Systesatle measuresent of electron drlet veloctey and study of sose proport les of rour gos al atures: $A-\mathrm{CH}_{4} \mathrm{~A}-\mathrm{C}_{\mathrm{B}} \mathrm{H}_{4}$, $A-C_{2} H_{0}, A-C_{2} H_{0}$. Nuel. Instrup. Hathods 159, 215 (1979)

Mukthersl, So: Noyak, A. K. Calculatl on or heavy ton ranges In coeplex tedt d. Nucl. Inetrua. Mothods 159. 421 (1979) 


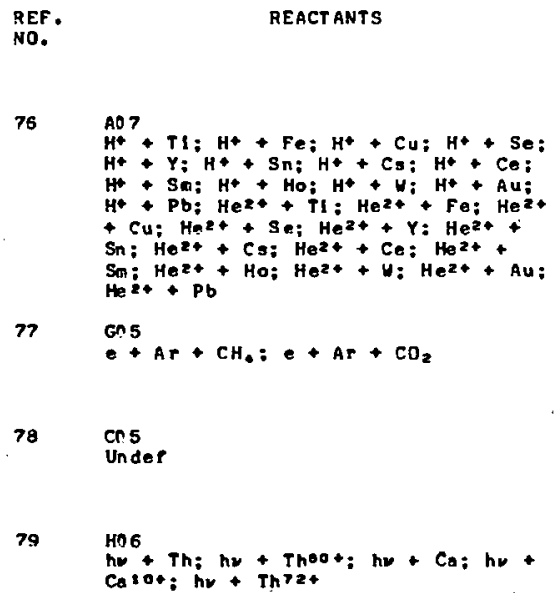

\section{$\begin{array}{cc}\text { EXP } & \\ \text { DR } & \text { ENERG } \\ \text { THEORGE }\end{array}$}
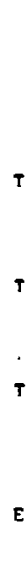

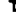

E
$4-30 \quad y \in V$

$0-10$ eV

Unde?

$0.58-81.6$

3-30 $\mathrm{keV}$

$1-2$ MeV

100 ov

30 kev

$100 \mathrm{kov}$

$12.7 \mathrm{MeV}$

$\therefore$

309 $\mathrm{K}$

is-60 keV

$0.5-2,0 \mathrm{kev}$

Under

$1.5 \mathrm{keV}$
Ponce?, H.: Engelsann;, C. Contrlbution a l'etude de lonlaotlon des olements par des protons et des perticules alpha de 4 a $30 \mathrm{MeV}$. Nuel. Instrum. Methods 159,455 (1979)

France

Mathleson, E.: El Hakeen, N. Calculation of electron transport coefficlents in counting gas at xtures. Nucl. Instrum. Mothods 159, 489 (1979) United Kirigdom

Suglyana, H. Snall-angle multiple scattering of lou-energy ions. Nucl. Instrum. Methods 159, S43 (1979)

Ong, A.: Manson, S. T.: Tseng, H. Ko: Pratt, R. H. Photoloilization of highiy stripped atonie lons: relatl

Unitad states

MIttmaack, K. On the mochanisn of cluster exlssion in sputtoring. Phys. Lett. 69A, 322 (1979) United states

Mukherdee, S. D.; Palaof, D. U: Planar channelling of hellue tons ang (100), (110) and (111) mgu. Phys. Lett. 69A, 356 (1979)

Unlted Kingdos

Das, J. N. An Iaproved second Born calculatl on or e-He olastic scattering at Intereedlate energles. Phys. Intt. 69A, 405 (1979)

Ind Ia

Ahaed, N. A. G.: Chrlatodoul Ides, C. E.: Carter, G. The depth distribution of disorder produced by roo temperature $30 \mathrm{keV}$ Art and Clt ion irradiation of sllicon. Phys. Lett, 69h, 4:1 (1979)

rea Ningdon

Burenkoy, A. F.; Komarov, F. F. Energy loss of charged partleles In nonuniform el ectron gos. Phys. Latt. $69 A, 439$ ( $19 / 9)$

sovier inion

Tenluura, 0.; Imanlohl, B. Effect of the adlabotle procesa on the absorption in heavy-ion seattering. Phys. Lett. BOB, 340 (1979) Japan

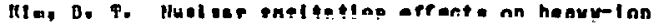
scattering. Phyg. Lett. 80B, 353 (1979) Franco

Chow, H. Aton-solld scattering: He t graphite basal plans. Furfase 001 . 20 , 157 (1979) inted states

Bruallovaky, B. A. Ton-al actran ealaston tron spherfcal erystali ine target. Surface Sel. 79, L337 (2078)

Soviet Unt on

Koshikawa, T,: Goto, K.: Saokl, No: Shlelzu, R. sugato, F. Prefarantlai soutterting of Cu-Ni alioys at low tomperature us Ing lover onergy Auger electron speetro. Surfaee Sel. 79,461 (1979) apoin

Coles, 3. N. A study of tho feaslbillty of aurface plosna Influencing secondary Ion and phot on ents ston under sedi umonergy i on bombardment. Surface Se 1.79 , $549(1979)$

Bellard, S. Wo: Utlllamis, E. M. Electron desorption cross aeftions with oxygon at tungaton (100). Surface Sel. 80, 450 (1979) 


\begin{tabular}{|c|c|c|c|}
\hline $\begin{array}{l}\text { REF. } \\
\text { NO. }\end{array}$ & REACTANTS & $\begin{array}{l}\operatorname{EXP} \\
\text { OR } \\
T H E D R\end{array}$ & $\begin{array}{l}\text { ENERGY } \\
\text { RANGE }\end{array}$ \\
\hline 92 & $\begin{array}{l}\mathrm{DO} ? \\
\mathrm{He}\end{array}$ & E & $300 \mathrm{~K}$ \\
\hline & $\because$ & ! & \\
\hline 93 & $\mathrm{HeB}_{\mathrm{He}}^{\mathrm{DOB}}+\mathrm{Ni}$ & $\varepsilon$ & $19 \mathrm{~S} \mathrm{keV}$ \\
\hline 94 & $\begin{array}{l}\text { pn? } \\
\text { Under }\end{array}$ & $T$ & Under \\
\hline 95 & $\begin{array}{l}\text { De7 } \\
\text { Under }\end{array}$ & $\mathbf{T}$ & Undet \\
\hline 96 & $\begin{array}{l}\mathrm{DOB} \\
\mathrm{Not}+\mathrm{Cu} \\
\mathrm{Not} \\
\mathrm{Ne}+\end{array}$ & E & $5-10 \mathrm{keV}$ \\
\hline 97 & 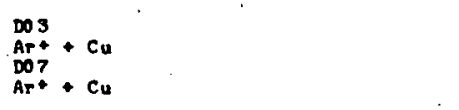 & $\mathbf{E}$ & $10-35 \mathrm{keV}$ \\
\hline 98 & $\begin{array}{l}\text { Dos } \\
\text { Under }\end{array}$ & $T$ & Unde? \\
\hline 99 & 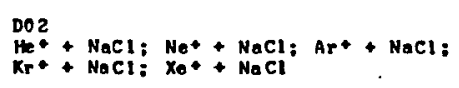 & $\mathbf{E}$ & $20 \mathrm{kev}$ \\
\hline 100 & $\begin{array}{l}\text { DOS } \\
\text { + TI } \\
+\mathrm{Tg}_{1}\end{array}$ & E. & $2-16 \mathrm{k} \in \mathrm{V}$ \\
\hline 101 & $\begin{array}{l}\text { Der } \\
\text { Under }\end{array}$ & $T$ & Under \\
\hline 102 & ${ }_{A F}^{D O 7}+$ LIF; Ne + Lif & $T$ & $300 \cdot K$ \\
\hline 100 & $\mathrm{Her}+\mathrm{Ag}: \mathrm{H}_{2}+\mathrm{Ag}^{\circ}$ & $F$ & $150-500 \cdot$ \\
\hline 104 & $\begin{array}{l}\mathrm{CO} 2 \\
\therefore+\mathrm{AI} \\
\mathrm{CO}+\mathrm{AI}\end{array}$ & $T$ & $1-108$ ov \\
\hline 105 & 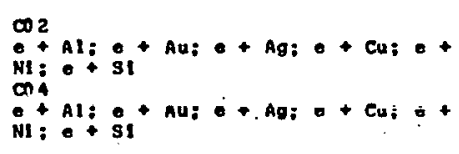 & $\mathbf{T}$ & $1-10-=$ \\
\hline 105 & 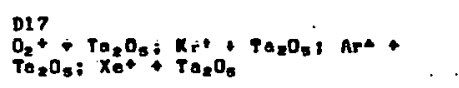 & $\mathbf{E}$ & $20-80^{\circ} \mathrm{keV}$ \\
\hline 107 & Dor & $\mathbf{T}$ & $500 x$ \\
\hline
\end{tabular}

Boato, G.; Cantlnt, P.: Tatarek, R.: folener, G. P. Diffraction of helius at ons by the basal $(0001)$ race of graphlte: a study of bound state resonances. Surface" sei. 8 , 518 (1979)

Italy

REFERENCE

Varalas, C.: Goltz, K.; SI zaonn, R. Optlcal exeltation of He profectlles durino raflectlon opt monoerystall ine surface undar channoling conditions. Surface sol, 80, 524 (1979)

Vest Germany

Armand, G.: Manson, J. R. El sot le and Inelastle scattoring of neutral atoma by a corrugated hard wall. Surface Sel. 80, 532 (1979)

Surrace
France

Benedek, Go; Garel o, N. Theory of the one-phonon seattering of atoms trom a hard corrugatod surface. Surface Sel. Bn, 543 (1979) Wat Germany

Lultjens, S. B.: Algra, A. J.: Boers, A. L. Ion tractlons of lou energy $\mathrm{Ne}\left(E_{0}\right.$ less than $\left.10 \mathrm{keV}\right)$ scattered from copper ingle erystal. Surfoce Scl. 80.566 (1979)

Balaghova, L. Lo: Bor 1 sov, A. M.: Maohkova, Eo so: Molchanov, V. A. Energy dlatributions of faot lonized. rocolls ejected prom cryat als under Ion boabardaent. :Surface Set. Bo, 573 (1979) Soviet Union

Lanac, Z.; Sunjle, M.; Sokcavle, D.; Brako, R. Low-energy scattering by al ecules ad sorbed on metal surfacos. Surface Sel. 80, 602 (1979) Yugosiavia

Hus Insky, W.: Bruekaull lor, R. Emeroy speetro of sputtered Na atons Prom boabardeent of MaCl ulth $20 \mathrm{keV}$ rare ges tons. Surface Sal. 80, 65? (2979) Austria

Viarts de Lesegno, P.: Hennequin, J.-F. Apt and or exclted argon. Auger ol ectron eel wo ton Pron Mo, Al, 81 , Gap and TI surfaces. Surface Sel. 80, 656 (1979) France

Ficocell I Varracenlo, E. Effectlve potentlal theory of at on--solld surface el astic acottering. Surfece sel. 81, 295 (1979)

Italy

Kleln, J. R.; Cole, M. Y. On the energy dependence of ratnbow paak in elassical aten--surface seattering. Surface sel. B1, LS19 (1979) Unted statos

Agede. H. Specul ar roflectlon of thellum and hydrogen polecul or beans froe the (11i) plane cr allver. Surtace Sel. 01 ; 386 (1979) Japan

Aohley, J. C.: Tung; C..J.: RItehle, R. H. Electron Inolostlc wean troo paths and energy lossos in oollda. I. Alual nue metal. Surface 801. 81, 409 (1979) Unttod stateo

Tung, C. J.: Ashloy, J. C. : Rltehio, R. H. Electron

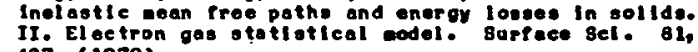
427 (1979)

Murt1, D. Ro: Relly, Ro: LLtau, 2. Lo: Pooto, Jo N. Struetural and compositional ehemes In I on-boibarded Taz $0_{3}$ : Surfoee Set. B1, S7i (1979)

Chow, H.: Thompson, E. D. The opt tcal potentlel In the scattering of hydrogen and deuterl ua atoms from LiF and Nufl 001 ): Surface Be1. 82, 1 (1979) 


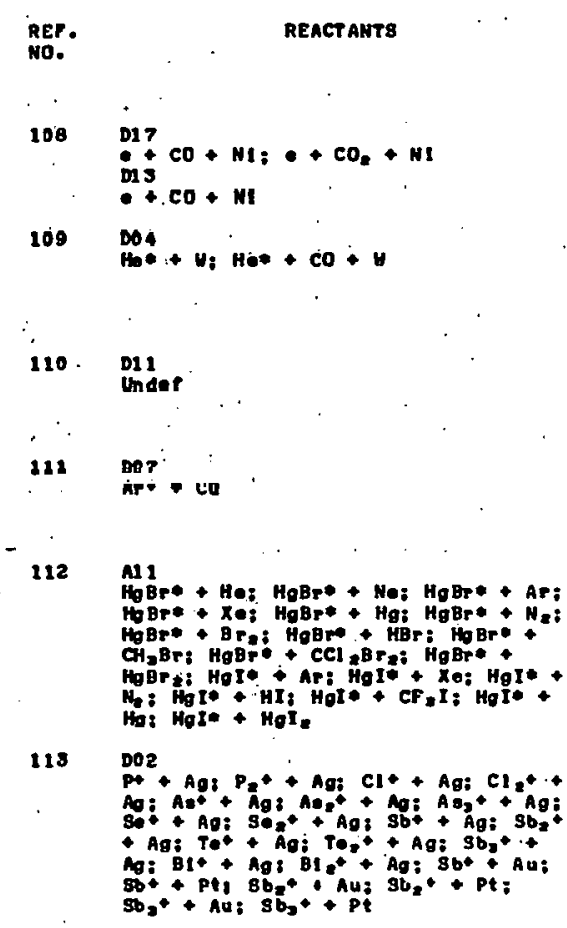

114

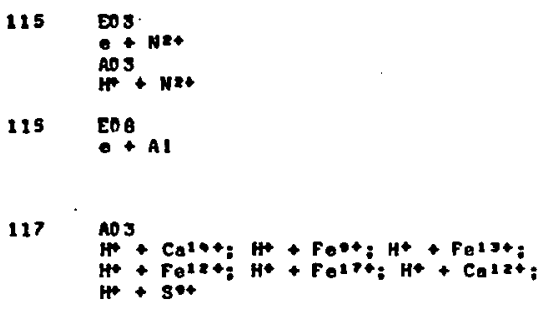

118 A05

119

Als 5 + He

tho 5 Hete

120

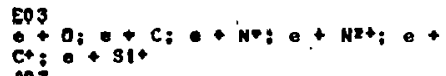

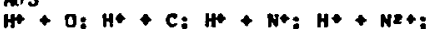

$\mathrm{H}+\mathrm{C}^{+} \mathrm{H}^{+}+\mathrm{Sl}^{+} \mathrm{H}+\mathrm{H}+\mathrm{H}: \mathrm{H}+\mathrm{O}_{2} \mathrm{H}$ $+\mathrm{C} ; \mathrm{H}+\mathrm{N}^{+}: \mathrm{H}+\mathrm{N}_{2+}: \mathrm{H}+\mathrm{C}^{+}: \mathrm{H}+$

121

\begin{tabular}{|c|c|}
\hline $\begin{array}{c}\text { EXP } \\
\text { OR } \\
\text { THEOR }\end{array}$ & $\begin{array}{l}\text { ENERGY } \\
\text { RANGE }\end{array}$ \\
\hline E & $0.5-1.5 \mathrm{kev}$ \\
\hline & . \\
\hline$\varepsilon$ & Under \\
\hline . & \\
\hline & . \\
\hline T & Undef \\
\hline $\mathbf{E}$ & $\begin{array}{l}\text { n. naks=1.n } \\
\text { bev }\end{array}$ \\
\hline & \\
\hline
\end{tabular}

$351-388 K$

15-136 kev

Thompson, D. A.; Johar, 8. S. Non 11 near sputterting efrects in thin motal tilas. Appl. Phys. Lett. 34 , $342(1979)$

$6142-5853$

Pollard, B. D.: Bl ackburn, M. B.: N1kdel, S.: Massaunt, A.: Winatordner. J. D. Atonlc Pluorescence spectronetry In the Induct Ively coupled plasmo ith coñt Inuvus dye 1 asar. Appl. Spect rosc. 13, s (1979)

Nussabcuner, H.: Storey, P. J. N III Ilnes for solor

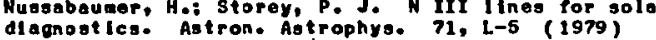
diagnoot les.

So ev in:-10: $K$

$E \quad 1 \times 10^{*} \pi$

Rondt ga, G.: Kusch, H. J. Electron Impact broaden Ing

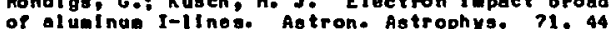
( 2079 ) Woot Germany

T

$1 \times 10^{00-4 \times 10^{\circ}}$

Kastrer, S. O.: Bhatla, A. K. Approxlaatlons for proton excitation: arrotue and extension. Astron. Astrophys. 71,211 (1979) United stateo

$105 x$

Tarter, C. B.: Velshelt, J. C: B Paloerno. A.

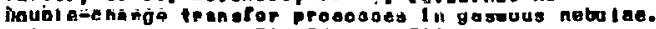
Astron. Astrophye. 71, 366 (1979) United states

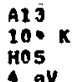

Flower, D. R.: Rougf, E. On the formation and destruetion of He $H$ in gaseovs nebul ae and the assoctated infra-red enlsglon llne spectrua. Astron. Astroonys. 72,361 (1070)

France

T $10 * k$

sanaling, A. G.1 Potlasch, 3. Rt Population ratios of Ine structure levels. . Astron. Astrophys., Suppl. Ser. $35,257$ ( 1979$)$
the Netherland:

$1000 \mathrm{~A}^{\circ}$
Federnan, 5. Ro: Glagegold, Aa Ea: Kumn, J, Atonto to wlecular hydrogen transit ion In Interstellar el ouds. Astrophys. J., Part 1 227, 466 (1979) 


\begin{tabular}{|c|c|c|c|c|c|}
\hline $\begin{array}{l}\text { REF. } \\
\text { NO: }\end{array}$ & REACTANTS & & $\begin{array}{l}\text { EXP } \\
\text { OR } \\
\text { THEOR }\end{array}$ & $\begin{array}{l}\text { ENERGY } \\
\text { RANGE }\end{array}$ & REFERENCE \\
\hline 122 & $\mathrm{HLO}_{\mathrm{H}}^{\mathrm{H} 2}+$. & $\cdot$ & $T$ & $1-20 \mathrm{keV}$ & 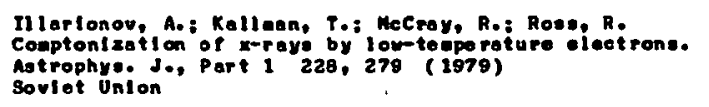 \\
\hline 123 & $\begin{array}{l}\text { Ex } 8 \\
\text { Under }\end{array}$ & & $T$ & $0.1-100 \mathrm{OV}$ & 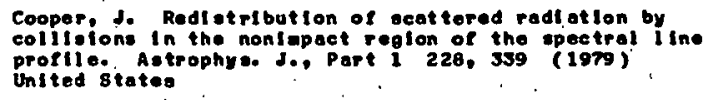 \\
\hline 124 & $\stackrel{\mathrm{Al} 3}{\mathrm{H}+\mathrm{H}-}$ & . & T & $102-20=K$ & 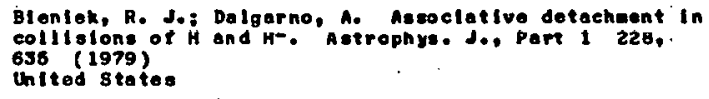 \\
\hline 125 & $\mathrm{EO}+\mathrm{FeSt}^{2}$ & & $T$ & $100 \mathrm{~K}$ & $\begin{array}{l}\text { Haug, E. Fo Ix IInes as density indl cotoris in coronal } \\
\text { plasios: Aotrophys. J., Part Iy } 228.903,(1979) \\
\text { unlted stotes }\end{array}$ \\
\hline 126 & $\mathrm{EOS}_{-}^{\mathrm{N}}+\mathrm{N}_{2} \mathrm{H}^{+}: \mathrm{e}+\mathrm{N}_{2} \mathrm{D}^{+}$ & & $\varepsilon$ & $\begin{array}{l}0.006-0.75 \\
\text { ov }\end{array}$ & 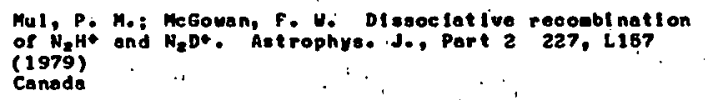 \\
\hline 127 & E05 Li seq: - + Be seq: & + B seq & $\boldsymbol{T}$ & $100 . K$ & 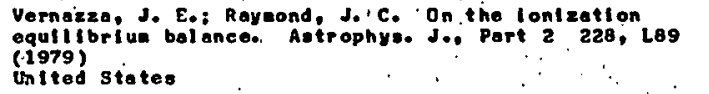 \\
\hline 128 & EOS + No & & $\boldsymbol{T}$ & $0.02-10 \mathrm{keV}$ & $\begin{array}{l}\text { Dayashankar Ion lzat ion y1 old in noon. due to oloctron } \\
\text { Impaet. Physlea B+c 96, 160 (1979) } \\
\text { Indla }\end{array}$ \\
\hline 129 & 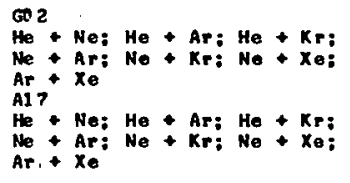 & $\begin{array}{l}H_{0}+X_{0}: \\
A r+K_{r}: \\
\mathrm{He}_{\mathrm{A}}+\mathrm{X}_{0} \\
\mathrm{Ar}\end{array}$ & $E T$ & $\begin{array}{l}275-323 k \\
\ddots\end{array}$ & 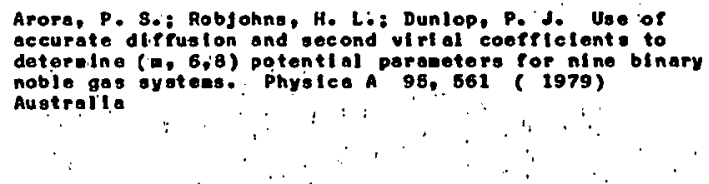 \\
\hline 139 & $\stackrel{\mathrm{Al}_{H} 2}{+}+\mathrm{He}$ & & $E T$ & $300 \cdot K$ & 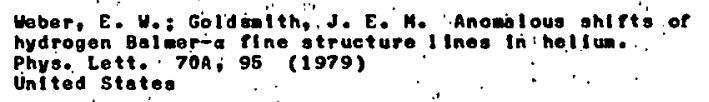 \\
\hline 131 & 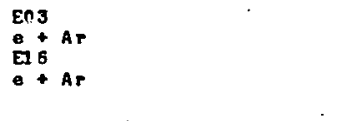 & & $E$ & $0.3-3.0 \mathrm{keV}$ & 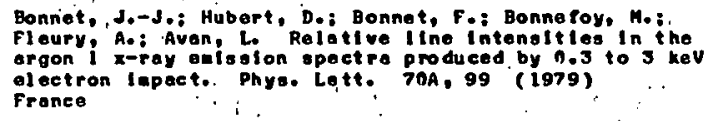 \\
\hline 132 & $\begin{array}{l}\mathrm{Cos} \\
\mathrm{O}+\mathrm{CO}=\mathrm{a}+\mathrm{CO}_{2} \\
\mathrm{On}+\mathrm{CO}=\mathrm{e}+\mathrm{CO}_{2}\end{array}$ & & E & $300^{\prime} K$ & 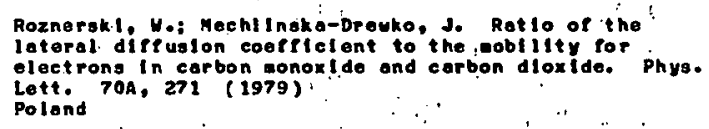 \\
\hline 133 & 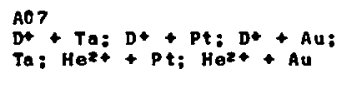 & : Hex+ & $E$ & 66 Mev/anu & 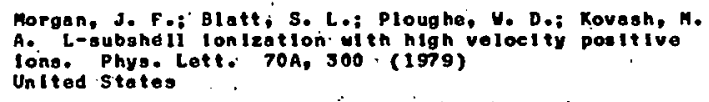 \\
\hline 134 & $\mathrm{~Pb}^{\mathrm{D}} 2+\mathrm{Pb}$ & : & $\varepsilon$ & $4.7 \mathrm{MeV} / \mathrm{asu}$ & 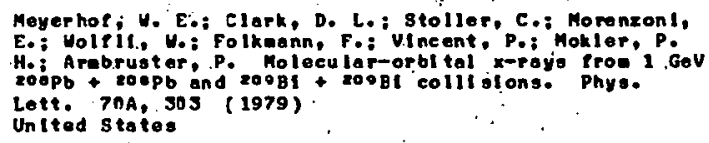 \\
\hline 135 & 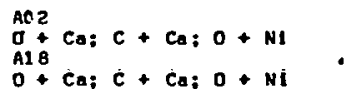 & - & $\varepsilon$ & $80-215 \mathrm{MeV}$ & 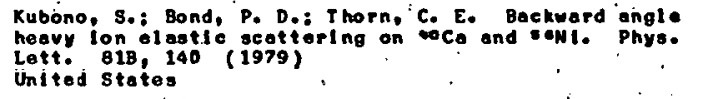 \\
\hline 136 & $\begin{array}{l}\mathrm{AOZ}_{\mathrm{H}} \\
\mathrm{A1B} \\
\mathrm{Al}^{+}+\mathrm{CO}\end{array}$ & & $\varepsilon$ & $\begin{array}{c}809 \mathrm{MoV} \\
. \quad .\end{array}$ & 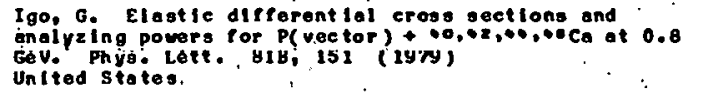 \\
\hline 137 & $\begin{array}{l}A B 2 \\
D^{2}=N I \\
A A B \\
D^{*}+N I\end{array}$ & & $\mathbf{T}$ & $\begin{array}{c}21-80 \text { MeV } \\
:\end{array}$ & 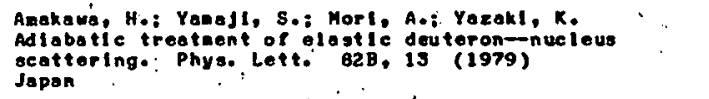 \\
\hline
\end{tabular}




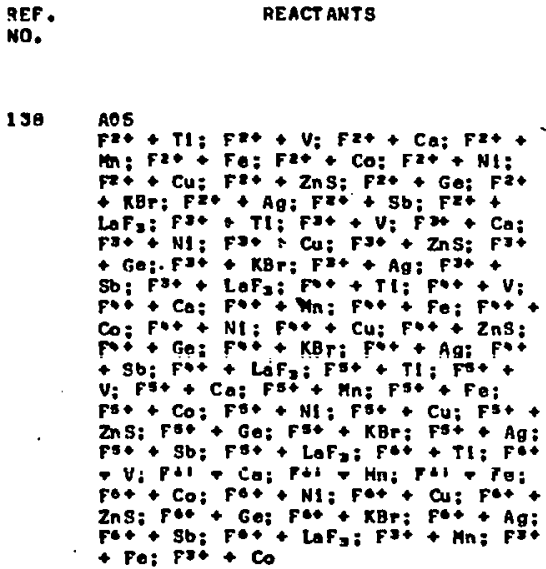

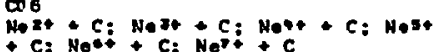

$\infty$ ?

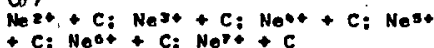

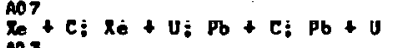

$\mathrm{X}_{0} \mathrm{~J}+\mathrm{C}_{1} \mathrm{X}_{0}+\mathrm{U}_{1} \mathrm{PO}_{0}+\mathrm{C}_{1} \mathrm{~Pb}_{6}+U$

143

An $+c ; v+v$

$U$ ? $C ; U+U$

144

$\operatorname{Cns}_{A s}+\mathrm{Xr}_{\mathrm{r} ;} \mathrm{As}_{\mathrm{s}}+\mathrm{X}_{0}$

195

$\operatorname{Ans}_{K}+\mathrm{N}_{2}: K+C O$
$\mathrm{~A}_{\mathrm{B}} \mathrm{B}+\mathrm{N}_{2}: K+\mathrm{CO}$

146

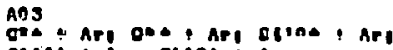
Silit+ Ar: $8128++$ Ar

AQ?

Ci. Ari $001+\operatorname{Ar} ; 9 t 101+A r$

167

no2

$2 n v+s F$.

148

Ho4

hot +8

thos
EXP ENERGY

ENERGY
RANGE

REFERENCE

TR

2-28 MeV Knap, B.: Presser, G.: Stahler, J. K-shell x-ray
production for Pluorine ions on targat el enonts Ti to production for eluorline lons on torg
La. 2. Phys. A 289, 131 (1979)

toot Getranny
$0.1-0.6$

a.u.

$1.4-5.9$
HeV

E $\begin{array}{r}\text { S.6-5.9 } \\ \text { MoV/anu }\end{array}$

E 5 oV

E $\quad 50-603.04$

2-4 MoV/anu

109

$\cos$ AO

$2300-2.100$

AO
Sarkar, K.: Basu, D.; Talukdar, B. Varlablo-phase nethod in post tron-hydrogen elastle scatterlng. Z Phys. A 289, 236 (1075)

Beyer, H. F.: H! ppler, R.; Sehartner, K.-H.; Albat, Ro An application of the branehing rotlo methed for ton Impoct cross section mossurements In the rar ul travlolet. 2. Phy o. A 289, 259 (1979) thest Goranny

Schumann, S.: Groeneveld, K. 0.: Nolto, G.: Frleke, B. Equilitbilum k-ehell excltation of highly Z. Phys. A 289, 245 (1979)

West Goradny

Behneke, Ho - - Ho ; Arabrust or, P.; Fo lkmann, F.; Haganann, S.: Hacbonal d, J. R.: Hokl er. F. H. Hessurements of $k$-shell fonizetion ith $832 x_{0}$ and 200 pb projectlles in the onorgy rango of 1.4-6. $3 \mathrm{MeV} / \mathrm{u}$. 2. Phys. A 289, 333 (1979)

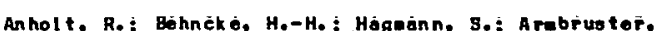

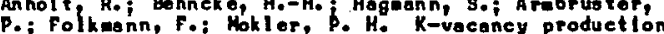
by 3.6-, 4.75-, and 5.9-HoV/anu zaeu lons. Z. Physe. A 289,349 (1979)

Seend, F.: Schueachor, H.: Rull husen, P.: Zlegelér, $C_{0}$ The slowing dom of atong ulth kinotle energles of $y$ less than or equal to Sov in noblo gasos: evldence for Inelastic interactions. 2. Phys. A 289, 365 (1979) Uest Gormany

Mertln. P, J,: Glomena, E, : Zehnle, La: Keopter, V. Appl lcat lon of aloa-photon colneidence techniquos to the deteral nation of the different lal crose section for

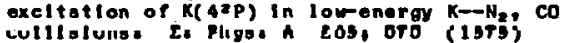
Cutll sturis:

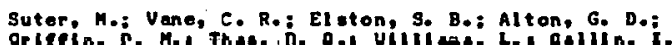

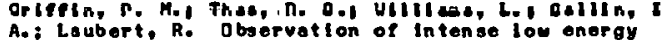
A.: Labert, Re Observatlon of Intense low onorgy autelonization linea in the ringa of the poryard peak (1907) faot

United States

Oufgley, G. P. Optoacoust ie stadl es of two-frequency Infrared-1 aser puapling of SF.. Opt. Lett. 4, B4 (1979)

Hynna, J. J.; Mereana, J. P. Rpentroennpy nf even-partity autotonizing 1 evels In Be. Opt. Lott. A, $106(1979)$ 


\begin{tabular}{|c|c|c|c|c|}
\hline $\begin{array}{l}\text { REF. } \\
\text { NO. }\end{array}$ & REACTANTS & $\begin{array}{l}\text { EXP } \\
\text { OR } \\
\text { THEOR }\end{array}$ & $\begin{array}{l}\text { ENERGY } \\
\text { RANGE }\end{array}$ & REF ERENCE \\
\hline 149 & 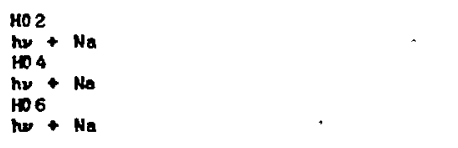 & $\varepsilon$ & $380-450$ AO & 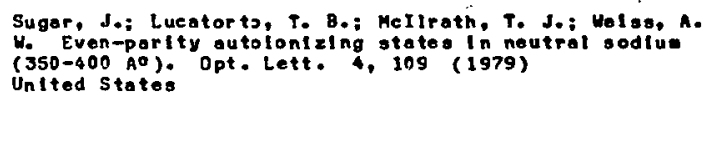 \\
\hline 150 & $\mathrm{Bl}_{\mathrm{B}}^{\mathrm{A} 2}+\mathrm{Kr}$ & E & $1200 k$ & 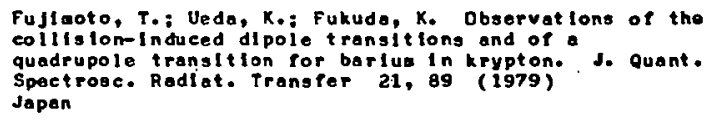 \\
\hline 151 & $\begin{array}{l}\mathrm{AlO} \\
\mathrm{Na} *+\mathrm{H}_{2} \mathrm{O}+\mathrm{Ar} ; \mathrm{Na} *+\mathrm{H}_{2} \mathrm{O} \\
\mathrm{All}+\mathrm{H}_{2} \mathrm{O}+\mathrm{Ar}: \mathrm{Na} *+\mathrm{H}_{2} \mathrm{O}\end{array}$ & E & $1800 \mathrm{~K}$ & 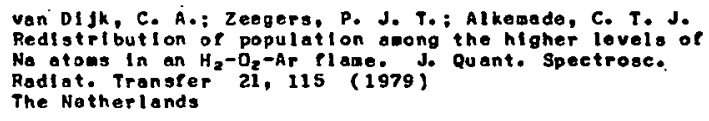 \\
\hline 152 & $\begin{array}{l}\text { A1 } 2 \\
\text { Under }\end{array}$ & $T$ & Unde? & 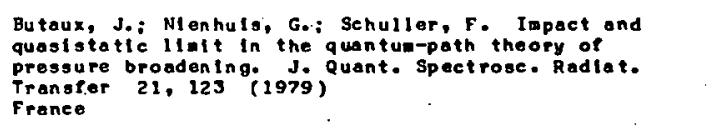 \\
\hline 153 & ${ }_{\text {he? }}^{\text {HOP }}+\mathrm{O}^{-}$ & E & $0.5-4$ ev & 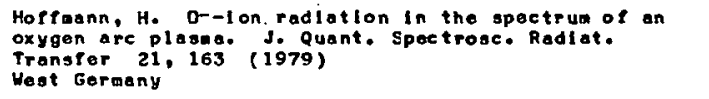 \\
\hline 154 & ${ }_{\mathrm{Cs}}^{\mathrm{A} 12}+\mathrm{Cs}_{3}$ & $\varepsilon$ & $120-250 \mathrm{C}$ & 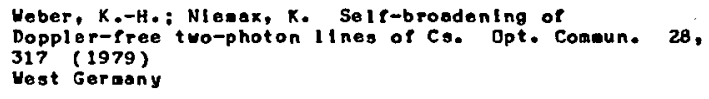 \\
\hline 155 & $\begin{array}{l}\text { Do } 3 \\
\mathrm{C}_{s}\end{array}$ & T & $15-600 \bullet V$ & $\begin{array}{l}\text { Liepinsh, A. Ko; Peterkop, R, K. Electron lapact } \\
\text { exeitation of autolonizing siates in alkall atoms. } \\
\text { opt. Spectrose. 44, 621 (1979) } \\
\text { Soviet Union }\end{array}$ \\
\hline 156 & 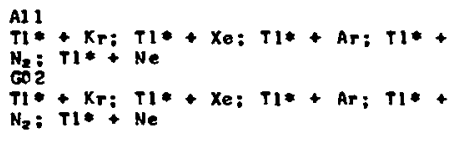 & E & 390 oc & $\begin{array}{l}\text { Aleksandrov, E. B.: Baranov, A. V.: Kulyasov, U. N. } \\
\text { Quenching, ditfugion, and spontoneous decay of } \\
\text { metostable thalliua at ons in bufter gases. Opt. } \\
\text { Spectrosc. } 44,624 \text { (1979) } \\
\text { soviot Union }\end{array}$ \\
\hline 157 & 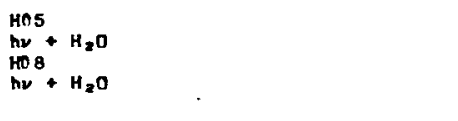 & E. & $900-1100$ AO & 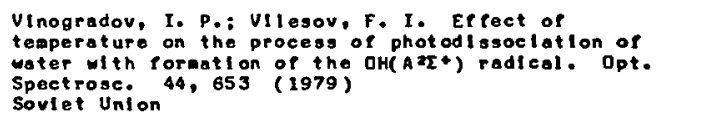 \\
\hline 158 & $e^{E 03}+A 1: e+G a ; e+I n ; e+T_{1}$ & 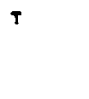 & $3-700 \mathrm{eV}$ & $\begin{array}{l}\text { Peterkop, R. K.; Ryabykh, Y. 1. Exeltatlon of Al, Ga, } \\
\text { In, and i1 atoas by electron lipact. Opt. Speet rose. } \\
45 \text {, } 111 \text { (1979) } \\
\text { Soviet Union }\end{array}$ \\
\hline 159 & 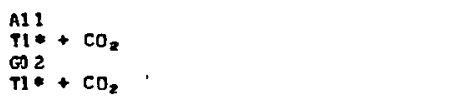 & $\varepsilon$ & $390.0 \mathrm{C}$ & 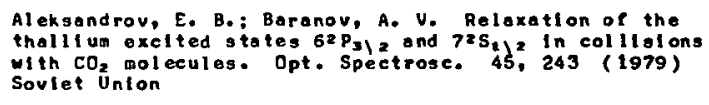 \\
\hline 160 & AI 7 F & $\varepsilon$ & Under & 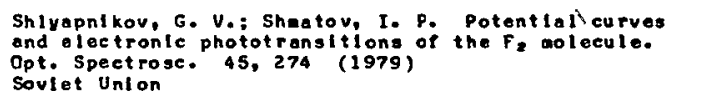 \\
\hline 161 & $\mathrm{HO}_{\mathrm{H}}^{\mathrm{HO}}+\mathrm{N}_{2} \mathrm{O}$ & $E$ & $\mathrm{eV}^{12} \cdot 7-16.5$ & 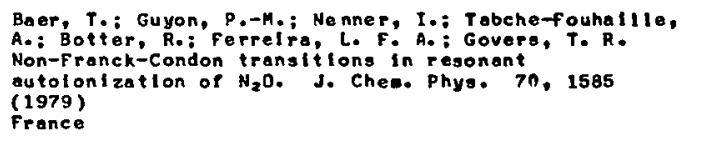 \\
\hline 162 & $\stackrel{\text { Al } 7}{0}+0^{\circ}$ & $\tau$ & Under & 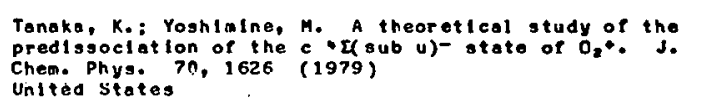 \\
\hline 163 & $\mathrm{ACO}_{2}+\mathrm{Ar}$ & $\varepsilon$ & $2800-3830 \mathrm{~K}$ & $\begin{array}{l}\text { Grlllo, A.; Reed, Re: Slack, M. Y. Infrared } \\
\text { measurements of sulfur dioxide the rmal decompost tion } \\
\text { rate In shoek waves. J. Chem. Phys. } 70,1634(1979) \\
\text { United states }\end{array}$ \\
\hline
\end{tabular}




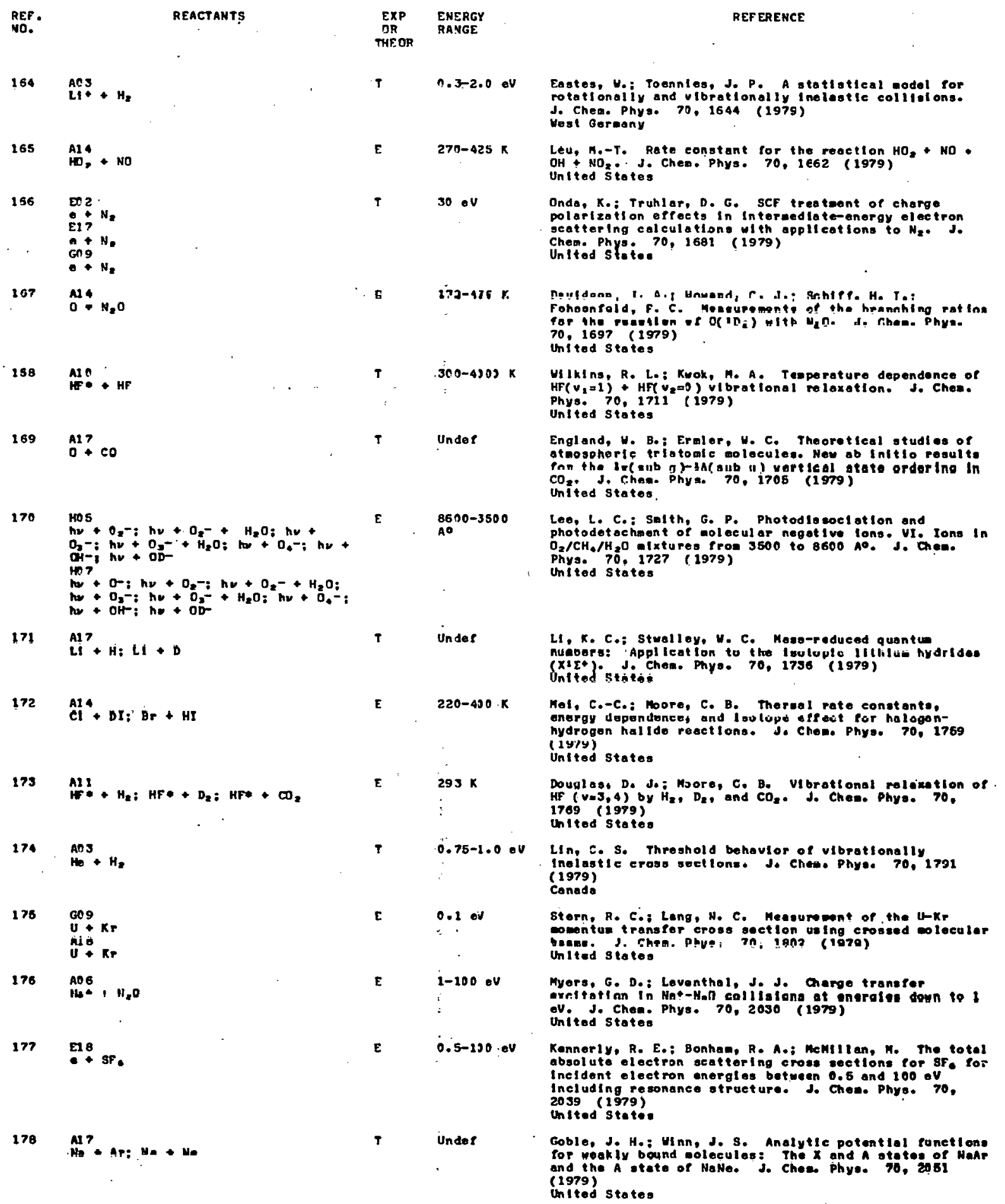




\begin{tabular}{|c|c|c|c|c|}
\hline $\begin{array}{l}\text { REF. } \\
\text { No. }\end{array}$ & REACTANTS & $\begin{array}{c}\text { EXP } \\
\text { OR } \\
\text { THEOR }\end{array}$ & $\begin{array}{l}\text { ENERGY } \\
\text { RANGE }\end{array}$ & REF ERENCE \\
\hline 179 & $\mathrm{HOG}_{\mathrm{H}}+\mathrm{H}_{2} ; \mathrm{HV}+\mathrm{D}_{2} \mathrm{i} \mathrm{hv}+\mathrm{N}_{2}$ & $\varepsilon$ & $15-10 \bullet v$ & 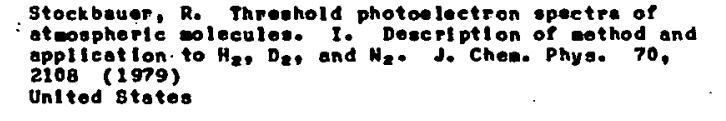 \\
\hline 180 & 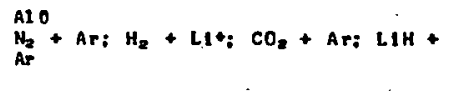 & $\begin{array}{lll} & \end{array}$ & $300 \cdot k$ & 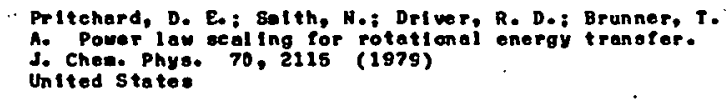 \\
\hline 181 & $\begin{array}{l}\mathrm{Al}^{2} 3 \\
\mathrm{C}^{+}+\mathrm{H}_{2}+\mathrm{He}_{2}: \mathrm{N}+\mathrm{H}_{0}+\mathrm{N}_{2}+\mathrm{H}_{0}: \mathrm{N}_{2}++\end{array}$ & $\mathbf{T}$ & $100-300 \ldots \mathrm{K}$ & 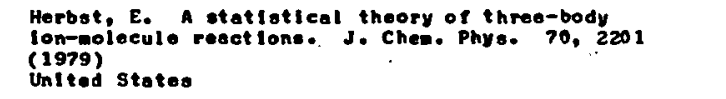 \\
\hline 182 & 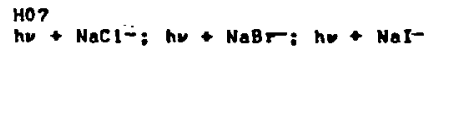 & $\mathbf{E}$ & $2.0-2.3 \mathrm{oV}$ & 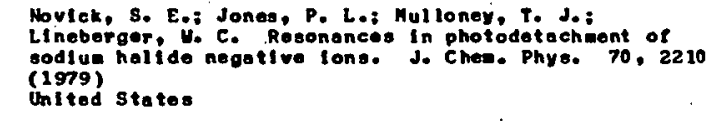 \\
\hline 183 & $\mathrm{AOP}_{\mathrm{A}}^{\mathrm{An}}+\mathrm{CS}_{2}$ & E & $17-80 \cdot .00 \mathrm{~V}$ & 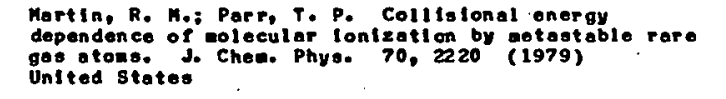 \\
\hline 184 & $\begin{array}{l}\mathrm{AlO}^{\mathrm{CO}} *+\mathrm{He}: \mathrm{CO}_{2} *+\mathrm{He}^{2} \mathrm{CO}_{2} *+\mathrm{Ar} ; \\
\mathrm{OO}_{2} *+\mathrm{Kr} ; \mathrm{CO}_{2} *+\mathrm{Xe}^{2}\end{array}$ & E & $153-533 \times$ & 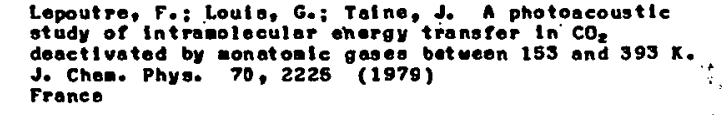 \\
\hline 185 & $\begin{array}{l}\mathrm{EO} 3 \\
\mathrm{O}+\mathrm{CO}_{2} \\
\mathrm{OD}+4 \mathrm{CO}_{2}\end{array}$ & $\varepsilon$ & $25-100-00$ & 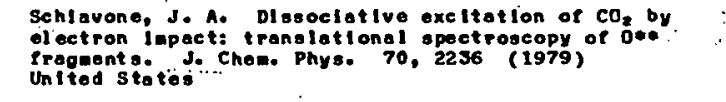 \\
\hline 186 & $\mathrm{HBr}_{\mathrm{HB}}+\mathrm{Cl}_{2}: \mathrm{HBr}+\mathrm{BrCl}$ & $\begin{array}{lll}\mathbf{T} & & \end{array}$ & $1-7$ eV & 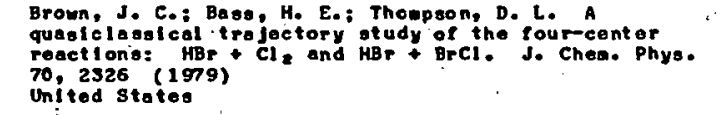 \\
\hline 187 & $\operatorname{Ar}_{2}=+N_{2}$ & . $\varepsilon$ & $294 K$ & 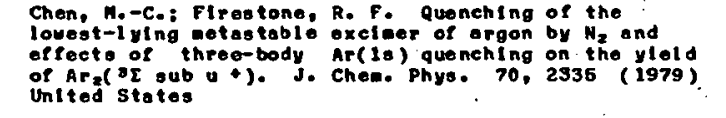 \\
\hline 188 & $\begin{array}{l}\mathrm{Al}_{4}+\mathrm{F}: \mathrm{D}_{2}+\mathrm{F}: \mathrm{H}+\mathrm{Cl}_{2} \\
\mathrm{H}_{2}+\mathrm{F}+\mathrm{F}: \mathrm{D}_{2}+\mathrm{F}: \mathrm{H}+\mathrm{Cl}_{2}\end{array}$ & $\begin{array}{llll}\mathbf{T} & & \end{array}$ & $0.06-3.14$ & 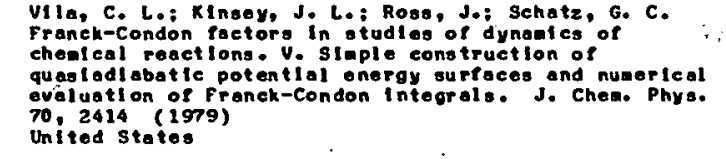 \\
\hline 199 & $\begin{array}{l}\mathrm{A03}+\mathrm{He} \\
\mathrm{CD}+\mathrm{He} \\
\mathrm{Al}+\mathrm{He}\end{array}$ & 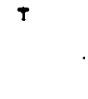 & $0-1.0$ ev & 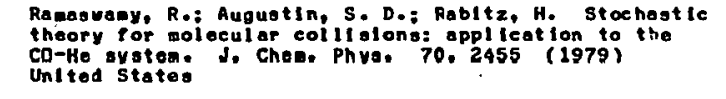 \\
\hline 190 & $\begin{array}{l}\mathrm{Al} 1 \\
\mathrm{He}+\mathrm{HDW}\end{array}$ & $\begin{array}{ll}\mathbf{T} \\
\end{array}$ & $300-530 \cdot K$ & 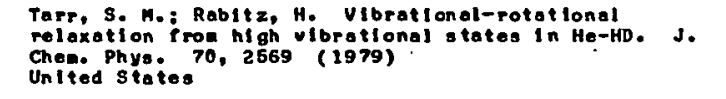 \\
\hline 191 & 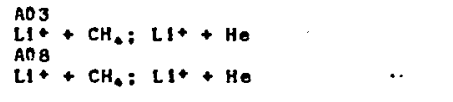 & $E_{1}$ & $250 \cdot k \in V$ & 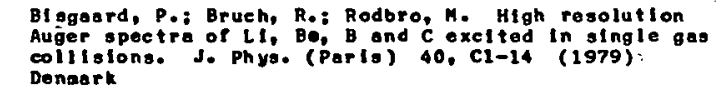 \\
\hline 192 & 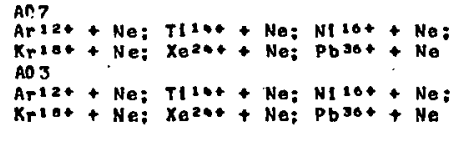 & $\mathbf{E}$ & $1.4 \mathrm{MeV} / \mathrm{amu}$ & 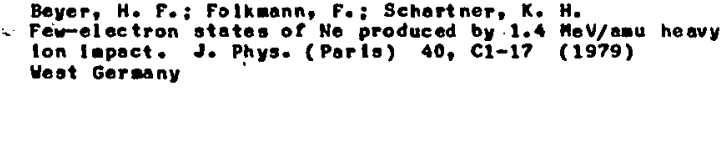 \\
\hline 193 & 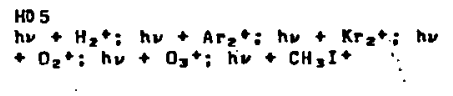 & E & $\begin{array}{l}5800-5500 \\
A O\end{array}$ & 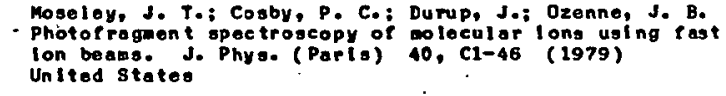 \\
\hline
\end{tabular}




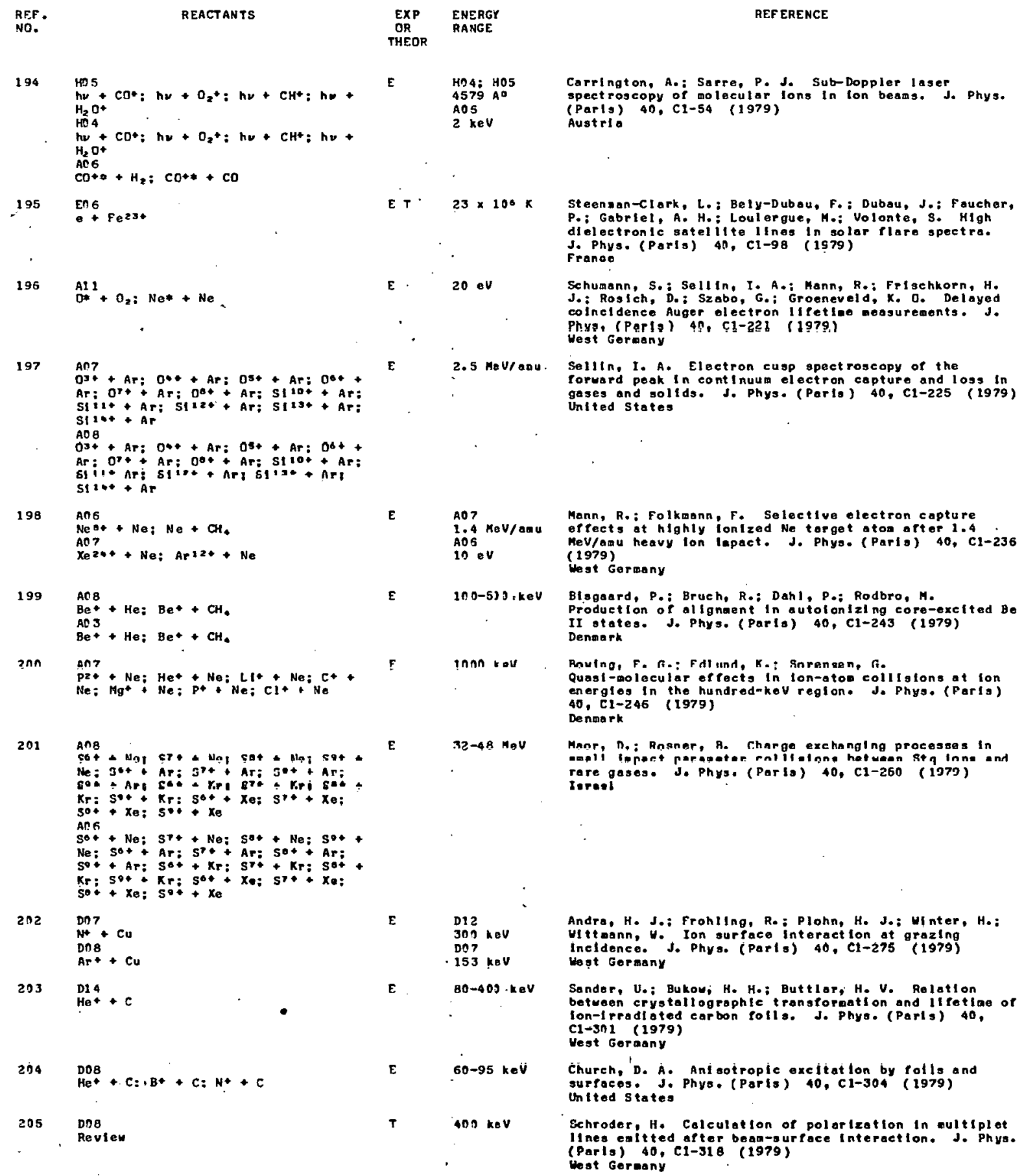

An?

O3t + Ar: $000+$ Ar: $05++$ Ar: $004+$ Ar: ort + Ar: $0^{\circ+}+$ Ar: Sl10\% + Ar: Slilt+ Ar: Slize + Ar: Silst + Ar: Silet+ Ar

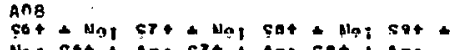

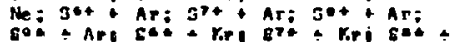

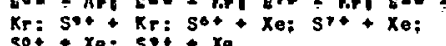

Ans + Ne: Sot + Ne; sot + Ne; Soet Sot Not Sit + Ne; Sot + Ne: Sot+ Ne: So+A Ar: Spor: Ar: sot Ar:

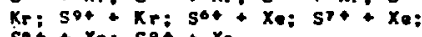

202

$\mathrm{Dn}_{\mathrm{Na}} \mathrm{P}+\mathrm{Cu}$

$\mathrm{DnB}^{+}+\mathrm{Cu}$

$\mathrm{Ar}+\mathrm{Cu}$

203

D14 + C

294

$\mathrm{He}^{+}+\mathrm{C}: \mathrm{BB}^{+}+\mathrm{C}: \mathrm{N}^{+}+\mathrm{C}$

205

E

HO4:
4579
AO

AOS

$2 \mathrm{keV}$

Carrington, A.: Sarre, P. J. Sub-Doppler laser spectroseopy of molecular lons in lon beams. J. Phys. (Parlg) 40, C1-54 (1979)

Aust rio

Steensan-Clark, L.: Bely-Dubou, F.: Dubau, J.: Faucher P.: Gabriel, A. H.: Loulergue, Moi Volonte, S. High dielectronle satellite lines in solar flare spectra. J. Phys. (Parls) 4A, C1-98 (19979)

E. $20 \mathrm{eV}$

Schumann, S.: Sellin, I. A.: Mann, R.: Frl gehkorn, H. J.: Roslch, D.: Szabo, G.: Groeneveld, X. O. Delayed colncldence Auger olectron llfetlee measurements. J. Phys, (Parla) 4h, C1-232! (1979)

E 2.5 Mov/ano

Sellin, I. A. Electron cusp spect roseopy of the Porward poak in cont I nuum el ectron capture and 1 ogs in
gases and solfds. J. Phys. (Parta) 40, c1-225 (1979) United States

E 10 eV

Mann, R.: Folkmonn, F. Selectlve electron capture erfects at hlghly ionized Ne torget oton ofter 1.4 . Mev/amu heavy lon lapact. J. Phys. (Parla) 40, C1-236 (1979) Hest Germany

100-5)jikeV Btsgaard, P.: Brueh, Ro; Dahl, P.; Rodbro, M. Production of alignaent in autoionizing core-excited Bo II otates. J. Phys. (Parls) 40, C1-243 (1979)
Denark

tonn $x$ เo

Raulng, F. fi.: Fdl und, K.: Snranean, fi. Quasi-molecular efracts in lan-aton colliglons at ion energles in the hundred-kev region. J. Phys. (Parig) $40, C_{1-246}(1979)$$$
\text { Denmark }
$$

$32-48 \mathrm{MeV}$

Manr, D.: Rasnar, A. Tharge exchanglng processes in

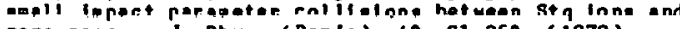
rare gaseg. Jo Phys. (Parla) 40, C1-250 (1079)

Andra, H. J.: Frohling, Re: Plohn, H. J.: Winter, H.: Hittann, W. Jon surface interact fon at grazlng
incldence. J. Phys. (Parls) 40, C1-zis (1979) Incidence.

Sander, U.: Bukou, H. H.; Buttlor; H. V. Relatton betuaen erystallographle transforation and lifetlae of betuaen crystallographic transforation (Parl s) 40 , c1-sni (1979)

Hest Geraany

E $\quad 60-95 \mathrm{keV}$

Ćhureh, 'D. A. Ant sotrople excitat ton by rolls and surfoces. J. Phys. (Paris) 40, C1-304 (1979) united states

T $400 \mathrm{keV}$

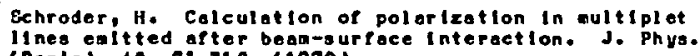
Revien (Parls) 40, c1-318 (1979)

West Germany 


\begin{tabular}{|c|c|c|c|c|}
\hline $\begin{array}{l}\text { REF. } \\
\text { No. }\end{array}$ & REACT ANTS & $\begin{array}{c}\operatorname{EXP} \\
\text { OR } \\
\text { THEOR }\end{array}$ & $\begin{array}{l}\text { ENERGY } \\
\text { RARGE }\end{array}$ & REF ERENCE \\
\hline 205 & ${ }^{806}$ & $T$ & . & 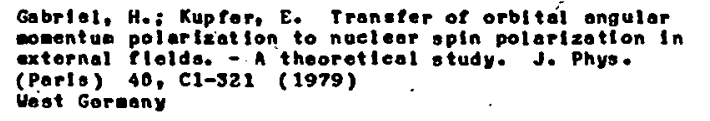 \\
\hline 207 & $\mathrm{BO}^{\mathrm{OCB}}+31 ; \mathrm{B}^{+\infty}+31$ & E & $140 \mathrm{k}=\mathrm{V}$ & $\begin{array}{l}\text { Church, D. A.: loo, C. S. Fast lon double colllstion } \\
\text { spectroseopy: J. Phys. (Parla) } 40, \text { C1-324 (1979) } \\
\text { United Stetes }\end{array}$ \\
\hline 208 & 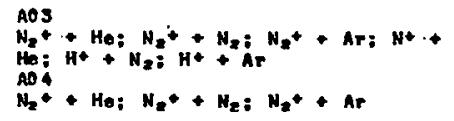 & E & $71 \mathrm{keV} / \mathrm{ous}$ & 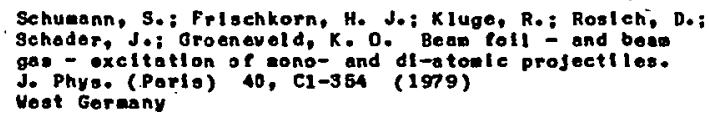 \\
\hline 209 & Ho6 + No & E & $30-300-v$ & 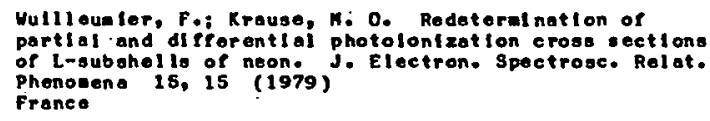 \\
\hline 210 & 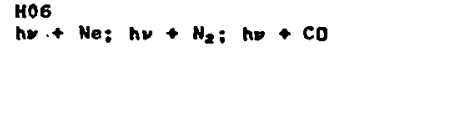 & $\mathbf{T}$ & $0-1500.0$ & 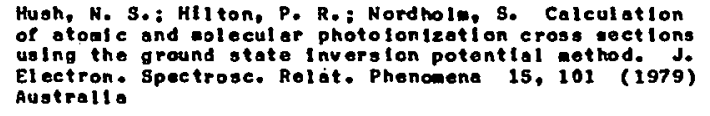 \\
\hline 211 & $\begin{array}{l}\text { HOS } \\
\text { Under }\end{array}$ & $\mathbf{T}$ & Unde? & 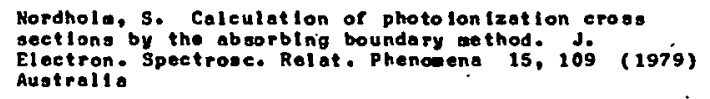 \\
\hline 212 & $\stackrel{\mathrm{EnS}}{e}+\mathrm{HCN}$ & $\mathbf{E}$ & $1.5-2.5 \mathrm{keV}$ & 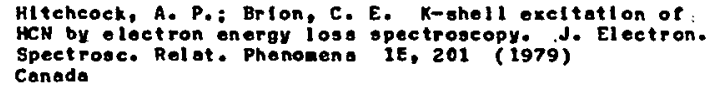 \\
\hline 213 & 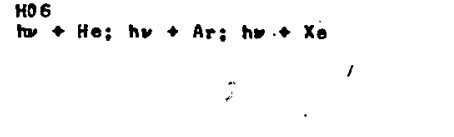 & $E$ & $30-110 \cdot 0 \mathrm{~V}$ & 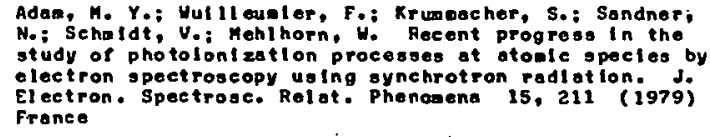 \\
\hline 214 & $\mathrm{HO}_{\mathrm{H}}+\mathrm{CO}_{2}=\mathrm{hv}+\mathrm{N}_{2} \mathrm{O}$ & E & $20-60$ ov & 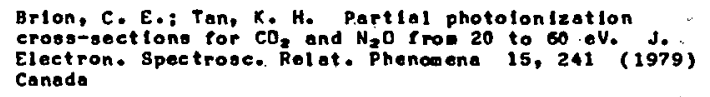 \\
\hline 215 & $\begin{array}{l}E 05 \\
E+H \\
E 17 \\
C+H\end{array}$ & E & $250-414$ ev & 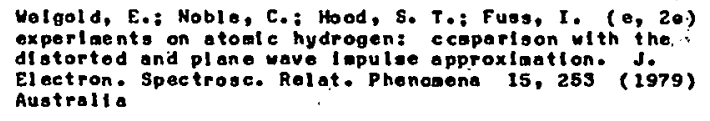 \\
\hline 215 & ${ }_{h O}^{H O G}+N_{2}+h_{2}+X_{0}$ & $\varepsilon$ & $14-150 \bigcirc V$ & 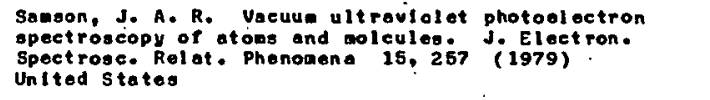 \\
\hline 217 & 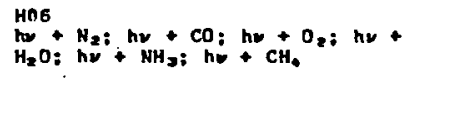 & $\varepsilon$ & $584 \mathrm{AO}^{\circ}$ & 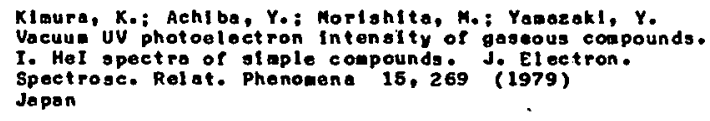 \\
\hline 218 & 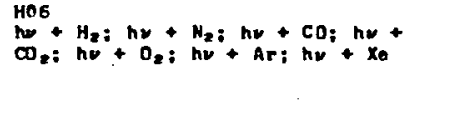 & E & ${ }_{o v}^{16.9-21.2}$ & 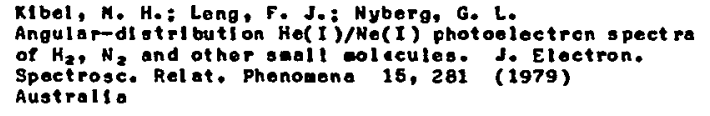 \\
\hline 219 & ${ }_{\mathrm{HO}}^{\mathrm{H} 06}+\mathrm{O}_{2}$ & E & $875-89$ A0 & 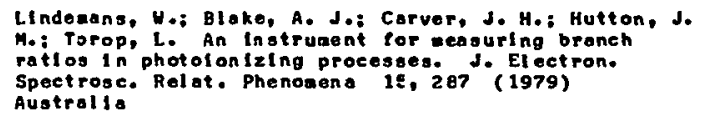 \\
\hline 220 & ${ }_{\text {ho }}^{\text {Ho }}+\mathrm{sb}$ & $\varepsilon$ & $30-805 \cdot 0 V$ & 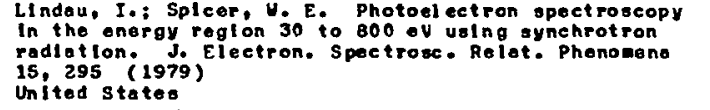 \\
\hline
\end{tabular}




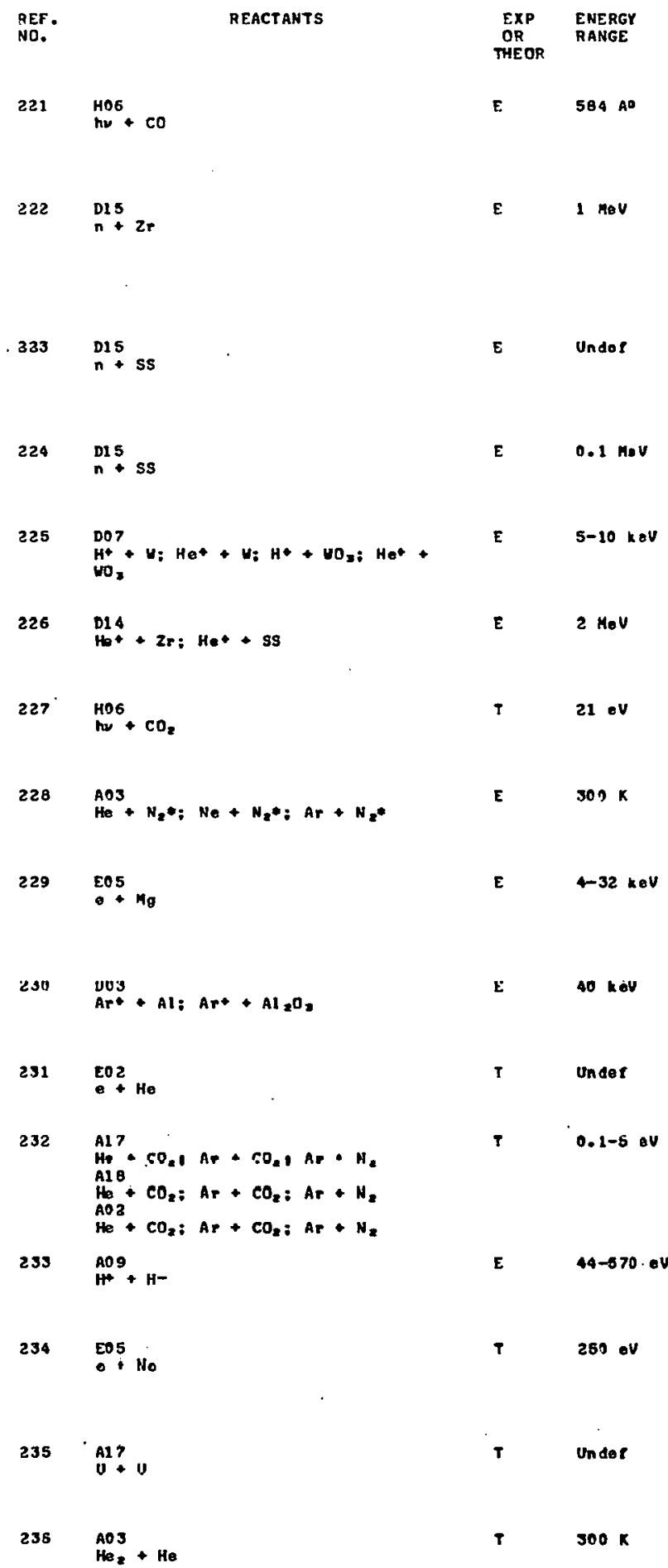

REFERENCE Sell, J. A.: Kupperaann, A.j Mintz, D. M. Angul ar
distributions in the photoel ectron spactroseopy of carbon monoxide. J. Ellectron. Spectrose. Rel phenomena 16, 127" (1979)

United states

Horthwood, D. O.: Gllbert, H. Wo: Bahen, L. E. Rolly. P. M.: Blake, R. G.: Jostsons, A.: Madden, P. K. foulkner, D.: Bell, H.: Adasson, R. B.

Characterization of neutron irradiotion daeage in 21 reonlue alloys - an internatlanal round-robln experínent. J. Nuel. Mater. 79, 579 (1979)

MoVay, G. L.: Malters, L. C.; Hudon, G. D. Neutron Irradiat ton-induced creep of hellue pressurt zed $304 \mathrm{~L}$ otalnless steel copsules. J. Nucl, Moter. 79, 395 (1979)

Unlted states

Porter, D. L. Ferplte formotion in neutrom-l rrodlated type 304L stotnleas steel. J. Huel. Moter. 79, 406 (1979) United states

Bhat tacharya, R. S.; Eeksteln, M.; Verboek, H. Backseat tering of $H$ and He Pros $H$ and $\mathrm{VO}_{3}, \mathrm{~J}$. Nuel. Mator. 70,120 (1979) West Germany

Krlohnan, R.: Mehta, M. K. Bllatering of el reonlum. Ineonel-71B and etalnlae otenl-3i6 by 3 MeV hellut lona. J. Nuel, Mater, 79, 423 (1979) Indio

Doncke, W. Vibrat lonal st ote dependence of the photoelectron angular asyosetry paraneter caused by vibronle coupling. Phys. Seriptn 19, 11 (1979) West Geruany.

Carlson, T: A.: Durle, N.: Ermań, P.: Larason, $M$. Colllslonal transfer to the $\mathrm{B}$ otate in $\mathrm{N}_{2}$. Phys. Serlpta 19,25 (1979) Sueden

Mikkol a, E,: Kogki-Rahkonen, O.: Kuoppala, R. Kaf uuper h) hyporsetellite epoctrum and electron double lontzotion cross sectlon of the K-shell In argneslue. Phys. Seripta 19, 29 (1979)

Braun. H. Spectral Investigation or exclted eputtered alualniug porticlea and the Inficonce of oxygen during lon Irradiation. Phys. Serlpto 19, 33 (1979)

Peach, G. The evaluation of phaseshires at very lou enorgles. J. Phys. B 12, L13 (1979) Unlted Ringdoen

Khromov, V. No: Leonas, V. H. The d rect tose of

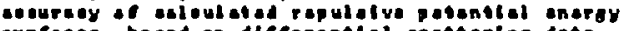
surfaces, based on differential ecatterling doto.. J. Phys. B 12, Lig (i979)

Peare, B.: Forrese, R. A, measurements or crocke Peart, B.: Forrose, R. A, Measurebents of crowe

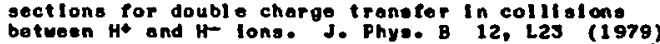
between $\mathrm{H}^{+}$and

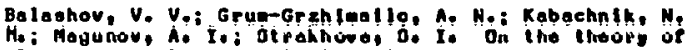
al octron angul ar correlations In olectron Iepact lonieation of noon. J. Phys. B 12, L27 (1979) Ionisation or Wletschorke, $K_{*}-H_{0}$ : Mull or, B.: Grelner, H.; Sot?, G. Self-coneletent dotoralnotion of orltioal two-oentro
distances. J. Physo $B$. L3i (1979)

Bates. D. R. Foreation of exerted hellun atons fros

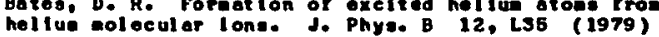
United Kingdoe 


\begin{tabular}{|c|c|}
\hline $\begin{array}{l}\text { REF. } \\
\text { NO. }\end{array}$ & REACT ANTS \\
\hline 237 & $\stackrel{H 06}{N}+N_{2}: h_{2}+C O$ \\
\hline 238 & $\mathrm{Al}_{\mathrm{H}}^{\mathrm{Al}}+\mathrm{He}$ \\
\hline 239 & 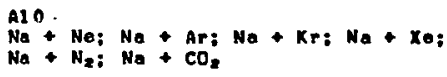 \\
\hline 240 & $\begin{array}{l}\mathrm{AD6} \\
\mathrm{H}+\mathrm{H} \\
\mathrm{AO} \\
\mathrm{H}+\mathrm{H}\end{array}$ \\
\hline 241 & $\mathrm{En2}$ \\
\hline 242 & $\mathrm{COS}^{\mathrm{E}}+\mathrm{He}$ \\
\hline & $\cdot$ \\
\hline 243 & 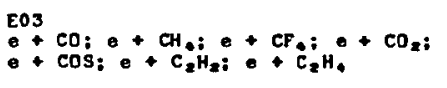 \\
\hline 244 & ${ }_{\text {hy }}^{H 06}+\mathrm{Ba}$ \\
\hline 245 & $\begin{array}{l}\mathrm{EO} 2 \\
:+\mathrm{H}_{2} \\
\mathrm{E} 3+\mathrm{H}_{2}\end{array}$ \\
\hline 246 & $\mathrm{Ens}+\mathrm{H}_{2}$ \\
\hline
\end{tabular}

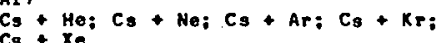

And

Aos

251

ADP

$\mathrm{Cot}+\mathrm{H} ; \mathrm{He}^{2+}+\mathrm{H}$
Un jer

\begin{tabular}{|c|c|}
\hline $\begin{array}{l}\text { EXP } \\
\text { OR } \\
\text { THEOR }\end{array}$ & $\begin{array}{l}\text { ENERGY } \\
\text { RARGE }\end{array}$ \\
\hline $\mathbf{E}$ & $20-46$ :V \\
\hline$T$ & Undef \\
\hline
\end{tabular}

E
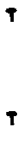

t. $\quad 1-10$ ov

E $\quad 50 \bullet V$

Under
$1.5 \mathrm{kov}$

$7.1-9.6$
$10 \cdot 6$
ces

$0.025-2 \mathrm{MeV}$

-10 ev

$21-2000.00$

$304 \mathrm{~A}^{\circ}$

45-90 ov

Marr, G. U.: Morton, :. M.: Holeae, R. Mo: MeCoy, D. G. Angul ar distribution of photosleot rong fros fres miecules of $\mathrm{Hz}_{2}$ and CO as. Iunction of phot on energy. J. Phye. B 12, 45 (1979) Untted KIngdom

Vall Iron, P.: Gayet, R.: MeCarroll, R.: Mas nou-So ouwa, F.: Phililippe, M. 'Model-potenti al bethode for tho calculation of atoerare-gat interactiona: application to the H-tho eystes. J. Phye. B 12, 83 (1979) France

Apt, J.: Pritehard, D. E. Veloetty dependanee of

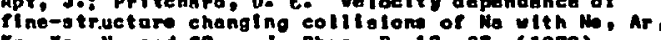

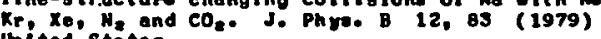
United statos

Roy,. A.: Ghosh. A. 8. The capture of electrons by fast protons troe hydrogen atose. J. Phys. B 12, 99

Barrott, R. F.: Robson, B. A. Metural boundary condition othods for lou energy electron-heltu seattoring. J. Phya. B 12, 108 (1979) Australlo

Westarveld, U. B.: Heldionon, H. G. M.: van Eek, J. Electron inpact exeltation of 1 18-2 ap and 1 is-3 ip of hellun: oxeltatlon crose sectlons and polarisation Practl ons obtalned frod XuV radioton. J. Phys. B 12, $116(1979)$

Trone, M.: KIng, G. C.: Read, F. H. Corbon K-aholl exclestion In sali il oocules by Migh-resolution electron Inpoct. J. Phys. B 12, 137 (1979) electron Inpoct.
United RIngdoen.

Connerade, J. P.; Rose, S. J.: Grant, I. P. Two-atep autotonl setion ond the double tont ast ion anonely in I. J. Phys. B 12, LES (1979) Unitad Kingdon

Klonover, A.: Kaldor, U. Zaro-point vibration In. low-energy dioctron-dolecule seattering theory. J. Phys. B 12, L61. (1979)

Malcol w, I. C.: MeConkey, J. U.

Electron-polarl sod-photon colncldence atudy of Heis aub g) L67 (1979)

Canada

Bruhn, R.: Sonntsg, B.: Volfs, H. Wo 3p oxcltatlons of atomle and netallle Fe, Co, NI, and Cu. J. Phys. B 12,203 (1979)

Weat Geraany

Chamboud, G.: MIllle, P.: Ridard, Ja; Levy, B. Proflle of the Lyenan a line in the presence of on argon plased. J. Phys. B 12, 221 ( (1979): rance

Sayer, Be: Ferray, H.: Loxingot, J.' Experinental deteralnation of the $C s(78$-rore-gas potentiol onergy curves and of the 6S-7s collis lon-induced osclll ator strengths. J. Phy 0.. B 12, 227 (1979) Franee

Kunc, J. Binary encounter opproxleation trotiment of collislons within the atomic structure. J. Phys. B 13,341 (1070)

Solop. A. Efchler, J. Sudden approxteat lon croos sections for Iontsation of $H$ otons by energetic Cot and Hezt inpact. J. Phys. B 12, 257 (1979) Untited States 
24

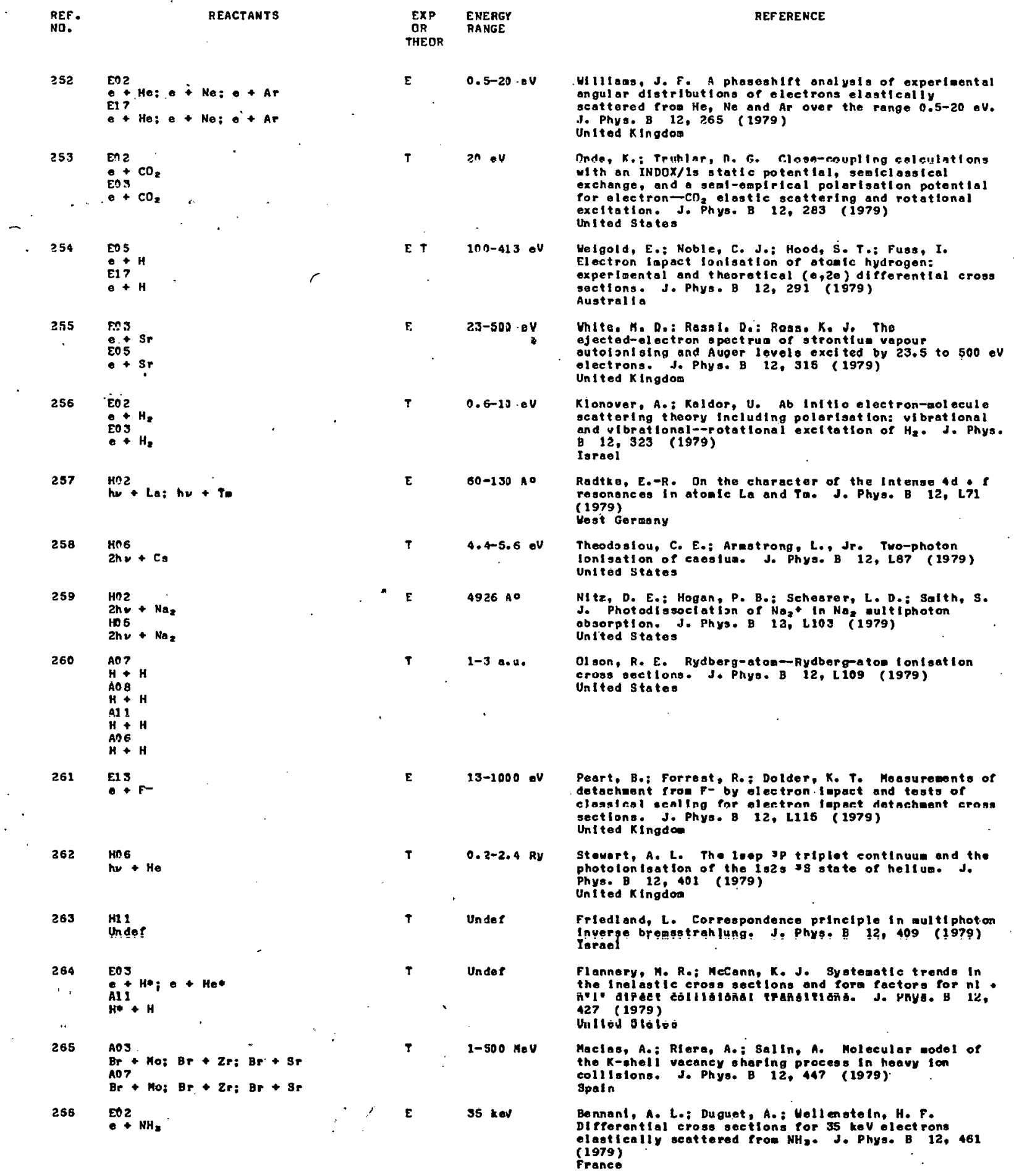




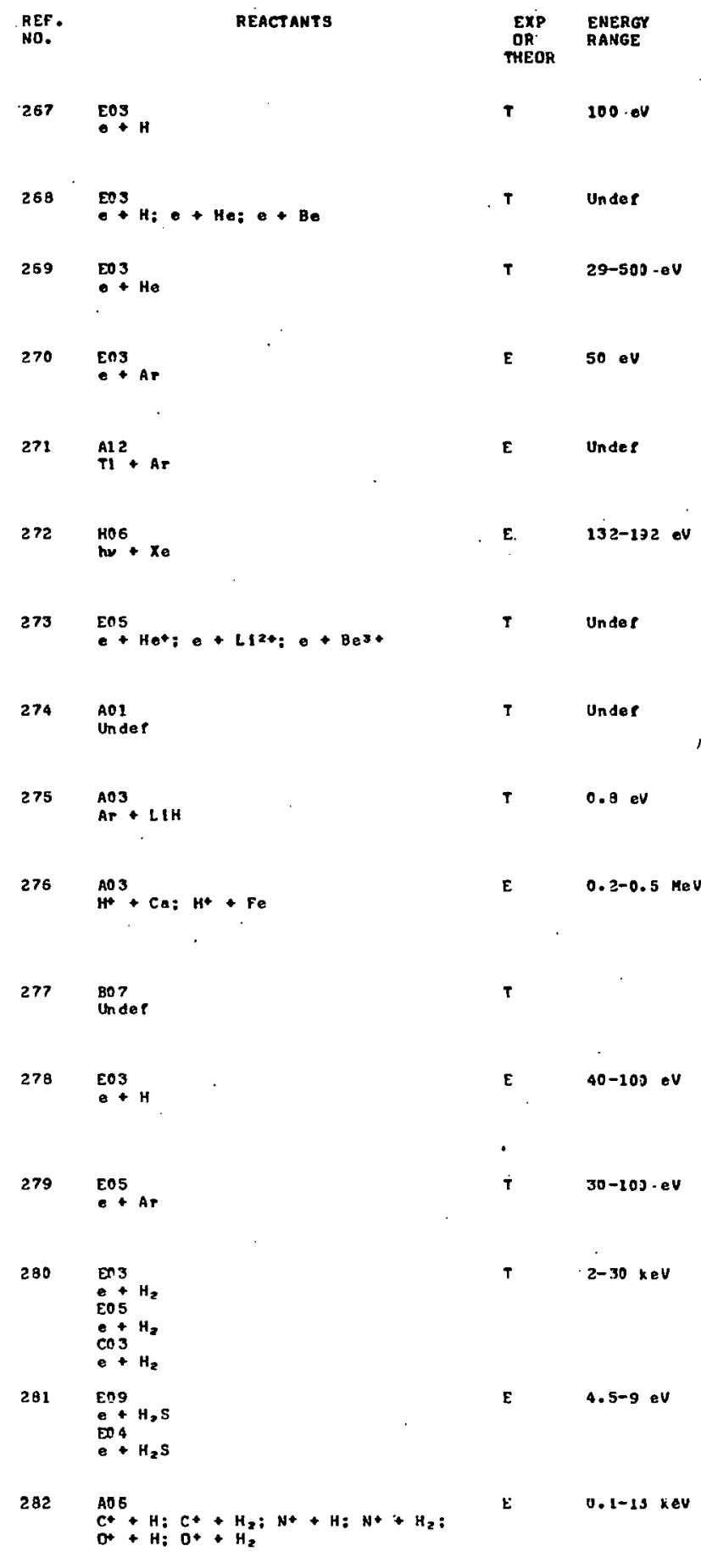

Vandorpoorten, R.: Ul nters, K. H. OfP-dl agonal

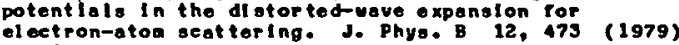
ol eetron-

REF EREMCE

Heddlo, D. W. O. The Bethe approx Iaction: whore to dray the ilne. J. Phys. B 12, 489 (1979) United KIngdoo

Roy, A. C.: S11, N. C. Excltation of $15(18,38)$ stote of hel lua by olect ron impact in the Glauber approxlation. J. Phys. B 12, 497 (1979)

Malcol 1., I. C.: MeConkay, J. U. Electron-photon coincidence studitos of the resol Electron-proton IInes. Je Phyoo D 22,513 (1979) Canado

Rayner, M. G.: Carlaton,. J. L.; Pl Chlar, G. Comparlson of collifional redistribution and exiselon line shapes. J. Phyo. B 12, L119 (1979) United States

Banna, M. S.: Krause, M. O.: Hullleuwler, F. the xenon $4 d^{3} z^{4 d} d_{12}$ photool ectron intensity ratio in the exglon or the Cooper ainteum. J. Phys. B 32, L125 (1979) Unlted States

Grufle, P. V. Angul or movento near 1 onlsatl on threshol da for electron--lon colllistong. J. Phya. B 12. L131 (1979) Yugosiavia

Saha, H. P.: SII, N. C. Evaluat tori of exehango matrix el enents with masentus transfer for ton--atos collistons. J. Phys. B 12, 5e5 (1979) India

Bhat tacharyyo, S. S.; Dlek inson, A. S.: Rlehards, D. Rotatlonal tranglt lons in Ar--LiH collislons. J. Phys. B. 12,595 (1979)
Unt ted Kingdos

Lopes, J. S.: Jesug, A. P.: Ramos, S. C.: Ferrelra, G. P. Prot on-Induced $k$ X-ray production from $C a$ to Fe for
0.2 to 0.5 HoV ineldent onergy. J. Phys. B 12,605 (1979)

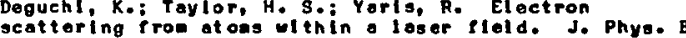
12,613 (1979)
United States

Hood, S. T.; Welgold, E.; Dixon, A. J.

Electron-photon angular corralaticns in the alect ron tapact excitation of $H(2 P)$. J. Phys. B 12, 631 (1979) Australl a

Aauste, M. Y.: Snoineraen, S. A. Single-olectron tons sation of $\mathrm{Ar} 3 \mathrm{~s}^{2}$ subshell by slow electrons. J. Phys. B 12,649 (1979) Soviet Union

Douthat, D. A. Enorgy dopositton and eloetron onergy degradation in molecular hydrogen. J. Phys. B 12,663 (1979)

Azrla, R-: Le Coat, Y.: Lefeure, G.: Stmon, D. Dl ssoclative electron at tachment of $\mathrm{H}_{2} \mathrm{~S}$ : energy and angular distributiong of $\mathrm{H}^{-}$lons. J. Phys. B 12,679 (1979)

Nutr, Y. L.: Relultougn, K. W.: Glitooy, n. g. El ectron capture by $0.1--13 \mathrm{keV} \mathrm{C}^{+}, \mathrm{N}^{+}$and $\mathrm{O}^{+}$lons in $\mathrm{H}$ and $\mathrm{H}_{2}$. J. Phys. B 12,1157 (1979) 


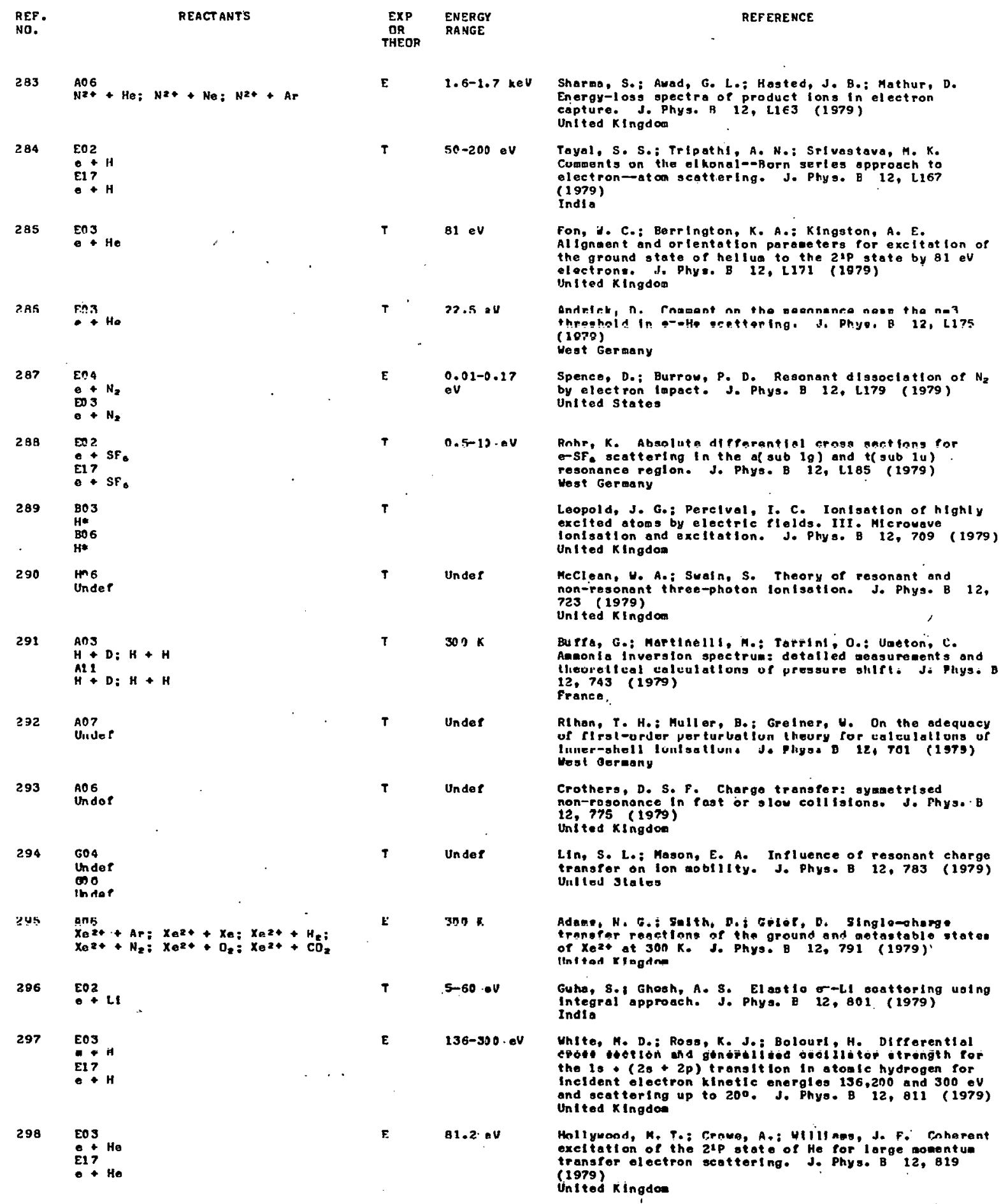




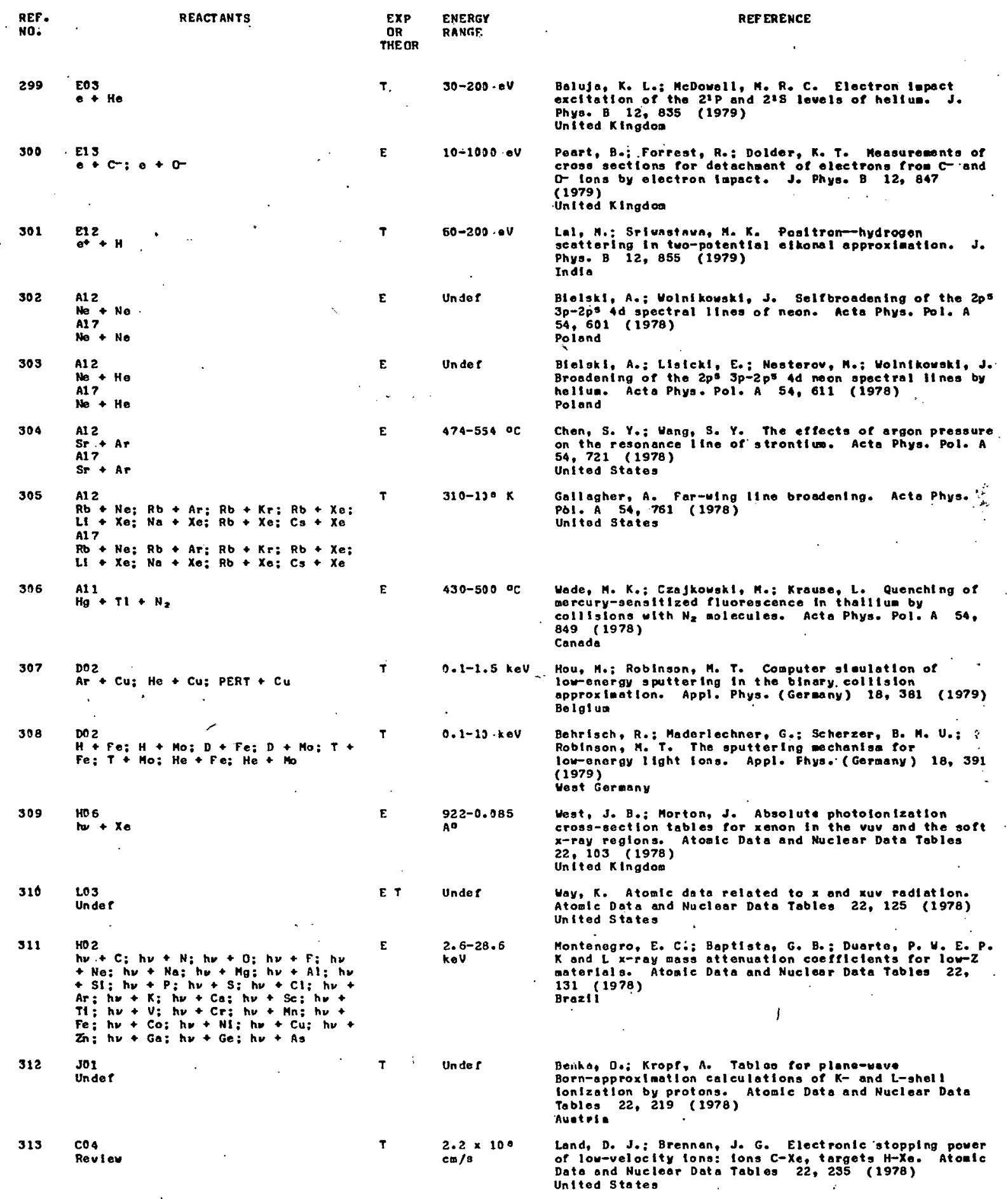


REF.
NO.

REACTANTS

314

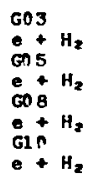

315

316

$\mathrm{HOS}_{\mathrm{H1O}}^{\mathrm{HO}}+\mathrm{NO}_{2}$

$\mathrm{HIO}^{\mathrm{N}}+\mathrm{NO}_{3}$

317

Al $4+\mathrm{N}_{2} \mathrm{O}$

318

$\mathrm{AO}_{2}+\mathrm{He}: \mathrm{I}_{2}+\mathrm{Ar}: \mathrm{I}_{2}+\mathrm{Xe}_{\mathrm{O}}: \mathrm{Br}_{2}+$

$\mathrm{He}$ : Brz $+\mathrm{Ar}_{2}$ : Bry $+\mathrm{Xe}_{\mathrm{e}}$

A1 4

$\mathrm{I}_{2}+\mathrm{He}$ : $\mathrm{I}_{2}+\mathrm{Ar}: \mathrm{I}_{2}+\mathrm{Xe}_{\mathrm{O}} \mathrm{Br}_{2}+$

319

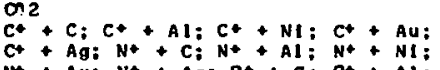

$\mathrm{N}^{+}+\mathrm{Au} ; \mathrm{N}^{+}+\mathrm{Ag}^{+} \mathrm{O}^{+}+\mathrm{C}^{+} ; \mathrm{O}^{+}+\mathrm{Al}$;

$\mathrm{O}$
$\mathrm{FH}$

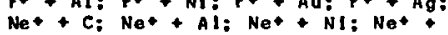

$\mathrm{Nu}: \mathrm{Ne}^{+}+\mathrm{Ne}_{\mathrm{g}}^{+} \mathrm{Na}^{+}+\mathrm{Ne}^{+} \mathrm{Na}^{+}+\mathrm{Nl}^{+}$:

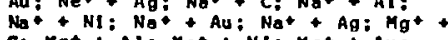

C: $\mathrm{Ha}^{+}+\mathrm{Al}^{2} \mathrm{Hg}^{+}+\mathrm{NI}^{+} \mathrm{Hg}^{+}+\mathrm{Au}$ :

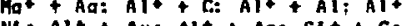

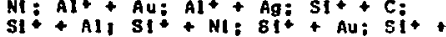

$\mathrm{Ag} ; \mathrm{Pt}+\mathrm{C}: \mathrm{Pt}+\mathrm{Al} ; \mathrm{Pt}+\mathrm{NI} \mathrm{Pt}+$

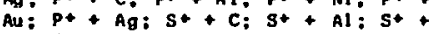

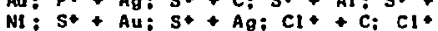

$+\mathrm{Al}: \mathrm{Cl}+\mathrm{NI}^{\mathrm{C}} \mathrm{Cl}+\mathrm{Au} ; \mathrm{Cl}+\mathrm{Ag}^{\circ}$

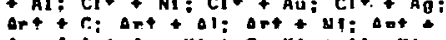

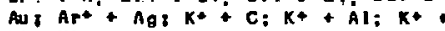

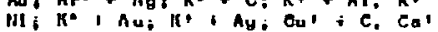

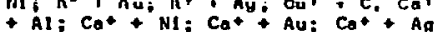

320

Ho: 3

$321 \quad \begin{aligned} & \text { D15 } \\ & \mathrm{He}+\text { + Al }\end{aligned}$

322
$\mathrm{HO}^{\mathrm{A}}+\mathrm{Zr}$
$\mathrm{An}^{+}+\mathrm{Z}$
$\mathrm{H}^{+}+\mathrm{Z}$
A1 8

323

804
14

324
$\stackrel{A 02}{H+H e}$
EXP ENERGY

DR
THEOR RANGE

T Under

Bl evin, H. A.; Fletcher, J.: Hunter, S, R. A Monte-Carlo simulation of the behavlour of electron swaras In hydrogen using an anisotrople scottering model. Aust. J. Phys. 31, 299 (1978) Australla
E $293 x$

$366 \mathrm{~nm}$

$5-180$
$k J / 001$

$200-1500 \times$

$20-2 B$
$k \in V / a q u$

$24.5-1908$

Gọ ReV

40 Meil

E T

Unde $\mathrm{f}$
Hack, W.: Preuss, A. H.: Hegner, H. G.: Hoyermann, K. Reactlons of hydrogen atoas ut th hydroperoxyl radicals II. Deteralnation of the rate constant of the overali reaction. Ber. Bunsenges, Phys. Chem. 83, 212 (1979) vest Bermany

Canosa, C.: Penzhorn, R.-D.: von Sonntag, C. Product quantua yields from the photolysts of NOz at $366 \mathrm{~nm}$ in the presence of ot hylone - the role of NÓz". Ber. Bunsengas, phys: that as, 21? (1979) Vest Germany

Marx, W.: Bahe, F.: Schurath, U. The No yleld of O(1D) $+\mathrm{N}_{2} \mathrm{O}$ as function of $k$ Inet le energy. Ber. Bunsenges, Phys. Ches. 83, 225 (1979) Uest Germany

Snider, N. Bound complex and triple colliston eechan is for fiaton dissoctiotion and recabination. can. J. Chem. 57, 1167 (1979)

Ward, D.: Andrews, H. Ro: Mitchell, I, V.: Lennard, H. N.: Walker, R. B.: Rud, No Systematics for the $z_{1}$-osclllation In slopping powers of varlous sol ld cotertal s. Can. J. Phys. S7, C4S (1979)
Ramanethen, Ne: Kenneth, T. J.: Prestutch, U. V. An experimental investlgation of suall angle photon
el astic seattering. Can. J. Phys. 57, 343 (1979) clastic

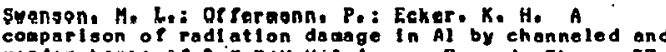
comparison of radiation dadage In Al by chonneled and $457(1979)$

vest Germany

de SulnlargkI, R.: Phoe, D.-L.: Begleu, G.: Geramb, H. V. Elastlc and inelastic seattering of $40 \mathrm{MoV}$ polarized protons fros $02 \mathrm{r}$ and $a z r$. Can. J. Phys. 67,540 (1979)

France

Scherk, L. R. An Inproved value for the eloctron affinity of the negatlve hydrogen ton. Can. J. Phya. 57,558 (1979)

Leung, S. H.t.: Shortf, H. S, Eerratue Optlcal model analyols of $p+$ + OHe olostic seattering at Interaediate energles. Can. J. Phys. 57, 601 (2979) 


\begin{tabular}{|c|c|c|c|c|}
\hline $\begin{array}{l}\text { REF. } \\
\text { NO. }\end{array}$ & REACTANTS & $\begin{array}{l}\text { EXP } \\
\text { OR } \\
\text { THEOR }\end{array}$ & $\begin{array}{l}\text { ENERGY } \\
\text { RANGE }\end{array}$ & REFERENCE \\
\hline 325 & $\hat{0}_{2}{ }^{14}+0_{2}$ & $\tau$ & $300-k$ & 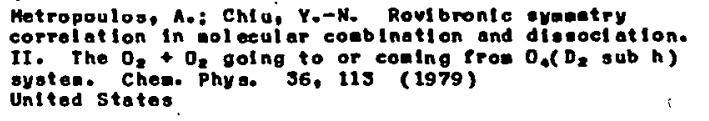 \\
\hline 326 & 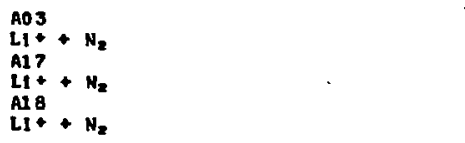 & $\tau$ & $4.2-7.1$ ov & 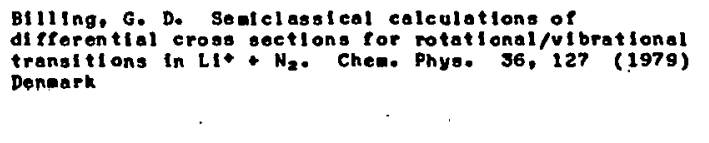 \\
\hline 327 & 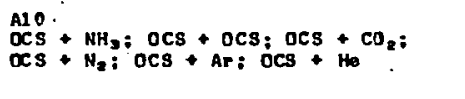 & E & $300 \times$ & $\begin{array}{l}\text { Williams, J. R.: Kukolich, S. G. Bean gaser rot at lonal } \\
\text { relaxati on messurements on ocs. Chean. Phys. 36, } 201 \\
\text { (1979) } \\
\text { Unlted states }\end{array}$ \\
\hline 328 & $\begin{array}{l}\mathrm{EOS}+\mathrm{H}_{2} \mathrm{O}=+\mathrm{D}_{2} \mathrm{O} \\
\therefore \mathrm{EO} \\
\because+\mathrm{H}_{2} \mathrm{O} ; \cdots+\mathrm{D}_{2} \mathrm{O}\end{array}$ & E & $18-200 \cdots .0 \mathrm{~V}$ & 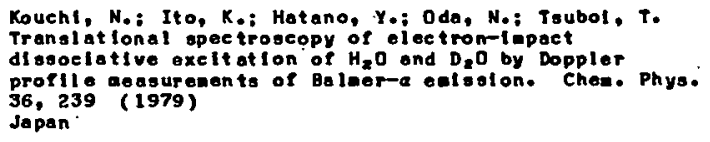 \\
\hline 329 & $\begin{array}{l}\mathrm{AOS} \\
\mathrm{He}+\mathrm{N}_{2} \mathrm{O} \\
\mathrm{AO} 6 \\
\mathrm{He}+\mathrm{N}+\mathrm{N}_{2} \mathrm{O} \\
\mathrm{DO} 5 \\
\mathrm{O}+\mathrm{N}_{2} \mathrm{O} \\
\mathrm{E1} 6 \\
\mathrm{O}+\mathrm{N}_{2} \mathrm{O}\end{array}$ & E & $\begin{array}{l}\text { AO5: } A 05 \\
0.05 \text { :V } \\
\text { E05; E15 } \\
16-100-0 V\end{array}$ & 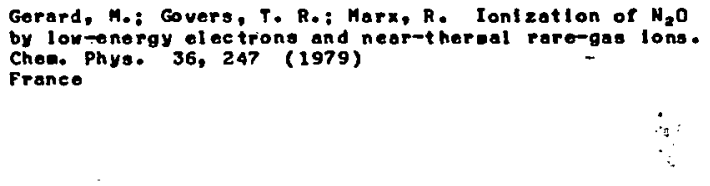 \\
\hline 330. & $\mathrm{O}_{2}^{\mathrm{An}}+\mathrm{Ar:} \mathrm{HCl}+\mathrm{Ar}$ & E & $3500-8500-K$ & 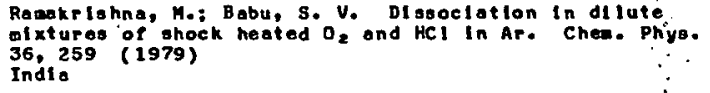 \\
\hline 331 & ${ }_{\mathrm{HF}}^{\mathrm{A} 11}+\mathrm{DF} ; \mathrm{DF} *+\mathrm{HF}$ & T & $300-3000 \cdot K$ & 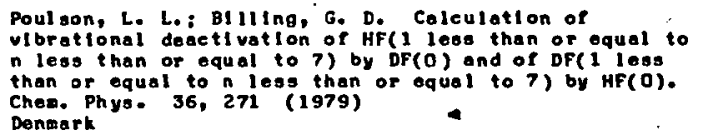 \\
\hline 332 & 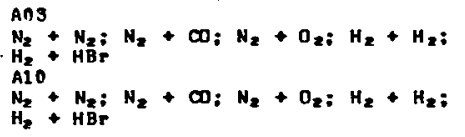 & $T$ & $3 n 0 \mathrm{~K}$ & 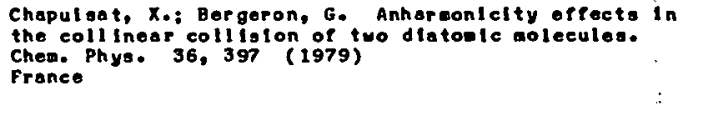 \\
\hline 333 & $\mathrm{H}_{\mathrm{H}}^{\mathrm{A}}{ }^{2}+\mathrm{CO}^{\circ}: \mathrm{He}+\mathrm{HF}$ & $\mathbf{T}$ & $0-10.0$ & 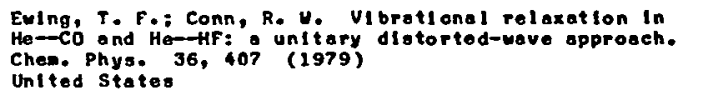 \\
\hline 334 & $\begin{array}{l}\mathrm{E} \cap 4 \\
\mathrm{EO}+\mathrm{H}_{2} \mathrm{O}:=+\mathrm{D}_{2} \mathrm{O} \\
\mathrm{O}+\mathrm{H}_{2} \mathrm{O}: O+\mathrm{D}_{2} \mathrm{O}\end{array}$ & E & $2-18$ ov & 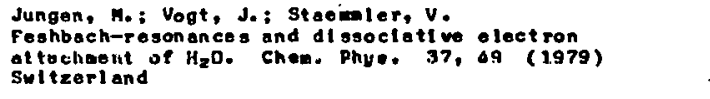 \\
\hline 335 & $\begin{array}{l}A 02 \\
H D+H o \\
A 11 \\
H D+H o \\
A 17 \\
H D+H e\end{array}$ & $T$ & $\begin{array}{l}\text { A02; }{ }^{A 11} \\
\text { 15-300 K } \\
\text { A17 } \\
\text { Undof }\end{array}$ & 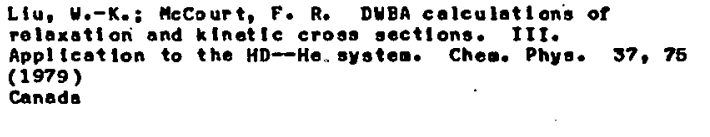 \\
\hline 336 & $\mathrm{Na}_{\mathrm{Na}}+\mathrm{Na}_{2}$ & $\varepsilon$ & $430-454 K$ & 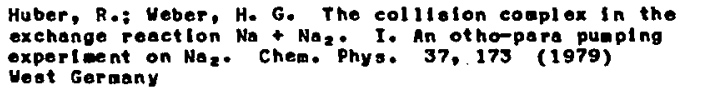 \\
\hline 337 & $\mathrm{NO}_{\mathrm{NO}}+\mathrm{Na}$ & T & Undef & 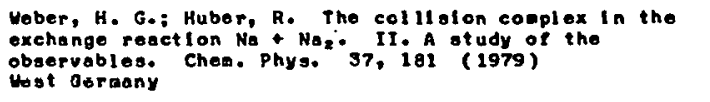 \\
\hline 338 & Al? ${ }_{\text {Ne }}+\mathrm{Ne*}$ & $\mathrm{T}$ & Unde? & 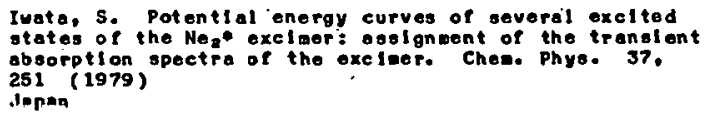 \\
\hline
\end{tabular}




\begin{tabular}{|c|c|c|c|c|}
\hline $\begin{array}{l}\text { REF. } \\
\text { NO. }\end{array}$ & REACTANTS & $\begin{array}{l}\text { EXP } \\
\text { OR } \\
\text { THEOR }\end{array}$ & $\begin{array}{l}\text { ENERGY } \\
\text { RANGE }\end{array}$ & REF ERENCE \\
\hline 339 & 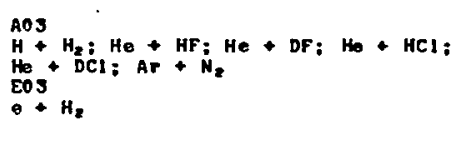 & $T$ & $\begin{array}{l}A 03 \\
0.5-1.5 \text { ev; } \\
0.01-9.1 \\
\text { ev; } 0.07 \text { ov } \\
\text { Eoj } 0.0 .0 \text { eV } \\
0.4-2.0 \text { ev }\end{array}$ & $\begin{array}{l}\text { Top, Z. H.: Kourl, D. J. Computational tests of the } \\
\text { factorization of cross seetlons In the sudden } \\
\text { approximation. Chom. Physe 37, } 265 \text { (1979) } \\
\text { United States }\end{array}$ \\
\hline 340 & HOS + HCN; HO + DCN & $\mathbf{T}$ & ov.5-14.5 & 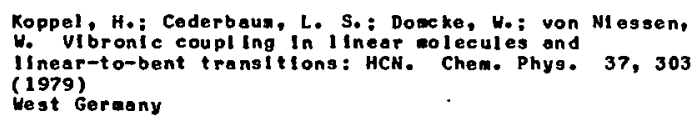 \\
\hline 341 & E15 $\mathrm{HCH}_{4}$ a $\cdot \mathrm{C}_{2} \mathrm{~N}_{2}$ & $\varepsilon$ & $1.5-2.5 \mathrm{kOV}$ & 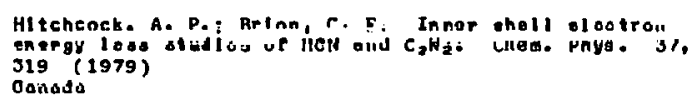 \\
\hline 345 & 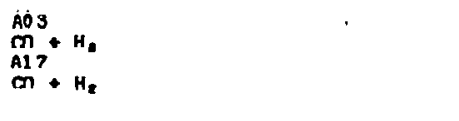 & $\mathbf{T}$ & $77-300 \cdot K$ & 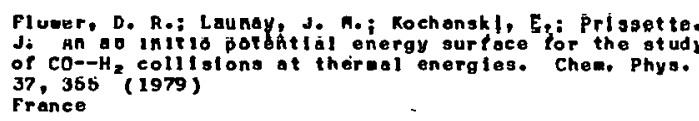 \\
\hline 343 & $\stackrel{A 11}{N^{*}}+N$ & E & 3no.k & 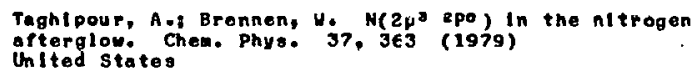 \\
\hline 344 & $\mathrm{HOOH}_{\mathrm{HO}}^{\mathrm{HOS}}+\mathrm{H}_{2}$ & $\mathbf{T}$ & $730-586$ AO & 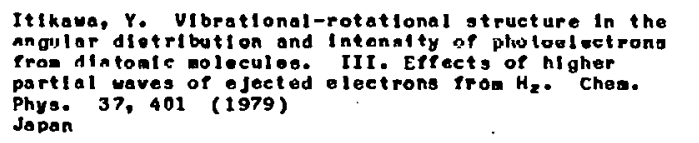 \\
\hline 345 & $\begin{array}{l}\text { Go4 } \\
\text { Under } \\
\text { Oo6 } \\
\text { Under }\end{array}$ & $\mathbf{T}$ & Under & $\begin{array}{l}\text { Lin, S. L.: Vtehland, L. A.: Meson, E. A. } \\
\text { Three-teaperature theory of gaseous ion transport. } \\
\text { Chom. Phyo-37, 411 (1379) } \\
\text { United States }\end{array}$ \\
\hline 346 & 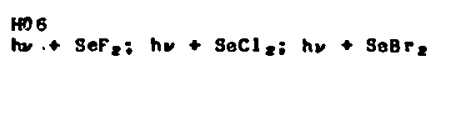 & $\varepsilon$ & $9-19$ aV & 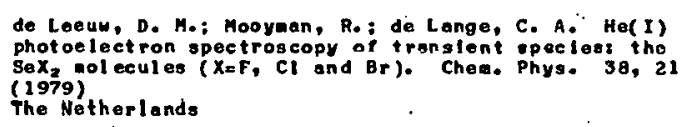 \\
\hline 348 & $\begin{array}{l}\mathrm{AOS}_{\mathrm{O}}+\mathrm{H}_{2} \\
\mathrm{AlO}_{2}+\mathrm{H}_{2} \\
\mathrm{He}_{2}+\mathrm{H}_{2} \\
\mathrm{Al}_{1}+\mathrm{Hz}_{2}\end{array}$ & $\mathbf{T}$ & $\begin{array}{l}\text { A0 3: } A 10 \\
0-5 \text { OU } \\
\text { A11 } \\
300-2500 K\end{array}$ & 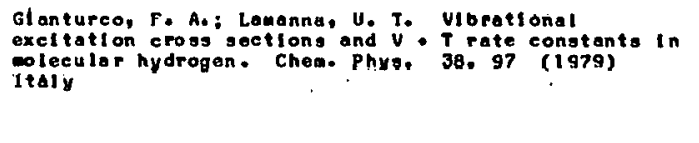 \\
\hline 348 & $\begin{array}{l}\mathrm{AOA}_{4}+\mathrm{HA}_{2}+ \\
\mathrm{He}+\mathrm{H} \\
\mathrm{Al}+\mathrm{HO}_{2}+\end{array}$ & $T$ & $2.7-20.7$ ev & 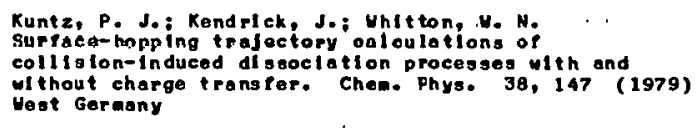 \\
\hline 349 & $\begin{array}{l}\mathrm{AOS}^{\mathrm{O}}+\mathrm{H}_{2}+ \\
\mathrm{HL}_{3}+\mathrm{H}_{2}+ \\
\mathrm{He}+\mathrm{H}_{2}+\end{array}$ & $T$ & $0.5-5.0 .0 \mathrm{~V}$ & 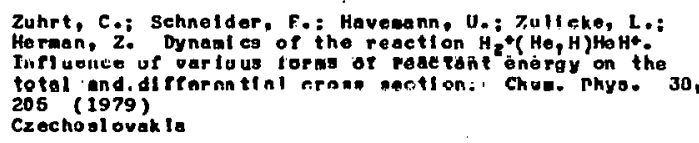 \\
\hline 350 & ${ }_{0}^{A l}+\mathrm{CHF}$ & E & $300 \mathrm{~K}$ & 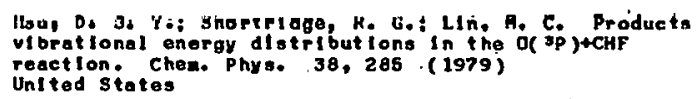 \\
\hline 351 & $\mathrm{AOA}_{\mathrm{Ar}}+\mathrm{O}_{2}: \mathrm{Ar}+\mathrm{H}_{2}: \mathrm{Ar}+\mathrm{D}_{2}$ & $\mathbf{T}$ & $3000-8000 x$ & 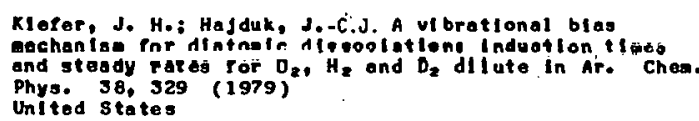 \\
\hline 352 & $\begin{array}{l}\mathrm{AlT}_{\mathrm{T}}+\mathrm{x}_{\mathrm{B}} \\
\mathrm{AlB}+\mathrm{x}_{0}\end{array}$ & $E$ & $\begin{array}{l}2.37-26.1 \\
k \in a 1 / m 01\end{array}$ & 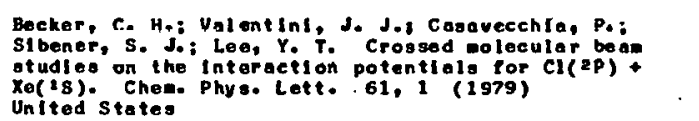 \\
\hline
\end{tabular}




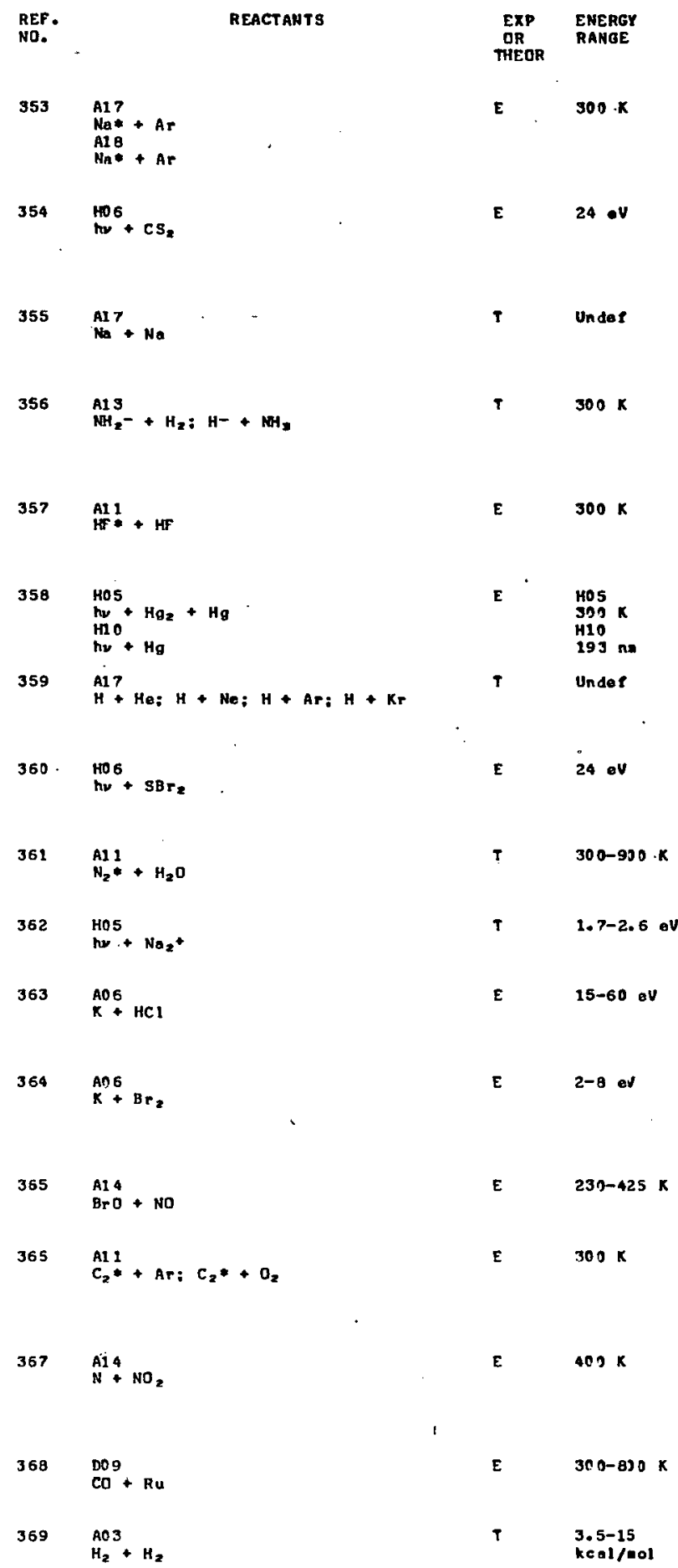

REF ERENCE

Duren, Ro: Groger, Ho Exporlaental doterminatlon of tho potenilal tor Ka(3 ep) + Ar trem distorential seattering croas seetions. Chez. Phys. Lett. 61, 6 (1979) wast Garasny

Schlrmer, Jo: Doecke, Mo: Coderbaum, L. Ss: von N1 ossen, W.: Aobrink, L. St rong corralati on effect In the lonl sation of CS.. Chea. Phys. Lett. 61, 30 (1979) Weot Gerwany

Lyyra, M.: Bunker, P, R. The potentlal onergy curve of the B 23 (sub u) state of Naze Chea. Phys. Lett. 62, Canada (1979)

Caraky, P.: Zahradnlk, Ro: Urban, Ho: Kello, V., AD Inltlo studies of chealcal equllibrla. A reflned approach to the reactlon $\mathrm{NH}_{2}-\mathrm{H}_{2}$ going to or cowing Pros NH, + H-. Ches. Phys. Lett. 61, BS (1979)

Macdonald, R. G.; Sloan, J. J. Ml croscople V-E onergy trangfor rates in the HF /NF systen. Chea. Phys. Lete. 61. 137 (1979) canada

Ehrlteh, D. J.; Oagood, R. M., Jr. Colliglon Induced prodlasoci at lón in photoas sociated Hg.. Chen. Phys. prodlasoct, 150 (1 pho) Unitod Statea

Brual, G., Jr.: Rothoteln, S. M. H Atom-rare gas Interaction potent lalo fros an eloctron gas oodel. Interaction potent fals from an alect
Chas. phys. Lott. 61, 167 (1979) canada

de Leauw, D. Mo: Hooyan, Ro: de Lange, C. A. He(I) photoelectron spectroscopy of transient specles: the photoelectron spectroscopy. of transiont speciess the
SBrz aol cullo. Chem. Phys. Lett. 61, 191 (1979) The Notherl ands

Nagel, J.: Rogovin, D. Deactl uatt on of ut brat lonally exclted $\mathrm{N}_{2}$ by $\mathrm{H}_{2} \mathrm{O}$. Ches. Phys. Lett. 61, 209 (1979) Unlted state:

Uzer, T.: Dalgarno, A. Photodlasoclatlon of $\mathrm{Na}_{2}+$. Chem. Phys. Lett. 61, 213 (1979)

United States

Okada, S. Measurements of energy loss spect ra in lon-pali pornation react tons for the $K+$ HCl systeme Chen. Phys. Lett. 61, 245 (1979) Japen

Mout Inno, A. M. C.; Kleyn, A. H.; Los, J. Ion-palr pormation in some potasstumeolecule collitstons. Dependence on the vibrat tonal energy. Chem. Phy 3. Lett. 61, 249 (1979) Portugal

Leu, M.-T. Rate constant for the reactlon BrU. + NO Br+ $\mathrm{NO}_{3}$ : Chen. Phys. Lett. 61, 275 (1979) United States

Flloeth, S. V.: Haneock, G.; Fournler, J.: Meler, K. quenching of $C_{2}($ a 3. sub u) produced in on Intense Infrared laser field: Cheo. Phys. Lett. 61, 288 (1979)

Porter, R. A. Ro: Broun, G. Ro: Grosser, A. E. Angular distribution of reaction product s fros the el eaentary reaction $\mathrm{N}+\mathrm{NO}_{2}-\mathrm{N}_{2} \mathrm{O}+\mathrm{O}$ by crossed molecular beams. Cheas phys. Lett. 61, 313 (1579). Canada

MeCarty, J. G.: Ulso, H. Dl ssoclative eheal sorpttion of co on riwthan lii.. Cham. Phys. Lett. 61, 323 (1979) Unlted States

Gelb. A. Classical trajectory study of rotational exclt ation. In collislong of hydrogen mol ecul es. Chea. Phys. Lett. 61, 333 (1979) Merice 


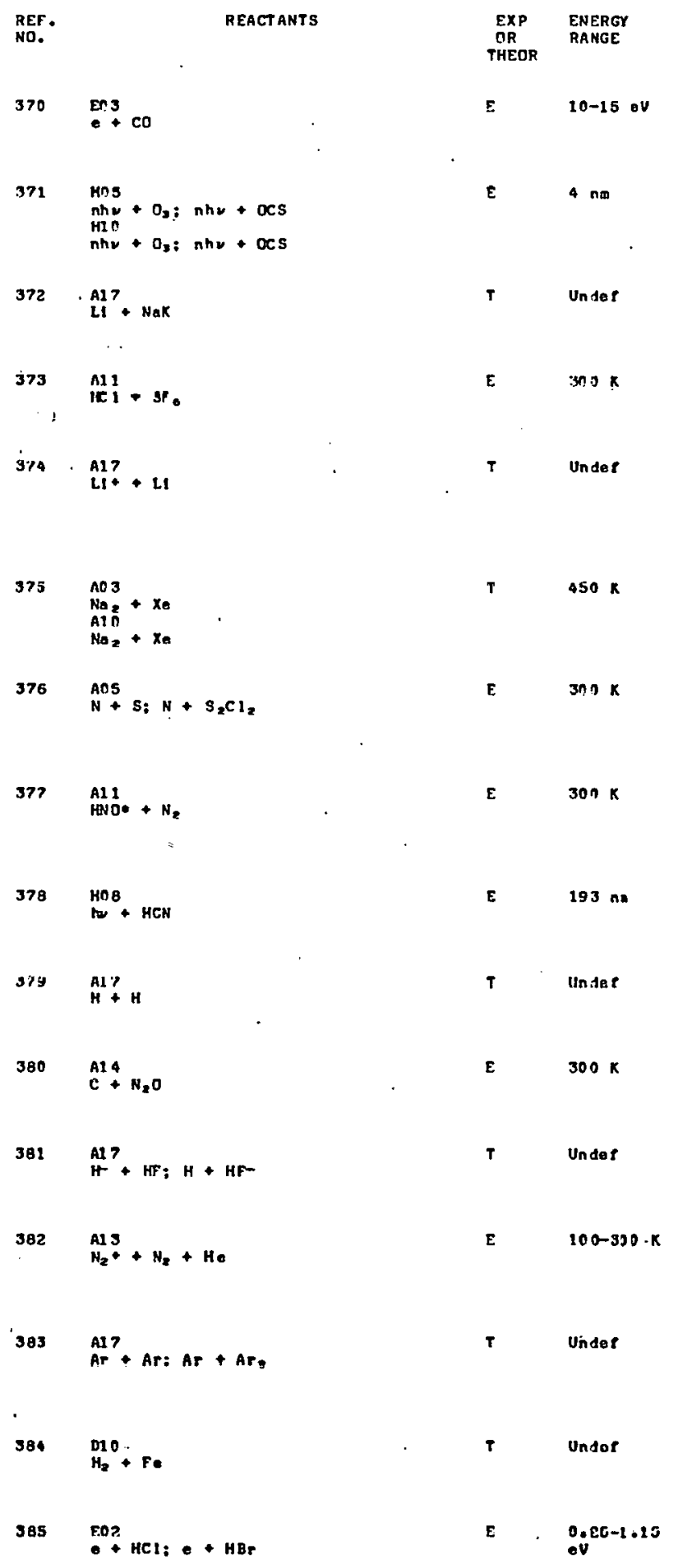

Bose, N. Influence of resonances in the el ectron

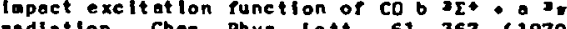
radiatlon. Ches. Phys. Lett. 61, 367 (1979) Hest Germany

Proch, D.: Schrodar, H. IR laser photocheolstry of $\mathrm{O}_{3}$ and nis. The Plrst aul tiphot on d Is ooclatt on of trlotomle molecules. Chen. Phys. Lett. 61, 426 (1979)

Varandes, A. J. C.: Tennyson, J.: Murrell, J. N. Chercher le erol seaent. Chen. Phys. Lett. 61, 431 (1979) $1 \mathrm{e}$ crol

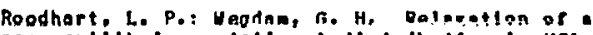
non-equilibrlua rotatlongl diotribution In HCl. Chea Phys Lelt. 81,449 (1979)

Konovalow, D. D, : Rosenkrantx, M. E. AD $\ln l t 10$ calculations of the oloctronle otructure and the vibrational spectra of the $X \& 2$ (sub g)t, $2 \Sigma$ (sub $u) t$,

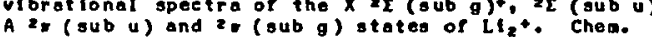

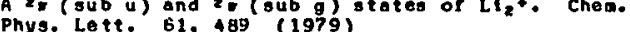

Romeswany, R.: DePrtsto, A. E.: Rabltz, H. On the Romeswamy, R.: Depristo, A. E.: Rabltz, H. On the theroti cal analysis. Chem. Phys. Lett. 61, 498 $(1979)$

Mural, H.: Yagl, T.: Obl, Ko: Tanakia, I. Chenliuminegcence of NS radical produced by the reaction of octive nitrogen uith sulfur vapor and

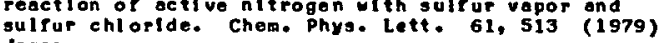
Japan

Yamado, F.: Ishlwata, T.: Kavasakl, N.: Obl, K.: Tanaka, I. St at e-gel ect ed $f l$ uores cence 1 lfot tres and colliaional quenching rates of HNo (A IA double prines). Chem. Phys. Lott. 61, sis (197s) Japan

Daronavokl, A. P. The rluareseence spectrua of HCN(A tllde $1 A$ double prime $x$ tilde $3 E$ ) using ArF laser excitatl on. Chem. Phys. Lott. O1, 832 (1979) unitad sthion

Kafri, n. Modiftod Moras Punotlon flttlig procedure for dlatonic potentlal g. Chem. Phys. Lett. 61, 538 (1979)

Costes, Mo: Dorthe, Fo: Destrlau, M. Vlbrattonal distribution In CN(A E, sub i) trom the reaction $C$ * $\mathrm{N}_{2} \mathrm{O}+\mathrm{CN}+\mathrm{NO}$. Chem. Phys. Lott. 61, 588 (1979)

Amos, R. D. An ab Int tlo potentlal energy surface for the reactlon between hydrogen 21 uorlde ond the hydrlde lon. Chem. Phys. Lott. 61, 597 (1979) United Kingdom

Adams, N. Go: Solth, D.: Lleter, D. G.i Rakohlt, A. Bu: Tulddy, N. D. A Purther study of the ternary

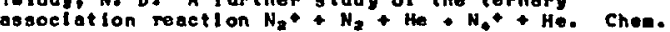

Phys. Lett. 51, 608 (1979)
Unl ted Kingdom

Doudey, J. P.: Novaro, O.: Berrondo, M. Uge of poeudepotontlal for the alsal yala of the nan-acisieturity of short-range intereolecular Interactions. Chem. Phys. Lett. 62, 26 (1979) Maxico

Purvto, G. D., III; Volken, G., JP. Model potentlal for the interact lon of eolecules with atorphous surfaces. Chem. Phys. Lett. 62, 42 (1979) unitad states

Mathur, D.: Hasted, J. B. Lov-lyling resonont states In HCl and HBr. Chee.: Phys. Lott. 62, 86 (1979)
unt ted KIngdion 


\begin{tabular}{|c|c|c|c|c|}
\hline $\begin{array}{l}\text { REF. } \\
\text { NO. }\end{array}$ & REACT ANTS & $\begin{array}{c}\text { EXP } \\
\text { DR } \\
\text { THEOR }\end{array}$ & $\begin{array}{l}\text { ENERGY } \\
\text { RANGE }\end{array}$ & REF ERENCE \\
\hline 386 & $\mathrm{AlO}_{2}+\mathrm{SO}_{2}$ & $T$ & $30 n-2000 \mathrm{~K}$ & $\begin{array}{l}\text { Chalapat1 Rao, Y. V. Vibratlonal relaxation in } \mathrm{SO}_{2} \text {. } \\
\text { Chem: Phys. Lott. 62, ing (1579) } \\
\text { Indla }\end{array}$ \\
\hline 387 & $\begin{array}{l}\text { Hos } \\
\text { ho }\end{array}$ & E & Under & 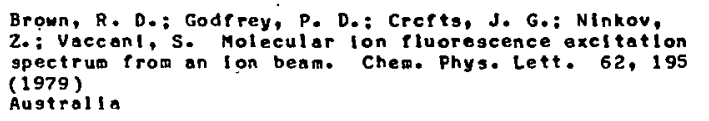 \\
\hline 338 & $\begin{array}{l}106 \\
2 n v\end{array}$ & $\varepsilon$ & $\begin{array}{l}6800-5000 \\
A^{\circ}\end{array}$ & 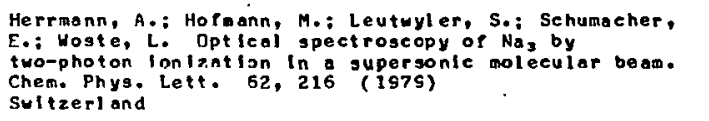 \\
\hline 389 & $\mathrm{AlO}_{1}+\mathrm{N}_{2} ; \mathrm{LI}++\infty$ & $T$ & $4.2-7.1 \mathrm{eV}$ & 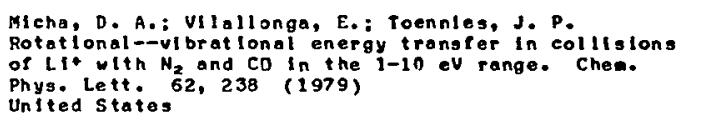 \\
\hline 390 & Al? & $T$ & Under & 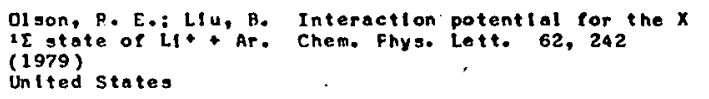 \\
\hline 391 & ${ }_{\text {How }}^{\text {HOS }}+\mathrm{H}_{2}$ & T & $3 n 4 A^{\circ}$ & $\begin{array}{l}\text { Itlkawa, Y. Vibrotlonal-state dependence of the } \\
\text { intensity ond angular distribution of photoelectrons } \\
\text { rrom } H_{2} \text { at the lapact of } 304 \text { Ao II ne. Chem. Phys. } \\
\text { Lete. 62, } 261 \text { (1979) } \\
\text { Japan }\end{array}$ \\
\hline 392 & $\begin{array}{l}\text { HOB } \\
\text { ho }+\mathrm{NH}\end{array}$ & E & $\begin{array}{l}3350-3050 \\
\mathrm{AO}^{\circ}\end{array}$ & $\begin{array}{l}\text { Anderson, U. R.: Crosley, D. R. Lasor-exefted } \\
\text { pluorescene in the A-X aystem of KH. Chem. Phys. } \\
\text { Lett. 62, } 275 \quad(1979) \\
\text { Unlted States }\end{array}$ \\
\hline 393 & $\begin{array}{l}\mathrm{A03} 3 \mathrm{~N} \\
\mathrm{Ar}+\mathrm{N}_{2} \\
\mathrm{AlO}+\mathrm{N}_{2}\end{array}$ & E & $769-12280 \mathrm{~K}$ & $\begin{array}{l}\text { Sanctuary, B. C. Energy dependence of rotatlonal cross } \\
\text { sect lons. Chea. Phys. Lett. } 62,378 \text { (1979) } \\
\text { Canada }\end{array}$ \\
\hline 394 & AIO $+\mathrm{NO}$ & $\varepsilon$ & $303 k$ & $\begin{array}{l}\text { Nutt, G. F.: Haydon, S. C.: MeIntosh, A. I } \\
\text { measurement of electronlc quenchlng roteg in nitrle } \\
\text { oxtde uging two-photon spectroscopy. Chem. Phys. Lett. } \\
62,402 \text { (1979) } \\
\text { Australla }\end{array}$ \\
\hline 395 & $\mathrm{EO}^{-}+\mathrm{HCN}$ & $\varepsilon$ & $8-40=V$ & 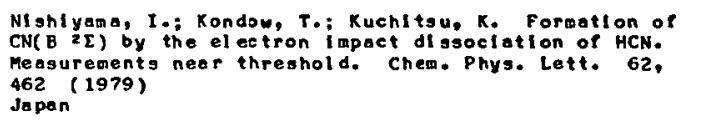 \\
\hline 396 & $\begin{array}{l}A_{1} 1 \\
H_{0} *\end{array}$ & E & $3 n 0 \mathrm{~K}$ & 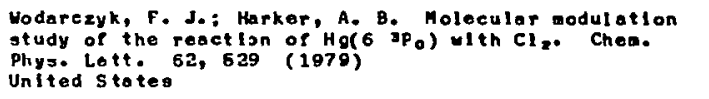 \\
\hline 397 & $\begin{array}{l}A_{0} 5 \\
X_{e}+X_{e}\end{array}$ & $\varepsilon$ & $10-16 \mathrm{eV}$ & 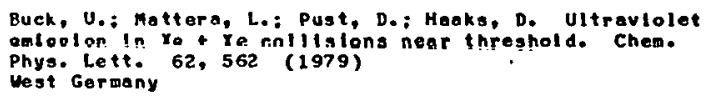 \\
\hline 398 & 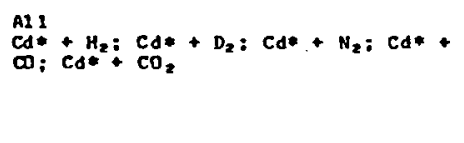 & E. & $393 \mathrm{~K}$ & 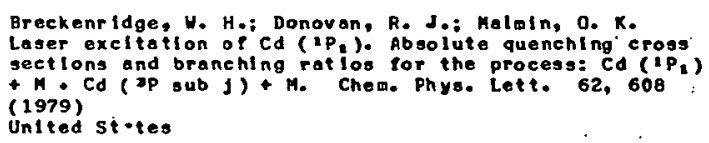 \\
\hline 399 & $\stackrel{\mathrm{AI}}{\mathrm{H}}+\mathrm{HCl}: \mathrm{H}+\mathrm{HBr}: \mathrm{H}+\mathrm{HI}$ & $\varepsilon$ & Under & 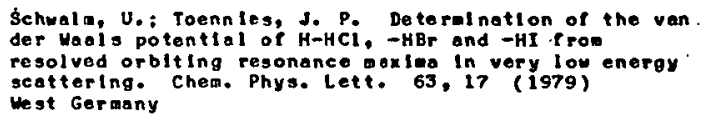 \\
\hline 400 & $\mathrm{HW}_{\mathrm{HW}}+\mathrm{CH}^{+}$ & $T$ & $\begin{array}{l}4751-3925 \\
A^{\circ}\end{array}$ & 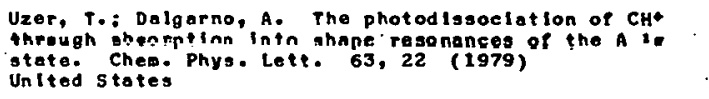 \\
\hline 401 & $\begin{array}{l}\mathrm{Al} 7 \\
\mathrm{He} * \mathrm{Ar}\end{array}$ & E. & Under & 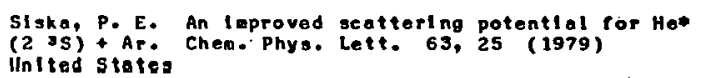 \\
\hline
\end{tabular}




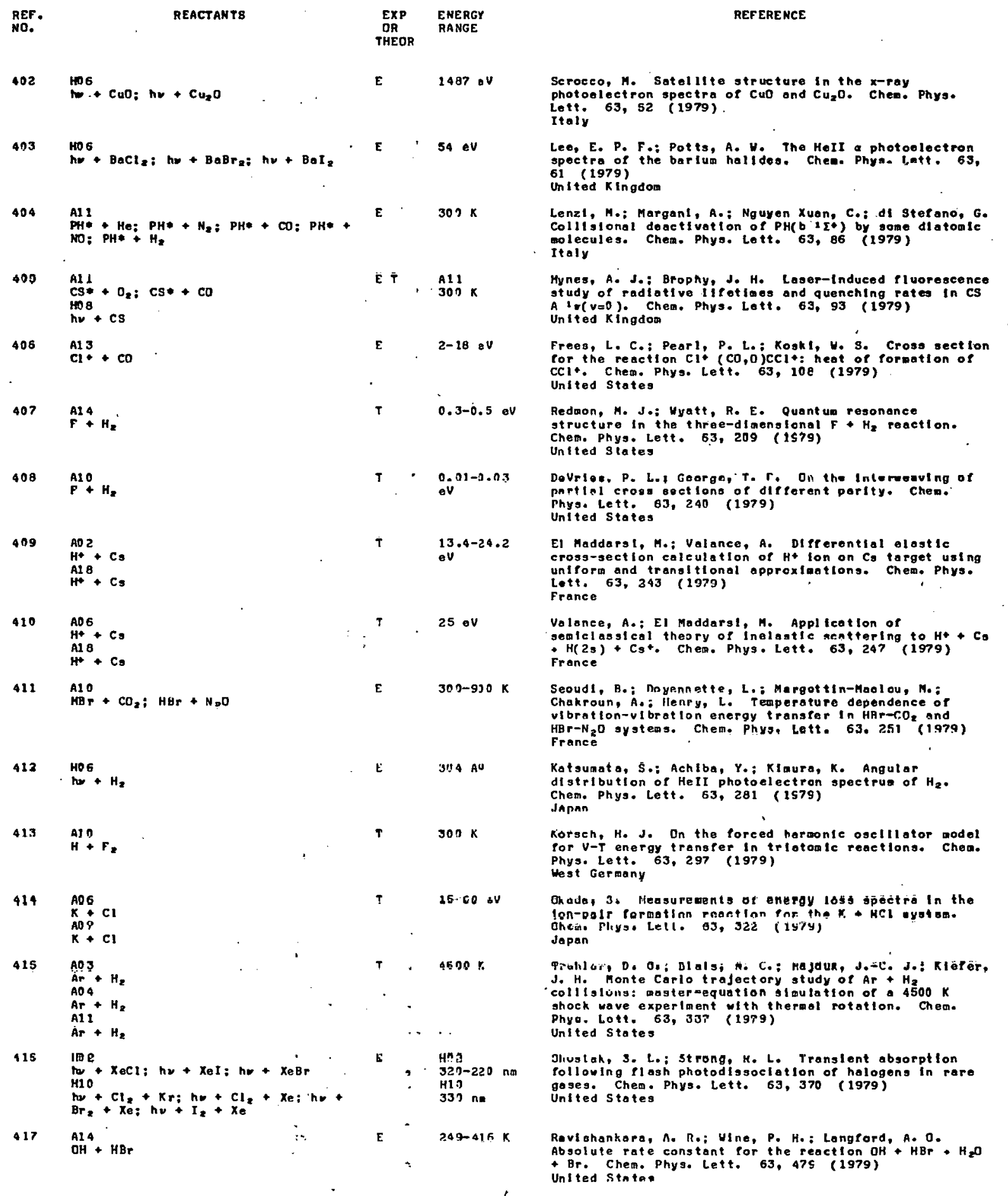




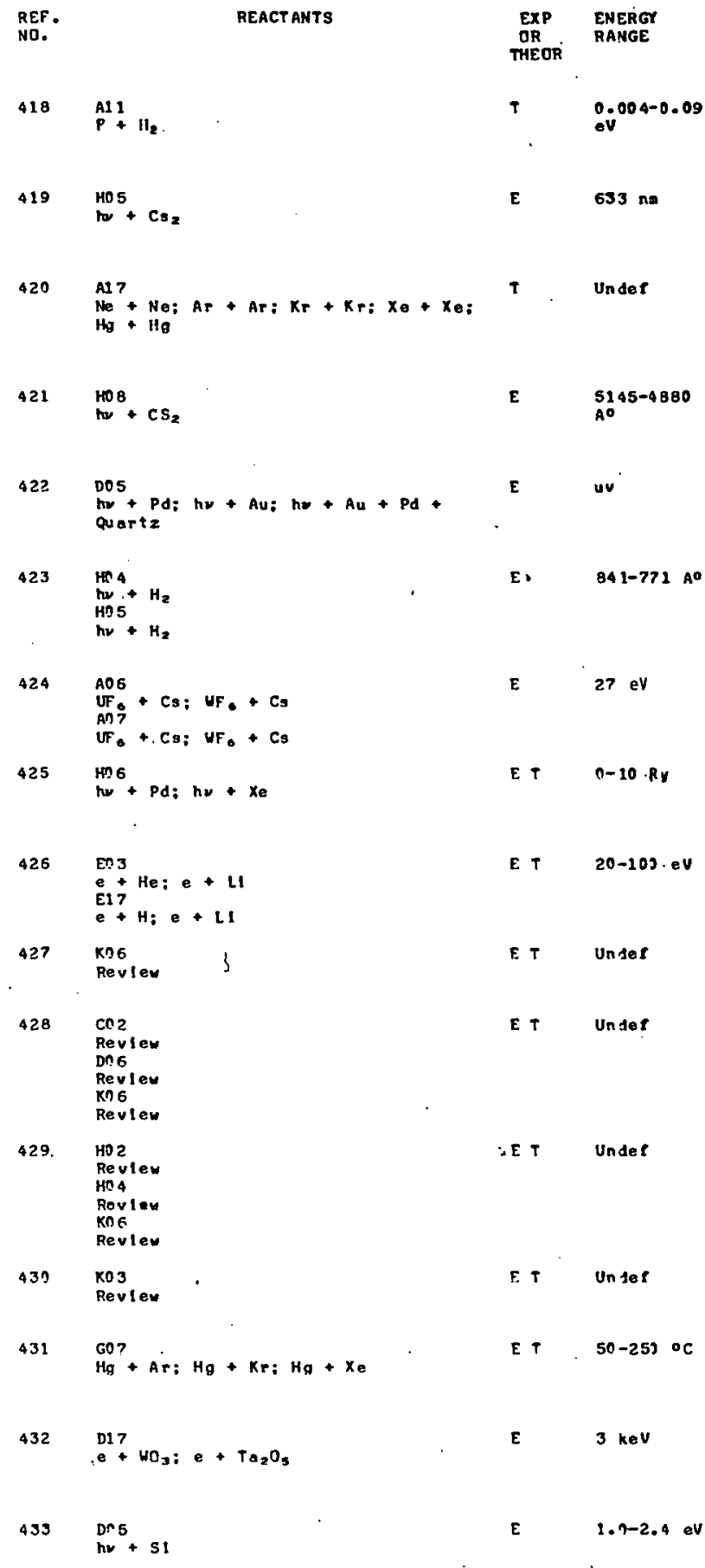

REFERENCE

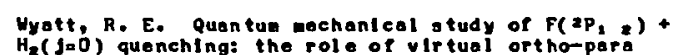
$\mathrm{H}_{2}(\mathrm{j}=\mathrm{O})$ quenching: the role of utr tual ortho-para Uniling. Chom. Phy a. Lett. 63, 503 (1979)

Kraul inya, E. K.; Papernov, S. M.; Janson, M. L. He- He 632.8 na radi at lon-induced dis intogratlon of caestum diaer. Chea. phys. Lott. 63, 531 (1979)

Clugston, M. J.: Pyper, N. C. A comparatlue gtudy of Gordon-k o applieat ion to the Inert gases and marcury. Chem.
Phys. Lott. 63, 549 (1979)
Unl ted Kingdom

Bhaskar, N. D.: Zouboulls, E.: Novak, R.: Happer, H. Laser-exclted eross fluorascent ew 1 sst on from ceslum wolecules. Chel. Phys. Lett. 63, 555 (1979) United Stotes

Slomos, K.: Unite, J.: Chrlstophorou, L. G.: Carter, I: G. Photoelectron injectlon In geses Proo thin metal Pilas on quartzo. Chem. Phys. Lett. 63, Se4 (1979)

Glass-Maujean, M.: Broton, J.: Guyon, P. M. A Fano-profile otudy of the.prediosocitiot ion of the 3p: (1979)

Stockdal e, J. A. D.: Harmack, R. J.: Compton, R. N. collistonat ionization of otopic ceslum by Uf, and UF. Chom. Phys. Lett. 63, 621 (1975) United states

Aausla, M. Y. The canifostation of Intershell intersctions in the process of otoule photolonization. Cosments At. Mol. Phys. B, 61 (1979) Sovlet Uni on

Winters, K. H. . Hl gher-order dl a torted-wave methods. Comments Ai. Mol. Phys. 8, 93 (1979) Unsted Kingdom

Stern, E. A. Atoale structure datermination by $x$-ray absorption. Conteap. Phys. 19, 289 (1979) United States

Willlows, R. H. Electron spectroscopy of surfaces :Conteap. Phys. 19, 389 (1979) Unlted $k$ Ingdom

Connerade, J. P. The non-Rydberg spectroscopy of oftomas. Contemp. Phys. 19, 415 (1979)
unlted Kingdon

Stenholm, S. Laser-induced multiphoton transitions. Conteap.'Phy. 20, 37 (1979) Finland

Partlou, U. D.: Kline, L. E. Prebreakdoun discharges Partlou,
in mercury-rare-gas mixtures. P. Appl. Phys. 50, 1250 (1979) United States

Lin, T. T.: Llehtaan, D. The effects of electron bombardment on the surface composltion of k0, oxidized bombardment on the surface compositlon of United States

Shahelary, I.: Sehwank, J. R.: Allen, F. G. Energy looo and acenpe depth of hot electrons (row shallow p-n Junctlons in 3 il 


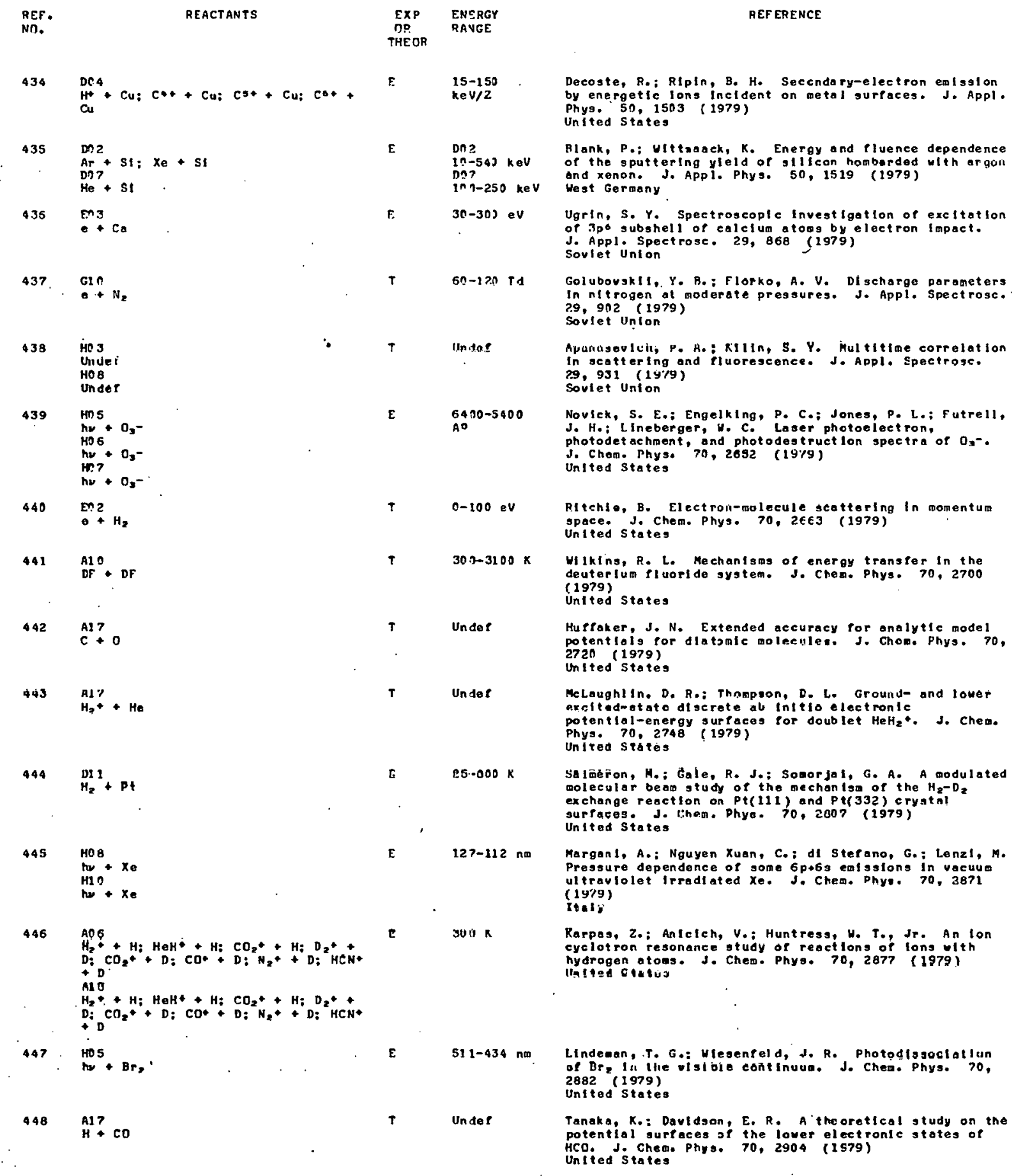




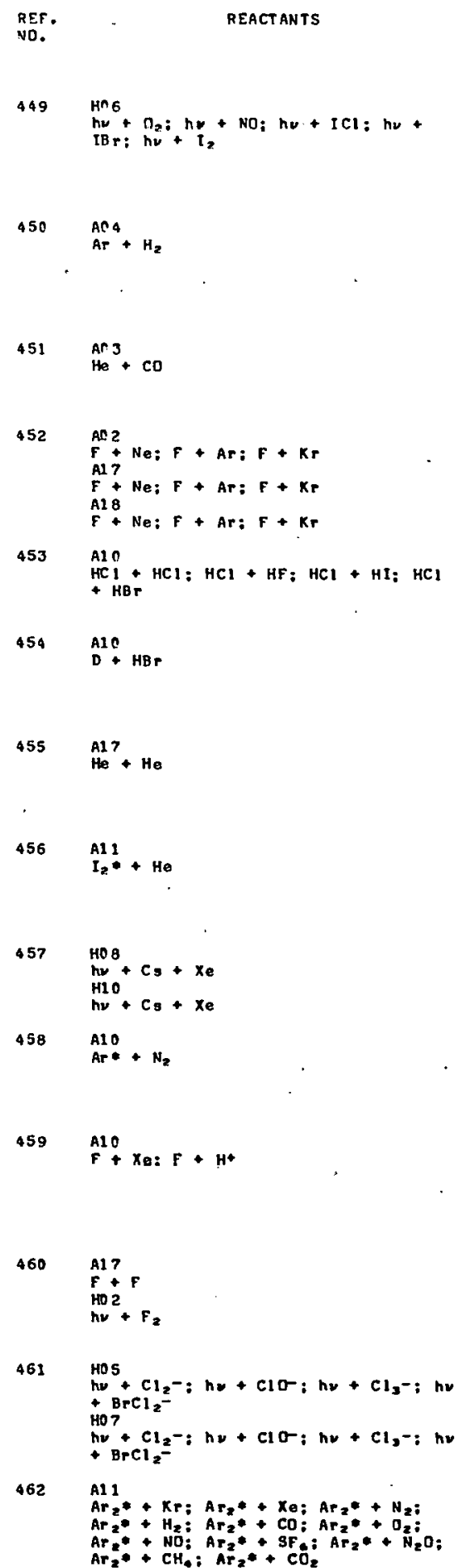

\section{Hos}

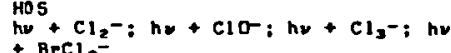
$+\mathrm{BrCl}_{2}-$

HO?

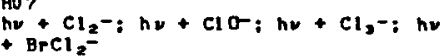

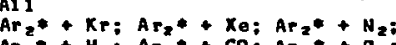

$\mathrm{Ar}_{2} *+\mathrm{H}_{2} ; \mathrm{Ar}_{2} *+\mathrm{CO} ; \mathrm{Ar}_{2} *+\mathrm{O}_{2}$

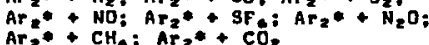

T

\section{Mecurdy, C. H.; Meyer, H. D.: HIller, H. H. Classical collision processes: poeudopotentiol analyot sond

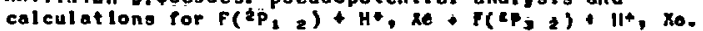 J. Chea. Phys. 70, 3177 (1979) Unted states}

Carturlght, D. C.; Hoy, P. J. Theoretieal studles of the valane electronic atates and the Ir (sub u) cosing From $X 1 \Sigma$ (sub $g$ ) absorption spectrua of the $F_{*}$ molecule. J. Chem. Phys. 70, 3191 (1979) United States Lee, L. C.: Solth, G. P.; Moseley, J. T.; Cosby, P. C.:

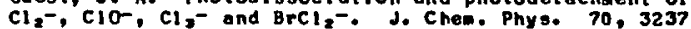
(1979) United States

$300 \times$

Kogane, M.: Inanura, M.: Aral, S.; Matanabe, T. Energy

Cutshall, E. R.: Musehlltz, E. E., Jr. Transfer of electronic excltation In collitolons of netastable argon atons ut th nltrogen molecules. Jo Chen. Phys 70, 3171 (1979) transfer of argon exclted diatomic molecules. J. Chem. Phys. 70, 3384 (1979) Japan

F.l and, J. H. D. Angular diserloutlons, energy disposal, and branchlng studied by NO+. ICI+, I Brt, and. I col fragmentetion. J. Chen. Phys. United states diseof AP + $\mathrm{H}_{2}$ : vibrational selectivity or characterlotics of dls soci ation under equllibriue Unitedser. Phys. 70, 2962 (1979)

Thomss, L. D. Solution of the coupled equations of inelastle at anmolecule scottering for a single West Germany

Becker, C. H.; Cosavecchla, P.: Lee, Y. T. Crossed for $F(2 P)+N e$, Ar, Kr(is). J. Chem. Phys. 7h, 2986 $(1979)$

Turfa, A. F.: Marcus, R. A. Rotational onergy trangfer In hydrogen hall de molecules at supersonite bean

Jollicard, G. The influence or the phase shift on the vibration energy transfer for a colilision between an

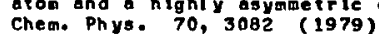

Burton, P. G. Design of basls sets for preclae Intermolecular force computation. Investigation of the Hez potentlal curve using, CEPA-PND correlated
wovefunctions. J. Chem. Phys. 70, 3112 (1979)
Awet

Tusa, J.: Sulkes, M.; Rlce, S. A. Low energy resonance enhanced vitorational deactivation. Jo Chem. Phys. 70, 3136 (1979)

Benedlet, R. P.: D rumond, D. L.: Sehlie, L. A. Phys. 70, 3155 (1979) 


\begin{tabular}{|c|c|c|c|c|}
\hline $\begin{array}{l}\text { REF. } \\
\text { NO. }\end{array}$ & REACT ANTS & $\begin{array}{l}\operatorname{Exp}_{\text {OR }} \\
\text { THEOR }\end{array}$ & $\begin{array}{l}\text { ENERG } \\
\text { RANOE }\end{array}$ & REF ERENCE \\
\hline 463 & $\mathrm{HE}^{\mathrm{A1O}}+\mathrm{H}_{2}=$ & $T$ & $0.8-1.6$ ov & 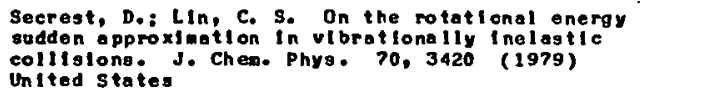 \\
\hline 464 & $\hat{0}_{2}^{11}+0_{:} 0_{2}+0_{3}$ & E & $500 \mathrm{~K}$ & 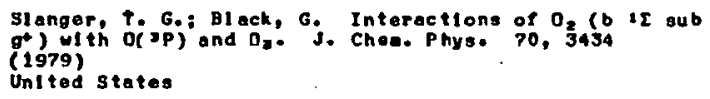 \\
\hline 465 & $\stackrel{G 08}{A R}$ & $T$ & Under & $\begin{array}{l}\text { Vervlach, P.: Terrier, M. Influence of inelastlc } \\
\text { collislong betwoen exclted stotes on the electron } \\
\text { energy diotribution in argon. J. Ches. Phys. } 70,3453 \\
\text { (1979) } \\
\text { France }\end{array}$ \\
\hline 465 & 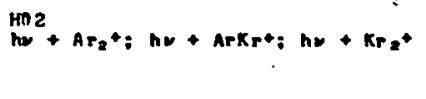 & E & 320-295 nm & 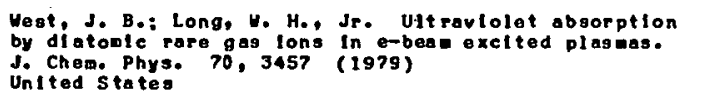 \\
\hline 467 & 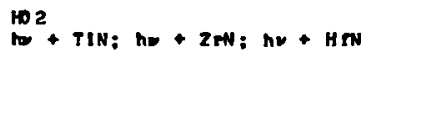 & E & $\begin{array}{l}890-1050 \\
c m-8\end{array}$ & 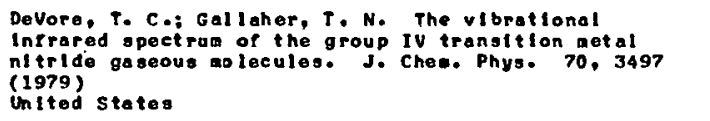 \\
\hline 468 & $\mathrm{~F}^{\mathrm{Ala}}+\mathrm{H}_{2}$ & $T$ & $:^{0.300-0.375}$ & 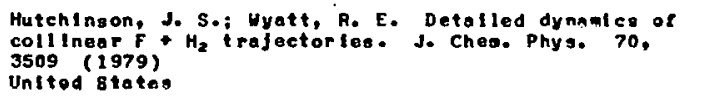 \\
\hline 469 & 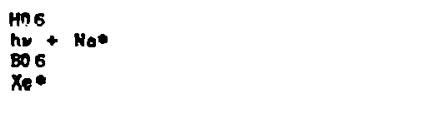 & E & $\lim _{3 \ln } 6 x$ & 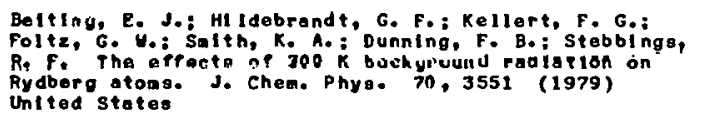 \\
\hline 470 & $\mathrm{HO}_{\mathrm{h}} 2$ & E & $616-570 \mathrm{~nm}$ & 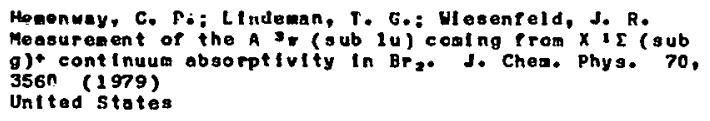 \\
\hline 471 & $N^{D 12}+C_{u} ; N++c_{U}$ & $\mathbf{E}$ & $300 \mathrm{~K}$ & 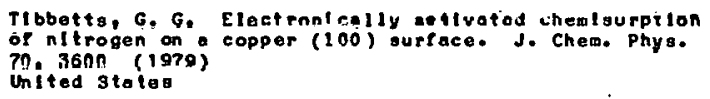 \\
\hline 472 & $\begin{array}{l}\text { Hos } \\
\text { Under }\end{array}$ & $T$ & Under & 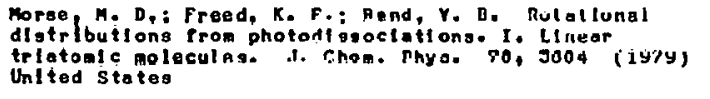 \\
\hline 473 & 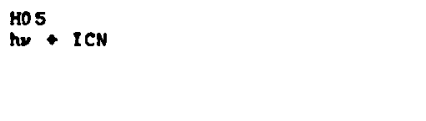 & T & $290-220 \mathrm{nE}$ & 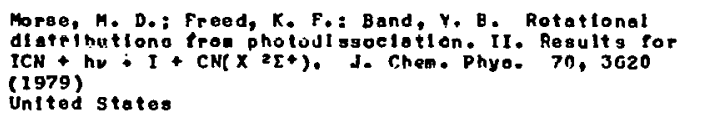 \\
\hline 474 & $\mathrm{He}_{\mathrm{Bn}}+\mathrm{LIF} ; \mathrm{Ne}+\mathrm{W}$ & $T$ & n.15-100. ev & 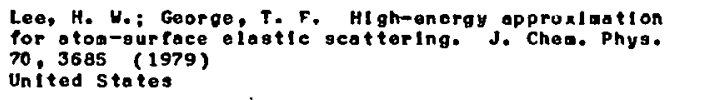 \\
\hline 475 & $\mathrm{H}_{\mathrm{H}}^{\mathrm{A}}+\mathrm{H}$ & $T$ & Undes & $\begin{array}{l}\text { Varandas, A. J. C. A LEPS potentlal for H, Prom force } \\
\text { lleld data. J. Chem. Phys. } 70,3786(1979) \text { Prom }\end{array}$ \\
\hline 476 & $\mathrm{O}_{2}^{\mathrm{Al} 7}+\mathrm{O}_{2} ; \mathrm{O}_{2}+\mathrm{Ar}$ & $T$ & Under & 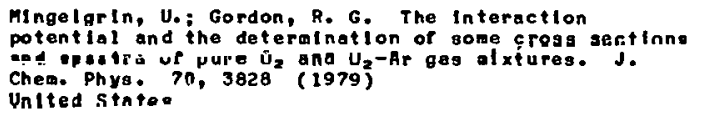 \\
\hline 477 & $\mathrm{C}_{2}^{\mathrm{Al}} 4+\mathrm{NO}$ & E. & $2510 \mathrm{x}$ & $\begin{array}{l}\text { Krause, H. F. A carbon reactlon studied by crosged } \\
\text { moleculart beang. J. Chem. Phys. } 70,3871 \text { (1979) } \\
\text { United Stateg. }\end{array}$ \\
\hline 478 & $\mathrm{AlP}^{\mathrm{He}}+\mathrm{I}_{2}$ & $\begin{array}{l}T \\
T\end{array}$ & $n-1$ ev & 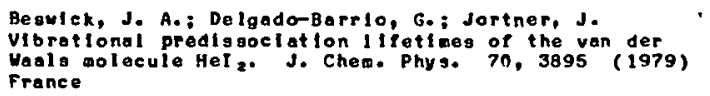 \\
\hline
\end{tabular}




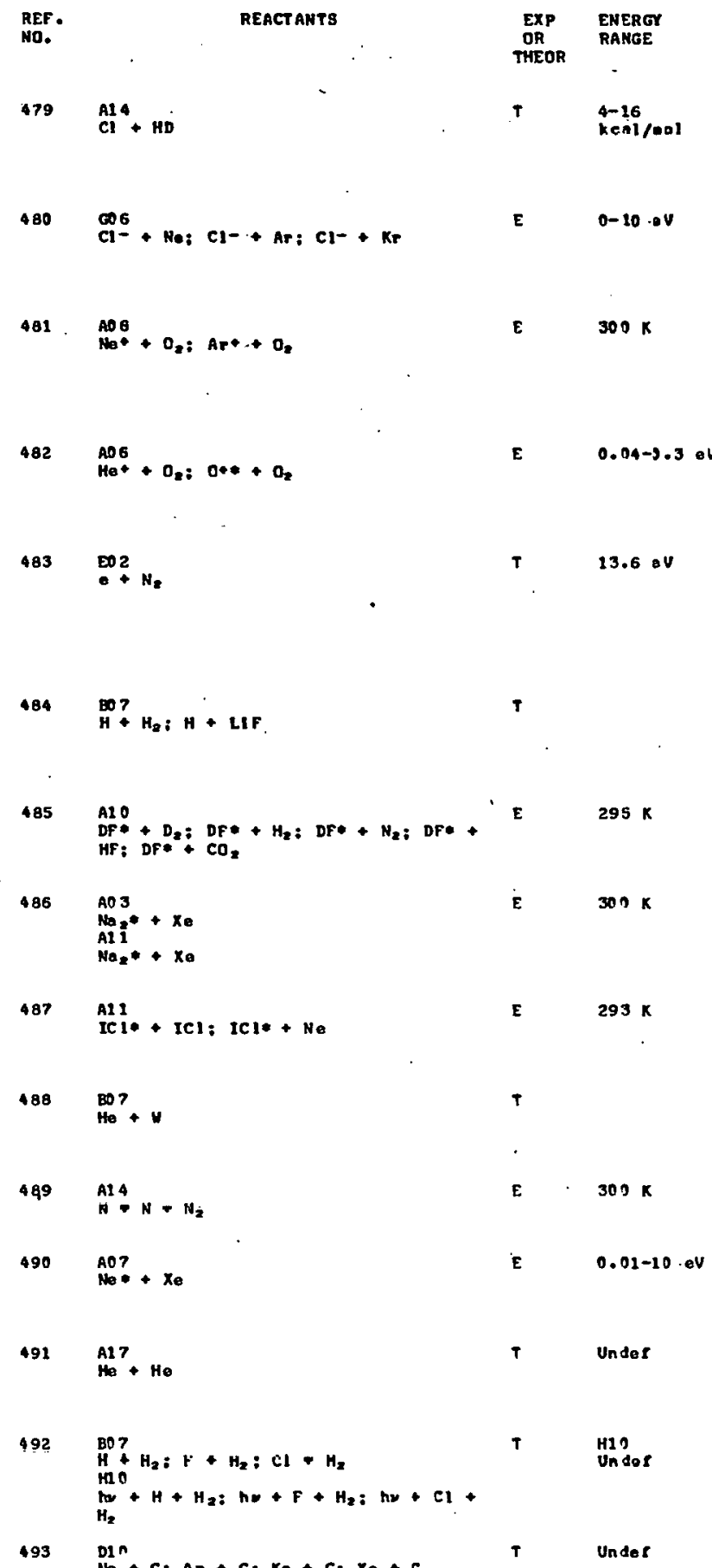

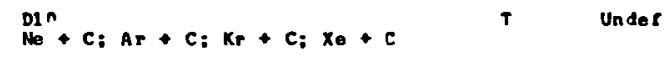

REP ERENCE

Persky. A. Quadelasalcal trojectory studies of the chlorlne-hydrogen systea. III. Branching ratlo, energy partitioning, and angular diotributlon in the reaction of CI at oas uith HD. J. Chen. Physe. 70, 3910 (1979)

Thacketon, H. G.: El sele, F, L.: Pope, W. M.: ; El 118, H. W.: Gatland, I. R.: McDaniel, E. $U$. Robility of $\mathrm{Cl}^{-}$ lons In Ne, Ar, and Kr. J. Chea. Physe 70, 3996 (1979)

Mauclalre, G.; Deral, R.: Fenlatelin, S.; Merx, R. Energy disposal In thereal-energy charge transfor reaction deternlaed by kinetic energy masurecents of produet lons: $\mathrm{Ne}^{+}+\mathrm{O}_{2}+\mathrm{O}+\mathrm{O}_{+}+\mathrm{No}$ and $\mathrm{Ar}^{*}+\mathrm{O}_{2} \cdot \mathrm{O}_{2}$ Ar.. J. Chean. Phya. 70, 4017 (1979)

Mauclalró, G.; Deral, Ro; Fenlsteln, S.: Horx, R.: Johnsen, R. Thernal energy charge trangfer from Het to $D_{0}$ : klnetle onergy nature, and reat product lons. J. Chow. Phys. 20, 4023 (1979)

Rubblo, J. R.: Truhlar, D. G. Low-energy

acosorison of couplod el ectron-mol ecule acatterling: Comparison of coupled channel troataents of e- scatering at $13.6 \mathrm{eV}$ using varlowa approxlats lons to the of at le and exchange potentials and an approxtmate polarization potential .
J. Chen. Phys. 70, 4101 (1975) J. Chen. Phys.

Llght, J. C.: Al tenberger-Siczek. A. Lasor-collision Induced chemical reactions: collinear exchange reaction Induced chomical reactlons: coll inear exchange reactlon

United states

Bott, J. F. vibratlonal rolaxation of DF $(y=1-4)$ In $\mathrm{D}_{2}, \mathrm{H}_{2}, \mathrm{H}_{2}$, HF, and $\mathrm{CO}_{2}$, J. Chea. Physe 70,4123 (1979) United stater

Brunner, T. A.: Drlver, R. De: Sulth, N.; Pritehard, D. E. Rotatlonal onergy transfer In $\mathrm{Na}_{2}$ * - Xe collislons: level to level dynapics. J. Chea. Phys. 70,4155 (1979)

United states

Harrls, S. J.; Natzlo, W. C.; Moore, C. B. Zero pressure itfot ines and pluoreocence quenching of $\mathrm{ICl}$ (A 3., J. Ches. Phys. 70, 4216 (1975).

Lee, H. H.: Goorge, T. F. Energy trangfer in gas-surface seattering in the presence of a laser fleld. J. Cham. Phys. 70, 4220 (1979) Untited states

Yamashita, $T$. Rat of reconblnotion of nftrogen atome. J. Chea. Phys. 70,4248 (1979)

Neynaber, R. H.: Tang, S. Y. Cheal-t onlzetlon In collialono of metastable neon with xenon. J. Chea. Phys, 70, 4272 (1979) United states

Az12, R. A.: Naln, U. P. S.; Carley, J. S.: Taylor, U. Li: Meconvilile, G. T. An accurate interaolecular potential for hellum. J. Chem. Phys. 70, 4330 (1979) Canado

Orel, A. E,; Miller, H. H. Intrared laser enhancement of rhealcal reactlong via collis ion Induced absorpt lon. J. Chen. Phys. 70, 4393 (1979) United states

Rybolt, T. R.: PlerottI, H. A. Mare gas-yraphlto interaction potentlala. J. Ches. Phys. 70, 4413 (1979) 


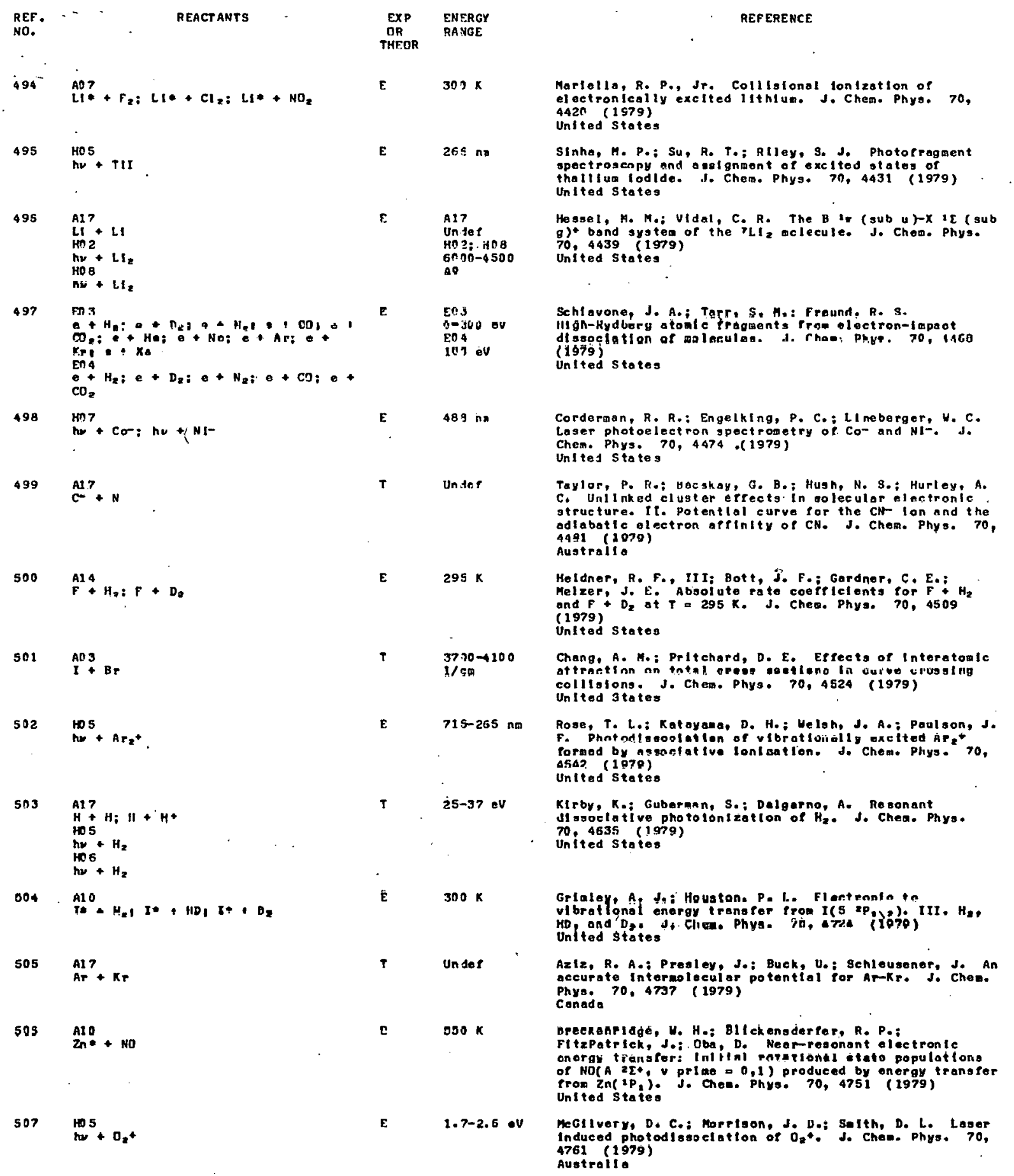




\begin{tabular}{|c|c|c|c|}
\hline $\begin{array}{l}\text { REF. } \\
\text { No. }\end{array}$ & REACT ANTS & $\begin{array}{c}\operatorname{EXP} \\
\mathrm{DR} \\
\text { THEOR }\end{array}$ & $\begin{array}{l}\text { ENERGY } \\
\text { RANGE }\end{array}$ \\
\hline 508 & $\begin{array}{l}\mathrm{Al} 4 \\
\mathrm{H}+\mathrm{O}, \mathrm{z}\end{array}$ & E. & $299 K$ \\
\hline 509 & ${ }_{\mathrm{OH}}^{\mathrm{A}}+\mathrm{O}_{3}$ & $\varepsilon$ & $239-357 x$ \\
\hline 510 & 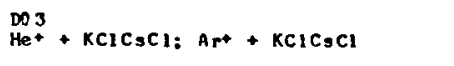 & $\varepsilon$ & $0.2-3.0 \mathrm{ke}$ \\
\hline 511 & $\mathrm{AO}_{4}+\mathrm{H}_{2}$ & $T$ & íngoo $\mathrm{x}$ \\
\hline 512 & $\begin{array}{l}\mathrm{Al} 7 \\
\mathrm{Ar}+\mathrm{N}_{2}\end{array}$ & $T$ & Under \\
\hline 513 & 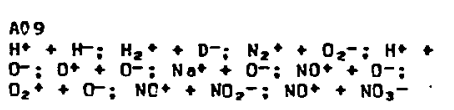 & $T$ & $300 \mathrm{~K}$ \\
\hline 514 & $\mathrm{Al}_{\mathrm{O}}^{\mathrm{A}}+\mathrm{H}_{2}$ & T & $\begin{array}{l}1-40 \\
k \operatorname{col} / \text { nOL }\end{array}$ \\
\hline 515 & $\begin{array}{l}\mathrm{AlO} \\
\mathrm{HCI}\end{array}$ & E & $192-632 \mathrm{~K}$ \\
\hline 516 & $\begin{array}{l}\mathrm{DOP} \\
\text { Ar }+ \text { U: } \mathrm{He}+\mathrm{WC}: \mathrm{Ne}+\mathrm{H} ; \mathrm{Ne}+\mathrm{VC} ;\end{array}$ & E & $298-1850 \mathrm{~K}$ \\
\hline 517 & 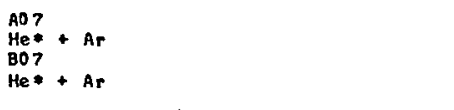 & $T$ & ${ }_{300}^{A 07} \mathrm{~K}$ \\
\hline 518 & $\mathrm{Al}_{\mathrm{H}_{2} \mathrm{O}}+\mathrm{D}_{2} \mathrm{O}$ & $T$ & $305 k$ \\
\hline 519 & $\begin{array}{l}\mathrm{AO3}_{3} \\
\mathrm{H}_{2}+\mathrm{H}_{2} \\
\mathrm{Al}_{1} \\
\mathrm{H}_{2}+\mathrm{H}_{2}\end{array}$ & $T$ & $\begin{array}{l}2000-8000 \\
1 / \mathrm{cm}\end{array}$ \\
\hline 520 & 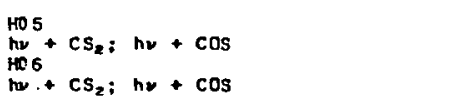 & E & $960-500 \mathrm{AO}$ \\
\hline$\$ 21$ & ${ }_{\mathrm{Br} *}^{\mathrm{AlO}}+\mathrm{H}_{2} ; \mathrm{Br} *+\mathrm{HD} ; \mathrm{Br} *+\mathrm{D}_{2}$ & E & $300 \mathrm{~K}$ \\
\hline $\mathbf{s 2 2}$ & $\stackrel{A 14}{N}+n_{2}$ & E & $298 K$ \\
\hline
\end{tabular}
reaction of hydrogen itoms ith ozone: evidence for a secend reactlon path produclng $\mathrm{HO}_{2}$ and $\mathrm{O}\left(\mathrm{JP}_{\mathrm{P}}\right)$. J. Chem. Phys. 70, 4804 (1979) United states

Ravt ghankara, A. R.; Wine, P. H.: Langford, A. O. - Erratum Absolute rate constant for the reaction $\mathrm{OH}(\mathrm{V}$ $=$ I) $+\mathrm{O}_{2}+\mathrm{HO}_{2}+\mathrm{O}_{2}$ over the temperature range 238-35? K [J. Chem. Phys. 70, 984 (1979)]. J. Chem. Phyg. 70, 4812 (1979)

Honda, F.: Fukudi, Y.; Rabal als, J. U. Clustering distances critleal to metal diser foration in the secondary lon tas opoctrai(sins) o . Cheme. Phys.

Lehr, T.: Blrks, J. W. Monte Carlo quaslclassical trajectory study of the collis lon-induced dissoclation trajectory study of the collis lon-induced dissoclatlon
of hydrogen by neon. J. Chem. Phys. 70, 4843 (1979) United States

Lee, S.: KIm, Y. S. Study of the Ar-Nz Interaction. II. Modification of the electron gas oodel potential at intermediate and $\mathrm{l}$ arge distances. Jo Chem. Phys. 70 . Korea (1979)

Hickman, A. P. Approximate scolling formula for ion-ton

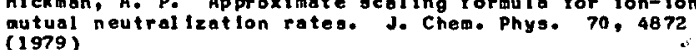

United state:

Schinke, R.: Lester, H. A., Jr. Trojectory otudy of 0 $+\mathrm{H}_{2}$ reactions on fitted $a b$ initlo surfaces. I. Triplet case. J. Chem. Phys. 70, 4893 (1979) Unted States

Horwitz, A. B.: Leone, S. R. Teaperature dependence of resonant Isotople vibratlonal energy transfer In
Hssc1-H33C1. J. Chem. Phy s. 70, 4916 (1979) Untted States

Bush, P. S.: Raft, L. M. At omle bean diferaction and scattering 8 ros A $(3 \times 5)$ y (110)-carbl de surfaces do Chem. Phys. 70, 5026 (1979) Unlted States

Bellum, J. C.: George, T. F. Collistonal lonizotion in the presence of intense laser radiatlon: quantua-mechan feal calculations for Ho*('s) + Ar plus' or ml nus h omega - He + Art (2P) P o - Jo Chem. Phy s: $7 n, 5059(1979)$

ta tes

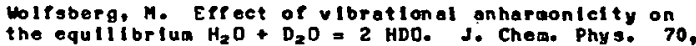
5322 ( 1979 )

colidrian, k. : Kourl, j. J. On angular momentum decoupling approxlmations and fact ort zation in diatan-diaton seat toring. J. Chem. Phys. 70, 5076 (1979) United States

Eland, J. H. D.: Berkowltz, J. Dl ssoclatlve photolion izatlon of carbon dlsulphide and carbonyl sulphide. J. Chem. Phys. 70, 5151 (1979) United States

Grimley, A. J.: Houston, P. L. El ectronle to vibrational energy transfor from Br(4 $2 \mathrm{P}_{2}$, i) to $\mathrm{H}_{2}$, HD, and $D_{2}$. J. Chem. Phys. 70, 5184 (1979) United states

Stler, L. J.: Payna, W. A. : Lee, J. H.: Michael, J. V. The reactlon $N\left(\right.$ S) $+0_{1}$; an upper linlt por the rate constant at $298 \mathrm{~K}$. J. Cheal. Phys. 70, 5241 (1979) United States 


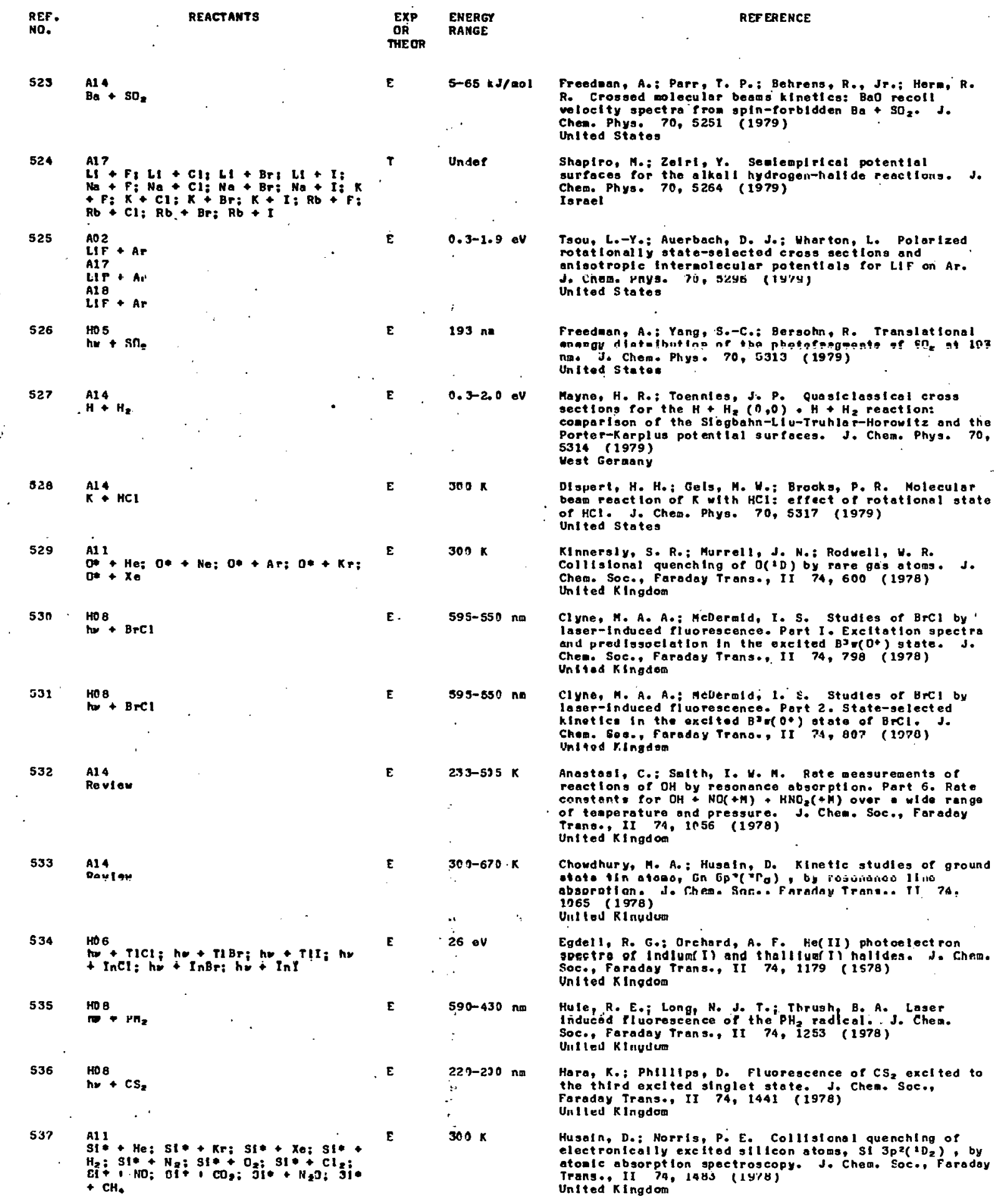




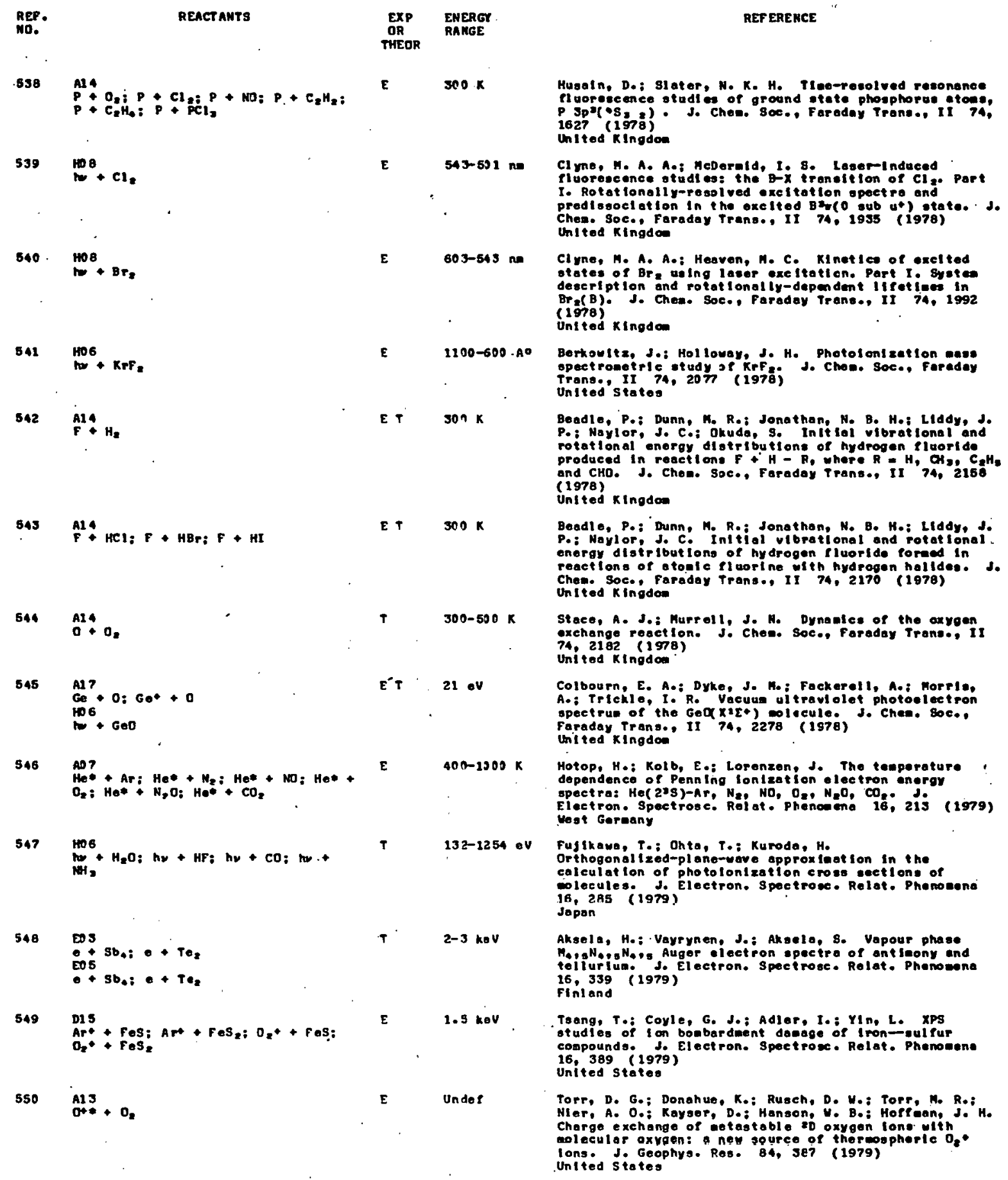




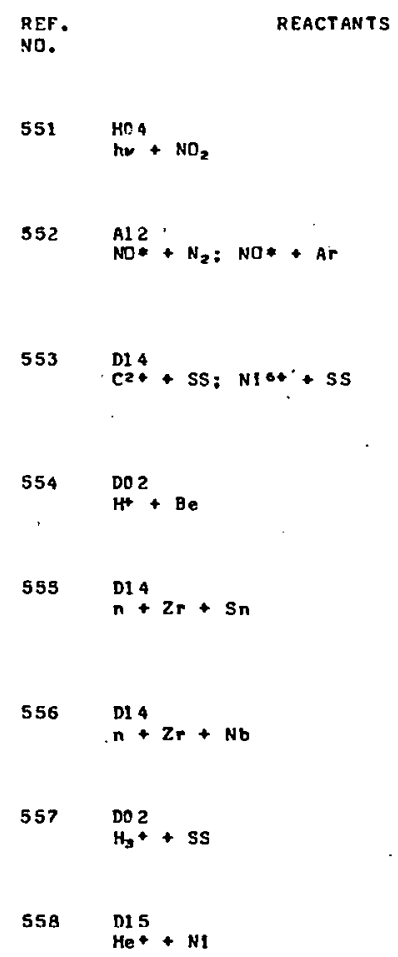

NO* + $\mathrm{N}_{2} ; \mathrm{NO} *+\mathrm{Ar}$

Do 2

$\mathrm{Dl}_{\mathrm{He}} \mathrm{5}+\mathrm{NI}$

566 Unde?,

D1 + + NO: N1P+ NO+

D1 6

Under

$\mathrm{CO3}^{\mathrm{C}} \mathrm{Hg}$

He 1
Under

Under

EOG
Indef

A1 ?

$\mathrm{Na}+\mathrm{He}$
EXP ENERGY

OP. RAVGE
50.7090

$297 \mathrm{~K}$

\section{E}

$T$

E

$22-46$ yev

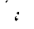

E Under$$
\text { : }
$$

E in kev

$500 k=0$
REF ERENCE

Brand, A. C. D.: Chlu, P. H. Spln-orblt-Indueed transitlons in the laser-exclted luoreseence of
nitrogen dioxide. J. Mol. Spectrose. 75,1 (1979)

Henry, A.: Severln, F.: Henry, L. Magnetle noment effect on pressure broadening: IInes from the $2_{11} / 2$ and ${ }^{2} r_{3} / z$ states of the $(1-0)$ band of NO colliding with $\mathrm{N}_{2}$ and Af. J. Mol. Spectrose. 75, 495 (1979)

VIllags, T. M.: Arkell, D. R. Voldowelling in 20 Willams, T. Mo: Arkell, D. R. Voldswell ing In 20 irrodlated with 22 MeV C24 Ions or 46.5 MeV Nies Ions. Irrodiated with 22 MeV C2* lons Untted $k \operatorname{lng}$ dow

Matfox, D. M.: Shsrp, D. J. Influence of surface gorphology on the lou energy hydrogen lon eroslon
ylelds of beryllitum. J. Nuel. Mater. 80,115 (1979)

Rogerson, A.; Murgatroyd, R. A. Extects of texture and

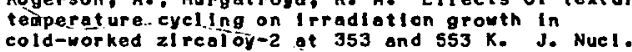
cold-worked zircoloy-2 at 353
Mater. 80,253 (1979)

Untered Kingdom

Rogergon, A.: Murgatroyd, R. A. Irradlatlon-grouth In annealed $2 r-2.5$ wt percent $N_{b}$ ot $353 \mathrm{~K}$. J. Nuel. United Kingdow $-1$

Salth, J. N., Jr. Angular erfects in the sputtering of Crt Proa stalnless steel by $10 \mathrm{keV} \mathrm{Hy+.} \mathrm{J.} \mathrm{Nuel.}$ Moter. 80,356 (1979) United States

Fonako, G.l Dao, S. K.! Kaningky, H. A tochnlque for deteralning the depth distribution or cavitles in Het-1 rradlated ntckel. J. Nucl. Mater. 80,373 (1979)

United states

Loom s, B. A, Berber, $\$$, B, Feduction of vald-volume Praction in Nib by Fi ailoying. J. Nucl. Mater. 80 , 383 (1979)

United 3 tates

Nasu, S.; Shlozava, K.; Ku ragaua, T, Channelling

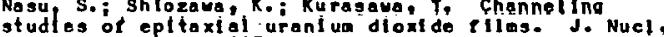
Hater. $80,3 \dot{B}$ (1979)

HIson, K. L.: Baskes, M. I. EErratum Deutertuon trapplng in Irradiat ed 316 etalnlene oteel [J. Nuol. mater. 76-77, 291 (1978)] J. Nuel. Mater. 80, 393 (1979)

Untted states

Kaul, R. D. Measurement of excltation cross gectlon of the 6 3p, state of $\mathrm{Hg}$ using self-eallbrating apparatus. J. Opt. Soc. Ann. 69, 150 (1579) United states

Letlkhov, V. S.: Minogin, V. G, Coolithg, trapping, and storage or atong by resonant laser flelds. J. Opt. suce Aus. is; 4 ij (1979) Soviet Unlon

Mendas, I. Varlatlonal principle equival ent to the

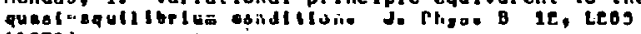
(1979) Yugoalavin

Hanssen, J.: MeCarroll, R.; Valiron, P. Mode potential calculations of the Ha-He oystea. J. Phys. B 12, 899 (1979) France

Souza, H. D.: Kuear, A Elactron opt n couplings in lon-atos colilislons. J. Phys. B 12, 909 (1979) Indi: 


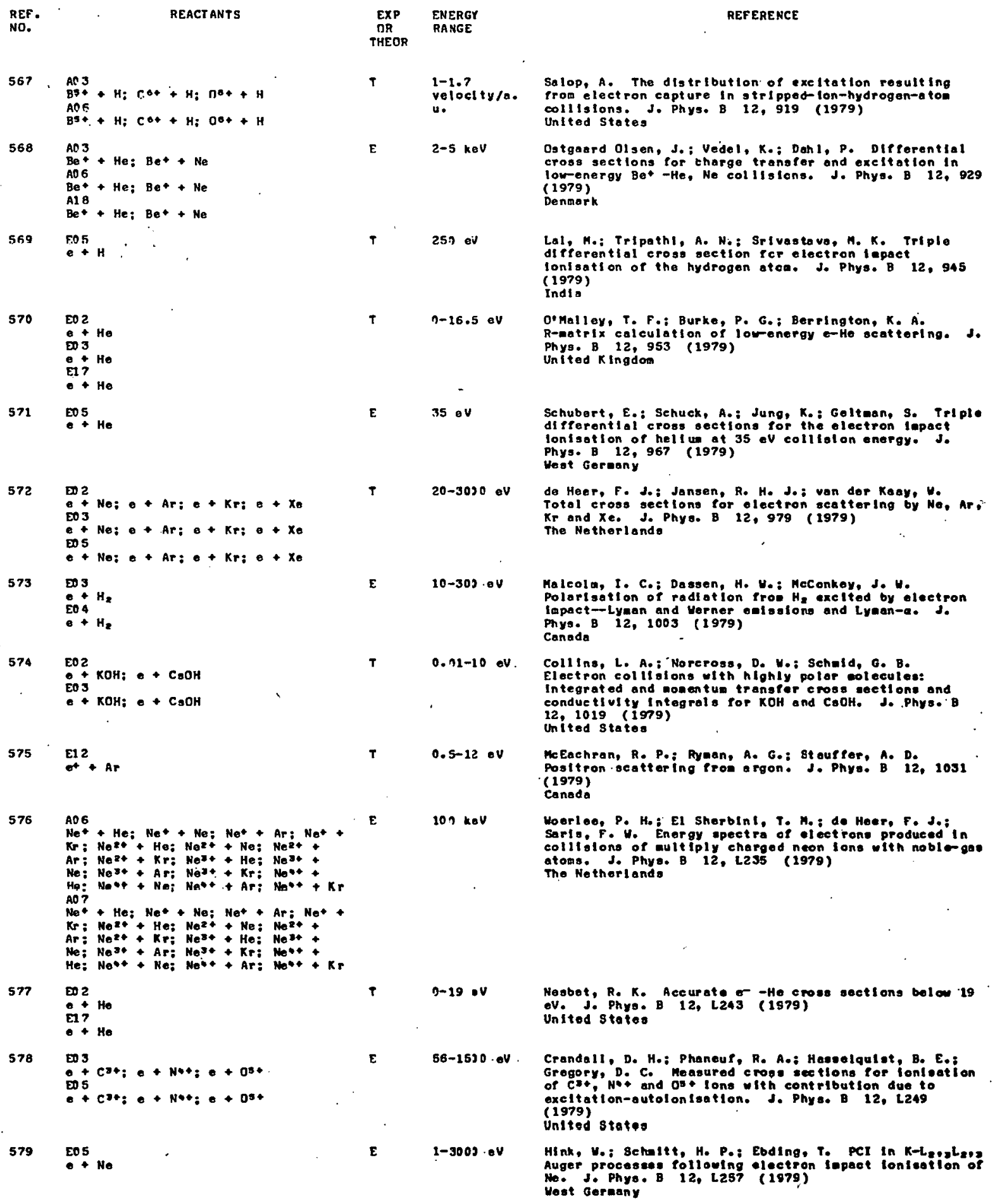




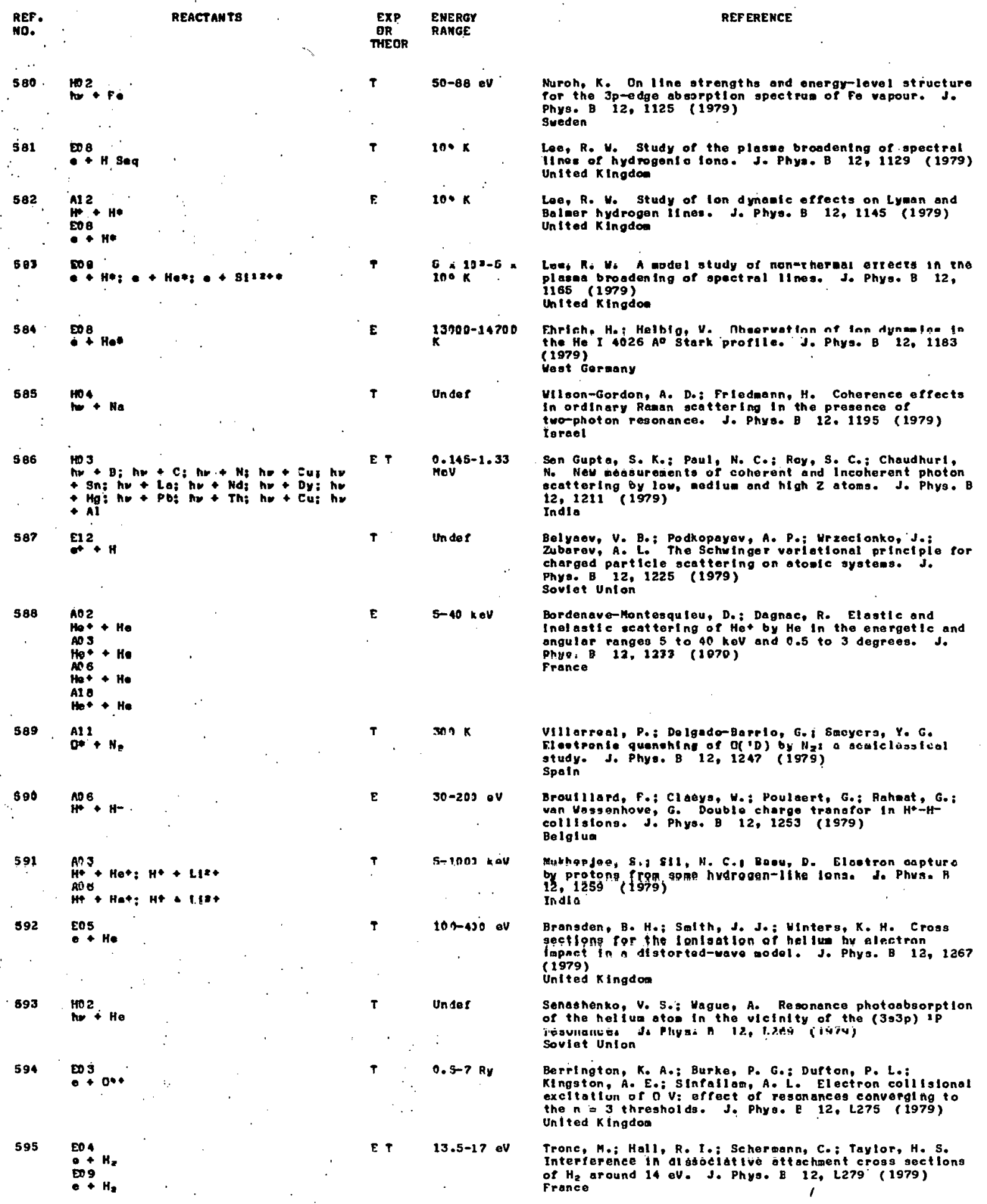




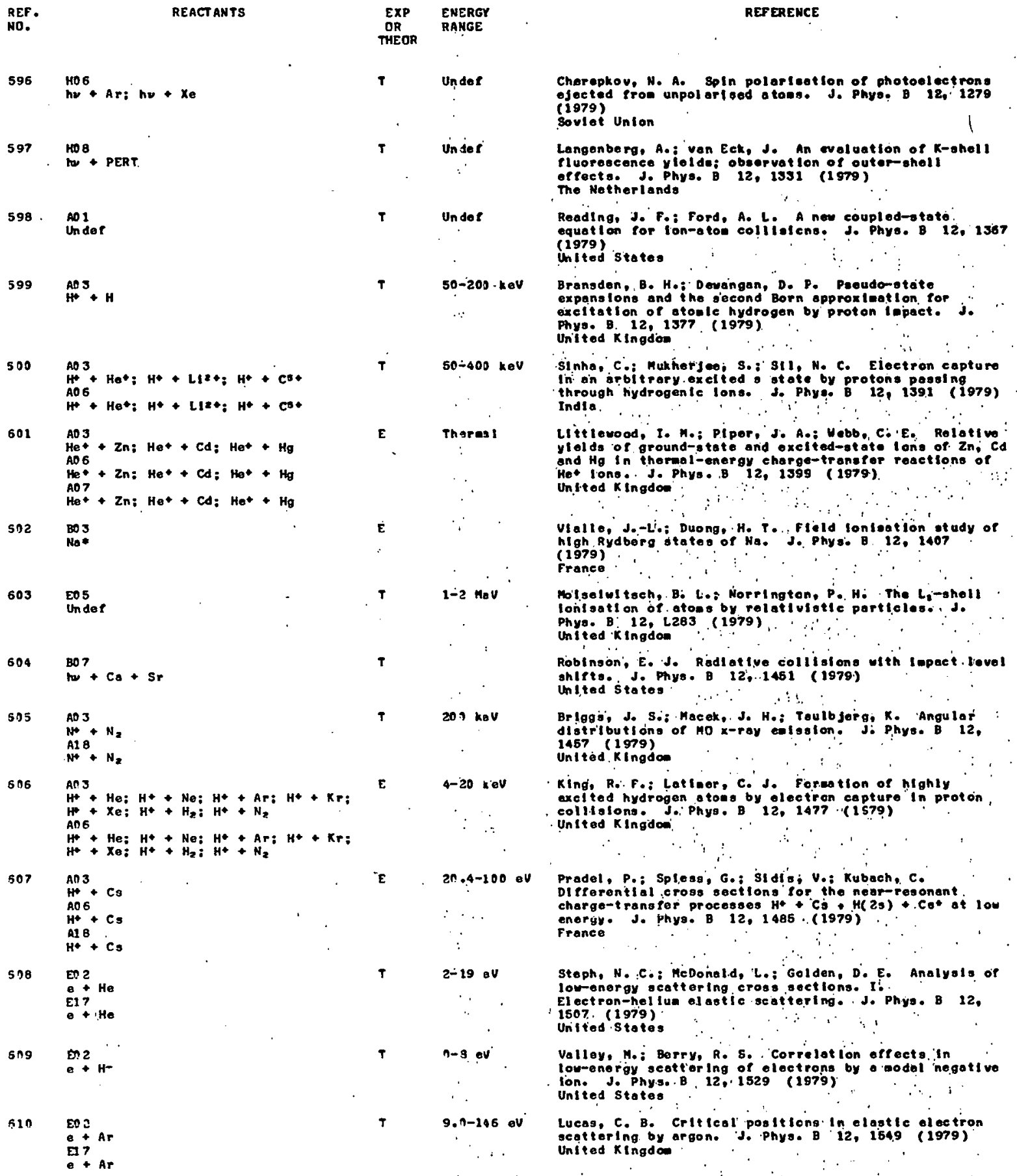




\begin{tabular}{|c|c|c|c|c|}
\hline & & : & 48 & \\
\hline $\begin{array}{l}\text { REF. } \\
\text { NO. }\end{array}$ & REACTANTS & $\begin{array}{l}\text { FXP } \\
\text { DR } \\
\text { THE.OR }\end{array}$ & $\begin{array}{l}\text { ENERGY } \\
\text { RAVGE }\end{array}$ & REF ERENCE \\
\hline 611 & 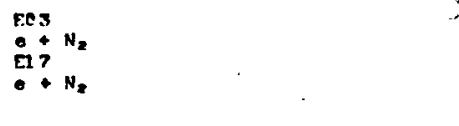 & $\varepsilon$ & $0.5-100 \mathrm{ev}$ & 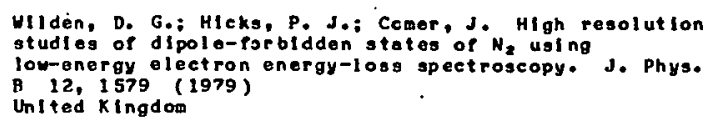 \\
\hline 612 & 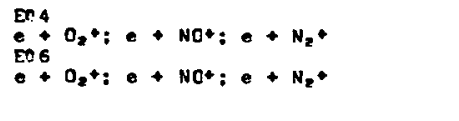 & E & $0.367-1$ ev & 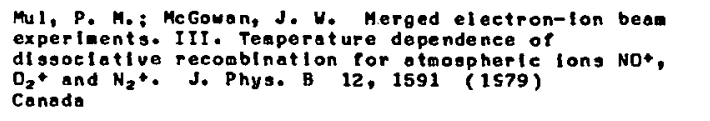 \\
\hline 613 & $F_{0+12}^{2}+H$ & $T$ & $8.7-2300 \mathrm{eV}$ & 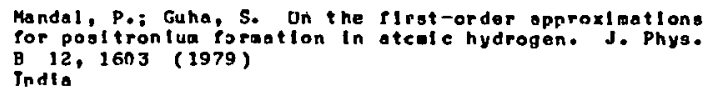 \\
\hline 614 & $\begin{array}{l}\text { Gn. } 6 \\
\text { Het+ }+ \text { He }\end{array}$ & $T$ & $77-294 \mathrm{~K}$ & 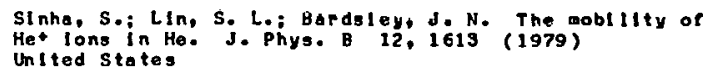 \\
\hline 515 & $\mathrm{DO} 6$ - Mos, & E & $151 \mathrm{ov}$ & 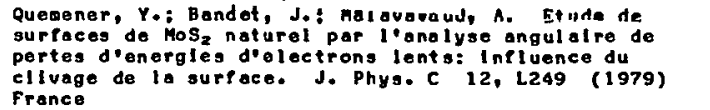 \\
\hline 616 & $\begin{array}{l}\mathrm{CO} 3 \mathrm{CO}_{2}+\mathrm{N}_{2}+\mathrm{He} \\
\mathrm{Crg} \mathrm{CO}_{2}+\mathrm{N}_{2}+\mathrm{He} \\
\mathrm{G}+\mathrm{CO}_{2}+\mathrm{CO}_{2}+\mathrm{N}_{2}+\mathrm{He}\end{array}$ & E T & $\begin{array}{l}1-1000 \\
e m^{-1} \\
\text { Torr }\end{array}$ & 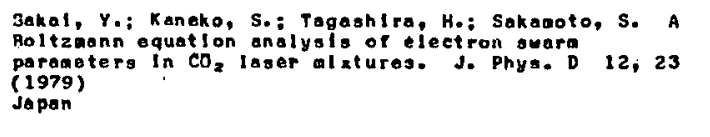 \\
\hline 617 & $\begin{array}{l}\text { D12 } \\
\text { Art }^{+}+S 1\end{array}$ & 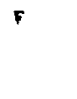 & $50-50) \mathrm{kaV}$ & 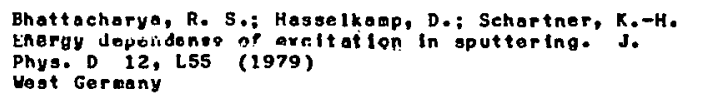 \\
\hline 818 & $\stackrel{\mathrm{Ong}}{\mathrm{CO}}$ & E & $14-5650$ Td & 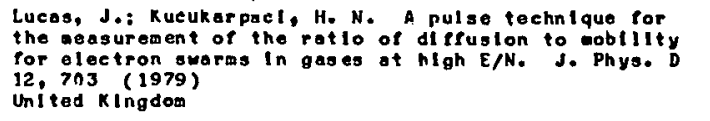 \\
\hline 619 & $\begin{array}{l}C D 2 \\
B+C ; 0+A I N \\
0 \\
0+C ; 0+A I N\end{array}$ & E & $80 \mathrm{keV}$ & 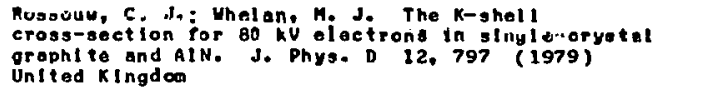 \\
\hline \$2.0 & 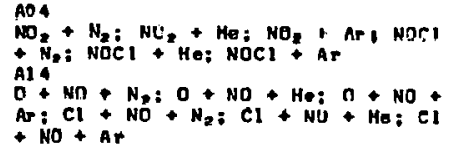 & E & $217-2506 k$ & 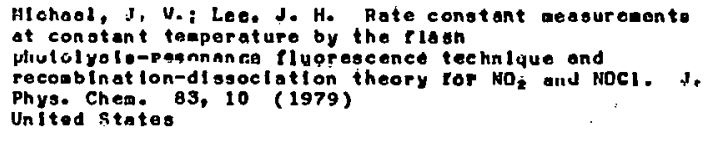 \\
\hline 621 & $\mathrm{OH}^{\mathrm{O}}+\mathrm{H}_{8}: \widehat{\mathrm{OH}}+\mathrm{CO}: \mathrm{OH}+\mathrm{OH}$ & E & $250-2000 \mathrm{~K}$ & 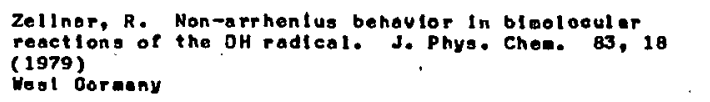 \\
\hline 622 & $\mathrm{HI}_{\mathrm{H}}^{\mathrm{A}} \bullet \mathrm{N}_{2} \mathrm{O}$ & E & $1473-2710 \mathrm{~K}$ & $\begin{array}{l}\text { Glass, G. P.: Quy, R. B. Moasurewent of high } \\
\text { temperature rate constants using d dischargo tlow shock } \\
\text { tube. Jo Phys. cheme B3, so (1979) } \\
\text { United States }\end{array}$ \\
\hline 623 & $\mathrm{H}_{4}+\mathrm{Cl}_{1}: \mathrm{H}+\mathrm{OCl}$ & E & $200-1200 \mathrm{~K}$ & 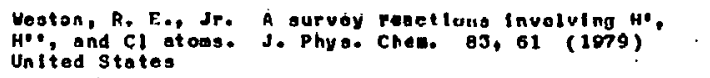 \\
\hline 624 & $\begin{array}{l}\text { Alf } \\
\text { Ald }+\mathrm{Cl} \\
\mathrm{Cl}+\mathrm{HCl}\end{array}$ & E T & $298 \mathrm{~K}$ & 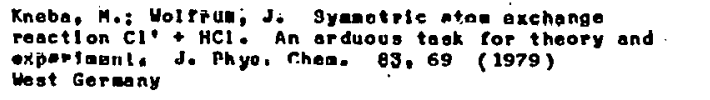 \\
\hline 625 & $F_{z}+H: F_{2}+D ; F_{2}+T$ & $i^{\top}$ & $300-900 \cdot K$ & 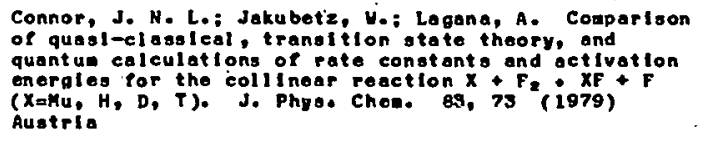 \\
\hline
\end{tabular}




\begin{tabular}{|c|c|c|c|c|}
\hline $\begin{array}{l}\text { REF. } \\
\text { NO. }\end{array}$ & REACTANTS & $\begin{array}{l}\text { EXP } \\
\text { OR } \\
\text { THIOR }\end{array}$ & $\begin{array}{l}\text { ENERGY } \\
\text { RANGE }\end{array}$ & PEF EREKCE \\
\hline 625 & $\stackrel{\mathrm{A}}{\mathrm{F}}+\mathrm{H}_{2}: \mathrm{Cl}+\mathrm{H}_{2}: \mathrm{O}+\mathrm{H}_{2}$ & 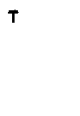 & $2 n 3-1300 x$ & 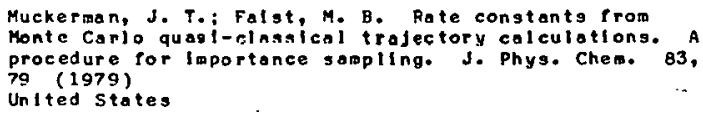 \\
\hline 627 & $\mathrm{AH}_{\mathrm{OH}}^{\mathrm{A}}+\mathrm{CO}$ & T & $303-1200 \mathrm{~K}$ & $\begin{array}{l}\text { Golden, D. M. Experimental ond theoretical examples of } \\
\text { the value and IImitations of transition state theory. } \\
\text { J. Phys. Chem. B3, Ing (1979) } \\
\text { Untted States }\end{array}$ \\
\hline 628 & $\begin{array}{l}\mathrm{AC}_{4} \\
\mathrm{H}_{2}+\mathrm{He} \\
\mathrm{Al}_{\mathrm{N}} \text {. He } \\
\mathrm{H}_{2}+\mathrm{He}\end{array}$ & $T$ & $1000-10000$ & $\begin{array}{l}\text { Dove, J. E.: Raynor, S. An ab initio ealculatlon of } \\
\text { the rate if vibratlonal relaxatlon and thermal } \\
\text { dissoclation of hydrogen by hellum at high } \\
\text { temperatures. I. Phys. Chem. BJ, } 127 \text { (1979) } \\
\text { Canada }\end{array}$ \\
\hline 529 & $\begin{array}{l}\mathrm{AO}_{4} \\
\mathrm{H}_{2}\end{array}+\mathrm{Ar}$ & $\mathbf{T}$ & $2000-5000 \mathrm{~K}$ & 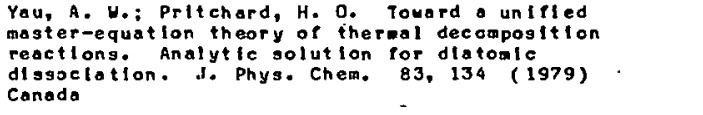 \\
\hline 630 & $\mathrm{H}+\mathrm{H}_{2}: \mathrm{F}+\mathrm{H}_{2}: \mathrm{F}+\mathrm{D}_{2}$ & $\mathbf{T}$ & $200-1000 \mathrm{~K}$ & $\begin{array}{l}\text { Kuppermann, A. An exact quantuw mechanical trangltion } \\
\text { state theory. 1. An overvilew. J. Phys. Chem. 83, } 171 \\
\text { (1979) } \\
\text { United States }\end{array}$ \\
\hline 631 & $\begin{array}{l}\mathrm{Al} 4 \\
\mathrm{H}+\mathrm{H}_{2} ; \mathrm{Cl}+\mathrm{H}_{2}: \mathrm{F}+\mathrm{H}_{2} ; \mathrm{I}+\mathrm{H}_{2} ; \mathrm{H}\end{array}$ & $\mathbf{T}$ & $300-1500 \mathrm{~K}$ & $\begin{array}{l}\text { Truhlar, D. G. Ac curacy of trajectory calculatlons and } \\
\text { trangltion state theory for therad rate constants of } \\
\text { atom tronster reactiong. J. Phyg. Chem. B3, } 188 \\
\text { (1979) } \\
\text { Untted States }\end{array}$ \\
\hline 632 & $\mathrm{H}_{\mathrm{H}}^{\mathrm{I}} 4 \mathrm{H}_{2}: \mathrm{Cl}_{1}+\mathrm{H}_{2}: \mathrm{D}+\mathrm{D}_{2} ; \mathrm{Cl}_{1}+\mathrm{T}_{2}$ & $\mathbf{T}$ & $300-1500 \mathrm{~K}$ & $\begin{array}{l}\text { Garrett, B. C.: Truhlor, D. G. Accuracy. of tunneling } \\
\text { corretions to transitionstate theory for thermal rate } \\
\text { constants of atom transfer reactions. J. Phys. Chem. } \\
83,200 \text { (1979) } \\
\text { United States }\end{array}$ \\
\hline 633 & 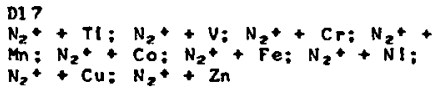 & $\boldsymbol{E}$ & $30-3000 \mathrm{ev}$ & $\begin{array}{l}\text { Lancagter, G. M.: Rabalais, J. W. Chemical reactions } \\
\text { of Nz+ Ion beams with (irst-row transition metals. J. } \\
\text { Phys. Chem. B3, } 209 \text { (1979) } \\
\text { United States }\end{array}$ \\
\hline 634 & $\begin{array}{l}\text { AI } 4 \\
\mathrm{H}+\mathrm{O}\end{array}$ & $E$ & $195-424 K$ & $\begin{array}{l}\text { Keyger, L. F. Absolute rate constant and temperature } \\
\text { dependence of the reaction between hydrogen (2S) atoms } \\
\text { and ozone. J. Phys. Chem. } 83,645 \text { (1979) } \\
\text { United States }\end{array}$ \\
\hline 635 & $\mathrm{O}+\mathrm{N}_{2}: \mathrm{O}+\mathrm{N}_{2} ; \mathrm{H}+\mathrm{NO}: \mathrm{D}_{2}+\mathrm{Ar}$ & E & $400-4300 k$ & 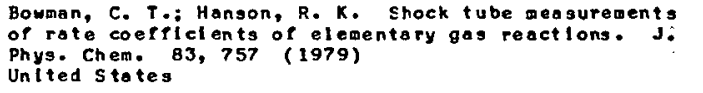 \\
\hline 636 & $\mathrm{Al}_{2}^{4}+\mathrm{O}_{3} ; \mathrm{HO}_{2}+\mathrm{OH}$ & $E$ & $\begin{array}{l}-43-+61 \\
{ }^{\circ} \mathrm{C}\end{array}$ & 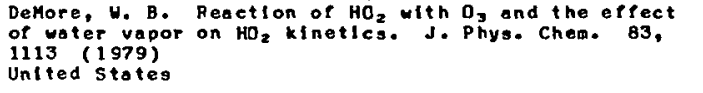 \\
\hline 637 & 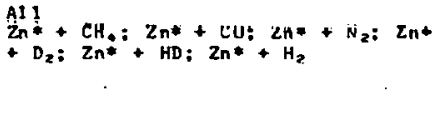 & $\mathbf{E}$ & $300 \mathrm{~K}$ & 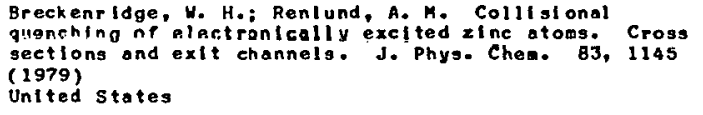 \\
\hline 638 & $\mathrm{Na}^{\mathrm{A} 7}+\mathrm{H}^{+} ; \mathrm{K}+\mathrm{H}^{+} ; \mathrm{Na}+\mathrm{H} ; \mathrm{K}+\mathrm{H}$ & $T$ & Unde $f$ & $\begin{array}{l}\text { Mellus, C. F.; Nunrleh, R. U.: Truhlar, D. G. } \\
\text { Calculations of potential energy curves Por the ground } \\
\text { states of NaHo ond KH* and II gtates of NaH and KH. J. } \\
\text { Phys. Chem. } 83,1221 \text { (1979) } \\
\text { United States }\end{array}$ \\
\hline 639 & $\begin{array}{l}\mathrm{AO} 7 \\
\mathrm{CH}+\mathrm{O} \\
\mathrm{CH}+\mathrm{O}+\mathrm{O}\end{array}$ & $\mathbf{E}$ & $295 \mathrm{~K}$ & 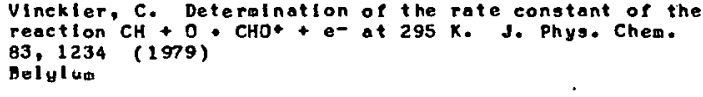 \\
\hline 640 & $\mathrm{HF}^{\mathrm{AO}}+\mathrm{HF}$ & $\mathbf{T}$ & $8000-1 / \mathrm{cm}$ & 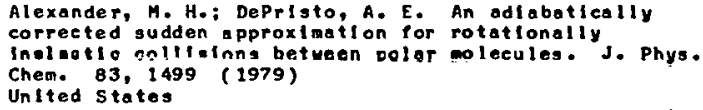 \\
\hline
\end{tabular}




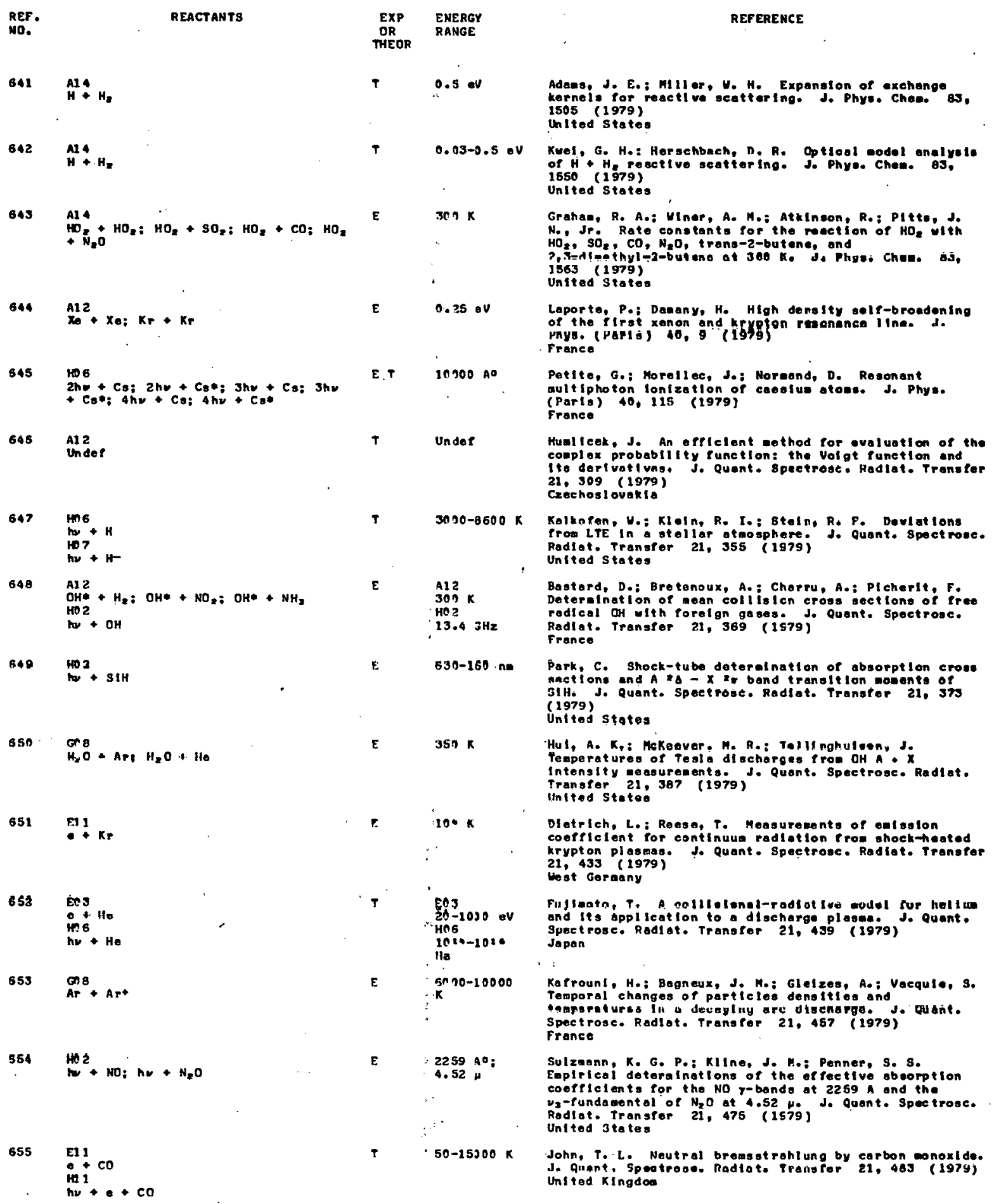




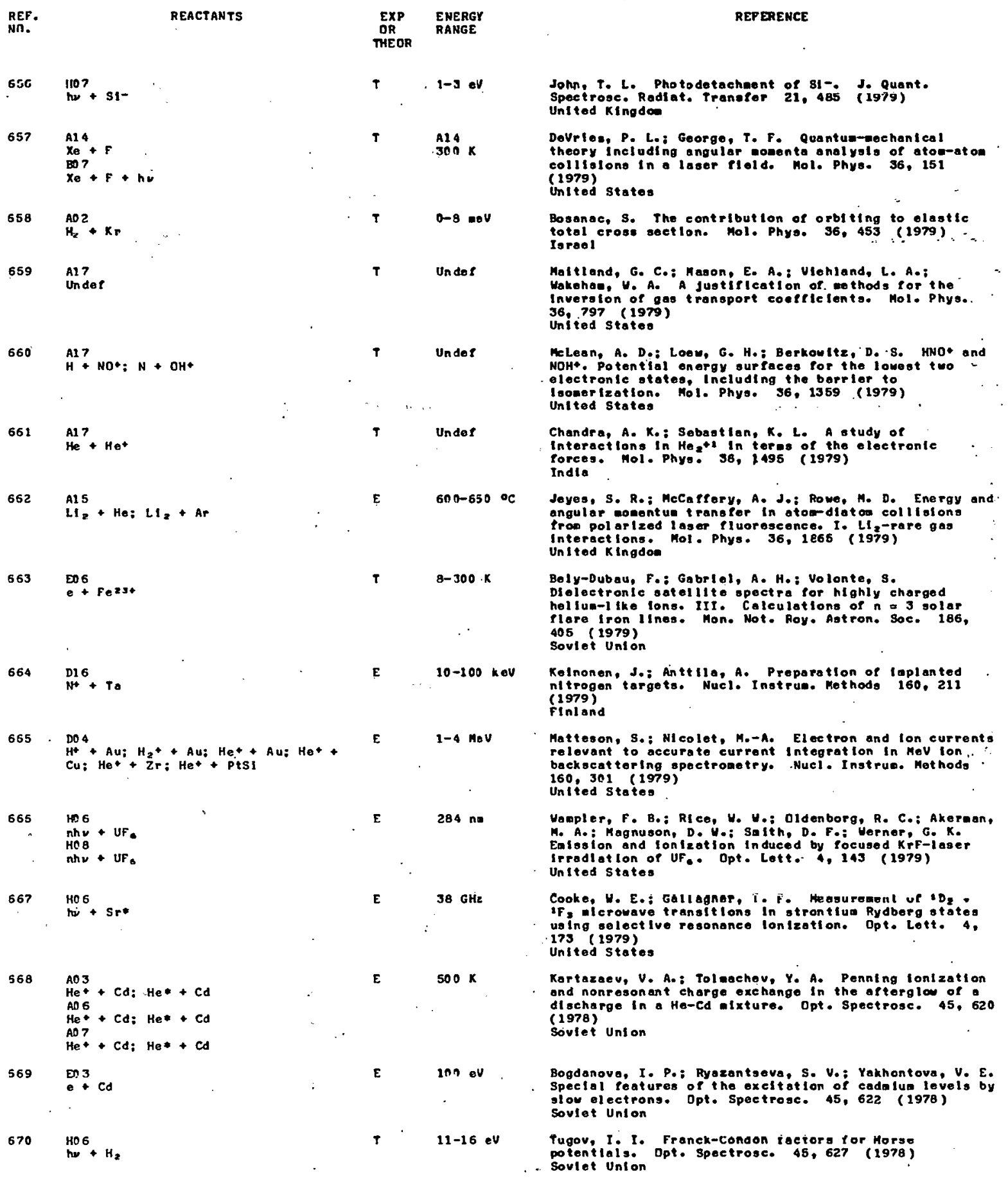




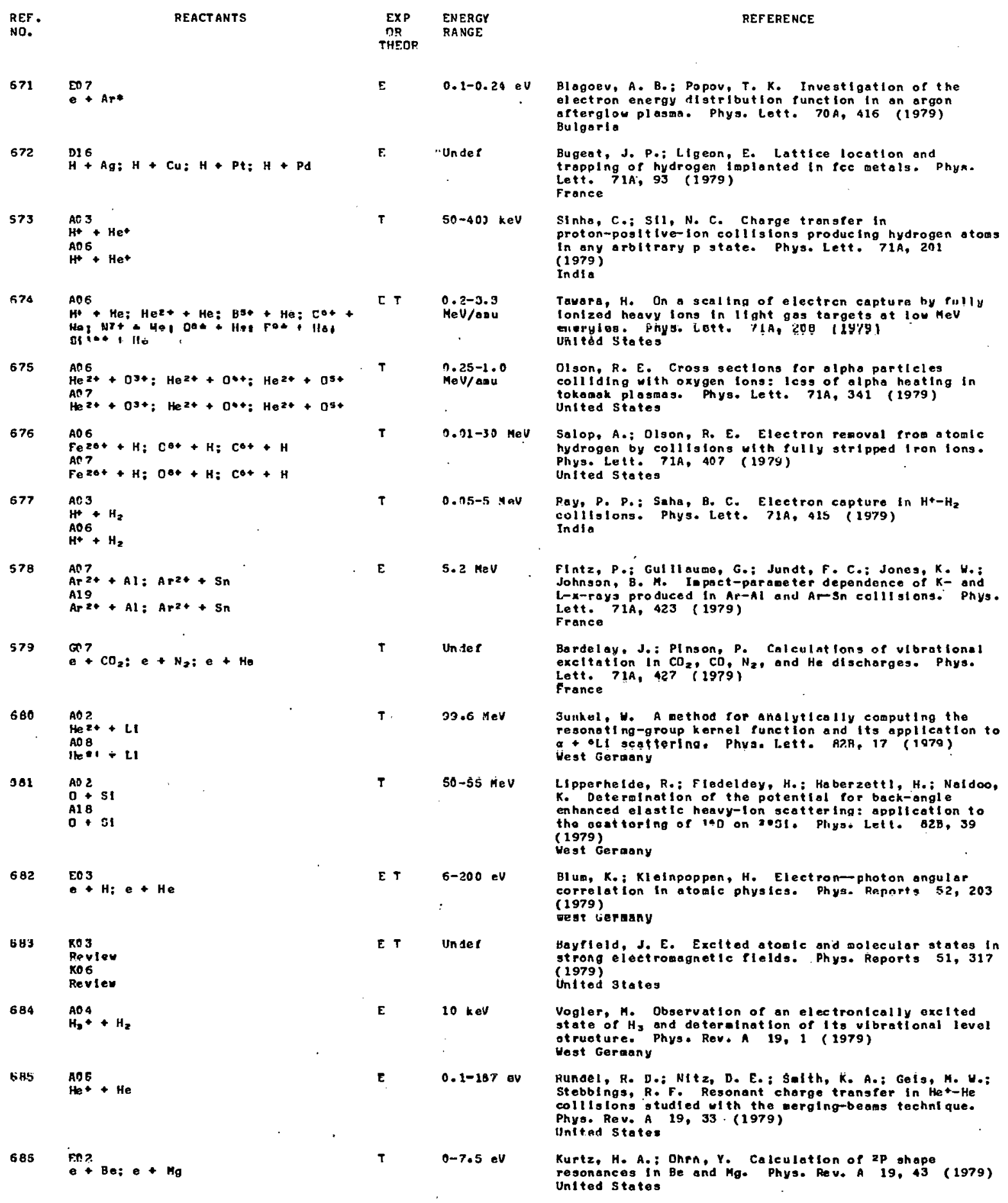




\begin{tabular}{|c|c|c|c|c|}
\hline $\begin{array}{l}\text { REF. } \\
\text { No. }\end{array}$ & REACT ANTS & $\begin{array}{l}\text { EXP } \\
\text { OR } \\
\text { THEOR }\end{array}$ & $\begin{array}{l}\text { ENERGY } \\
\text { RANGE }\end{array}$ & REF ERENCE \\
\hline 587 & $\begin{array}{l}\text { A0? } \\
\text { H }+ \text { Ar } \\
\text { A16 } \\
H+\text { Ar }\end{array}$ & E & $0.5 \mathrm{~m} \in \mathrm{V}$ & 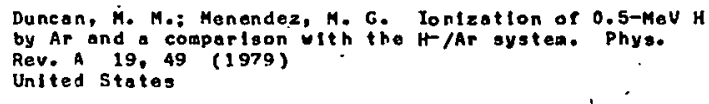 \\
\hline 588 & 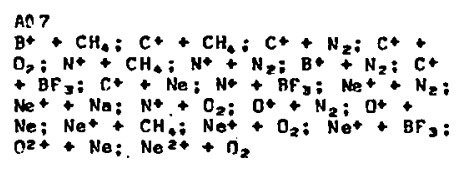 & $E$ & $40-603 k \in V$ & 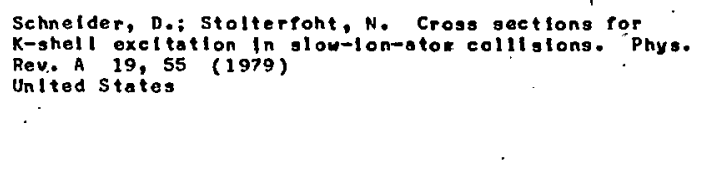 \\
\hline 689 & $\mathrm{H}^{+} 5+\mathrm{H}^{2} \mathrm{H}^{+}+\mathrm{He} ; \mathrm{H}^{+}+\mathrm{Ar}: \mathrm{He}^{2+}+\mathrm{He}$ & $T$ & $0-3.0 \mathrm{MeV}$ & 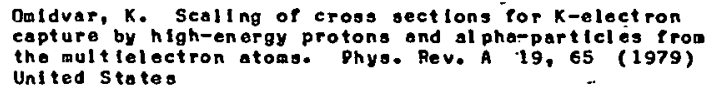 \\
\hline 590 & $e^{200} 3+0 \times 4$ & $\mathrm{~T}$ & $579-650$ ev & 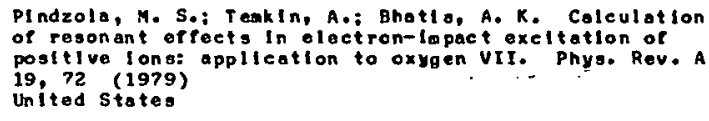 \\
\hline 691 & $\mathrm{NI}_{\mathrm{N}}^{\mathrm{Al}}+\mathrm{Mn}: \mathrm{N1}++\mathrm{Sn}: \mathrm{N}_{1}++\mathrm{Pb}$ & E & $45-133 \mathrm{nev}$ & 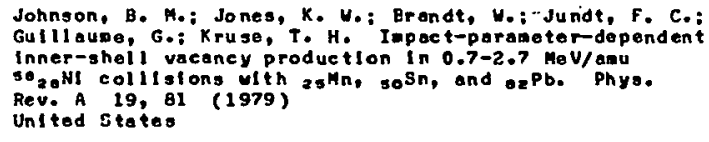 \\
\hline 592 & $\operatorname{sen}_{2}+\mathrm{Not}: 0+02$ & 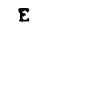 & $30 \mathrm{eV}$ & 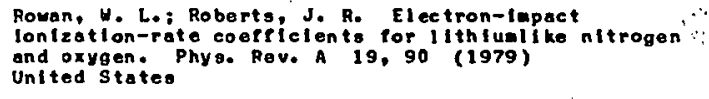 \\
\hline 693 & $\begin{array}{l}\text { Bo7 } \\
\text { Under }\end{array}$ & 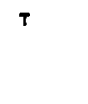 & & 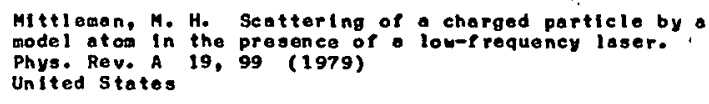 \\
\hline 694 & $\begin{array}{l}\text { ED } 1 \\
\text { Under }\end{array}$ & $T$ & Untef & $\begin{array}{l}\text { Kaldor, U. L2-basls-set ineory of electron-molecule } \\
\text { scattering uith vibratlonal and rotatlonal coupling. } \\
\text { phys. Rev. A } 19,105 \text { (1979) } \\
\text { lorael }\end{array}$ \\
\hline 695 & 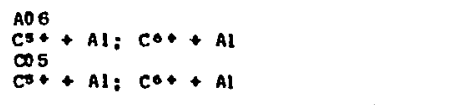 & $\varepsilon$ & $3-36 \mathrm{seV}$ & 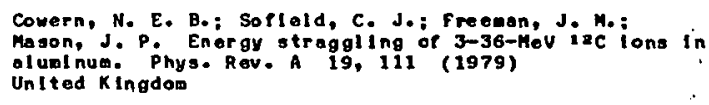 \\
\hline 696 & 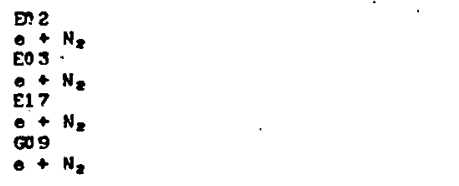 & $\boldsymbol{T}$ & $50-500$ eV & 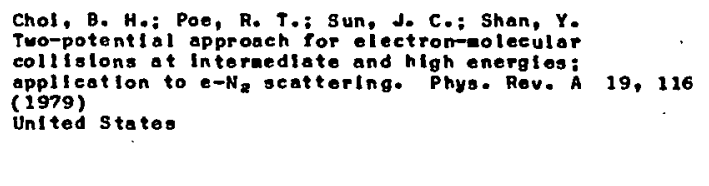 \\
\hline 697 & $\begin{array}{l}\text { AO } 2 \\
H+H y \\
A 18 \\
H+H g\end{array}$ & $T$ & $0.1-1.25 .0$ & 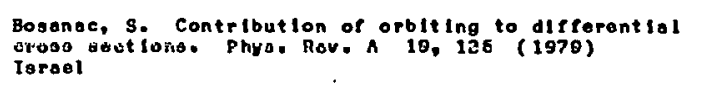 \\
\hline 698 & $\begin{array}{l}807 \\
\text { Undor }\end{array}$ & $T$ & ." & $\begin{array}{l}\text { Mittleasn, M. H. Potentlal acottering of eharged } \\
\text { partleleg in the pleld of a low-frequeney laser. phys. } \\
\text { Rav. A } 19 \text {, 134 (1979) } \\
\text { United states }\end{array}$ \\
\hline 699 & hos +9 & $\top$ & & 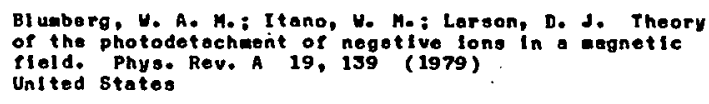 \\
\hline 700 & $\mathrm{HOF}_{\mathrm{H}}+\mathrm{FO}$ & $\boldsymbol{E}$ & $4880 \cdot A 0$ & 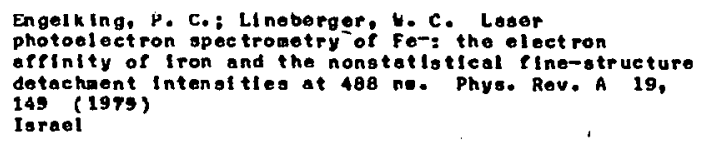 \\
\hline 701 & $\mathrm{All}_{0}=\mathrm{He}$ & E & $292 \times$ & 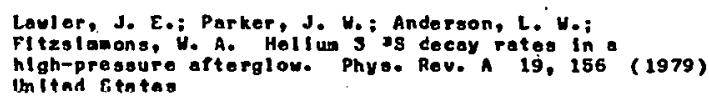 \\
\hline
\end{tabular}




\begin{tabular}{|c|c|c|c|}
\hline $\begin{array}{l}\text { REF. } \\
\text { NO. }\end{array}$ & REACTANTS & $\begin{array}{l}\text { EXP } \\
\text { OR } \\
\text { THEOR }\end{array}$ & $\begin{array}{l}\text { ENERGY } \\
\text { RANGE }\end{array}$ \\
\hline 702 & $\begin{array}{l}A 12+A r \\
A r+A r \\
B O S+A r+A r\end{array}$ & T & Under \\
\hline 203 & 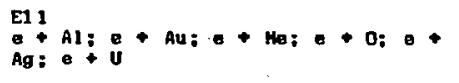 & $\begin{array}{l}\top \\
\end{array}$ & $1-500 k \in \mathrm{V}$ \\
\hline 704 & $\underset{\mathrm{Ar}^{+}}{\mathrm{AOS}}+\mathrm{H}_{2}$ & E & $0.5-43=v$ \\
\hline 705 & $\begin{array}{l}\text { Ho6 } \\
3 h v+C s: 4 h v+C s\end{array}$ & $T$ & $e_{e m-1}^{9443-14414}$ \\
\hline 705 & $\begin{array}{l}\text { Hor } \\
\text { Under }\end{array}$ & T & Under \\
\hline 707 & $\begin{array}{l}\mathrm{Al} ? \\
\mathrm{H}\end{array}$ & $\mathbf{T}$ & Under \\
\hline 708 & $\begin{array}{l}\text { Ao } 1 \\
\text { Unde? }\end{array}$ & $\mathbf{T}$ & Under \\
\hline 709 & 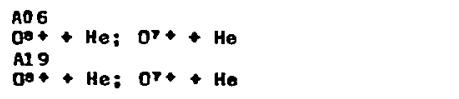 & $\varepsilon$ & $12-22 \mathrm{MOV}$ \\
\hline 710 & 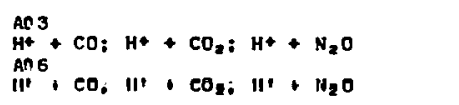 & $\varepsilon$ & $2.3-8.2 \mathrm{~kg}$ \\
\hline 711 & $\begin{array}{l}\mathrm{COT} \\
\mathrm{Het}+\mathrm{C} \\
\mathrm{OOA} \\
\mathrm{Pl}+\mathrm{C}\end{array}$ & $\mathbf{E}$ & $60-189 \mathrm{kel}$ \\
\hline 718 & $\begin{array}{l}\mathrm{AQP} \\
\mathrm{CI}+\mathrm{He}\end{array}$ & e & $10-50 \quad k=V$ \\
\hline 713 & $\mathrm{Ar}_{\mathrm{Ar}}^{\mathrm{AO}}+\mathrm{Ar}: \mathrm{Kr}+\mathrm{Kr}$ & E. & $0-400 \mathrm{eV}$ \\
\hline 714 & 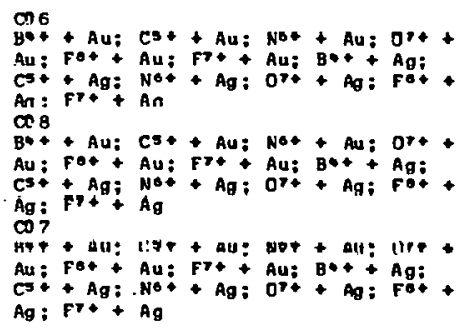 & ET & $31-44 \mathrm{keV}$ \\
\hline 715 & 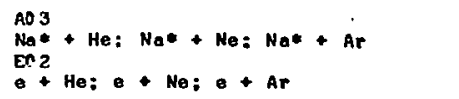 & $T$ & $0-9.8=v$ \\
\hline
\end{tabular}

REFERENCE

Balucanl, U.: TognettI, V.: Vallauri, R. LI ne-shape theory and aolecular dynasico in colitolon-i nduced light scattering. Phyo. Rev. A 19, 177 (1979) Itoly

Toeng, H. K.: Pratt, R. H.; Lee, C. M. Electron bremsotrahlung ongular distritutlons in the 1-500 keV energy range: Phys. Rev. A 15, 187 (1979) United states

Brandt, D.: Ottinger, C. $H_{2}$ oxz (sub g)+ $b$ is (sub u) + continum enission exclted by lou-energy rare-gas lon collislons. Phys. Rev. A 19, 219 (1979) West Gerany

Gontler, Y.: Trahin, M. Theory of resonant ault Iphoton Ionization: applteation to the ceslue aton. Phys. Rev. Arance 1964 (1979)

Contria Vasconcellos, J.'I. Nonll near absorption of monochronatie radiation noar a gas-aedlua resonance. Phys. Rev. A 19, 280 (1979) BrazII

Luyckx, R.: Delbaen, $F_{P}$ : Coul on, P.: Lekkerkerker, $H$. N. Y. New bounds for Von der Vaal a coefficlents. Phys. Rev. A 19, 324 (1979) Bolglue

Fano, U. Adlabatic analys is of collislone. III Remorks on the spln model. Phys. Rev. A 19, 410 (1979) Unlted states

Ellsworth, L. D.: Doyle, B. L.: Sentebel, U.; Macdonal d, J. R. p-atate allignaent of one- and two-electron oxygen tons exclted by collislons in hellute. Phys. Rev. A 19,943 (1979) United states

Loyd, D. Ho: Dauson, H. R. Electron eapture Into the $n=3$ states of hydrogen by proten inpact on $\mathrm{CO}_{3} \mathrm{CO}_{2}$, and $\mathrm{N}_{2} \mathrm{O}$. Phys. Rev. A 19,948 (1579) Uillied. jiales

Gay. T.' J.: Berry, H. G. Temperature dependence or alignment production In He I by beam- poll excitation. Phys. Rev. A 19, 952 (1979) Uiil ted 9 tates

Elslun, 3. Bd: Schumani, 34: Vant, C. Re Hang-eloctron aspects of eolecular promotion in lon-aton collisions: production of core-exclted states of LI In Lit-Ho collisions. Phys. Rov. A 13,963 (1979)

Rothwell, H, L., Jr.: Aase, R. C.: van Zyl, B. vitravloiet radiatlion produced In low-energy Ar + Ar
and $K r+K r$ collisions. Phys. Rev. A 19,970 (1979) thited states

Moak, C. D.: Datz, S.: Crawford, O. H.: Krause, H. F.: Dittner, P. F.: Gonez del Conpo, J.: Blggerstaff, J. A.: MI ller, P. D.: Huelplund, F.: Knudsen. H. Resonant coherent excltation of channeled lons. Phys. Rov. A United seatos

HI ekman, A. P. Relatlon between lourenergy-el eetron

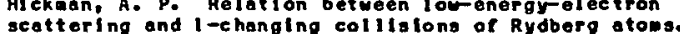
scattering and 1-ehanging col
Phys. Rev. A 19, 994 (1979) United Stotes 


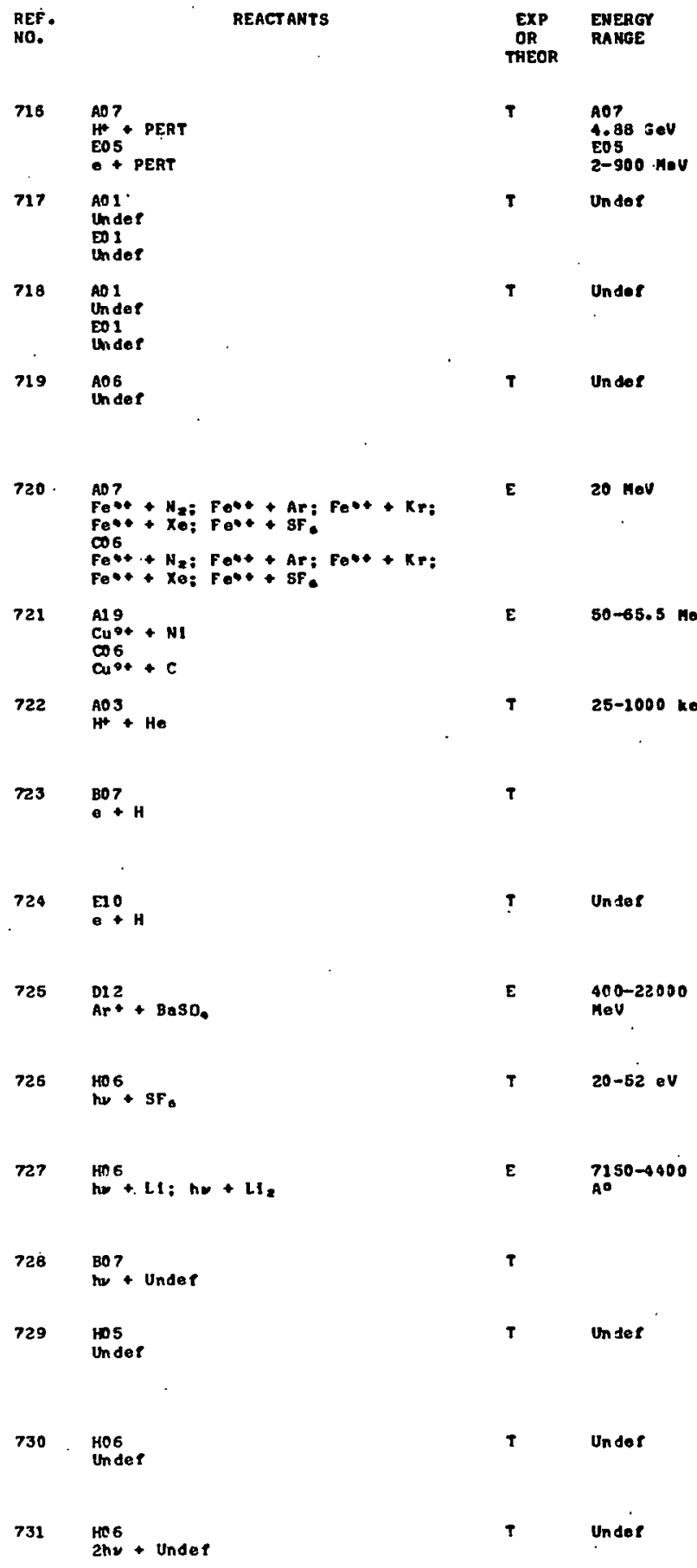

Anholt, R. Calculation of k-waeancy product Ion by rolativiotic projectllos. Physe Rev. A 19,1004 (1979)

ted stetes

Taketsuka, K.: fueno, T. Optielzed Kohn eothod for ocattering. I. Bingic-enonnel ecattering. Phys. Rev. icattering: I. 81ng

dapan

Takot anka, K., Fueno, T. Optiat eod Kohn mothod for seatterling. II. Mult Ichannel seat ter Ing. Phys. Rev. A 19, 1018 (1979)

Japan

Spruch, L.: Shakeahaft, R. Ctassleal erose sect ion for charge tronsfer via knoek-on cepture pros high-Rydberg states at asyept ot lealiy hlgh ippet velocitios. Phys. Rev. 19, 1023 (1979)

Unitied statos

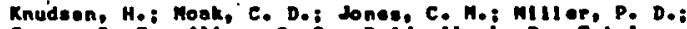
Sayer, R. D.: Alton, B. D.: Bridweil, L. B. Total ol ectron-loss cross dectlons and absolute chorge-state Ialds of $20 \rightarrow$ oU Fo lons trangeitt ed through gascous targots. Phys. Ret. A 19, 1029 (1979) United states

Annett, C. H.: Curnutte, B.; Coche, C. L. Inpact-paraneter dependence of $\mathrm{R}$-iecency product ion In CutNI collitions. Phys. Rov. A 19, 1058 (1979) Untiod states

Sur, S. K.: Mukherjee, B. C. Colculation of proton-l apoct excl tation of holl un weing the Glouber opproxisation. Physe'Rev. A 19, 1046 (1879) Indio:

Brandl. H. So; Kolller, B.Z LIne de Barros, H. G. P. Electron hydrogen-at os colilistons in the prosence of a laser. flelds. Born-Opponhel eor approximotlon. Phys. Rov. A. 19, 1068, (1979)

Kelsey, E. J, Electron ecettering fros hydrogen: collisions. In which the total epin quantom nubbers change. Phyd. Rev. A. 19, 1062 (1979) Unitad states

Ahlen, S. P.: Solason, H. H. Optl cal Cerenko radiatión Proo ilcroscople Bes0, gralns. Phys. Rev. A 19,1084 ( 1979 ) United states

Levinson, H. Je: Gustafssón, T. : Soven, P.' Calculat ed photolonization crost aect lons. Ror. 8f.- Phys. Rev. A 19,1089 (1979) united states.

Koch, H. E. Collins, C. B. Space-charge Ion' detection of cultiphotion absorption phenomena In iltht un vapor. Phys. Rev. A 19, 1098 (1979)

Yeh, S.; Borman, P. R. Theory of colllolonally al dod radiativo oxeltation.: Physe. Rev. A 19, 1106 (1979) United 's te tes

Lov, A. M. F. Losier-Anduced molecular predissoctation by stiaulated ingle-photon or witiphoton absorption or enfisston of Infrared photons. Phys. Rev. A 19, 1117 (1979)

zolier, P. Reoonant aultiphoton Ionlzation by pinito-tanduldeh chaotic plelde. Phys. Rev. A 19, 1151 (1979)

Eberly, J. H. OHeli, 8. V: Coherenee vorisus incoherence: tinetwo-photon ion Ization. Phys. Fev, A 19, 1161 (1979) Unlted States 


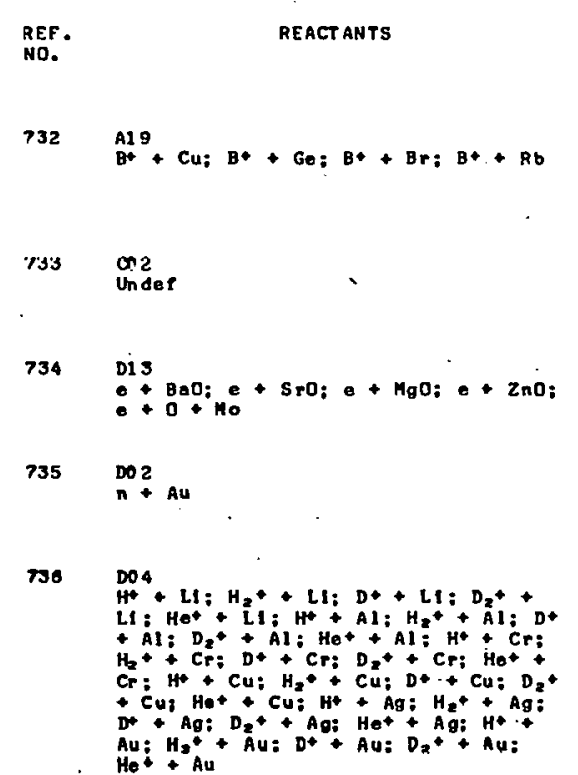

EXP ENERG

EXP ENERG

THEOR

$4-38$ yeV

1

Under

Under

E $\quad 2-60 \mathrm{kOV}$

$10-303 \cdot \mathrm{eV}$

(n)

(n)

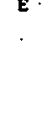

$30-6000-$ kev/anu
REFERENCE

Mehta, R.: MeDanlel, F. D.: Duggan, J. L.: MIllor, P. D. X-sheil lonlzation of el enents zic to mRb for D. 4-3.8-HeV/anu 10 B-ion boubardnent. Phys. Rev. A 19,1363 (1979) United states Drlce, D. K. Erratue Three-parametor rormula for the
electronle stopplng eros section [Phys. Rev. A 6,1791 (1972)]. Phys. Rev. A 19, 1367 (1979) , United States

Phlll ipg, J. C. Slow-el ectron-atl aulated desorptlon

Pros oxldes: theoretleal oodels of se lected
experinenta. Phys. Rev. B 19, 47 (1979) United Statea

KIrk, M. A.: Conner, R. A.: Hoznlak, D. G.: Groenwood, L. R.: Haleulckl, R. L.; Helnrich, R. R. Sputtering of

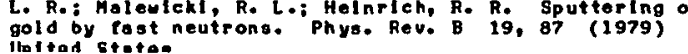

Boraglola, R. A.; Aloneo, E. V.: Oliva Florlo, A. Electron eal solion from clean motal aurfaces induced by lowenergy IIght Ions. Phys. Rev. B 19, 121 (1979) Argent In a 


\begin{tabular}{|c|c|c|c|c|}
\hline & & 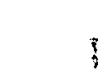 & 57 & \\
\hline $\begin{array}{l}\text { REF. } \\
\text { NO. }\end{array}$ & REACTANTS & $\begin{array}{c}\operatorname{EXP} \\
\text { OR } \\
\text { THEOR }\end{array}$ & $\begin{array}{l}\text { ENERGY } \\
\text { RANGE }\end{array}$ & REF ERENCE \\
\hline 747 & $\mathrm{HO}_{4}+\mathrm{He}^{*}$ & $E$ & 4 & $\begin{array}{l}\text { Panock, R.: Rogenbluh, M.: Lax, B.: Mlller, T. A. } \\
\text { Laser-driven forbldden trangitions to high-L itates in } \\
\text { He. Phys. Rev. Lett. 42, } 172 \text { (1979) } \\
\text { United States }\end{array}$ \\
\hline 748 & 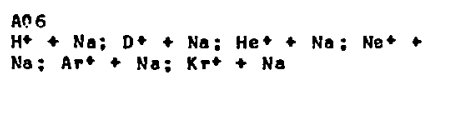 & $\varepsilon$ & $309 x$ & 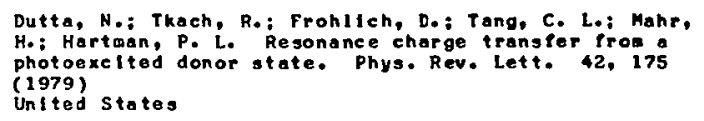 \\
\hline 799 & $\begin{array}{l}\text { Dog } \\
\mathrm{OH} \mathrm{H}^{\circ}+\mathrm{C}\end{array}$ & E & $10 \mathrm{MeV}$ & 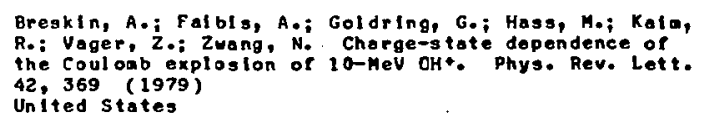 \\
\hline 750 & 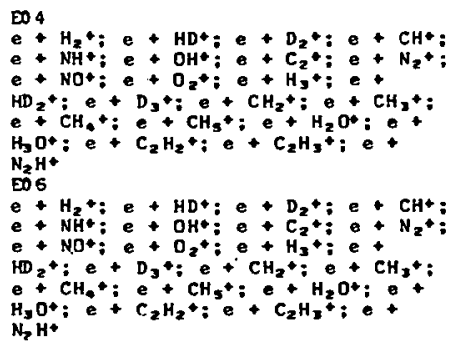 & $\varepsilon$ & $0-0.1=V$ & 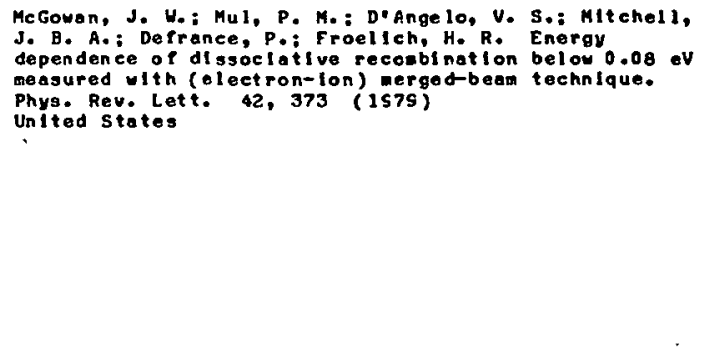 \\
\hline 751 & 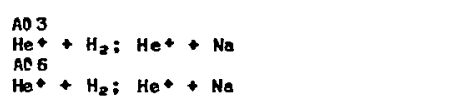 & $\varepsilon$ & $25-90 \mathrm{keV}$ & $\begin{array}{l}\text { Pedersen, E. H. Matastablo-atos popul ation of rast, } \\
\text { neutral heli un beals. Phys. Rev. Lett. } 42,440 \\
\text { (1979) } \\
\text { Denmark }\end{array}$ \\
\hline 752 & $\mathrm{CO}_{\mathrm{He}}^{\mathrm{CO}}+\mathrm{H}$ & E & $3 n 0-475 \mathrm{ev}$ & 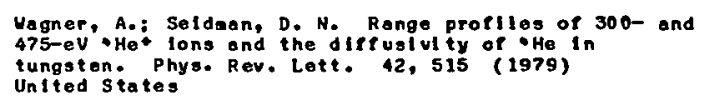 \\
\hline 753 & $\begin{array}{l}\mathrm{Ae} 3 \\
\mathrm{H}^{+}+\mathrm{He}: \mathrm{H}^{+}+\mathrm{Ar}: \mathrm{H}^{+}+\mathrm{O}_{2} \\
\mathrm{AOS}^{+}+\mathrm{He}: \mathrm{H}^{+}+\mathrm{Ar} ; \mathrm{H}^{+}+\mathrm{O}_{2}\end{array}$ & E & 2-3 a.u. & 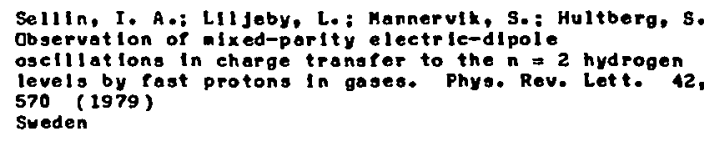 \\
\hline 754 & $\begin{array}{l}\text { A1 } \\
K+K \\
\text { Ho } 2 \text { K } \\
\text { hy }+K_{2}\end{array}$ & $\varepsilon$ & $1.6-1.1 \mu \mathrm{s}$ & 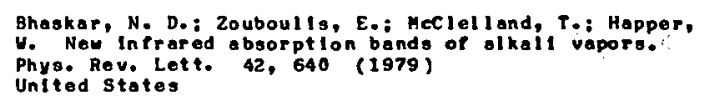 \\
\hline 755 & $\mathrm{AO}_{\mathrm{He}}^{+}+\mathrm{H}$ & $T$ & $25-3000 \mathrm{keV}$ & 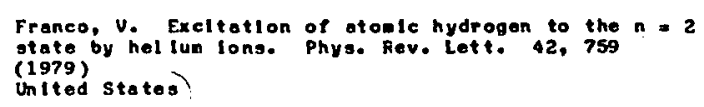 \\
\hline 756 & $A 06+C ; S+C: S+A l$ & $\varepsilon$ & 93-123 YeV & $\begin{array}{l}\text { Splndier, E.: Betz, H.-D.; Bell, F. Influence of } \\
\text { retardation on the angular distribution of radiative } \\
\text { electron capture. Phys. Rev. Lett. 42, } 832 \text { (1979) } \\
\text { Wegt Geraany }\end{array}$ \\
\hline 757 & $\begin{array}{l}B 06 \\
h h_{2}+L I \\
h D 2 \\
h L L I\end{array}$ & E T & $\begin{array}{l}\text { Hn2 } \\
43237-43250 \\
1 / \mathrm{Ca}\end{array}$ & 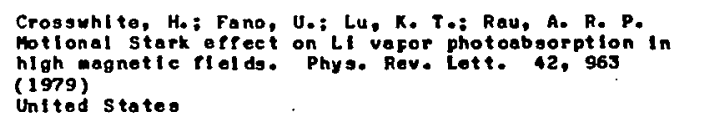 \\
\hline 758 & $\begin{array}{l}\mathrm{AlO} \mathrm{C}^{-} \\
\mathrm{Sr}+\mathrm{Ca} \\
\mathrm{Br} ?+\mathrm{Ca}\end{array}$ & E & $\begin{array}{l}\text { A10 } \\
300 \mathrm{~K}\end{array}$ & 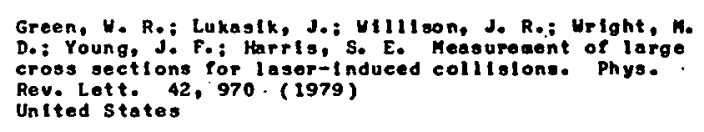 \\
\hline 759 & 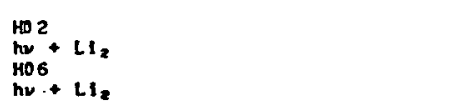 & $E$ & $\begin{array}{l}6400-5700 \\
A^{\circ}\end{array}$ & 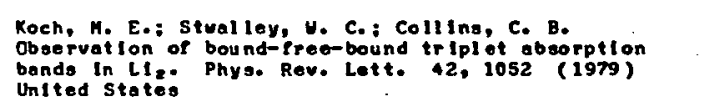 \\
\hline
\end{tabular}




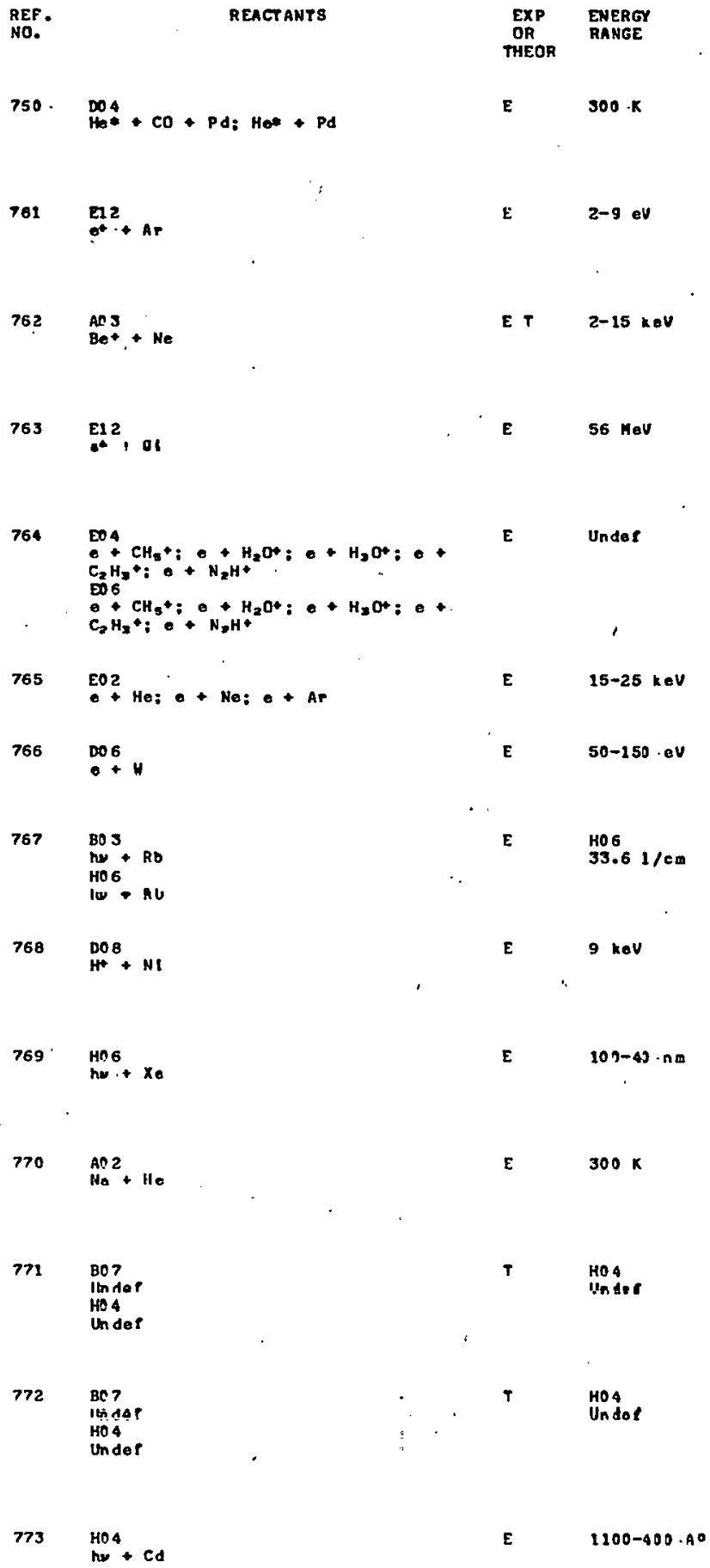

Conrad, H.: Ert1, Go: Kuppors, J.: Hang, S. H.: Gerard, Ko: . Haborland, H. Ponning-lonlzat lon ol octron spect roseopy of chenisorbed CO. Phys. Rev, Lett. 42, Mest Germany

Coleman, P. G.: NeNutt, J. D. Heasurement of difforontial cross soctlons for the elastic acat tering of positrons by orgon atoms. Phys. Rev. Lett. 42. unlead statos

Andersen, N.: Anderson, T.: Ol sen, J. O.: Pedersen, E. H.: Niolion. S. Ea : Dahler. J. S. Quantun-merhnnlent phase Juaps In col llslon-1 ndu ced cong
Phys. Rev. Lelt. 42, 1134 (1979)

Demmark

Alguard, M. J.: Swent, R. Lo: Pentell, R. Ho: Bermon,

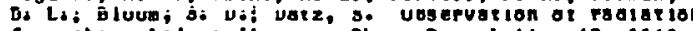
Pros channeled positrons. Phys. Rev. Lett. 42, 1148 (1979)

Unitod States

MeGowan, J, M.: Mul, P. M.: D*Angelo, V. S.; MItehell, J. B. A.: Defraince, P.: Froelfch, H. R. Erratue Energy dependence of dissoclative recombl nat lon below 0.08 eV measured ulth (el ectron-tion) morged-beas
teehnique [Phys. Rev. Lett. 42, 373 (1979)] Phys. Rav: l.at t. S?, 11 as (1979) Japan

Gelger, J.: Moron-Lean, D. Electron-atom shador seattering. Phys. Rev. Lett. 42, 1336 (1979) Weat Gormany

Wang, G.-C.: Dunlap, B. I.: Celotta, R. J.: Plerce, D. Tisymatry In lowenergy-polerizod-oloot ron diffraction. Phys. Rev. Lett. 42, 1349 (1979) Unitod States

Feneut11e, S.: Ll beraan, S.; PInard, J.: Taleb, A Observation of Fano profll as in photolonizat ion of rubidiun in the presence of a de Pfeld. Phys. Rev.

Frtt. 42,1404 (1979)

To1k, N. H.: Tully, J. C.: Kraus, J. S.: Hellond, M.: Nerf, S. H. Polarizatlon Promgrazing-incidonce Phys. Rev. Lett. 42, 1475 (1979) United States

Helnzaann, U.: Sehonhense, G.: Kosaler, J.

polarization of phatasiactrans ajected hy unpolerizod llght from xenon atong. Phys. Rev. Lett. 42, 1603 (1979)

West Germany

Hossberg, T.: Flusberg, A.: Xochru, R.; Hartann, S. R. Total-beattering erisss section for $\mathrm{Na}$ on He measurad by stimulated photon eehoes. Phys. Rev. Lett. 42,1665 (1979)

ted States

Sulth, R. A. Excltation of transl tions between atoule

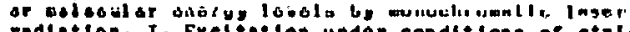
radiation. I. Exeltatlon under conditlons of strietly homogeneous ll ne brodenlng. Proc. Roy. Soc., London A

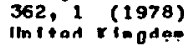

Salth, R. A, Excltatlon of tronaltlona between atoalc

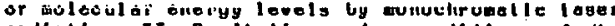
radiation. II. Fexcltation under conditlons of strictly inhomogeneous 1 ine broaden ing and ol ixed homogeneous and inhorogeneous bro Unlited Kingdos

Mansfleld, M. H. D. The simultaneous excitation of two Monsfield, M. W. D. The simultanaous excitation of tuo
electrons in atosic cadafua. Proc. Roy. Soc., London A elect rons in ato
$362.129(1978)$ United $k$ ingdom 


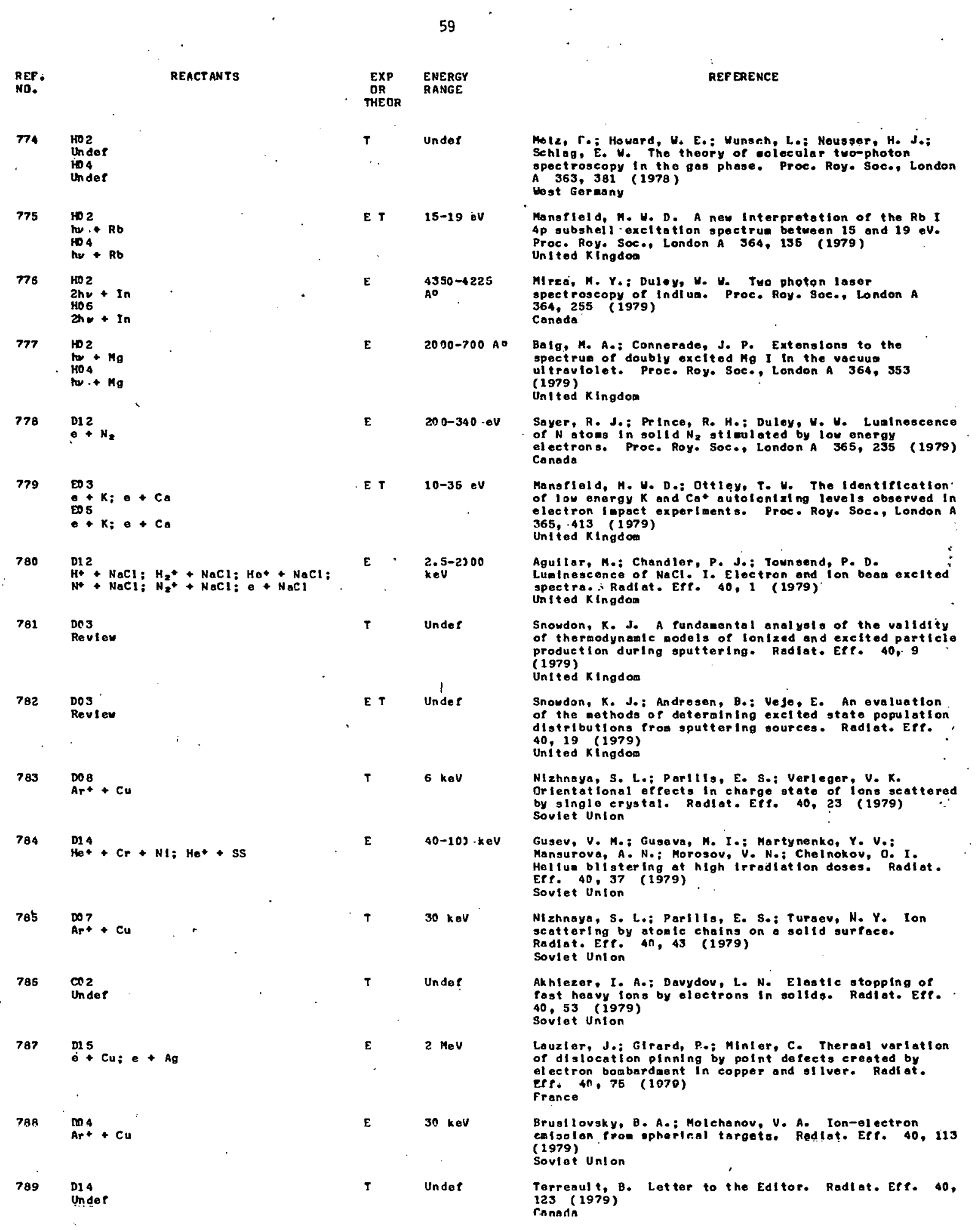




\begin{tabular}{|c|c|c|c|c|c|}
\hline $\begin{array}{l}\text { REF. } \\
\text { NO. }\end{array}$ & REACT ANTS & & $\begin{array}{c}\operatorname{EXP} \\
\text { OR } \\
\text { THE.OR }\end{array}$ & $\begin{array}{l}\text { ENERGY } \\
\text { RANGE }\end{array}$ & REFERENCE \\
\hline 790 & $\begin{array}{l}\mathrm{COB} \\
\mathrm{He}^{+}+\mathrm{V}_{3} \mathrm{St}\end{array}$ & & $T$ & $2 \mathrm{VeV}$ & 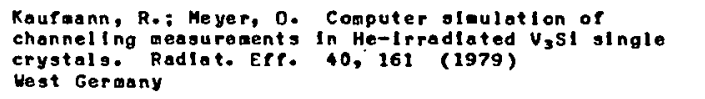 \\
\hline 791 & 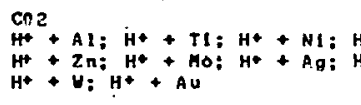 & $\begin{array}{l}\mathrm{H}+\text { Cu: } \\
\mathrm{H}+\end{array}$ & E & $0.3-1.5 \mathrm{MeV}$ & 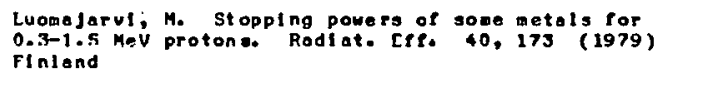 \\
\hline 792 & $\begin{array}{l}\text { D12 } \\
\text { Review }\end{array}$ & & E. T & Under & 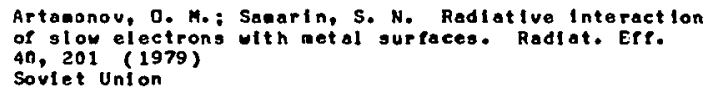 \\
\hline rys & Uns & & E & Under & 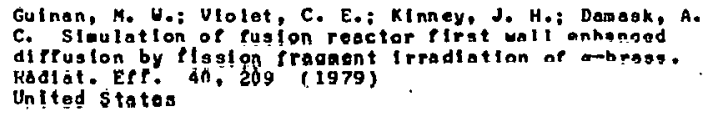 \\
\hline 794 & 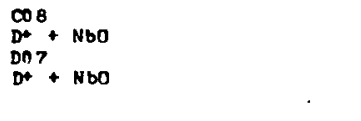 & ' & E & $1 \mathrm{MeV}$ & 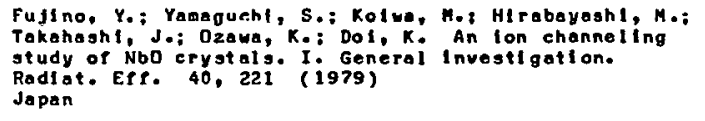 \\
\hline 795 & 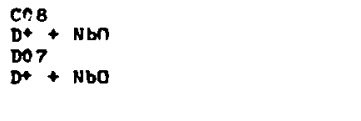 & & E & $1 \mathrm{MeV}$ & 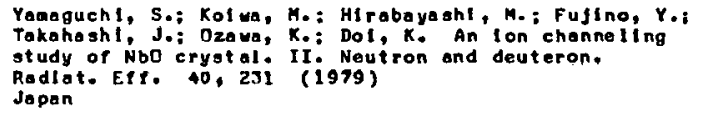 \\
\hline 796 & $\mathrm{Ar}^{+}+\mathrm{Au} ; \mathrm{N}++\mathrm{Au}^{2} \mathrm{~N}_{2}+\mathrm{Au}^{+}$ & & $E$ & $70 \mathrm{keV}$ & $\begin{array}{l}\text { Lebedov, S. Y.: Lysova, G. V. Angular dlstribut lon of } \\
\text { replected kev ions under boubardaent of single erystals } \\
\text { of gold. Radlat. Erf. 40, } 249 \text { (1979) } \\
\text { Soviet Unlon }\end{array}$ \\
\hline 797 & $\begin{array}{l}\text { D1 } 5 \\
\text { Under }\end{array}$ & & T & Undof & 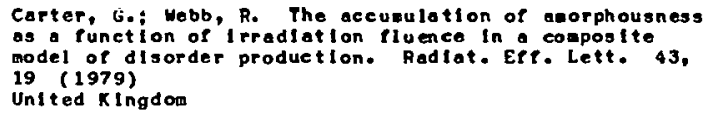 \\
\hline 798 & $\begin{array}{l}\text { A1? } \\
\text { Undef }\end{array}$ & & $T$ & Under & 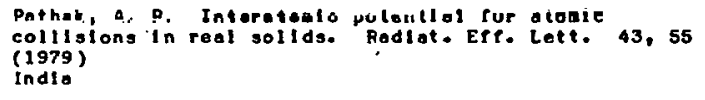 \\
\hline 799 & 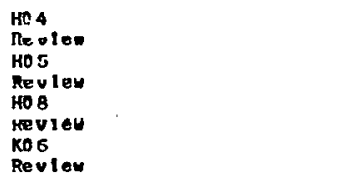 & & ET & Under & 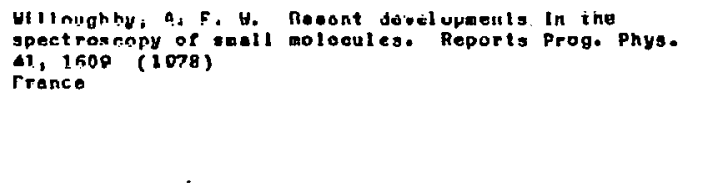 \\
\hline 800 & $\begin{array}{l}\text { ADI } \\
\text { Review } \\
\text { Col } 2 \\
\text { Revilev } \\
\text { Do } 1 \\
\text { Revieu }\end{array}$ & : & $T$ & Under & 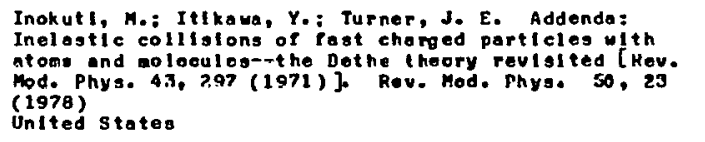 \\
\hline 801 & 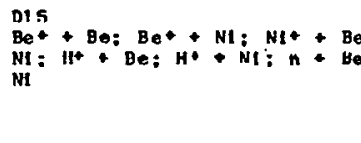 & $\mathrm{Nit}^{+}$ & T & $2.5-20$. MeV & 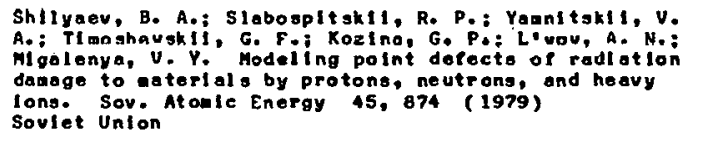 \\
\hline 802 & 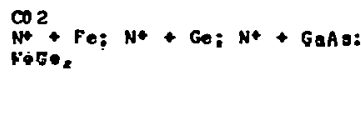 & $=1++$ & E & $\begin{array}{l}0.75-7.4 \\
\operatorname{lov}\end{array}$ & 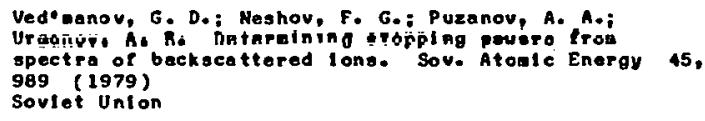 \\
\hline 803 & $\mathrm{H}^{\mathrm{CO}}+\mathrm{PERT}$ & - & T & 1-100 MeV & 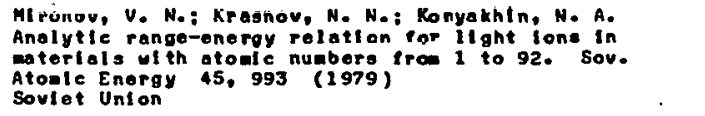 \\
\hline
\end{tabular}




\begin{tabular}{|c|c|c|c|c|}
\hline $\begin{array}{l}\text { REF. } \\
\text { NO. }\end{array}$ & REACTANTS & $\begin{array}{l}\operatorname{EXP} \\
\text { OR } \\
\text { THEOR }\end{array}$ & $\begin{array}{l}\text { ENERGY } \\
\text { RANGE }\end{array}$ & REF ERENCE \\
\hline 804 & 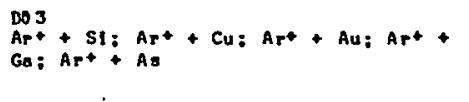 & $\varepsilon$ & $25-30 \cdot \mathrm{keV}$ & $\begin{array}{l}\text { MIlliams, P.: Evana, C. A., Jr. Anowaloug enhancement } \\
\text { of nagative gputtered ion enisolon by oxygen. Surface } \\
\text { Sel, } 78,324 \text { (1978) } \\
\text { United States }\end{array}$ \\
\hline 805 & $\mathrm{AOJ}_{\mathrm{Ar}+}^{\mathrm{DOS}}$ & $\mathbf{T}$ & $43-74$ keV & 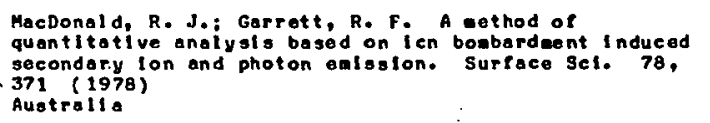 \\
\hline 806 & $\mathrm{Bnz}_{\mathrm{Ar}+}+\mathrm{Cu}$ & $\mathbf{T}$ & $600^{\circ} \mathrm{eV}$ & 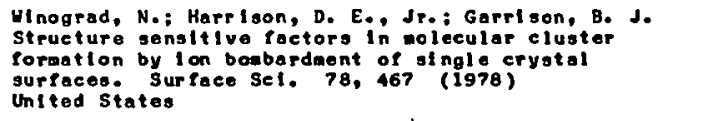 \\
\hline 807 & 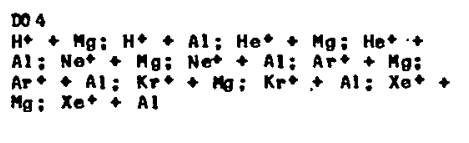 & $\varepsilon$ & $10-100-k e V$ & 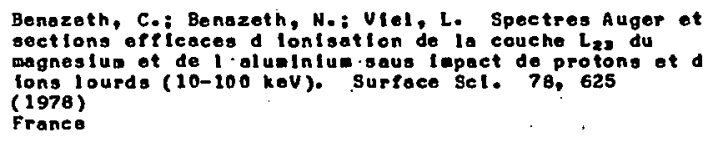 \\
\hline $808^{\circ}$ & 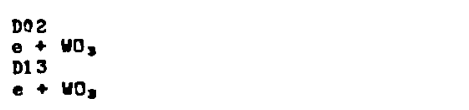 & $\cdot \varepsilon$ & $100-530 \mathrm{eV}$ & $\begin{array}{l}\text { Ni ehus. H. El Eectron otinulated desorption Prop ordered } \\
\text { wo, layers. Surface Scl. } 78,667 \text { (1978) } \\
\text { West Germany }\end{array}$ \\
\hline 809 & $\mathrm{DO}^{\circ}+\mathrm{MgO} ;=+\mathrm{N} 1 \mathrm{O}$ & $\varepsilon$ & $5 \mathrm{keV}$ & 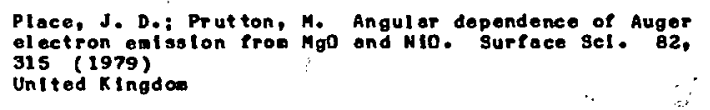 \\
\hline 810 & $\begin{array}{l}\mathrm{DOA}_{\mathrm{A}}+\mathrm{Al} \\
\mathrm{A}\end{array}$ & E & $50 \mathrm{keV}$ & 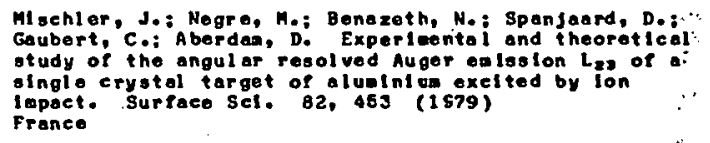 \\
\hline 811 & 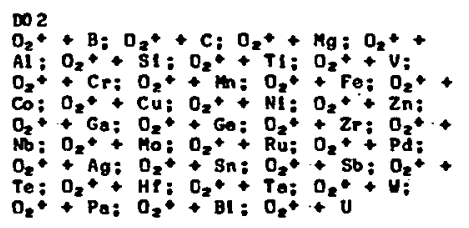 & E & $5.5 \mathrm{kaV}$ & 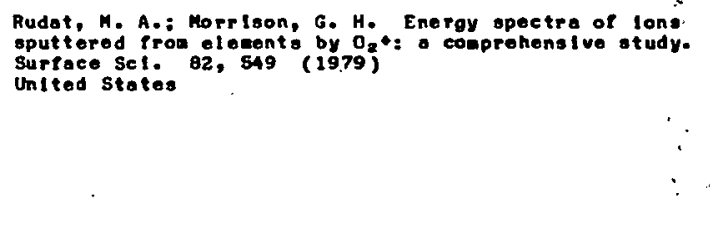 \\
\hline 812 & $\begin{array}{l}\text { Do7 } \\
\text { Unde? }\end{array}$ & $T$ & Under & $\begin{array}{l}\text { Manaon, J. R.: Araand, G.: Lapujoulado, J. On the } \\
\text { Fourfer couponents of the otom-gurface seattering } \\
\text { potentlal. Surtace Scl. B2, L593 (1979) } \\
\text { Unlted States }\end{array}$ \\
\hline 813 & $\stackrel{\mathrm{DO}}{0}+\mathrm{MgO}$ & $\mathbf{E}$ & $3 \mathrm{keV}$ & 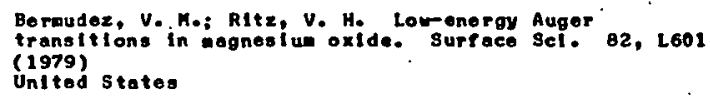 \\
\hline 814 & $\begin{array}{l}\text { Dor } \\
\text { Under }\end{array}$ & $\tau$ & Under & 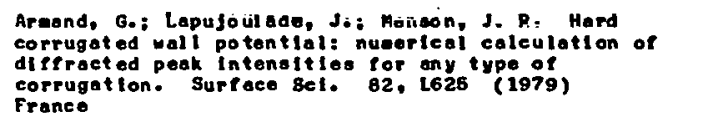 \\
\hline 815 & $\stackrel{D O G}{\mathrm{Dn}}$ & $\mathbf{E}$ & $100-1000 \cdot 0 \mathrm{~V}$ & 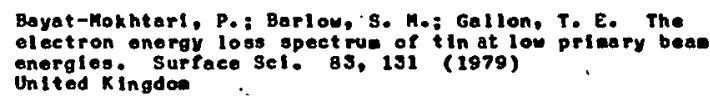 \\
\hline 816 & $\begin{array}{l}\text { Do } \\
\text { Undor }\end{array}$ & T & Undor & 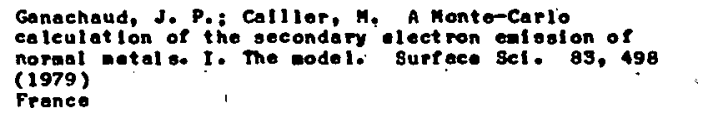 \\
\hline 817 & $\begin{array}{l}\text { DO4 } \\
\text { DOB } \\
\text { DOA } \\
- \text { AI }\end{array}$ & $\begin{array}{l}\top \\
\end{array}$ & $0-1000.0 \mathrm{~V}$ & 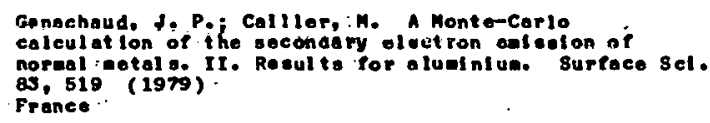 \\
\hline
\end{tabular}




\begin{tabular}{|c|c|c|c|}
\hline $\begin{array}{l}\text { REF. } \\
\text { NO. }\end{array}$ & REACTANTS & $\begin{array}{c}\text { EXP } \\
\text { OR } \\
\text { THEOR }\end{array}$ & $\begin{array}{l}\text { EHERGY } \\
\text { RAHGE }\end{array}$ \\
\hline a18 & $\begin{array}{l}\mathrm{DO}^{\mathrm{Ar}}+\mathrm{Cu} \\
\mathrm{Cu}\end{array}$ & E & $3-15 \leqslant e v$ \\
\hline 819 & $\begin{array}{l}A 12 \\
H+A C+; D+A C^{*} \\
B O H\end{array}$ & E & $9600-k$ \\
\hline 820 & $\begin{array}{l}\text { A05 } \\
\text { S1 } 8+\text { C C }\end{array}$ & E & 21-24 Mov \\
\hline B21. & 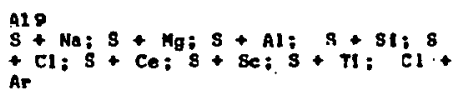 & E & 3. 9-48 MeV \\
\hline 822 & $\begin{array}{l}A 03 \\
I+Y b ; I+A u: I+P b ; I+T h: I+ \\
A 19 \\
I+Y b ; I+A U: I+P b ; I+T h ; I+\end{array}$ & T & $6.3-45 \mathrm{MeV}$ \\
\hline 823 & $\begin{array}{l}A 17 \\
K+N_{2} ; K+C O \\
A 1 B+N_{2} ; K+C D\end{array}$ & E & $a_{o v}^{34-1.24}$ \\
\hline 824 & 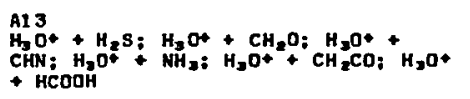 & E & $298 \mathrm{~K}$ \\
\hline 825 & $\begin{array}{l}A^{A 06} \\
\mathrm{Ne}+\mathrm{Kr}_{\mathrm{r}} ; \mathrm{Ar}^{+}+\mathrm{Ar}^{+}+\mathrm{Kr}_{\mathrm{r}} ; \mathrm{Kr}^{+}+\mathrm{Kr} ; \mathrm{Xe}^{+}+\end{array}$ & E & $60-115 \mathrm{keV}$ \\
\hline 826 & 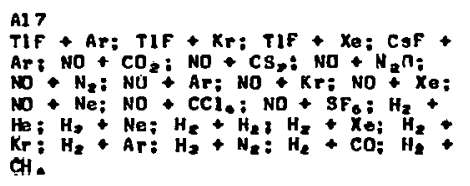 & E $T$ & Undaf \\
\hline 827 & 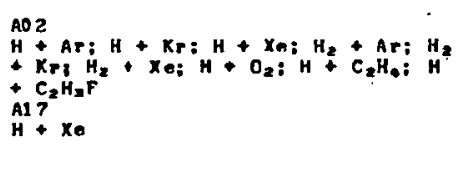 & ET & 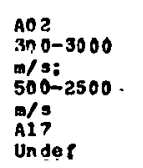 \\
\hline 829 & $\begin{array}{l}\mathrm{Al}^{3} \\
\mathrm{Cl}_{+}-\mathrm{SO}_{2}+\mathrm{SO}_{2} \mathrm{Cl}_{2}: \mathrm{SO}_{2} \mathrm{Cl}_{2}-+\mathrm{SO}_{2} \mathrm{Cl}_{2} ; \mathrm{SO}_{2}-\end{array}$ & $\varepsilon$ & under \\
\hline 829 & 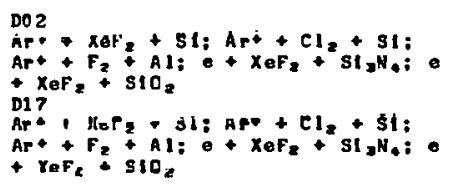 & n & $\begin{array}{l}+050 \mathrm{uv} ; \\
1500 \mathrm{ov}\end{array}$ \\
\hline 30 & $\mathrm{Pl}_{\mathrm{He}}^{\mathrm{D}+}+\mathrm{Nb}$ & E & $20-502 \mathrm{keV}$ \\
\hline
\end{tabular}

Abat Ino, C.: Luxz1, G.: Papagno, L. Sput torlng ylel d coerticlents of thin copper T11.s boubardod by Art, at an angle
56,291 (1979)

Italy

Ehrleh, H. Experlmental study of Baleer-a Stark broodoning. Z. Naturiorseh. A 34,188 (1979) Vest Germany

Trabert, E.: Heekmann, P. Ho: Buttlar, H. U. HIghly resolved EUU bean-foll spectra of sllicon. 2. Phys. A 290, ? (1979)
Je if Gerany

Schuch, Ro: Gouk10r, G.: Bchaldt-B ocking, H. a) -branching of the $K$ (sub a) (sup $h$ ) $/ K$ (sub double

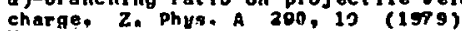
Hest Ginreany

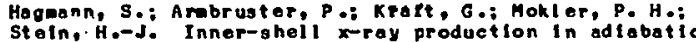
lodine- $Z_{2}$ collist ons with $Z_{z}+Z_{2}$ greater than or equal to 100 . II. Molecular vacancy productlon 290 , 25 (1979)

Sehepper, H.; Ross, U,; Beck, D, Anlootropy of the Topulsive Intertol eeular potentinl Prad potationaliy
inelastle seattering. 2. Phyo. A 290. 131 (1979) Hest Germany

Mackav, G. I.: Tanner, S. D.: Hopk Inson, A. C.: Bohme,

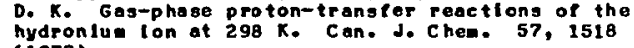
(1979)

Canade

Hird, B.: All, S. P. Search for long-lived states of the heavior rare gas negetive lons. Can. J. Phys. 57, 867 (1979)

Thuts, H.: stolte, S.; Reuss, J. Nol acul orrbeam Investigations of ani isotrople potential s. Comments at. Hol. Phys. O, 123 (1979)

The Netherl ands

Toennles, J, P. Rogononces In the henttering of otous and nolecules. Coments At. Hol. Phys. 8, 137 (1979) thest Germany

Robblenl, R.; Franklln, J. L. Negetive Innmoroloeulo.

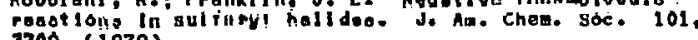
3709 (1979)

Coburn, J. H.: Mlnters, H. F. Ion- and

el ectron-assigted ga s-8urface chenlstry-an Inportant offect In plasma etehing. J. Appl. Phys. 50, 3189 (1979)

unirea states

Das, S. K.: Kaningky, M.: Fengke, G. On the

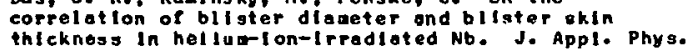
50, 3304 (1979)

united states 


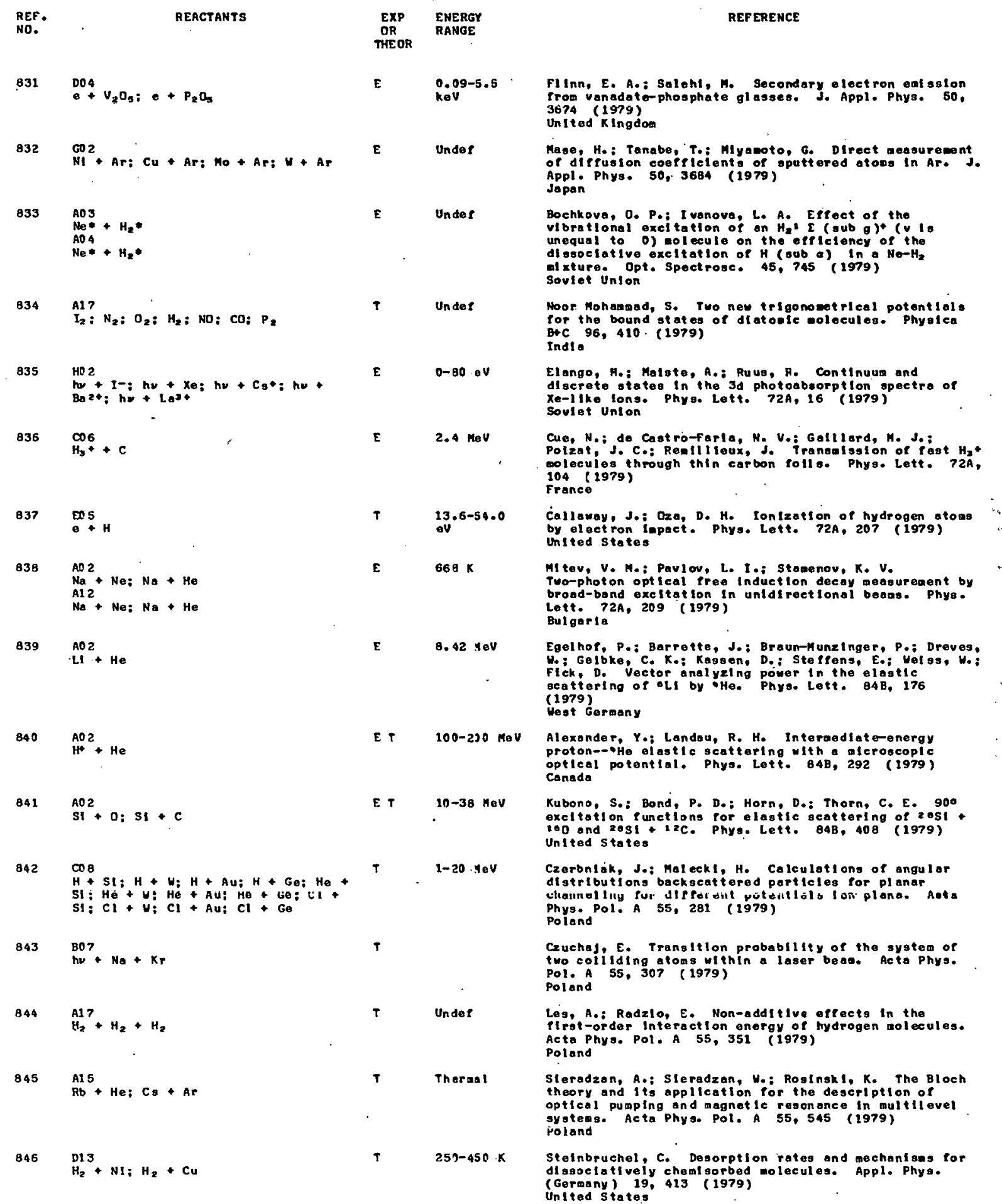


64

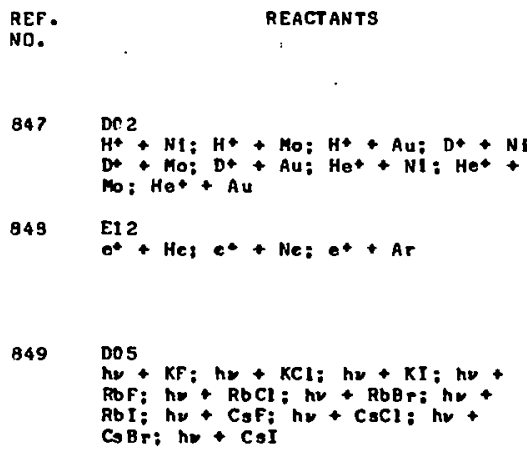

850

${ }_{\mathrm{H}^{+}}^{\mathrm{OOO}}+\mathrm{Zn} ; \mathrm{H+}+\mathrm{Cd} ; \mathrm{He}^{2+}+\mathrm{Zn} \mathrm{He}^{2+}+$

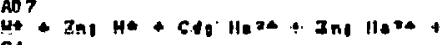

851

$\mathrm{Na}+\mathrm{CO} ; \mathrm{Na}+\mathrm{NO}: \mathrm{Na}+\mathrm{CO}_{2}: \mathrm{Na}+$

$\mathrm{C}_{2} \mathrm{H}_{4} ; \mathrm{Na}_{\mathrm{O}}+\mathrm{CH}_{4}$

852

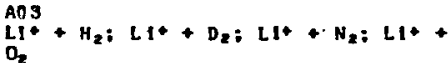

853

$\mathrm{He}^{\mathrm{AO}}+\mathrm{He}^{2+}$

He 6 + $\mathrm{He}^{2}$

854

$\mathrm{He}^{\mathrm{AO}} \mathrm{B}+\mathrm{He}$

855

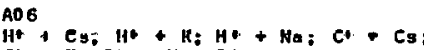

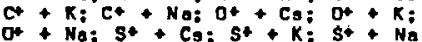

CO 6

$\mathrm{H}^{+}+\mathrm{Cs}^{2} \mathrm{H}^{+}+\mathrm{K}_{\mathrm{Z}} \mathrm{H}^{+}+\mathrm{Na} \mathrm{C}_{+}+\mathrm{Cs}^{\circ}$

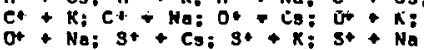

855

$\mathrm{H}^{+}+\mathrm{H}(\mathrm{n})$

857

${ }_{\mathrm{LI}}^{\mathrm{AO}} \mathrm{3}+\mathrm{H}_{2}$

85:

$204+\mathrm{H}_{2}^{+}: \mathrm{O} \rightarrow \mathrm{HD}^{+}=-\mathrm{D}_{2}+$

EOG $\mathrm{H}_{4} *: \div+\mathrm{HD}: \mathrm{C}^{\circ}+\mathrm{D}^{\circ}$

859

$\mathrm{HOC}^{*}+\mathrm{H}(\mathrm{n})$

860 .

$\mathrm{C}^{\mathrm{AO}} 6+\mathrm{Cs} ; \mathrm{C}+\mathrm{K} ; \mathrm{C}+\mathrm{Na} ; \mathrm{O}+\mathrm{Cs}^{\circ}$

$\mathrm{O}^{+}+\mathrm{K} ; \mathrm{O}^{+}+\mathrm{Na} \mathrm{S}^{+}+\mathrm{Ca}_{;} \mathrm{S}^{+}+\mathrm{K}$;

Cs: O $+K$ : C + No: $S+C a: S+K$ S
EXP ENERGY. REFERENCE

$1-8$ keV

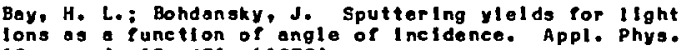
(Germany) 19, 421 (1979) (Gerting)

E $20-1030 \mathrm{eV}$

Grifflth, T. C.: Heyland, G. R.: Lines, K. S.; Twomey, T. R. Total eross-sections fop the scattering of positrons by hellua, neon, ond orgen at intermediate energles. Appl. Phys. (Gereony) 19, 431 (1979) Unlted Kingdom

E $\quad 10-40 \mathrm{eV}$

Sugawara, H.: Sasakl, T. Photoelectrie yleld speetra of $k-$, $R b$ - and $C s-h a i l d e s$ in the photon energy range 10

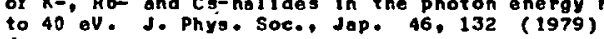

Japar

$0.5-2.75$

KoJlaa, H.: Kobayashl, No: Moeda, No: Sakt saka, M. Auger ol ectrons from $z$ Ine and codofue vapors by proton and al pha partlcle bombardnents. J. Phys. Soc., Jap. 46,198 (1979)

Javen

E $6.3 \times 19 *$

Ray, 3.: Barua, A. K. Total eross sectlons of Na seattered by different gases. J. Phys. Soc., Jap. 46, 205 (1979)

$100-1290$ ov

Kobayashl, No: Itoh, Yo: Kaneko, Yo Vibratlonal exctitation of $\mathrm{H}_{2}, \mathrm{D}_{2}, \mathrm{~N}_{2}$ and $\mathrm{O}_{2}$ by $\mathrm{LI}_{1}$ + impact In the energy range eran jód av to 1200 u. d. Phye. Soc.. Jap. $46,208(1979)$,

T Under

Sato, Ho; Hara, S. The radlal and rotational coupling teras between the quasi-molecular states of Hest+. J. Phys. Soc., Jap. 46, 215 (1579)

E $\quad 10-103 \cdot \mathrm{keV}$

Arlkawa, T.: Narushlaa, K.: Inoue, M. Electron loss cross section of $\mathrm{He}^{+}$on He in 10-100 keV. J. Phys. Soc., Jap. 46,353 (1979)

E $\quad 0.5-5.0 \mathrm{keV}$ Nagata, T. Charge changling ccllls long of at omic beass S. Ions. J. Phys. Soc., Jap.. 46, 919 (1979) Japan

$12.5 \mathrm{keV} / \mathrm{n} *$

Toshlas, W. charge trangfer processes involuing highly exclted hydrogen atons. I. The Born anplltude In Japan

E $15 \%-750$ oV

Itoh, Y.: Kobayasht, N.: Kaneko, Y. Rotational

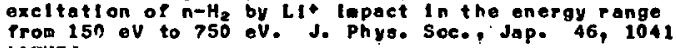
(19\%9)
dapall

T.5-2.7. 0

Ral Dastldar, K.; Ral Dastldar, T. K. Dlssoclative recoubination of $\mathrm{H}_{2}+$, $\mathrm{HD}^{*}$ and $\mathrm{D}_{2}$, molecular lons. J. Phys. Soc., Jap. 46, 1288 (1979) inti:

T $\quad 0.1-23 \cdots \mathrm{keV}$

Toshlea, N. Charge transfor processes involving highly exclted hydrogen atoms. II. Numerl cal results by the

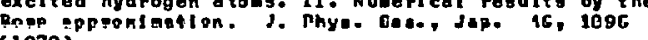
(1979) Japen

E $\quad 1-5 \mathrm{keV}$

Nogota, T. Charge chonglng cellistons of atonle beans In alkal i-netal vapors. Ir. Total cross sectlons for one-electron capture by $\mathrm{C}^{*}, \mathrm{O}^{*}, \mathrm{~S}^{*}$ lons and $\mathrm{C}, \mathrm{O}, \mathrm{S}$ atoms. J. Phys. Soc., Jap. 46, 1302 (1979) Japan 


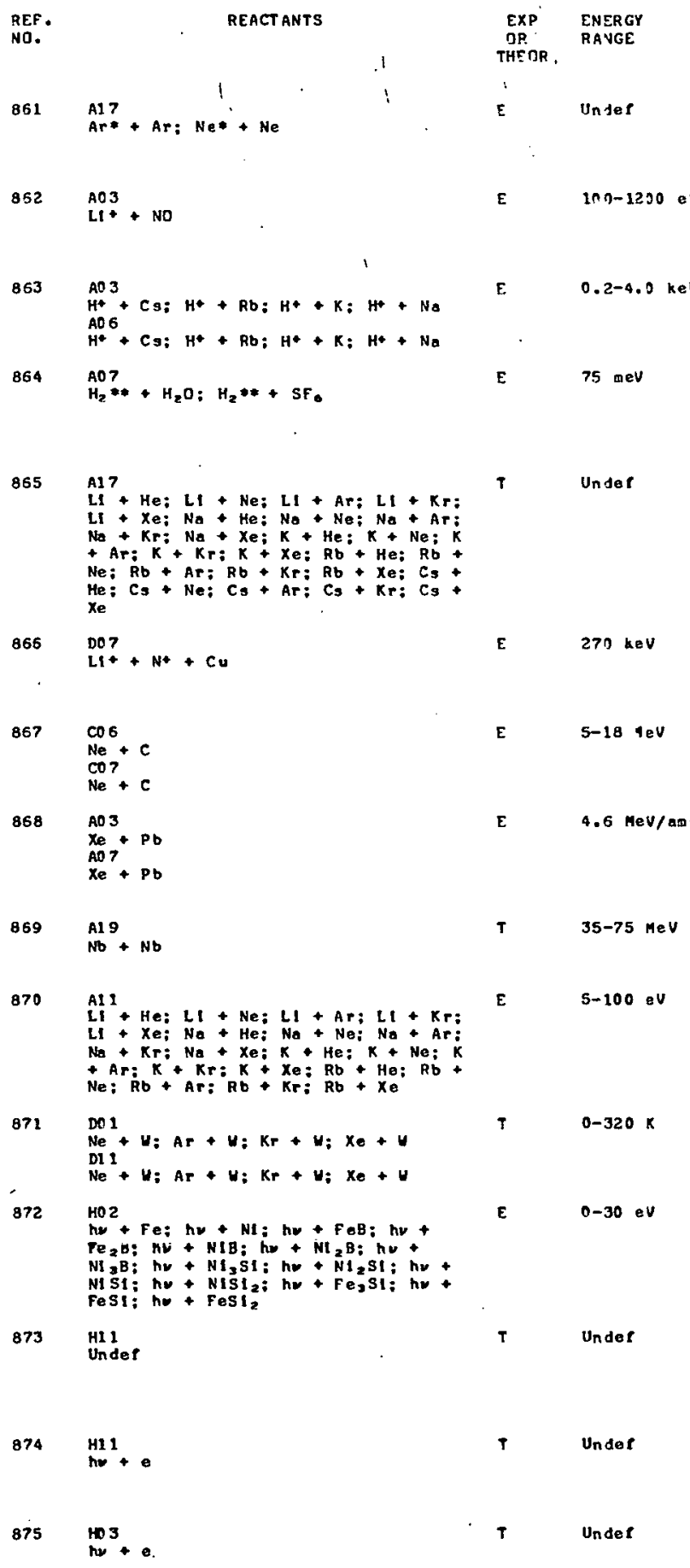

Matsuara, Y.; Fukuda, K. Neu oscl llatory struct ures of Nez and $\mathrm{Ar}_{2}$ emlssion contl nue in the vacuum (1979) Japan

Itoh, Y.; Kobayashi, -N.: Kaneko, Y, Vibrational excitation of ND by LI* Impact 1 rom $190 \mathrm{eV}$ to $1200 \mathrm{eV}$. J. Phys. Soc., Jap. 46, 1399 (1579) Japan

REF ERENCE

Nagata, T. Charge changing collis lons of at omic beams In alkal i-metal vapor3. III. Formation of $H(2 s)$ atoas from $\mathrm{H}^{+}$ions. J. Phys. Soc., Jap. 46, 1622 (1979) Japon

HIralght, H.: Kondow, T.: Fukuyaara, T.: Kuchltsu, K. Iontzing colitsions of long-lived highily exelted hydrogen moleculeg with $\mathrm{H}_{2} \mathrm{O}$ and $\mathrm{SF}_{6}$. 2. Phys. Soc., Jap. 46,1628 (1979)

Japan

Czuchaj, E.: Slenklewlez, J. Adlabatic potentlals of the alkali-rare gas atom pairs. Z. Naturforseh. A 34, $594(1979)$

Poland

Winter, H.: Andra, H. J., Nuclear polarlzed 'Ll and in $N$ lons $v i$ a ion beam surface interaction at grazing incidence. 2. Phys.. A 291, 5 (1579) West Germany

Schule, R.: Stafast, H.: Bethge, K., Charge-state distributions and the population of x-ray emltting states. Z. Phys. A 291, 117 (1979) West Germany

Anholt, R.: Moyerhot, H. E.: Stoller, C.

Impaet-parameter dependence of 1 s $\Sigma$ vaconey productlon in 4.6-MeV/amu Xe + Pb collistong. Z. Phys. A 291, $287 \quad(1979)$ United States

Antolt, R. Theory of the ongular distributton of $2 p \Sigma$ molecular orbltal x-rays. Z. Phys. A 291,289 (1979)
Republic of China

Speller, E:: Steudenmayer, B.: Kempter, $V$. Quenching cross sections for olkall-inert gas colilisions. 2. Phys. A 291, 311 (1979) Nest Germany

Muller, H.: Brenlg, W. Kinetle theory of gas-surface lnteraction. Z. Phys, B 34, 165 (1979) West Garmany

Koval', A. V.; Karal'nik, 3, Me X-ray k-1Ino absorption spectra of certain tronsition matal thelr bl nary alloys with metallolds. Sov. Phys. 3 21,992 (1978)

Danlshevskl1, Y. A.; Shuartsman, S. M. Green's Danlshevskll, Y. A.; Shuartsman, S. M. Green's functlon of charged scalar particles doving in the 1021 (1978)

Sovlet Union $\because ; "$ zadorozhnyl, V. N.: Shvartsaan, S. M. Green's eunction

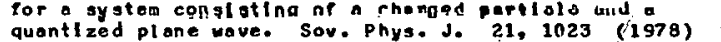
quantized plane wave. Sov. Phys.J. 21, 1023 (1978)
Sovlet Union

Bozrikov, P. V.: Kopytov, G. F. Splitetng of polarlzed photon by an unpol arized el ectron. Sov. phys.J. 21, 1087 (1978) Soviet Unton 


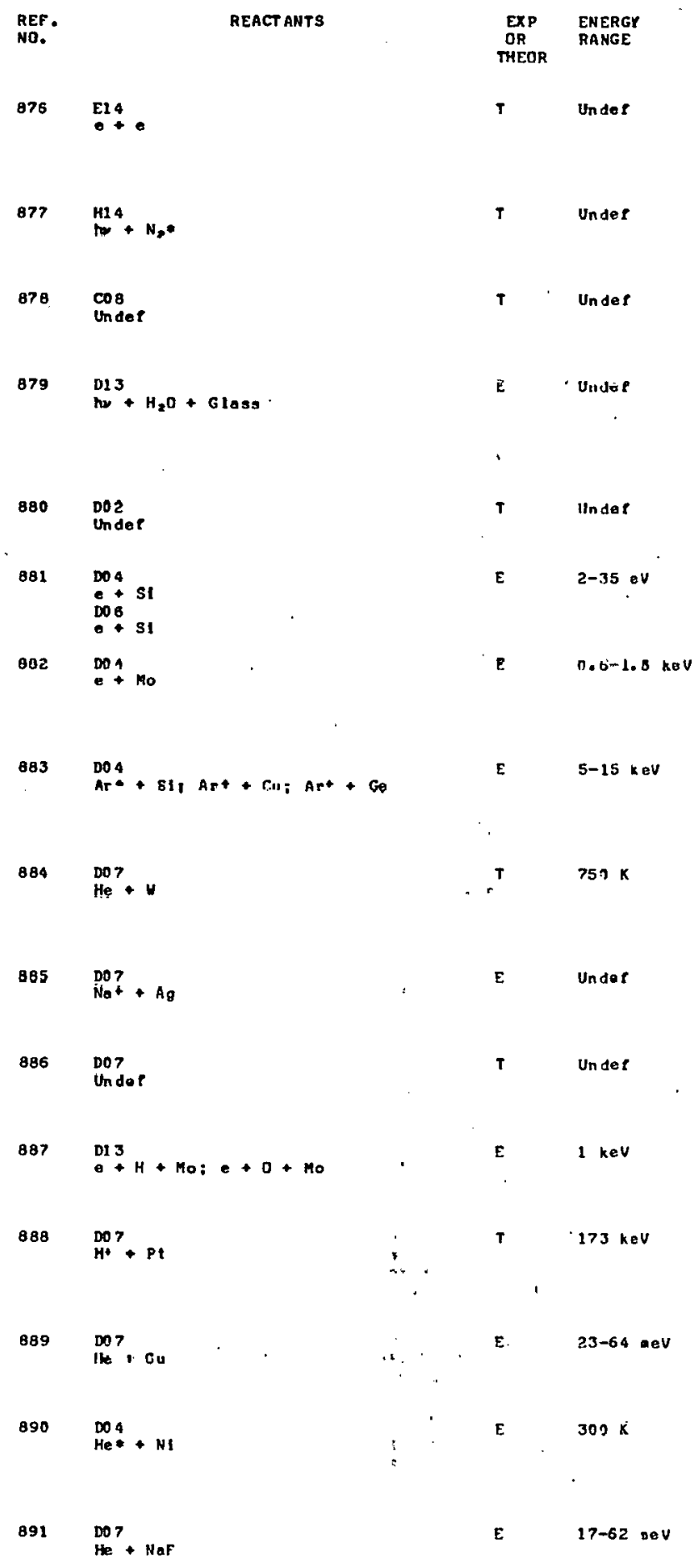

Gnedov, Y. A.; Romanov, Y. I. Pos slble offocts of wook lepton Interactlon on olectron-olectron and el ectron-auon seat tering. Sov. Fhys. J. 21, 1219 (1978)

Soviet Union

Lyutyl, A. I.: Mel'nlkova, L. D. XInottes of radiation-induced relaxation of vibrational onorgy of gaseous systea. Sov. Phyg. J. 21, 1253 (1978) Soviet Unlon

2101 , N. I.: Voroblev, S. A. Posslbllity of resonence radiotion during channelling of relativistlc particles. Sov. Phys. J. 21, 1359 (1978) union

Tagl pnu, R. R.: Kessel', N. I.; Shubalova, N. H.: Kuksinskt1, N. $I$, Role of photodlenoclation and desorption of water molecules Prom surface of glass
under pulged irradlation. Sov. Phys. J. 21, 1385 . (1978) Sovlet Unlon

Lusnikov, A, V. Mechanism of sputtering of surface at oms. Sov. Phys.-Solla Stale 20, 1440 (1978) Soviet Unton

Bronshteln, I. M.: Novltskil, M. G. Secondary el eetron ealssion $\mathrm{P}$ rom sill con bombarded with low-energy prinory electrans. Sov. Phyg.-Solld State 20, 1467 (1978) Soviet Union

Bopoyunova, A. 1.; 7.s slavskll, S. L.; Pronln, I. I. oricntation effects In Auger el ect ron spectroscupy of single-crystal mol ybdonum. Sov. Phys.-Solld state 20 , 1607 ( 1978 )
Soviet Uni on

Dorozhkin, A. A.: Petrov, A. A.; Petrov, N. N. Structure of the energy spectro of the Auger electrons oblalited by lrradiation nr solids uith moderate-energy lons. Sov. Phys.-Solld State 20, 1660 (1978) Soviet Unt on

Goodman, F. O.: Garela, N.: Cabrera, N. Scattering of at ons by surfaces with one-dlatensicnal perlodiclty on the gtatlonary sinusotdal hard-uall model. Surface Scl.iptra11: 94 (1978)

Terzlc, I.: Cirlc, De: Peroulc, B. On the 'observatlon of the double-scattoring effect tron polycrystalline targets. Surfoce Sci. II, LIT (1970) Yugosiavia

Masel, R. I. On the Debye-waller Pactor for atom--surface seatterling. Surface Sclence 77, L179

United States

Hock, J. L.: Lleht aan, D. El ectron st laul at ed desorption of negatlue long rroon gases adsorbed on gaolvbdenum, Surface Scl. 77,4184 (1978)

von der Veen, J. F.: Sanders, J. B.; Sarlo, F. W. Two-atom model for the cal eulation of surface blocklng in medlut and high enorgy lon seat ter ing. Surface Sel. $77,337(1978)$

Mason, H. F.; Whlllass, B. R. The Inglnatle gcattering of He trom curdoi) at $20 \mathrm{~K}$. Surface Sel. 77, 385 (1978)

Johnison, ip. D.: Delcliar, T. A. Dl taptent a jpet lun bv hellum metastable atomg incldent on the elean and chel cogen cove unlted $\mathrm{kingdon}$

Krl glinoswamy, 8. V.i Dorry, G.; Hesner. D.: OCGorman, T. J: Franki, D. Re Debye-woller effect: In atom-surface ) 
67

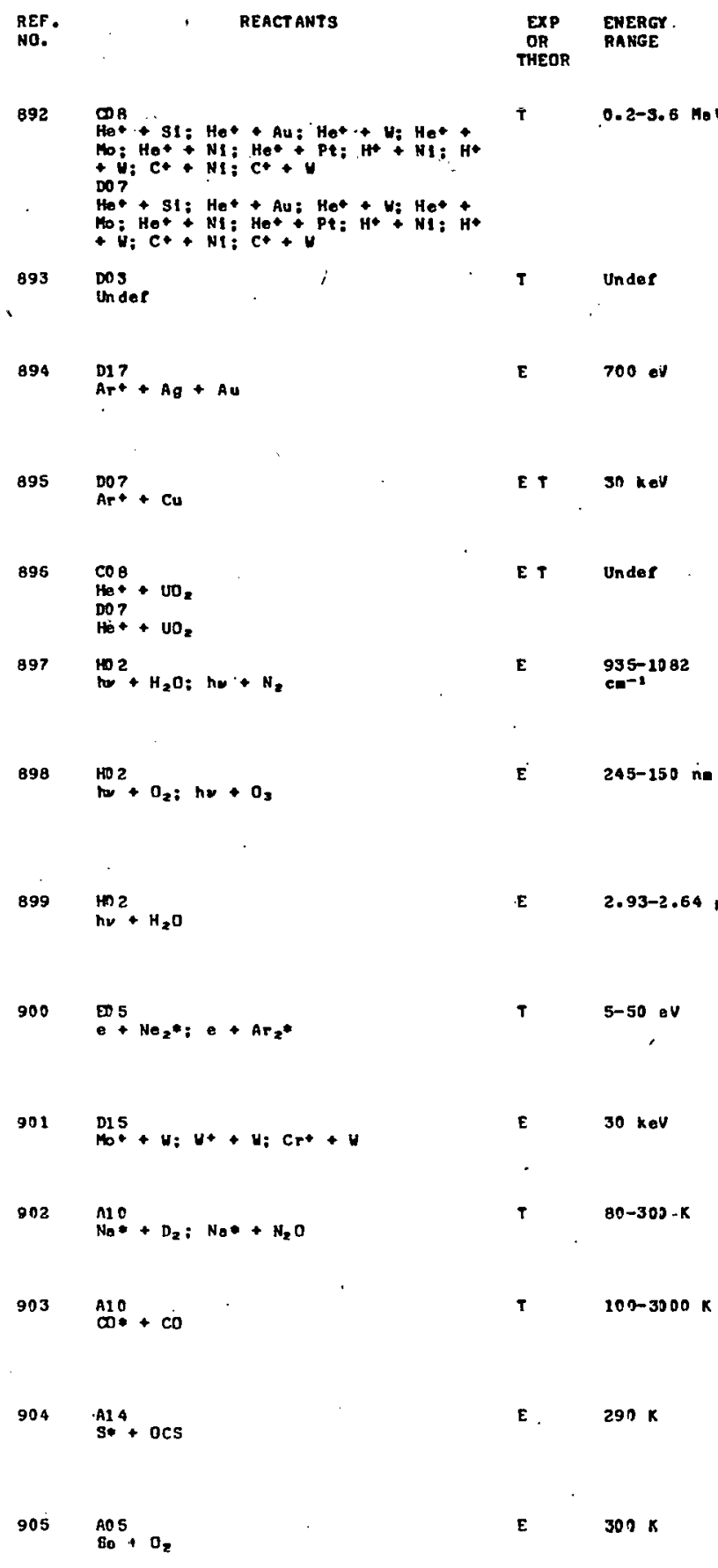

REF ERENCE

Stenggea rd, .I.; Foldmen, L. C.; SI Iverman, P. J. Calculat lon of the backseattering-channeling surface peak. Surface sel. 77,613 (1978)
untited states

Sroubek, 2.: ZavadIl, J.: Kubec, F.: Zdansky, K. Model of lonization of atods aputered tros sollids. Surface Sel. 77, 603 (1978) Czechosl ovakla

Yabusoto, M.: Hatanabe, K.: Yamashina, T. An AEs study of aurface segregetion of Ag-All alloys vith lon-bombardaent and onneal ing. Surface Scl. 77,615 (1978) Japan

Balashova, L. L.: Borlsov, A. M.: Mashkova, E. S.: Molehanov, V. A. Bloeking opleets tor fast lonlzéd recolls surface sel. 77, LeA3 (1978) Sovfet Union )

Ellls, Y. P.; Taylor, T. N. Erratue Ion-scottering structure studles of $\mathrm{NO}_{2}$ surfaces. [Surface Scl. 75, 279 (1978)]. Surface Sel. 77,658 (1978)

peterson, J. C.: Thonas, H. E.: Nordstros, R. J.: Dason, E. Ko: Long, R. K, Water vapor-nltrogen
absorption i $\mathrm{CO}_{2}$ laser Prequencles. Appl. Opt. 18,
B34 (1979) (it)

Cann, M. U. P.; N1 cholla, R. Y.; Evans, W. F. J.; Kohl, Cann, M. U. P.: N1 cholls, R. U.; Evans, We. F. J.: Koh HIgh resolution at eosherle trangalssion calculation. down'to $28.7 \mathrm{k}$ in the 200-243-na apectral $r$ ungo. Appl. Opt. 18, 964 (1979) United states

Watkins, W. R.; Spellley, R. Le; Uhlte, K. O.; Sojka, B. 2.: Bower, L. R. vater vapor absorption coefticlents at MF laser vavelengths $(2.64-2.93 \mu)$. Appl. Opt. 18, 1582 (1979)

United States

MeCann, K. J.: Flannery, M. R.: Hazl, A. Theoretlcal eross sectlons for lonizot lon of netastable excloers Nez* and Ar, by lectron inpoct. Appl. Phys. lott. 34,543 (1979) United states

Hel, C.-Y.; Seldaan, D. N. Dlrect observati on of the vacaney structure of depleted zones in tungsten ion irradiated at $10 \mathrm{~K}$. Appl. Phys. Lett. 34,622 (1979) United states

Taylor, H. S. Electronle to vibratlonal energy transfer in Na(32p) with siople a leeules. Chea. Phys. Lett. 64,17 (1979)

Unted state.

Shin, H. K. Temperature dependence of

vibrat ion-vibration energy exchange probabilities among hlghly exelted levels: $\operatorname{co}(v-1)+\operatorname{co}(1) \cdot \operatorname{co}(v)+\operatorname{co}(0)$. Chem. Phys. Lett. 64, 21 (1579) United states

AddIson, M. C. : Byrne, C. D.: Donovan, R. J. Di reet observation of $S\left(3_{12} D_{2}\right)$ and deteralination of the absolute rate of reaction ulth OCS. Chem. Phys. Lett. 64,57 (1979)

medonmot, U. Eu: Dorrard, D. J. SeU chemlluml nescence Induced by the reaction of selentue vapors with di scharged oxygen. Chen. Phys. Lett. 64, 60 (1979) United states 


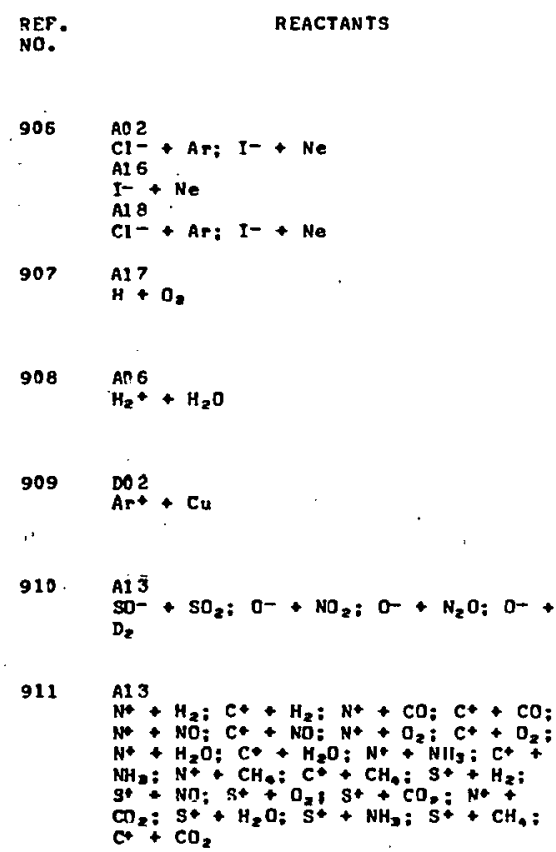

ENERGY and nitrogen adgorption on secondary fon onergy spectra. Int. J. Haso Spectron. Ion Phys. 90,233 (1979)

de Vreugd, C.: Wignaendts van Resandt, R. Wo: Los, J. Electron-dotachment In differential $I_{-}$- Ne cross

Melfus, C. F.; Blint, R. J. The potentlal energy surfoce of the $\mathrm{Hn}_{2}$ molecular systen. Chen. Phys. Lett GA, 183 (1979)

Parker, J. E.: Mllner, R. G. Charge-transter reactlons of $\mathrm{Hz}^{*}$ with $\mathrm{H}_{2} \mathrm{O}$. Int. J. Mass Spectrom. Ion Phys. 29 , United Kingdoi

Snoudon, K. J.: Hacdonald, R. J. Secondary lan energy par unt t mass spectro troo polyerystalline copper Auatroilig

moten, T.: Klain. F. S. Negotlve ton-niolecule reactions at 1 ow collibion energles. Int. J. Ráss Spectrow. Inn Phys. 29, 137 (1979)

TIChy, M.: Rakshlt, A. B.: LIster, D. G.: TwIddy, N. Do: Adans, N. G.: Salth, D. A study of the reactions of the ground and metastabla states of $\mathrm{C}^{*}, \mathrm{~N}^{*}, \mathrm{~S}^{*}$ and $\mathrm{N}_{2}$. hit $300 \mathrm{~K}$. Int. J. Mass Spectron. Ion Phys. 29, Unsted kin

United states

LIndinger, Ho; Alge, E.; Storl, Ho: Varney, R. No; Helm, Ho: Holzaann, P.: Pahl, Ho Investlgation of Ion-wolecule reactlons uging a drift tube with geparated lon source. Int. J. Mas s Spactrow. Ion Phys. 30,251 (1979)

Tatevakl, H.: Tanaka, K.: Sasahl, Fe: Obara, S.: Onno, $K_{0:}$ Yosh imine, M. Configuratlon-1 nteractl on study of lower exclted st at es of $\mathrm{O}_{2}$ : valonee and Rydberg charaotoro of the tys loment JI(sub ut states. Int. J. Quantum Chem. 15, 533 (1979) Japuin

Inoue, K.: Hireo, To: Yaegashl, Y.: Takayanagt, S. Aeymatricol proft les of ton Iaplanted phosphorus in sllicon. Jap. J. Appl. Phys. 18, 367 (1079) Japan

E $\quad 1.29$ Arikawa, T.; kaneko, S, Mult1-crossod-baan method. Jap. J. Appi. Phys. 18, 413 (1979) Japan

E $\quad 9-25$ iv

Mashlzume; Ad: Hayada, Mo Tho oleptran lapant Ionlzation effleleney curve of zince Jap. J. Appl. Phys. 18, 429 (1979) Jopan

Maeda, M.: Nlohitarunlzu, T.; Mlyazoo, Y. Formation and quenching of exel mers in lou-pressure rare-gas/halogen -ixtures by e-bena excitation. Jap. J. App1. Phys. 18, 439 (1979) Japan

E $\quad 2-25 \mathrm{keV}$

Okuda, S.: K Inura, T.: Akl sune, H. Depth prottles or laplanted $H$ and $H e$ in metal Ho det er-Ined with backscat tored protons. Jap. J. Appl. Phys. 18, 465 (1979) 


\section{9}

\begin{tabular}{|c|c|c|c|c|}
\hline $\begin{array}{l}\text { REF. } \\
\text { NO. }\end{array}$ & REACT ANTS & $\begin{array}{l}\operatorname{EXP} \\
\text { OR } \\
\text { THEOP. }\end{array}$ & $\begin{array}{l}\text { ENERGY } \\
\text { RANGE }\end{array}$ & REF ERENCE \\
\hline 920 & $\mathrm{RnO}+\mathrm{Rh} ; \mathrm{LI} *+\mathrm{Ta}$ & E & $\operatorname{sno} 0=$ & $\begin{array}{l}\text { Marlella, R. P. Jr. A study of losor-asslsted surface } \\
\text { lonization of iithlum. J. Chem. Phys. } 71,94 \text { (1979) } \\
\text { Unfted states }\end{array}$ \\
\hline 921 & ${ }_{h y}^{M e} B$ & $\varepsilon$ & $1508 \mathrm{AO}$ & 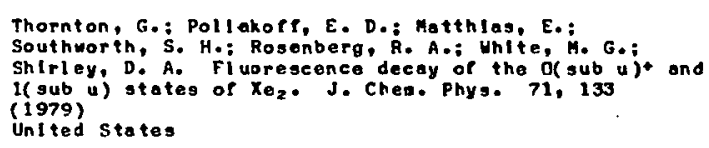 \\
\hline 922 & 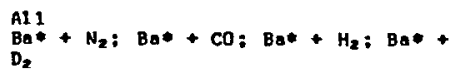 & E & $385 \mathrm{~K}$ & 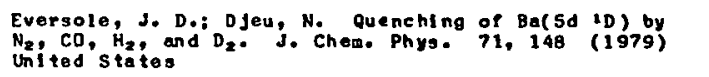 \\
\hline 923 & $\mathrm{AC}+\mathrm{He}$ & T & $0.01-1.0 \bigcirc 0$ & 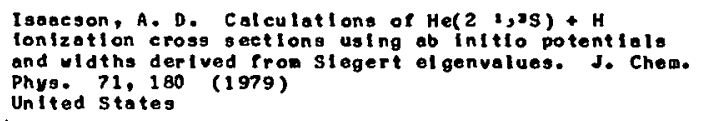 \\
\hline 924 & 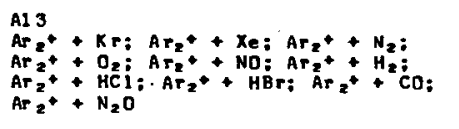 & E & $30 \cap K$ & 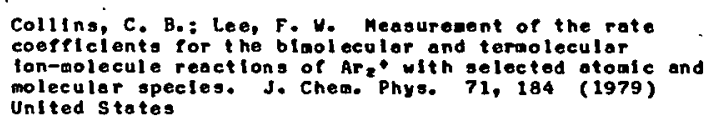 \\
\hline 925 & $\mathrm{NH}_{2}+\mathrm{H}_{\mathrm{i}} \mathrm{H}_{2}+\mathrm{Rh} ; \mathrm{N}_{2}+\mathrm{V}: \mathrm{N}_{2}+\mathrm{Rh}$ & E & $298 \mathrm{~K}^{\circ}$ & $\begin{array}{l}\text { Pollizeottl, R. S.: Ehrlleh, G. Cheal sorption on } \\
\text { perfect surraces: hydrogen, arid nit rogen on tungsten and } \\
\text { thodlua. J. Chem. Physe. 71, } 259 \text { (1579) } \\
\text { United states }\end{array}$ \\
\hline 925 & $\stackrel{\text { Al }}{\mathrm{H}}+\mathrm{H}+\mathrm{O}$ & 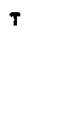 & Under & 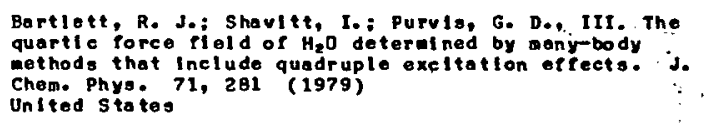 \\
\hline 927 & $\mathrm{LI}^{\mathrm{A} 7}+\mathrm{LI}$ & $T$ & Under & 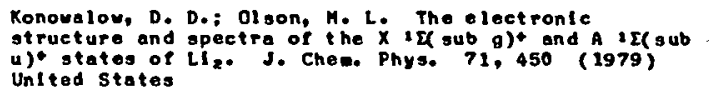 \\
\hline 928 & 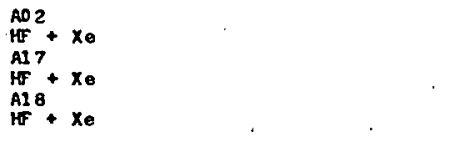 & ET & $0.04-3.15$ & 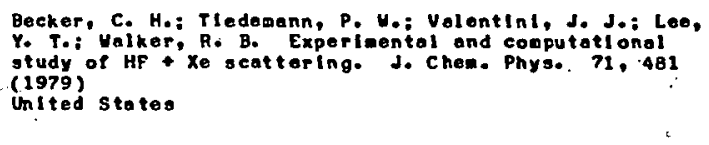 \\
\hline 929 & $\stackrel{\mathrm{A}^{*} 1}{\mathrm{H}^{*}}+\mathrm{H}_{2} \mathrm{O} ; \mathrm{O} *+\mathrm{H}_{2} \mathrm{O}$ & E & $294 K$ & 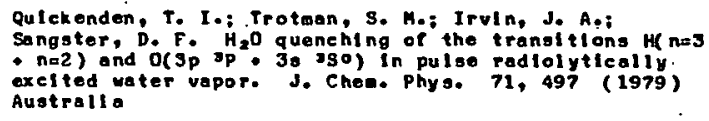 \\
\hline 930 & $\stackrel{\mathrm{A} 14}{\mathrm{O}}+\mathrm{CS}_{2}$ & $T$ & $0.1-0.25 \mathrm{av}$ & 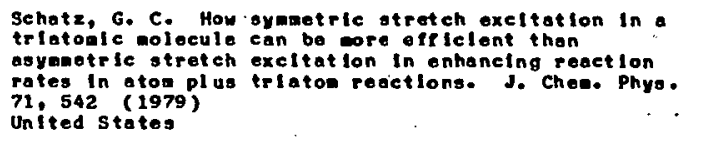 \\
\hline 931 & $\stackrel{A_{1}}{\mathrm{H}_{y}} \cdot \mathrm{My}$ & $\tau$ & Under & 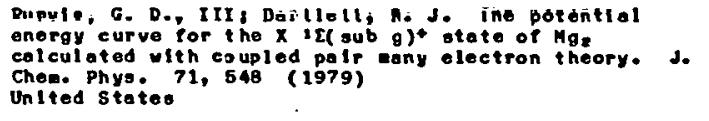 \\
\hline 932 & $\mathrm{HOS}_{\mathrm{H}+\mathrm{H}}^{\mathrm{H}} \mathrm{H} \mathrm{O}+\mathrm{H}_{2} \mathrm{O}$ & E & 675-255 na & 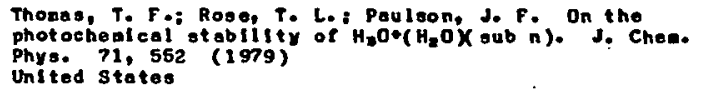 \\
\hline $\mathbf{9 3 3}$ & $\begin{array}{l}\mathrm{HO} 6 \\
\mathrm{hy}\end{array}$ & E & $780-740$ A $0^{\circ}$ & 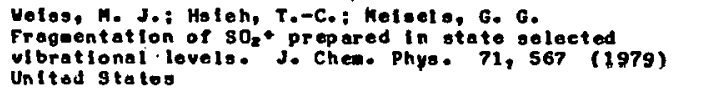 \\
\hline 934 & 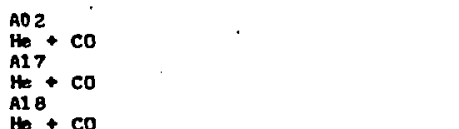 & $\top$ & $200 \mathrm{~cm}-2$. & 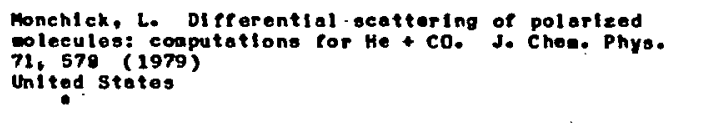 \\
\hline
\end{tabular}


70

\begin{tabular}{|c|c|c|c|c|}
\hline $\begin{array}{l}\text { REF. } \\
\text { NO. }\end{array}$ & REACT ANTS & $\begin{array}{l}\operatorname{EXP} \\
\text { OR } \\
\text { THEOR }\end{array}$ & $\begin{array}{l}\text { ENERGX } \\
\text { RANGE }\end{array}$ & REFERENCE \\
\hline 935 & 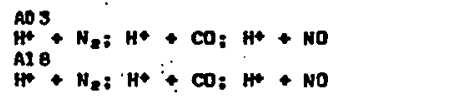 & E & $30-80 \cdot 8 \mathrm{~V}$ & 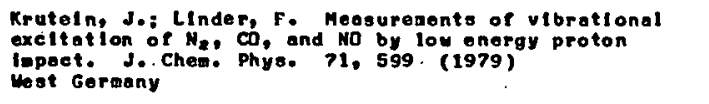 \\
\hline 936 & 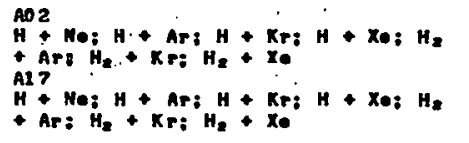 & E & $0.5-100-0.0 v$ & 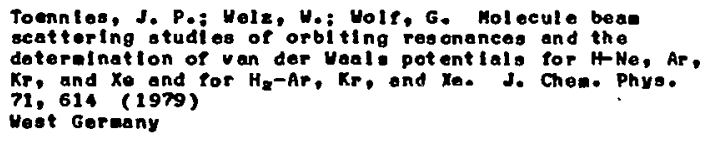 \\
\hline 937 & 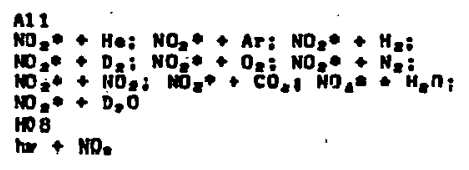 & $\varepsilon$ & $\begin{array}{l}\text { A12 } \\
300 \\
100 \\
689-473 n=\end{array}$ & 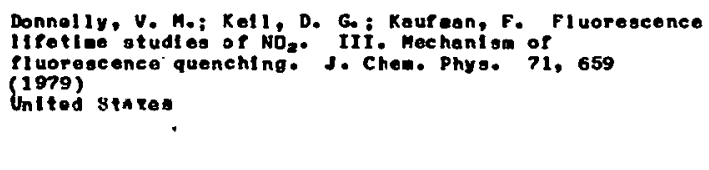 \\
\hline pap & 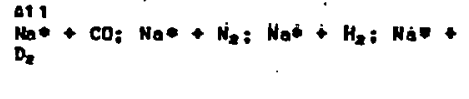 & z & $00-803-k$ & 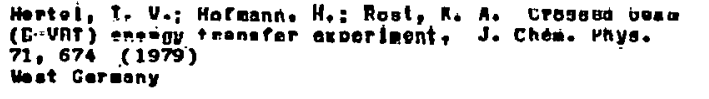 \\
\hline 939 & ins 5 + HBr* & E & $259 \mathrm{nn}$ & 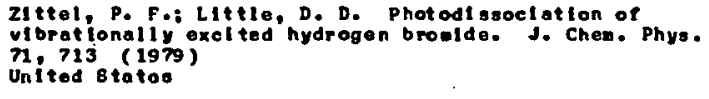 \\
\hline 940 & $\mathrm{Br}_{\mathrm{C}}+\mathrm{NO} ; \mathrm{Br}+\mathrm{Ar}$ & $\varepsilon$ & $298 \mathrm{~K}$ & 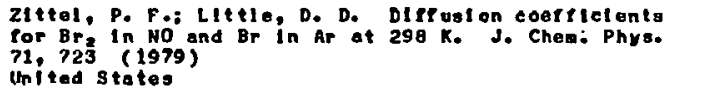 \\
\hline 941 & $S_{2}^{A 1 O}+H_{0}: S_{2}+$ Ar: $S_{2}+X_{0}$ & E & $900 \mathrm{~K}$ & 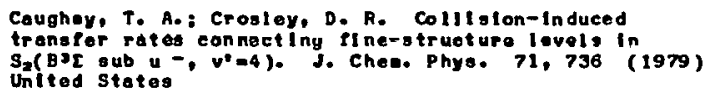 \\
\hline 942 & 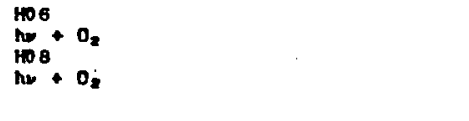 & E & $740-175$ A0 & 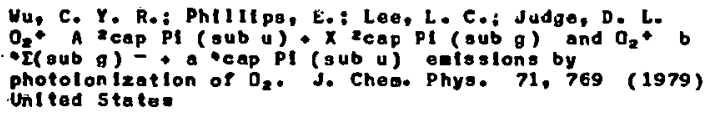 \\
\hline 943 & $m^{2}+0$ & E & $\begin{array}{l}3600-9350 \\
A^{\circ}\end{array}$ & 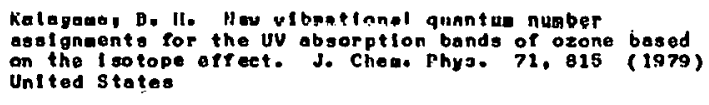 \\
\hline 244 & 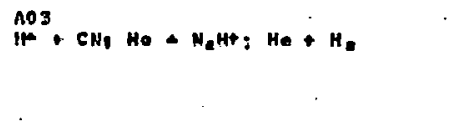 & $\uparrow$ & $0.04-100.0 V$ & 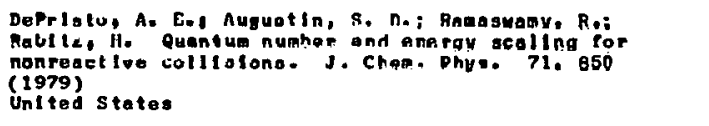 \\
\hline $945^{\circ}$ & $\cos _{2 n}+H_{2}: Z n+H_{0}: Z n+A r$ & $\varepsilon$ & $720-1120 \cdot K$ & 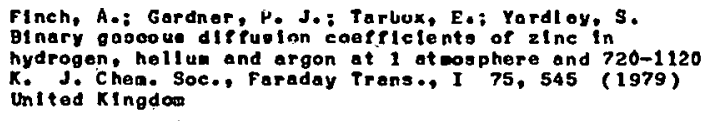 \\
\hline 946 & 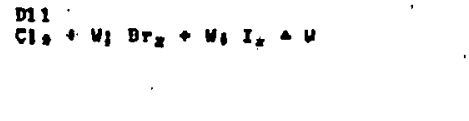 & E & $300-490 \cdot x$ & 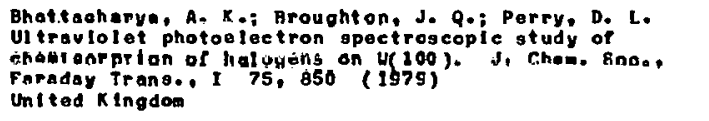 \\
\hline 947 & 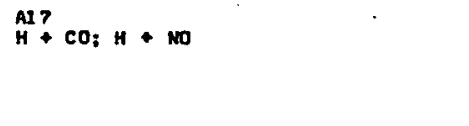 & $T$ & Under & 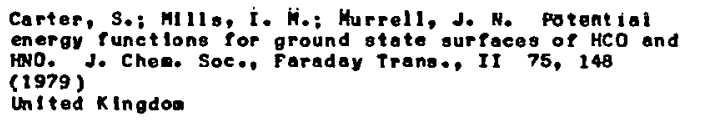 \\
\hline 948 & $\operatorname{mos}^{\mathrm{B}}+\mathrm{Cl}_{2}$ & E & $505 \mathrm{ne}$ & 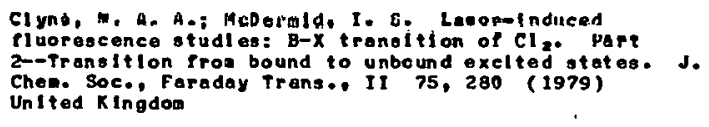 \\
\hline 949 & ${ }_{n+\infty}^{100}+\mathrm{Cs}_{2}$ & E & $120-60 \mathrm{~nm}$ & 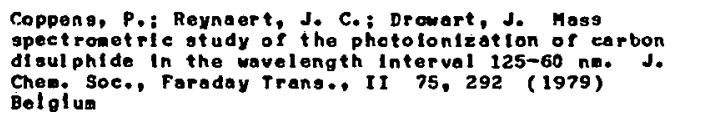 \\
\hline
\end{tabular}


71

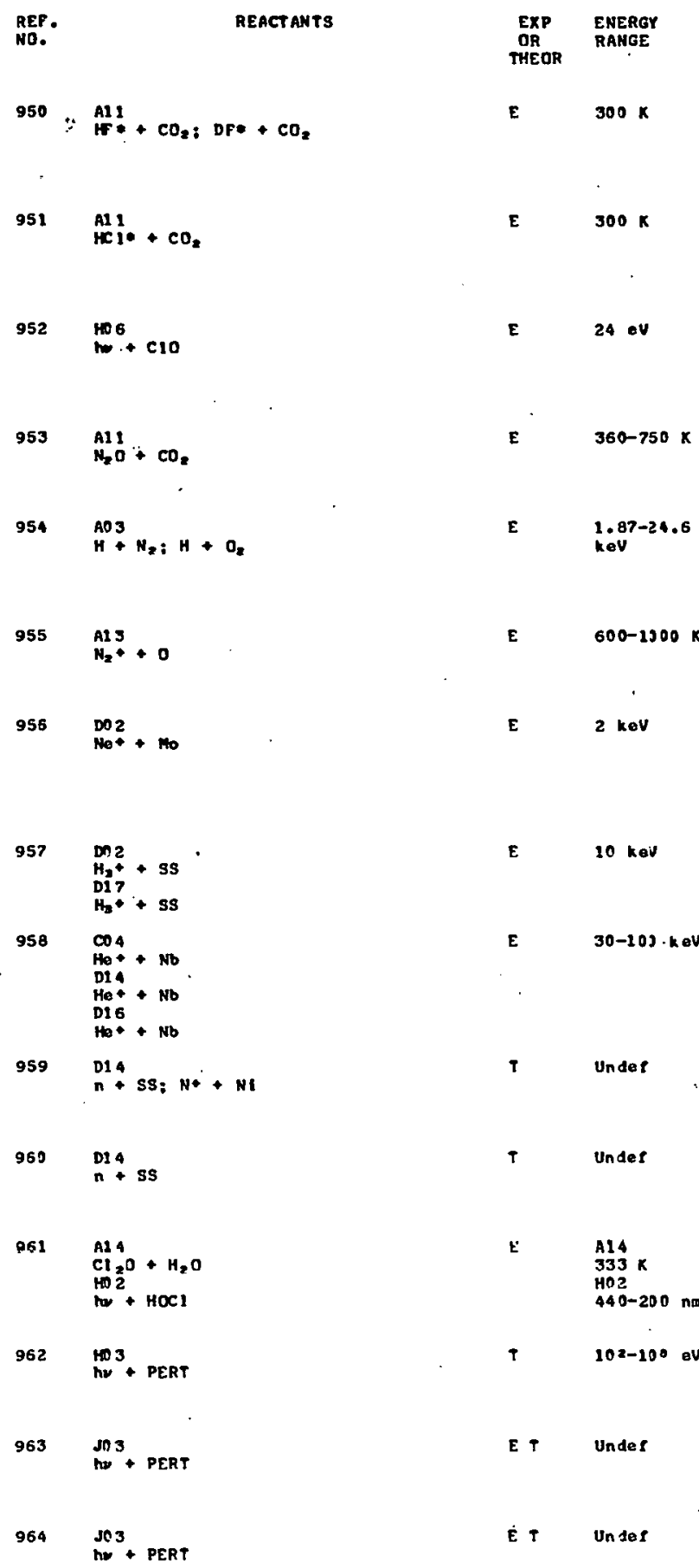

REF ERENCE

Brown, R. D. H.: Douglas, D. J.: Polanyl, J. C. Chemfl un Inescence-depletion of vibratlonal relaxation. Part 1.--HF( $\mathrm{V}=2-6) * \mathrm{CO}_{2}$, DF $(\mathrm{V}=2-4)+\mathrm{CO}_{2}$. J. Chea. Soc., Faraday Trans., II 75,422 (1979)

Bartoszek, F. E.: Brown, R. D. Ho: Polanyl, J. C. Chemil um Inescence-depletion of vibrational relaxat lon. Part 2.- $\mathrm{HCl}(\mathrm{v}=2-4)+\mathrm{CO}_{2}$, J. Chen. Soce, Faraday Trans., II 75,434 (1979)

Bulgin, D. K.: Dyke, J. M.: Jonathan, N.: Morefs, A. Vocuut ultraviolet photoelectron spectroscopy of transient specles. Part 9.-Clo( $X^{2}$ sub I ) radical. J. Chea. Soe., raraday Trans.. II 75,4

Foster, H. G.: SImpaon, C. J. S. M. Vibrotlonal energy trang fer between $\mathrm{N}_{2} \mathrm{O}$ and $\mathrm{CO}_{2}$. J. Chea. Sac., Foraday Unitod $x \operatorname{lng}$ don Sher Idan, J. R.: VIdaar, R. J. Exeltatlon of Balaer alpha and Lyean al pha om Issions in collisl ons of 1.87to $24,6-k e V$ hydrogen atong with $\mathrm{H}$, and $\mathrm{O}_{2}$. J. Geophys. Untied states

Torr, D. G. Refinement of the cerononlcally deteralned rate coefficlent for the reaction of $\mathrm{N}_{2}+$ uth $\mathrm{O}$. J. Goophy. Res. 84, 1939 (1979) United states

Yamads, R.: Saldoh, Mo; Sone, K.: Oht suka, H. Dose and al crostruetural of reets on surface topography change and oputtering field in poly crygtelit oomardaent il th $2 \mathrm{keV}$ No* lons. J. Nuel. Mater. 82 , 155 . (1979)

Japan

Salth, J. N.. Jr. Surface cleanup and sputtered lon production: observat lons with AES and SIMS. J. Nuel. Moter. 82, 179 (1979)

Unitod states

Rl seh, M. R.: Roth, J.: Scherzer, B. M. U. Dependence of bligter-deckeldicke and of depth protlles of Implanted He Ions In Nb on engle of Incidence. J. Nucl. Moter. 82, 220 (1979)

West Gerwany

Maydet, S. I.: Russell, K. C. . Numerlcal simulation of vold nucleation In Irradiated metalo. J. Nucl. Mater. 82,271 (1979)
Unitad states

Ghonten, N. Mo; Kuleinskl, G. L. The effect of damage rate on vold growth in metals. J. Nucl. Mater. 82 , $392(1979)$

Knauth, Ho-D.: Albertl, Ho: Clausen, H. Equllibrlun constant of the gas reaction $\mathrm{Cl}_{2} \mathrm{O}+\mathrm{H}_{2} \mathrm{O}=2 \mathrm{HOCl}$ and the ultravlolet spectrum of HOCl. $\mathrm{H}$. Phys. Chem. 83, 1604 (1979) West Germany

Hubbell, J. H.; Overbo, I. Relativistlc atomlc form Pactors and photon coherent scattering cross sections.

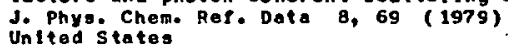

Krause, M. O. Atomic radiative and radiatloniess ylolds for $K$ and $L$ shells. J. Phy a. Chen. Ref. Data B. 307 (1979) Unlted states

Krauge, M. O.: Ollver, J. H. Natural uldths of atomle

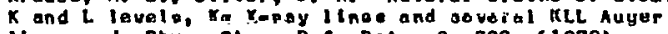
IInes. J. Phys. Ches. Ref. Dota O, 329 (1979) Untiod states 


\begin{tabular}{|c|c|c|c|c|}
\hline $\begin{array}{l}\text { REF. } \\
\text { No. }\end{array}$ & REACTANTS & $\begin{array}{l}\text { EXP } \\
\text { OR } \\
\text { THEOP. }\end{array}$ & $\begin{array}{l}\text { ENERGY } \\
\text { RANGE }\end{array}$ & REFERENCE \\
\hline 965 & $\mathrm{H}^{+2}+\mathrm{Te}_{\mathrm{O}} \mathrm{H}^{+}+\mathrm{MO}: \mathrm{D}^{+}+\mathrm{Ta}_{\mathrm{O}} \mathrm{O}+\mathrm{HO}^{2}$ & E & $0.6-15 \mathrm{keV}$ & 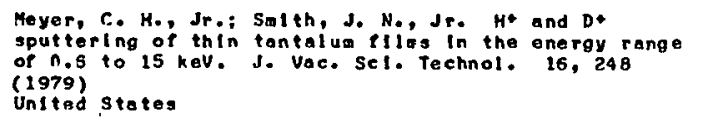 \\
\hline 956 & $\underset{A_{r}+}{D r^{+}}+T S: D+T I$ & $\varepsilon$ & $0.5-2.0 \mathrm{keV}$ & 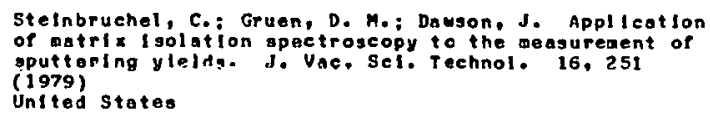 \\
\hline 967 & $D_{0}^{\mathrm{D}}+\mathrm{O}+\mathrm{NO}$ & $E$ & Under & 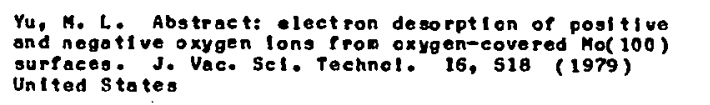 \\
\hline 958 & ${ }_{n=5}^{\infty}+T_{10}$ & ET & $\operatorname{lv}^{16,8-? h \cdot 2}$ & 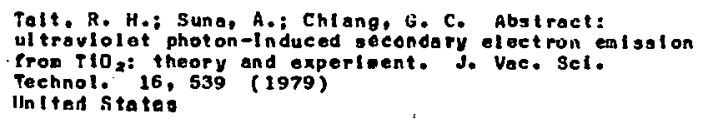 \\
\hline 969 & $\sum_{n=0}^{\infty 5}+\mathrm{Cu}$ & E & Undal & 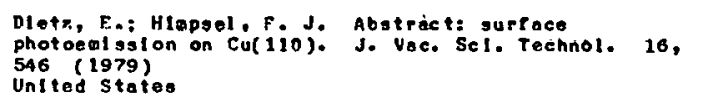 \\
\hline 970 & $\operatorname{DD}_{\text {In }}^{\mathrm{DI}}+\mathrm{In} ; \mathrm{CO}_{2}+\mathrm{In} ; \mathrm{H}_{2} \mathrm{O}+\mathrm{In} ; \mathrm{O}_{2}+$ & $\varepsilon$ & Under & 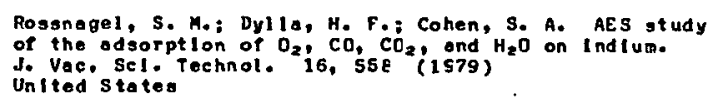 \\
\hline 971 & 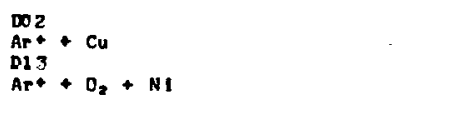 & E T & $600 \mathrm{ev}$ & 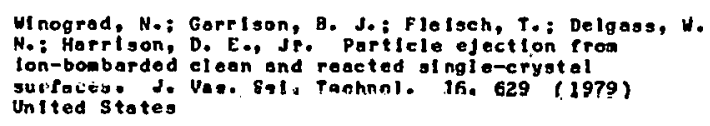 \\
\hline yirz & 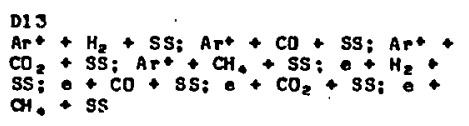 & $E$ & $\operatorname{sno-1000} 00$ & 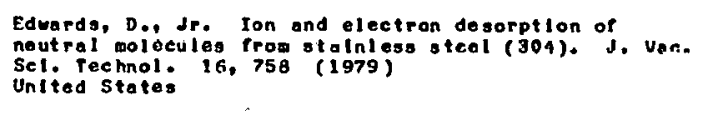 \\
\hline 973 & $\begin{array}{l}\text { nn? } \\
\text { Undor }\end{array}$ & $T$ & Under & 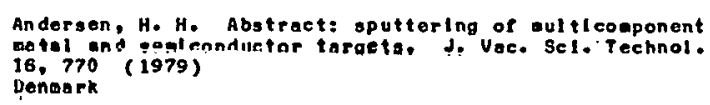 \\
\hline 970 & $\mathrm{Mr}^{\mathrm{P}}+\mathrm{Al}+\mathrm{Pd}+\mathrm{St}$ & E & $1 \mathrm{kaV}$ & 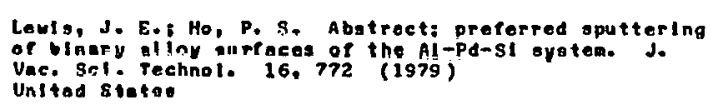 \\
\hline 975 & 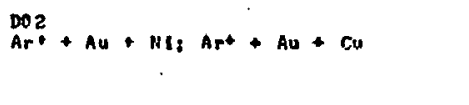 & $\varepsilon$ & $1 \mathrm{keV}$ & 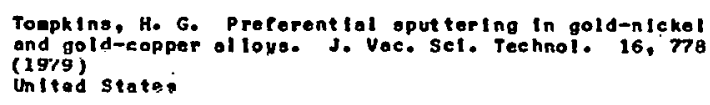 \\
\hline 976 & 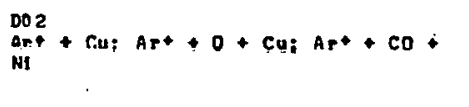 & $\mathbf{T}$ & ons ev & 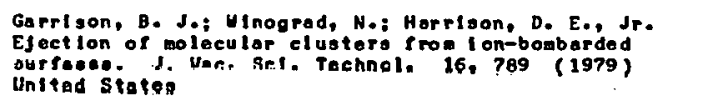 \\
\hline 977 & 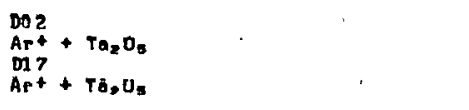 & B & $0,5=5.0 \mathrm{an}$ & 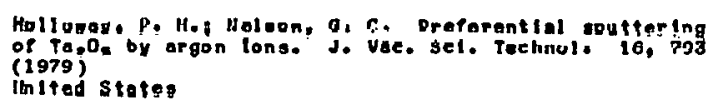 \\
\hline 978 & $\mathrm{DAr}_{\mathrm{Ar}+\mathrm{F}}+\mathrm{Cr}+\mathrm{NI} ; \mathrm{Art}+\mathrm{SS}$ & f. & $3 \mathrm{kev}$ & 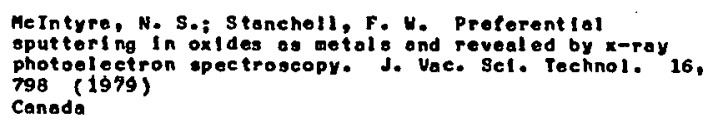 \\
\hline 979 & $\begin{array}{l}\mathrm{DO} 2 \\
\mathrm{Ar}+\mathrm{NB} \\
\mathrm{DA} \mathrm{N}^{+}+\mathrm{NBO} \\
\mathrm{Ar}+\mathrm{NBO}\end{array}$ & $\varepsilon$ & $15 \mathrm{keV}$ & 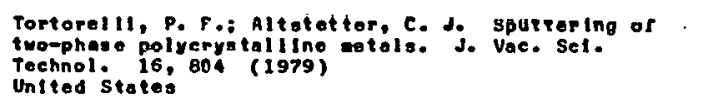 \\
\hline 980 & $\begin{array}{l}\mathrm{Dn}_{2} \\
\mathrm{Ar}_{2}+\mathrm{Cu}+\mathrm{NI} \\
\mathrm{DI}_{1} ?+\mathrm{Ag}+\mathrm{Au}\end{array}$ & $\varepsilon$ & Undar & 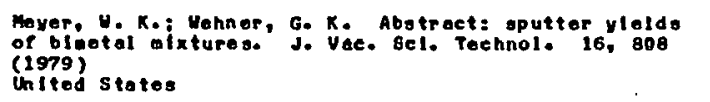 \\
\hline
\end{tabular}




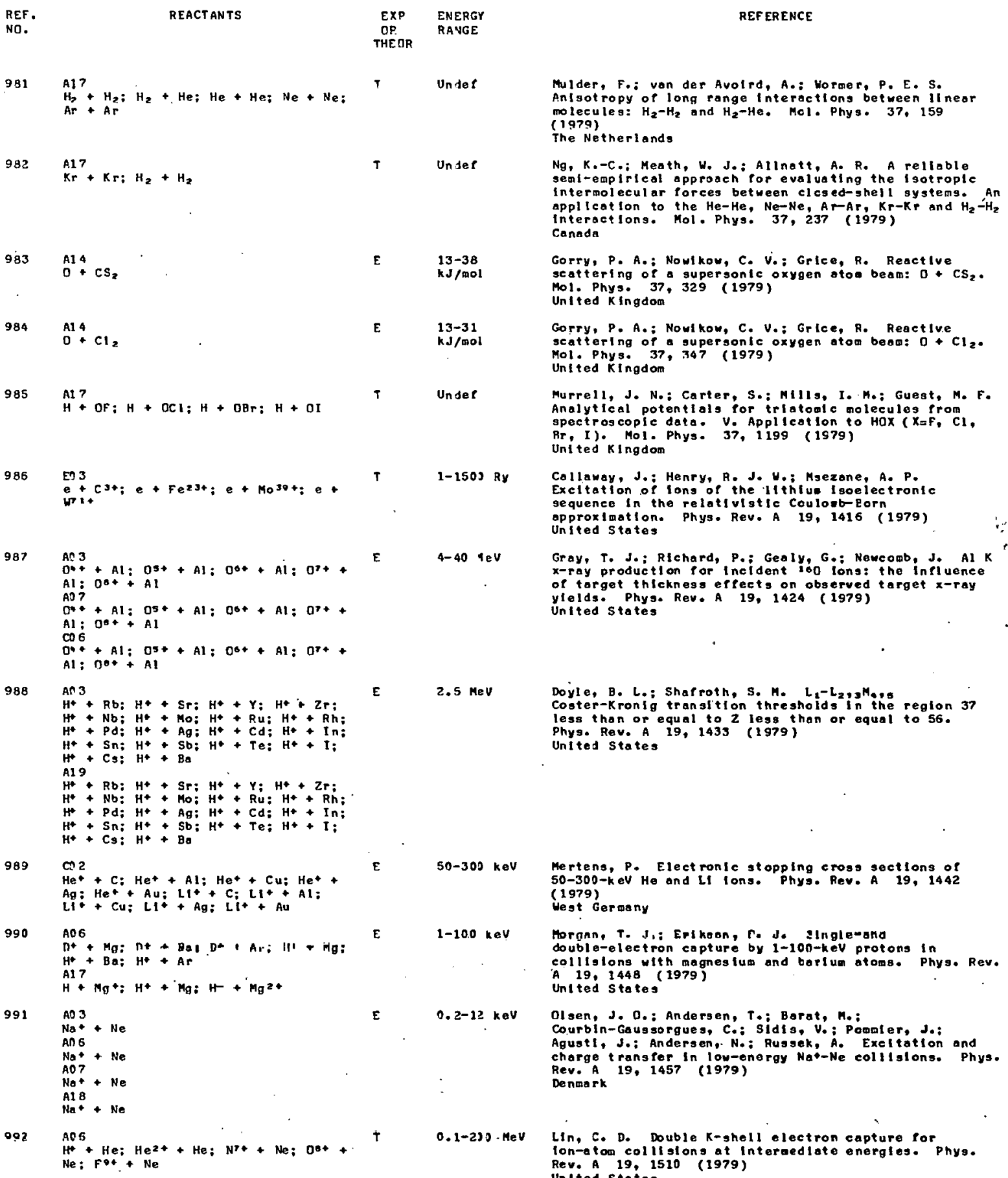

$0^{A+3}+\mathrm{Al}^{3}: \mathrm{O}^{3+}+\mathrm{Al}: \mathrm{O}^{3+}+\mathrm{Al}: \mathrm{O}^{2+}+$ Al: $0^{\circ+}+A_{1}$

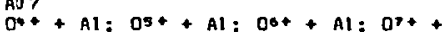
A1; $\mathrm{O}^{\circ+}+\mathrm{Al}$

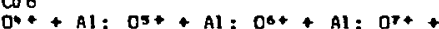
A1: $000+$

$\mathrm{H}^{+}+\mathrm{Rb}_{\mathrm{O}} \mathrm{H}^{+}+\mathrm{Sr}: \mathrm{H}^{*}+\mathrm{Y}_{\mathrm{Z}} \mathrm{H}^{+}+\mathrm{Zr} ;$

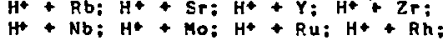
$\mathrm{H}^{+}+\mathrm{Nb}_{\mathrm{O}} \mathrm{H}^{+}+\mathrm{Mo} \mathrm{Hg}^{+} \mathrm{H}^{+}+\mathrm{Ru}^{+} \mathrm{Hd}^{+}+\mathrm{H}^{+}+\mathrm{Rh}_{3}$ $\mathrm{H}^{+}+\mathrm{Sn}_{\mathrm{O}} \mathrm{H}^{+}+\mathrm{Sb}^{+} \mathrm{H}^{+}+\mathrm{Te}_{\mathrm{O}} \mathrm{H}^{+}+\mathrm{I}$; $\mathrm{H}^{*}+\mathrm{Cs}_{3} \mathrm{H}^{+}+\mathrm{BO}^{+}$

A1 9

$\mathrm{H}^{*}+\mathrm{Rb} ; \mathrm{H}^{+}+\mathrm{Sr} ; \mathrm{H}^{+}+\mathrm{Y}_{3} \mathrm{H}^{+}+\mathrm{Zr} ;$ $\mathrm{H}^{+}+\mathrm{Nb}: \mathrm{H}^{+}+\mathrm{Ho}^{+} \mathrm{H}^{+}+\mathrm{Ru}: \mathrm{H}^{+}+\mathrm{Rh}^{+}$ $\mathrm{H}^{+}+\mathrm{Pd}_{3} \mathrm{H}^{+}+\mathrm{Ag}^{+} \mathrm{H}^{+}+\mathrm{Cd} ; \mathrm{H}^{+}+\mathrm{In}^{+}$ $\mathrm{H}^{+}+\mathrm{Sni}^{+} \mathrm{H}^{+}+\mathrm{Sb}^{+} \mathrm{H}^{+}+\mathrm{Te} ; \mathrm{H}^{+}+\mathrm{I} ;$

989

990

991

$\mathrm{Cn} 2$

$\mathrm{He}^{+}+\mathrm{C} ; \mathrm{He}^{+}+\mathrm{Al} ; \mathrm{He}^{+}+\mathrm{Cu}_{\mathrm{H}} \mathrm{He}_{+}^{+}$

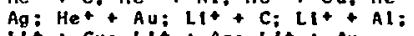

${ }^{A+}+\mathrm{Mg}_{\mathrm{g}} \mathrm{nt}+\mathrm{Bar} \mathrm{D}+$ A Ar; II + $\mathrm{Hg}_{\mathrm{g}}$

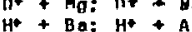

A1 7

$\mathrm{H}^{+} \mathrm{Mg}^{+} \mathrm{O} \mathrm{H}^{+}+\mathrm{MO}^{+} \mathrm{H}^{+}+\mathrm{Mg}^{2}+$

E $\quad \begin{aligned} & 13-38 \\ & \mathrm{~kJ} / \mathrm{mol}\end{aligned}$

E 13-31

kJ/mial

Under

1-1503 Ry

E

$4-40$ ieV

$$
2.5 \mathrm{MeV}
$$

$1-10.0 \mathrm{keV}$

$0.1-230 \cdot \mathrm{MeV}$$$
\mathrm{Na}+\mathrm{Ne}
$$

AQ 5 $\mathrm{Ne}: \mathrm{Fot}^{2}+\mathrm{He}$

Mulder, F.: wan der Avolrd, A.: Wormer, P. E. S. Anlsotropy of long range Interactions between lineor molecules: $\mathrm{H}_{2}-\mathrm{H}_{2}$ and $\mathrm{H}_{2}-\mathrm{He}_{0}$. Mol. Phys. 37, 159 (1979) The Netherlands

Ng, K.-C.: Meath, W. J.: Allnatt, A. R. A rellable seai-empirical approach for evaluating the fsotrople intermolecular forces between clcsed-shell systems. An tnteractions. Mol. Phys. 37, 237 (1979) Canada

Gorry, P. A.: Nowlkow, C. V.: Grlce, R. Reactlve scattering of a supersonic oxygen otoa beam: $0+\mathrm{Cs}_{2}$. Mo1. Phys. 37, 329 (1979) United $K$ ingtom

Gorry, P. A.: Nowikow, C. V.: Grlce, R. Reactlve scatterling of aupersonic oxygen oton beas: $\mathrm{O}+\mathrm{Cl}_{2}$. Mol. Phys. 37, 347 (1979)

Murrell, J. N.: Carter, S.; Mills, I. M.; Guest, M. F. Analytical potentials for triatomic molecules from spectroscople data. V. Application to HOX ( $X=F, C l$, Ar, I). Mol. Phys. 37, 1199 (1579) United KIngdon

Collaway, J.: Henry, R. J. U.: Msezone, A. P. Excltation of lons of the lithiue Isoelectronic sequence in the relativistle Coulonb-Ror approximation. Phys. Rev. A 19,1416 (1979) Gray, T. S.: Rlchard, P.: Gealy, G.: Neweomb, J. Al $K$ $x$-ray production for Incident 100 lons: the influence of target thlekness effects on observed target $x-r a y$ ylelds. Phys. Rev. A 19, 1424 (1979)

Doyle, B. L.: Shaf roth, S. M. $L_{2}-L_{2}, 9 M_{0}$, s Coster-Kronig transition threshold in the region 37 less than or equal to 2 less than or equal to 56 . Phys. Rev. A 19, 1433 (1979)

United States

Mertens, P. Electronle st opping eross sectlons of 50-300-keV He and LI lons. Phys. Rey. A 19, 1442 (1979)

West Germany

Morgnn, T. Ju: Erikeon, r. Je slngle-sho double-eleetron capture by $1-10 n-k e v$ protons in colllstons with magnestum and batium atoms. Phyg. Rev. A 19,1448 (1979)

United States

Olsen, J. O.: Andersen, T.: Barat, M.:

Courbin-Gaussorgues, C.: Sidis, V.; Pommler, J.: Agust1, J.; Andersen,. N.: Rusgek, $A_{\text {. Exeltat }}$ : En and charge transfer in low-energy Nat-Ne collisions. Phya. Rev. A

Lin, C. D. Double K-shell electron capture for ton-ator collistons at Interaed late energles. Phyg. Rev. A 19, 1510 (1979) 
REF.
NO.

REACTANTS

993

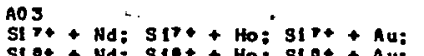

Slot + Nd: siot + Ho: slou + Au:

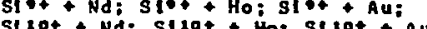

Sll1t Nd; Sllat: Ho: SI

Sl 12+ + Nd: Slaze + Ho: Sl12t: Au:

Fot + Nd; Fot + Ho: Fot Sl Au; Fou:

Nd Fot

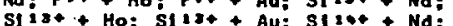

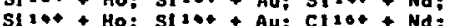

Cliot + Ho: Cl $100+$ Au

A0 6

SI

Slot + Nd: slot Ho: slot: Au;

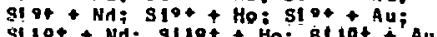

Sl $10+$ + Nd; Sllot + Ho; 81 lat + Au

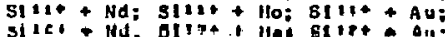

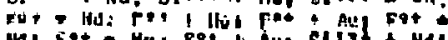

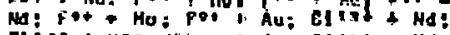

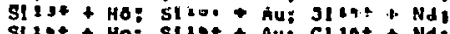

Sil $20++\mathrm{Ho}$ : Silot + Au; Cl $10+$ + Nd:

AD?

SI

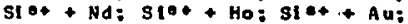

Slot + Nd: Slot + Ho: Slot + Aus

Sl $10+$ + Nd; Sllot + Ho: Sl $10+$ + Au

Sllt+ + Nd: SIlle + Ho; sl llt + Au

S1224 + Nd; Sl22t Ho: S122+ + Au:

Fot + Ndt Foot t Ho: Fot t Ay: Fot

$\mathrm{Nd}: \mathrm{FO}+\mathrm{Ho}$ : Fot + Au: Slist + $\mathrm{Nd}$

Slist + HOZ Gl130 + Auj Sllot + Nd;

Sl120t + Ho: Sllot + Au; Cl10+ + Nd;

Al19

Ho: $517+$ + Au:

Sl ot + Nd: S10+ Ho: Slot + Au:

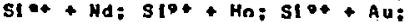

$\mathrm{SI}^{20+}+\mathrm{Nd}$ : Sl $10+$ + Ho: Sllot + Au

Sl11+ + Nd: Sl11+ + Ho: SI11+ + Au

SI 12t + Nd; SII2t + Ho; Sli2t + Au

Fot + Nd; Fot + Ho: Fot + Au: Fot

Nd: Fot + Ho: Fot + Au: Silst + Nd

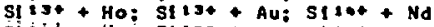

silibt + Ho: Slive + Au: Cliad + Nd

Cllot + Ho; Cl10+ + Au

994

995

yys

997

998

999
E11

e+A1; e+U

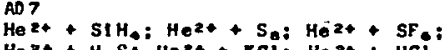

$\mathrm{He}^{2+}+\mathrm{H}_{2} \mathrm{~S} ; \mathrm{He}^{2+}+\mathrm{KCl}$ : $\mathrm{He}^{2+}+\mathrm{HCl}$

A19

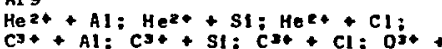

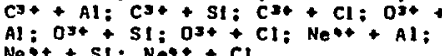

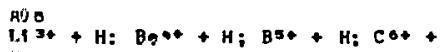

(

En 1
Under

AL 3. $+112,115+A T$

E) 1

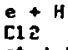

$0^{*}+H$
EXP ENERGY

THEOR

RANGE

REFERENCE

E $\quad 33-65 \mathrm{MeV}$

MeDantel, F. D.; Toten, A.; Peterson, R. S.; Duggan,

L.j Hilson, S. R.; Gressett, J. D, i Mlller, P. D.;

target $\mathrm{L}$-shell Ionlzation by 1.86 - Mev/anu fluorine and

silicon tons and 1.8-mev/amu chlorine lons. Phys. Rev. A 19, 1517 (1979)

Untied States
5-10 $\mathrm{reV}$

6. 4-114 MeV

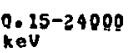

Undat

$0.05-0.30$

$-1$

$r$

Under

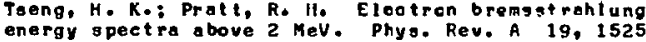
(1979)

Wotson, R. L.: Sonobe, B. I.: Deworest, J. A.: Langenberg, A. Systematics of the overage $L$-shel lonization probability in K-shell lonlzing collisions by light lono. Phys. HAu. A 19. 1529 (1570) United states

Ryufuku, H.: Watanabe, T. Charge-trangler erass

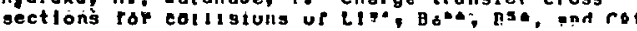

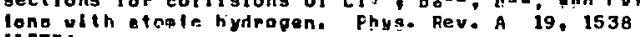
(1979)

Yatés, A. C. Mrgin-eneryg lilgliormarder ponen approx Imat lons: theoretical developalent. Phys. Rev. A 19,1550 (1979)

Baer, M.; Beswick, J. A. Electronlc transitlons In the toa-aglarimla reantlon (Art + Ha golng to or comling from

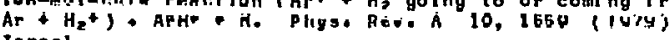
Arongon, 1.: Blau, R.: Klelnmon, C. J.: Rosenberg, L.;
Bpruah, L. Application of a verlational prinelple Cor the geattering length for the target wave function 1mpreelsely knuwh. Phys. Hov. A 19, 1sha (1979) 


\section{5}

\begin{tabular}{|c|c|}
\hline $\begin{array}{l}\text { REF. } \\
\text { NO. }\end{array}$ & REACTANTS \\
\hline 1000 & ${ }_{2 n v}^{\text {HOG }}+C_{s ;} 3 h v+X$ \\
\hline 1001 & $\mathrm{HOB}_{\mathrm{H}}^{\mathrm{No}}+\mathrm{Na} \mathrm{hr}+\mathrm{Ba}$ \\
\hline 1002 & $\begin{array}{l}\text { Hod } \\
\text { ho }\end{array}$ \\
\hline
\end{tabular}

1003 A11

1004 Hod

$1005 \cdot$ Ho8

1006 HOS

$1007 \quad 502+40$

1008

$\mathrm{Pl}^{\mathrm{Al}}+\mathrm{Pb} ; \mathrm{Sn}+\mathrm{Sn}$

1009

EO 2
E. Ho
ED 3 H०
E. Ho
E17

E17 He

1010. A03

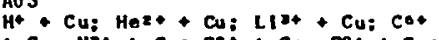

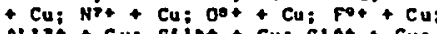

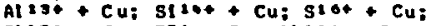

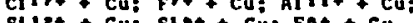

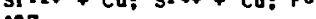

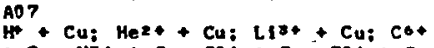

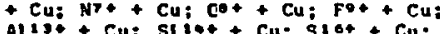

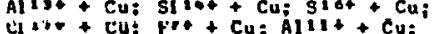

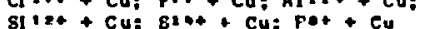
Alg + Cu; Brot + Cu; por + Cu A19

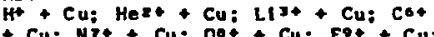

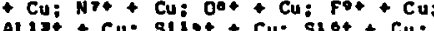

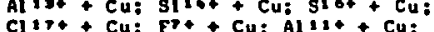

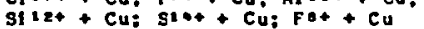

1011 Al?

Positronlum $+H$

$1012 \quad \begin{array}{ll}E 03 \\ \vdots \\ E 17 \\ B+H\end{array}$

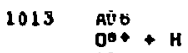

A07 $+\mathrm{H}$
EXP

THEOR

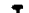

Under

Under

Under

$0-16$ ov

$0-1.5 \mathrm{eV}$

$30-103-e V$

$\operatorname{MeV}^{1.7-59.5}$

E

Under

$300 \mathrm{~K}$

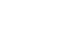

ENERGY

$20550-33240$ -

$35012 \mathrm{~cm}^{-1}$

$0.28-0.30$

Ry

n-tnn) . ov

$1-5003 \cdot \mathrm{keV}$
REF EREMCE

DIxIt, S. M.: Laboropoulos, P. LI no-proflle considerations of rosonont puit linotoproptie Phys. Rev. A 19, 1576 (1979) Unyitod Stotos

Leuls, D. A.: Tonn, J. F.: Kaufaan, S. L.: Greenlees, G. 4 . Photon-burst cethod in hl gh-resolution laser G. Photon-burst method in h1 gh-resolution
spectroscopy. Phys. Rev. A 19, 1580 (1979) onited States

Dlels, J.-C.: Georges, A. T. Coherent two-photon resonant thi rd- and fi fth-haraonle vacuu-ultrov folet generation in motal vapors. Phys. Rev. A 19, 1589 United

Flusberg, A.: Kachru, R.: Mossberg, T.: Hortmann, S. R. Forelgn-gas-induced rolaxation of Fydberg $S$ and $D$ states

United States

Kelsey, E. J. Cal culation of certain of ngle-photon double-excit at ion amplitudes of high-Rydberg statee in wheh long-range corrolation effecta dodinate. Phys. Rev. A 19, 1645 (1979)

United stater

Mayfeh, M. H.: Nayfeh, A. H. Double resonance with pulsed flelds. Phys. Rev. A 19,1666 (1979) United States

Robln, Y.: Ben-Reuven, A. Theory of resonance excltation of $\mathrm{N}-\mathrm{level}$ atoale systeng by strong coherent rediation. Phys. Rev. A 19, 1697 (1979) Iorael

Sinfallad, A. L.: Nosbet, R. K. Erratue Vartatlonal calculat lons on ol ectron-hel ius ecttering [Phys. Rev. A $6,2118(1972)$ ]. Phys. Rov. A 19, 1808 (1979) United states

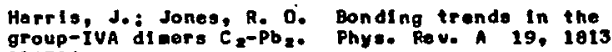
(1979) West Gorwany

Ehadra, K.; Callaway, J.; Honry, R. J.U. Electron-lapact excliation of $n=2$ level s op hell un a interaediate onerglog. Phys. Rev. A 19, 1841 (1979)
Untted States

Jamlaon, K. A.: Hall, J. H. Cu K xray production as a funetion of projectile atolle number, energy, and incldent charge st at 0 . Phys. Rev. A 19, 1896 (1979) United stateo Drachman, R. J. Aut odlasoci ot Ing Rydberg setates of
positron iun hydride. Phys. Rev. A 19,1900 (1979) positron Iun hydr

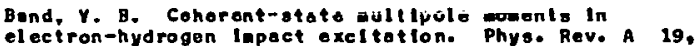
el ectron-hydrog United States

Salop, A.: Olson, R. E. Electron renoval frow atoale hydrogen by collisiong utth fully strlpped oxygen lone. hydrogen by collislong with fully
Phys. Rev. A 19, 1921 (1979) United States 


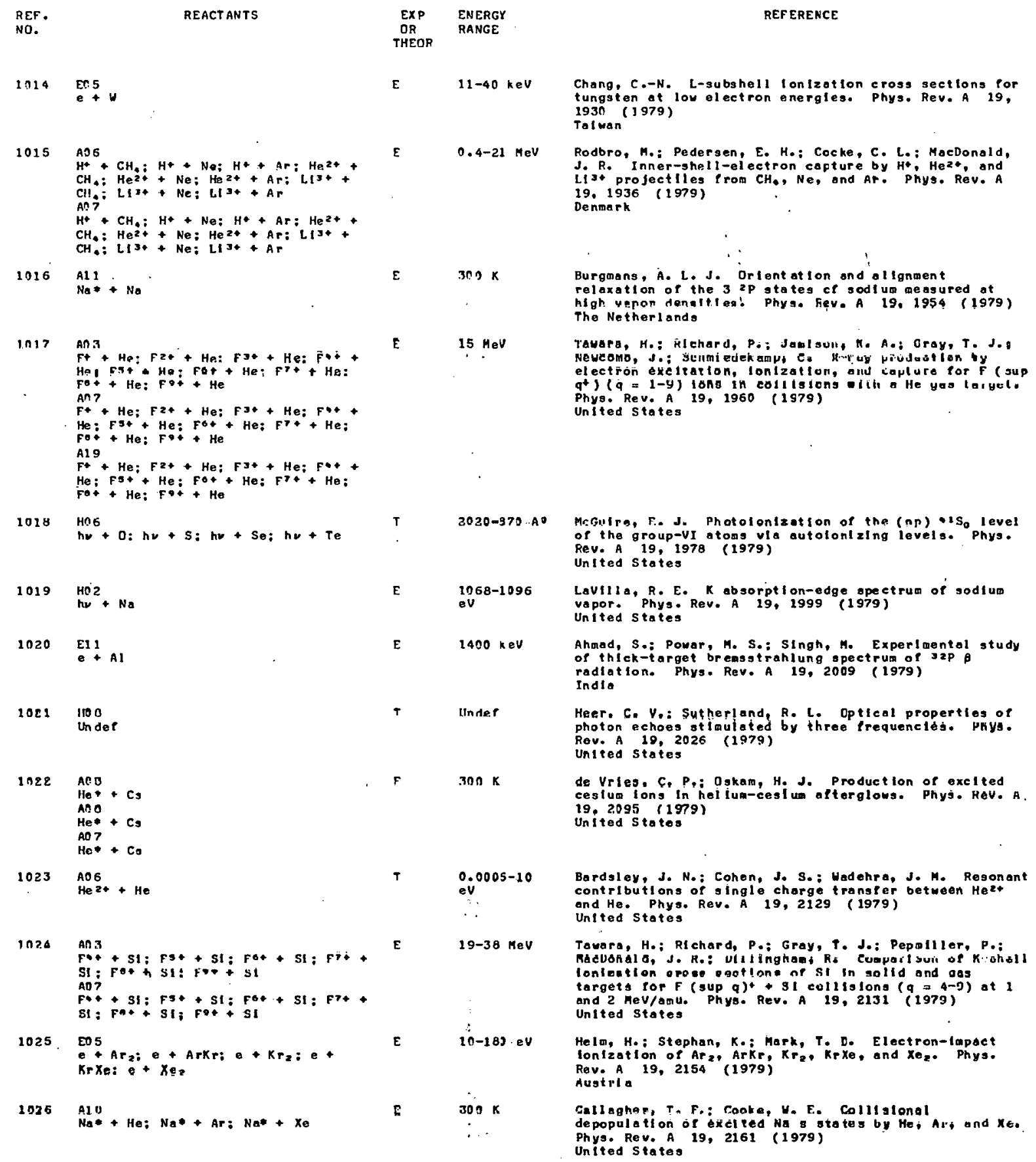

REF ERENCE

Chang, C.-N. L-subshell lonizotion cross sectlons for tungsten at lou electron energles. Phys. Rev. A 19, 1930 ( 1979 )

Tol wan

Rodbro, M.; Pedergen, E. H.; Cocke, C. L.: MocDonald, J. R. Inner-shell-electron capture by $\mathrm{H}^{+}$, $\mathrm{Hez}^{+}$, and List projectlles from CH., Ne, ond At. Phys. Rev. A 19. 1936 (1979) Denmark Burganas, $A$. L. J. Orlent at I on and allgnment
relaxation of the 3 ip gtateg cr sod I um measured at relaxation of the 3 2P states ef sodt um measured at
high wepor denalfies! Phys. Fẹ. A 19, 1954 (1979) The Netherlands

Tawser, H.: Flehard, P.: Jamlsull, M. A.: Oray, T. J.S

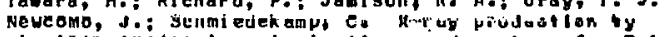
el ectrón excizazion, tontzation, an caulure for $f$ (sup

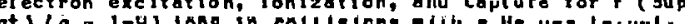
Phys. Rev. A 19, 1960 (1979) United States

MrGuirs, F. J. Photolionlaetion of tha $(n p)$ is of the group-VI atoms vila autolonlelng levels. Phys. Rev. A 19, 1978 (1979) United states

LaVilla, R. E. K absorption-edge spectrum of sodium vapor. Phys. Rev. A 19,1999 (1979)
United States

Ahmad, S.: Powar, M. S.; SIngh, M. Experl mental study of thick-target bremsstrahlung spectrum of $32 p$ radiation. Phys. Rev. A 19, 2009 (1979) India

Heer. C. V.: Sutherland, R. L. Optleal propertles of photon echoes st inulated by three Prequencids. PKys. Rev. A 19, 2026 (1979) United states

de Vries, C, P,; Oskam, H. J. Production of exclted cestum lons in hel ium-cesl un afterglows. Phys. HeV. A. $19.2095(1979)$

Bardsley, J. N.: Cohen, J. S.: Wadehra, J. M. Regonant contributions of single charge transfer between $\mathrm{He}^{2+}$ and He. Phys. Rev. A 19, 2129 (1979) United States

Tovara, H.: RIchard, P.: Gray, T. J.: Pepolller, P.:

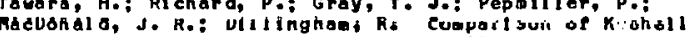
Conieation orees eqotione ne si in solld and gas targets for $F($ sup $q)+31$ collistons $(q=4-9)$ at 1 and 2 mev/anu. Phys. Rev. A 19, 2131 (1979) United States

Hela, H.: Stephan, K.: Mark, T. D. Electron-lapact conleation of Ar, ArKr, Kre, $k r X e$, and $X e_{z}$. Phys. Rev. A 19,2154 (1979) Austria

GallagheF, T. F.: Caake, H. E. Colllgtonal

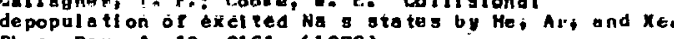
Phys. Rev. A 19, 2161 (1979) United States 


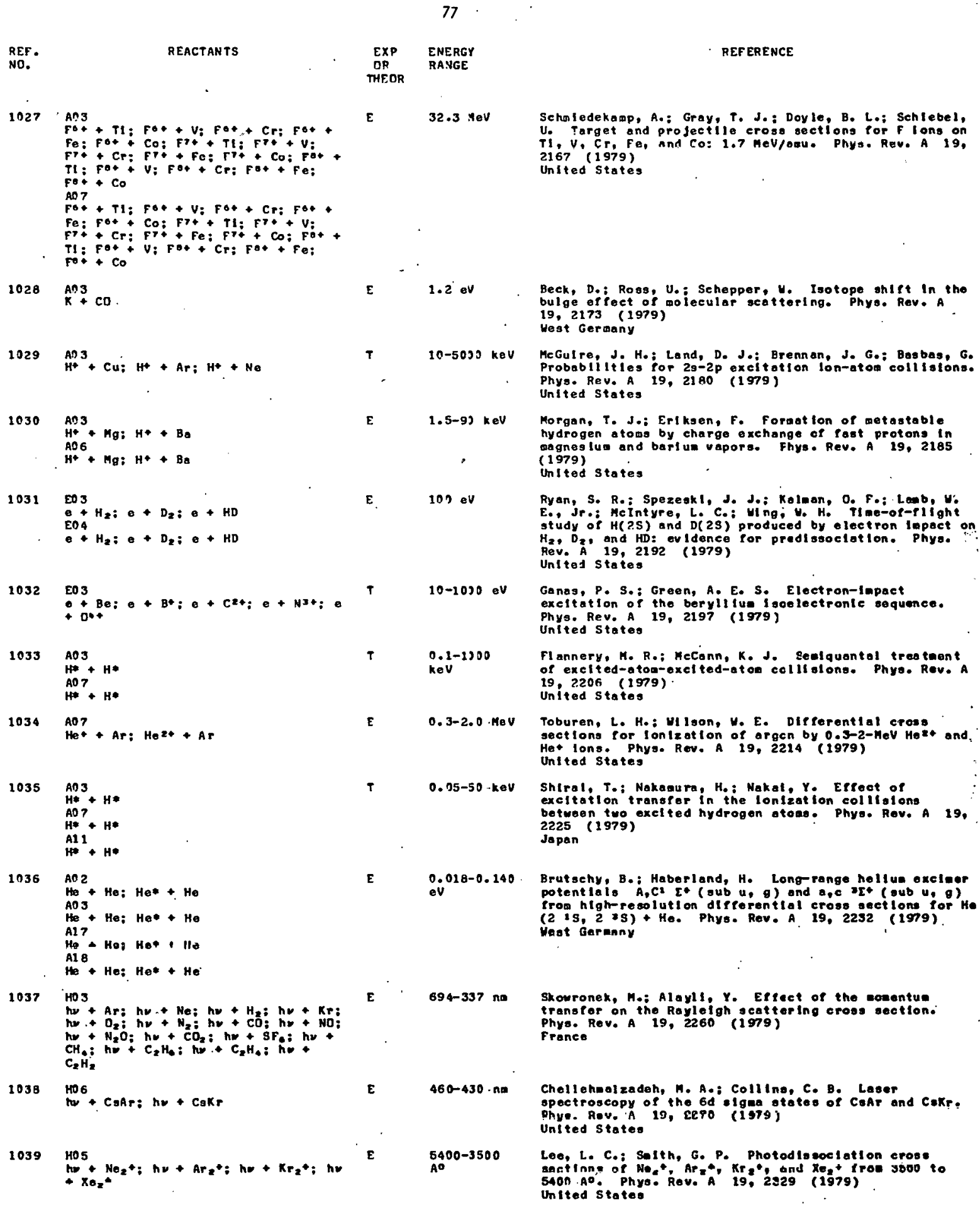

HO5 $\mathrm{HOS}_{4}+\mathrm{Ne}_{2}+\mathrm{HD}+\mathrm{Ar}_{2}^{+*} \mathrm{hl}+\mathrm{Kr}_{2}+\mathrm{HV}$

$0.1-1300$

$0.3-2.0 \cdot \operatorname{MeV}$

$0.05-50-k e v$

Beck, Do: Ross, U.: Sehepper, Y. Isotope shlet In the bulge of feet of molecular scettering. Phys. Rev. A $19,2173$ ( 1979$)$

Scholedekamp, A.: Gray, T. J.: Doyle, B. L.: Sehlebel, U. Target and pro Jectlle eroso sectlong for F lons on 2167 (1979)

MeGulra, J. H.: Land, D. J.: Brennan, J. G.: Bosbas, G. Probabilities for 2g-2p excitotion lon-atom collistons. Phys. Rev. A 19, 21 BO (1979)

Morgan, T. J.: Erikgen, F. foraot ton of metastable hydrogen atoms by charge exchange of past protong in wagnes lue and barlum vapors. Fhys. Rev. A 19, 2185 (1979)

Unlted States

Ryan, S. R.: Spezeokl, J. J.: Rolmon, O, F.: Low U: E.: Jr.: HeIntyre, L. C.: en United states

Ganas, P. S.: Green, A. E. S. Electron-iapact excltation of the berylliug i goelectronle sequence. Phys. Rev. A 19, 2197 (1979) United States

FIannery, M. R.; MeConn, K. J. Seliquantol treateent of exclted-atoo-exel tad-aton collislono. Phyo. Rev. A $19,2206(1979)$

United 5 tates

Toburen, L. Ho: HI Ison, W. E. Differentlal eross sectlong for lon lzat Ion of argen by 0.3-2-MeV Hezt and, Het Ions. Phys. Rov. A 19, 2214 (1979) United states

Shtral, T.: Nakasura, H.: Nakat, Y, Effoct of betweon on transfor In the lonization colls ofons 19, 2225 (1979)

Jopan

Brutsehy, B.: Haberland, H. Long range hellue oxelar

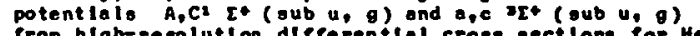
from $\mathrm{hlgh}-\mathrm{resoluti}$ on dipterentlal croso sectlons for Ho $(2$ is, $2 * 3)+$ He. Phys. Rev. A. 19, 2232 (1979) Nast Geranny

Skowronex, M.: Alayll, Y. Effect of the coeentue transfor on the Rayleigh sattering cross section. Phya. Rev. A 19, 2260 (1979) France

Chell ehwol zadeh, M. A.; Collino, C. B. Laser opectroscopy of the 6d Igna states of CaAr and CaKr: Phye. Rov. A 19, cero (1sts) United States

$\varepsilon \quad A_{A 0}^{5400-3500}$

Lee, L. C.: Salth, G. P. Photodiageclation erose

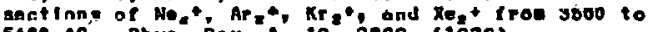
5400 A ${ }^{\circ}$. Phys. Rev. A 19, 2329 (1979) United states 


\begin{tabular}{|c|c|c|c|c|c|}
\hline $\begin{array}{l}\text { REF. } \\
\text { No. }\end{array}$ & REACTANTS & & $\begin{array}{l}\operatorname{EXP} \\
\text { OR } \\
\text { THEOR }\end{array}$ & $\begin{array}{l}\text { ENERGY } \\
\text { RANGE }\end{array}$ & REF ERENCE \\
\hline 1040 & ${ }^{H 05}+C_{0}$ & & I & $3.5-6.0 .0 \mathrm{~V}$ & 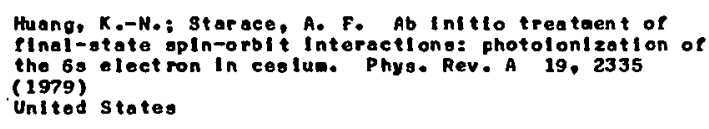 \\
\hline 1041 & 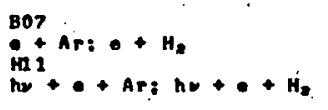 & $\cdot$ & $\varepsilon$ & $\begin{array}{l}\text { H11 } \\
117 \text { aed }\end{array}$ & 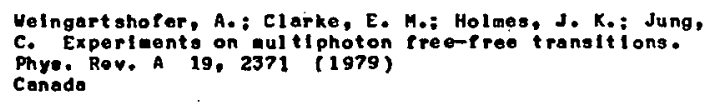 \\
\hline 1042 & 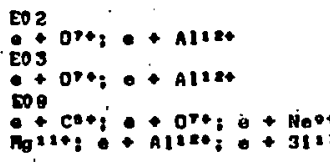 & i: & $\mathbf{T}$ & $\begin{array}{l}5 \times 108-2 \times \\
100 \times\end{array}$ & 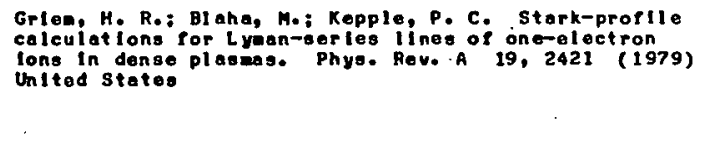 \\
\hline 1043 & $\operatorname{cov}_{\text {Art }}+c$ & & $F T$ & Indes & 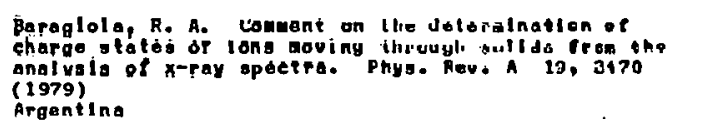 \\
\hline 1044 & $\begin{array}{l}\cos \\
\text { Art }\end{array}+C$ & & E T & Under & $\begin{array}{l}\text { Fortner, R. Joj Garcla, J. D. Che racteristle } x \text { roys } \\
\text { due to projectiles coving through sollds. Phys. Rev. A } \\
19,2474 \text { (1979) } \\
\text { united states }\end{array}$ \\
\hline .2045 & $\begin{array}{l}\mathrm{D07} \\
11,+\end{array}$ & - & $\varepsilon$ & $0.2-2.0$ keV & 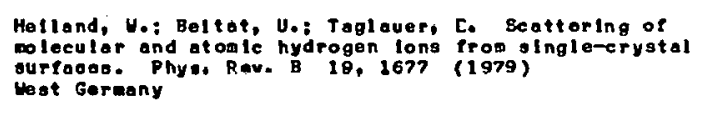 \\
\hline 1046 & $\mathrm{DOS}_{\mathrm{H}}^{\mathrm{DOS}}+\mathrm{SO}+\mathrm{NI}$ & & T & $79-213 \cdot 0 v$ & 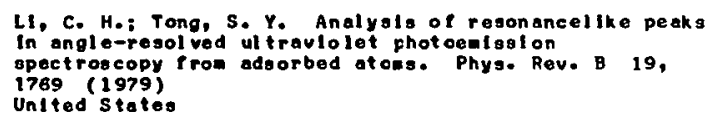 \\
\hline 1047 & $\mathrm{DOP}_{\mathrm{Ha}}^{\mathrm{DO}}+\mathrm{Cu}$ & . &. & Under & 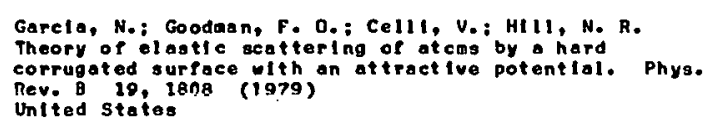 \\
\hline 1049 & $m_{n v}^{m s}+s i$ & & E & $6.5-7.1 \mathrm{eV}$ & 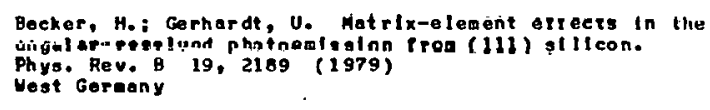 \\
\hline 1049 & ?ou si & & E & 60 ov & 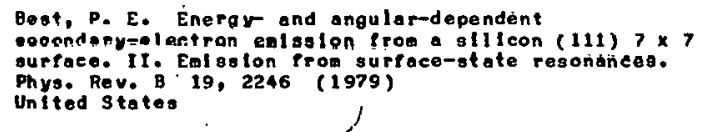 \\
\hline 1050 & $e^{\mathrm{DOS}}+\mathrm{Cr}_{\mathrm{r}_{2} \mathrm{O}} \mathrm{i} e+\mathrm{N1O}=e+\mathrm{C}$ & & $E$ & $600-2300=0$ & 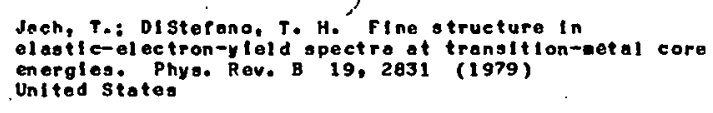 \\
\hline 1051 & 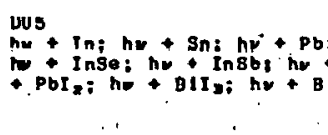 & 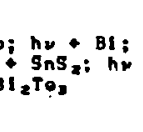 & r & $26-f i n$ ay & 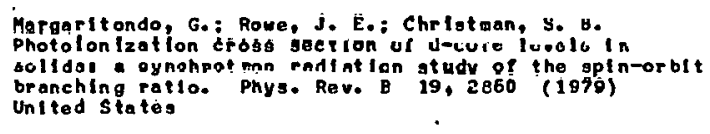 \\
\hline 1052 & $\mathrm{HeS}^{\mathrm{DO}}+\mathrm{H}_{2}+\mathrm{NI}$ & . & E & $B-33 \cdot V$ & 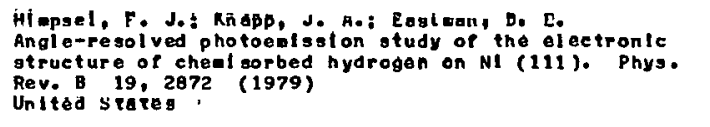 \\
\hline 1053 & 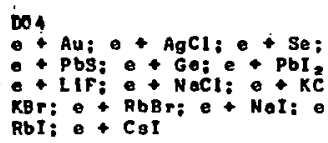 & 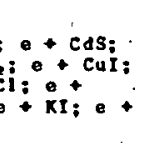 & ET & 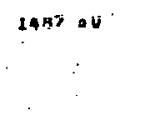 & 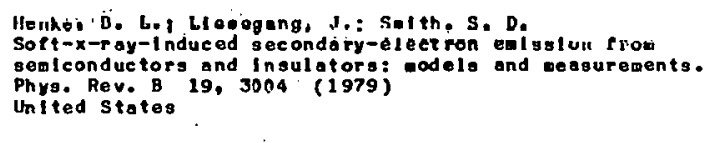 \\
\hline
\end{tabular}




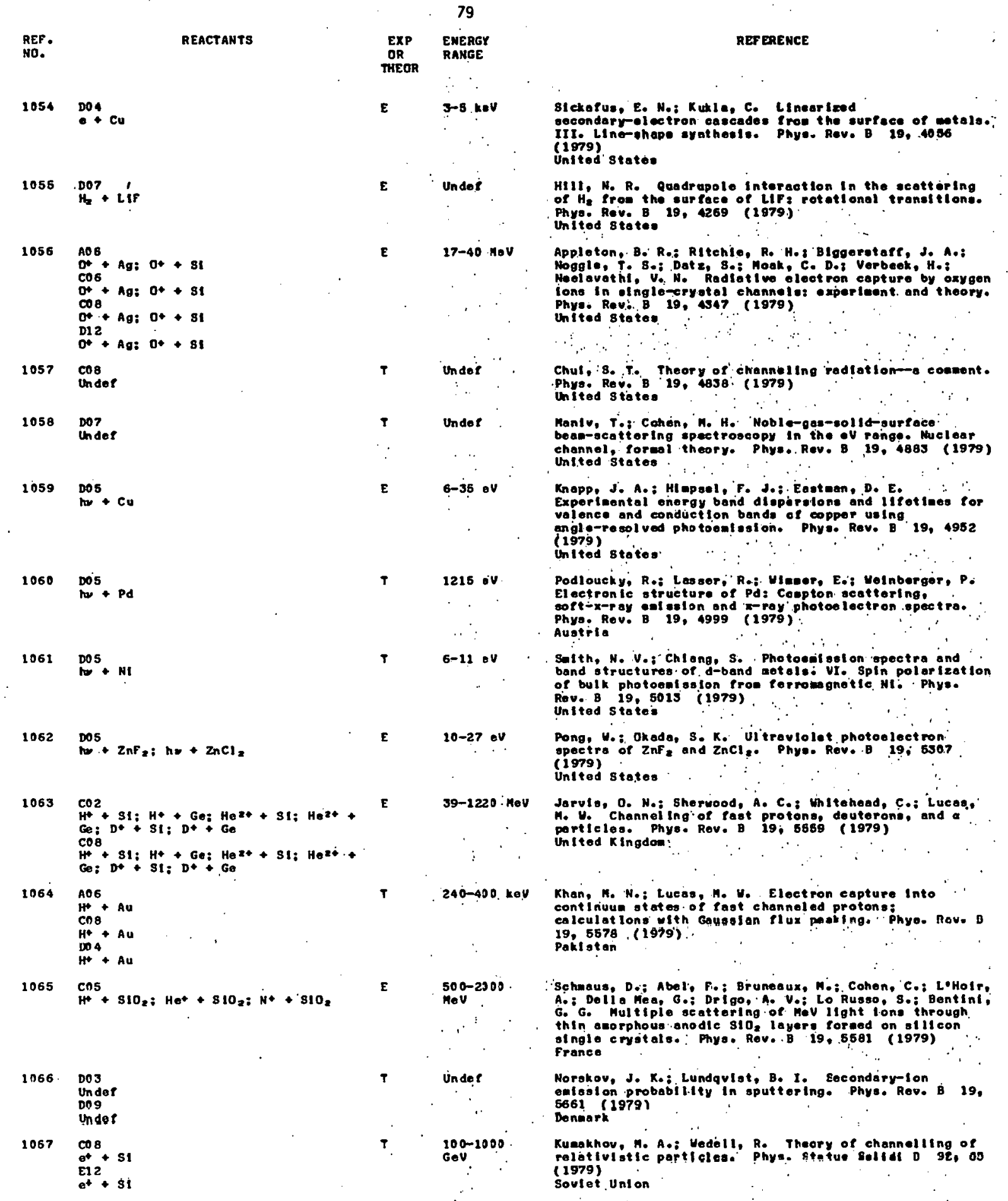




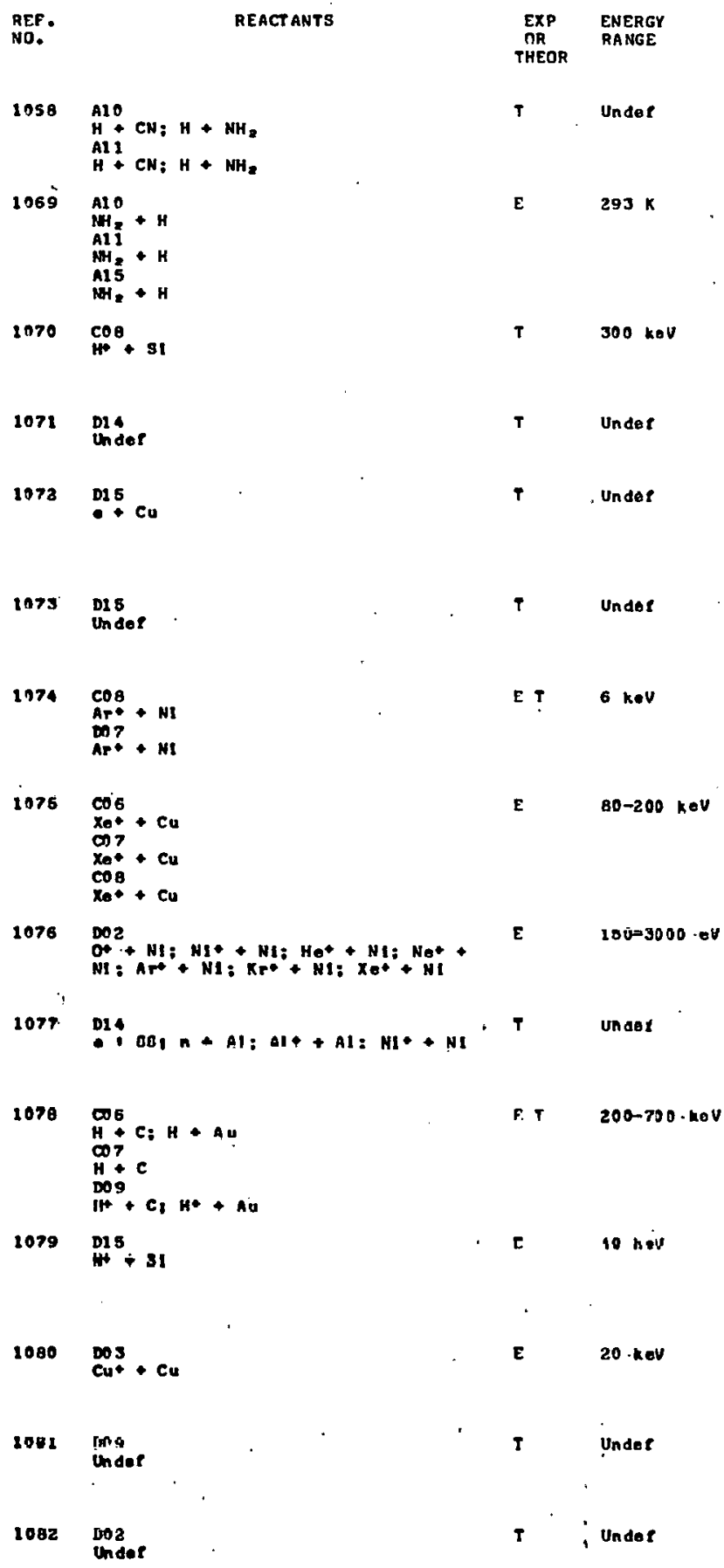

DIxon, R. N.; Fiel d, D. Rotational energy transfer In Dyllisions between orbitally non-degenerste open-shell United KIngdoc.

Dixon, R. N.: Fiel d, D. Rotetional energyt trangfer within the A tllde $2 A_{2}$, tate of $\mathrm{NH}_{2}$ : absolute rate coofflotents and the influence of spin exehange in collis

$366,247$ ( 1979$)$
United $K$ Ingdom

Kunak hov, M. A.: Kuhrt, E.: Wedel1, R. The theory of channelling at saall depths. III. Radlat. Eef. 41, (1979) Soviot Union

Martynenko, $Y_{.} V$. The role of cascados in vold generattion. Redlat. ERf. 41, 7 (1979) soviet union

thite, R. J-i Fishor, S. B.; Mlller, R. H. The of tod of Poll surfaces on the loss of point dotect, Frop (1979)

United KIngdom

mite, R. J.i Fieher, S. B. The effect of autual racoabination on dislocat ion polnt dofect sink strengths. Radiot..EfP, 41, 25 (1979) United Kingdoe

Evdoklnov, I. N.: van den Berg, J. A.: Armour, D. G. Proper surface channelling of low energy orgon lons incldent on a ntekel (110) cryatal. Radlat. Eff. 41 , $35(1973)$ United Kingdoen

Kudella, F.: Kerkou, H.: Wodell, R. Enhancaments of $X_{e}(H)$-radlation during chorinelling of $X_{0}-10 n$ in in capper crystals. Radlat. Ef?. 41, 4 (1979) Vest Geraany

Day, II. L. I Rastenaky, Je: Hecht 1, E. Lav-onergy sputtering ylolds of nlekel os a function of lon aass--a rolnuast Igat ion. Radilat. Erf, 41, 77 (1979) Wost Geraany

otiont on, H. Mis Kuletnakt. G. L. the use of the ruily dynanlo rato theory to prodict vold grouth in weralu: Ródlat. Err. 41, A1 (1979) Unlted states

Kt nura, X.: Kyosht ea, A.: It oh, A.: Mannasl, M. Neutrailzation of taergotio light ion at a solld surface. Rediat. Eff. 41, 91 (1979)

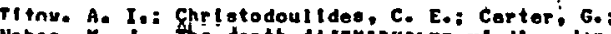
Nobes, M. J. The depth distriousi on uf Jl ousdori

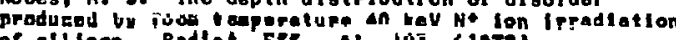
ar allicion. Radiat. Ept. 41, 107 (19r9) Unit ted Kingdoe

Kl rlakldle, G.: Colligon, J. B.I Lnensklm, Q. P. Secondary ion acss apeetromet rie study of self-sputtared coppor. Radiat. Ef 1 . 41,119 (1979) Unlted KIngdan

Pazdzerskil, V. A.: Tolplnyuk, B. A. Ionlzatlon of an PYos near. Molal ourfece: San. Phys.-Solld State 20 , $1893(1978)$

Pletnev, U. v: Angular distribution of atoms sputtorod Trow the sur face of an amophoug taproet. Sov. Phys.-Solld State 20, 1950 (1978) 
81

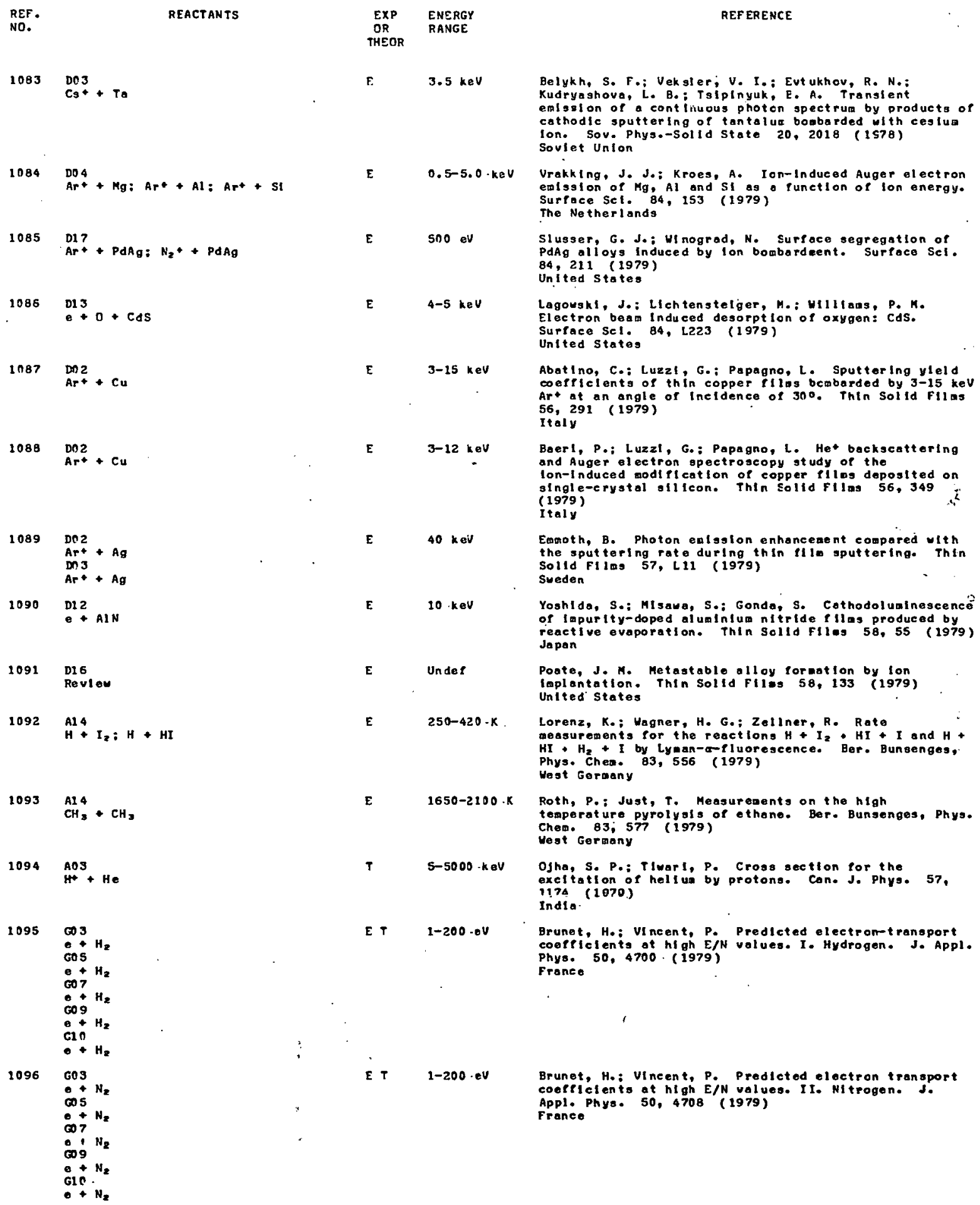




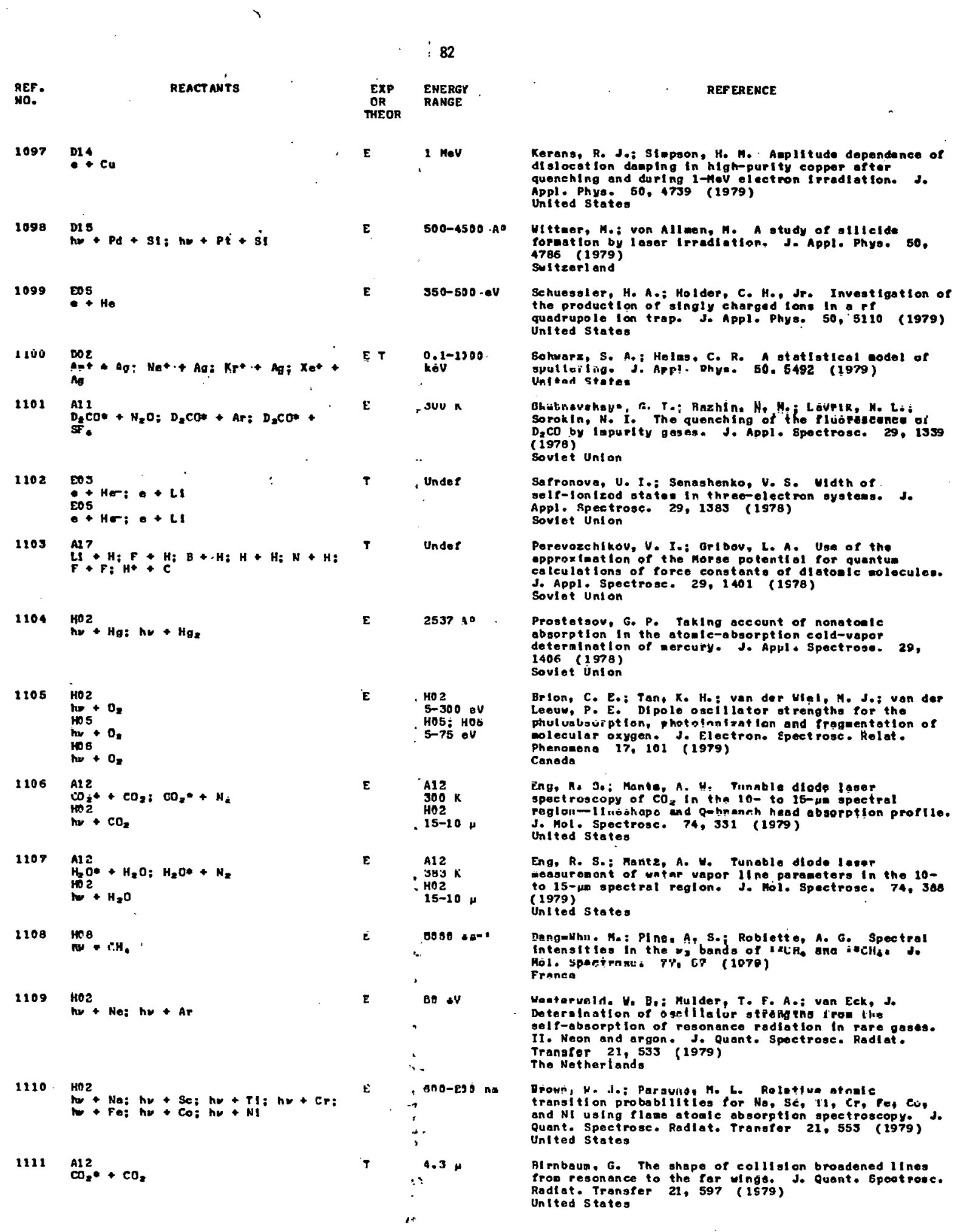




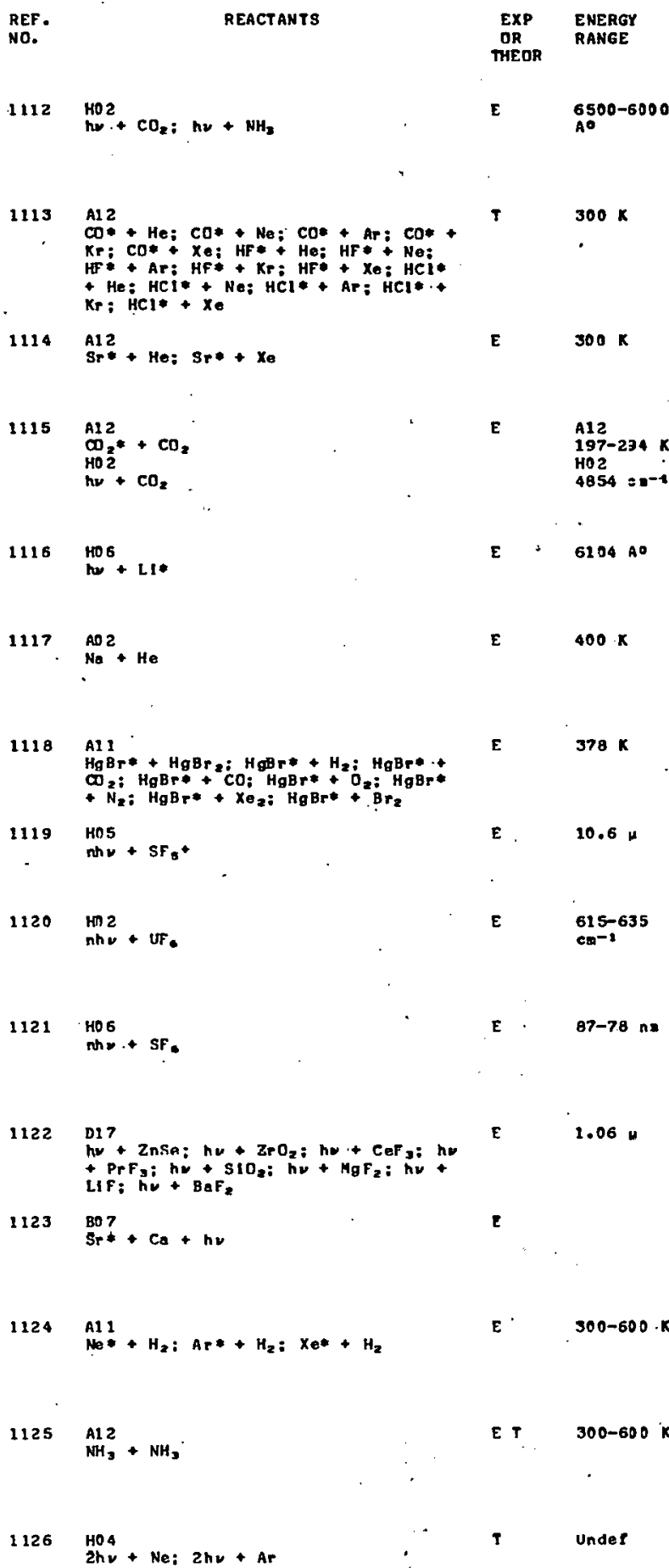

Antonov, E. No: Berik, E. Bo; Koloshn1kov, V. G. Intracovity laser dotection of amonla and methane absorption spoctro In the regtion 6000-6500 AO. J. Quant. Spectrose. Radlat. Transfor 22, 45 (1979)
Soviet Unt on

de Pristo, A. Eo; Rabltz, H. The erfect of elastic and reorlentation coilitsione on vibrot lon-rotation 11 neshapes: a sesi-omplrical approach. J. Quant. Spectrosc. Radiat. Transfer 22, 65 (1979) Unit ted states

Uang, S. Y.: Ch'on, S. Y. Pressure offects of He and to on the resonance line of stront lua J. Juant. Spoetroac. Rodiat. Transfor 22, 87 (1979) Unitod States

Valero, F. P. J.: Suaroz, C. B.: B'oese, R. W. Intensitles and half-uldth at different teaperatures for the 201 III coning frow 000 tand of $\mathrm{CO}_{2}$ at 4854 cor. J. Quant. Spectrosc. Radist. Transfor 22, 93

United States

Kramer, S. D.: Young, J. P.: Hurst, G. S.: Payne, M. G. Resonance lonization spectroscopy of ilthium. Dpt. Comaun. 30,47 . (1979)

Kachru, R.: Mossberg, T. Y.: Hartmann, S. R. Stimul at ed photon echo study of $\mathrm{Na}(32 \mathrm{~g}, 2,2)-\mathrm{CO}$ eloclty-changing collisiona. Opt. Cominn. 30, 57

Helvalian, Ho: Hittlg, C. Collist onal quenehing of

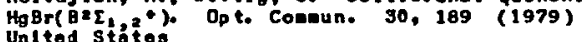

von Hell Pold, A.; fel dmann, Do: Holge, K. Ho.; Fournt er,

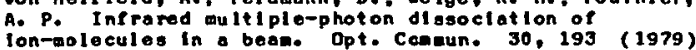
lon-moloculog

Lucht, R. A.: Beardall, J. S.: Kennedy, R. C.:

Sullivan, G. 'U.: R Ink, J. P. Mult Iple-photon

absorption of $16-\mu m$ radiction in Uf a $300 \mathrm{~K}$. Opt.

lott. 4,216 (1979)

Unitod States

Sudbo, A. S.: Schulz, P. A.; Kra Jnoulch, D. J.: Lee, Y. T.: Shen, Y. R. Photolonizat fon otudy of -uitiphoton-exeltad SF, in a colecular bead. Opt. Lett. 4,219 (1979)

United stotos

Bettle, J. T.: Guenther, A. H.: House, R. A., II Refractl va-Index dependence of pul sed-laser-induced damage. Opt. Lett. 4, 256 (1979)

Unsted States

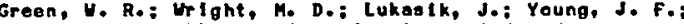
Marrls, S. E. Observation of a laser-induced dipole-quadrupole collilaton. Opt. Lett. 4, 265 (1979) Unlied States

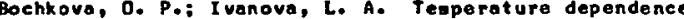
of the cross sections for the breakdown of the

metastable states of Ne, $A r$, and $x_{e}$ by the hydrogen molecule. Opt. Spectroso. 45, 926 (1979) Soviet Unlon

BulonIn, M. O.: Bulyehev, V. P.: Ladvishehenko, Y. H. Teapotature dopoindeatce of tho belf-broodening coefflelent for the as $R(1,1)$ IIne of the $v_{2}$ bond of anonla. Opt. Spectrose. 45, 930 (1979) Soviet Union

Lebedev, I. U.: Yakovleva, N. S. Varlatlonal method for calculating probablilitioe of two-phot on oxelt at lons In Inert gas atoes with considerat fon of Interel octron corrolation. Opt. Spectrose. 46, 3 (1979) Sovlat Uni on 


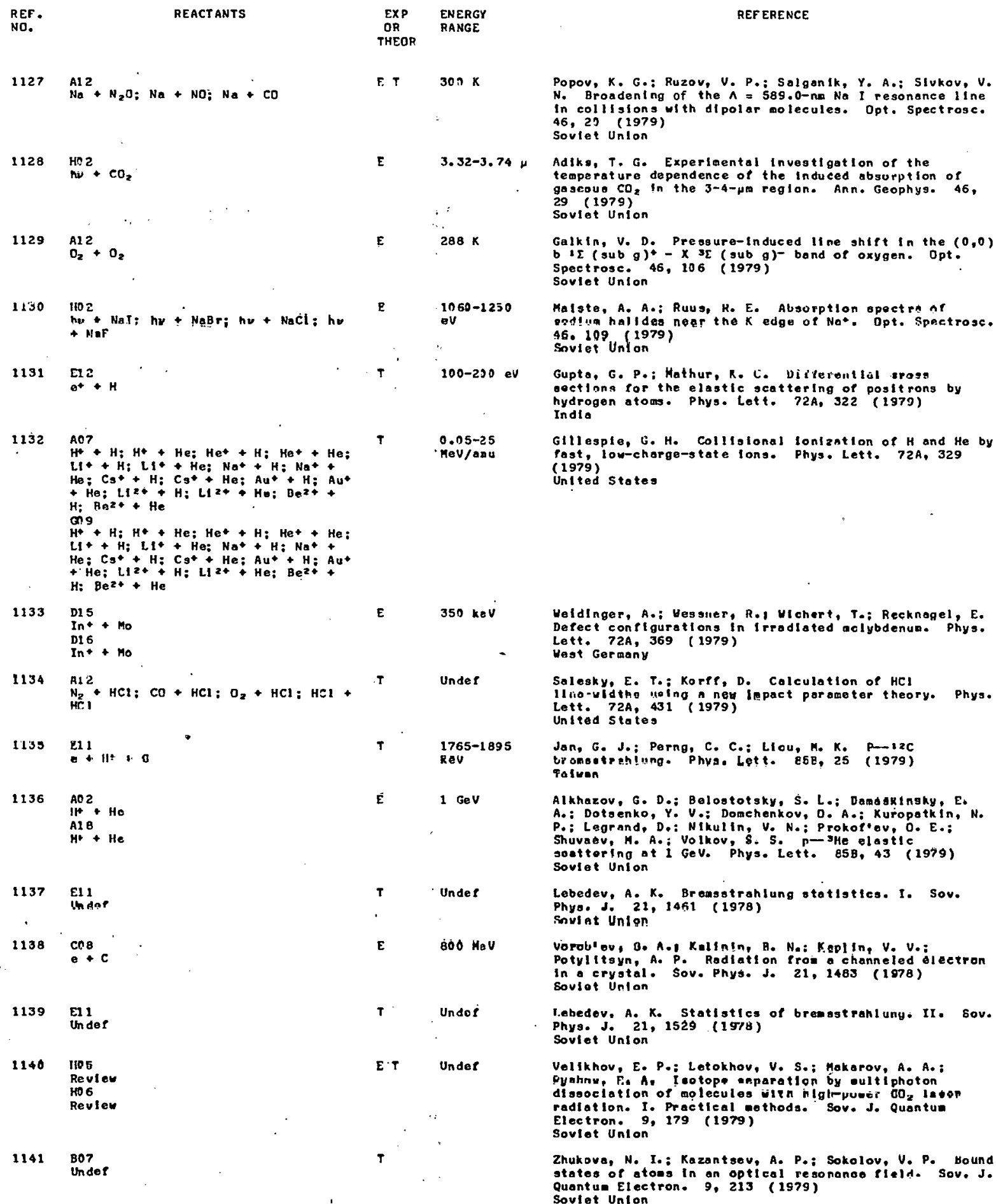

$0.05-25$
MeV/anu

MeV/apu

$350 \mathrm{keV}$

Galkin, U. D. Preasure-induced line shift in the $(0,0)$ b IE (sub g)* - $X{ }^{3} \sum$ (sub $g$ )- band of oxygen. Opt. Spectrosc. 46,106 (1979)

Malste, A. A.: Ruus, H. E. Absorption opect rh af ost!un halldes noar the $K$ edge of No*. Dpt. Spectrose. S6. 109 Un (1979)

Gupto, G. P.: Mathur, R. C. Dicterantiál zross coctinns for the elastic geattering of positrons by
hydrogen atoms. Phys. Lett. $72 A, 322$ (1979) hydrogon

GIllesple, B. H. Collifional Ionlantlon of $H$ and $M e$ by fast, low-charge-state lons. Phys. Lett. $72 \mathrm{~A}, 329$

Yeldinger, A.: Vessuer, R., Vichert, To; Reeknogel, E. Defect conflgurations in Irradlated aclybdenun. Phys. Lett. $72 A, 369$ (1979)

West Germany

Under

Salesky, E. T.: Korff, D. Calculatlon of HCI ll lio-uldtho Moing ney impact porameter theory. Phys. lett. $72 A, 431$ (1979)

United States

Jan, G. J.: Perng, C. C.: Llou, M. K, P-12C troneitrah!ung. Phva. Lett. 858, 25 (1979)

$\operatorname{Rev}^{1765-1895}$ Foluen

E $1 \mathrm{GeV}$

Alkharov, G. D.; Belostotsky, S. L.; Dandspinsky, E.

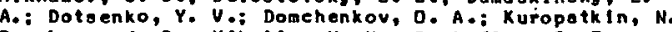
P.: Legrand, D.: Nikulin, V. No: Prokol'ev, O. E.: Shuvaev, H. A. Volkov, S. S. p- JHe elastle
soettoping at $i$ GeV. Phys. Lett. $85 B, 43$ (1979) Soviet Union

Lebedev, A, K. Bremsotrahlung totlotics. I. Sov. Phyo, J. 21, 14651 (1978)

Snviat Untor?

vo robl ou, D. A.l Kalln!n, B. Na: Kaplin, V. Vo: Potvltisyn, A. P. Radiation Proi a channeled electron In a cryotal. Sov. Phys. J. 21, 1483 (1978) Soviot Untan

l.ebedev. A. K. StatIstlcs of breastrahlung: II. Bov. Phys. J. 21, 1529 (1978) Soviet Union

Velikhov, E. P.: Letokhov, V. S.: Makarov, A. A.: Cyahnu. F. A. I eotope exparation by oultiphoton

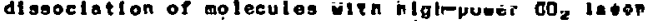
radiatlon. 1. Practical nothoda. Sov. Jo Quantua Electron. 9, 179 (1979) Soviet Uni on

Zhukova, N. I.: Kazantsev, A. P.: Sokalov, U. P. Baund states or atoss In an optlical resonanos Pleld. Sov. J. Quont un Electron. 9,213 (1979) 


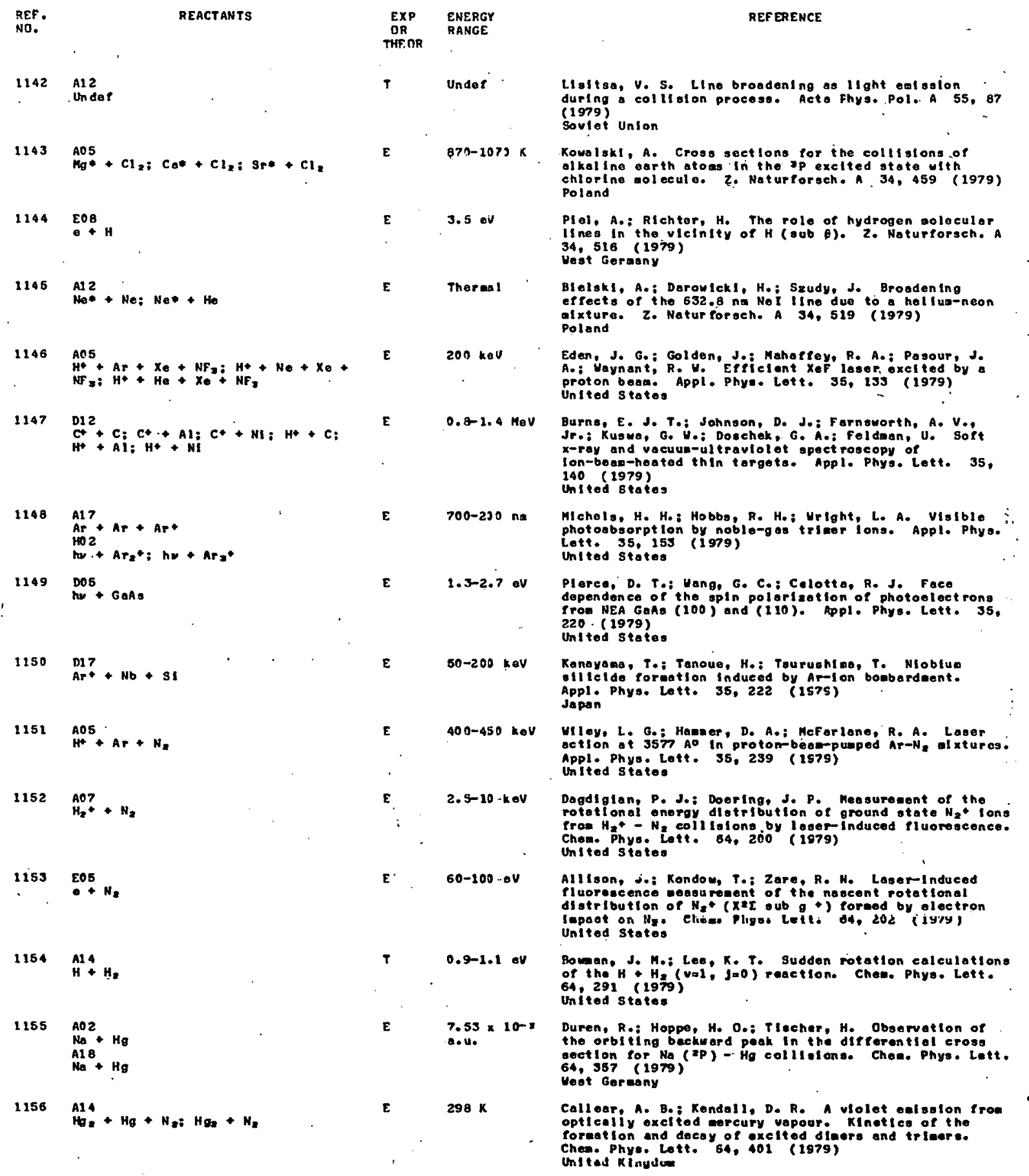


86

\begin{tabular}{|c|c|c|c|c|}
\hline $\begin{array}{l}\text { REF. } \\
\text { NO. }\end{array}$ & REACT ANTS & ? & $\begin{array}{l}\text { EXP } \\
\text { OR } \\
\text { THEOR }\end{array}$ & $\begin{array}{l}\text { ENERGY } \\
\text { RANGE }\end{array}$ \\
\hline 1157 & $\mathrm{O}_{2}^{\mathrm{A} 11}+\mathrm{O}_{2}: \mathrm{O}_{2} *+\mathrm{N}_{2}: \mathrm{O}_{2} *+$ & Ho & $\varepsilon$ & $500 \mathrm{~K}$ \\
\hline 1158 & $\mathrm{NO}_{\mathrm{NO}}^{\mathrm{NO}}+\mathrm{NO}$ & & E & $2700-3500$ \\
\hline & 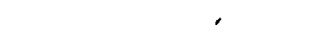 & & & 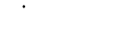 \\
\hline 1159 & $\mathrm{~S}+\mathrm{OH} ; \mathrm{SO}+\mathrm{OH}$ & & $\varepsilon$ & $298 \mathrm{~K}$ \\
\hline 1150 . & $\mathrm{II}_{2}^{4}+\mathrm{H}_{2} \mathrm{~S}$ & & E & $555-895 K$ \\
\hline 1161 & 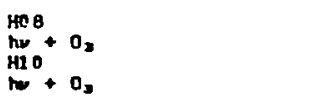 & & E & $280-230 \mathrm{ne}$ \\
\hline 1162 & $\begin{array}{l}\mathrm{Al}_{4} \\
\mathrm{HO}\end{array}$ & & E & $284 \mathrm{~K}$ \\
\hline 1163 & $\begin{array}{l}\mathrm{A} 14 \\
\mathrm{Cl}\end{array}$ & & $\varepsilon$ & $300 \mathrm{k}$ \\
\hline 1164 & $\mathrm{Al}_{\mathrm{H}}^{\mathrm{A}}+\mathrm{H}_{2}$ & & $T$ & $300-1300 \cdot k$ \\
\hline 1165 & $\stackrel{A 14}{B r}+0$, & & $E$ & $234-360 \cdot K$ \\
\hline 1186 & $\mathrm{AD}^{\mathrm{Al} 1}+\mathrm{NO} ; \mathrm{CO}+\infty$ & & $\varepsilon$ & $300 \cdot k$ \\
\hline 1257 & $\mathrm{H}_{\mathrm{H}}+\mathrm{H}+\mathrm{Th}$ & & $\mathbf{E}$ & $300 \mathrm{~K}$ \\
\hline 1158 & $\begin{array}{l}\mathrm{Dos} \\
\mathrm{He}+4 \mathrm{Mg} \\
\mathrm{Dl} 5 \\
\mathrm{He}++\mathrm{Hg}\end{array}$ & & ET & $2 \mathrm{keV}$ \\
\hline 1159 & $\mathrm{PO}_{2}^{2}+\mathrm{Ho}_{0}: \mathrm{He}^{*}+\mathrm{Ho}^{2}$ & . & $\varepsilon$ & $15-40 \mathrm{keV}$ \\
\hline 1170 & $\mathrm{H}_{\mathrm{H}}^{\mathrm{A}}+\mathrm{H}_{2}: \mathrm{F}+\mathrm{H}_{2}: \mathrm{H}+\mathrm{Cl}_{2}$ & & $T$ & $\begin{array}{c}0.1-0.4 \text { ov } \\
:\end{array}$ \\
\hline $11 \% 1$ & $\mathrm{~B}_{\mathrm{B}}^{\mathrm{A}}+\mathrm{O}_{2}: \mathrm{B}+\mathrm{SO}_{2}: \mathrm{B}+\mathrm{CO}_{2} ;$ & $B+\mathrm{N}_{2} \mathrm{O}$ & ᄃ & $30 n$ \\
\hline 1172 & $\stackrel{A 17}{H}+H_{z}$ & & $T$ & Unde? \\
\hline
\end{tabular}

REF ERENCE

Chatho, J. P. S.: Arora, P. K.: Ralln! Raja, 8. M. T.: Rulkarnl, P. B.: Vohra, K. G. Collielonal deact Ivetion of $\mathrm{O}_{2}\left(\mathrm{I}\right.$ sub $\left.g^{*}\right)$. Int: J. Chen. Kinoties 11,176 (1979) Inda

Koshl, M.: Asoba, T. Bhock-tubo otudy of thermal deconposition of nitif e oxide between 2700 ond $3500 \mathrm{~K}$. Int. J. Cheil. Kinetleg 11, 308 (1979) Japan'

Jourdaín, J. L.: Le Bras, G.: Combourleu, d. KInetice study of tone el enentary reactions of sulfur conpounds Ineluding reactions of $S$ and SO with of rodicalo. Int. J. Chem. KInotles 11, 669 (1979) Franee

Huang, R. J.J Bonoon, S. U. KIngt les of lod lnat Ion of hydregen eui flde by lodine and the heet of roreatton of

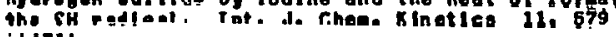
ilst3: Unillud oluló

Kajlmoto, O.: Cuetanovlc, R. J. Abeolute quontur yteld of $0\left(\mathrm{ID}_{2}\right.$ ) In the photolysto of ozone In the Hartley band. Int. J. Chea. Rinetice 11, 605 (1979) Canoda

Cox, R. A.: Patrlek, K. KInetles of the reaction of $\mathrm{HO}_{2}+\mathrm{NO}_{2}(+\mathrm{H})=\mathrm{HO}_{2} \mathrm{NO}_{2} \cdot \mathrm{ust} \mathrm{ng}$ molecular modulation Spectronetry. Int. S. Criou. Kinet les 11, 635 (1979)

Baseo, N.: Himt, J. E. Hutual coabination of $\mathrm{ClO}$ radicalo. Int. J. Chel. Kinetics 11,649 (1979)
Canada

Chrlstov, S. Ga: Parlapanaki, M. Quantur effects and laotope effects in chenical kinetlcs. Int. Jo Chen. Kinat Ies 11, 665.(1979) Bulgarlo.

Mlchael, J. V.: Payne, H. A.: Absolute rate constente for the reaction of bronineictoss ulth ozono trom 23

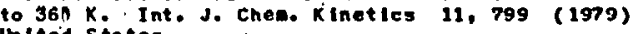

HorlguehI; H.: Tsuchlyá, 's.: Vibrational rel axat Ion of NO $\left(X^{2} Y\right)$ in the states of 2 difterence between 10 . Jap. J. Appl. Phys. 18, 1207 (1979) Jopan

KIW, H. W.: Guno, $K_{\text {. }}$ Coedsorptlon or thorl un and hydrogen on a tung ot en surface. Jap. J. Appl. Phy, 18,1223 (1979)

Aral, Di: Tazaya, Y.: Shlmanuro, T.: Kobayasht, $K_{\text {. }}$ Isotople fractlonation of aggnoblue eputterad by $2 \mathrm{keV}$ Isotople fractlonation of eagnoslue eputtorad by
Het Ions. Jap. J. Appl. Phya. 18, 231 (1979) Het Ions

Okuda, S.: Aki uune, $H$. Surtace erostion of metal

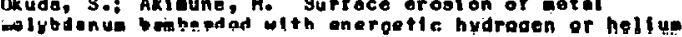
cons. Jap. J. App 1. Phys. 18, 1355 (1979) Japan

VI 1a, C. L.; Zul jac, D.. J.; Ross, J. Franek-Condon theary of chealcal dynatiog. VI. Angular distributions

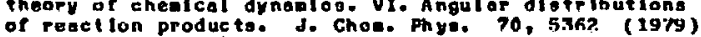
United Btatoo

Seltharan. 11. S.e: DiGtusoppe, T: G.j Mefadden, D. L.: Davidoults, P. Rate constants for the rodet Idits or atomle boron with $\mathrm{O}_{2}, \mathrm{SO}_{2}, \mathrm{CO}_{2}$, and $\mathrm{N}_{2} \mathrm{O}_{4} \mathrm{~d}$. Chea. Phyo, 0,6422 (3U\%) United stater

Garela, Ro: Ro'ss 1, A. Ro: Russek, A. Dissoctatting states of the Hz- systes. J. Ches. Physa P0, 5463 (1979)

United stotas 


\begin{tabular}{|c|c|c|c|c|}
\hline $\begin{array}{l}\text { REF. } \\
\text { NO. }\end{array}$ & REACT ANTS & $\begin{array}{l}\text { EXP } \\
\text { OR } \\
\text { THEOR }\end{array}$ & $\begin{array}{l}\text { ENERGY } \\
\text { RAKGE }\end{array}$ & REF ERENCE \\
\hline 1173 & 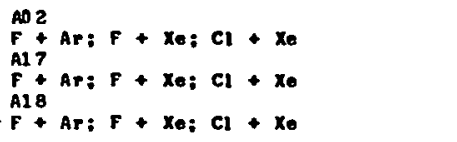 & $T$ & $e^{0,003-0.5}$ & 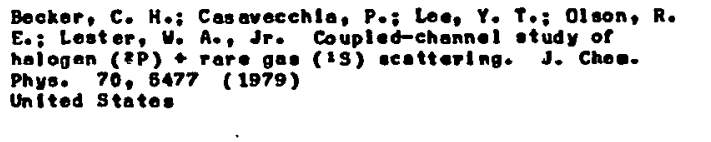 \\
\hline 1174 & 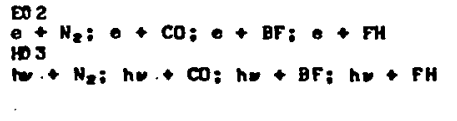 & $\mathbf{T}$ & Under & 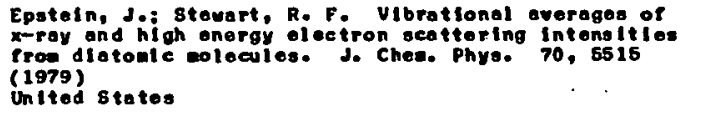 \\
\hline 1175 & 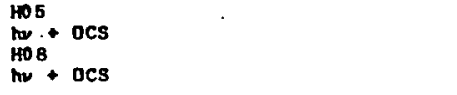 & E & $170-142 \mathrm{nw}$ & 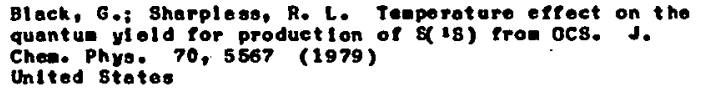 \\
\hline 1176 & $\begin{array}{l}A 05 \\
S+H_{e}: S+A r: S+H_{z}: S+H_{2}: S+ \\
K r: S+X_{e}\end{array}$ & $E$ & $232-425 K$ & 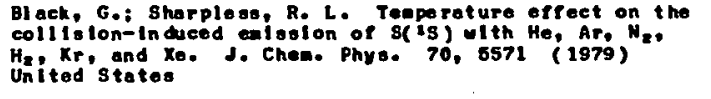 \\
\hline 1177 & $\begin{array}{l}\text { Al1 } \\
\text { Se }+ \text { ocse; } \operatorname{se}_{2} *+\text { ocse }\end{array}$ & E & $300 \mathrm{~K}$ & 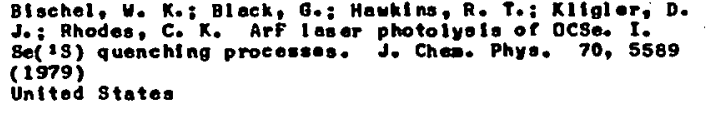 \\
\hline 1178 & $\begin{array}{l}\text { mo } 2 \\
h y+\text { ocse } \\
108+\text { ocse }\end{array}$ & E & $\begin{array}{l}\text { Hn2 } \\
953-914 \text { Ao } \\
\text { HOB } \\
193 \text { ns }\end{array}$ & 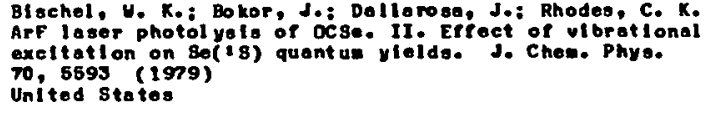 \\
\hline 1179 & A06 + No & $\mathbf{T}$ & $10 . u$ & 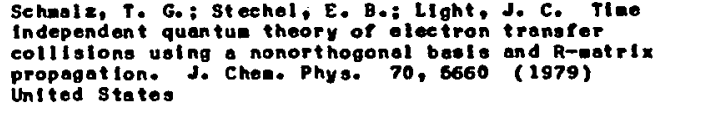 \\
\hline 1180 & $\mathrm{AO}^{\mathrm{GO}}+\mathrm{N}_{2}$ & $\mathrm{~T}$ & Unde? & 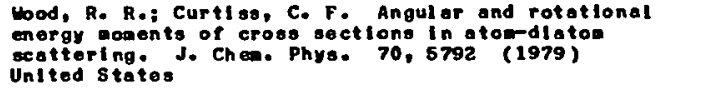 \\
\hline 1181 & $\begin{array}{l}\mathrm{GO} 2 \\
\mathrm{Ar} \\
\mathrm{CO}\end{array}$ & 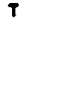 & $250-650 \mathrm{~K}$ & 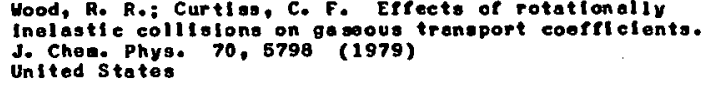 \\
\hline 1182 & $\mathrm{O}_{\mathrm{A}}^{\mathrm{A}}+\mathrm{H}_{2}$ & E T & $300 \mathrm{~K}$ & 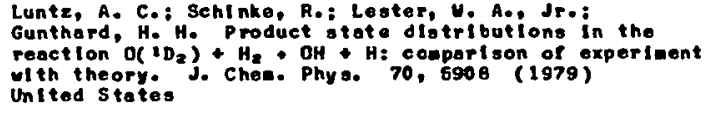 \\
\hline 1183 & $\mathrm{H}_{\mathrm{H}}^{\mathrm{A}+\mathrm{NO}_{2}}$ & E & $3 n 0 \mathrm{~K}$ & 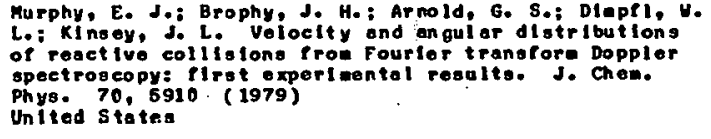 \\
\hline 1184 & $\mathrm{HCl}^{\mathrm{Al1}}+\mathrm{HCl}: \mathrm{HF}+\mathrm{HF}$ & E & $215 \mathrm{~K}$ & $\begin{array}{l}\text { Koura, K. On rotational. relaxatlon of hydrogen } \\
\text { halldes. J. Ches. Phys. } 70,5913 \text { (1979) } \\
\text { Japon }\end{array}$ \\
\hline 1185 & $\begin{array}{l}\mathrm{Cl}_{4}^{4} \\
\mathrm{Xl}_{\mathrm{e}}\end{array}$ & $\mathbf{E}$ & $0-4 \in V$ & 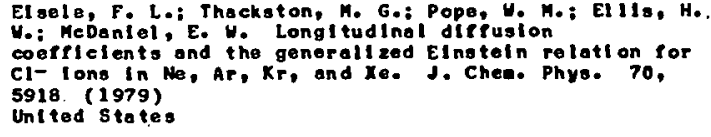 \\
\hline 1186 & ${ }_{\mathrm{H}_{2}}^{\mathrm{A}}+\mathrm{I}: \mathrm{H}+\mathrm{HI}$ & $\mathbf{T}$ & $300-1500 \mathrm{~K}$ & 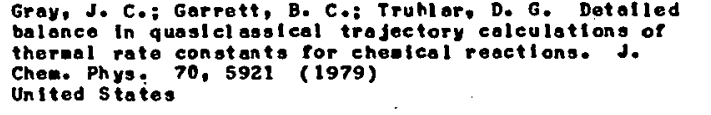 \\
\hline 1187 & $\mathrm{CO}_{2}+\mathrm{N}_{2}: \mathrm{NO}++\mathrm{N}_{2}$ & $\mathbf{E}$ & $300-640 \mathrm{~K}$ & 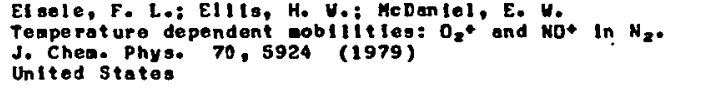 \\
\hline
\end{tabular}




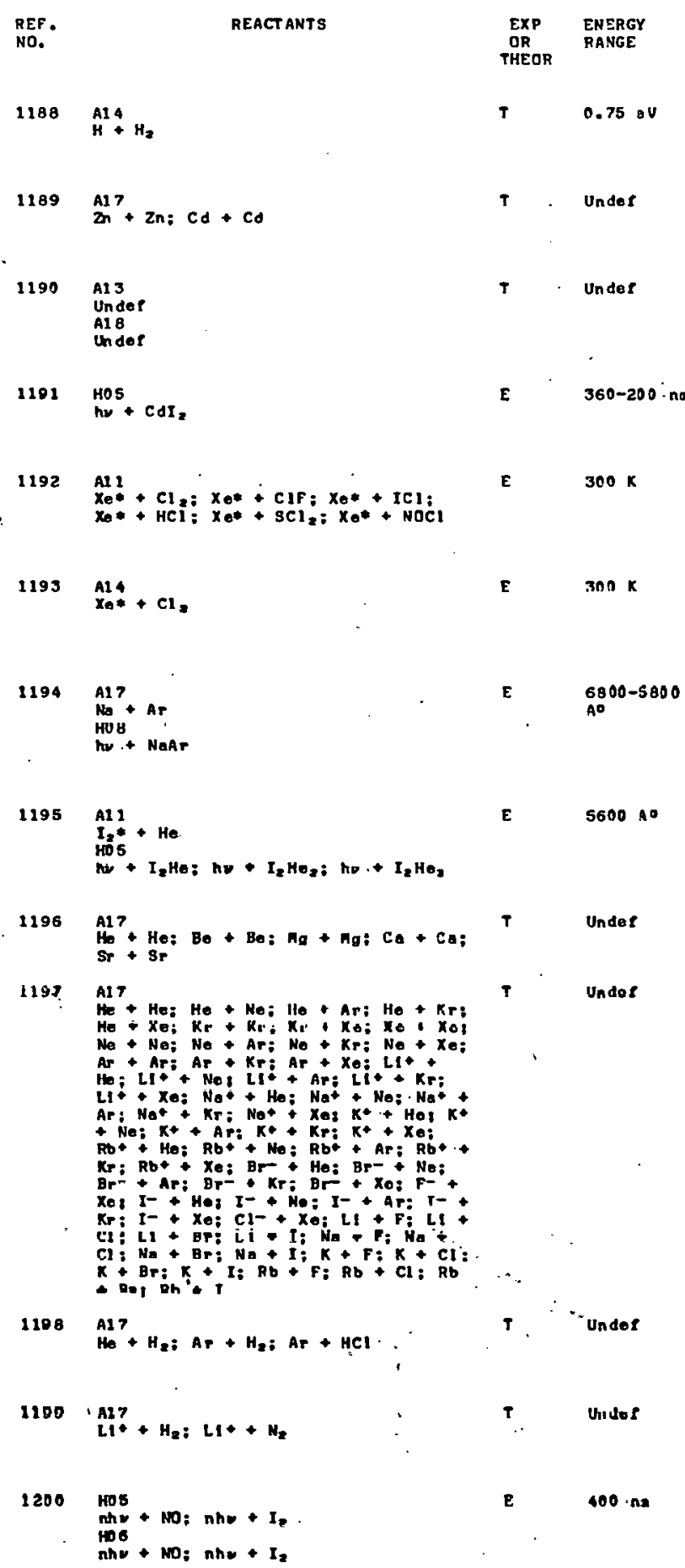

E $\quad{ }_{A O}^{6800-5800}$

E 5600

T Under

T Undor

$\sqrt{2}$




\begin{tabular}{|c|c|c|c|c|}
\hline $\begin{array}{l}\text { REF. } \\
\text { NO. }\end{array}$ & REACTANTS & $\begin{array}{l}\text { EXP } \\
\text { aR } \\
\text { THEOR }\end{array}$ & $\begin{array}{l}\text { ENERGY } \\
\text { RANGE }\end{array}$ & REFERENCE \\
\hline 1201 & $\mathrm{He}_{\mathrm{He}}+\mathrm{H}_{2}$ & $\boldsymbol{T}$ & Under & 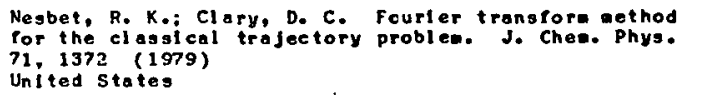 \\
\hline 1202 & $\begin{array}{l}\mathrm{AOJ}_{\mathrm{He}}+\mathrm{H}_{2}: \mathrm{H}+\mathrm{Br}: \mathrm{He}+\mathrm{HBri} \mathrm{N}_{2}+ \\
\mathrm{N}_{2} \\
\mathrm{All}_{2} \\
\mathrm{He}+\mathrm{H}_{2}: \mathrm{H}_{2}+\mathrm{Br}_{2}: \mathrm{He}+\mathrm{HBr} \mathrm{N}_{2}+\end{array}$ & $T$ & Undef & 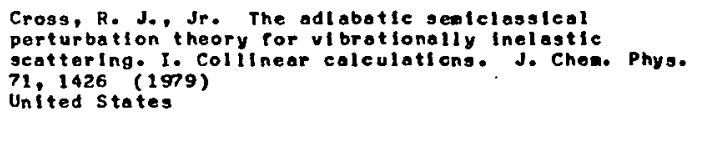 \\
\hline 1203 & $\begin{array}{l}\mathrm{An3} 3 \\
\mathrm{He}+\mathrm{H}_{2} \\
\text { Al1 } \\
\text { He }+\mathrm{H}_{2}\end{array}$ & $\boldsymbol{T}$ & $0.6-5.5 \mathrm{eV}$ & 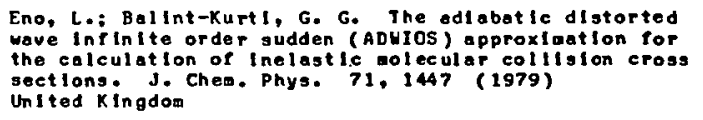 \\
\hline 1204 & $\begin{array}{l}\mathrm{Al}_{1} \mathrm{~F}^{+}+\mathrm{H}_{2} \\
\mathrm{BOF}+\mathrm{H} \\
\mathrm{F}+\mathrm{H}_{2}\end{array}$ & 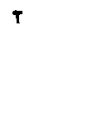 & $a_{0.01}^{A 11} \cos ^{-01-3.04}$ & 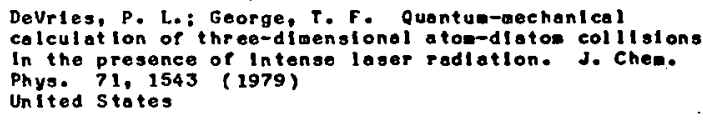 \\
\hline 1205 & 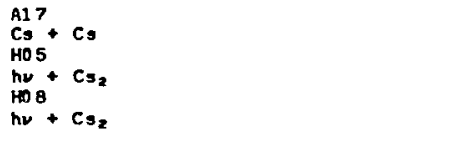 & E & ${ }_{A 0}^{5145-4658}$ & 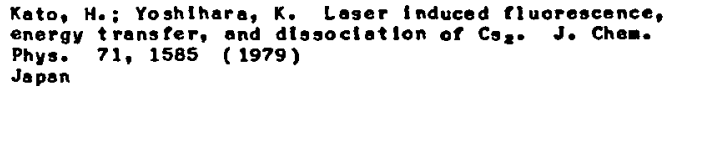 \\
\hline 1208. & $\stackrel{A 1}{C N}+\operatorname{Ar}$ & E & $300 \mathrm{~K}$ & 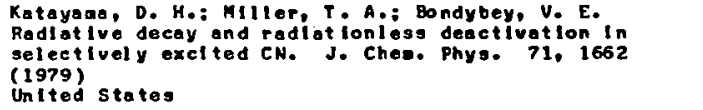 \\
\hline 1207 & $\begin{array}{l}\text { AO3 } \\
L I H+H C 1: L H+D C 1 \\
\text { Al1 } \\
L H H+H C 1 *: L I H+D C 1 *\end{array}$ & E T & $0.74 \mathrm{BV}$ & 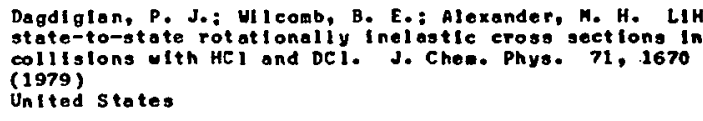 \\
\hline 1208 & $\begin{array}{l}A 03 \\
L 1 H+H C 1: L I H+D C 1 \\
A 11 \\
L I H+H C 1 *: L I H+D C 1 *\end{array}$ & 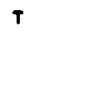 & 0.78 .6 & $\begin{array}{l}\text { Al axander, M. H. Sudden theorles of rotatlonally } \\
\text { Inolastic LiH-HCl and LIH-DCI coll islons. J. Chas. } \\
\text { Phys. TI, } 1683 \text { (1979) } \\
\text { United States }\end{array}$ \\
\hline 1209 & 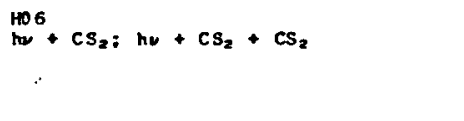 & E & ${ }_{A 0}^{1292-1005}$ & 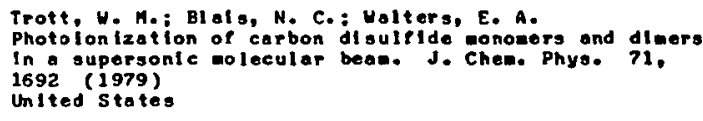 \\
\hline 1210 & 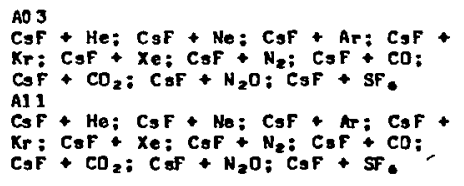 & E & $800-1000 \cdot K$ & 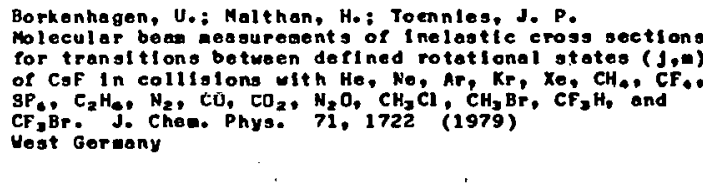 \\
\hline 1211 & $\begin{array}{l}\mathrm{AiOS} \\
\mathrm{Cl}-+\mathrm{H}_{2} \\
\mathrm{AO} 4 \\
\mathrm{Cl}-+\mathrm{H}_{2}\end{array}$ & E & $4-11$ ov & 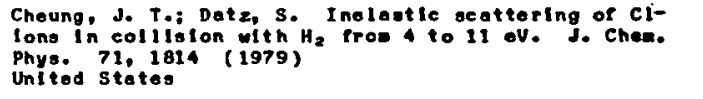 \\
\hline 1212 & $\begin{array}{l}\mathrm{Al}_{\mathrm{H}} 3 \\
\mathrm{H}+\mathrm{H}^{*} \\
\mathrm{BO}+\mathrm{H}^{*}\end{array}$ & T & $\begin{array}{l}\text { A13 } \\
2000 \mathrm{~K}\end{array}$ & 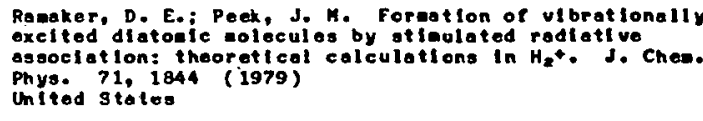 \\
\hline 1213 & $\mathrm{Gl}_{1}+\mathrm{I}_{2}+\mathrm{N}_{2}$ & E & $1-50$. Id & 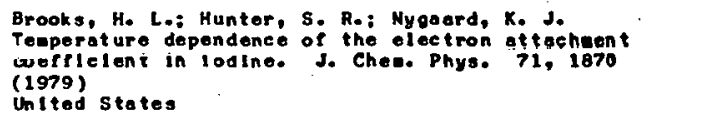 \\
\hline 1214 & $\mathrm{Ar}_{2}+2 \mathrm{Ar}$ & F. & $144-217 \mathrm{~K}$ & 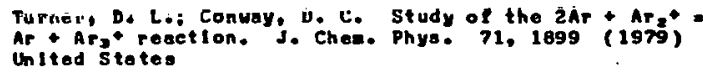 \\
\hline
\end{tabular}




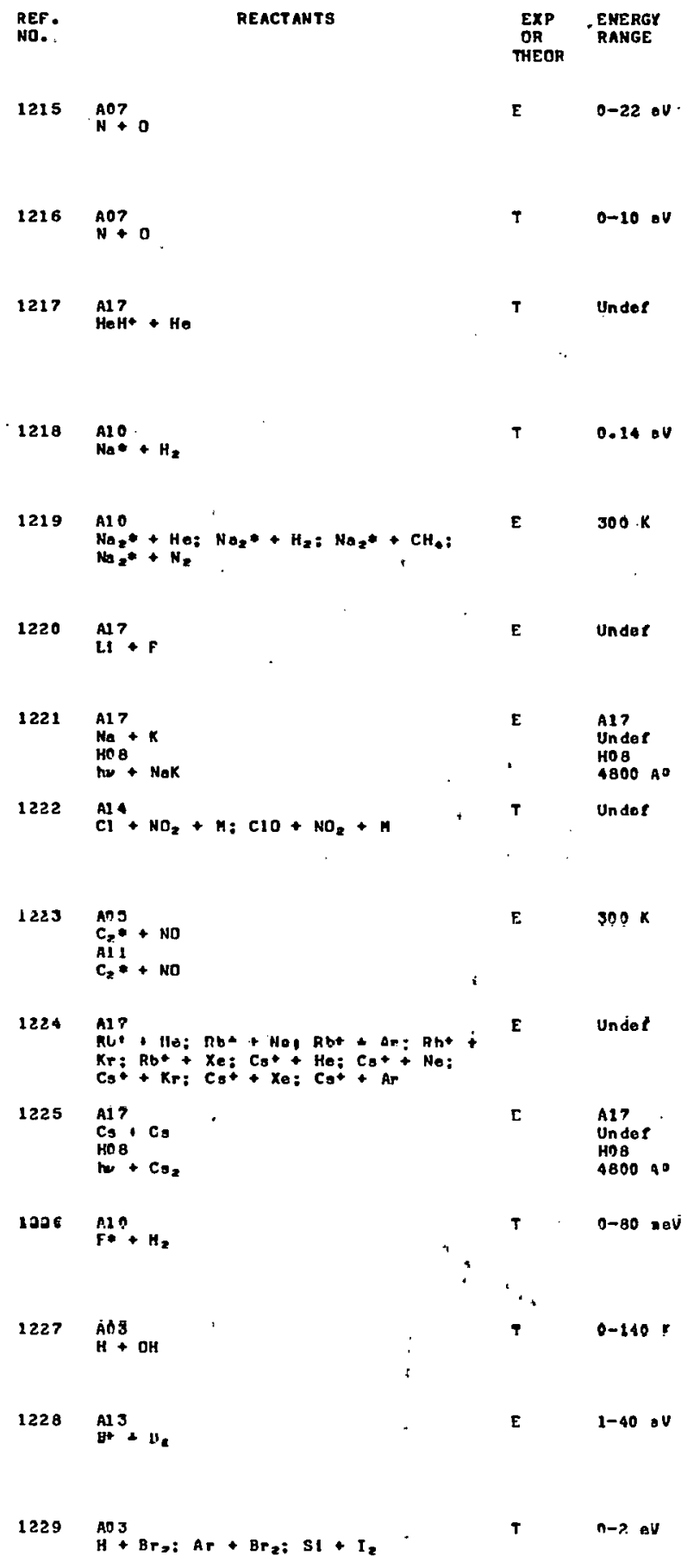

REF EREHCE

Ringer, Go: Contry, H. R. A meroed molocular beaa study of the ondoergle assoclative ionizat lon roacti on

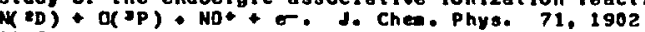
(1979)

Unleod Stotes

Niol gen, S. E.: Dahlor, J. S. Endoergle
cheal-fonization in N-O collistons. J. Chea. Phys. 71,1910 - (1979)

Denseark

Meler, P. F.: Hayes, E. F.: Ell1100n, F. O. A diatoefcs-intuol ecules (DIM) formalise for the react lon AH+ + A prise + A + A prine $\mathrm{H}^{+}:$a ground atate potentlal onergy gurface lor collinear $\mathrm{He}_{2} \mathrm{H}^{+}$. J. Chem. Phys. 71,1948 (1979)

McGul re, P.: Bel luin, J: C. El eetronle-to-vibratlonal energy transfor in colligi ons of exclted sodlun uith molecular hydrogon. Jo Chem. Phys. 71, 1975 (1979) Veat doizaity

Weinger, Mo: Al-Ag11, I.: Brunner, T. A,: Karp, A. Wo: Solth, N.: Prltehard, D. E. Power law seall ng of rotational onergy transfer In $\mathrm{Na}_{2}^{*}(\mathrm{AE})+\mathrm{He}_{2} \mathrm{H}_{2}, \mathrm{CH}_{0}$, and $\mathrm{N}_{2 .}$ J. Chem. Phys. 21, 1977 (1979) Unitod states

Dreford, E. J.: Engelia, F. The a sEt state of Nak. HI gh rosoluttion spectroscopy using laser-I nducéd Plinnascence (LIF). J. Chea. Phys. 71. 1994 (1979) Veat Germany

Elsel, D.: Zeugolls, D.: Dentroder, $\psi$. Sub-Doppler laser apectroscopy of the Nak molecule. J. Chen. Phys. 71. 2005 (1979)

Cheng, J. S.; Balduln. A. C.; Golden, D. H. An explanation of the preferential toreatlon of tes stable isomers In three-body reactlons: $\mathrm{Cl}+\mathrm{NO}_{2}+\mathrm{M}$; $\mathrm{ClO}^{\mathrm{O}} \mathrm{NO}_{2}+\mathrm{M}$. J. Ches. Phys. 71, 2021 (1979) United States

Relsler, H.: Mangl $r$, Mo: UIttlg, $c$. The kinetlcs of Pres rodeais generaled tig in loen photolyet.. T. Reasflons of $C_{3}$ (as sub u ) with NO, VInyl cyand de, and othylone. J. Rhe. Ynys. 11. E10s (19T9) United States

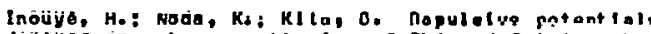
derived ipon beam scallorting of Rbt and fot lane by rare gas btots. j. Eheas Pliga. 71 , 2136 (1970) Jopan

Hontng, G.: Czajkousk1, M-l Stock, $M_{+}$: Dantroder. $W$ : High resolution laser spectroscopy of $\mathrm{CS}_{2}$. I. Orüuind state constants and potentlal ourve. J. Chase. Phys. 71,2138 (1979) Hest Gormany

Heyer, H.-i.: milier; $V_{1} 11$, Olúoolan medele mo

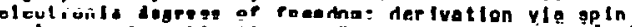
analogy and appilication to $\mathrm{PQ}+\mathrm{H}_{2}+\mathrm{F}_{+}+\mathrm{H}_{2}$. JP." Cheer. pliyso 71,3166 (10\%0) Unitod states

Shapirn. He: Kaplen, H. On the theory of $H+O H(2 x)$ collislons and interstellap OH abetr antion. J. Clies. Phys. 71, 2182 (1979)

Sonderga ard, N. A.: Savierg, I.; Jones, A. C.: kaurman, J. J, : Koski, $U$. $S$. React lve scottering of $B+(M P$ sub

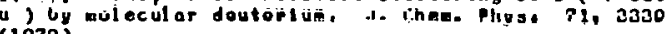
United States

Bowaan, J. M.; Drolghagen, G.; Toennles, J. P. A test of the quantum mechanical suoden vibrallutiol approximation Pne collinear inelastlc collilstons. J.

chea. Phys. 71, 2270 (1979) 


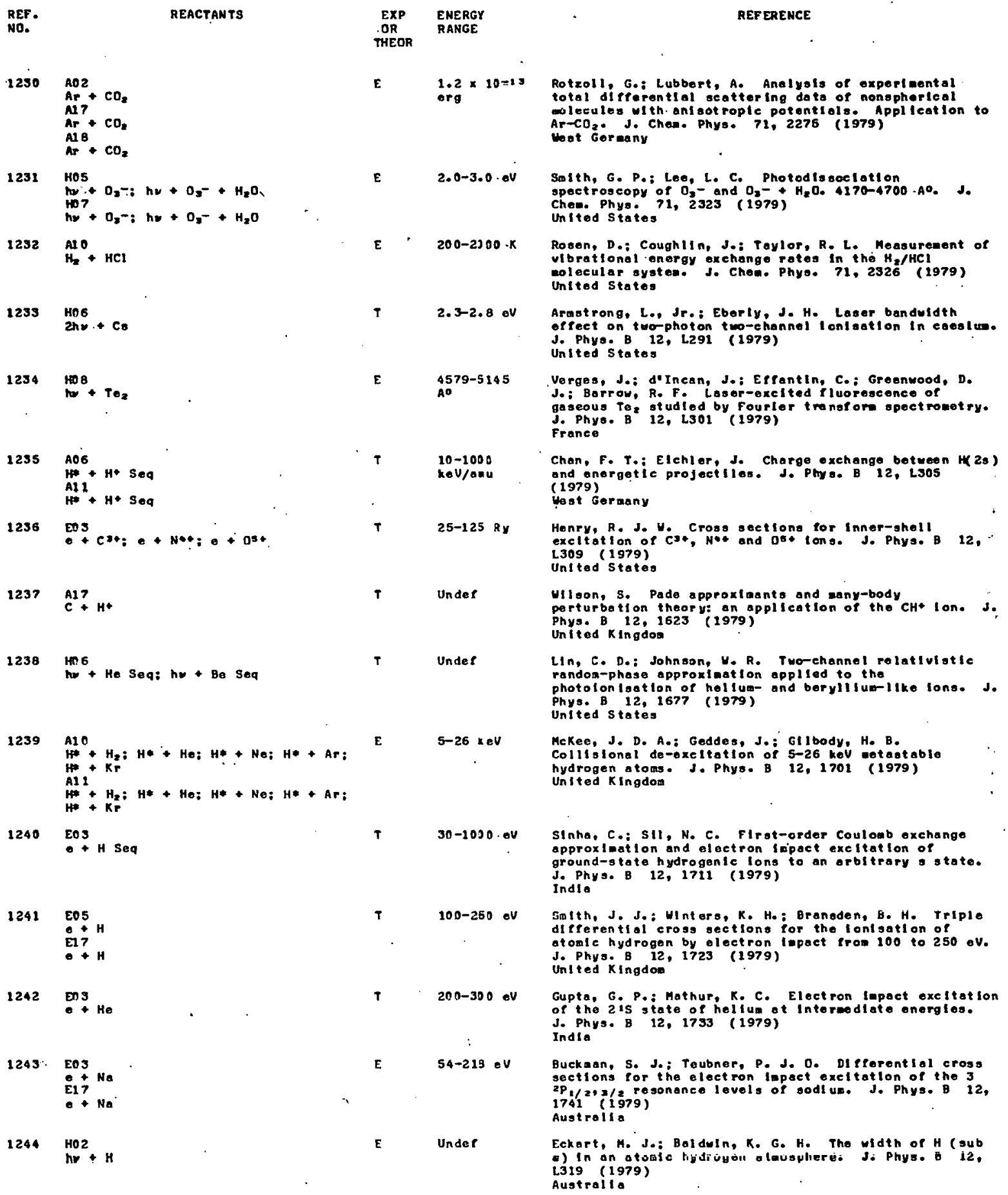


92

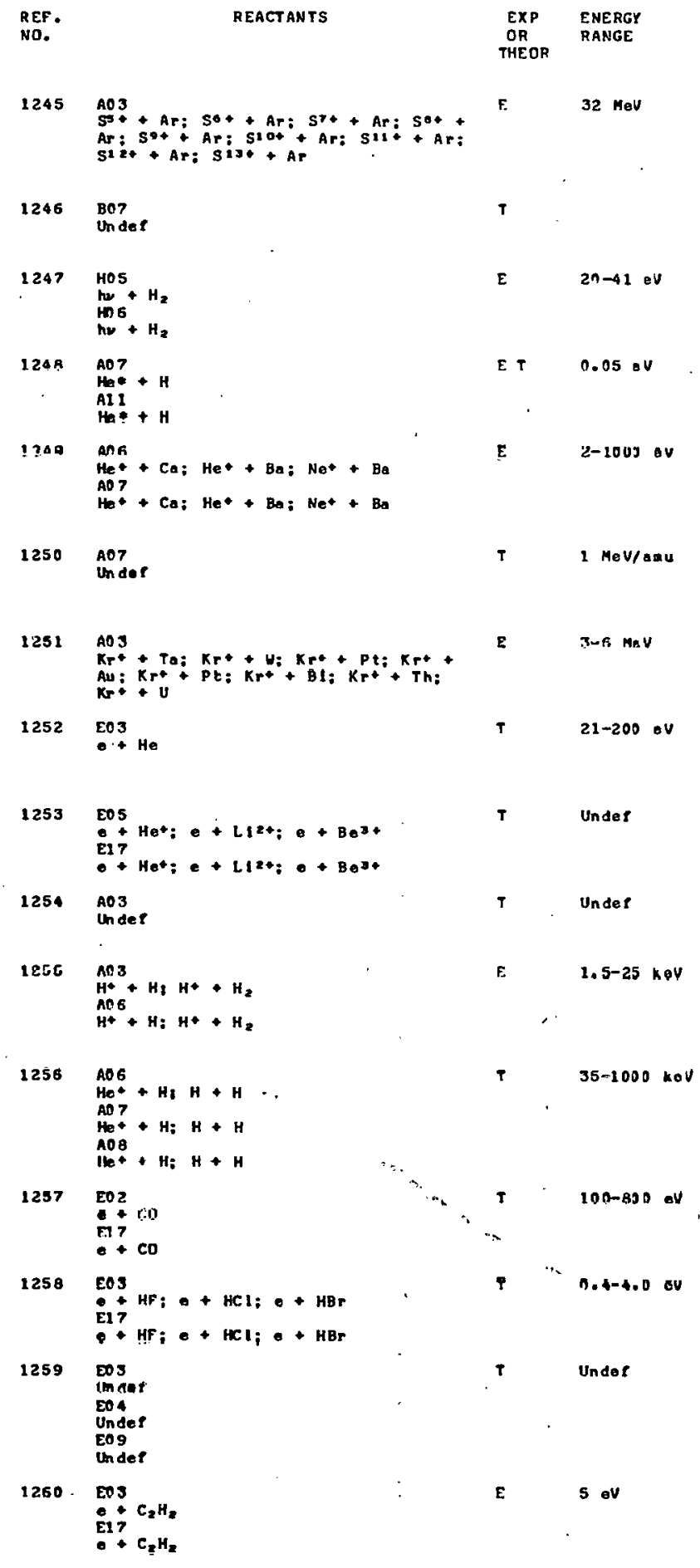

REFERENCE

Volpp, J,: Schuch, R.: Nolte, G.: Sehaldt-Boeklng, $H_{\text {, }}$ $K($ sub a sup $h) / K(s u b \alpha \alpha)$ branching ratlos of $S$ and $A r$ In dependence on 5 project 11 e-chorge state. J. Phys. B $12,1325$ ( 1979$)$

Mittleman. M. H. Resonant laser Induced negative Ion Mittlepan, M. H. Resonant laser Induced
production. J. Phys. B 12,1781 (1979) states

Strathdee, S.; Brownting, R. DIssoclatlve photolon isation of $H_{2}$ : proton kinetle energy spectra. J. Phys. B 12, 1789 (1979)

Morgner, H.: Ntehaus, A. Experi aental and theoretical Morgner, H.: Niehaus, A. Experi aental and theoret atudy of the Penning lonls of on of H (itom: West Germaing

Hultzgeh, Ho: Kivinast, U.: Mlohoue, A.: Rur, W. U.

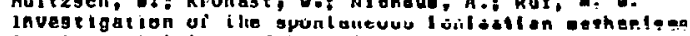
in t t ow collisions of Het with $C_{0}$ and Ba, and of Net? uth Ba. J. Phys. B 12, 1821 (1579)

West Germony

Olson, R. E. Multiple-ton lsation erose sect lons. Por highly stripped lons colliding with He, Ne and Ar. J. phys, B 12, 1843 (1979)

United states

Holta, C.: Hegmann, S.; Kratt, G.: Kuadow, M. X-ray

measurements of $K-L$ levol matching in heavy ion collistons. J. Phys. B 13, 1851 (1979)

colitisto

Fon, H. C.: Berrington, K. A.: Burke, P. G.: KIngston, A. E. The 1 is-2 ss and 1 is-2 ip exctitation of hel (um by Unitod Kingdant. J. Phys. B 12, 1861 (1979)

DIeltrijavic, M. S.; GruJle, P. V. On mutual angle and energy distributlons near lontsati on thresholds Por olectrontion colllslons. 2. Phys. B 12,1873 (1979) Yugosiavia

Fritseh, W.: Ville, U. Un the rneory of molervlar-orbltal x-ravs. J. Phya. B 12, L335 (1979) West Gerasny

HI 11, J.; Geddes, J.: Gllbody, H. B. Inproved measurenents of eross sections 1 or $H(2 \mathrm{~g})$ formall un III electron capture by $1.5-25$ keV protong in $H$ and $H_{z}$. Jo Phys. B 12, L341 (1979) United KIngdom

Becker, R. Lo: MaeKellar, A. D. Clasalcal Pour-body calculations of Het- H and $\mathrm{H}+\mathrm{H}$ collistons. J.

Phys. B 12, L345 (1979)
United States

Khare, 5. P.: Haj, D. Elast te seattering of intaraarinta anergy eloctrons by hoteronuclear nolecules. J. Phys. B 12, LJ5i (1995)
India

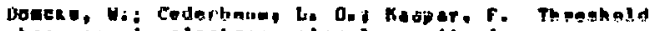

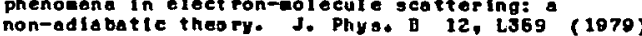
West Geraany

Schnelder, B. I.: LeDourneur, M.: Burke, P. G. Theory of Ulbratinnal oxcltation and di gsocl atf ve aftachent: an k-datplL epproach. J. Physe, B 12, L3CE (1070) France

Wilden, D. G.: Coner, J. High-resolution el ectron energy-1 osg spectroscopy of ethylonor analyeis of

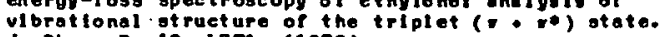
J. Phys. B 12, L371 (1979) United ringdom 


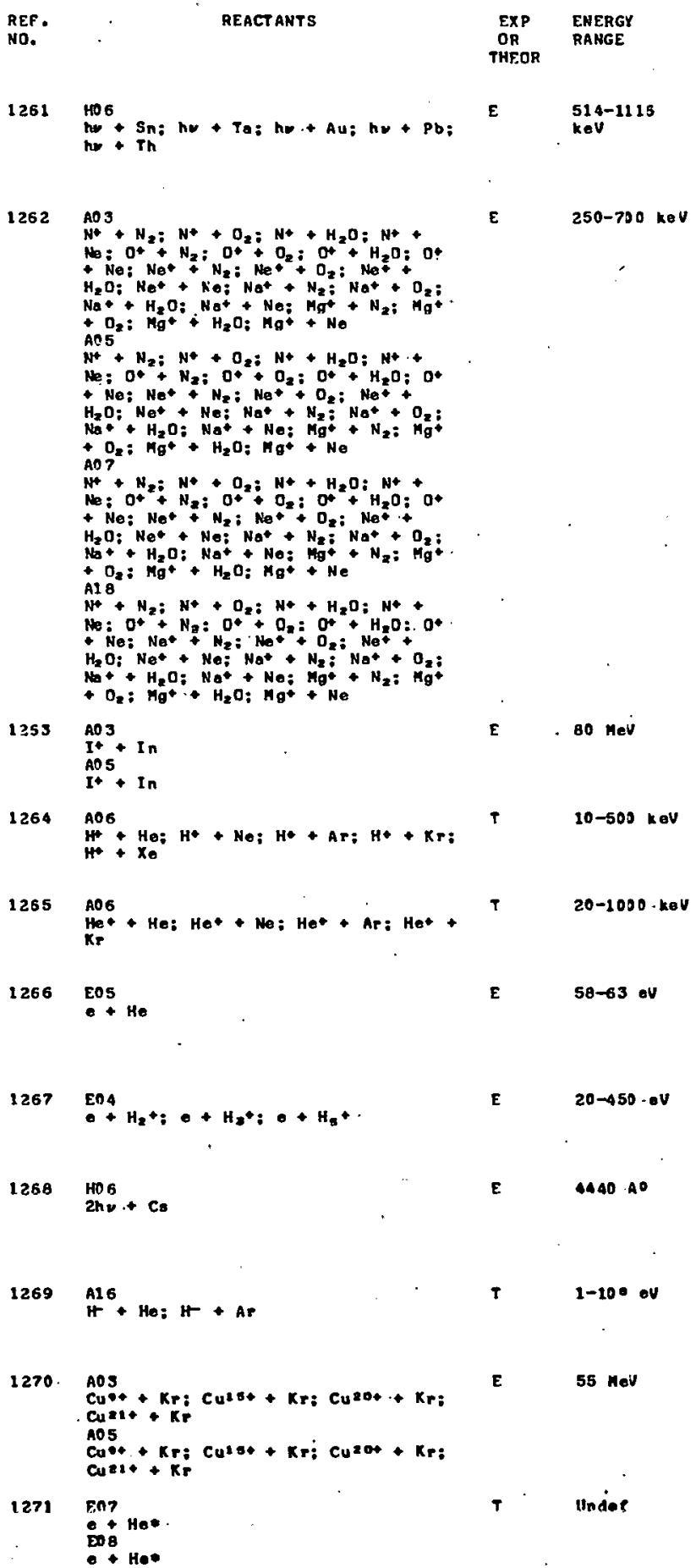

514-1113 $k \in v$

$250-700$ keV

Indes

$58-63$ ov

$20-450 \cdot \cdot v$

$\$ 440 \cdot 10$

Ponganathalah, C.; Gowda, R.; Sanjeevalah, B. K-ahell photolon lsat lon cross sact lons por $514,661.6,765.8$ and $1115.5 \mathrm{keV}$ gamas rays. J. Phys, B 12, 1965 (1979)

Luz, N.: Sackmann, S.: Lutz, H. O. Iapact parometer dependence of $R$-shell exct tation $t n$ stow lon-aton. collistons.. J. Phys. B 12, 1973 (1979) Hest Germany
Stott. U. R.: Yadd Ington, J. C. Double K-shell vacancles produced in noor-syametric collialons with 2 opproxtanately 5J. J. Phys. B 12, 1995 (1979) Canoda

10-50s keV Roy, B. N.: Ral, D. K. Modifled blnarymeounter calculat lons for elect ron capture from noblo-gas atons by protons: I. J. Physe. B 12, 2015 (1978) Ind I a

20-1000-keV Kunar, A.: Roy, B. N. Modirled bl nary-encounter calculations for electron capture froen noble-gas atos by He* I ons. II. J. Phys. B 12, 2025 (1979)

Baxter, J. A.: Coser, J.: HICks, P. J.: MeConkey, J.i H. Studles of post-collision interact lons for electrond efected from autolonising levelo of hellum exelted by low-energy electrons. J. Phys. B 12, 2031 (1979) Unlted Kingdoa

Mathur, D.: Hasted, J. B.; Khan, B. U. Colliston procesos of elect rons with aclecular hydrogon tons. J. Phys. B 12, $2 n 43$ (1979)

Kawlnski, H.: Kegeler, J.: Kolloth, K. J. The 6t=6d quadrupole resonances in tro-photon Ionlsation of cassiua. Heasuresent of lon osyacetry and epl Host Geríany

Gaoyacg. J. P. A siaple approxieation for low-energy totel dotachment eroses asetlons: J. Phys. B 12, 2387 (1979) Hagaann, S.: Cacke, C: L.; Justl nl ano, E.; HacDonald,

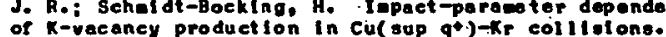
of R phaces $B$ 12, L395 (1979) United states

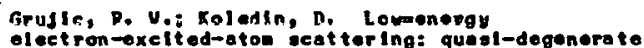
electron-oxclited-at on seat ter ing: qua
case. J. Phys. B 12, L399 (1979) Yugool avie 


\begin{tabular}{|c|c|c|c|c|}
\hline \multirow{2}{*}{$\begin{array}{l}\text { REF. } \\
\text { NO. }\end{array}$} & \multicolumn{4}{|c|}{$94^{\circ}$} \\
\hline & REACTANTS & $\begin{array}{l}\text { EXP } \\
\text { OR } \\
\text { THE OR }\end{array}$ & $\begin{array}{l}\text { ENERGY } \\
\text { RANGE }\end{array}$ & REF ERENCE \\
\hline 1272 & $\begin{array}{l}\text { Ho6 } \\
\text { She + Cs: } 4 \text { he }+C_{8} \text {. }\end{array}$ & $\boldsymbol{T}$ & $\begin{array}{l}9443-14414 \\
c x^{-1}\end{array}$ & 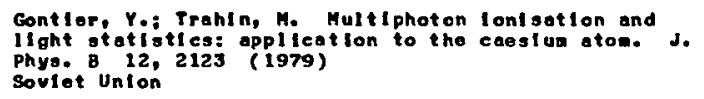 \\
\hline 1273 & 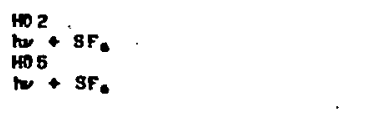 & $E$ & $5-63 \otimes V$ & 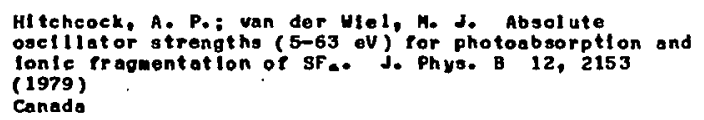 \\
\hline 1274 & $\begin{array}{l}\text { A07 } \\
\text { Ne: }+H \\
\text { A11 } \\
H_{0}+H+H\end{array}$ & E & Thermal & $\begin{array}{l}\text { Morgnar, H. Poinning ionlsotton of at onte hydrogen by } \\
\text { otastable neon at ons. J. Phys. B } 12,2171 \text { (1979) } \\
\text { west Germany }\end{array}$ \\
\hline 1275 & $\mathrm{H}^{*}+\mathrm{Au}_{\mathrm{U}} \mathrm{H}^{+}+\mathrm{Ag}_{\mathrm{g}}: \mathrm{H}^{+}+$PERT & 9 & 1-15 $\mathrm{MeV}$ & 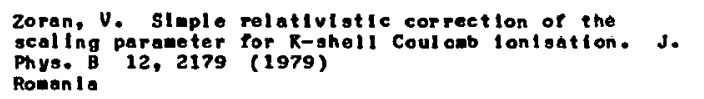 \\
\hline 1276 & 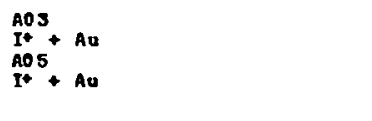 & $\varepsilon$ & 6-25 $\mathrm{YeV}$ & 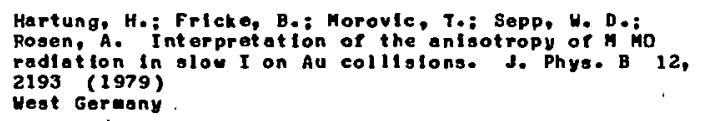 \\
\hline 2277 & 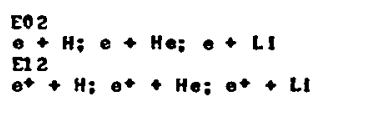 & $T$ & $10-503 \cdot 0 \mathrm{~V}$ & 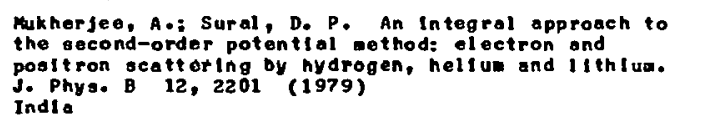 \\
\hline 1278 & $\begin{array}{l}\mathrm{EO} 2 \\
\mathrm{E}+\mathrm{Hz} \\
\mathrm{E} T+\mathrm{H}_{2}\end{array}$ & $\tau$ & $50-200 \cdot \mathrm{eV}$ & 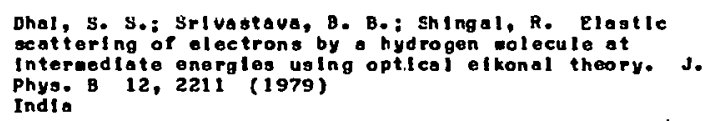 \\
\hline 1279 & $\begin{array}{l}\mathrm{EOz} \\
\mathrm{E}+\mathrm{Ne} \\
\mathrm{ES} \\
\mathrm{B}+\mathrm{Ne}\end{array}$ & $\mathbf{T}$ & $16-19 \mathrm{eV}$ & $\begin{array}{l}\text { Noro, T.: Basakl, ro: Tatewakt, I1. R-motrix otudy of } \\
\text { resonance spectra of the neon at om. Jo Phys. B 12, } \\
2217 \text { (1979) } \\
\text { Japan }\end{array}$ \\
\hline 1280 & 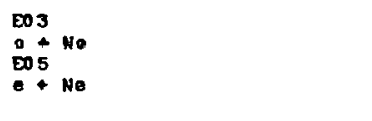 & $T$ & $50-1020-\mathrm{eV}$ & 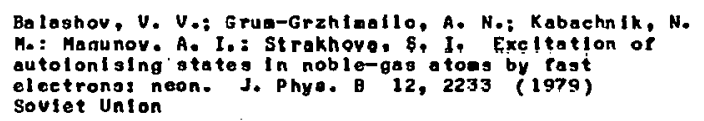 \\
\hline 1381 & 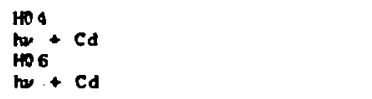 & ? & $27 \bullet 4$ & $\begin{array}{l}\text { Klar, H. Analysis of allgnaent produced by } \\
\text { photolonigation of Cd. J.Phys. B 12, L409 (1979) } \\
\text { West farmany }\end{array}$ \\
\hline 1282 & $\mathrm{hu}_{\mathrm{hy}}^{\mathrm{m}}+\mathrm{Ba}$ & E & $21-25 \mathrm{eV}$ & $\begin{array}{l}\text { Potta, A. W.: Lee, E. P. F. Thie He IIa Sp-1 } \\
\text { photoalectron spootrum of Ba. J. Phyc. B 12, L413 } \\
\text { (1979) KIngdo. } \\
\text { Unlted KIngd }\end{array}$ \\
\hline 2283 & $\mathrm{HO}_{\mathrm{H}}^{\mathrm{H}}+\mathrm{N}_{2}=\mathrm{hN}+\mathrm{CO}$ & $T$ & $0.1-10 \cdot R y$ & 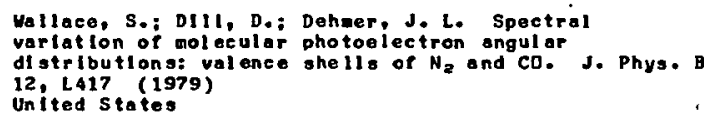 \\
\hline 1284 & 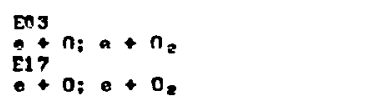 & $\hat{\varepsilon}$ & $100-500 \mathrm{eV}$ & 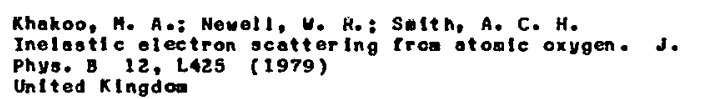 \\
\hline 1285 & 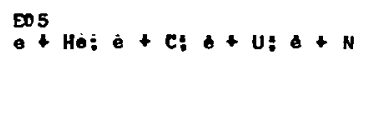 & E & $20-153-e V$ & 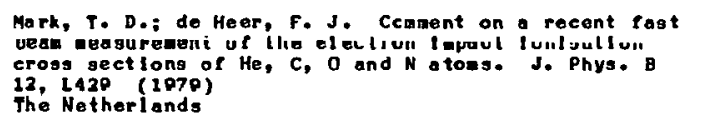 \\
\hline 1286 & $\mathrm{eOS}_{\mathrm{e}}^{\mathrm{HO}} \mathrm{O}+\mathrm{C}: \mathrm{e}+\mathrm{N}: \mathrm{O}+\mathrm{O}$ & $\varepsilon$ & $20-15) \cdot e V$ & 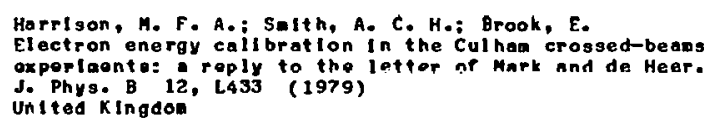 \\
\hline
\end{tabular}




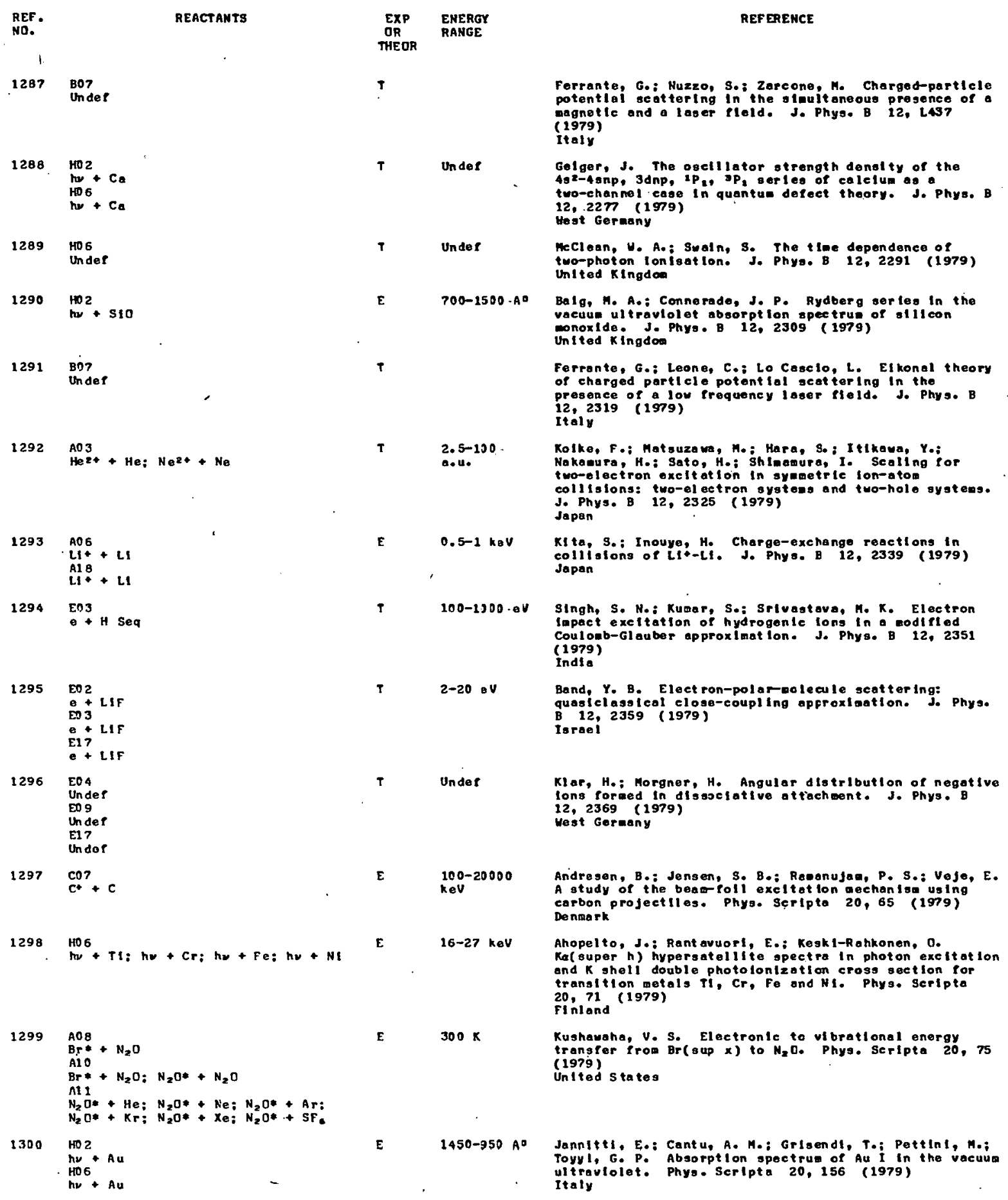




\begin{tabular}{|c|c|c|c|}
\hline $\begin{array}{l}\text { REF. } \\
\text { NO. }\end{array}$ & REACT ANTS & $\begin{array}{l}\text { EXP } \\
\text { OR } \\
\text { THEOR }\end{array}$ & $\begin{array}{l}\text { ENERGY } \\
\text { RANGE }\end{array}$ \\
\hline 1301 & $\begin{array}{l}\mathrm{Al}_{2} 2 \\
\mathrm{He}+\mathrm{He} \\
\mathrm{HO} 3 \\
\mathrm{~h},+\mathrm{He}\end{array}$ & $\mathbf{E}$ & $\begin{array}{l}\text { A12 } 2 \\
300 \mathrm{~K} \\
\text { H9 } 3 \\
5145\end{array}$ \\
\hline 1302 & $\mathrm{D}^{\mathrm{CDG}}+\mathrm{Hg}_{\mathrm{g}} \mathrm{D}+\mathrm{Ca} ; \mathrm{D}^{+}+\mathrm{Sr} ; \mathrm{D}+\mathrm{Ba}$ & $\mathbf{E}$ & $\begin{array}{l}1.25-100 \\
k \in y^{-10}\end{array}$ \\
\hline 1303 & $\begin{array}{l}\mathrm{EO} 2 \\
\mathrm{E}+\mathrm{He} \\
\mathrm{E} 17 \\
\mathrm{E}+\mathrm{He} \\
\mathrm{C} 5 \\
\mathrm{O}+\mathrm{He}\end{array}$ & $\mathbf{T}$ & $0-19.6 \mathrm{eV}$ \\
\hline 1304 & $\begin{array}{l}\text { ter } \\
\text { te }\end{array}$ & $\mathbf{E}$ & $1 y-300 \cdot 6 v$ \\
\hline 1305 & 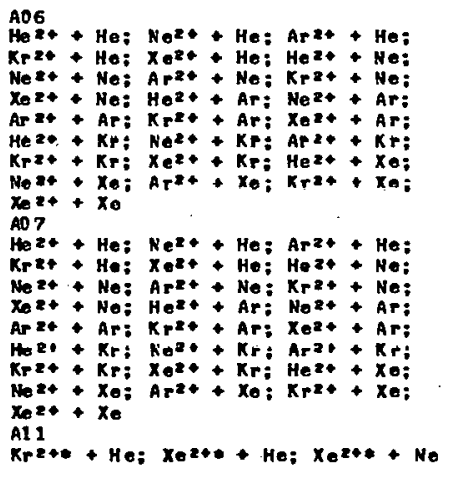 & $\mathbf{E}$ & $300 \mathrm{~K}$ \\
\hline 1306 & 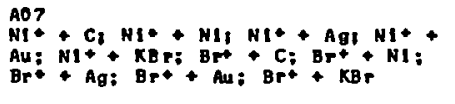 & $\mathbf{E}$ & $40-120 \cdot . \mathrm{HeV}$ \\
\hline 1307 & $\begin{array}{l}A 06 \\
C^{\circ}+H\end{array}+\mathrm{H} ; \mathrm{PERT}+\mathrm{H}: \mathrm{PERT}+\mathrm{He}$ & $\mathbf{T}$ & $\begin{array}{l}200-150000 \\
k \in V\end{array}$ \\
\hline 1300 & Ander & $\mathbf{T}$ & Under \\
\hline 1309 & 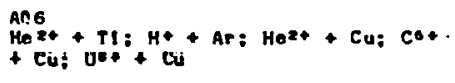 & $T$ & $2.5-95 \mathrm{HeV}$ \\
\hline 1310 & 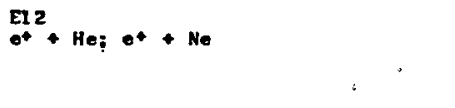 & $\mathbf{E}$ & $2-50.0 v$ \\
\hline 1311 & 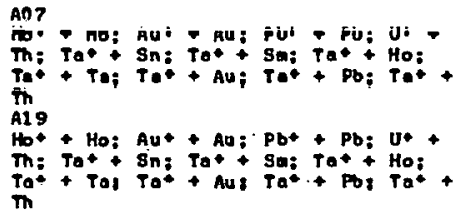 & $\mathbf{E}$ & $17-60$ HeV \\
\hline 1312 & 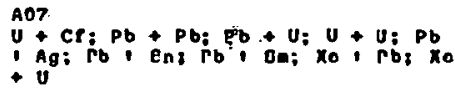 & $\mathbf{T}$ & $\begin{array}{l}973-1600 \\
\text { MeV }\end{array}$ \\
\hline
\end{tabular}

REF ERENCE

LeDuP, Y. Collislon-induced seot tering in hellun. Phys. Rev. A 20, 48 (1979)

France

Morgan, T. J.: Stone, J.: Mayo, Mo: Kurose, J. Dproduetion by autlpie eharge-trangrer colilsions in alkall ne-barth-net al vapors. Phys. Rev. A 20,54 (1979)

United states

Nesbet, R. K. Varlatlonal calculatlons of accurato (1979)

United states

Seniavone, J. Á:; Tarr, So H.: Freund, R. S.

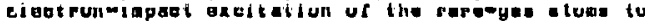
high-Hyaberg stares. Hhys. Hev. A $20, " 1$ (1979) Unlted státés

Johnsen, R.: Blondt, M. A. Theraal-energy charge transfer, quenching, and assoclatl on reactlons of doubly chorged tons in the rare gases. Phys. Rev. A 20, 87 (1979)
Mecilntock, j. A.; Lee, Y. K.: Madansky, L. Production

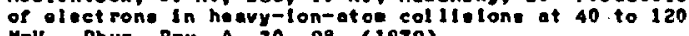
Mev. Phys. Rev. A 20, 98 (1979) United Statas

Elchler, J.; Chan, F. T. Approach to electron capture Into arblitrary orineloel shells of energotlls
projectilies. Phys. Rev. A 20,104 (1979) projectiles.
Heat Germany

Ruaseh. A. Diefraotion and angular nomontua offoote In semiclassicol otoale scattering theory. Phys. Rev. A 20,113 (1979) United states

Reading, J. F.: Ford, A. L.: Swatford, G. L.: FItchard, A. Inner-sheli charge transfer in osymotilc ton-otod colissions, Phys. ReV. $A$ LU, lav (Iy/y)

Coleman, P. Go: meNutt, J. D.; Drano, L. Mo: Burelaga, J. R. Scattering of 1 ow-energy posttrons by hel lue ond noon azous. Pnys, Rov. A dU, lto (14/0)

Meyerhor, $V_{0}$ E.; Ruetseht, A.: Stoller, C.; stoek11,

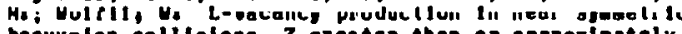
heavy-ion collisions 2 greater than or approxinately equal to 35 : Phyo. Rev. A 20, 1 s4 (1979)

Soff, G.: Grotnar, M.: Batz, W.: Mullor, B. Electrons in superhoavy guasimoleculos. Phys. Rev. A 20,169 (1070) United states 


\begin{tabular}{|c|c|c|c|c|}
\hline $\begin{array}{l}\text { REF. } \\
\text { No. }\end{array}$ & REACTANTS & $\begin{array}{c}\operatorname{EXP} \\
\text { OR } \\
\text { THEOR }\end{array}$ & $\begin{array}{l}\text { ENERGY } \\
\text { RANGE }\end{array}$ & REF ERENCE \\
\hline 1313 & 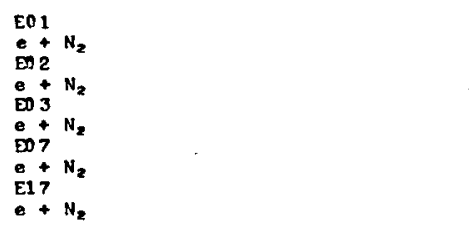 & $\mathbf{T}$ & $0.5-4.5 \mathrm{eV}$ & $\begin{array}{l}\text { Dube, L.: Herzenberg, A. Absolute cross sections from } \\
\text { the boomerang nodel ior resonant electron-molecule } \\
\text { seatering. Phys. Rev. A } 20,194 \quad(1979) \\
\text { United States }\end{array}$ \\
\hline 1314 & $\underset{H^{+}}{\mathrm{DCB}}+\mathrm{SI}_{\mathrm{O}}: \mathrm{H}^{+*}+\mathrm{Hu}^{+}+\mathrm{Pt}^{+}+\mathrm{Ge}: \mathrm{H}^{+}+\mathrm{MO}$ & $E$ & $\begin{array}{l}6.5-12.5 \\
k \in V\end{array}$ & 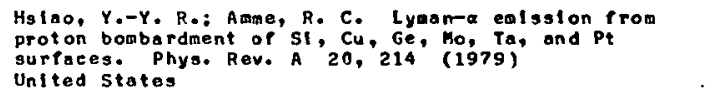 \\
\hline 1315 & 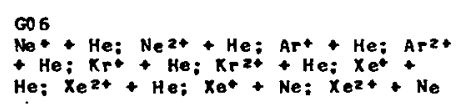 & E & $5-10 \mathrm{eV}$ & $\begin{array}{l}\text { Johngen, R.: Blond, M. A. Mobllitles of singly and } \\
\text { doubly charged trare-gas lons in thellua and in neon. } \\
\text { phys. Rev. A } 20,221 \text { (1979) } \\
\text { United States }\end{array}$ \\
\hline 1316 & $\begin{array}{l}\mathrm{Al2} \\
\mathrm{HF} * \mathrm{Ar} ; \mathrm{HF} *+\mathrm{Kr} \\
\mathrm{H} 2 \mathrm{C} \\
\mathrm{h} 2+\mathrm{HF}\end{array}$ & $\varepsilon$ & $\begin{array}{l}\text { A12 } \\
300 \\
\text { H04 } \\
2.2-3.3\end{array}$ & 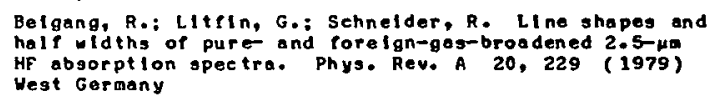 \\
\hline 1317 & $\begin{array}{l}\text { Bn? } \\
\text { Unde? }\end{array}$ & $T$ & & $\begin{array}{l}\text { Rosenberg, L. Genarallized low-trequency approxtactlon } \\
\text { for seattering in a laser tield. II. Phys. Rov. A } \\
20 \text { 275 (1979) } \\
\text { United states }\end{array}$ \\
\hline 1318 & 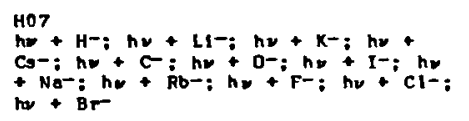 & $T$ & $0.5-4.0 \mathrm{eV}$ & 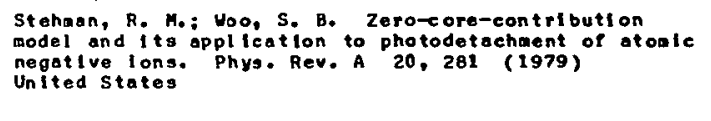 \\
\hline 1319 & 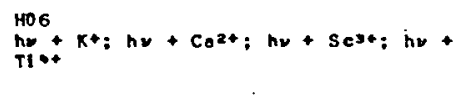 & $T$ & $40-203 \mathrm{eV}$ & 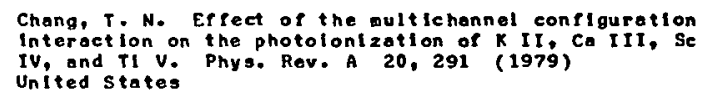 \\
\hline 1320 & $\mathrm{ML}^{2} \mathrm{2}+$. & $T$ & Under & $\begin{array}{l}\text { Chan, Y. H.: Tsul, W. L. Closalcal theory of } \\
\text { seattering of an electron beas by a laser standing } \\
\text { wave. Phys. Rev. A } 20,294 \text { (1975) } \\
\text { Japan }\end{array}$ \\
\hline 1321 & $\mathrm{CO}_{\mathrm{He}}^{\mathrm{CO}}+\mathrm{C}$ & E & $200 k=V$ & 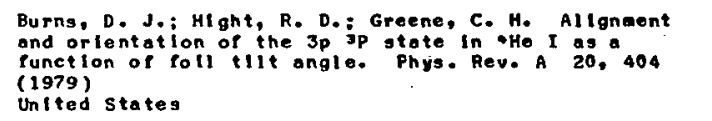 \\
\hline 1322 & $e_{0}^{-3}+$ No. & $\varepsilon$ & $4-50$ eV & 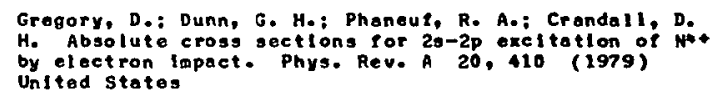 \\
\hline 1323 & ${ }_{H}^{A 07}+\mathrm{H}$ Seq & $t$ & Under & 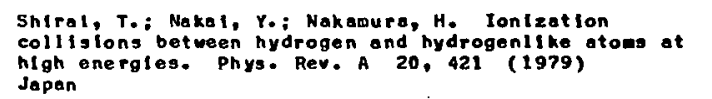 \\
\hline 1324 & $E^{E 12}+H$ & T & Under & $\begin{array}{l}\text { Drachan, R. J.: Sucter, J: Annithilatlon in } \\
\text { positron-atom colliglons: A new epprooch. Phys. Rev. A } \\
20 \text { A42 (1979) } \\
\text { United States }\end{array}$ \\
\hline 1325 & 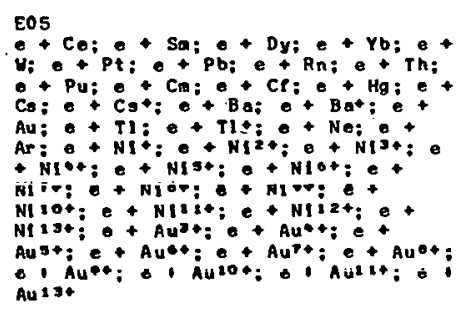 & $\boldsymbol{T}$ & $5-10+e v$ & $\begin{array}{l}\text { MeGutre, E. J. Sealed electron tonization eross } \\
\text { sections in the Born approximotlon ror at ons with ss } \\
\text { less than or equal to } 2 \text { less than or equal to } 102 \text {. } \\
\text { Phys. Rev. A } 20,445 \text { (1979) } \\
\text { Unlted States }\end{array}$ \\
\hline 1326 & $\begin{array}{l}807 \\
\text { Undef }\end{array}$ & $T$ & & $\begin{array}{l}\text { Rosenberg, L. Coulosb seatterl'́ng in laser'́liold. } \\
\text { Phys. Rev. A } 20,457 \text { (1979) } \\
\text { United States }\end{array}$ \\
\hline
\end{tabular}


98

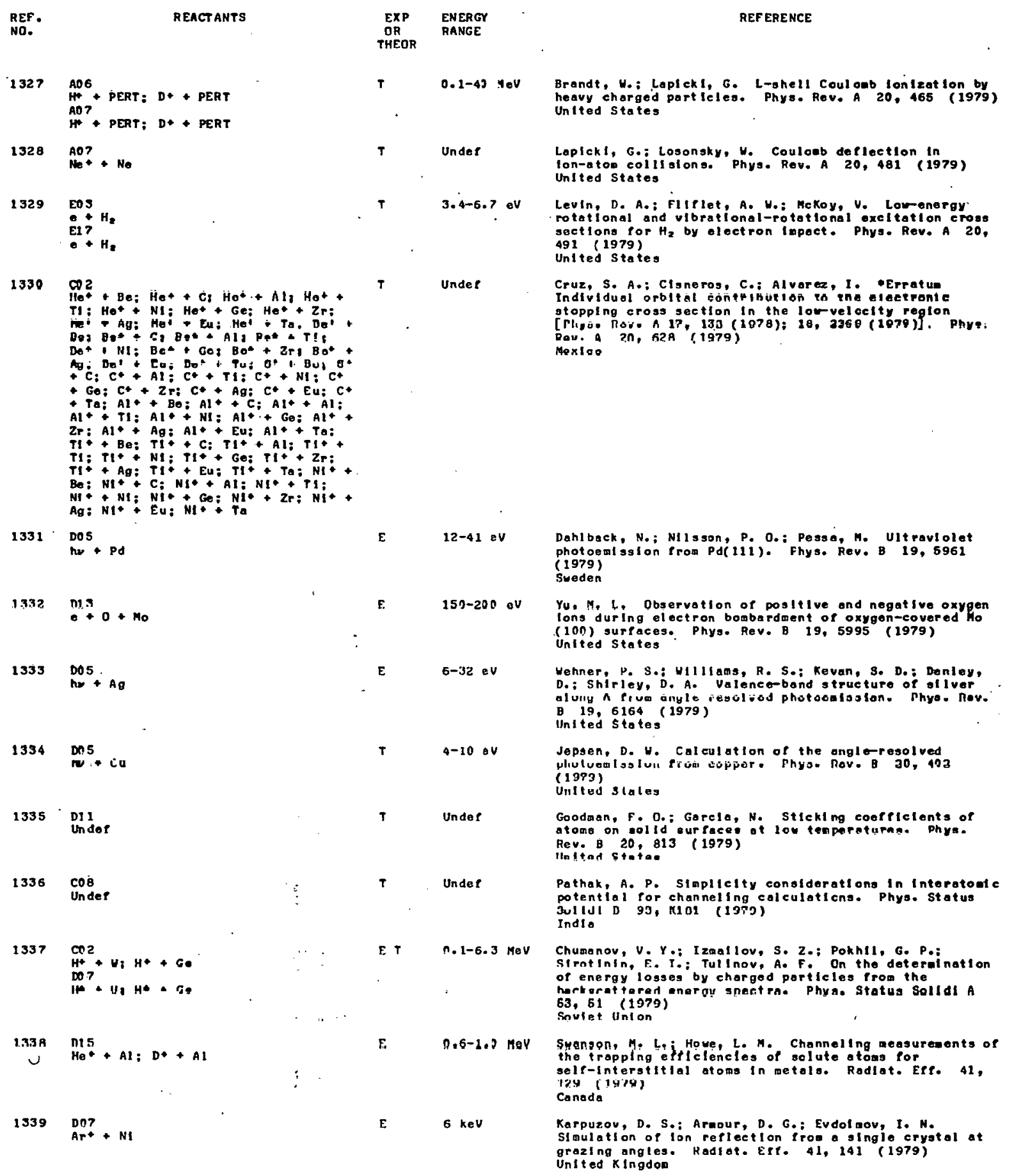


99

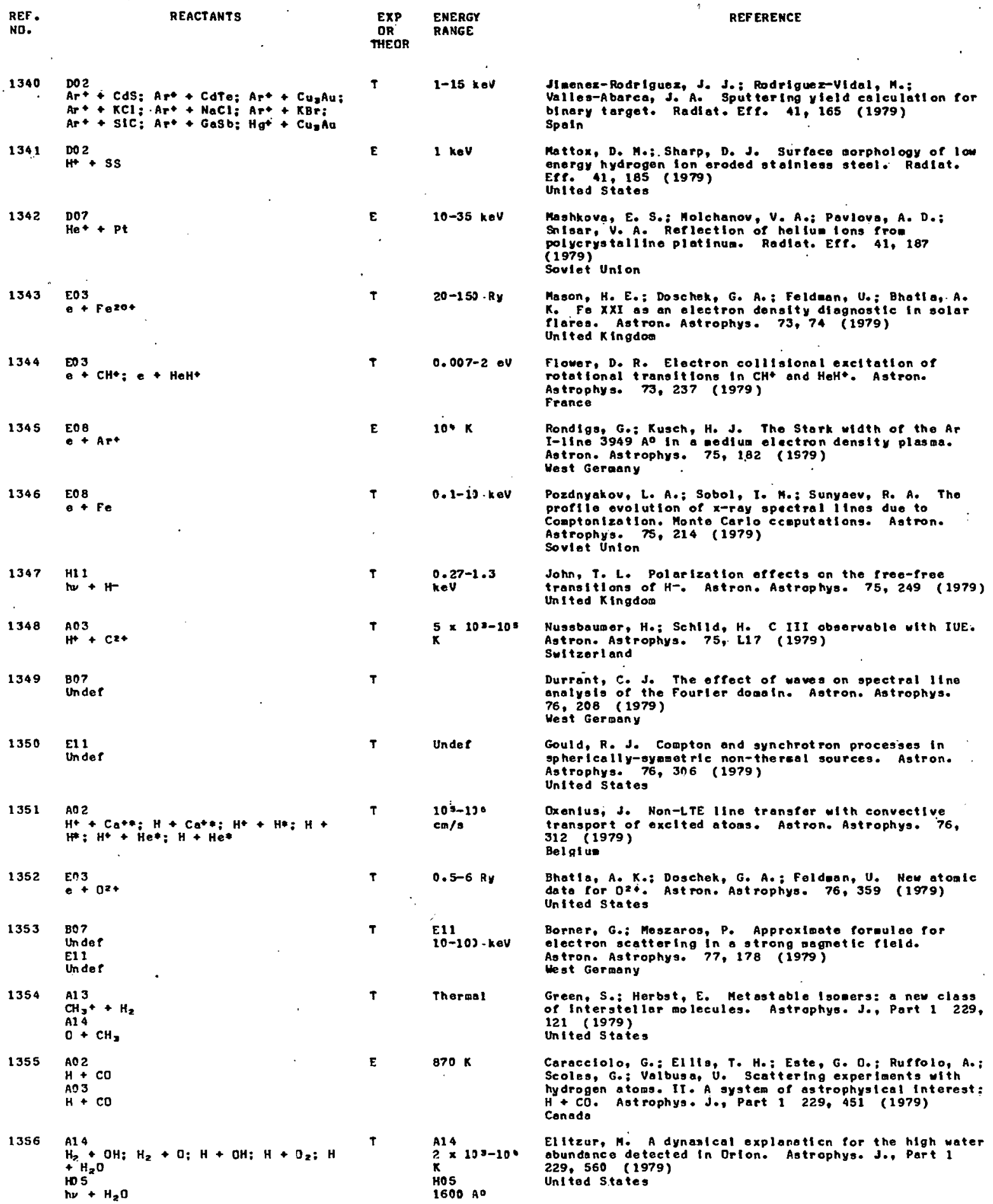




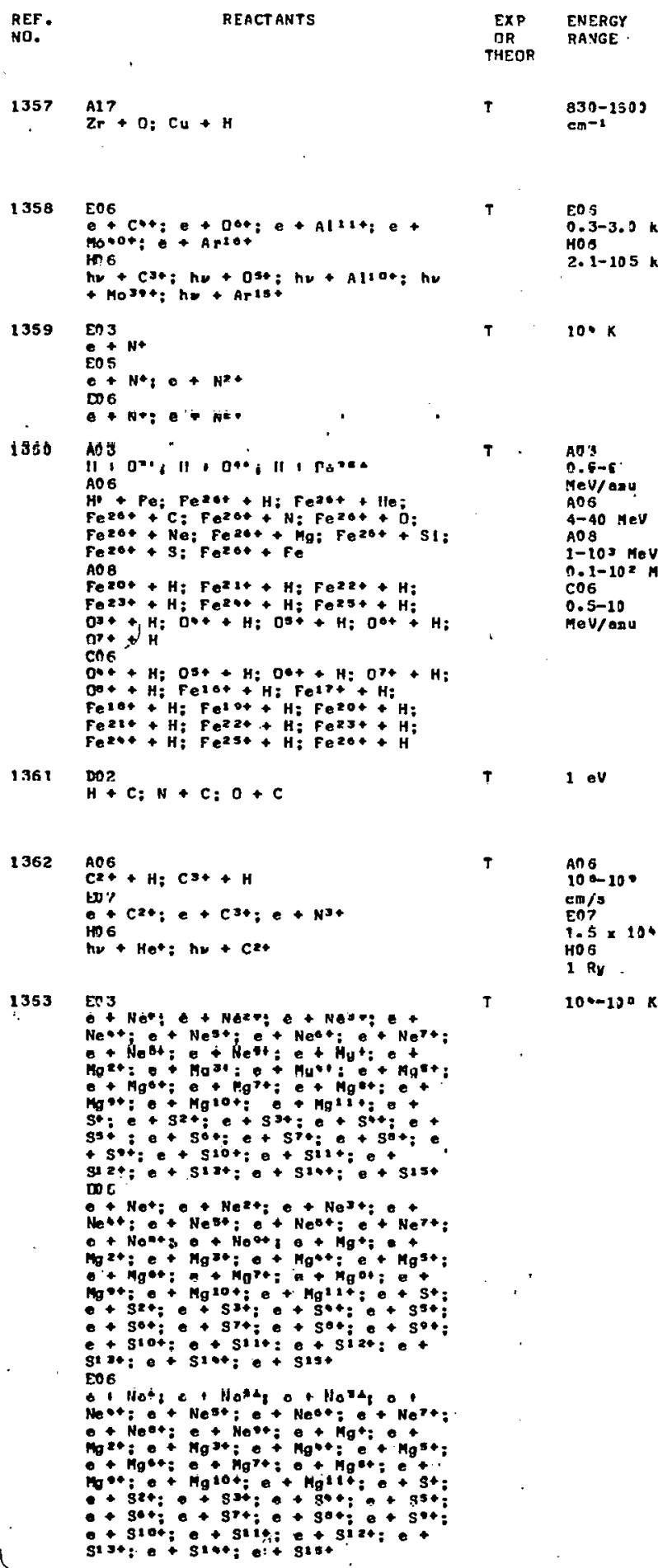




\begin{tabular}{|c|c|c|c|}
\hline $\begin{array}{l}\text { REF. } \\
\text { NO. }\end{array}$ & REACTAN TS & $\begin{array}{l}\text { EXP } \\
\text { ORP } \\
\text { THEOR }\end{array}$ & $\begin{array}{l}\text { ENERGY } \\
\text { RANGE }\end{array}$ \\
\hline 1364 & E1! $\mathrm{H}^{+}$ & $\tau$ & $\ln \times k$ \\
\hline & . & & \\
\hline 1365 & ${ }_{h 2}^{H 12}+\mathrm{e}$ & T & $\begin{array}{l}\text { n.05-5 MeV } \\
:\end{array}$ \\
\hline & . & & \\
\hline 1366 & 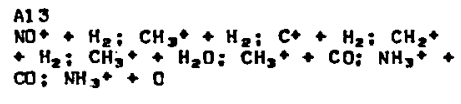 & $\begin{array}{lll} & \end{array}$ & $100 K$ \\
\hline 1367 & $\mathrm{C}_{3+}^{\mathrm{An} 6}+\mathrm{H}: \mathrm{N}^{3+}+\mathrm{H}$ & .7 & $0.003-4 \mathrm{eV}$ \\
\hline 1358 & $\mathrm{Fl}^{\mathrm{F}}+\mathrm{H} ; \mathrm{Fe}+\mathrm{D}$ & $\mathrm{T}$ & $\begin{array}{l}1670 \mathrm{~cm}: 1: \\
1200 \mathrm{~cm}: \mathrm{s}\end{array}$ \\
\hline 1369 & ${ }_{\text {Ho }}^{\text {HOS }}+\mathrm{MgH}$ & T & $4-4.7 \mathrm{eV}$ \\
\hline 1370 & $\mathrm{En}^{\mathrm{E}}+\mathrm{C}$ & T & $10-100 x$ \\
\hline 1371 & 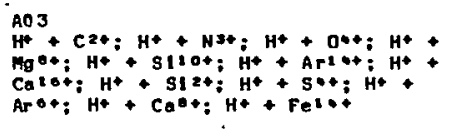 & $\mathbf{T}$ & $\begin{array}{l}50-12 \times 103 \\
\text { eV }\end{array}$ \\
\hline 1372 & $\begin{array}{l}\text { A1 } 3 \\
\text { Revileu }\end{array}$ & $\varepsilon \mathrm{T}$ & $300 \mathrm{~K}$ \\
\hline 2373 & $\mathrm{H}_{\mathrm{H}}^{\mathrm{H} 2} \mathrm{2}+\mathrm{e}$ & 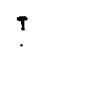 & $7-50 \mathrm{keV}$ \\
\hline 1374 & $\begin{array}{l}\text { Eos } \\
\text { pet: Feist: }\end{array}$ & $\boldsymbol{T}$ & $10-5000$ \\
\hline 1375 & $\begin{array}{l}\mathrm{AO}_{3} \\
\mathrm{H}_{2}+\mathrm{NH}= \\
\mathrm{AI}_{2}=\mathrm{NH}_{3}=\end{array}$ & $T$ & $0.5-100 \mathrm{~K}$ \\
\hline 1376 & $\stackrel{\mathrm{AO} 3}{\mathrm{H}}+\mathrm{OH}$ & 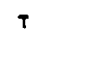 & $10-120-K$ \\
\hline 1377 & $\mathrm{EO}^{\mathrm{E}}+\mathrm{HO}$ & $\mathbf{T}$ & $1-11 \bullet V$ \\
\hline 378 & $\stackrel{\mathrm{AO} 6}{\mathrm{O}^{2}+}+\mathrm{H} ; \mathrm{Ne}^{2+}+\mathrm{H}$ & $\begin{array}{lll} & \end{array}$ & $100 k$ \\
\hline
\end{tabular}

REF ERENCE

Hoyng, P.: Melrose, D. B.: Adans, J. C. Reloxatlon and bremgstrahlung of thick-torget electron streams: a sl mple oppll cation of the Legendre expansion aothod. Astrophys. J., Part 1 230, 950 (1979) United stotes

Gould, P. J. Soft-phot on exlsolon effects and radlative corrections for electromagnetle processes of very high onergles. Astrophys. J., Port 1 230, 967

Unlted stotes

Huntress, W. T., JP.: Mitehell, G. F. The synthegis of cosplex molecules in interateliar elouds. Astrophys. J.p Part 1 231, 456 (1979)

Watson, w. D.: Chrlotenson, R. B. Quantal colculatlons for charge transfer in colilisions of Clt and wat ulth atoms. Astrophys. J., Part 1 231, 627 (1979)

Dendramls, A.: van zee, R. J.: Ueltner, W., Sr. The FeH molecule at $4 \mathrm{~K}$. Astrophys. J., Part 1 251, 632 (1979)

stotes

KI rby, K.; Saxon, R. P.: LIu, B. Oselllator strengths and photodlssoclation cross sectlons in the $x$. A and $x$

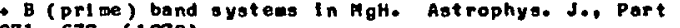
Uni, 637 (1979)

Flannery, B. P.: Pross, Y. H. An ionizat lon-coupled acougtle ingtabill ty of the intergtellar nedlum. acoustle. Ingtabillty. of the intergtellar United States

Landaan, D. A.: Broun, T. Proton callistonal exeltation in the lowest-lying $3 p$ terns of lons in the Be and $\mathrm{Mg}$ isoelectronte sequenees. Astrophys. J., Part 1232,636 (1979) Unlted States

Schirt, H. I.: Bohme, D. K. An ton-aolecule seheme for the synthesis of hydrocarbon-etain and organonlt rogen moleeules in dense interst allar el ouds. Astrophys. J., part 1 232, 740 (1979) conada

Langer, S. H. A Purther gtudy of the Coaptontzotton of x-rays by lourtemperature olectrong. Astrophys. J... Part 1 232, 891 (1979)

United States

Couan, R. D.: Mann, J. B. Contrlbution of autolonization to total lonlzation rotes. Astrophys. J., Part 1 232, 94n (1979) United States

Sehwartz, P. R. Estlant ion of colllstonally Induced transition rates of Interstallor nolecules froe laboratory dat a. Astrophys. J., Port i2 2Z2y, Las (1979)

United States

Kaplan, H.: Shaptro, H. The role of $H+O H$ collistons In punpling the OH 18 centlater aser lines. Astrophys. J., Part 2 229, LS1 (1979) Isroel

Tully, J. A. : Sumers, H. P. On the rate coofflelent for eloctron Impact oxcltation of the 2 ss 2 sp transtition In hell un. Ast rophya, J., Part 2 229, L113 (1979)

United Kingdon

Butler, S. E.; Bender, C. F.; Dalgarno, A. Chorge transfer of Ot. and Not+ with. H. Astrophys. J., Pert 2 230 : 59 (1979) 


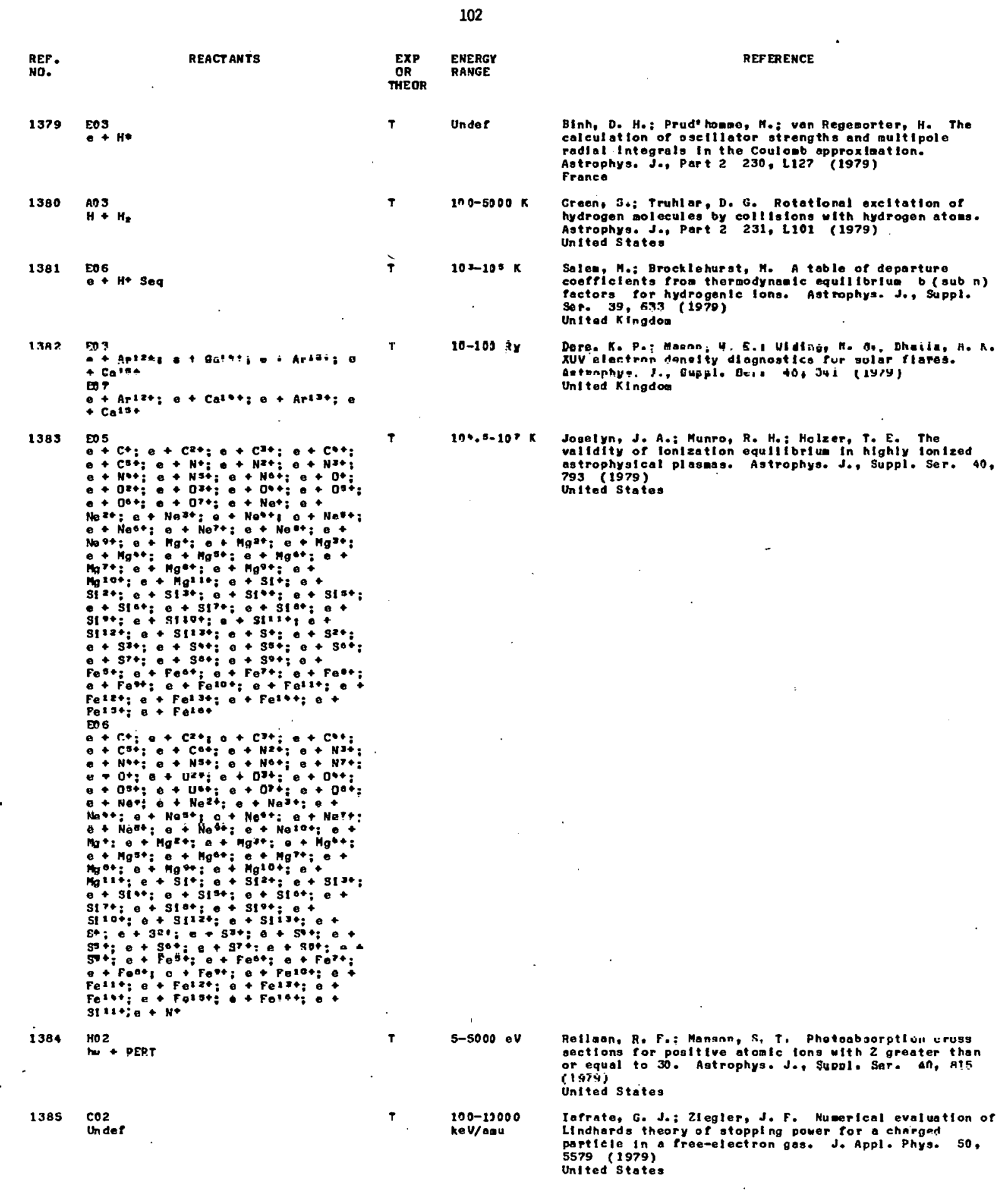




\begin{tabular}{|c|c|c|c|c|}
\hline $\begin{array}{l}\text { REF. } \\
\text { NO. }\end{array}$ & REACT ANTS & $\begin{array}{l}\text { EXP } \\
\text { OR } \\
\text { THEOR }\end{array}$ & $\begin{array}{l}\text { ENERGY } \\
\text { RANGE }\end{array}$ & REFERENCE \\
\hline 1386 & $\frac{\mathrm{HO} 2}{\mathrm{Sh} p}+\mathrm{CdS}: 3 \mathrm{hr}+\mathrm{PbI}$ & $E$ & $\begin{array}{l}5100-5140 \\
A^{\circ}\end{array}$ & 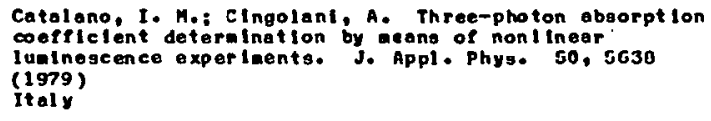 \\
\hline 1387 & $\begin{array}{l}\text { Cos } \\
\text { Under }\end{array}$ & $\mathbf{T}$ & $\begin{array}{l}50-20000 \\
\text { Mev }\end{array}$ & $\begin{array}{l}\text { Khelfets, S.: Knight, T. Full overage radtatlon of } \\
\text { el ectrong and positrong channeled bet ween the planes of } \\
\text { a crystal. J. Appl. Physe 50,5937. (1979) } \\
\text { United States }\end{array}$ \\
\hline 1388 & D17 +910 & $\mathbf{E}$ & $7-15 \mathrm{keV}$ & 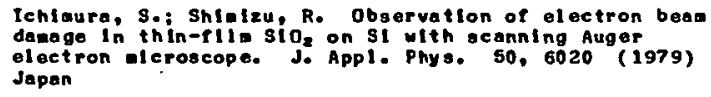 \\
\hline 1389 & $\begin{array}{l}\mathrm{AO}^{\prime} 3 \\
\mathrm{Ne} * \mathrm{Ne}\end{array}$ & $\mathbf{E}$ & $300 \times$ & 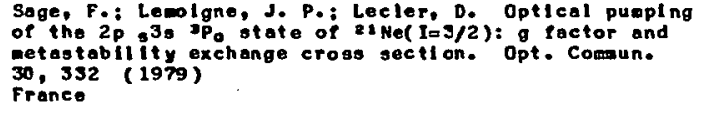 \\
\hline 1390 . & 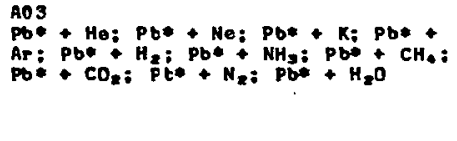 & $\mathbf{E}$ & $550-650 \circ \mathrm{C}$ & 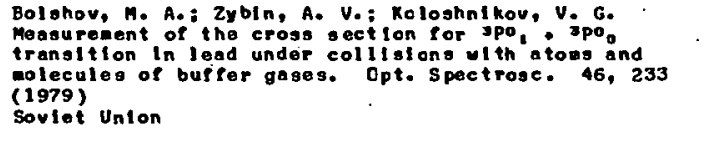 \\
\hline 1391 & $\begin{array}{l}\mathrm{AD} 3 \\
\mathrm{Na} *\end{array}$ & $T$ & $0 . n 01$ a. u. & $\begin{array}{l}\text { Dashevskaya, E. } t \text {. Effect of short-range forces and of } \\
\text { tulsting on Intranultiplet ining In collislons of } \\
\text { alkali-wetal atoas. Opt. Spectrose. } 46,236 \text { (1979) } \\
\text { Soylet Union }\end{array}$ \\
\hline 1392 & $\mathrm{HI}_{2}^{2+} ; \mathrm{BO}_{2}{ }^{2+} ; \mathrm{C}_{2} \mathrm{2t} ; \mathrm{O}_{2} 2+; \mathrm{He}_{2}{ }^{2+}$ & $T$ & Under & $\begin{array}{l}\text { Penkl na, N. N.: Robane, T. K. Deterginat lon of the } \\
\text { potentlal eurves of homonuclear eolecular lons. Opt. } \\
\text { Spectrose. } 46,253 \text { (1979) } \\
\text { Soviet Union }\end{array}$ \\
\hline 1393 & $\operatorname{lno}_{2 \ln }+$ H & $T$ & $20-2800$ AO & 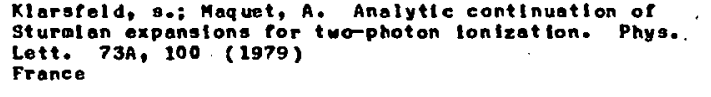 \\
\hline 1394 & $\begin{array}{l}\mathrm{AOS} \\
\mathrm{He}+\mathrm{H}_{2} \\
\mathrm{AO} ? \\
\mathrm{He}=\mathrm{H}_{2}\end{array}$ & E. & $30 \cap K$ & 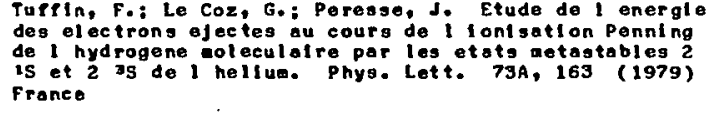 \\
\hline 1395 & $\mathrm{H}+\mathrm{Cs}+\mathrm{Ho}_{0}$ & E & $3000 \mathrm{~K}$ & $\begin{array}{l}\text { Graham, W. G. Negat Ive hydrogan fon production by } 1 \text { ow } \\
\text { energy hydrogen afoon bombardaent of gurfaces. Phys. } \\
\text { Lett. } 73 A \text {, } 186 \text { ( } 1979) \\
\text { United States }\end{array}$ \\
\hline 1396 & $\begin{array}{l}\text { Ens } \\
\text { E15 Au } \\
\text { E Au }\end{array}$ & $\mathbf{E}$ & $0.5-100 \mathrm{MeV}$ & 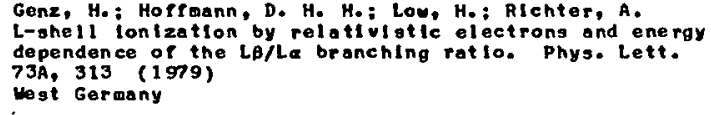 \\
\hline 1397 & $\begin{array}{l}\text { Ho } 2 \\
\text { Under } \\
\text { Ins } \\
\text { Under }\end{array}$ & $T$ & Under & $\begin{array}{l}\text { Knight, P. L. The role of tranalents in resonant } \\
\text { multiphoton absorption. J. Phys. B } 12 \text {, L449 (1979) } \\
\text { United Kingdom }\end{array}$ \\
\hline 1398 & 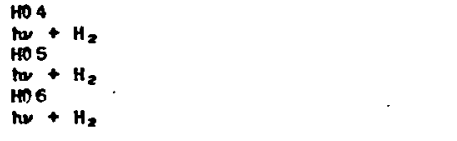 & $\mathbf{E}$ & $620-350 \cdot A^{0}$ & 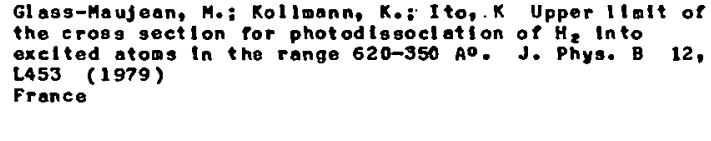 \\
\hline 1399 & $\begin{array}{l}\text { Dres } \\
\text { He* + Undef: } H^{*}+\text { Undef }\end{array}$ & $T$ & $\begin{array}{l}0.05-0.5 \\
0.0 .\end{array}$ & 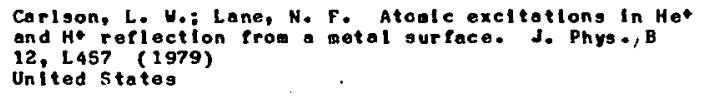 \\
\hline 1400 & $\mathrm{~K}+\mathrm{He}$ & $\mathbf{E}$ & $0.07-0.4 \mathrm{eV}$ & 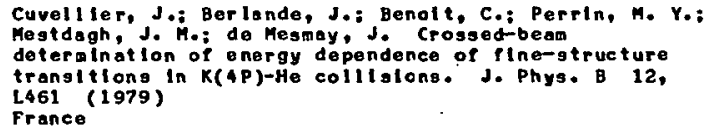 \\
\hline
\end{tabular}


104

REF.
ND.

REACT ANTS

EOg $\mathrm{HCl}$

OHC1
-4 HCI

1422

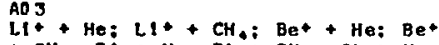

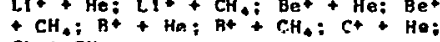

$\mathrm{Ct}+\mathrm{CH}_{*}$

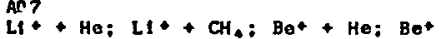

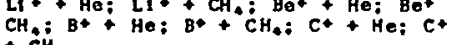

1403106

now 6

1404 HOS

hos + He; he + He: hy + Ar: he + krs

1405

$\mathrm{hom}_{\mathrm{H}}^{\mathrm{HO}}+\mathrm{H}_{2}$

$\mathrm{hu}+\mathrm{H}_{2}$

$1406 \quad$ A17

No + No

1407

No + He; Na* + $\mathrm{Ne}$; No* + Ar: Na* +

$\mathrm{Kr}_{r}$ : Na*: $\mathrm{Xe}: \mathrm{Ne*}+\mathrm{N}_{2}$ : $\mathrm{Rb}$ : $\mathrm{He}:$ $\mathrm{Rb}+\mathrm{Ne}: \mathrm{Rb}_{+}+\mathrm{Ar}_{2} \mathrm{Rb}+\mathrm{Kr} ; \mathrm{Rb}^{+}+$

1408 $\mathrm{Bb}+\mathrm{N}_{2}$

$\mathrm{MO}^{+}+\mathrm{He}^{\circ} \mathrm{Mg}^{+}+\mathrm{Me}^{\mathrm{O}} \mathrm{Mg}^{+}+\mathrm{Ar}$

$\mathrm{Hg}^{*}+\mathrm{He}: \mathrm{Hg}+\mathrm{He}^{\circ} \mathrm{Hg}^{+}+\mathrm{Ar}$

$1409 \stackrel{\mathrm{AO} 3}{\mathrm{C}}+\mathrm{Ho}$

Ans ${ }^{+}+$He

1410

$\stackrel{\mathrm{AO} 6}{\mathrm{H}+\mathrm{He}}$

$\mathrm{AOP}^{\mathrm{B}} \mathrm{P}+\mathrm{HE}$

1411503

$\begin{aligned} 1412 & \text { EOS } \\ & E+N o \\ & =\mathrm{N}\end{aligned}$

$1413 \quad \mathrm{POS}_{\mathrm{PO}}+\mathrm{Pb}$

1414 $\stackrel{A 06}{H^{+}+\mathrm{Ar}}$

1415
ExP

DR

ENERGY

E $\quad$ G-12 ov

$10 n-500 \mathrm{kol}$

1-3 Ay

$\ddot{E}$ SQ-2甘Q . OV

Undor

3-15 keV

$\mathbf{T}$

Under

$60-153 \odot V$

200-3600 ov

$645-1230$

A06 6 Ar

A0? + Ar

REFERENCE

Trone, M.: Azrla, Ro: Le Call, Y.; Stmon, D. In Hel: H formation. J. Phys. B 12, L46? (1979)

Rodoro, Me: Bruch, R.: Bisgaard, P. High-resolution projectlle Auger pectroscopy for $L$, , Be, B and $C$

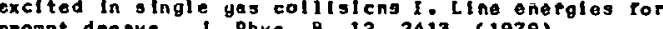

prompt decays. J. Physe. B 12, 2413 (1979)

Le Dournouf, H.: Lan, V. K.: Zelppen, C. J. The phosalonisAtion of the go ground atoto of atomle photalonisAtion of the go ground otato
nitrogen. J. Phys. 12,2449 (1979)

France Hollgnd, N. M-: CaAling, K. Heat, J. B. Marr, C. V. threshold to 280 ev. J. Phys. B 12, 2465 (1979) Unt tad Kingdom

Komarov, I. V.:-Dstrovsky, V. N. Production of exclted hydrogen by photodissoclation of the $H_{z}$ molecule near Soviet Union

Phllippe, M.: Masnou-Seeurs, F.: Valtron. P. Model-potential wethod for the colculation of at of-rape-gas interactions: application to the $\mathrm{Na}-\mathrm{Ne}$ oystem. J. Phys. B, 12, 2493 (1979)

France

de Prunele, E.: Pascale, J. Theoretical lodel for the colliglon of high Rydberg atons with noutral atons or molecules. J. Phys. B 12, 2E11 (1979)

France

Andersen, No: Anderson, T.: Coeke, C. L.: Pedorsen, E. H. Differential-exclition studies of

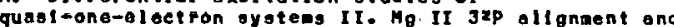
orlentat ton in $\mathrm{Mg}^{+}-\mathrm{He}$, No, Ar colltialong. J. Phys. B 18, 2641 (19r3)

Denmark

500-2000 eV Dowek, D.: Kruteln, J.: Thlelmenn, U.: Fayeton, J.: Barat, M. Collision spectroscopy of open-shell systens I. Ci-He eullislons. J. Phys, i 12, 25s3 (1979) Tratico

figgs, J. S.; Taulbjorg, $K$. Charge transfer by a double-scettering mechanion Involving target electrons. J. Phyo. B 12, 2666 (1979)

nIted KIngdoa

Gerveld, H. B.: Kots, F, B.: Heldewan, Ho G. M.: ven Eck, J. Electron inpoct oxcltat fon of the (2Pz) dp doubly exclted ste te of hellua. J. Phys. B 12, 2575 (10i9)

The Natherlands

Steronl, G.: Cabllionl, R.: GSardint Gutdont, A. Abanlute $(0,20)$ coplenar symet plo orogo dectlono ceasured for valence orbltals of Me. J. Phys. B 12, $25 a 3(1070)$

Italy

Tholg, J.: Relnhardt, J.: Nuller, B. How good to the adiabatic basla In hesuy Ian ralliofone in. Phy ?. B 12, 4479 (1979)

Garany

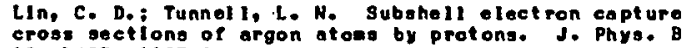
12. L485 (1979) ed statos

Ford, A. Lo: Boeker, R. L.: Swefford, a. L.t Roading, J. F. Proton Iapact argon Inner-ahell charge tranafor

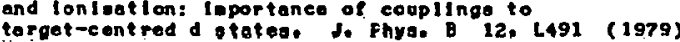
United $s$ tatos 


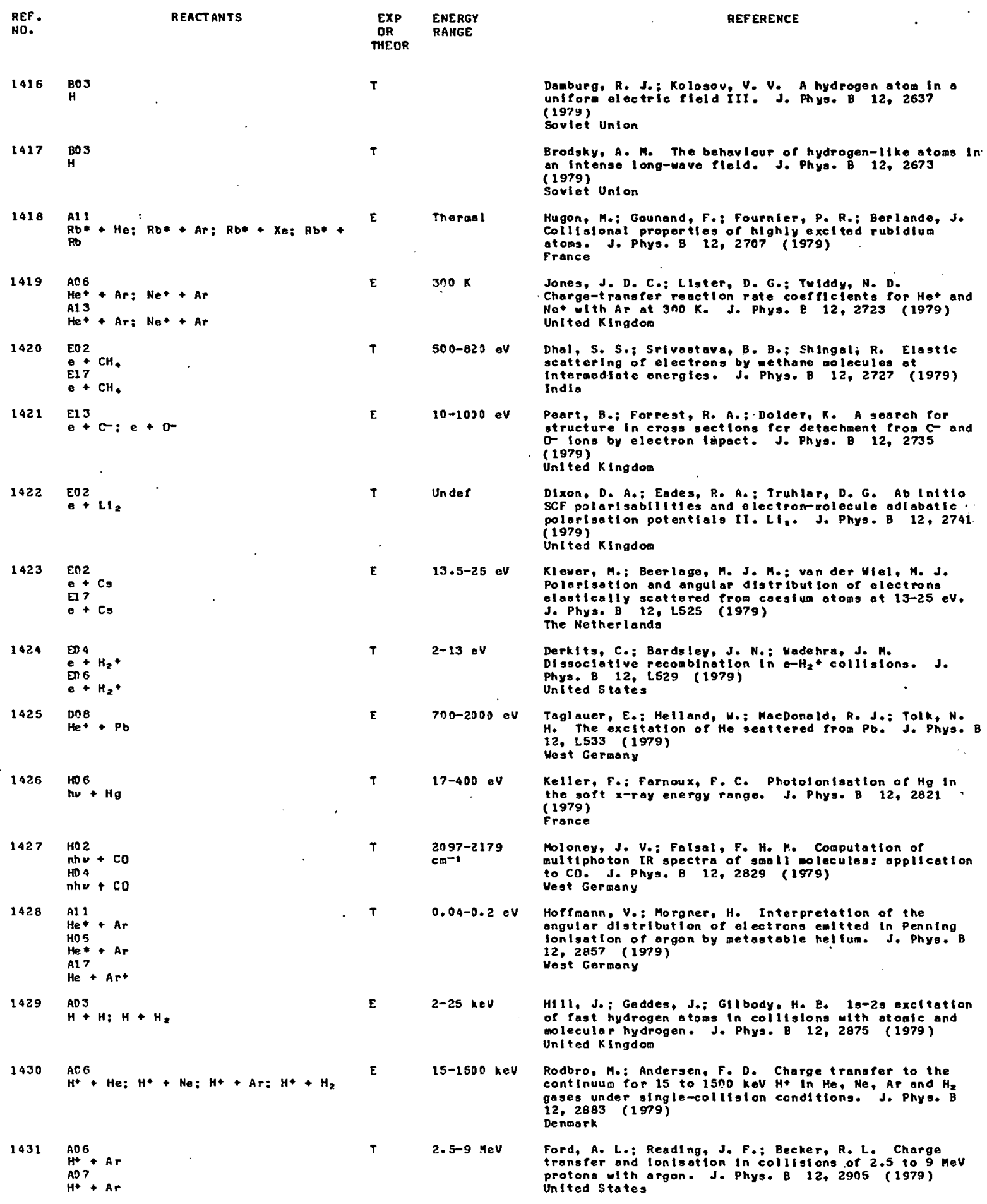




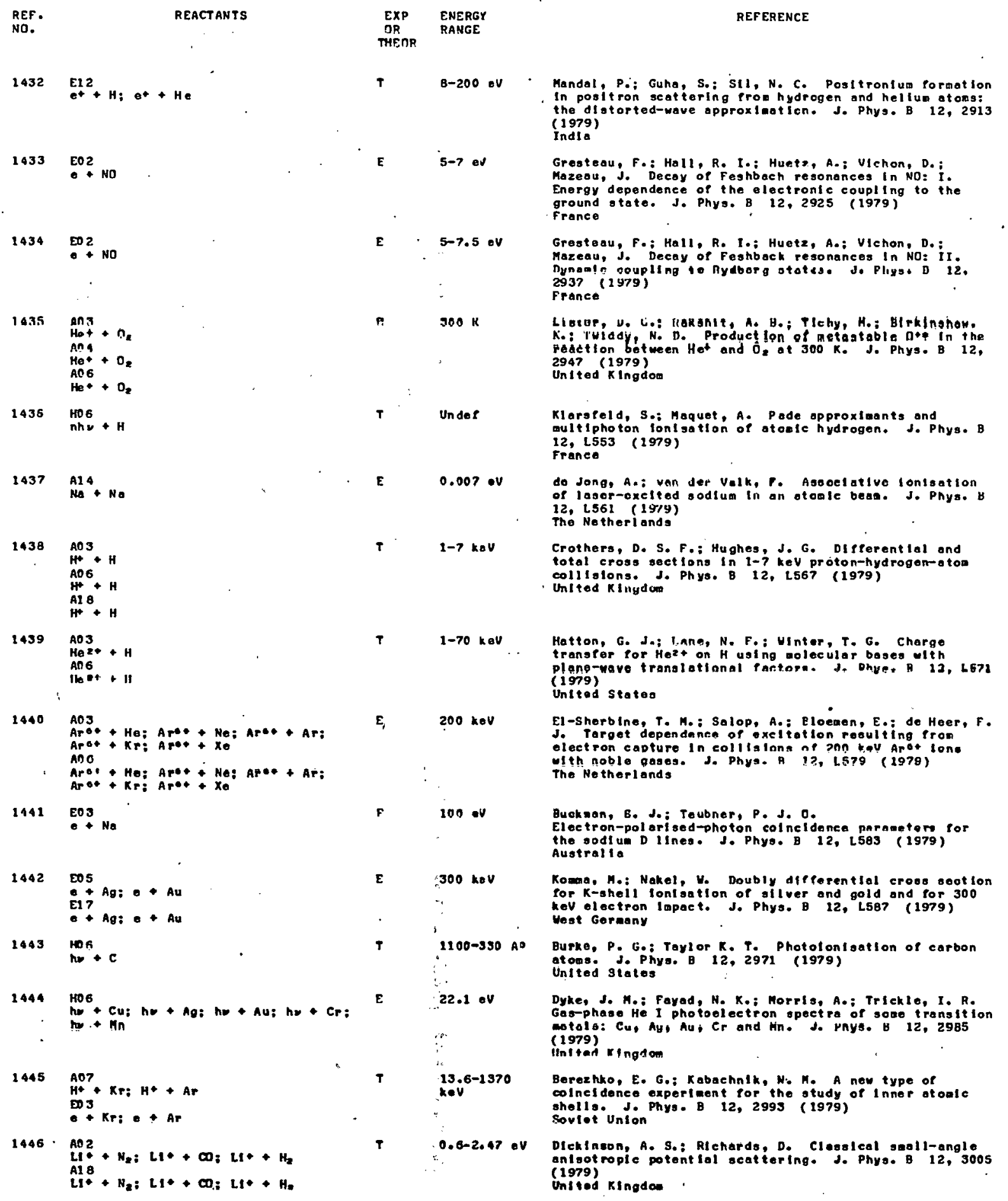




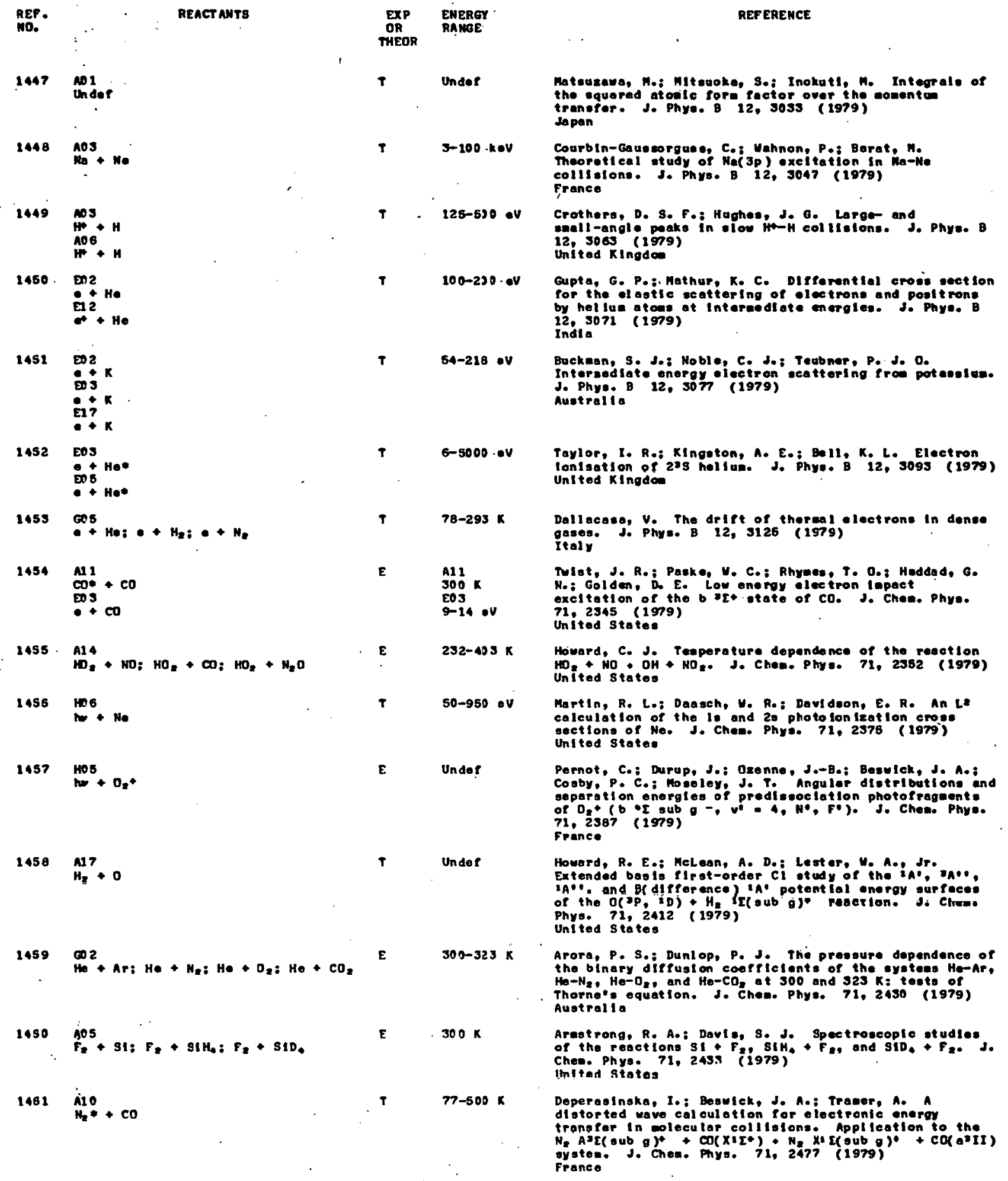




\begin{tabular}{|c|c|c|c|c|}
\hline $\begin{array}{l}\text { REF. } \\
\text { NO. }\end{array}$ & REACT ANTS & $\begin{array}{l}\operatorname{EXP} \\
\text { OR } \\
\text { THEOR }\end{array}$ & $\begin{array}{l}\text { ENERGY } \\
\text { RANGE }\end{array}$ & $\begin{array}{l}\text { REF ERENCE } \\
\vdots\end{array}$ \\
\hline 1462 & 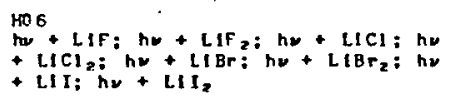 & \&. & $21 \mathrm{eV}$ & 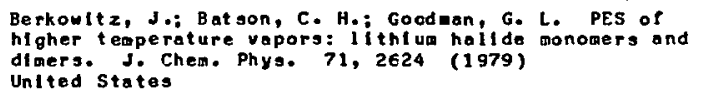 \\
\hline 1463 & $\begin{array}{l}\mathrm{Al} ? \\
\mathrm{He}+\mathrm{Ar}\end{array}$ & $\varepsilon$ & Under & 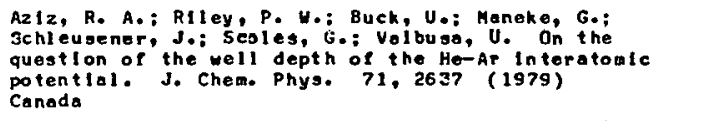 \\
\hline 1454 & $\mathrm{Al}+\mathrm{NaCl}: \mathrm{H}+\mathrm{ICI}$ & T & $\begin{array}{l}3-42 \\
k c a l / 401\end{array}$ & 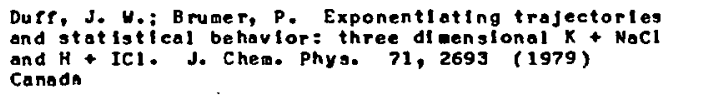 \\
\hline 1465 & 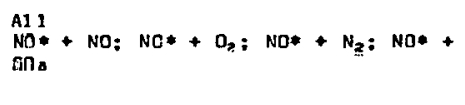 & F. & $300 \mathrm{~K}$ & 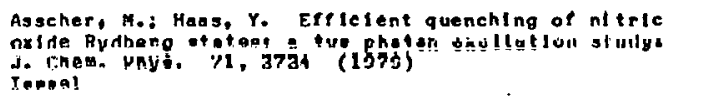 \\
\hline 1456 & $\begin{array}{l}\mathrm{AOC}^{2} \\
\mathrm{He}+\mathrm{Na}_{2} \\
\mathrm{AO3} \\
\mathrm{He}+\mathrm{Na}_{2}\end{array}$ & $\varepsilon$ & $0.1 \mathrm{eV}$ & 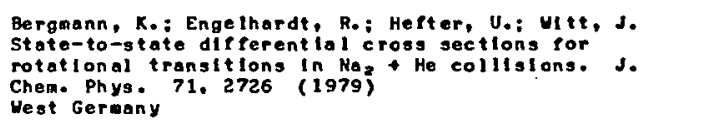 \\
\hline 1457 & $\mathrm{~s}^{+} 3+\mathrm{O}_{2} ; \mathrm{s}^{*}+\mathrm{NO}$ & E & $0.04-2.0 \mathrm{eV}$ & 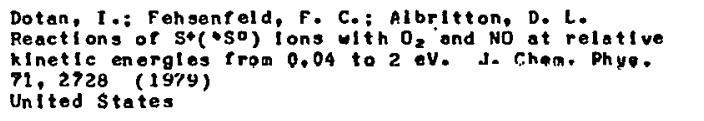 \\
\hline 1468 & $\begin{array}{l}n D S \\
h y+P b\end{array}$ & $E$ & $41 \mathrm{eV}$ & $\begin{array}{l}\text { Suzer, S. Photoenlssiton Prom ot omle Rb } 4 p \text { and } A g \text { ad } \\
\text { levels. J. Chem. Phys. P1, } 2730(1979) \\
\text { Turkey }\end{array}$ \\
\hline 1459 & 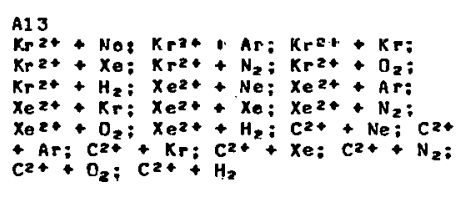 & $\varepsilon$ & $300 \mathrm{~K}$ & 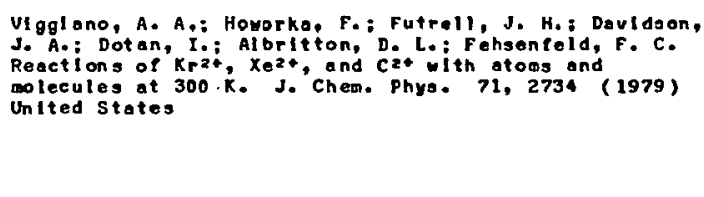 \\
\hline 1470 & $\stackrel{\text { A1 }}{\mathrm{I}} \mathrm{Cl}$ & $T$ & Under & 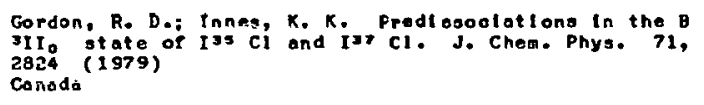 \\
\hline 1471 & $\mathrm{H}_{2}^{A 10}+C d ; \mathrm{N}_{2} *+2 \mathrm{Zn} ; \mathrm{N}_{2} *+\mathrm{Sr}$ & $E$ & $300 \mathrm{~K}$ & 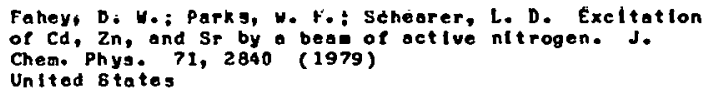 \\
\hline 1472 & 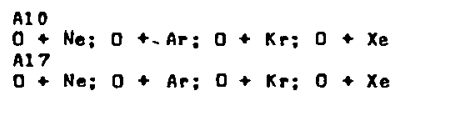 & T & $0.001-10$ ov & 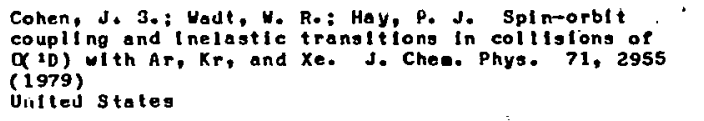 \\
\hline $1+70$ & $\mathrm{H}_{2}=\mathrm{H}+\mathrm{H}=\mathrm{r} \ddot{n}+\mathrm{rn}$. & $\uparrow$ & $\begin{array}{l}100-300 \cdot k \\
\therefore \\
\therefore\end{array}$ & 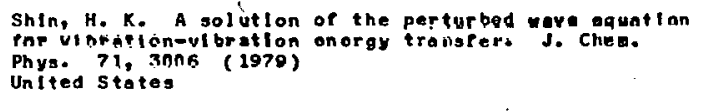 \\
\hline 1474 & $\mathrm{COg}^{\mathrm{CO}} \mathrm{N}_{2} \mathrm{O} ;+\mathrm{N}_{2} \mathrm{O}+\mathrm{CO}_{2}$ & E & $299 \mathrm{~K}$ & $\begin{array}{l}\text { Shlmagorl, H.: Fessenden, R. U. Election at achment to } \\
N_{2} \text { ot pressures near one atmosphere. J. Chem. Phys. } \\
71 \text {, } 3009 \text { (1979) } \\
\text { United Stotes }\end{array}$ \\
\hline 1475 & 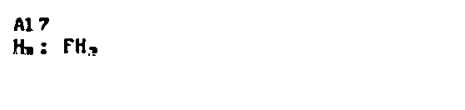 & T & Under & 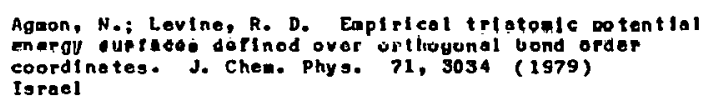 \\
\hline 1476 & 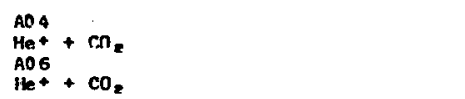 & E & $9-200=0$ & 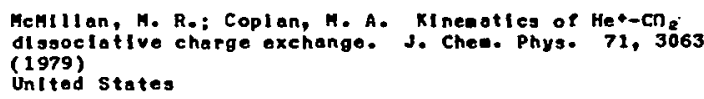 \\
\hline
\end{tabular}


109

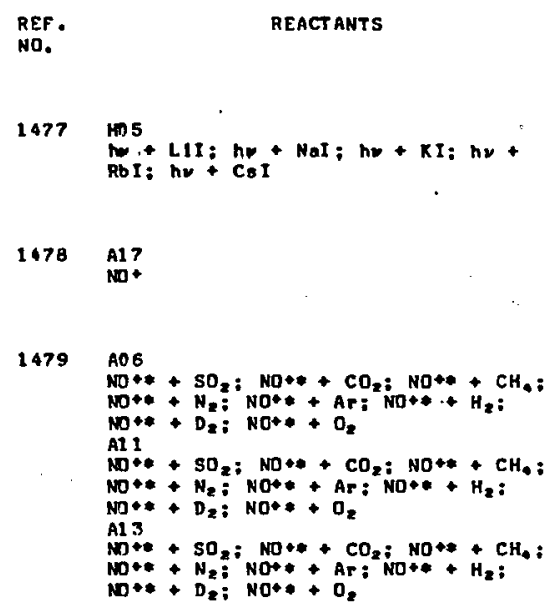

REFERE NCE

Su, T.-H. R.: RIley, S. J. Alkall hallde photofragaent opectro. I. Alkall lodide bond energles and oxelted at ate symetifos at $266 \mathrm{~nm}$. J. Chem. Phys. 71, 3194 (1979) Unitad states

Albrition, D. L.: Schmeltekop P, A. L.: Zare, R. N. Potentlal energy eurveg Por Not. J. Chem. Phys. 72 , 3271 (1979) Unlted states

Dotan, I.: Fehsenfeld, F. C.: Albritton, D. L. Rate congtants for the reactions of entaotabie $N O \cdot(a j \Sigma+)$ long with $\mathrm{SO}_{2}, \mathrm{CO}_{2}, \mathrm{~N}_{2}, \mathrm{Ar}, \mathrm{H}_{2}, \mathrm{D}_{2}$, and $\mathrm{O}_{2}$ at relotive kInetic energies $0.04-2.5$ ov. "J. Ches. Phys. 71, 3280 (1979)

United states

300

$\mathrm{K}$

$8 \mathrm{~K}$

$700 \mathrm{~K}$

Unde?

Undor

E Under

$E \quad 0.1-3 k \in V$

E I Under

E $t$ Undel
Dotan, I : Fehsenteld, F. C.: Aloritton, D. $L_{4}$ On the product rotlos of the roactions cP Art with NO and NO $\left(a^{2} \Sigma+\right)$ wl th Ar of $300 \mathrm{~K}$. J. Ches. Phys. 71, 3289 (1979)

Albrltton, D. Lo: Vlgglano, A. A.: Dotan, Io:

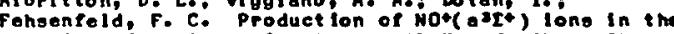

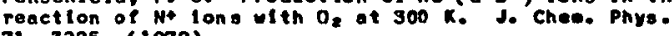
71, 3295 (1979) United states

Petersen, A. B.: Smleh, I. H. M. Melaxotion of HCW (001) using stiaulated el ectronic Rasen scatterling C. vapor as a tunable infrared source. S. Chese. Phys. 71.3546 ( 1979 )

Sllver, J. A.: Blalo, N. C.: Kual, G. H. Crossod bece Sllver, d, A.; Blala, N. C.: Kuel, G. H. Crossod bece otudies of the dynamice of electronte onergy tranefer:

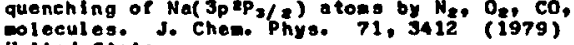
United states

Coul on, P.: Luyekx, R.; Lekkerkerkar, H. N. V. Disperstion porces betueen linear aolecules. J. Chase Phys. 71, 3462 (1979) Beligi us LIn, S. L.: Robson, R. E.; Mason, E. A. Mesent theory of dectron drift and dtefuslon in noutral gases in an Unitod statos

Taohtbana, K.: Pholpo, A, V. Excltation of the coll sub u) stote of $\mathrm{H}_{2}$ by low enser olectrons. J. Cheat Phys. 71,3544 (1979) United states

Aqullantl, V.: Casavec chla, P.: Oruesl, O, Gmo soctlong for exclt ot ion of cd( $\left.\mathrm{g}_{2}\right)$ and $C d\left(S^{2} \mathrm{P}_{2}\right)$ and of thelr agnotic sublevels in Mat-cd colliglons. J. Ches. Phys. P1, 3546 (1979)

traly

Bates, D. R. Other nen's flowers. Phyo. Reports 35 ,

Loreng, A, Atonlc and fol ceular deta for cont rolled

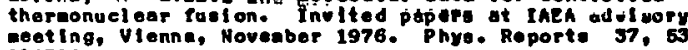
(1976) 
110

REF.

REACT ANTS

1490

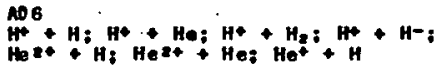

1491

Ens

bos

1492

Cos $\mathrm{CO} ;+\mathrm{HCl}_{\mathrm{O}} \mathrm{O}+\mathrm{H}_{2} \mathrm{O}$; $+\mathrm{CaF}$

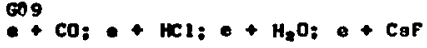

1493

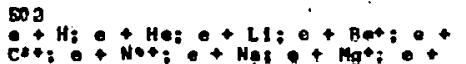

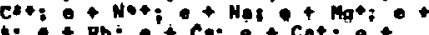

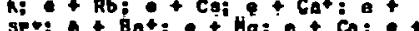

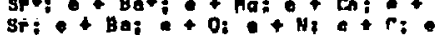

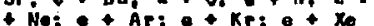

कons:

C+ H; O + He; + + LI: O+ Be+; O

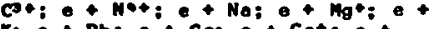

R:

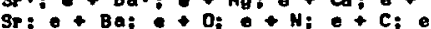

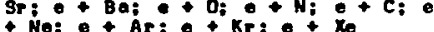

1494

$\mathrm{CO} 2$

Under

tois

1495

$\mathrm{Al} 2+\mathrm{Ne}: \mathrm{No}+\mathrm{He}$

Al? $+\mathrm{Ne}$; $\mathrm{Ne}+\mathrm{He}$

2496

A17

$\mathrm{H}_{\mathrm{H}} \mathrm{H}^{\mathrm{L}} \mathrm{LI} ; \mathrm{He}+\mathrm{Na}_{2} ; \mathrm{He}+\mathrm{K}_{2} \mathrm{He}+$

$\cos ^{2}$

$\mathrm{He}+\mathrm{H}_{2} ; \mathrm{He}+\mathrm{Na}_{2}$ : $\mathrm{He}+\mathrm{K}_{2} ; \mathrm{He}+$

1407506

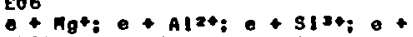

ctor:

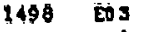

e+ Ar

1499 HO6

HO6 +4

$1500 \quad$ A06

Under

1501 KO3

1 PO2 E11

1533 E11

O+Ar: O + Fe: O+ Fest: O+

Forot: e+ Cu: e + Cust: e + Culot

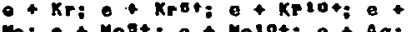

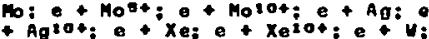

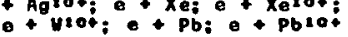

$\operatorname{Exp}$

THEOR

T

T
ENERGY
RAMGE

REFERENCE

T $\quad 0-1$ mov

$0-100=v$

$0.01-10 \mathrm{eV}$

$0-2$ kov

$0.5 \mathrm{mov}$

E T $330 K$

$0.01-3.1 \mathrm{eV}$

Takubov, I. T.; Pollshchuk, A. Y.: Shpllraln, E. E. The Infi uence of an anlaot ropy of the Interaction potentlal at phystca B+C 97, 299 (1979) Soviat Unian

$2-1003$ eV

Mltal, H. P.: Naraln, U. Dielectrontc recosblnation In sodlun loo-elect ronle soquence. Physlea B+C $9 \%, 305$ (1979) India

23-37 eiv

Marehand, P.: Cardinal, Jo Metastable yleld of argon between 23 and 37 ov by ol ectron I apact. Can. J. Phys. 57, 1624 (1979)

Canada

Vagner, G.: Isenor, N. R. Multlphoton tonizatton of LI at the ruby laser wavelength. Con. J. Phys. 57, 1770 (1979)

Dusan, E. L.: Men'shlkov, L. I. Rodiative capture of on electron of a target atom by aultiply charged lons. Sov. Phys,-Doklady 24, 116 (1979) Soviet Union

Latbkhöi, V. S. Sal ective action of laser radiatlon on antter. Sov. Phys.-Usp. 21, 405 (1878)

T $8000 k$

$0.4-5 \mathrm{keV}$
Kag' yonov, V. A.: Starostin, A. N. Bremsstrahlung In resonant scattering In molecular gas. Sov. J, Plasma Phyo. 4,6? (1980)

Zhdanov, $V$. P. Brensstrahlung opectra of electrons at 0.4-5 keV in colllatons wh neutral and veakly lonlzed atono. Sou. J. Plasma Phys. 4, 71 (1978). 


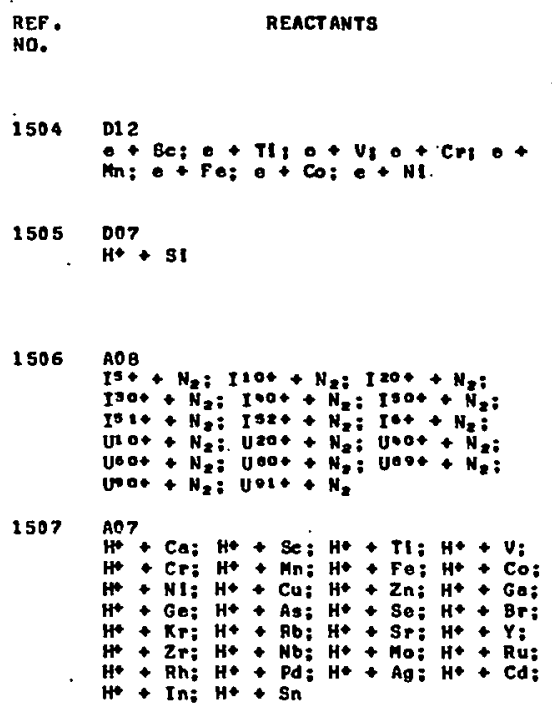

gxp THEOR

$\mathbf{E}$

Under

E T 350 kol

\section{$0.2-2.0 . \mathrm{HoV}$}

Lopes, J. S.: Jesus, A. P.: Ranos, 8. C. Fitted inner-shell proton ionizat ion cros seetlons froa Ca to
Sn for $0.2-2$ MeV Inetdont onergy. Nuel. Inat rum. Methods 184, 369 (1979)

Portugal

Muroh. $K$. Theory for the Leis aot $x$ roy oppearanco potential spectra for the 3d tranition atal se Jo Phya. F 9, 1321 (1979)

Miothe, K.: Schlebel, U.: Houfort, A.: Clauentizer, $0_{0}$ Sall angle scattering of 350 kev protons In elilcon at graxing wast Geraany

2x100-2x104 Daterlov, I. S.: Zalkov, V. P.: Tatheev, Y. A. A senteepifical iethod for doterifint the cros ections for the loss of el ectrons by tost uultiply charged lons. Nuel. Instrai. Mothod. 164,329 (1979) sovict Union
$300-1200$

Under

$1.5-2.5 \mathrm{MoV}$

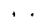

$50-503$ ov

$0.4-23 \cdot e V$

Tharas I

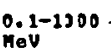

Under

$0.8-1.5 \mathrm{keV}$

Under
Mi r, D.; Regenstrelt, Mo: Mann, Ao: Rosner, Bo: Liran, B. Correlations In the notion of coleculor Pragments traveraling thlek folls. Nuel. Instrum. Methode 164, 553 (1979)

Iarael

Czuchad, E. Collisionally induced tranaltion dipole monts of alkal t-rore gos atoes oysteins. z. Phys. 292, 1.09 (1979) Poland

Kobayash 1, N.: Iri e, T.: Maeda, N.: Kojlan, Ho: Akanusa, S.-T.: Sakisaka, H. Angular distributions of K-shell Auger electrons from carbon, nitrogen, oxygen and neon excited by energettc lone. J. Phys. Soce. Jap. 47, 234 (1979)

Jopan

Fujtita, T.; Imat, T.: Ogura, K.: Uotanabe, S.: Hotanabe, $Y$. Dlasociative excltation of water aolecule by electron inpact. IV. The effect of uultiple scattering. J. Phys. Soc.. Jap. 47, 240 (1979) Japan

Toshlaa, N. Charge transfor processes Involuing highly exclted hydrogen atoms. III. The perturbed stationary state cothod. J. Phys. Soc.. Jap. 47, 267 (1979) Japan

Yabuzak I, T.; Monabe, T.: Ogovo, T. Allgneent destrovina crogs seet fons of the 2 pe state of neon tor collilstons with Hes and Ha. J. Phys. Soc.. Jap. 47, 343 ( 1979 ) Molselultset, B. L.: Stockaen, S. G. Eloctron capture.
at relativistic energleo. J. Physo B 12, (591 (1979) at rel at ivistle

Blshop, D. M.: Cheung, L. M. Static higher polarloobilities of $\mathrm{H}_{2}+$. J. Physe B 12,3135 (1979)

Band, I. M.; forilehev, V. I.: Trzhoskovokaya, M. B. The influence of the hole and exchange teras in the Dirac-slater potential on the photefontsation cross tections and internal convors Phys. B 12, 3187 (1979)

Soviet Unl en

Swaln, S. A gimle nodal for aultiphoton lon Isation. J. Phys. B 12, 3201 (1979) 


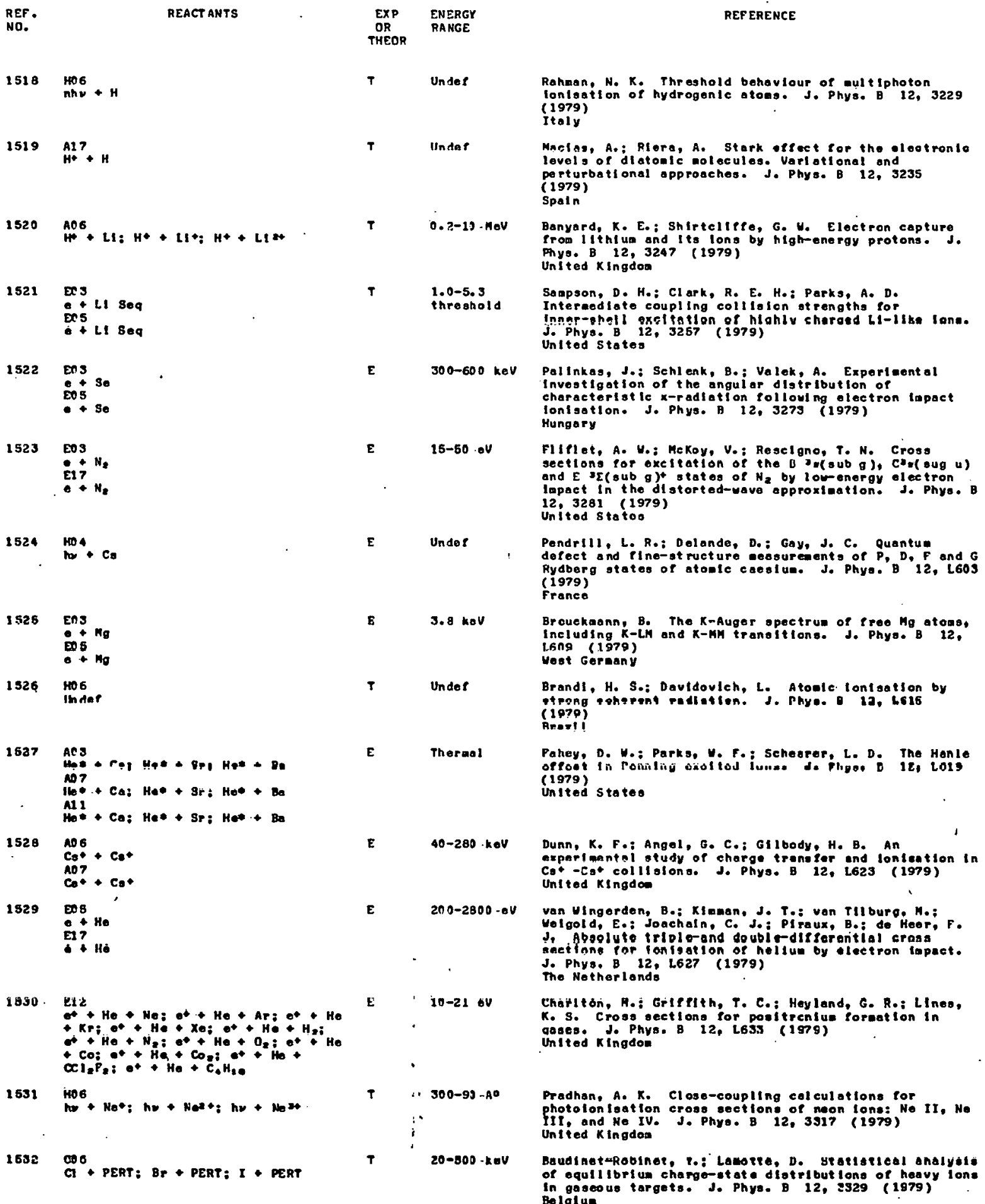

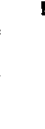

$10-2160$

Under

$1.0-5.3$ threshold

$300-600 \mathrm{keV}$

$15-50 \cdot \cdot V$

Unde?

3.8 kov

Undet

Theraal

$40-280 \cdot k e V$

$200-2800-0 V$

$20-800-k 00$

REF ERENCE

Rahman. N. $R$. Threshold behavlour of eult tphoton lontsation of hydrogenic atoas. J. Phys. B 12, 3229 (1979)

Naclas, A.: Rlera, A. Stark offect tor the elootronto levels of diatonic molecules. Varl atlonal and perturbati onal approsehes. J. Phys. B 12, 3235 (1979) Spain

Banyard, K. E.: Shirteltefo, G. W. Electron capture from lithl un and Its Ions by high-energy protons. Jo Phys. B 12, 3247 (1979)

Sanpson, D. H.: Clark, R. E. H.: Parks, A. D.

Interaed late coupling collialion atrengths for Inngr-grall pxettotion of hlahiv sharaed bi-like lana. J. Phys. B 12, 3267 (1979)

Pollinkes, J.: Sehl enk, 8.: Valek, A. Exporteont ol Pallinkes, J.: Schl enk, B.: Valek, A. Export
Investigot lon of the angui ar distribution of characterfot ic $x-$ adiation folloulng olectron lapact $12.3273(1979)$ ngary

Fltelet, A. U.: MeKoy, V.: Reselgno, T. N, Cross sectlons for oxcltation of the B m(sub $g$ ), Casoug $u$ ) and $\mathrm{E}$ 'L (sub $\mathrm{g})^{+}$states of $\mathrm{N}_{2}$ by lou-energy electron lapact In tho distorted-uave approxtaation. J. Phys. $12,3281$ ( 1979$)$ Pendrill, L. R.: Delande, D.: Gay, J. C. Quantua

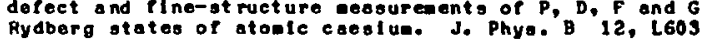
(1979)

France

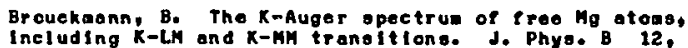
Ineluding RLest Gerang

Brandl, H. S.: Davtdovleh, L. Atonle tontalls on by

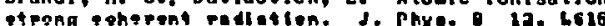
(1070)

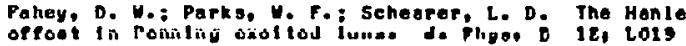
(1979)

United states

Dunn, K. F.: Angel, G. C,: Gilbody, H. B. An expertmantal ofudy of eharge trenefer and lonteatlon in Cat-cat collifolons. J. Phys. B 12, L623 (1979) United Kingdoe

van Uingerden, B.: KImman, J. T.: van TIllburg, M.: Volgold, E.: Joachaln, C. J.: PI raux, B.: do Heor, F. J, Aboglute triplo-and doubi -differential crosa sectinne for lonigation of hellue by olectron inpact. J. Phys. B 12, 6627 (1979)

The Netherlonds

Chajlt bri, H.: Gilffith, T. C.: Heyland, G. R.: LInes, K. S. Cross sectlons for positrenlus porantion in gases. J. Phys. 8 12, L633 (1979)

United Kingdon

Pradhan. A. K. Cl ose-coupling cal culattons for photolon laction cross sections of neon tone: Ne II, Ne fII, and Ne IV. J. Phys. B 12, 3917 (1979) Untted Kingdon

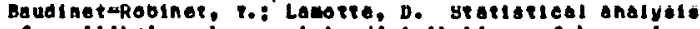
of equillbriun eharge-etate distribut tons of heavy lone in goseous targets. J. Physe. B 12, es29 (1979) 


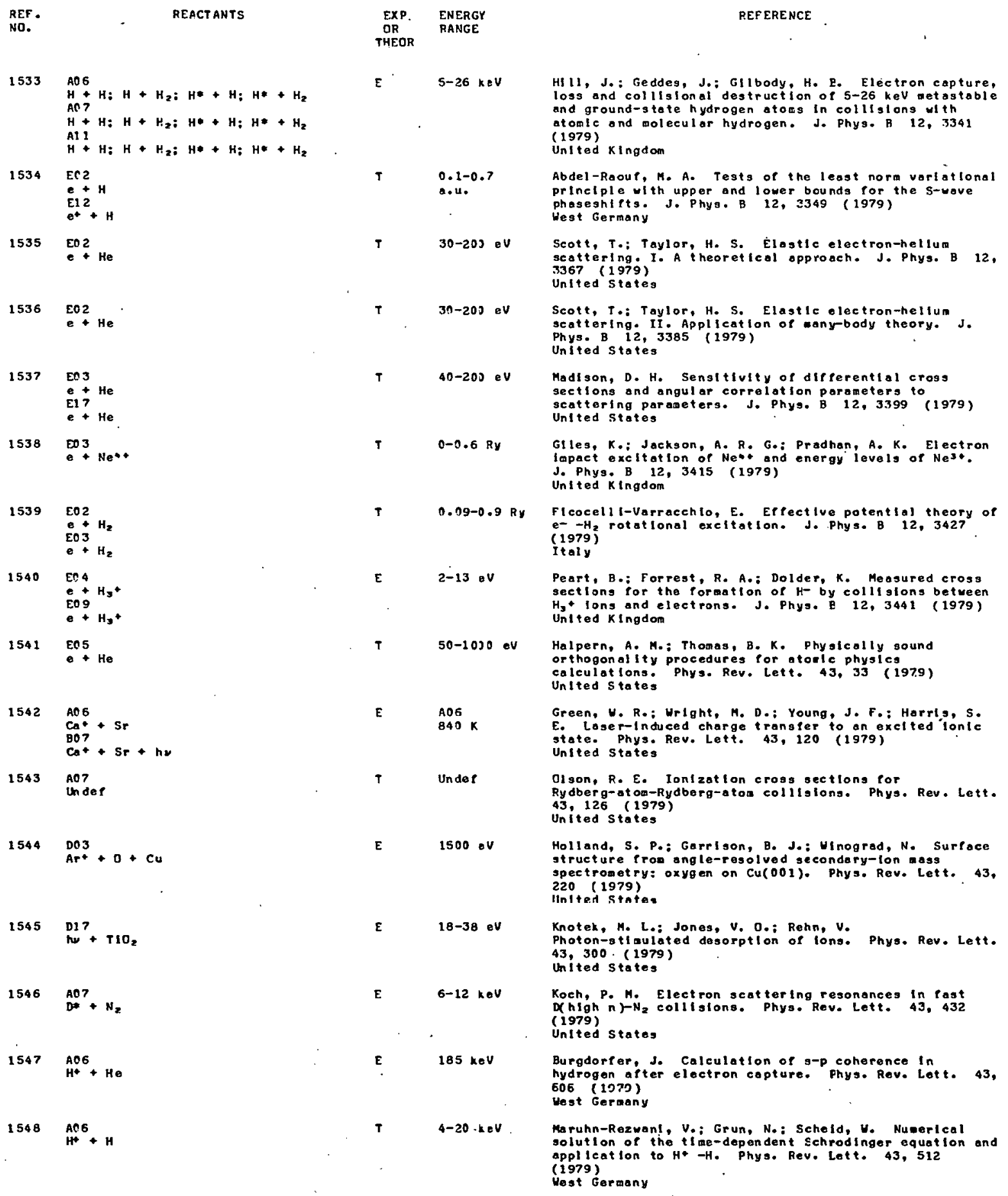




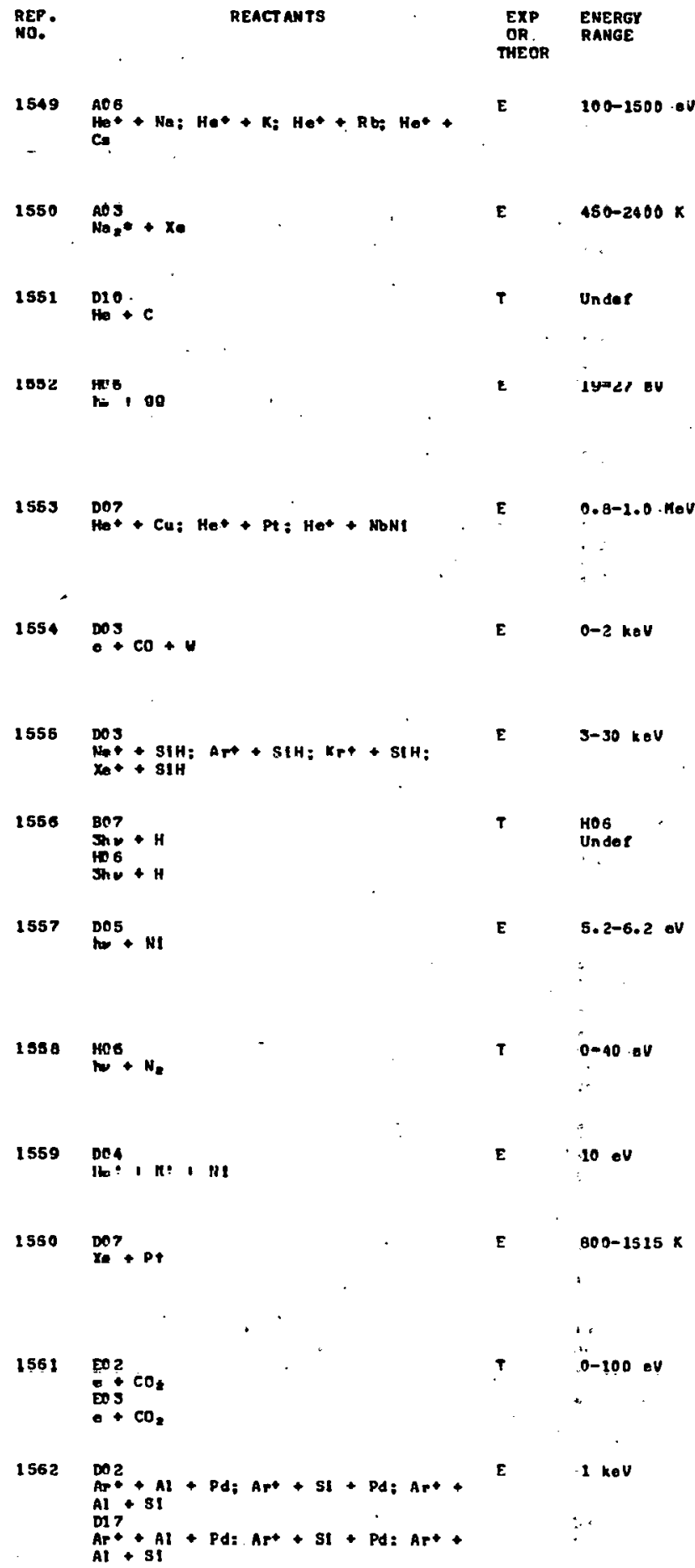

Reynaud, C.: Pount or, J.: Tuan, V. N.: Barat, Mo Composition of Ho motastable beens tormed by charge exchange in Het -alkoll-eotel coll lelons ot cedl ul energy. Phya. Rev. Lett. 4J, 579 (1979)

Solth, N.: Brunnor, T. A.: Korp, A. U.: Priteherd, D.

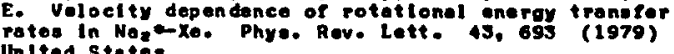

Carlos, W. E.; Cole, M. Y. Anfsot rople He-c palr Interact lon for hi the ston near o graphite our sace.

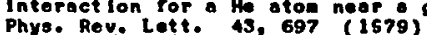
United stotes

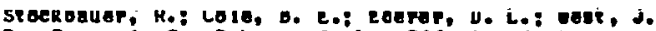

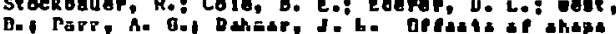

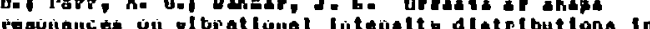

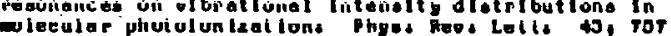
(1979)

United states

Pronko, P. P.: Applaton, B. R.; Holland, O. H.; Hilaon, S. R. Anomalous yleld anhoncesent for highly

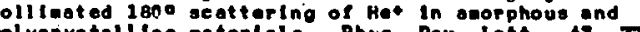
polycrystalline material a. Phye. Rev. Lett. 45, 79

inled. States

Franchy, R.: Menze, D. Adsortate core lonizat lon as primary prooeso in el eot ron-and photon-atieulated desorption Pron motal surfaces. Physe. Rev. Lett. 43, 865 (1979)

Weot Germany

Vittaoack, $K$. Ionlzotlon aechanise of $H^{*}$ sputtored frow hyd rngennted sili can. Phys. Rey. Lett. 45, g7? (1979) Unlted stotes

Luc-KoenIg, E.: Bachellor, A. Interpretetlon or electrlc-pleld-Induced oselliatlons th the eross section of a one-olectron at on above the Ionization ileit. Phyo. Rov. Lote. 43, giz (1979) Frane:

KI skor, E:; Gudat, W: : Caapagna, M.: Kuhleann, E.: Hopster. H.: Moore. I. D. Crossover Iron negatl ve to

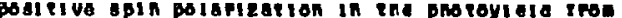

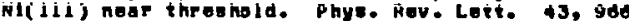
(1979) Uest Geraany

Dehner, J. L.; DIII. D.: Vallace, 8. Shape-resonance-enhanced nucleartootion offecto in elecular photolonization. Phye. Rev. Lett." 45, 1005 (1979)

United states

Hagstrun, H. D. Exclted-aton deexcltation spectroscopy

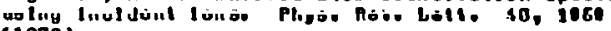
(1979) United states

Hurot, J.'E*; Bocker, C. A.: Couln, J. P.: Janda, K.

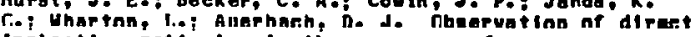
Inelastic getetering in the presence of trapping-desorptlon ocottering: $x_{e}$ on Pt(111). Phye. Rev. Lett. 43, 1175 (1979)

Dill, D.; Weleh, Jo; Dehmer, J. L.; slegel, J.

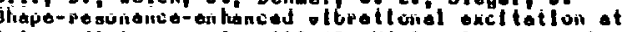
Intermedlate energles $(10-40$ ev $)$ In eleet ron-mol ecule seatter! ng. Phys. Rev. Lett. 43, 1236 (1979) Unlted states

Ho, P. S.: Lewls, J. E.: Chu, U. K. Preforred eputtering on binary Cil oy urefoces of the Ai-pd-s systea. Sur Pace ScI. 85, 19 (1979)

Unted states 


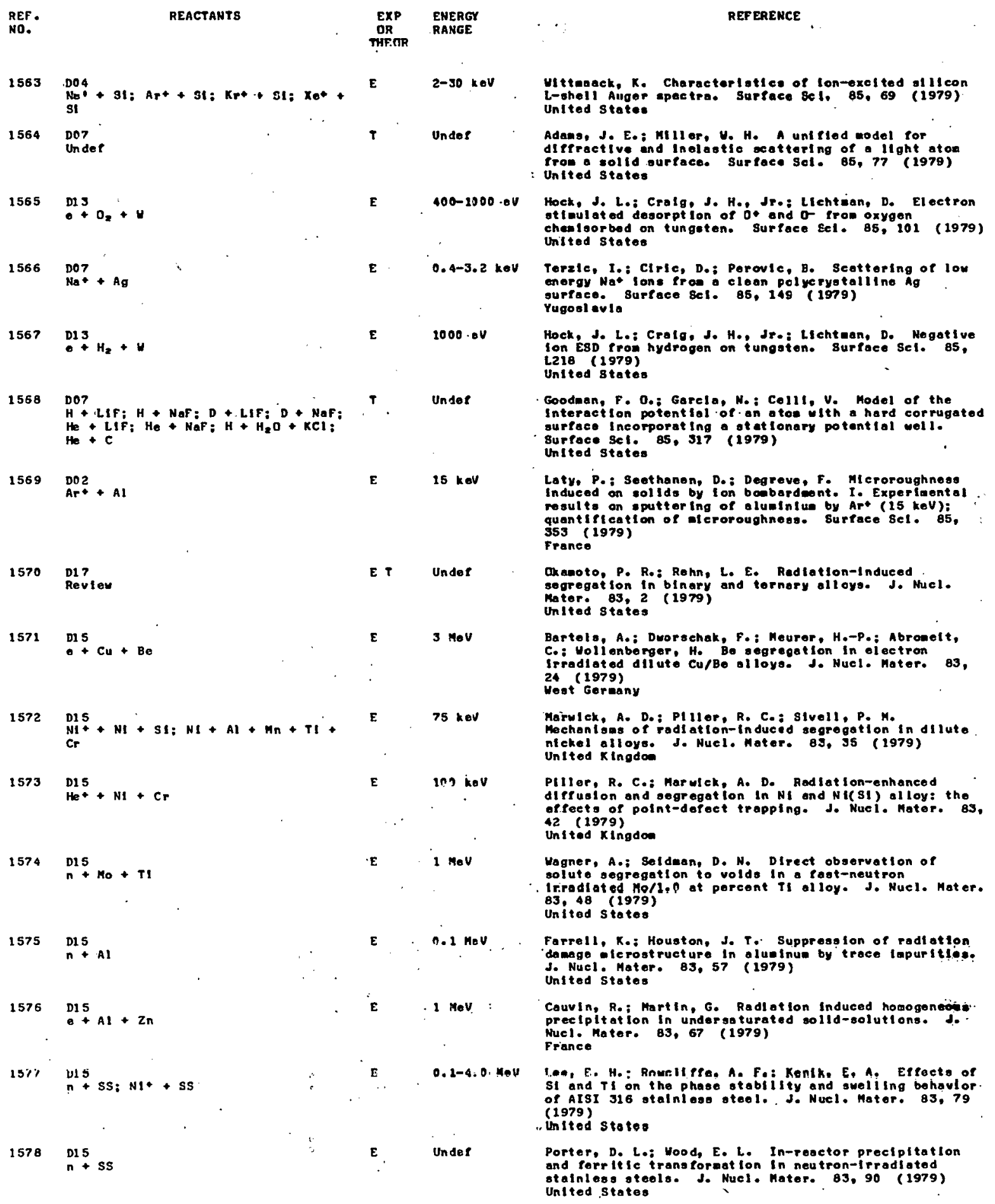




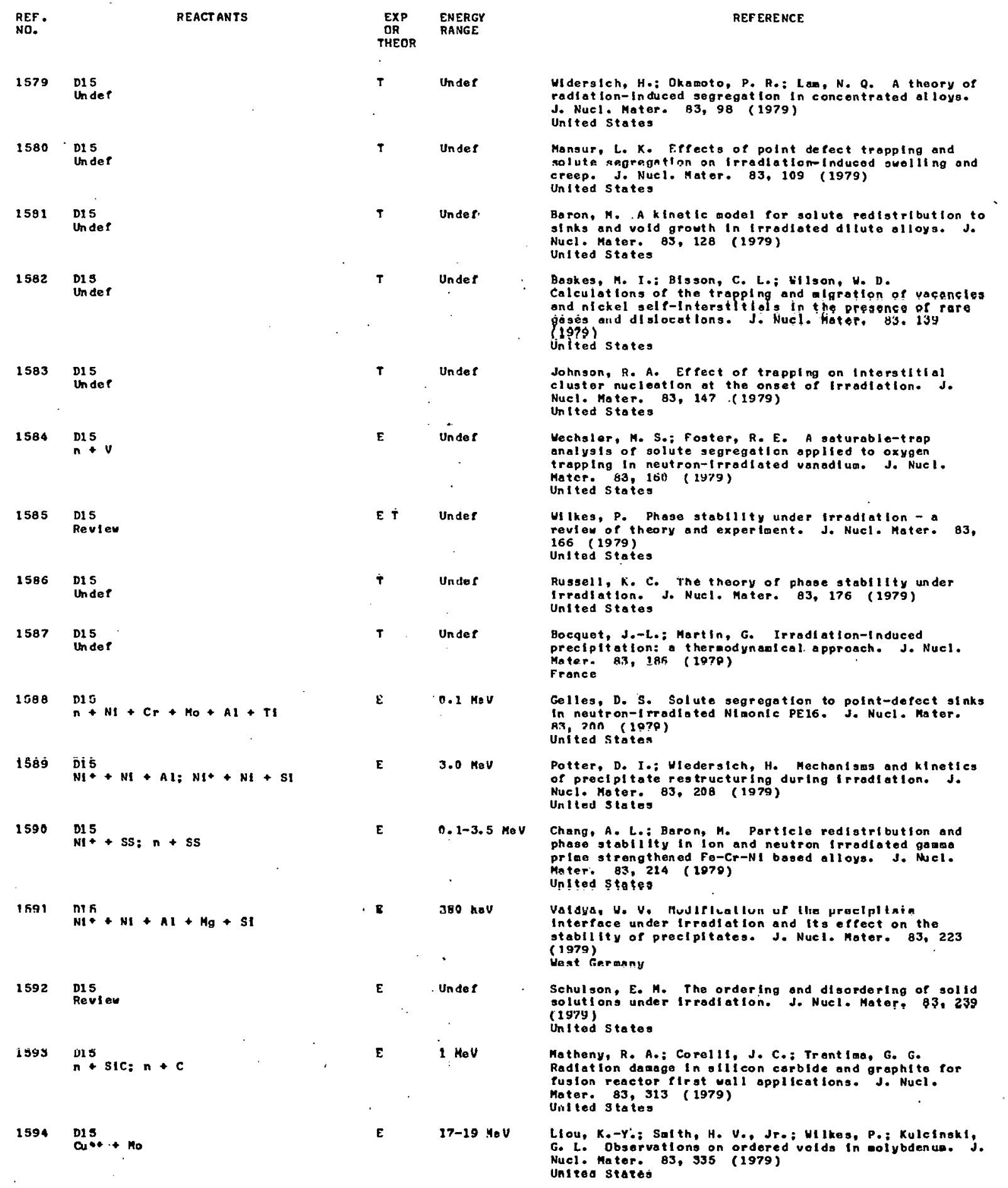




\begin{tabular}{|c|c|c|c|}
\hline $\begin{array}{l}\text { SEF. } \\
\text { NO. }\end{array}$ & REACTANTS & $\begin{array}{l}\text { EXP } \\
\text { OR } \\
\text { THEOR }\end{array}$ & $\begin{array}{l}\text { ENERGY } \\
\text { RANGE }\end{array}$ \\
\hline $\begin{array}{l}1595 \\
+\end{array}$ & $\mathrm{AOE}_{\mathrm{He}}^{+}+\mathrm{NO} ; \mathrm{H}_{2}++\mathrm{NO}$ & $\varepsilon$ & $1-5 \mathrm{k} \in \mathrm{V}$ \\
\hline 1596 & ${ }_{A r^{+}}^{A B 2}+C u: A C^{+}+C u D^{\circ}$ & $E$ & $5 \mathrm{keV}$ \\
\hline 1597 & $\mathrm{HOS}_{\mathrm{H}}+\mathrm{H}_{2} \mathrm{~S}_{\mathrm{H}} \mathrm{H}_{2}+\mathrm{O}_{2} \mathrm{~S}: \mathrm{H}_{2}+\mathrm{C}_{2} \mathrm{H}_{2}$ & E & $58.4 \mathrm{~nm}$ \\
\hline 1598 & 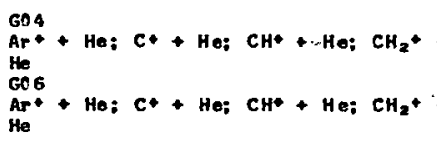 & + & $5-100 \mathrm{rd}$ \\
\hline 1599 & $\begin{array}{l}A 06 \\
A r\end{array}=+N+N=$ & E & $0-81$ ov \\
\hline 1600 & $\mathrm{ALO}_{2} \mathrm{Al}_{2}$ & $\varepsilon$ & 20 oc \\
\hline 1601 & $\stackrel{608}{\circ}+\mathrm{H}_{2} ; \mathrm{O}+\mathrm{H}$ & 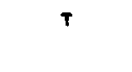 & $10-60 \mathrm{Td}$ \\
\hline 1602 & $\begin{array}{l}\text { Gou } \\
\text { Under }\end{array}$ & $\mathrm{T}$ & Undef \\
\hline 1603 & $\mathrm{AOG}^{+}+\mathrm{SF}$ & E & - \\
\hline 1604 & 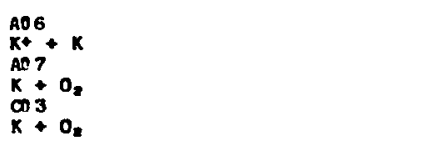 & $\varepsilon$ & $15-40$ ov \\
\hline 1605 & 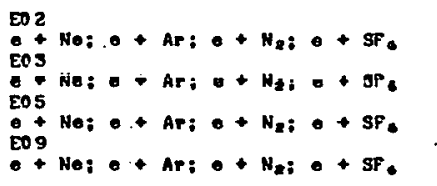 & $\begin{array}{c}\varepsilon \\
1\end{array}$ & $10-603.0 \mathrm{~V}$ \\
\hline 1606 & $\begin{array}{l}\cos \\
i+c s \\
\operatorname{sog} c_{8} \\
0+c_{8}\end{array}$ & E & $4-100 . \mathrm{Td}$ \\
\hline 1607 & $\begin{array}{l}\operatorname{Cos} 2 \\
i+A 1 N \\
\cos \\
0+A 1 N\end{array}$ & $T$ & an $\mathrm{keV}$ \\
\hline
\end{tabular}

Parker, J. E.: Haddad, R. Y. Chorge-transtor of nititic oxlde with Hat and $\mathrm{H}_{3}$. Int, I. Mass Spectron. Ion United Kingdom

Kloppel, K. D.; Seldel, H. Invest Igatlon of monolayers by oecondary ion ass spectroseopy. Int. J. Hago by aecon dary lon ass spectrcocopy.
Spect rom. Ion Phys. 31, 151 (1979) Spect Gom. Iony

El and, J. H. D. Dissoclations of state-selected $\mathrm{C}_{2} \mathrm{H}_{2}{ }^{+}$, $\mathrm{H}_{2} \mathrm{~S}^{4}$ ind $\mathrm{H}_{2} \mathrm{~S}$. ions of udled by photoel ectron-photolon $\mathrm{H}_{2} \mathrm{~S}^{4}$ and $\mathrm{D}_{2} \mathrm{~S}^{+}$ions ot udled by phot oel ectron-photo ion
coincidenes opectrogcopy. Int. J. Mass Spectron. Ion phys 31,161 (1979)

United States

Thoosas, R.: Barassin, J.: Barasaln, A. Mobfllttos et coofriclents do diffugion longltudinale dos lons: Ar et CH(oub $x)^{+}$dens 1 lehel lum. Int. J. Mass Spect ros. Ion Phys. 31, 227 (1979)

France.

Neuschaser, D.: Dttlinger, C.: 21 emeraann, S.: Lindinger, W.: Howorke, f.: Stor 1, Ho Drift tube and beas atudios of reactiona of $\mathrm{Ar}^{2} \mathrm{w}^{4}$ ulth $\mathrm{N}_{2}$. Int. J. Maso Hest Germany

Chatel ot, M.: WIdentocher, G.; Oksengorn, B. Et ude experinentale du temps de rel oxetion vibrationnelle $(V-T)$ do 1 hydrogene compriae o hauto dens ite. coapt. Rend. 287, 195 (1978)

France

Capltelli, Mo: Dilonardo, M. Electron energy

distribution Punctions of hydrogen: the orfect of superelostlc vibratlonal colligiong and of the disooctation process. Z. Naturforseh. A 34,585

(1979)

Henson, B. L. Ions in water vapour: liquld droplet model follure. J. Phys. D 12, L65 (1979) United States

Sehnaldt, H. F.: Jungblut, Ho Ion mobllity and reconbination in compressed sulphur hexarluoride. J. Phys. D 12, L67 (1979)

United Kingdos

Okada, S. Genorat lon of a fast alkall atomle bean by charge-transfer aethod Por fon-pol r Poration studies. J. Phys. D 12, B25 (1979)

Japan

Christophorou, L. G.: Jases, D. R.: Mathts, R. A. On the rols of the electron impact fonlsotion and electron ecottering cross-bection in the breakdown st rength of diolortmir gasel. J. Phys. D 12. 1223 (1979) United states

Saelee, H. T.: Lucas, J. Eloctron drlet veloclty and momentum trangfor crososection in caesium vopour. Phys: D in, 1275 (1979)
United Kingdoes

Rossoun, C. J.: Whalion, M. J. The orientation dependonce of mossured inolaptle scattering probabil ities for fast olectróns in el ingle crystals. J. Pligo: $\theta$ 10, 16a2 (2070) United $k$ ingdon 


\begin{tabular}{|c|c|c|c|c|}
\hline $\begin{array}{l}\text { REF. } \\
\text { NO. }\end{array}$ & REACTANTS & $\begin{array}{c}\text { EXP } \\
\text { OR } \\
\text { THEOR }\end{array}$ & $\begin{array}{l}\text { ENERGY: } \\
\text { RANGE }\end{array}$ & REF EREMCE \\
\hline 1608 & 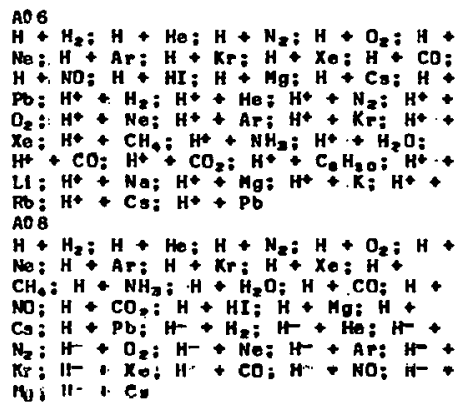 & E & $10-10000.0 V$ & 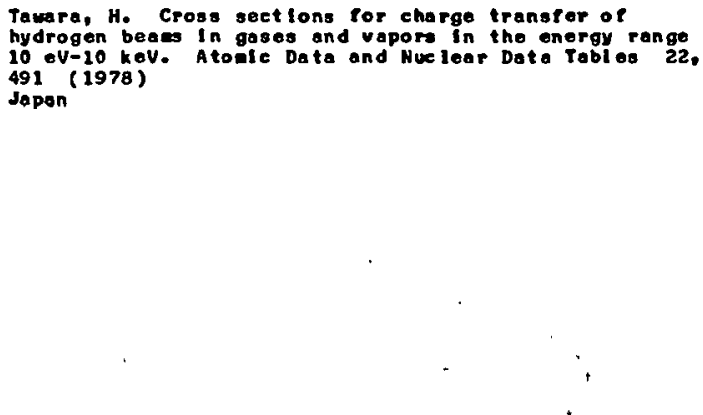 \\
\hline 1309 & eos He & $T^{*}$ & $\begin{array}{l}\text { 1-4 } \\
\text { threshold } \\
\text { energy }\end{array}$ & 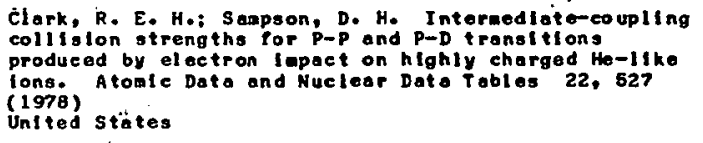 \\
\hline 1610 & $\mathrm{EO}^{\mathrm{E}}+\mathrm{KrF} ; \vdots$ Xer* & $\begin{array}{l}\top \\
T\end{array}$ & $5-100$ ev & 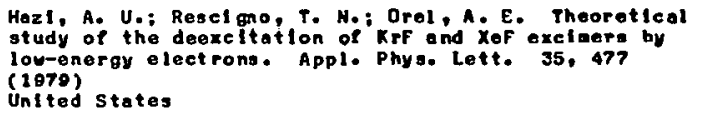 \\
\hline 1611 & $\begin{array}{l}\mathrm{A05} \\
\mathrm{H}+\mathrm{Br} \\
\mathrm{AH} \\
\mathrm{H}+\mathrm{Br}\end{array}$ & $\mathbf{E}$ & $300 \mathrm{~K}$ & 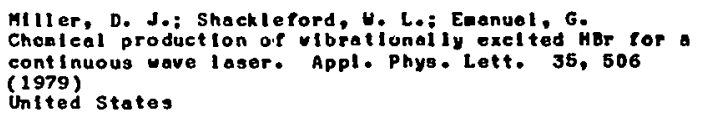 \\
\hline 1612 & 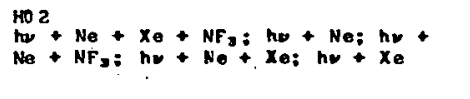 & E & 3511 40 & $\begin{array}{l}\text { Chompagne, L. F. Temperature-dependent dboorption } \\
\text { processes in the Xef laser. Appl. Phys. Lett. S5, } 516 \\
\text { (1979) } \\
\text { United states }\end{array}$ \\
\hline 1613 & $\begin{array}{l}\text { MO4 } \\
\text { Undet }\end{array}$ & T & Unde? & 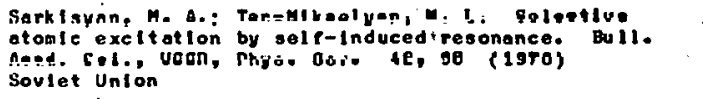 \\
\hline 1614 & $\begin{array}{l}\text { HoS } \\
t_{\infty}+\mathrm{H}_{2} \\
\mathrm{HOS} \\
\mathrm{H}_{2}+\mathrm{H}_{2}\end{array}$ & $T$ & Under & 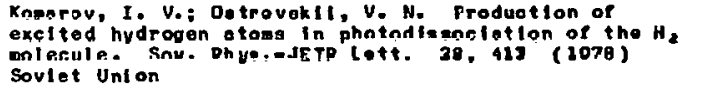 \\
\hline 1615 & E06 $+x+$ & $\begin{array}{llll}\varepsilon & & \end{array}$ & $10-20 \mathrm{eV}$ & 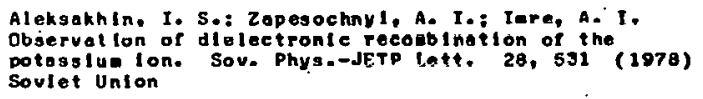 \\
\hline 1616 & CO 2 PERT & $\mathbf{T}$ & $17 \mathrm{keV}$ & 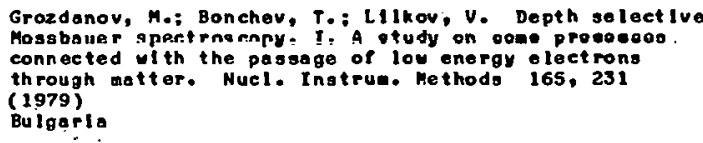 \\
\hline 1617 & DOG $+\mathrm{NI}$. & $\varepsilon$ & $19 \mathrm{kev}$ & 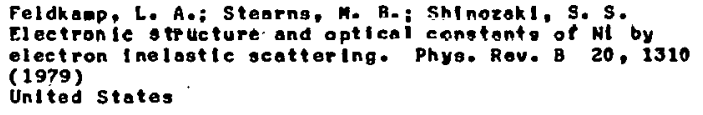 \\
\hline 1618 & 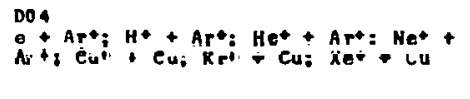 & $\mathbf{T}$ & $20-400 \cdot k e V$ & 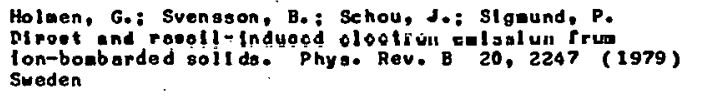 \\
\hline 1619 & $\begin{array}{l}\text { Ao } 1 \\
\text { Under }\end{array}$ & $\begin{array}{l}T \\
T\end{array}$ & Unde? & 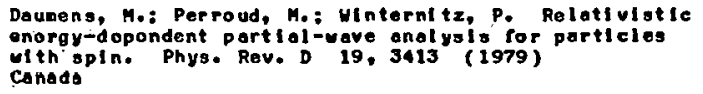 \\
\hline
\end{tabular}




\begin{tabular}{|c|c|c|c|c|c|}
\hline $\begin{array}{l}\text { REF. } \\
\text { NO. }\end{array}$ & & REACT ANTS & $\begin{array}{l}\text { EXP } \\
\text { OR } \\
\text { THEOR }\end{array}$ & $\begin{array}{l}\text { ENERGY } \\
\text { RAHGE }\end{array}$ & REFEREKCE \\
\hline 1620 & 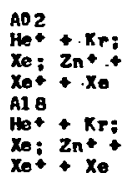 & 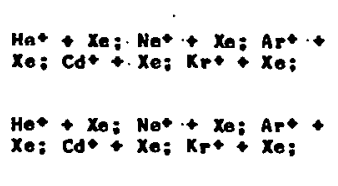 & $T$ & $26-500 \cdot \mathrm{keV}$ & 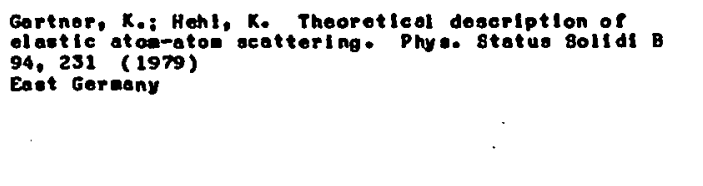 \\
\hline 1621 & 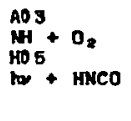 & & $\boldsymbol{\varepsilon}$ & $\begin{array}{l}\text { A03 } \\
\text { SDO K } \\
\text { KOS } \\
193 \mathrm{nN}\end{array}$ & 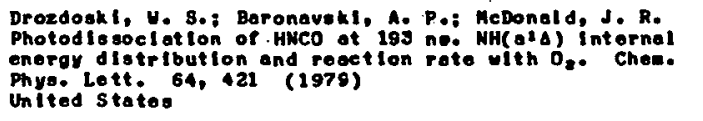 \\
\hline 1522 & Pod $+\mathrm{PbF}$ & & E & $450-410 \cdot n=$ & 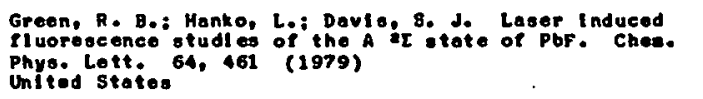 \\
\hline 1623 & $\begin{array}{l}\text { Nod } \\
\text { Under }\end{array}$ & & $\mathbf{T}$ & Under & 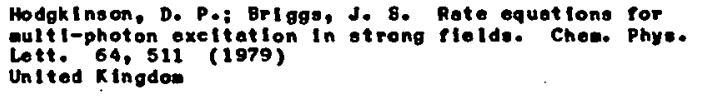 \\
\hline 1624 & $\mathrm{H}_{\mathrm{N}}^{\mathrm{Ho}}+\mathrm{H}_{2} \mathrm{O}$ & & $\mathbf{T}$ & $12-50$ \&V & 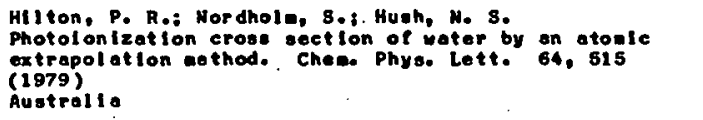 \\
\hline 1625 & AO $* B 1:$ & $0_{2} *+1$ & $T$ & $300 k$ & 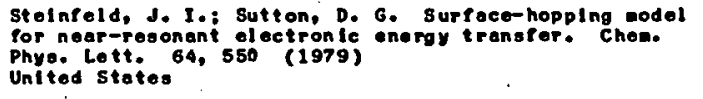 \\
\hline 1626 & $\operatorname{los}_{h 2}+\mathrm{Ll}_{2}+$ & & T & $700-530 \cdot \mathrm{nm}$ & 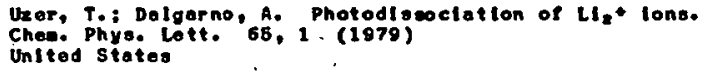 \\
\hline 1627 & $\stackrel{\mathrm{EO} 3}{\mathrm{COO}}$ & $+\mathrm{NO}_{1}+4+\mathrm{N}_{2}$ & E & 300 ov & 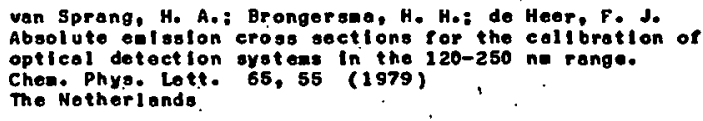 \\
\hline $162 \theta$ & $\mathrm{AnJ}_{\mathrm{N}}^{\mathrm{An}}=+\mathrm{CO}$ & & E & $300 x$ & 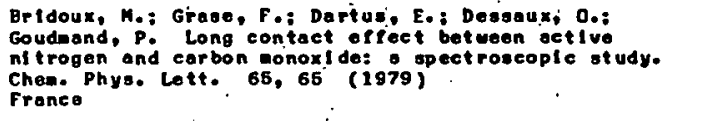 \\
\hline 1629 & $\mathrm{FO}^{\mathrm{AO}}+\mathrm{X}_{0} ; \mathrm{C}$ & $c_{1}-+x_{e}$ & $\varepsilon$ & $250-500$ eV & 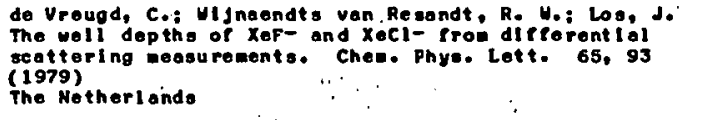 \\
\hline 1630 & $\mathrm{O}^{\mathrm{A}} 4+\mathrm{H}_{2}$ & & E & $300 x$ & 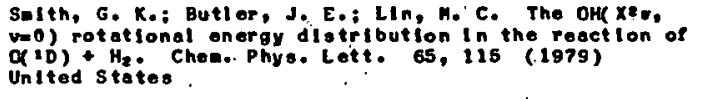 \\
\hline 1631 & $\begin{array}{l}\mathrm{AOS} \\
\mathrm{K}_{\mathrm{r}}^{+}+\mathrm{Cs}_{3}\end{array}$ & & E & $3.5-5.5 \mathrm{kgV}$ & 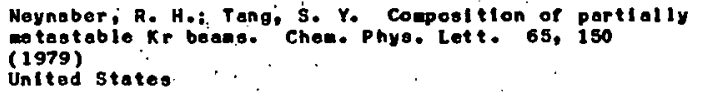 \\
\hline 1652 & $\begin{array}{l}\text { Ho5 } \\
\text { Under }\end{array}$ & & $\mathbf{T}$ & Undef & 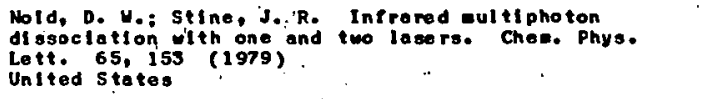 \\
\hline 1633 & $\begin{array}{l}A 11 \\
\mathrm{CF}_{2}=+\mathrm{HQ}^{2}: \\
\mathrm{CF}_{\mathrm{z}}=\mathrm{BF}\end{array}$ & $: \mathrm{CF}_{2} *+\mathrm{He}: \mathrm{CF}_{2} *+\mathrm{N}_{2}$ & E & $295 \mathrm{~K}$ & 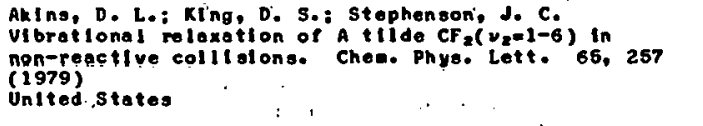 \\
\hline 1634 & $\begin{array}{l}\mathrm{AOA}_{4}+\mathrm{Hg}_{3} \\
\mathrm{H}_{3}+\mathrm{Mg} \\
\mathrm{H}_{3}+\mathrm{Mg}\end{array}$ & & E & $3-4 k \in V$ & 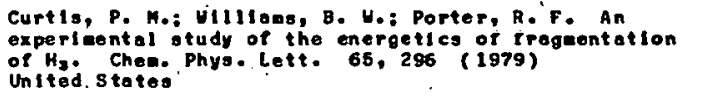 \\
\hline
\end{tabular}




\begin{tabular}{|c|c|c|c|c|c|c|}
\hline $\begin{array}{l}\text { REF. } \\
\text { NO. }\end{array}$ & & REACT ANTS & & $\begin{array}{l}\text { EXP } \\
\text { OR } \\
\text { THEOR }\end{array}$ & $\begin{array}{l}\text { ENERGY } \\
\text { RANGE }\end{array}$ & REFERENCE \\
\hline 1635 & $\mathrm{~A}_{\mathrm{H}}^{\mathrm{A}}+\mathrm{Br} \mathrm{r}_{2}: \mathrm{H}$ & $\mathrm{H}+\mathrm{HBr}$ & & E & $300 \mathrm{~K}$ & 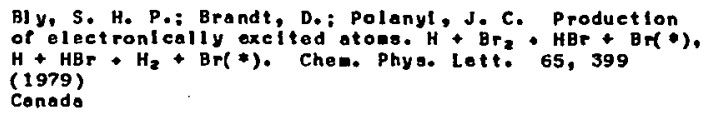 \\
\hline 1636 & $\begin{array}{l}\mathrm{HO}_{4} 4 \mathrm{ICN} \\
\text { hy } \\
\text { ho } 5 \text { ICN } \\
\text { he }+\mathrm{ICN}\end{array}$ & & & $\varepsilon$ & $260 \mathrm{nn}$ & 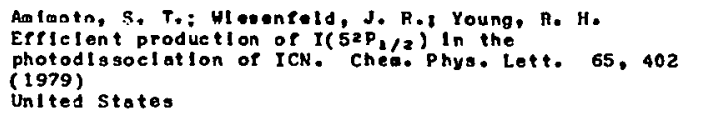 \\
\hline 1637 & $\begin{array}{l}\mathrm{hO}_{\mathrm{h}}^{\mathrm{HO}}+\mathrm{ICN} \\
\mathrm{HOB}, \mathrm{ICN} \\
\text { hW }\end{array}$ & & & E & $\begin{array}{l}1700-1100 \\
{ }_{A O}\end{array}$ & 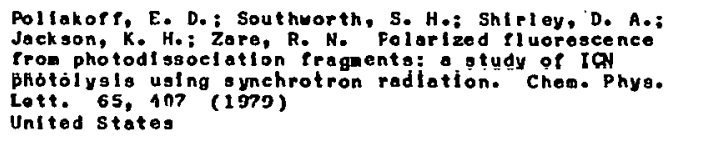 \\
\hline 1638 & $\mathrm{~N}^{\mathrm{A}} 3 \mathrm{H} \cdot \mathrm{n}$ & & . & $T$ & 0. 23-1.6 & 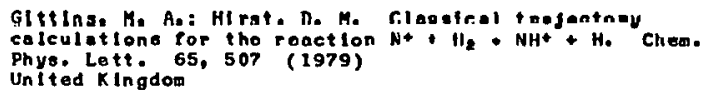 \\
\hline 1639 & $\stackrel{A_{11}}{\omega_{2} *+N_{2}:}$ & $\mathrm{CO}_{2} *+\mathrm{CO}$ & & $\varepsilon$ & $170-370 \cdot K$ & 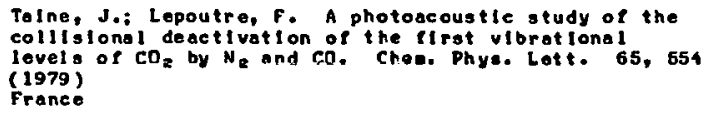 \\
\hline 1640 & $\begin{array}{l}\text { Al n } \\
\text { NaF }+\mathrm{HF} \text { : }\end{array}$ & $\mathrm{MgF}+\mathrm{HFF}$ & & $E$ & $500 \mathrm{~K}$ & 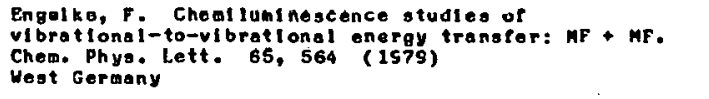 \\
\hline 1541 & $\begin{array}{l}\mathrm{AO7} \\
\mathrm{H}_{2} *+\mathrm{He}: \\
\mathrm{Kr} \\
\mathrm{Al}_{4} \\
\mathrm{H}_{2} * \mathrm{He}: \\
\mathrm{K}_{\mathbf{r}}\end{array}$ & $\begin{array}{l}\mathrm{H}_{2} *+\mathrm{Ne}_{\mathrm{O}}: \mathrm{H}_{2} *+ \\
\mathrm{H}_{2} *+\mathrm{Ne}_{\mathrm{E}}: \mathrm{H}_{2} *+\end{array}$ & & E & $330 \mathrm{~K}$ & 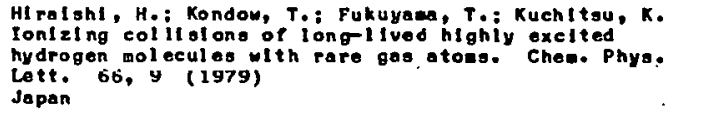 \\
\hline 1642 & 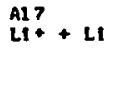 & & ' & $\begin{array}{l}\top \\
\end{array}$ & Undef & 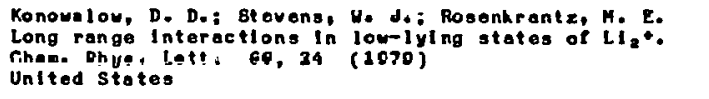 \\
\hline 1643. & $\mathrm{HA}_{\mathrm{He}}^{\mathrm{A}}+\mathrm{He}$ & & & T & Under & 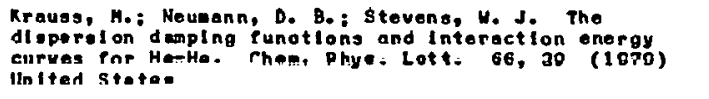 \\
\hline 1644 & $\mathrm{CH}_{\mathrm{CH}}^{\mathrm{Al}}+\mathrm{O}_{2}$ & & & $\begin{array}{l}\top \\
\end{array}$ & $298 \mathrm{~K}$ & $\begin{array}{l}\text { Messing, I.: Sadouskl, C. M.: Filseth, S. V. Absolute } \\
\text { rate constant for the reaction of } \mathrm{CH} \text { ulth } \mathrm{O}_{2} \text {. Chem. } \\
\text { Phys. Lett. 66, } 95 \text { (1979) } \\
\text { Canado }\end{array}$ \\
\hline 1645 & $\mathrm{H}_{2}^{\mathrm{H} 6}+\mathrm{N}_{2}$ & & & $T$ & $15-50$ oV & 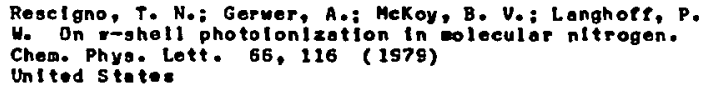 \\
\hline 1040 & $\mathrm{HOJ}+\mathrm{HCH}$ & & & $\mathbf{T}$ & $0.1-11.0 \mathrm{~V}$ & 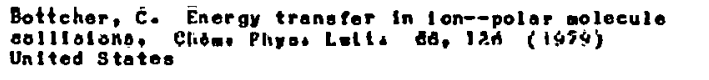 \\
\hline 1647 & $h_{h y}+x_{0}$ & & ; & E & $1470 A^{\circ}$ & 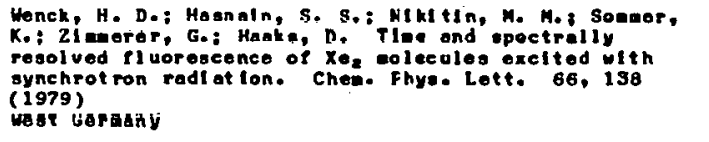 \\
\hline 1648 & ${ }_{\text {HF }}^{A 2} \div$ HF & & $:$ & T. & $\begin{array}{l}1000-2000 \\
\mathrm{~cm}-1\end{array}$ & 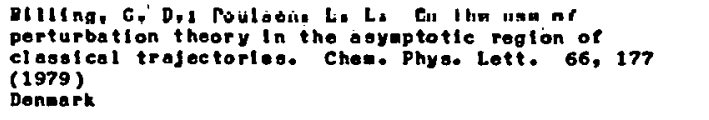 \\
\hline 1649 & ${ }_{0}^{\infty} \cdot 4$ & art & . & $T$ & $100-1300 \cdot 00$ & 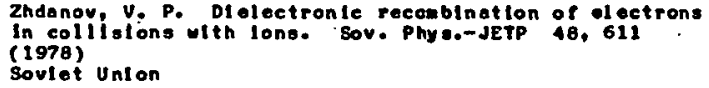 \\
\hline
\end{tabular}




\begin{tabular}{|c|c|c|c|c|}
\hline $\begin{array}{l}\text { REF. } \\
\text { No. }\end{array}$ & REACT ANTS & $\begin{array}{l}\text { EXP } \\
\text { OR } \\
\text { THEOR }\end{array}$ & $\begin{array}{l}\text { ENERGY } \\
\text { RANGE }\end{array}$ & REF ERENCE \\
\hline 1650 & $\begin{array}{l}\text { Ang } \\
\text { Unde? }\end{array}$ & $T$ & $2 \times 10^{\circ} \mathrm{cos} / \mathrm{s}$ & 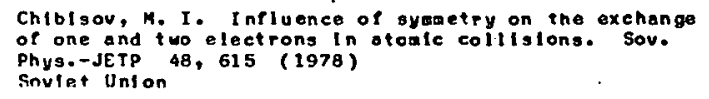 \\
\hline 1651 & 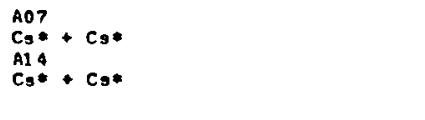 & $E$ & $500-730 \mathrm{~K}$ & 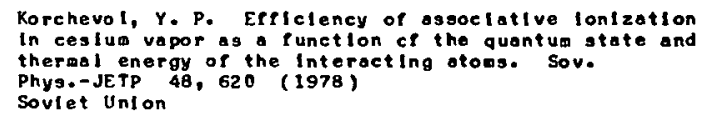 \\
\hline 1652 & $\mathrm{CO}+\mathrm{NoCl}$ & E & $15-50 \mathrm{keV}$ & 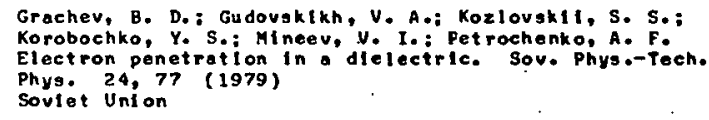 \\
\hline 1653 & 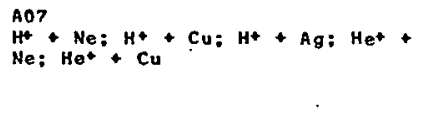 & T & $102 \times 10 \mathrm{~s} \mathrm{keV}$ & 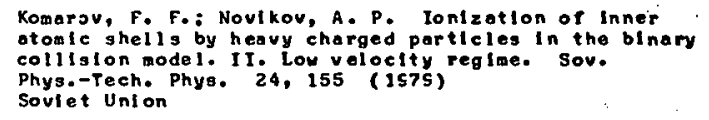 \\
\hline 1554 & $\begin{array}{l}\operatorname{Cog} \\
\text { under } \\
\text { Ex } 5 \\
\text { Under }\end{array}$ & T & Undet & 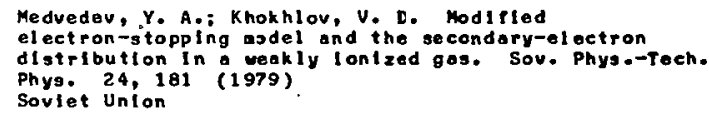 \\
\hline $1655^{\circ}$ & 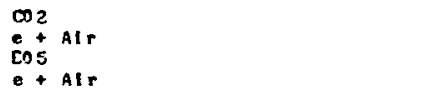 & $\tau$ & $\begin{array}{l}10-1-10-8 \\
\text { eV }\end{array}$ & $\begin{array}{l}\text { Medvedev, Y. A.: Khokhlov, V. D. Secondary olectron } \\
\text { distribution in weakly ionized air. Sov. Physe-Toeh. } \\
\text { Phys. } 24,185 \text { (1979). } \\
\text { Soviet Uni on }\end{array}$ \\
\hline 1656 & $\begin{array}{l}D_{12} \\
\text { Art }^{+}+M_{0}\end{array}$ & $\varepsilon$ & $40 \mathrm{keV}$ & 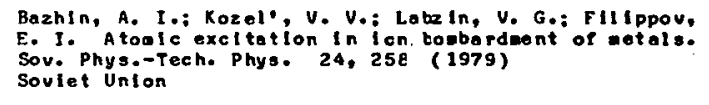 \\
\hline $165 ?$ & $\mathrm{HO}_{\mathrm{H}} 2 \mathrm{CH}$ & E & 3.39 m & 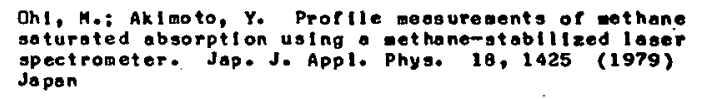 \\
\hline 1658 & $\underset{\mathrm{Ne}+}{\mathrm{DO} 2}+\mathrm{Ho}_{\mathrm{O}}$ & E & $0.6-1.5 \mathrm{keV}$ & 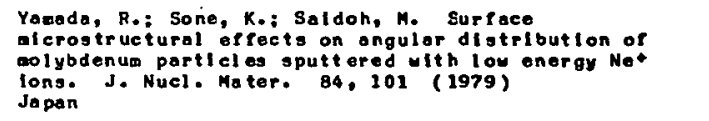 \\
\hline 1659 & $D_{D+C}^{D 14}+C u$ & $\varepsilon$ & $200 \mathrm{keV}$ & $\begin{array}{l}\text { Arastrong, T. R. The onset of rodlotton-blistering in } \\
\text { low-teaperature D*-irradloted copper. J. Nucl. Moter. } \\
\text { aA, ils (1979) } \\
\text { Nev Zeal and }\end{array}$ \\
\hline 1660 & $n+2 r: n+2 r+N b$ & E & $0.1 \mathrm{MeV}$ & 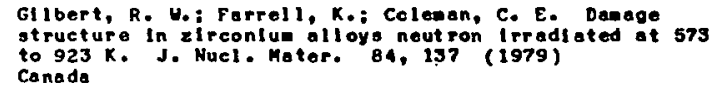 \\
\hline 1661 & 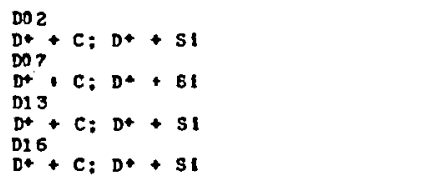 & E & $50-100 \in V$ & 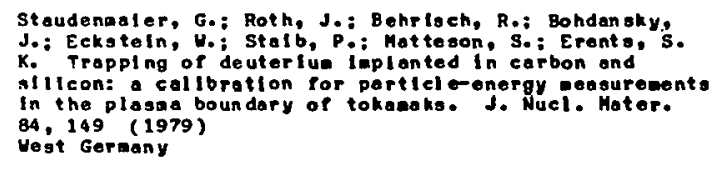 \\
\hline 1662 & 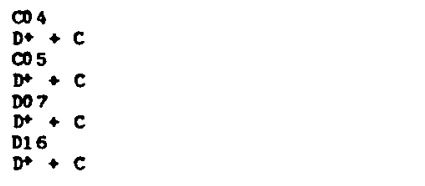 & $\mathrm{T}$ & $20-6700 \mathrm{aV}$ & 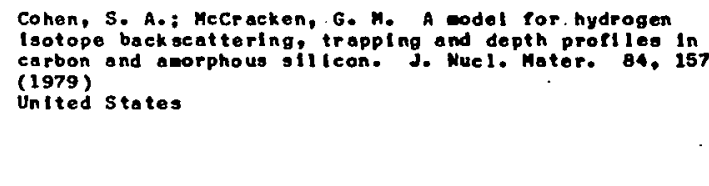 \\
\hline 1663 & $\begin{array}{l}\text { D1 } 4 \\
\text { Undep }\end{array}$ & T & Undor & $\begin{array}{l}\text { Brolls sord, A. D. The vacancy dlslocatlon } 1 \text { loop } \\
\text { me crostrueture formed during heavy-partlelo } \\
\text { bombardaent. J. Nuel. Mater. B4, } 245 \text { (1979) } \\
\text { United States }\end{array}$ \\
\hline
\end{tabular}




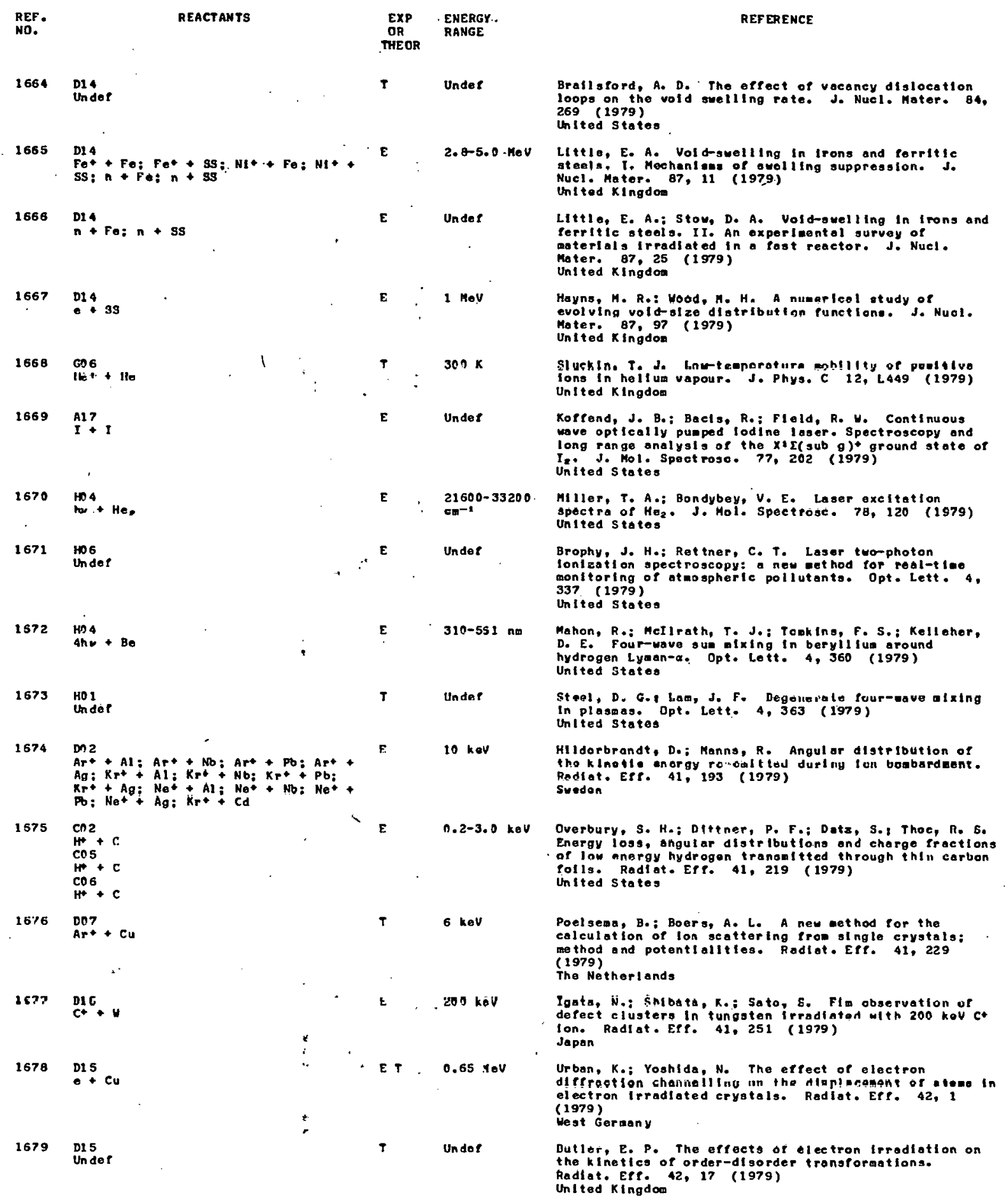




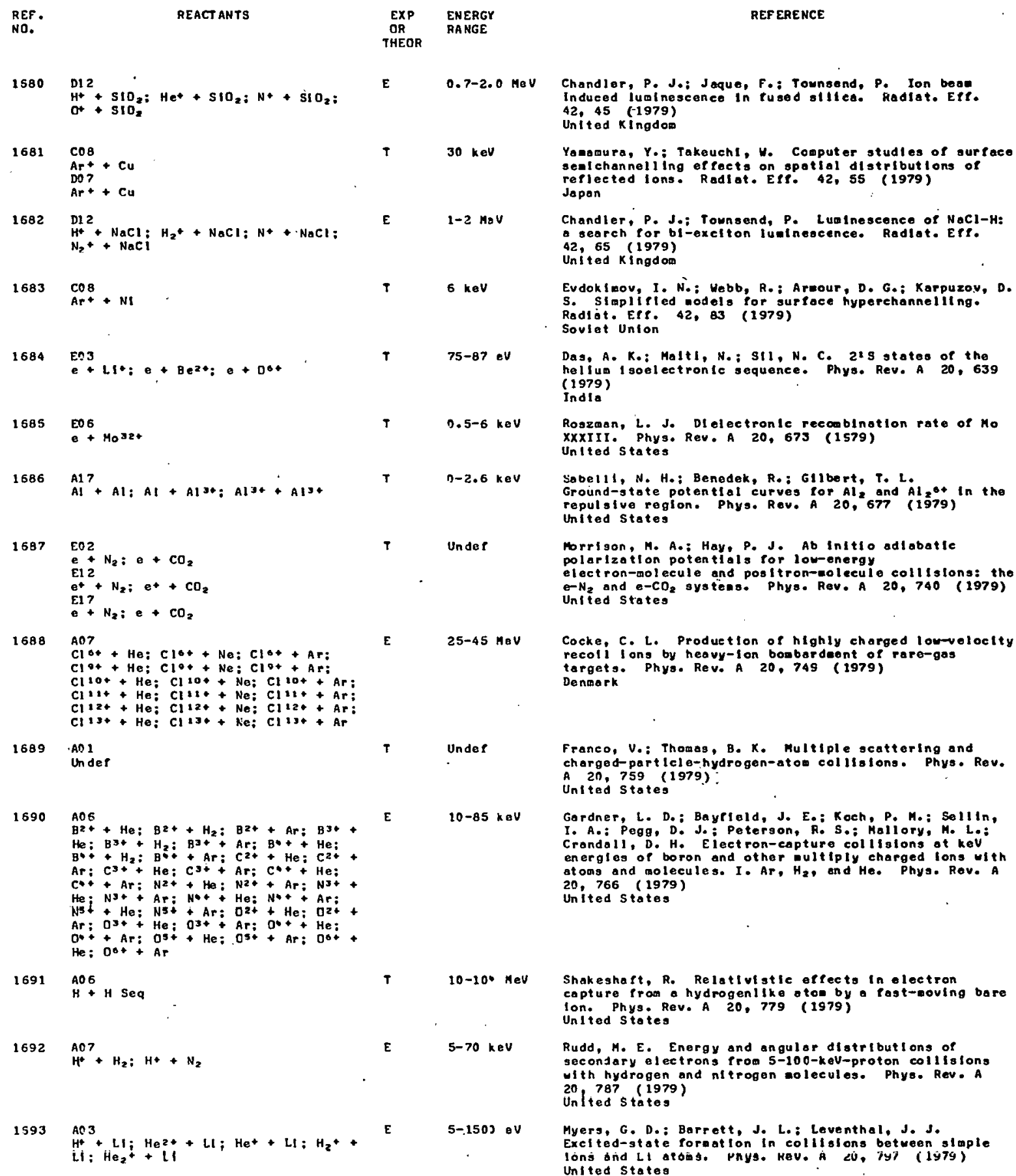

E

Under

$10-85 k \in V$

E

$10-10 * \mathrm{NeV}$

$0.7-2.0 \mathrm{MBV}$

Chandlar, P. J.; Jaque, F.: Tounsend, P. Ion beal $42,45(-1979)$

Yomamura, Y.: Takeuchl, W. Computer studles of surface senlchannelling of pects on spotlial distrlbutlons of reflected lons. Radiot. ElP. 42, 5S (1979) Japan

Chandler, P. J.; Tounsend, P. Lualnescence of NaCl-H: - seareh for bl-exelton luetnescence. Radlat. Erf. 42,65 United Kingd om

Evdoklnov, I. N.: Hebb, R.: A reour, D. G.: Karpuzov, D. S. Simplifled models for surface hyperehannelling. Radijt. Eef. 42, 83 (1979) Soviat Union

Das, A. K.: Maltl, N.: Sil, N. C, 2:s states of the helium isoelectronic sequence. Phys. Rev. A 20,639 (1979)

Roszan, L. J. Dlelectronle recomblnation rate of Mo XXXIII. Phys. Rev. A 20, 673 (1579) Unt ted states Sobel11, N. H.: Benedek, R.: Gllbert, T. L. repulsive rogion. Physo Rev. A 20, 677 (1979) United States

Norrigon, M. A.: Hay, P. J. Ab Initio adlabatle polarlzotlon potentials for low-energy electron-molecule and positron-aolecule collistons: the $e-\mathrm{N}_{2}$ and $\mathrm{e}-\mathrm{CO}_{2}$ systens. Phys. Rev. $\mathrm{A} 20,740$ (1979) United States

Cocke, C. L. Produetion of highly charged low-voloctty recoli long by heavy-ion bosbardeont of rare-gas targets. Phys. Rev. A 20,749 (1979) Dennerk

Franco, V.: Thomas, B. K. Multiple scattering and charged-partlcle-hydrogen-atow collisions. Phys. Rev. charged-partlcle-hydro A 20,759 (
Unted States

Gordner, L. D, : Bayfield, J. E.; Koch, P. M.; Sellitn, I. A.: Pegg, D. J.: Petergon, R. S.: Mallory, M. L.; energles of boron and other pultiply charged lons with 20,766 (1979) United statos

Shakeghaft, R. Relattulstle effects th olectron capture proma hydrogenlike atos by a Pat-eoving bare lon. Phys. Rev. A 20, 779 (1979) United States

Rudd, H. E. Energy and angular distrlbutlons of secondary electrons from 5-100-keV-proton collistons uecth hydrogen and iterogen oleculas. Phys. Rey. 20,787 (1979) Unlied states

Myers, G. D.: Barrett, J. L.: Leventhal, J. J. Exclted-state formation in collislons between simple lons ond $L i$ atốs. Pnys. Hev. $\dot{A} 20,7 y 7$ (1979) United stotes 


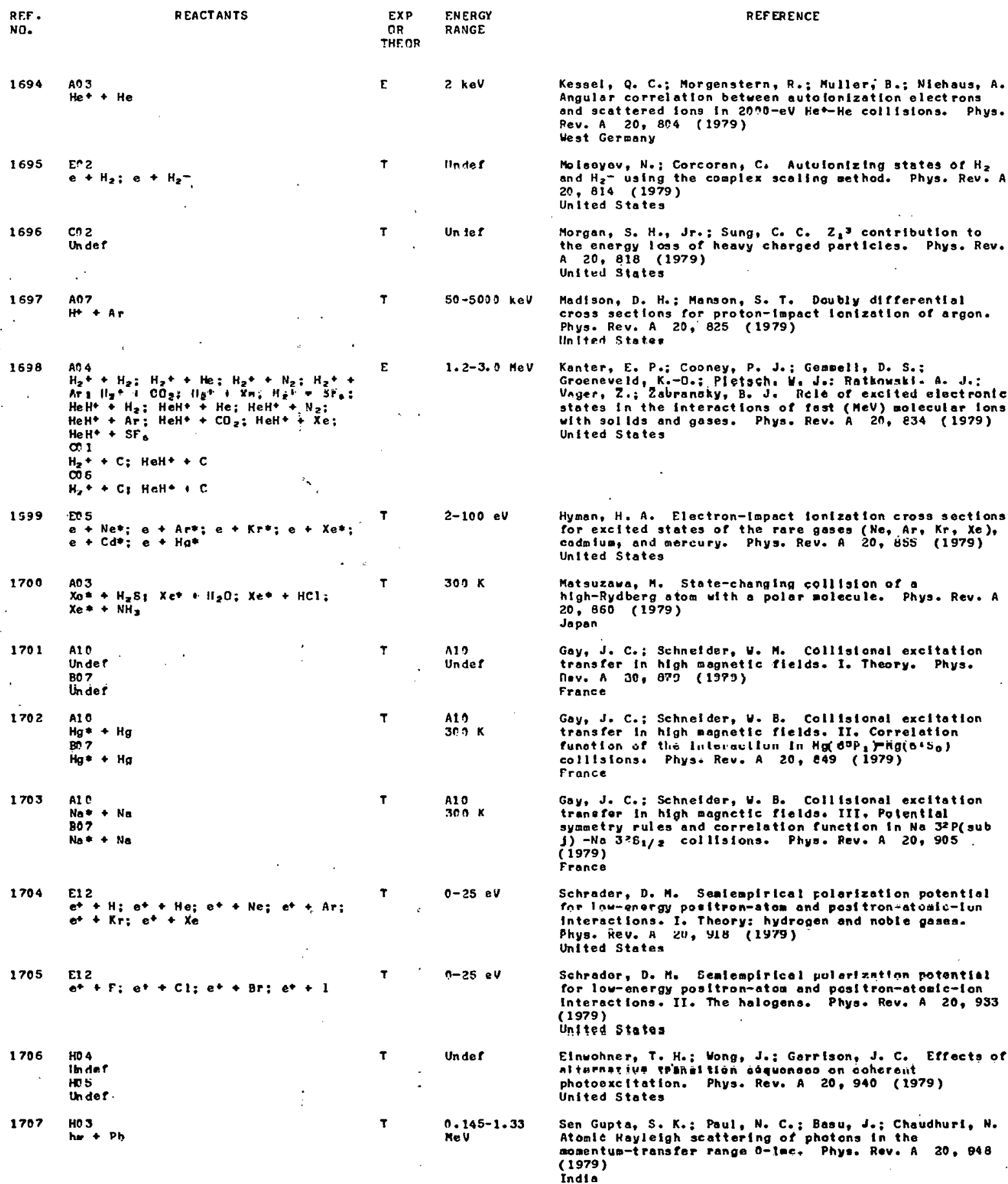

1706

hos

thdoe.

1707

Kessel, Q. C.: Morgengtern, R.: Muller; B.: Nlehaus, A. Angular correlation between autolonization electrons and scat tered lons in $2090-\mathrm{eV}$ Het-He collisions. Phys. Rev. A $20,804(1979)$ West Germany

Molsoyov, N.: Corcoron, C. Autulonlzing states of $\mathrm{H}_{2}$ and $\mathrm{H}_{2}-$ using the complex scalling aethod. phys. Rev. A $20,814(1979)$

Morgan, S. H., Jr.: Sung, c. c. $2_{2} 3$ contribution to the energy loss of heavy charged portlcles. Phys. Rev. A 20,818 (1979) Unlted slates

Madison, D. H.: Manson, S. T. Daubly dleterentlal eross sections for proton-timpact lonization of argon. Phys. Rev. A 20, 825 (1979)

Kanter, E. P.: Cooney, P. J.: Gemoell, D. S.: Groeneveld, K.-O.: Pletseh. Y. Jo: Ratknuaki: A. J.: anger, 2.; Zabranoky, B. J. Melo ox exclted electronte states in the interactions of last (MeV) wolecular lons with gol lds and gaseg. Phys. Bevo A 20,834 (1979) Unlted States

Hyman. H. A. Electron-lapact lontzat lon e ross sectlons

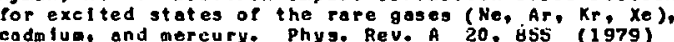
United States

Matguzawa, M. State-changing collligion of high-Rydberg atom ith a polar molecule. phys. Rev. A 20,860 (1979) Japan

Gay, J. C.: Sehnelder. H. M. Colltstanal excitation trangfer in high magnetic tilelds. 1. Theory. Phys. nev. A 30. orj (1973) Franee

Goy, J. C.; Schnelder, H. B. Colllstonal excitation transfer in high magnetic fields. II. Correlation

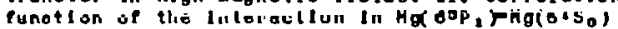
colllsions. Phys. Reu. A 20, 849 (1979) France

T A10

Gay, J. C.: Schnelder, H. B. Collistonal excitation trangtor in high magnetic pields. IIT. Potentlal

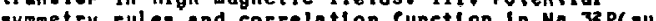

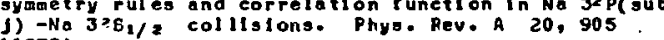
(1979)

Schrader, D. H. Sealeapirical folorization potentlal fnr lnu-onergy poeltron-aton and positron-atolic-t un Interactlons. I. Theory: hydrogen and noble gases. Phys. Rev. A 20,918 (1979) United States

Schrador, D. M. Eemlemplr leal pul orlantion potential for lou-anergy positron-aton and posltron-otonle-ton interactions. II. The halogens. Phys. Rev. A 20,983 (1979)

United states

Elnwohner, T. H.: Vong, J.: Garrlson, J. C. Effeets of

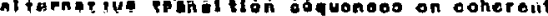
photoexcitation. Phys. Rev. A 20,940 (1979) United states

T $\quad 0.145-1.33$
Sen Gupta, S. K.: Paul, N. C.; Basu, J.; Chaudhuri, N. Atomle Kayleigh seattering of photons In the 20,048 (I979) 


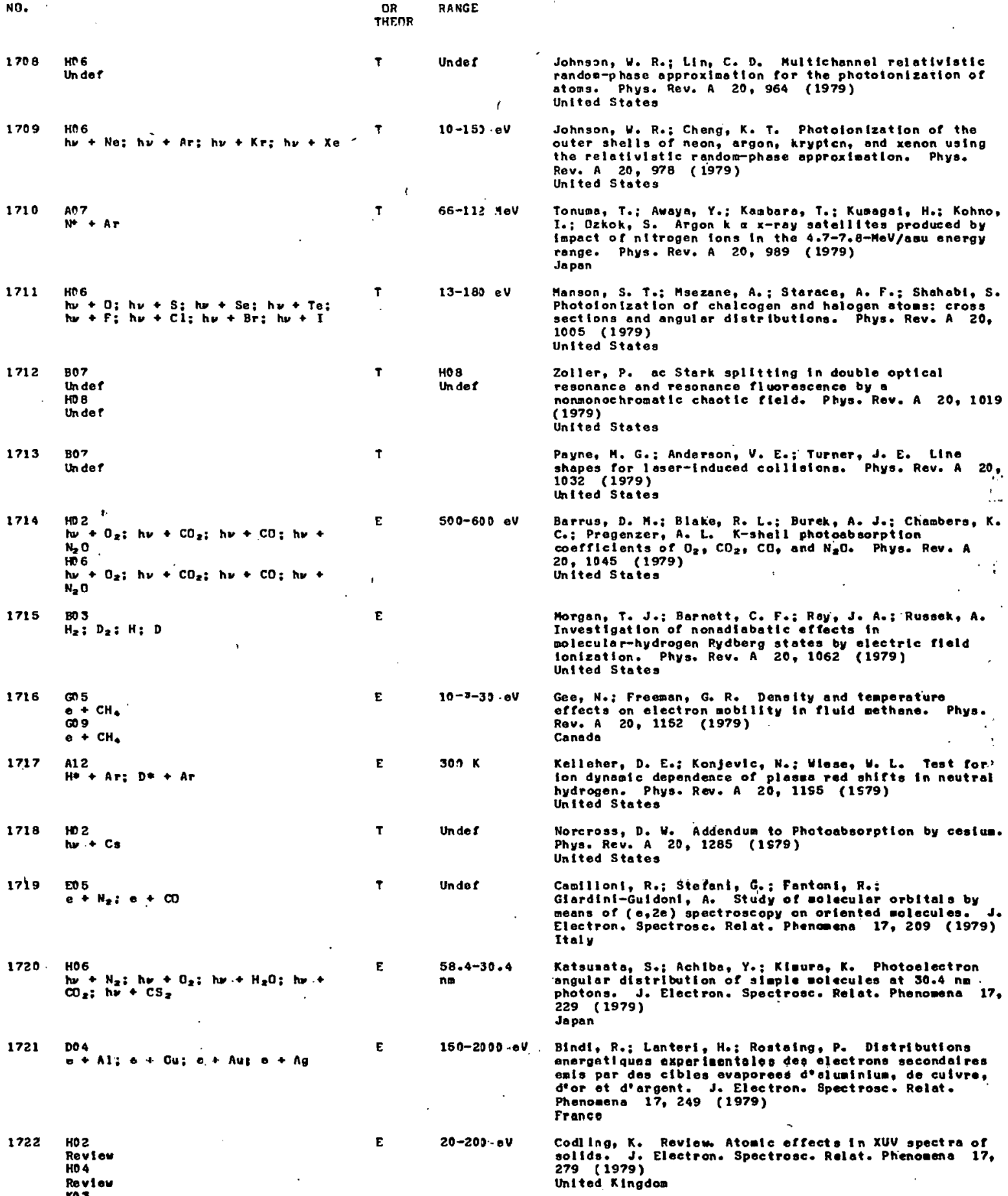

1711 HOG

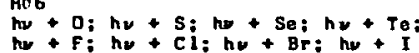

$\mathrm{HO}_{2}$

tow $+\mathrm{O}_{2}: \mathrm{hv}+\mathrm{CO}_{2} \mathrm{ihv}+\mathrm{CO} \mathrm{hw}+$ $\mathrm{NoO}$

$10-8-30 \cdot 06$

$309 k$

Under

Under

$8.4-30.4$

nim

$160-2000+00$

$13-180$ ev

108

der

$0-600$ ov R. L.: Burek, A. J.; Chawbers, $K$. C.: Pregenzer, A. L. K-shell photoabsorption coepflelents of $\mathrm{O}_{2}, \mathrm{CO}_{2}, \mathrm{CO}$, and $\mathrm{N}_{2} \mathrm{O}$, Phys. Rev. A 20,1045 (1979)

United states

Horgan, T. J.: Barnatt, C. F.: Rey, J. A.; Russek, A. Investigation of nonadiabatic eptects in molecular-hydrogen Rydberg stotes by electric pleld lonization. Phys. Rev. A 20, 1062 (1979) United states

Gee, N.: Freeman, G. R. Denolty and temperoturo offects on electron moblility in pluld dethone. Phys. Rev. A 20, 1152 (1979) Kelleher, D, E.: Konjevic, No: Hiose, H. L. Test for: lon dynamic dependence of plases red shlf
hydrogen. Phys. Rev. A 20,1155 (1579)
United States

Norcross, D. W. Addendum to Photoabsorption by cestum. Phys. Rev. A 20, 1285 (1979) United states

Caolllonl, R.: Sietanl, G.: Fantonl, R.: Glardinl-Guldonl, A. Study of nolecular orbltal s by means of (e,2e) spectroscopy on oriented molecules. J.
Electron. Spectrosc. Rel at. Phencent 17, 209 (1979)

Katsusata, S.: Achlba, Y.: Ktaura, K. Photoelectron angular distribution of iaple aol ecules at $30.4 \mathrm{~nm}$. photons. J. Elect ron. Spectrose. Relat. Phonomena 17 , 229 (1979)

Japan

Bindi, R.: Lanteri, H.: Rostalng, P. Dlstributlons enargatl ques experieentelos des elect rons socondal res enls par des clbles evaporees do ol uninlum, de culvre, do or ot d'argent. J. Electron. Spect rose. Relat. Phenouena 17, 249 (1979) France

$20-200 \cdot 0 V$

Codl Ing, $K$. Revlem. Atonle er fect I In XUV apect ro of solids. J. Electron. Spectrosc. Relat. Prienonens 17 United $x \operatorname{lng}$ dom 


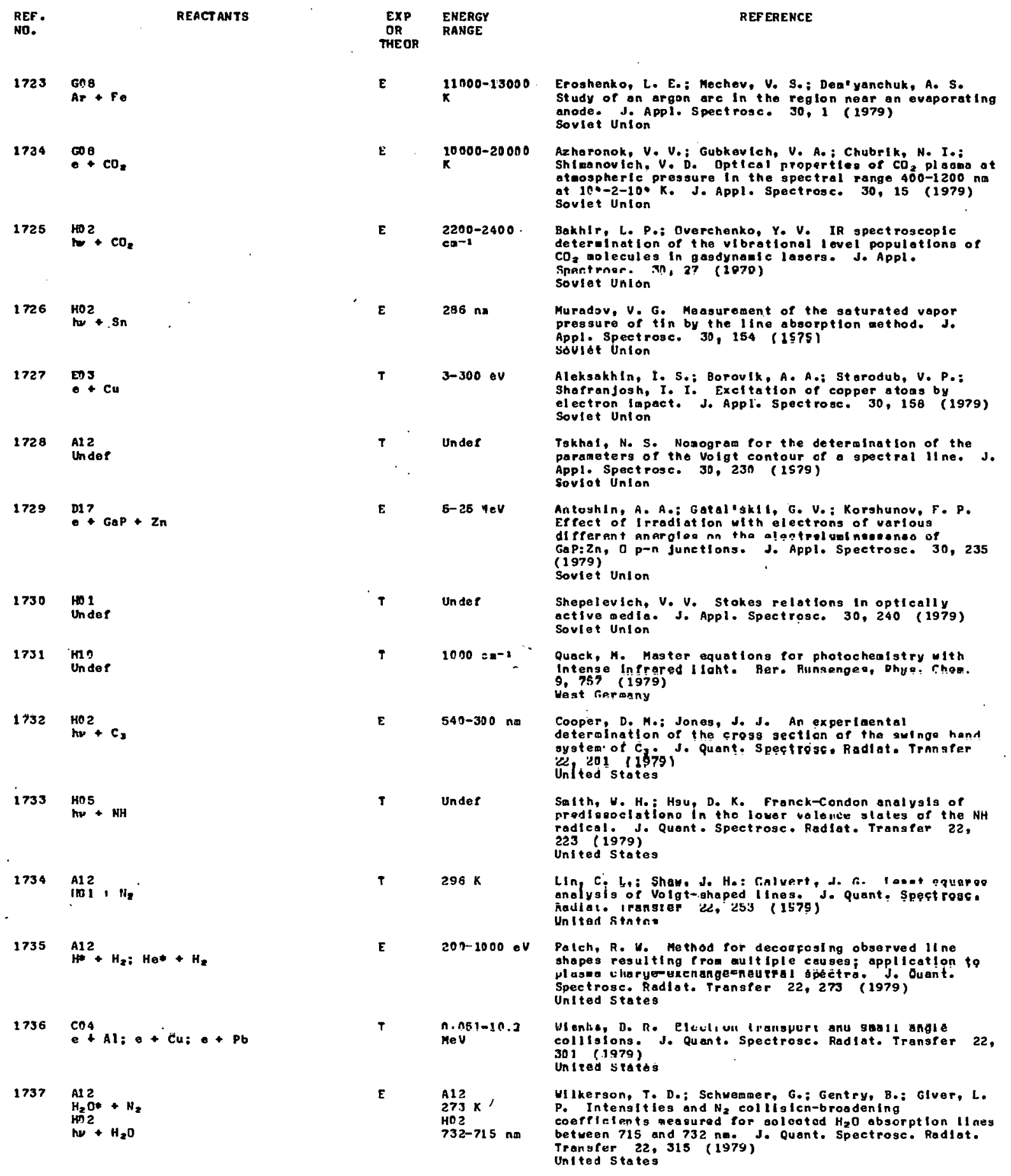




\begin{tabular}{|c|c|c|c|c|c|}
\hline $\begin{array}{l}\text { REF. } \\
\text { NO. }\end{array}$ & & REACT ANTS & $\begin{array}{c}\text { EXP } \\
\text { OR } \\
\text { THEOR }\end{array}$ & $\begin{array}{l}\text { ENERGY } \\
\text { RANGE }\end{array}$ & REF ERENCE \\
\hline 1738 & 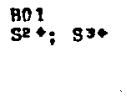 & & E & & 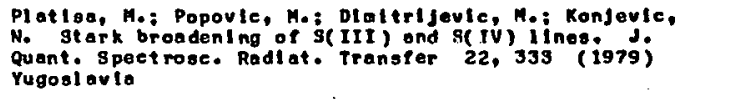 \\
\hline 1739 & $t_{t}^{\text {Ho } 1}+\mathrm{Cs}_{3}$ & & $T$ & $\begin{array}{l}0.1-1030 . \\
0.0 .\end{array}$ & 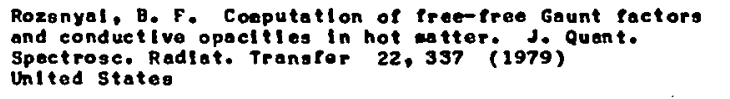 \\
\hline 1740 & $\begin{array}{l}\mathrm{Al}^{\mathrm{CO}} 2 \\
\mathrm{CO}_{2} *+\mathrm{N}_{2}\end{array}$ & & E & $203-325 \mathrm{~K}$ & 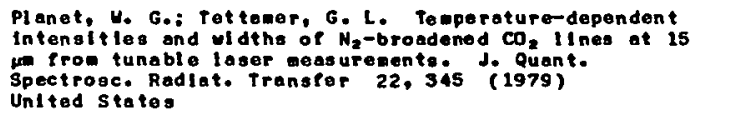 \\
\hline 1741 & $\mathrm{HO}_{\mathrm{H}}^{\mathrm{HO}}+\mathrm{H}_{2} \mathrm{O}$ & & $\varepsilon$ & $820-620 \mathrm{~nm}$ & 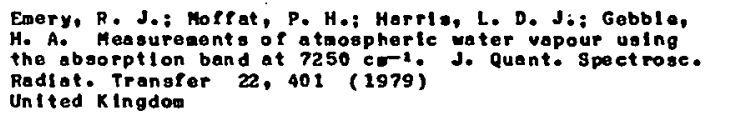 \\
\hline 1742 & $\begin{array}{l}\text { Hn 1 } \\
\text { Unde? }\end{array}$ & & $T$ & Under & $\begin{array}{l}\text { Cipolla, J. U., Jr.: Morse, T. F. K1 netle offects In } \\
\text { an optlcaliy exclted gaso; J. Guont. Spectrose. Rodiat. } \\
\text { Trangfer } 22,365 \text { (1979) } \\
\text { United States }\end{array}$ \\
\hline 1743 & 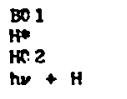 & & $E$ & $\begin{array}{l}\text { HO2 } \\
122 \mathrm{nd}\end{array}$ & 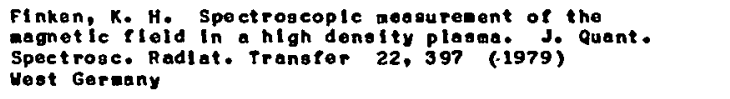 \\
\hline 1744 & $\begin{array}{l}\text { Cn2 } \\
\text { Under } \\
\text { cos } \\
\text { Under }\end{array}$ & & $T$ & Undet & 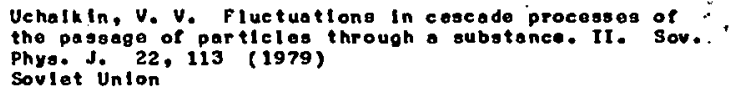 \\
\hline 1745 & hov $_{\text {Ho }}+$ e & & $T$ & Under & $\begin{array}{l}\text { Bozrikov, P. Y.: Tsaregorodtsev, L. I. Splltting of a } \\
\text { phot on into three by on ol ectron. Sov. Physe J. 22, } \\
117 \text { (1979) } \\
\text { Sovlet Unlon }\end{array}$ \\
\hline 1746 & ${ }_{\text {hL }}^{\text {H12 }}+$ e & & $T$ & Under & $\begin{array}{l}\text { Lysikov, Y. I. Compton seattering of a opectrally } \\
\text { narrou, radiation flux by Proe eloctrons. Sov. Phya. J. } \\
22,187 \text { (1979) } \\
\text { Soviet Union }\end{array}$ \\
\hline 1747 & $\mathrm{COB}^{\mathrm{CO}}+\mathrm{NaCl}$ & & $\varepsilon$ & $1.0-3.8 \mathrm{MeV}$ & 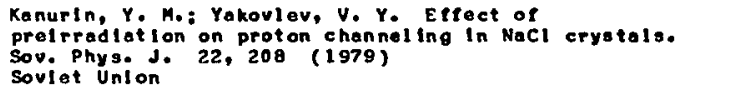 \\
\hline 1748 & $\mathrm{HOS}_{\text {ho }}+\mathrm{SF}$. & & $T$ & $944 c z-1$ & 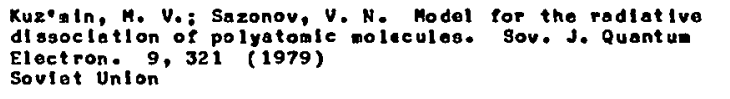 \\
\hline 1749 & $\begin{array}{l}\text { Bn? } \\
\text { Under }\end{array}$ & & T & & 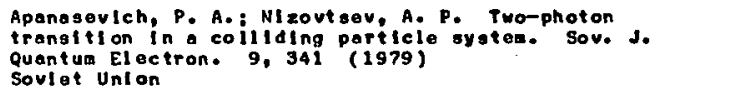 \\
\hline 1750 & $\begin{array}{l}\text { Ho } 4 \\
\text { 4he }\end{array}$ & & E & $354-530 \mathrm{~nm}$ & 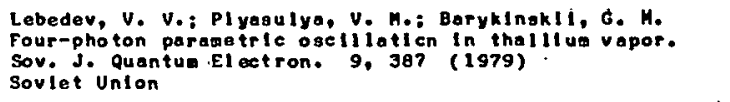 \\
\hline 1751 & 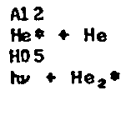 & & $T$ & $\begin{array}{l}\text { A12 } \\
\text { Under } \\
\text { H05 } \\
81.5 \text { na }\end{array}$ & 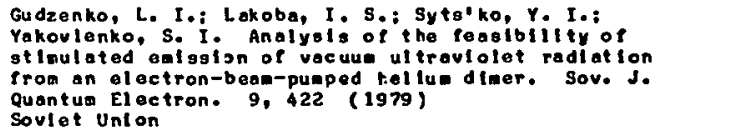 \\
\hline 1752 & $\begin{array}{l}\text { Hos } \\
\text { nhe }\end{array}$ & & $\varepsilon$ & $10.6 \mathrm{H}$ & 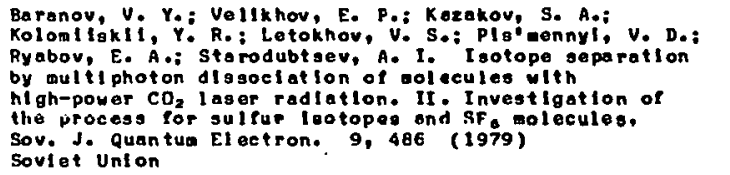 \\
\hline
\end{tabular}




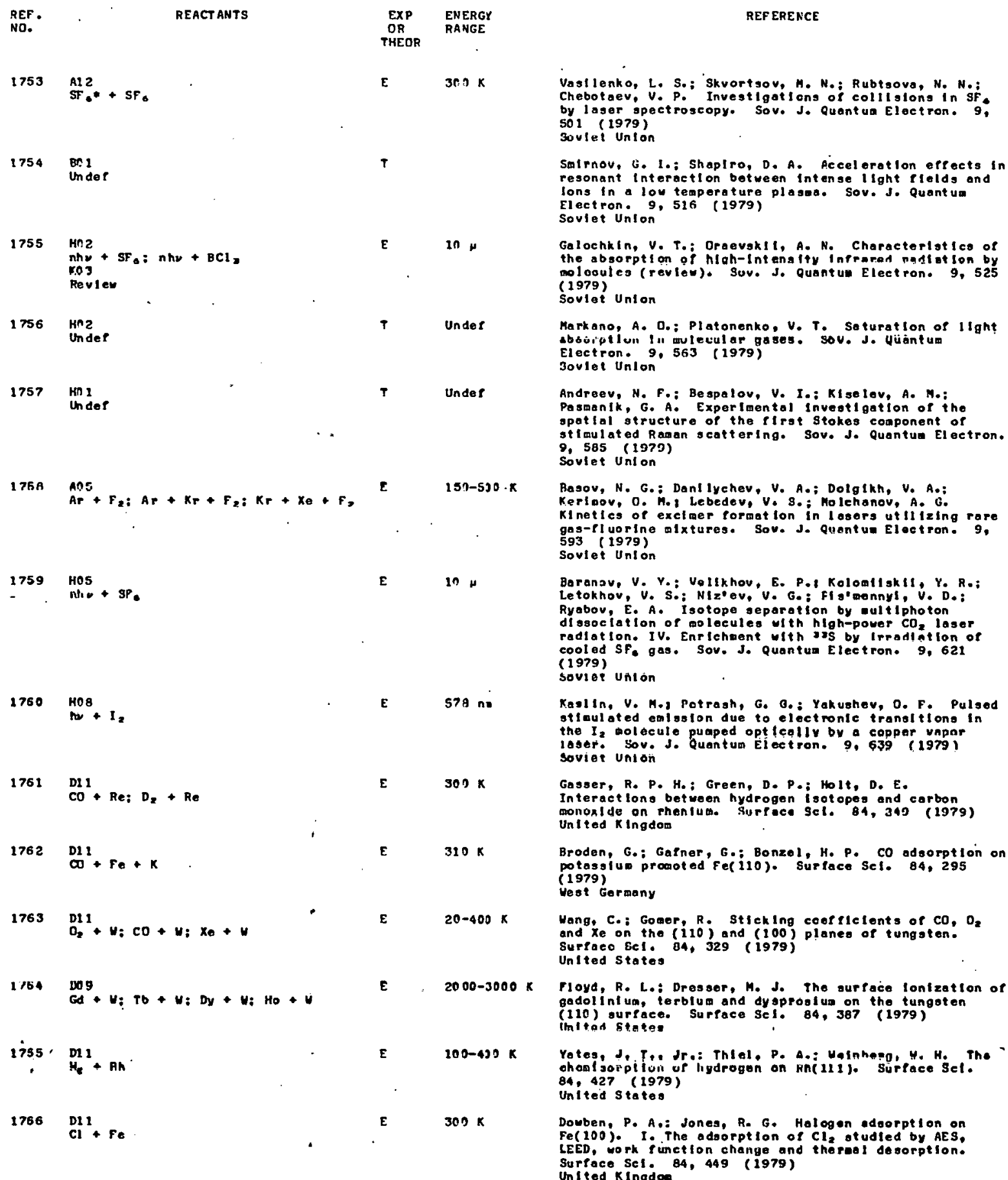

REF.
NO.

REACT ANTS

1753

$\stackrel{\mathrm{Al2}}{\mathrm{SF}} \cdot \mathrm{SF}_{\mathrm{B}}$

1754

En 1 Un

1755

$\ln _{n \rightarrow 2}+\mathrm{SF}_{a}: \mathrm{nh} v+\mathrm{BCl}_{2}$

Revier

1756

under

1757

Kn 1
Under

1768

Ane

$\mathrm{Ar}_{\mathrm{r}}+\mathrm{F}_{2}: \mathrm{Ar}+\mathrm{K}_{r}+\mathrm{F}_{2}: \mathrm{Kr}_{\mathrm{r}}+\mathrm{Xe}_{\mathrm{e}}+\mathrm{F}_{2}$

1759

HOS SP.

$1760 \frac{108}{100}+I_{2}$

1761 D1

CO + Re: $D_{2}+$ Re

1762 D1

D1 1

1763

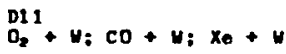

124009

GQ U: TO + W: DY + W: HO + V

$2755, \mathrm{DL}_{\mathrm{H}}+\mathrm{Ah}$

1766

$\operatorname{EXP}$
OR

THEOR

E $300 \mathrm{~K}$

T

in

Under

Under

E

$150-530 \cdot x$

10

$\mathrm{Dl}^{\mathrm{Cl}}+\mathrm{Fe}$

ENERGY

ANGE

s

679 n.

$300 \times$

$310 K$

$20-400 x$

$2000-3000 \times$

$100-439 k$

$309 \mathrm{~K}$

REF ERENCE Vastlenko, L. S.: Skvortsov, M. N.: Rubtsova, N. N.: by laser apectroscopy. Sov. J. Quantua Electron. 9, Sol ( 1979 )

Sulrnov, 6. I.: Shaplro, D. A. Accelerotlon effects in cesonant interaction between intense light flelds and lons in a lov temperature plasaa. Sov. J. Quant um Electron. 9, 516 (1979)

Soviet Unton

Galochkin, V. T.: Draevokit, A. N. Characteristlco of the absorption of hlah-lntena ity infrarad radt ot lon by molooules (revleu). Suv. S. Quantua Electron. 9, 525 (1979)

Soviat Unton

Markano, A. D.: Platonenko, V. T. Seturatlon of IIght Abobictlun !" unlecular gases. Sov. J. Uüantum Electron. 9, $563(1979)$

Bovlet Unlon

Andreev, N. F.: Bespalov, V. I.: Klse tev, A. M.: Pasmenlk, G. A. Exper Imental Inveotlgation of the opatial structure of the first stokes coaponent of st Imulated Rasan scattering. Sov. J. Quantua El ectron. 9. 585 (1979)

Soviet Unl on

Basov, N. G.: Danl lychev, V. A.; Dolglkh, V. A.: Kerlaov, O. H. : Lebedev, V. S.; Molehanov, A. $G$. Kinetles of exclmer format lon in losers utllizing rare gas-tl uorine Sovtet union

Baranav, V. Y.: Uallkhov, E. P.t Kolomllskll, Y. R.; Letokhov, V. S.: Nlz'ev, V. G. : Fl g'mennyl, V. D. Ryobov, E. A. Isotope separation by multiphoton disoctation of nolecules with high-power $\mathrm{CO}_{2}$ laser radlat lon. IV. Enrlehaene with a gh by lrradition of Sovier Unt on

Kaslin, V. Mol Potrash, G. O.: Yakushev, O. F. Pulsed stinulated emission due to electronic transltions In the $I_{2}$ molecule puaped opt teally by a copper vapar laser. Sov. J. Quantun El ectron. 9, 639 (1979)

Gasser, R. P. H. ; Green, D. P.; Holt, D. E. Interactions between hydrogen isotopes and corbon
monoslde on rhanlut. Surfece Scl. 84, 349 (1979) Unitad Kingdom

Broden, G.; Gafner, G.: Bonzel, H. P. CO adsorption on potassiun promoted fe(i10). Surface sel. 84, 295 potass 1979 ) Uest Germany

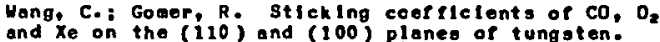
Surfaco Gel. B4, 329 (1979) United states

Floyd, R. L.: Dresser, M. J. The aurface Iontzation of gadolint um, terblum and dysprosf us on the tungoten
(110) ourface. Surface Sel. 84, 387 (1979) initod gtates

Yotes, J, Th Jre: Thial, P. A.: Uainhege, H. H. The ohonl jorptiui of ligdrogen on Hail11). Süreace Set. 84, 427 (1979)

United States

Douben, P. A.: Jones, R. G. Hologen edoorption on Fe(100). I. The adoorption of $\mathrm{Cl}_{2}$ otudied by $A E S$. Fe( 100 . $\mathrm{I}$. The adsorption of $\mathrm{Cl}_{2}$ ot udied by AES. Surface Sci. 84, 449 (1979) 


\begin{tabular}{|c|c|c|c|c|}
\hline $\begin{array}{l}\text { REF. } \\
\text { NO. }\end{array}$ & REACTANTS & & $\begin{array}{l}\text { EXP } \\
\text { OR } \\
\text { THF.OR }\end{array}$ & $\begin{array}{l}\text { ENERGY } \\
\text { RANGE }\end{array}$ \\
\hline 1767 & ${ }_{a}^{D 13}+0+M_{0}$ & & $\varepsilon$ & $2 n 3 \mathrm{eV}$ \\
\hline 1758 & $D_{21}^{D 1}+A_{1}$ & & $E$ & $300 \mathrm{~K}$ \\
\hline 1769 & $\mathrm{DH}_{+}^{\mathrm{DH}}+\mathrm{NI}_{1}$ & & E & $303 \mathrm{~K}$ \\
\hline 1770 & $e^{D 13}+\mathrm{CO}+u$ & .. & $\mathbf{E}$ & $1000 \geq 0$ \\
\hline 1771 & $\begin{array}{l}D_{11} \\
O_{2}+S 1\end{array}$ & . & E. & $303 x$ \\
\hline 1772 & $\mathrm{Anz}_{\mathrm{Ar}}+\mathrm{Cu}: \mathrm{Ar}+$ CuO & & $T$ & $600 \mathrm{ev}$ \\
\hline 1773 & $\begin{array}{l}\text { Der } \\
\text { Under }\end{array}$ & & $T$ & Under \\
\hline 1774 & $e^{D 04}+$ AI & & $\top$ & $350 \mathrm{ev}$ \\
\hline 1775 & $X_{e}^{D 11}+C u$ & & $\varepsilon$ & $300 K$ \\
\hline 1776 & $\mathrm{D} 11+\mathrm{Fe}$ & & E. & $300 \mathrm{~K}$ \\
\hline 1777 & $\stackrel{013}{0}+0+4$ & & $\varepsilon$ & $10-90 \mathrm{eV}$ \\
\hline
\end{tabular}
Yu, M. L. Effect of Cs on electron stimulated
desorption of negotive ions. Surface Sel. 84, L493 (1979)

Unitej states

Michel, R.: Jourdan, C.: Castald, J.: Derrien, J. On the Initlai interaction of oxygen with the (111) and
(19n) oluminium gurfaces. Surfoce Set. $84,(509$ $(1979)$

Schouten, r. C.: Gijzeman, O. L. J.: Bootsma, G. A. Interaction of methane with NI(111) and Ni(100): dirfugiton of surface

The Netherlands

Hock, J. L.: Cralg, J. H., Jr.: L L chtwan, D. A comparat lue study or ESD of posltive ond negative ions Prom co on ungsten. Surface scl. 87, 31 (1979)

Wheringe, P. E.; van Sllfhout, A.; Sparnaay, M. J. Reflectometric study of dangling-bend surface states and oxygen adsorpt lon on the clean $51(111) 7 \times 7$ surface. surfoce sel. 87,43 (1579)

The Netherlands

Garrlson, B. J.: Vinograd, N.: Herrison, D. E., Jr.

Classlcal trajectory calculaticns of the energy distribution or ejected at oms ircm ion bomborded single crystals. Surface Sc1. 87, 101 (1979)

United States

Celil, V.: Garcia, N.: Hutchinson, J. Interpretatl on of selectlve adsorption in atow-surface scatterlng. Surface Sci. 87, 112 (1979) United states

Ganachaud, J. P.; Colller, M.: Aberdam, D.: Blanc, E.: Gaubert, C. New theoretical results boout the anlsotropy of the gecondary emisgi on of $A l(001)$ and Al(110). Surface Sel. 87, 129 (1975)

France

Glachant, A.; Bard $t$, U. Thermodynalles and klnetles of $X e$ monol ayer odsorptlon on Cu(100) by LEED and AES. Surfoce Scl. 87, 187 (1979) France

Johnson, D. W.: Roberts, M. W. Nitrogen chenlsorption by Iron: Surface Sel. 87 , L255 (1979) Unlted Kingdom

Kirsehner, J.: Menzel, D.; Stalb, P. Electron gt imulated desorption of o lons: energy dependence of the desorption efficlency from adsorbed oxygen on meta surfaces and Its bearing on the dechanlsm. Surface Sel. 87, L267 (1979) West Germany

1778 D10. He $+\mathrm{C}: \mathrm{H}+\mathrm{C} ; \mathrm{H}_{2}+\mathrm{C}$

1779 D11 $X_{e}+P d$

1780 $\mathrm{D}_{2} 1 \mathrm{Cu}: \mathrm{N}_{2} \mathrm{O}+\mathrm{Cu}$

1792. ก11
$\mathbf{T}$

$300 \mathrm{~K}$

$300 \mathrm{~K}$

$300 \times$

Vidall, G.: Cole, M. W.: Schwortz, C. Asyaptotle interaction beteeell lle, II, $H_{2}$ and ornphite surfoce. Surface Scl. 87, L273 (1979) Unlted States

Kuppers, J.: Nltschke, F.: Uandelt, $K_{0} ;$ Ertl, G. The adsorption of $x e$ on Pd(1in). Surface Scl. 87, 295 (1979)

Hest Germany

Habraken, F. H. P. M.: Bootsan, G. A. The klnotles of the Interactions of $O_{2}$ and $N_{2} O$ with a cu(11n) surface and of the resction of $C O$ ol th adsorbed oxygen studied by means of ellipsonetry. AES ond LEED. Surface Sel. A7 333 (1979)
The Netherlands

Sehwarz, J. A.: Kelemen, S. R. Adsorptlon-desorption KInetics of co Prom clean and sulfur covered Ru(001). Surface Sc1. 67,310 (1270) 


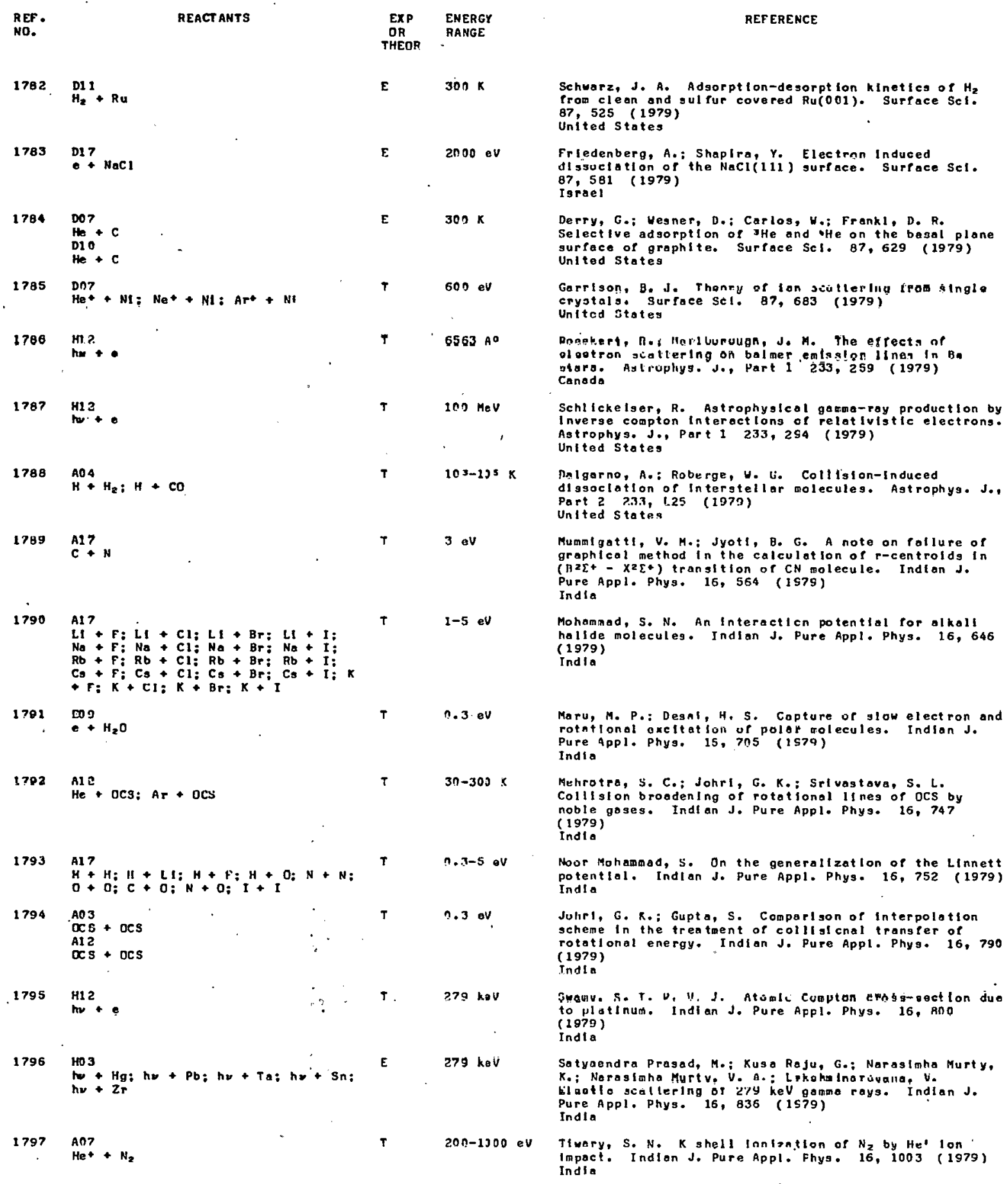




\begin{tabular}{|c|c|c|c|}
\hline $\begin{array}{l}\text { REF. } \\
\text { No. }\end{array}$ & REACT ANTS & $\begin{array}{l}\operatorname{EXP} \\
\text { OR } \\
\text { THEOR }\end{array}$ & $\begin{array}{l}\text { ENERGY } \\
\text { RANGE }\end{array}$ \\
\hline 1.798 & 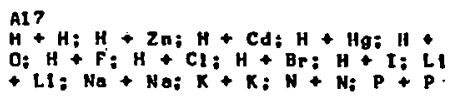 & $T$ & 3 ov \\
\hline 1799 & 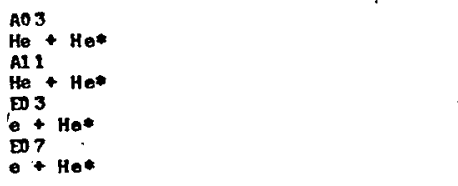 & $\varepsilon$ & $\begin{array}{l}\text { A03: }{ }^{\mathrm{A} 11} \\
300 \mathrm{~K} \\
803: \mathrm{E07} \\
300-3000\end{array}$ \\
\hline 1800. & D15 $\mathrm{Cr}$ & $\mathbf{E}$ & $\begin{array}{l}0.48-1.7 \\
\operatorname{MeV}\end{array}$ \\
\hline 1801 & $\underset{\mathrm{He}}{\mathrm{Con}}+\mathrm{C}$ & E & $1 \mathrm{MeV}$ \\
\hline 1802 & 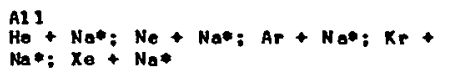 & E & $300 \cdot \mathrm{K}$ \\
\hline 1803 & 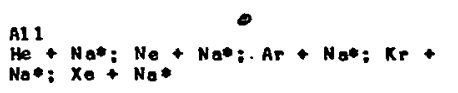 & T & $300 k$ \\
\hline 1804 & ${ }_{\text {Ho }}^{\text {HOS }}+\mathrm{Cl}$ & $T$ & $0.9-3$ Ry \\
\hline 1805 & $\begin{array}{l}104 \\
\text { nhy } \\
\text { A Atom }\end{array}$ & T & Under \\
\hline 1806 & 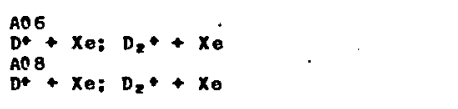 & E. & $1-14 \mathrm{keV}$ \\
\hline 1807 & $\begin{array}{l}\mathrm{AD2} \\
\mathrm{HCl}+\mathrm{Ar} \\
\mathrm{A} 12 \\
\mathrm{CO}+\mathrm{N}=\mathrm{CO}+\mathrm{CO} \mathrm{CO}+\mathrm{CO}_{2} \\
\mathrm{A17}+\mathrm{N} ; \mathrm{C}+\mathrm{C}\end{array}$ & $T$ & $\begin{array}{l}A 02 \\
398-83 B K \\
A 12 \\
100-300 \cdot K \\
A 17 \\
2 \mathrm{CQ}^{-1}\end{array}$ \\
\hline $18 U_{\theta}$ & $\stackrel{\mathrm{A} 1 T}{\mathrm{H}_{\mathrm{g}}}+\mathrm{Xe}_{\mathrm{e}}$ & E & $\cos _{\cos -1} 0000$ \\
\hline 1809 & $\mathrm{Hg}_{\mathrm{Hg}}^{\mathrm{AlO}}+\mathrm{Cd}$ & E & $300 \mathrm{~K}$ \\
\hline 1810 . & $\begin{array}{l}\text { Hh } 2 \\
\text { 3hy }+ \text { atow }\end{array}$ & T & Under \\
\hline 1811 & 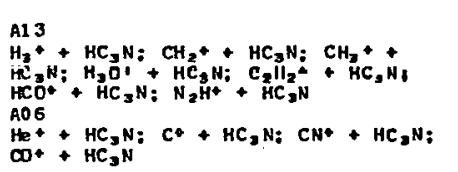 & ET & $30 n k$ \\
\hline
\end{tabular}

Raghuwanshl, S. S.: Shoraa, L. K. Potentlal enorgy function for diatomic wolecules. Indian J. Pure Appl. Phy. 16, 1071 (1979) Indi.

Devos, F.: Boulaer, J.: Delpech, J.-F. Transitlions Devos, F.: Boulner; J.: Dolpech, J,-F. Transitions and nout ral collislons. J. Phys. (Parts) 40,215 (1979). france

Bl get, M.: Maury, F.: Y VaJda, P.: Lucasson, A.: Lucasson, P. At on le displacesents in 1 or temperaturo irradioted ehrosiue eryotals. $J$. Phys. (Parts) 40 , 293 (1979)

293 France

Denlo, A.: Desesquelles, J. Quant um beets study of the Hot bean-earton roll interactlon. J. Phys. (Paris) 40,437 (1979)

France

FIntz, P.: RledInger-Ordonez, I.: Gull llaume, G.: Jundt, F.: Gall aann, A. Lifotlues of shin levels excited by the $\operatorname{soc}(a, p r)^{3} 3 n_{n}$ reaetl on. J. Phys. (Par1s) 40, 511 (1979)

France

Grynberg, G.: Glacoblno, E.: Blraben, F.: Beroft, K. Rolaxatlon of the sodlus atom in the $4 D$ level by collialons with noble gases. II. Theoret leal aspects. J. Phys. (Parts) 40, 533 (1579) France

Lanoureux, M.: Conbet Farnoux, f. Photol onlzati on cross section of the open 3p subshell in atomic chlorlne. S. Phys. (Por!s) 40,545 (1979) France

Grynberg, G. Doppler-free mult1-photon excltation: itght shift and saturation. J. Phys. (Parls) 40,657 (1979)

Bliman, S.: Dousson, S.: Geller, R.: Jaequot, B.: Jacquot, C.: Tsekeris, J. Experimental study of the production of negative deuterons by double charge-exehenge in xenon. J. Phya. (Parts) 40, 841 (1979)

Robert, D.: Bonasy, J. Short range rorce erfects in semiclagsfcal oolecular II ne broadening calculations. J. Phys. (Parls), 40, 923 (1s79) franes

Brag, N.; Bousquet, C. Spectral proflle of the mercury $1849 A^{6}$ absorption 1 ine perturoed by nulle yasos. $\mathrm{Hg}-x_{e}$ interaction potentials dertued (rom teaperature effect. J. Phys. (Parls) 40, 945 (1979) France

Evang, W. D.: Gough, H. Observation of modulat ion In the transfer of orlentation by collis lons of the second kind. J. Phys. (Parls) 40,961 (1979)

Grynberg, G. Three-photon absorpt ion: selectlon rules and line intensitles. J. Phys. (Poris) 40, 965 (1979)

Freeman, C. G.: Marland, P. W.; MeFwan, M. J.

Freeman, C. G.: Marland, P. W.: Me Ewan, M. J. Laboratory. I nuestigation of lon-aolecule reactions
HC N In dense interatellar clouds. Mon. Not. Roy. HC N In dense interatellar clouds. Hon. Not. Roy. Astron. Soc. 287, sal 1 (agry 


\begin{tabular}{|c|c|c|c|c|}
\hline $\begin{array}{l}\text { REF. } \\
\text { No. }\end{array}$ & REACTANTS & $\begin{array}{l}\text { EXP } \\
\text { OR } \\
\text { THEOR }\end{array}$ & $\begin{array}{l}\text { ENERGY } \\
\text { RANGE }\end{array}$ & REFERENCE \\
\hline 1812 & Ee 3 Nent & T & $5030-20000$ & $\begin{array}{l}\text { Giles, K. Coll1slon strengthg for Ne V. Mon. Not. } \\
\text { Roy. Astron. Soc. 187, } 49 \mathrm{p}(1979) \\
\text { United KIngdom }\end{array}$ \\
\hline 1813 & mon $+4-$ & $\begin{array}{l}\top \\
\text { t }\end{array}$ & ${ }_{A 0}^{16300-1250}$ & $\begin{array}{l}\text { Wishart, A U. The bound-tree photo-detachment } \\
\text { cross-section of H-: Mon. Not. Foy. Astron. Soc. 187, } \\
\text { S9p (1979) } \\
\text { United Kingdom }\end{array}$ \\
\hline 1814 & 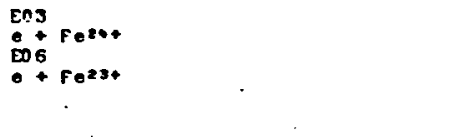 & $T$ & $\begin{array}{l}E 03 \\
1 \times 107-5 \times 107 \\
K \\
E 06 \\
1 \times 10^{2}=-5 \times 10^{2} \\
K\end{array}$ & 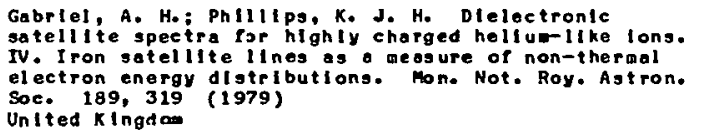 \\
\hline 1815 & 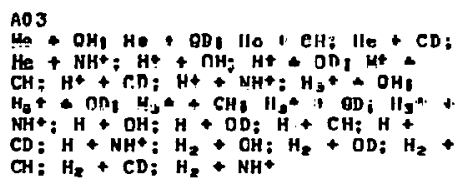 & $T$ & $(0-15)-K$ & 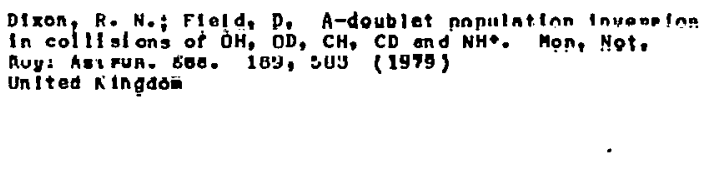 \\
\hline 1816 & $\mathrm{AnZ}_{\mathrm{H}}+\mathrm{CO} ; \mathrm{H}_{2}+\mathrm{CS}: \mathrm{H}_{2}+\mathrm{S1O}$ & $T$ & $20-100 \mathrm{~K}$ & 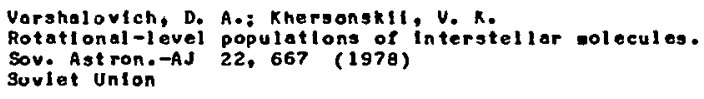 \\
\hline 1817 & Ho & $T$ & Intef & 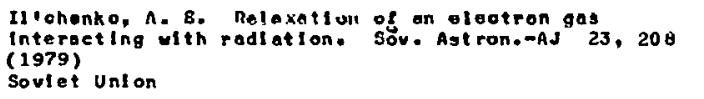 \\
\hline 1818 & $\mathrm{HOZ}_{\mathrm{H}}^{\mathrm{HO}}+\mathrm{CH}_{0}$ & $\varepsilon$ & $8-12=$ & 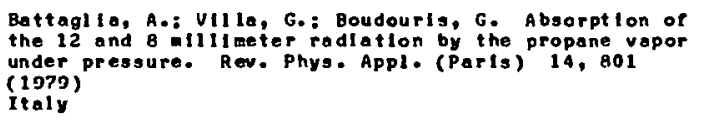 \\
\hline 1019 & 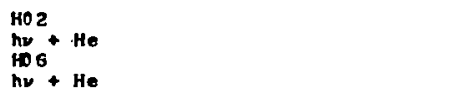 & $\tau$ & $10.6 \mu$ & $\begin{array}{l}\text { Gauthier, J.-C.: Gelndre, J.-P. Laser-Induced } \\
\text { gas-breakdoun montel. Rev. Phys. Appl. (Parls) } 14,007 \\
\text { (1979) } \\
\text { France }\end{array}$ \\
\hline 1820 & $\stackrel{\mathrm{AO} 3}{\mathrm{H}+\mathrm{H}_{2}+}$ & T & $10 \cdot k$ & 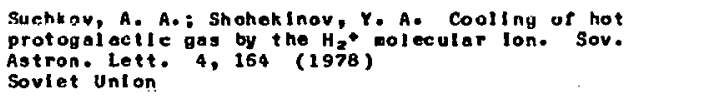 \\
\hline 1821 & $\ln _{n \rightarrow+0}$ & $\tau$ & $0.5-103 \mathrm{eV}$ & 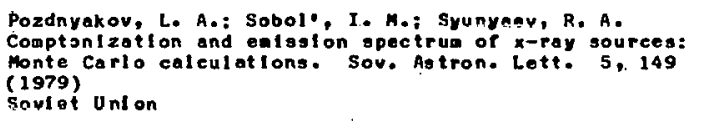 \\
\hline 1822 & $\begin{array}{l}D 04 \\
0+\text { mo } \\
\text { Do } \\
0+\text { mo }\end{array}$ & E & $0.6-1.5 \mathrm{keV}$ & 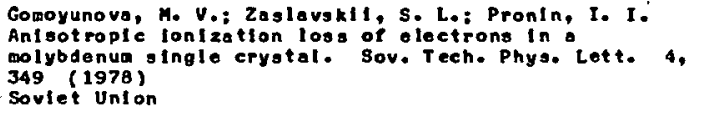 \\
\hline 1023 & ${ }_{-O C+A 1}^{E 05} \cdot A 1=O_{2}$ & E & In $\mathrm{keV}$ & 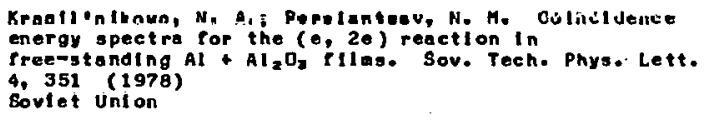 \\
\hline 1824 & $\begin{array}{l}\mathrm{DO3} \\
\mathrm{Ar}+\mathrm{Cu}\end{array}$ & E & $10-35 \mathrm{keV}$ & 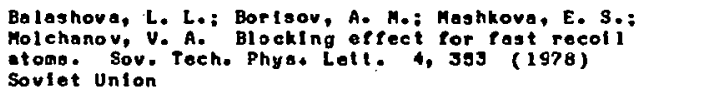 \\
\hline 2823 & $m_{\text {tom }}{ }^{2}+\mathrm{TI}$ & E. & 10.012 & 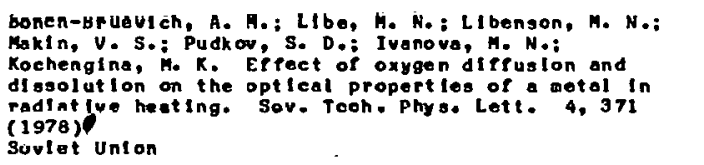 \\
\hline 1826 & $\mathrm{DOS}^{+}+\mathrm{Cu}$ & E & $30 \mathrm{keV}$ & 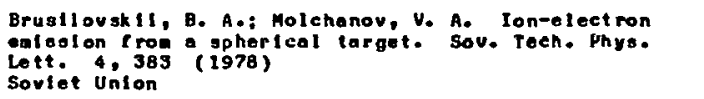 \\
\hline
\end{tabular}




\begin{tabular}{|c|c|c|c|}
\hline $\begin{array}{l}\text { REF. } \\
\text { NO. }\end{array}$ & REACT ANTS & $\begin{array}{l}\text { EXP } \\
\text { OR } \\
\text { THERR }\end{array}$ & $\begin{array}{l}\text { ENERGY } \\
\text { RANGE }\end{array}$ \\
\hline 1827 & $\mathrm{CO}_{\mathrm{CO}}+\mathrm{Co}_{8}: \mathrm{HO}+\mathrm{H}$ & E T & $502 x$ \\
\hline 1828 & $\mathrm{H}^{+B^{*}}+\mathrm{Cu}: \mathrm{D}^{+}+\mathrm{Cu}$ & $\varepsilon$ & $2-40$ kev \\
\hline 1829 & $\mathrm{He}_{\mathrm{He}}^{\mathrm{An}}+\mathrm{He}$ & E & $220 \mathrm{~K}$ \\
\hline & . & . & \\
\hline 1830 & ${ }_{n=0}^{H 06}+\mathrm{Hn}$ & $\boldsymbol{T}$ & so ev \\
\hline 1831 & $\mathrm{~B}^{*} \mathrm{D}+\mathrm{VO}_{2}$ & $\varepsilon$ & $50-100-\mathrm{keV}$ \\
\hline 1832 & $\begin{array}{l}\mathrm{DO}_{4} \\
\mathrm{Ar}^{+}+\mathrm{GaAs}: \mathrm{Ar}^{+}+\operatorname{InP}\end{array}$ & $\varepsilon$ & $3 \mathrm{keV}$ \\
\hline 1833 & $\mathrm{HC}^{+}+\mathrm{Gd} ; \mathrm{H}^{+}+\mathrm{H} ; \mathrm{H}^{+}+\mathrm{Th} ; \mathrm{H}^{+}+\mathrm{U}$ & $\varepsilon$ & $\begin{array}{l}6.8-10.1 \\
\operatorname{mev}\end{array}$ \\
\hline 1834 & 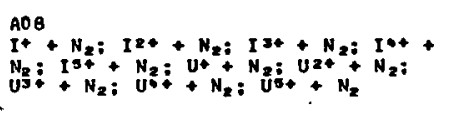 & $T$ & $\begin{array}{l}0.6-11900 \\
\text { MeV }\end{array}$ \\
\hline 1835 & 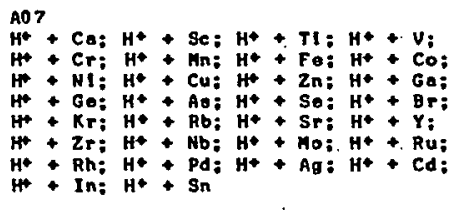 & $E T$ & $0.2-2.0 \mathrm{MeV}$ \\
\hline 1836 & $\underset{\mathrm{He}}{\mathrm{Bos}}$ & E & \\
\hline 1837 & $\begin{array}{l}\mathrm{AOZ} 2 \\
\text { Unde? }\end{array}$ & $T$ & Under \\
\hline 1838 & 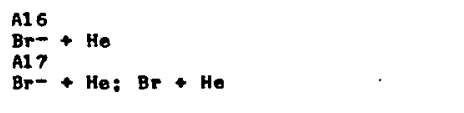 & T. & $6.0-8.4$ eV \\
\hline 1039 & $\mathrm{Fe}^{\infty}+\mathrm{C}$ & E & $0.3-1.6 \mathrm{MeV}$ \\
\hline 1840. & $\begin{array}{l}\text { Bo7 } \\
\text { Undee }\end{array}$ & $i$ & \\
\hline 1842 & $\mathrm{H}_{\mathrm{H}}^{\mathrm{CO}}+\mathrm{He} ; \mathrm{H}++\mathrm{Ne}+\mathrm{O}+\mathrm{He} \mathrm{e}+\mathrm{Ne}$ & $\varepsilon$ & $\begin{array}{l}\text { O. 25-2.5 } \\
\text { GeV/c. }\end{array}$ \\
\hline
\end{tabular}

Devdarialn, A. Z.: Klyueharev, A. N.: Lazarenko, A. V.: Sheverav, V. A. Collisional Ionlzotion of Rydberg states of alkeil atoms. Sov. Tech. Phys. Lett. 4, 408 1978

(laviot unt on

Zhabrev, G. I.: Kurnaev, V. A. Negat ive cosponent in hydrogen and deuterl ue beaus acalted by a copper Sorget. Sov Znitnikou, R. A.: Kartoshkin, V. A.'; Kleaent 'av, G. V. Determinatlon of the cross gections for metastabllity

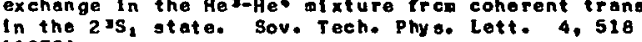
(1978) Amus'ya, M. Y.: Do lmatov, Y. K.: I vonov, V. K.: Sov. Glant-resonance au tolonization in Sovitet Union.

Suvorov, A. V.: Terukov, E. I. Ef reet of ton Suvorov, A. V.: Terukov, E. I. Et rect of lon
bombardment on the met ai--delectrle phase transition
in vanadlum dioxide. Sov. Teeh. Phys. Lett. 4, 537 In vanadi Soviet Unt on

Kuznetsov, A. A.: Protopopov, O. D. El eetron enorgy spectra in the exe ltat ton of GoAs and InP by argon spectra in the exe ltat lon of GoAs and Inp by argon
lons. Sov. Teeh. Phys. Lett. 5 , 40 (1979) Soviet Union

Celler, A.: Kantel f, J.: Luontawa, Mo: Zyllez, J. Detergination of Ion-induced k-she 11 ionizot lon cross
sectlons by compartson with nucl car couloub exeltotion. Nucl. Instrua. methode 163, 221 (1979) Polond

Domltrlev, I. So: Zalkov, V. P.: Tashoev, Y. A. A sealeapirical ue thod for doteribl ni ng the cross sectlons

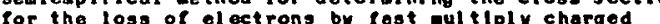
(or the Nic1. I electrons by Soviet Unton

Lopes, J. S.: Jesus, A. P.: Romos, S. C. Fitted Inner-shall proton lonization croso sectlons pros co to Sn for 0.2-2 MeV I neident energy. Nucl. Inatrus. Methods 164, 369 (1979)

Portugal

Drake, G. U. F.: Goldman, S. P.; van VIJngaarden, A. He* Laab-shift weasurement by the quenching-radi at ton onlsotropy wethod. Phys. Rev. A 20, 1299 (1979) Canada

Frenco, $v$. Thenry of diract acattering of neutral ond charged atons. Phyo. Rev. A 20, 1327 (1979) Unitod states

Olson, R. E.: LIu, B. Electron detachment In Br- * He collislons: potentlal enorgles for BrHer ond BrHe. Phyo. Rev. A 20,1344 (1579) United States

Dumont, P. D.: Baudinet-Robinet, Y.: Garnlr, H. P.: Blemont, E.: Grevesge, N. Bear- toll lifetiles 20,1347 (1979)

Rosenberg, L. Sun rule and elossleal linlt tor scattering In a low-prequency loser rield. Phyo. Rev. A 20, 1352 (2979) Unitod stetes

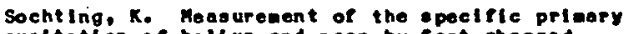
excltation of hell un and neon by fast charged particlos. Phys. Rev. A 20 , 1559 (1979) vest Gerainy 


\begin{tabular}{|c|c|c|c|c|}
\hline $\begin{array}{l}\text { REF. } \\
\text { No. }\end{array}$ & REACTANTS & $\begin{array}{l}\text { EXP } \\
\text { OR } \\
\text { THEOR }\end{array}$ & $\begin{array}{l}\text { ENERGY } \\
\text { RANGE }\end{array}$ & REF ERENCE \\
\hline 1842 & 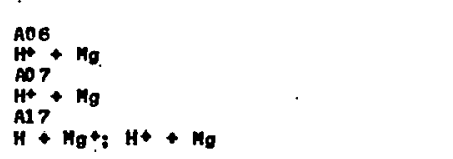 & $\mathbf{T}$ & $0.1-500.00$ & $\begin{array}{l}\text { Dl aon, R. E.: Llu, Be Potentlal onergfes and cross } \\
\text { gectlons por Hght. Physe Reve A } 20,1366 \text { (1979) } \\
\text { United States }\end{array}$ \\
\hline 1843 & $\mathrm{AOP}_{\mathrm{Ho}}+\mathrm{Sr}$ & $\varepsilon$ & 66 nov & 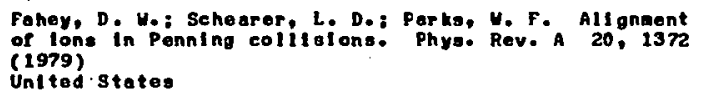 \\
\hline 1844 & $\mathrm{HOB}_{\mathrm{HO}}^{\mathrm{HO}}+\mathrm{MH}_{\mathrm{z}}$ & $\mathbf{E}$ & $1 n \cdot \mu=$ & 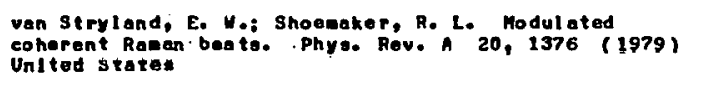 \\
\hline 1845 & 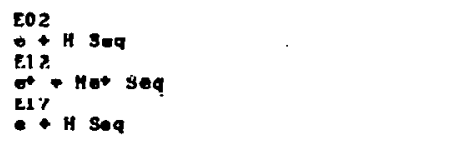 & + & $400-100^{\circ} \cdot 0$ & 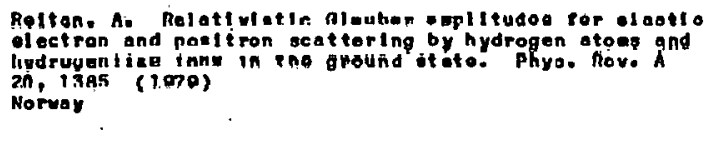 \\
\hline 1846 & $\because 24$ & $\varepsilon$ & 3-1200.0V & 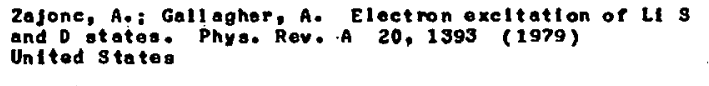 \\
\hline 1847 & $\begin{array}{l}\mathrm{CO} 3+\mathrm{H}_{2}:+\mathrm{D}_{2} \\
\mathrm{EOA}+\mathrm{H}_{21} \circ+\mathrm{D}_{2} \\
\dot{\mathrm{EO}}+\mathrm{H}_{2} \mathrm{O}+\mathrm{D}_{2}\end{array}$ & $\mathrm{~T}$ & $2.4-6.0 \bullet 0$ & 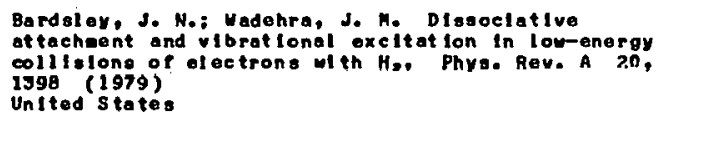 \\
\hline 1848 & $\begin{array}{l}\text { NoG } \\
\text { Ho: }\end{array}$ & T & $10-1030 \mathrm{kOV}$ & 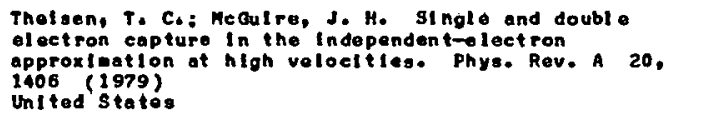 \\
\hline 1849 & 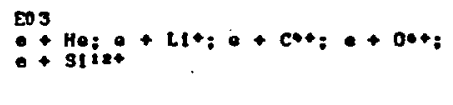 & $\mathrm{T}$ & $65-8000.0 \mathrm{~V}$ & 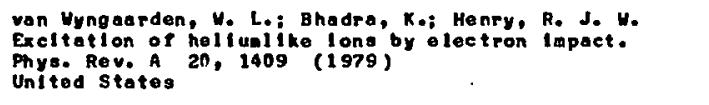 \\
\hline 1850 & Eos & E & $2-500 \mathrm{ov}$ & 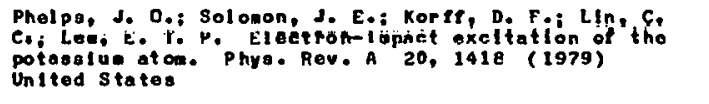 \\
\hline 1851 & 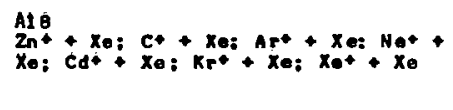 & E T & $2.5-400 \mathrm{keV}$ & 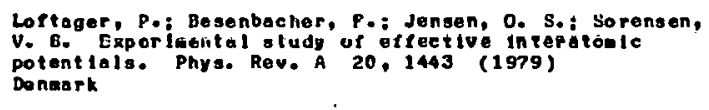 \\
\hline 2852 & $\mathrm{NE}_{\mathrm{N}+}^{\mathrm{A}+}+\mathrm{O}: \mathrm{NeO+}+\mathrm{Ne}$ & $\top$ & Under & 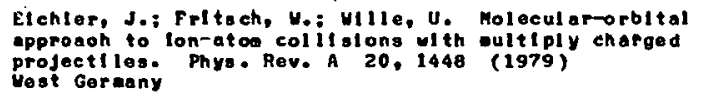 \\
\hline 1853 & 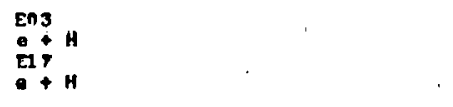 & 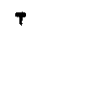 & $100-300.0 \mathrm{~V}$ & 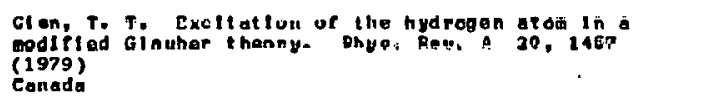 \\
\hline 1854 & 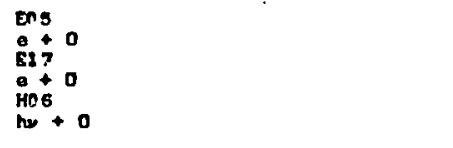 & T & $\begin{array}{l}\text { Ens; } E 17 \\
100-2000-00 \\
\text { nOS } \\
900-400 \text { AO }\end{array}$ & 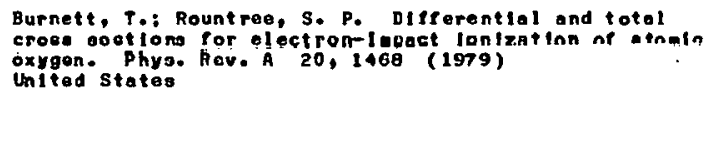 \\
\hline 1855 & $\mathrm{EO2}+\mathrm{HO}$ & $\top$ & Under & 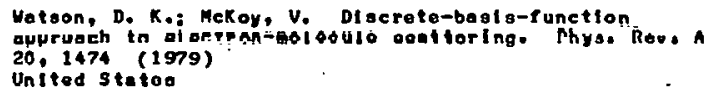 \\
\hline 1856 & $: \mathrm{H}^{+}+\mathrm{F}_{4}: \mathrm{H}^{+}+\mathrm{N1}: \mathrm{H}^{+}+2 \mathrm{Z}$ & E & $50-250 \cdot \mathrm{kaV}$ & 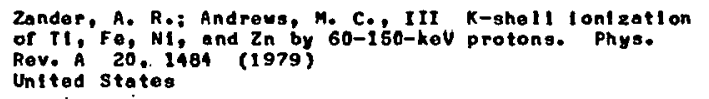 \\
\hline
\end{tabular}




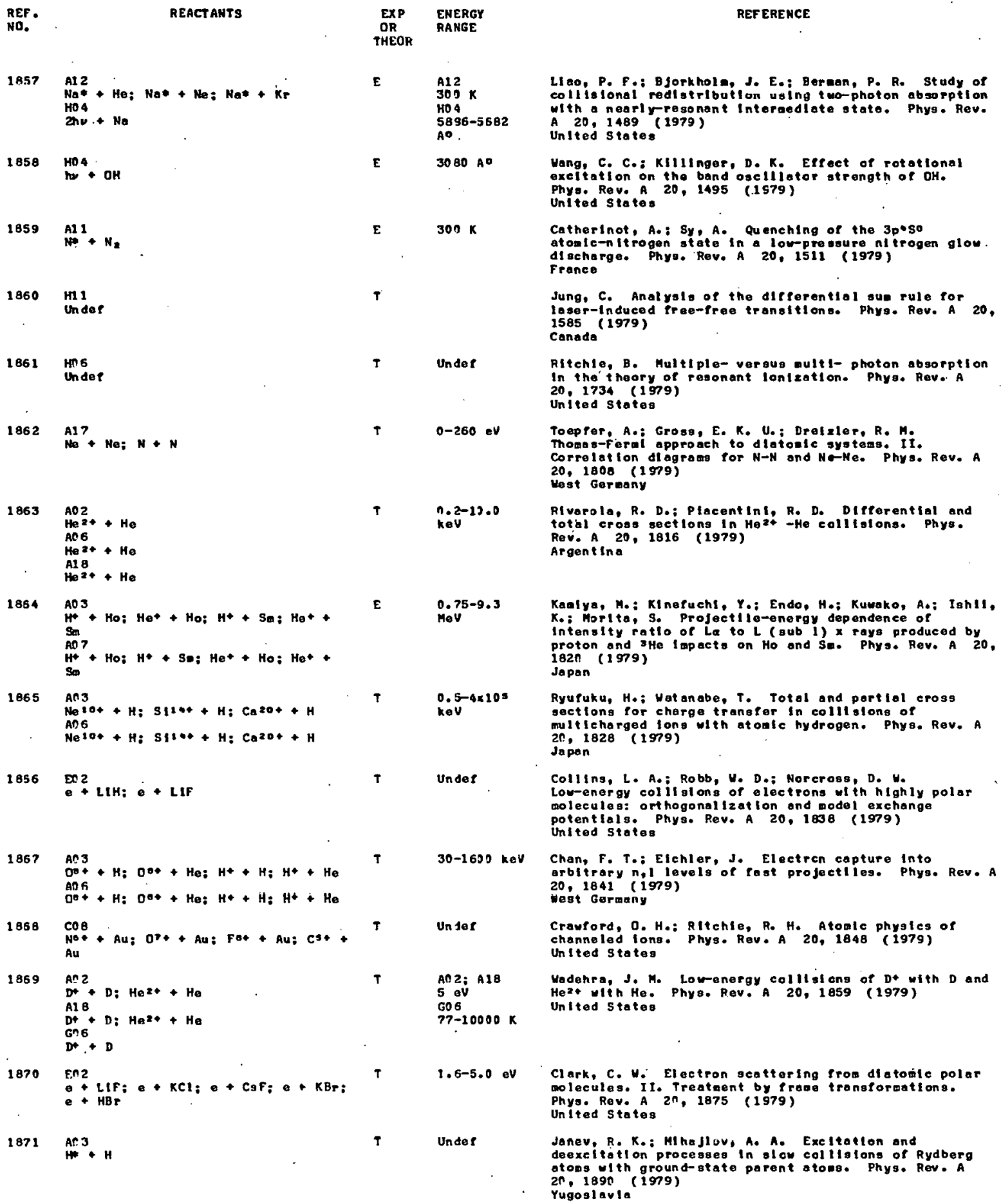

REF.
NO.

REACT ANTS

1857

$\mathrm{Na}_{2}$ + $\mathrm{He} ; \mathrm{Na} *+\mathrm{He} ; \mathrm{Ha} *+\mathrm{Kr}$

$\mathrm{HO} 4$

2hu . + Na

1858 HD4: OH

$1859 \quad \mathrm{Al}_{\mathrm{NO}}^{1}+\mathrm{N}_{2}$

$1860 \quad$ H11

1861 Hns

1862

$\mathrm{Al}^{\mathrm{H}} \mathrm{7}+\mathrm{Ne} ; \mathrm{N}+\mathrm{N}$

1863

$\mathrm{AO}^{\mathrm{A}} \mathrm{2}+\mathrm{He}$

AC6 6

$\mathrm{He} 2+\mathrm{He}$

Al 8
$H^{2+}$

1864

1865

$\mathrm{An3}$

$\mathrm{Ne} 10++\mathrm{H}: \mathrm{SPllt}+\mathrm{H}: \mathrm{Ca}_{20}+\mathrm{H}$

$1856 \quad 5$

EOL LIH;

1867

$\mathrm{O}^{\circ}+\mathrm{H} ; \mathrm{OO}+\mathrm{HeO} \mathrm{H}^{+}+\mathrm{H}_{3} \mathrm{H}^{+}+\mathrm{He}$

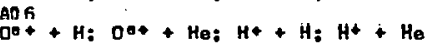

1858

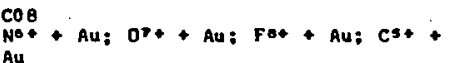

1869

$\mathrm{D}^{\mathrm{An}} 2+\mathrm{D}: \mathrm{He}^{2+}+\mathrm{He}$

Al 8 .

$\mathrm{DL}^{8}+\mathrm{Di} \mathrm{He}^{2+}+\mathrm{He}$

G) 6 . D

1870

En2

Enz $L I F ; e+K C l: e+C g F ; e+K B r ;$

$1871 \stackrel{\text { Af. } 3}{*}$

OR

ENERGY

$0-260$ ev

$n \cdot 2-12=0$
$k \in v^{-0}$

E $\quad \operatorname{Mov}^{\text {T.75-9.3 }}$

$0.5-4 \times 10 s$

kov

Unde?

$0-1600 \mathrm{keV}$

AL 2; A18

5 ev

77-10000 K

$1.6-5.0 \mathrm{eV}$

Under

REFERENCE

Llao, P. F.: B jorkhole, J. E.: Berman, P. R. Study of collistonal redistribullion using wo-photon absorption with a nearlyrisonant Interaediate stato. Phys. Rev. A 20,1489 (1979)

Unlted states

Hang, C. C.: Kllllinger, D. K. Effect of rotattonal exeltation on the band oselliator strength of $\mathrm{OH}$. Phys. Reve A 20, 1495 (.1579) United statos

Catherinot, A.: Sy, A. Quenching of the 3p.so atosic-nitrogen state in a lowpressure nitrogen glou. discharge. Phyo. Rev. A 20, 1511 (1979)

Jung, $C$. Analysls of the dicferentiol sue rule for leser-induced freo-free tronsitions. Phya. Rev. A 20, 1585 (1979)

Canada

Fitchle, B. Multlple- versus nulti- photon absorptlon In the theory or resonant Ionization. Phyo. Rev. A $20.1734(1979)$

United State

Toepfer, A.: Grosa, E. K. U.; Draleler, R. M.

Thomas-reral approach to dia

Correlation dlagrous for $N-N$ ond $N$-Ne. Phys. Reve A 20,1808 (1979)

Hest Gerasny

RIvarola, R. D.: Placentint, R. D. Differential and total cross sectlong in Hezt -He callislons. Phys. Rev. A 20,1816 (1979)

Argentina

Kaalya, M.: Klnofuchl, Y.: Endo, Ho: Kuwako, Ao: Iahil, K.: Horlta, S. Projectlla-energy dependence of

Intensity ratto of La to $L$ ( 806 1) $x$ rays produced by proton and 3 He Impacts on Ho and Se. Phys. Rev. A 20 , $1820(1979)$

Ryufuku, H.: Wat anabe, T. Total and partlal eross gectlong for charge transter in colltstons of multleharged tong with atoulc hydrogen. Phys. Rev. A 2ก, 1828 (1979)

Collins, L. A.; Robb, W. D.: Norerass, D. Wo Low-energy colil al ons of electrons wl th hlghly polar molecules: orthogonalizatlon ond model exchange potentlals. Phys. Rev. A 20, 1608 (1979)

Chan, F. T.: Elchler, J. Electron capture Into

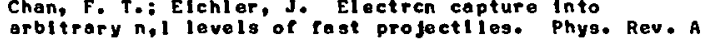
arbltrary n,l levol

20,1841 (1979)

Crawford, O. H.: Rltehle, R. H. Atonle phygice of channoled ions. Phys. Rev. 20,1848 (1979) Untied states

Wodehra, J. M. Low-energy eollliteng of $D^{\text {t }}$ with $D$ and Hezt ulin He. Physe Rev. A 20, 1859 (1979) United stateo

Clark, C. W. El ectron scattering from diatoile polar molecules. II. Treateent by frage trangformationg. molecules. II. Treataent by Prode
Phys. Rey. A 2 , 1875 (1979) Untied States

Janev, R. K.: Ml hajlov, A, A. Excltation and deexcitation processeg In slow collistons of Rydberg otoms ulth ground-state parent atons. Phys. Rev. A $2 n, 1890$ (1979)

Yugoslavia 


\begin{tabular}{|c|c|c|c|c|c|c|c|}
\hline $\begin{array}{l}\text { REF. } \\
\text { NO. }\end{array}$ & & REACT & TANTS & . & $\begin{array}{l}\operatorname{EXP} \\
\text { OR } \\
\text { THEOR }\end{array}$ & $\begin{array}{l}\text { ENERGY } \\
\text { RANGE }\end{array}$ & REF ERENCE \\
\hline 1372 & $\begin{array}{l}\text { Als } \\
H+H e \\
A^{A 1}+\mathrm{He} \\
\mathrm{H}+\mathrm{H}\end{array}$ & H- + He & & & $\begin{array}{l}\top \\
\text { t }\end{array}$ & $\begin{array}{l}\text { A15 } \\
0.3-2.0 \text { keV } \\
\text { A1? } \\
?-5 \text { eV }\end{array}$ & $\begin{array}{l}\text { Herzenberg, A.; Ofha, P. Electron detachment in } \\
\text { collisions of negative ions: } H+\text { He. Phys. Rev. A } \\
\text { 2n, } 1905 \text { (1979) } \\
\text { United States }\end{array}$ \\
\hline 1873 & Hno & & & & $\varepsilon$ & 5930 A & 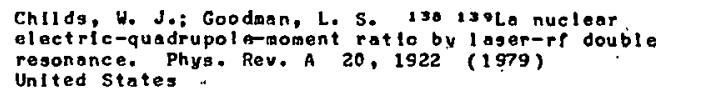 \\
\hline 1874 & $\begin{array}{l}\text { Hns } \\
\text { Under }\end{array}$ & & & & $\mathbf{T}$ & Under & $\begin{array}{l}\text { Nayfeh, M. H. Cod lls ion-1 nduced three-photon } \\
\text { lontzation. Phys. Rev. A } 20,1927 \text { (1979) } \\
\text { Unlted States }\end{array}$ \\
\hline 1875 & ${ }_{h N O}^{H 1}{ }^{2}+O$ & & & & $T$ & $2 n ?-2103$ ed & 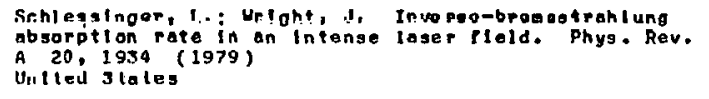 \\
\hline 1876 & $\begin{array}{l}\text { nez } \\
\text { Undap }\end{array}$ & & & & $T$ & Un:ler & 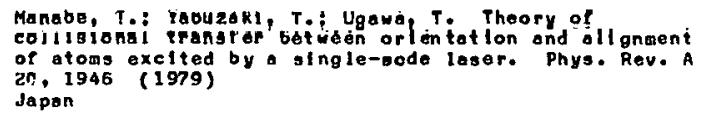 \\
\hline 1877 & $\begin{array}{l}\text { Mr } 1 \\
\text { Unde? }\end{array}$ & & & & $\begin{array}{l}\top \\
T\end{array}$ & Unter & 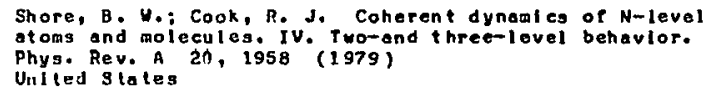 \\
\hline .1878 & $\begin{array}{l}\text { BC? } \\
\text { Under }\end{array}$ & - & & & $T$ & & 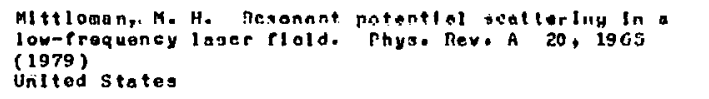 \\
\hline 1879 & $\begin{array}{l}\text { HnB } \\
\text { Under }\end{array}$ & & & . & $\top$ & Undef & $\begin{array}{l}\text { Mossberg, T. W.: Kachru, R.: Hartmann, S. R.; Flugberg, } \\
\text { A. M. Echoes in goseous media: a generalized theory or } \\
\text { rephosing phonomena. Phys. Reve. A } 20,1976 \text { (1979) } \\
\text { Unltod Etatos }\end{array}$ \\
\hline 1890 & $\begin{array}{l}\text { Hng } \\
\text { Undef }\end{array}$ & & & & $T$ & Under & 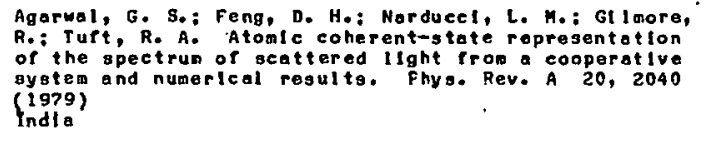 \\
\hline 1981 & $\begin{array}{l}\text { Mna } \\
\text { Under }\end{array}$ & & ) & & 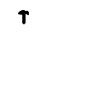 & Under & 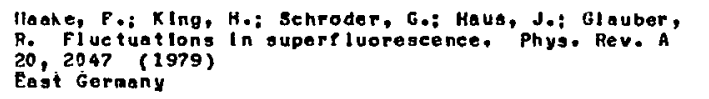 \\
\hline 1882 & $\begin{array}{l}\text { Hod } \\
\text { Undef }\end{array}$ & & & & $T$ & Undef & $\begin{array}{l}\text { Hopf, F. A. Phase-vove Rl uetuat lons In } \\
\text { superfluorescenee. Phys. Rev. A } 20,2064 \text { (1979) } \\
\text { Wegt Germany }\end{array}$ \\
\hline 1883 & $\begin{array}{l}\text { Bn? } \\
\text { Unde? }\end{array}$ & & & & $T$ & & 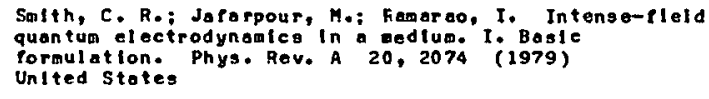 \\
\hline 1884 & $\begin{array}{l}A 07 \\
A r+, ~ B n:\end{array}$ & : NI $+3 \mathrm{H}$ & $\mathrm{NI}+\mathrm{Pb}$ & & $\mathbf{E}$ & $45-139 \mathrm{MaV}$ & 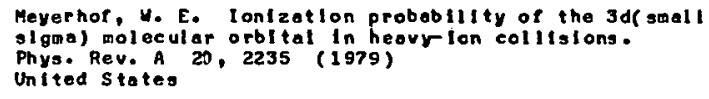 \\
\hline 1885 & $\begin{array}{l}\text { H06 } \\
\text { 3hy }-118\end{array}$ & & . & & $T$ & so ${ }^{50-5036}$ & 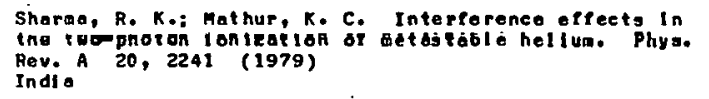 \\
\hline 18AK & $\mathrm{mr}+\mathrm{He}$ & $\mathrm{Kr}+\mathrm{Ne} ;$ & $K r+A r ;$ & $K_{r}+K_{e}$ & $E$ & 38071200 & 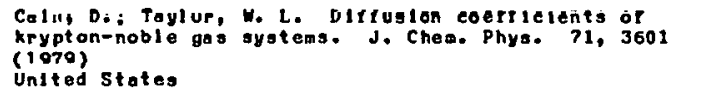 \\
\hline 1887 & $\begin{array}{l}\text { A07 } \\
\text { Ar* }+\mathrm{Kr} * \\
\text { Al4 } \\
\text { Ar* }+\mathrm{Kr} *\end{array}$ & & & & $\varepsilon$ & $0.11-10.00$ & 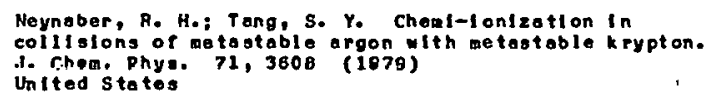 \\
\hline 1988 & 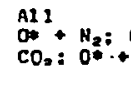 & $+\mathrm{OH}_{4}^{+}=\mathrm{O}_{2} ;$ & 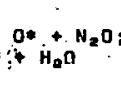 & ; $0 *+$ & E & $295 \mathrm{~K}$ & 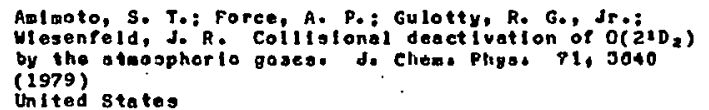 \\
\hline
\end{tabular}




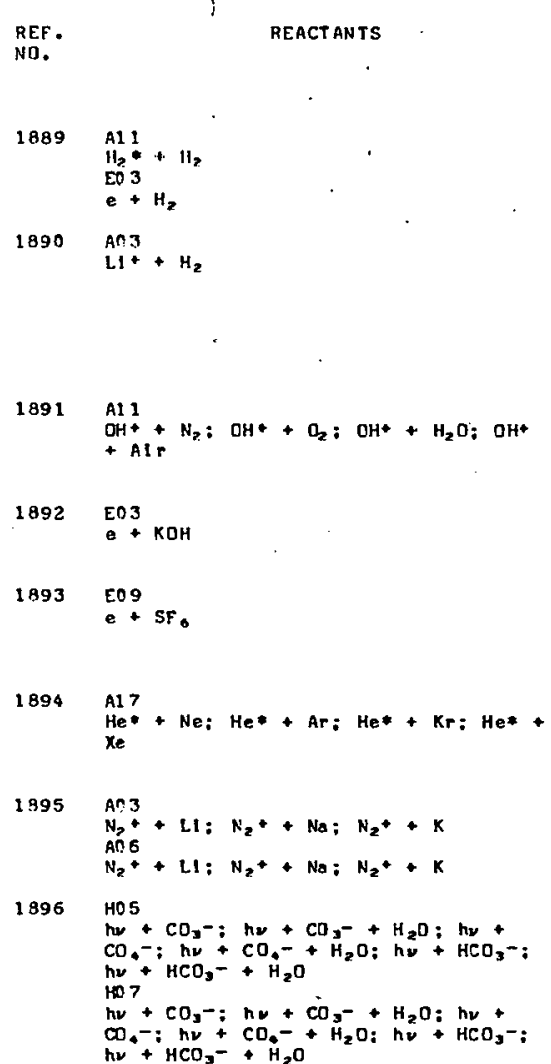
ENERGY
RANGE

REFERENCE

OP

$\operatorname{lng} 5$

Enj $15-300$ eV

E

$0.5 \mathrm{ev}$

E

$300 \mathrm{~K}$

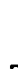

E $\quad 0-1.0 \mathrm{eV}$

$40 \mathrm{ev}$

Under

$50-1030 \mathrm{eV}$

$5300-3500$

E. AO

(10)

(1)

$300 \mathrm{~K}$

$1.9 \mathrm{eV}$

$20-1030 \cdot \mathrm{ev}$

$\sin k$.

0.1 ev

E $\quad 3-8$ ev

T $\quad 0-100 \mathrm{eV}$ Chem. Phys. 71, 3786 (1979)
Day, R. L.: Anderson, R. J.: Shappton. F. A. Electron excltation of the singlet-g atotes of $H_{2}$. $\mathrm{J}$. Chem. Phys. 71,3683 (1970)

Faubel, M.: Toenntes, J. P. Tlme-or-rllght measurements of the rotational excltation of para- and ortho- $H_{2}$ by colllstons with Ll+ lons. I. Angular dependent transition probablittles for the $j=0,2,2$. 0,2 4, and 1 . 3 transtitions at $\mathrm{E}(\mathrm{sub} \mathrm{com})=0.6 \mathrm{eV}$. J. Chem. Phys. 71, 3770 (1979)

Selzer, P. M.: Hang, C. C. Cuenchlng rates and rluoreseence efflciency in the $A=\Sigma^{+}$state of $O H$. $J$. Vuskovlc, Le: Trajmar, S. Electron Impact gtudy of
potassium hydroxlde. J. Chem. Fhys, 71, 3887 (1979) potasilum hydrox

Chen, C. L.: Chantry, P. J.' Photon-enhanced dssoclative electron attachment in $S_{b}$ and its isotopic selectivity. J. Chem. Phys. 71, 3857 (1979) Isotoplc selecti
Unlted States

siska, P. E. One-electron model potentlal calculations of van der Haals forces. I. He*( $\left.21 \leq, 2^{3 S}\right)+\mathrm{Ne}, \mathrm{Ar}, \mathrm{Kr}$, of von der waals forces. 1. He*( 215,2
Xe. J. Chem. Phys. 71,3942 (1979) United States

Barrett, J. L.: Leventhal, J. J. Electron trangfer and, excltation in iow energy $\mathrm{N}_{2}$-olikali ateo collifolons. J. Chem. Phys. 71, 4015 (1979) United States

Smlth, G. P.: Lee, L. C.: Moseley, J. T. Photodissociat ion and photodetachaent of moleculor negative long. VII. Ions formed In $\mathrm{CO}_{2} / \mathrm{O}_{2} / \mathrm{H}_{2} \mathrm{O}$ mixtures, Unlted States

Zlmerman, I. H.: Buar, M. ; George, T. F. F $+\mathrm{H}_{2}$ collislons on two electronle potentlal energy surfaces: quantum-mechanical study of the collinear reaction. J. Chem. Phys. 71, 4132 (1979) Untted states

Preuninger, F. N.: Bllotta, R, M.; Farrar, J. M. Crossed-beam study of the reactlon $\mathrm{H}_{2}+(\mathrm{CO}, \mathrm{H}) \mathrm{HCO}^{+}$at $1.89 \mathrm{ev}$. J. Chem. Phys. 71, 4166 (1979) United States

Genas, P. S. Electron-lmpact excltation cross gect 1 ons for NIII. J. Chem. Phys. 71, 4169 (1979)

Venzl. G.: Figcher, S. F. On the dynamics of exothermic trlat omfe exchange reactlong: semlelásslcal model for final state VRT distributions. J. Cheme

Phys. 71, 4175 (1979)
West Germany

Schinke, R.: MeGulre, P. Rotatlonal ralnbow oselllat lons in $\mathrm{He}-\mathrm{Na}_{2}$ colll sions: coeparison between coupled states and Infintte order sudden approxtations. J. Chem. Phys. 71, 4201 (1979) West Garmany

Kusunokl, I.: OttInger, C. Chemlluminescent lon-molecule reactions: rotational-vibrationol population distributions nt $C H^{+}\left(A^{2} r\right)$ and $\left.C D^{*}\left(A^{2}\right)^{2}\right)$ fron $\mathrm{C}^{+}+\mathrm{H}_{2}$ and $\mathrm{C}^{+}+\mathrm{D}_{2}$ collils lons. J. Chem. Phys. ?1, 4227 (1979) Japan

Lynch, M. G.: Dll1, De; Slegel, J.: Dehmer, J. L. Elastic electron scattering by $\mathrm{CC}_{2}$, OCS, and $\mathrm{CS}_{2}$ from 0 to 100 ev. J. Chem. Phys. 71, 4249 (1979) 


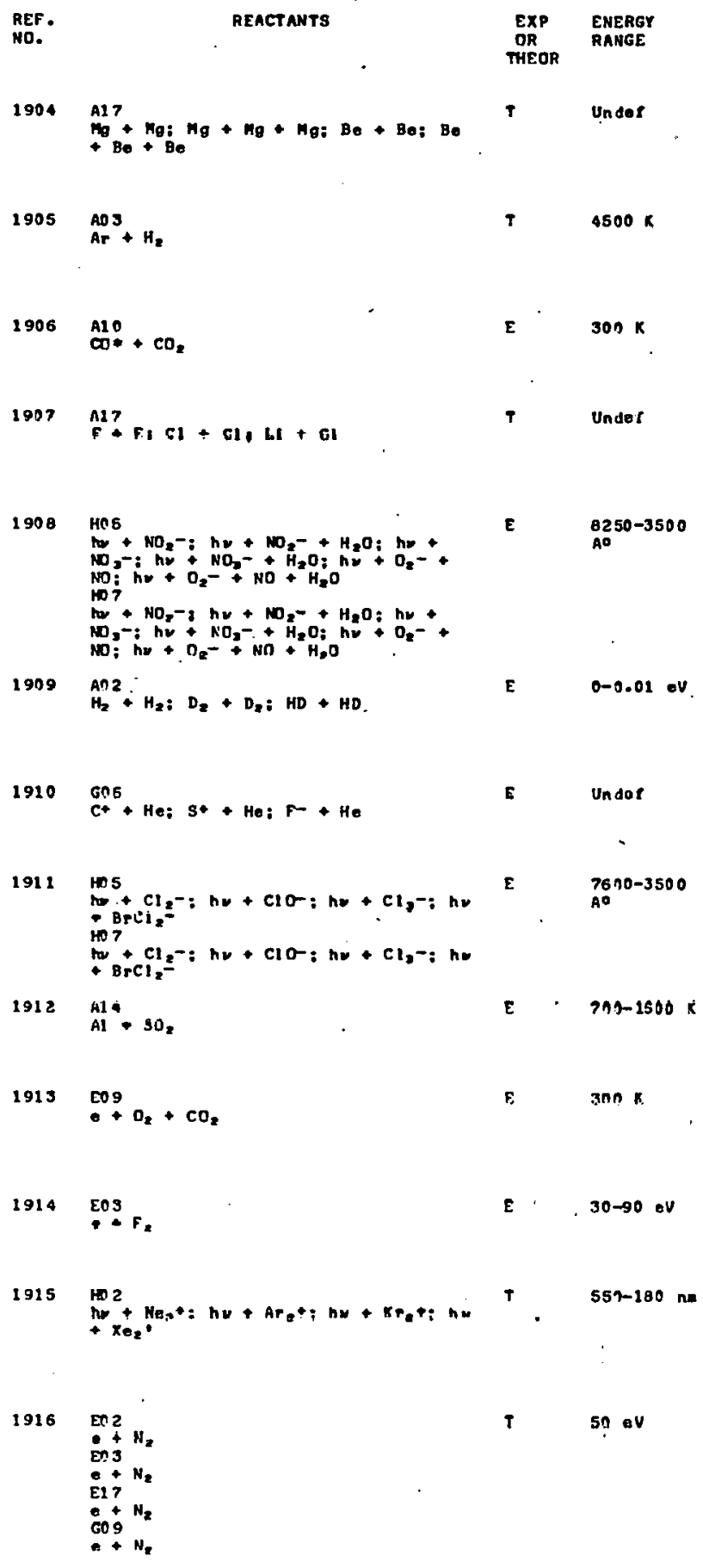

Daudey, J. P.: Novaro, O.: Kolos, H.: Berrondo, M. Convergence of aultibody oxpansions for alkall ine earth metals. Cont rast between magnes lua and beryll 1 um el usters. J. Chea. Phys. 71, 429? (1979) Mexico

Duef, J. Y.: Blals, N. C.: Truhlar, D. G. Monta Carlo trajectory atudy of Ar + $\mathrm{H}_{z}$ collilafons: theraally averaged vibrational transition rates at $4500 \mathrm{~K}$. J. Chea. Phys. 71,4304 (1979)

Coledonla, G. E.: Green, B. D.: Murphy, R, E. A study of the vibrational lovel dependent quenehing of
$\mathrm{CO}(\mathrm{v}=1-16)$ by $\mathrm{CO}_{2}$. J. Chome fhys. $71,43 \in 9$ (1979)

cnrisziansen, P. A.: Lèe, Y. S.: Pltzer, K. S. Improvod ab iniet? aprective curo vatentials lar molecular calculations. J, Çhert. Phrs. 71. 4445 (1979)

Stith, G. P.: Lee, L. C.: Cosby, P. C.

Photodissociation and photodetachent of polecular negative lons. VIII. Nitrogen oxldes and hydrotes. negat lve Ions. VIII. NItrogen ox Ides and hydrates, United States

Johnson, D. L.: Grace, R. S.: skofronlck, J. G. The total scattering eross sections tor $\mathrm{H}_{2}+\mathrm{H}_{2}, \mathrm{D}_{2}+\mathrm{D}_{2}$ total scattering e ross sections for $\mathrm{H}_{2}+\mathrm{H}_{2}$, $\mathrm{D}_{2}{ }^{+} \mathrm{D}_{Z}$, mev. J. Chem. Phys. 71,4554 (1979)

united states

Dotan. I.: Petigenfeld, P. C.: Albritton, D. L. Mobllitles of $C^{*}, S^{*}$, and $F$ ions in helium. J. Chea. Phys. 71,4762 ' (1979)

United states

Lee, L. G.: Solth, G. P.: Moseley, J. T.: Cosby, P. C.: Guest, J. A, Erratum Photndisannintinn and

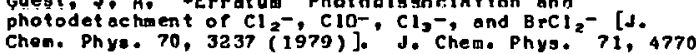
(1979)

Unted states

Fontijn, A,: Felder. Ho HTfFR kInetlcs aturlies ne tho

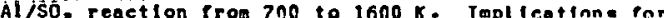
$D(A 1-5)$. J. Chem. Phys. 71, 4854 (1979)

Unlted Stotes

Kokaku, Y.: Hatano, Y.: EhIaomorl, II.: Fessendell, R. $W$. Mechanlem of theraal electron et tachment in $\mathrm{O}_{3}-\mathrm{C}_{2} \mathrm{H}_{1}$ $\mathrm{O}_{2}-\mathrm{CO}_{2}$, and $\mathrm{n}_{2}$-neopentane mixtures. J. Chem. Phys. 71,4883 (1979)

Nishlauro, Ho: Carturlght, D. C.; Trajaar, S. Electron

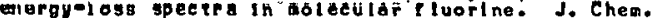
Phya. P1, Knsa (9970) United states

Mlehels, H. H.: Hobbo, R. H.: Uright, L. A. Electronic etruature of tho notlo yia Jimer lulng. II

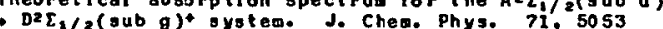
(1979)

Hilliod nlateo

Onda, K.: Truhlen, D, G. Eloetrenzel ooulo ooat tort hy at lintofíedlate energy. Centrifugol-dominent channel decoupling and the INDOX polarlzed SCF model applled to $\mathrm{N}_{2}$ at 50 ev. J. Chen. Phys. 71, 5097 (1979) 
REF.
No.

REACTANTS

1917

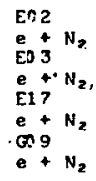

1918 A14

$\mathrm{ClO}^{\mathrm{A}}+\mathrm{HO}_{2}$

1919

An 3
$\mathrm{Ar}+\mathrm{N}_{2}$

1920

EN 1
EI
EI

1921

$\mathrm{El}^{\mathrm{l}}+\mathrm{CO}_{2} \mathrm{O} \mathrm{e}+\mathrm{CH}_{4} \mathrm{O} \mathrm{e}+\mathrm{CF}$

1922

$\underset{\mathrm{e}}{\mathrm{E}} \mathrm{e}+\mathrm{N}_{2} ; \mathrm{e}+\mathrm{CO}_{2} ; \mathrm{e}+\mathrm{Kr} ; \mathrm{e}+\mathrm{CH}$.

1923

$\mathrm{AlO}^{\mathrm{Al}}+\mathrm{CO}_{2}$

1924

$\stackrel{\mathrm{Al}}{\mathrm{H}} \mathrm{\textrm {O }}+\mathrm{H}_{2} \mathrm{O}: \mathrm{H}_{2} \mathrm{O}+\mathrm{Ne}$

1925

$\mathrm{H}_{2}+\mathrm{H}_{2}$

$1926 \quad \stackrel{\text { Al } 2}{N I}+\mathrm{NI}$

$1927 \quad \mathrm{AO3}$

1928

${ }^{A 06}+\mathrm{He} ; \mathrm{H} *+\mathrm{Ne} ; \mathrm{H} * \mathrm{Ar} ; \mathrm{H} *+\mathrm{Kr}$

$\mathrm{H}+\mathrm{He} \mathrm{H}+\mathrm{He} \mathrm{H}+\mathrm{Ar} ; \mathrm{H}+\mathrm{Kr}$

$1929 \quad \begin{array}{ll}\mathrm{HO} \\ \mathrm{hV}\end{array}$

1930

$\mathrm{An}:$ + $\mathrm{He}$

$\mathrm{Al} 1$ ? $\mathrm{He}$

1931

AO 3

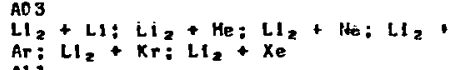

Ali

$\mathrm{LI}_{2}+\mathrm{LI}_{2} \mathrm{LL}_{2}+\mathrm{Ha}_{2} \mathrm{LL}_{2}+\mathrm{He}: \mathrm{LI}_{3}+$ Ar: $\mathrm{LI}_{2}+\mathrm{Kr}: \mathrm{LI}_{2}+\mathrm{Xe}^{4}$
ENERGY

RANGE

$10-50 \mathrm{eV}$

$10-50$

Onda, K.: Truhlar, D. G. State-ta-gtate cross geetlons for electron Impact on $N_{2}$. Clase coupling and

polarlzed Born calculations for rotatlonal and vibrational excltatlon and pure elagtic scattering at
nanresonant energleg. J. Chea. Fhys. 71,5107 (1979)

235-393 K

Stlmpfle, R. M.: Perry, R. A.: Heward, C. J. Temperature dependence of the reectlon of $\mathrm{ClO}$ and $\mathrm{HO}_{2}$ radicals. J. Chen. Phys. 71, 5183 (1979)

unter

Alexander, M. H. Tensorlal ractorization and rotationaliy Inelastic collisicrs. J. Chem. Phys. 71, 5212 (1979)

Fink, M.: Moore, P. G.; Gregory, D. Preelse

E. 30-50 keV

determination of diferential electron scattering eross sectiong. I. The apporatus and the $N_{2}$ results. J. Chem. Phys. 71,5227 (1979) United states

E: $\quad 50 \mathrm{keV}$

Flnk, M.: Schmledekamp, C. H.: : Gregory, D. Preelse determination of differential electron seattering cross gectIons. II. $\mathrm{CH}_{4}, \mathrm{CO}_{2}, \mathrm{CF} .$. J. Chem, Phys, 71,5238 (1979)

United states

Fink, M.: Sehmledekamp, C. W. Preclse determinatlon of dieferentlal differential electron scotterling crogs sectlons. II I. Exchange Phys. 71,5243
Untited States

Schatz, G. C.: Aultoney, T. Colltsional energy

trang fer in pol yatomle ooleculas:

onhorian lelty effects in $\mathrm{Kr}+\mathrm{CO}_{\mathrm{a}} \mathrm{J}$. Cheq. Physo 5257 (1979)

United states

van Heaert, Mo; van der Avolrd, A. Ab inltlo calculat lon of the firgt order interaction energy in excited dlwers. The $\mathrm{H}_{2} \mathrm{O}-\mathrm{H}_{2} \mathrm{O}$ and $\mathrm{H}_{2} \mathrm{O}-\mathrm{Ne}$ dimers. $\mathrm{J}$. Chem. Phys. 7i, 5310 (1979)

The Netherlands

Ree, F. H.: Bender, C. F. Repulsive Intermolecular potential between two $\mathrm{H}_{z}$ molecules. J. Chem. Phys. 71,5362 (1979)

United states

E $\quad 12.6-31.5$

VIncent, P.: Greenberg, J. S, Colltis lon broadening of continuum radiation Prom NI + Ni colliglong. J. Phys. B. $12, L 641$ (1979)

Bited states

$0.02-15 \mathrm{MeV}$

Fritsch, W.: Hllle, U. On the use of Nikitin's

two-state wodel in the description of vacancy-sharing orocesses. J. Phys. B 12, Le45 (1979)

germany

E $\quad 4-25 \cdot k \mathrm{eV}$

HIII, J.: Geddes, J.; Gltbody, H. B. H Poraation In electron capture by $4-25 \mathrm{keV}$ netastable hydrogen atoms In the Inert gases. J. Phys. B 12, L653 (1979) United KIngdom

Unde?

Ishart, A. W. The bound-free photodetachment cross sectlon of H-. J. Phys. B 12, 3511 (1979)

Unted Kingdom

Webster, A. J.; Shav, M. J. Excttotlon transfer in the 2p level s of Ne due to collistong ulth ground-state $\mathrm{He}$ etomp. A. Phys. B 12. 3521 (1975) United KIngdom

Under Ot tinger, C.: Schroder, M. Fote constants for

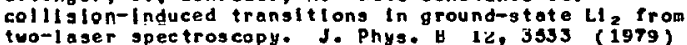
Wast Germany 


\begin{tabular}{|c|c|c|c|c|}
\hline $\begin{array}{l}\text { REF. } \\
\text { NO. }\end{array}$ & REACT AN TS & $\begin{array}{l}\text { EXP } \\
\text { OR } \\
\text { THEOR }\end{array}$ & $\begin{array}{l}\text { ENERGY } \\
\text { RANGE }\end{array}$ & REFERENCE \\
\hline 1932 & $\begin{array}{l}\mathrm{HOS} \\
\mathrm{hy}\end{array}$ & $T$ & $800-5800$ AD & 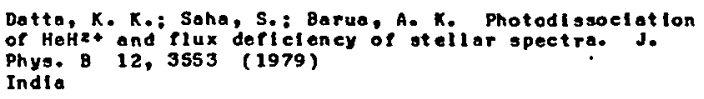 \\
\hline 1933 & $\mathrm{AO}^{+}+\mathrm{H}$ & $T$ & $50-200-k=V$ & 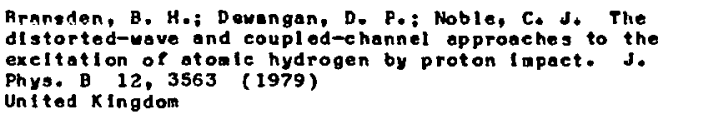 \\
\hline 1934 & $\mathrm{C}^{+}+\mathrm{C}^{+} \mathrm{N}^{+}+\mathrm{N}: \mathrm{O}^{+}+\mathrm{O}$ & $\varepsilon$ & $20-1530$ keV & 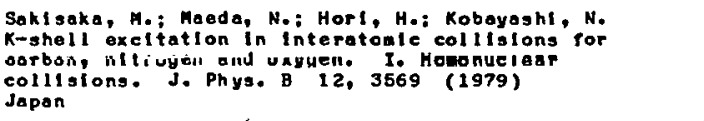 \\
\hline 1935 & 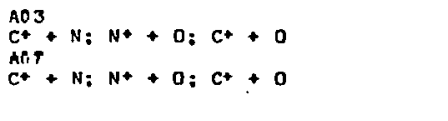 & $\varepsilon$ & $\begin{array}{l}0.4-2.5 \mathrm{MeV} \\
\vdots\end{array}$ & 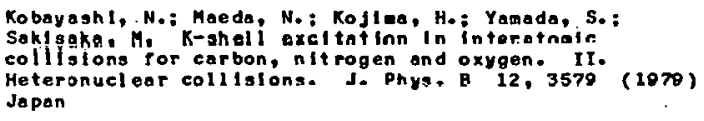 \\
\hline 1936 & $\begin{array}{l}\mathrm{COS} \\
\mathrm{He}+\mathrm{C}: \mathrm{He}^{+}+\mathrm{Al}^{+}: \mathrm{He}^{+}+\mathrm{NI}: \mathrm{He}^{+}+ \\
\mathrm{Ag}^{+} \mathrm{He}^{+}+\mathrm{Au}\end{array}$ & $\varepsilon$ & 6-24 $\mathrm{HeV}$ & $\begin{array}{l}\text { Gladleux, A.: Chat eau-Thierry, A.: Delaunay, B. } \\
\text { Experlmental charge Practions, in hellumbooms emerging } \\
\text { from solldy. J. Phys. B 12, 3591 (1979) } \\
\text { France }\end{array}$ \\
\hline 1937 & AI5 & $T$ & $10-10000 \mathrm{~K}$ & $\begin{array}{l}\text { Shizgal, B. A couparison of Mu-H and H-H el ectron spin } \\
\text { exchango eross seetions. J. Phys. B 12, 3611 (1979) } \\
\text { Conada }\end{array}$ \\
\hline 1938 & $\begin{array}{l}\mathrm{Go} 2 \\
\mathrm{Ar}+\mathrm{N}_{2}: \mathrm{Ar}+\mathrm{CO}_{2}: \mathrm{He}+\mathrm{H}_{2} ; \mathrm{He}+ \\
\mathrm{N}_{2} \mathrm{O} ; \mathrm{Kr}+\mathrm{N}_{2} \mathrm{O} ; \mathrm{He}+\mathrm{NH}_{3}\end{array}$ & E & $300-530 \mathrm{~K}$ & 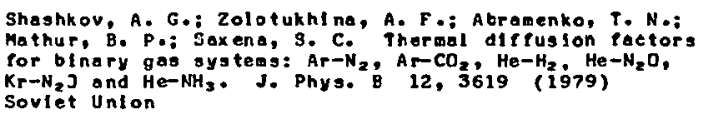 \\
\hline 1939 & $\begin{array}{l}\text { Hes } \\
\text { 4he + Cs }\end{array}$ & $T$ & Under & 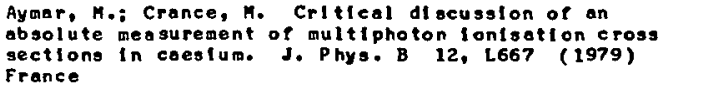 \\
\hline 1940 & $\mathrm{HOS}_{\mathrm{HeO}}^{\mathrm{H}}+\mathrm{Cd}$ & $T$ & Under & 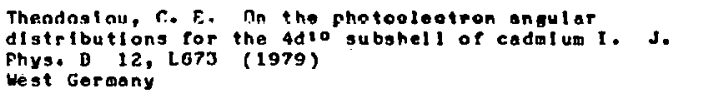 \\
\hline 1941 & $\begin{array}{l}\operatorname{Hn} 6 \\
\text { hy }\end{array}+x_{e}$ & $E$ & $90-50 \mathrm{~A}^{\circ}$ & 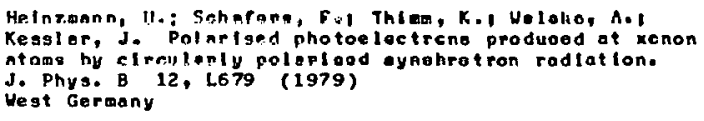 \\
\hline 1942 & $\begin{array}{l}\mathrm{AES} 3 \\
\mathrm{H}^{+}+\mathrm{H} \\
\mathrm{AOS} \\
\mathrm{H}^{+}+\mathrm{H}\end{array}$ & $\mathbf{T}$ & $\begin{array}{l}48-135 \mathrm{kav} \\
\text {. }\end{array}$ & 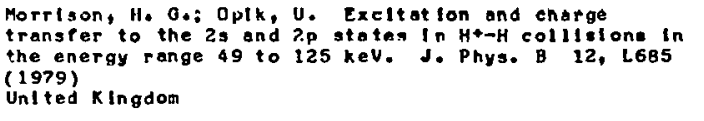 \\
\hline 1943 & 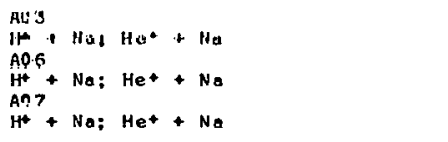 & $\bar{E} \tilde{T}$ & $5-30 \mathrm{keV}$ & 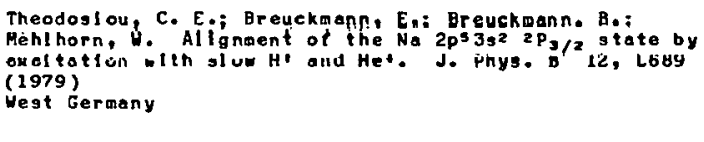 \\
\hline 1944 & $\stackrel{\text { ACs }}{H^{+}+11}$ & $T$ & $\begin{array}{l}100-10000 \\
\text { mev }\end{array}$ & 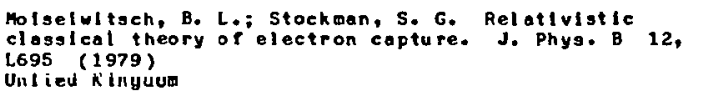 \\
\hline 1945 & $\begin{array}{l}\text { En } 2 \\
0111 \\
E_{1} 12 \\
e^{+}+4\end{array}$ & $T$ & IInder & 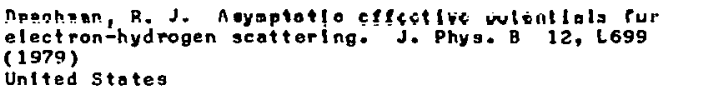 \\
\hline 1946 & $\begin{array}{l}\text { Hns } \\
\text { 4hy }+\mathrm{Cs}\end{array}$ & $T$ & "ndas & $\begin{array}{l}\text { Crance, N. Interpretatlon of shurt-pulse resonant } \\
\text { lontsation in Cs } 1 . \text { J. Phys. B } 12,3655 \text { (1979) } \\
\text { France }\end{array}$ \\
\hline 1947 & $\begin{array}{l}\text { HCS } \\
\text { 3hy }+ \text { Cs* }\end{array}$ & $T$ & Undef & $\begin{array}{l}\text { Crance, M.; Aymar, Mo. Three-photon } 16 \text { p resonant } \\
\text { tonisotion of cs } 1 \text {. J. Phys. } 12,3665 \text { (19ry) } \\
\text { Franee }\end{array}$ \\
\hline
\end{tabular}


REF.
NO.

REACTANTS

1948

Al1 ${ }^{1}+\mathrm{He}: \mathrm{X} *+\mathrm{Ar} ; \mathrm{K} *+\mathrm{Ne}: K *+\mathrm{X}_{t}$

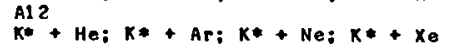

1949

$\mathrm{An} 3$
$K$
$Z$

$2 n+$ Ne; $2 n++A r$

1950

AC 3

$\mathrm{LI}+\mathrm{He}: \mathrm{LI}+\mathrm{Ne}: \mathrm{LI}+\mathrm{Ar} ; \mathrm{Be}^{+}+$

$\mathrm{He}: \mathrm{Be}^{+}+\mathrm{Ne}: \mathrm{Bo}^{+}+\mathrm{Ar} ; \mathrm{Na}+\mathrm{He}$ : $\mathrm{No}$

$+\mathrm{Ne}: \mathrm{No}+\mathrm{Ar:}^{\mathrm{Ng}} \mathrm{Ng}^{+}+\mathrm{He} \mathrm{Mg}^{+}+\mathrm{NeO}$

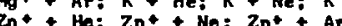

1951

$\mathrm{Ha}^{+3}+\mathrm{H}$

${ }_{A 06}+H$

1952

Ans 3

$\mathrm{No*}+\mathrm{He} ; \mathrm{Na*}+\mathrm{Ne}$ : $\mathrm{Na*}+\mathrm{Ar}$

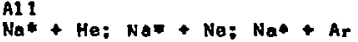

1953

AC 6

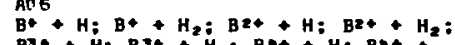

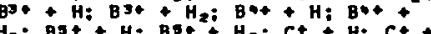

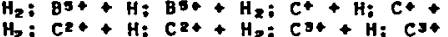

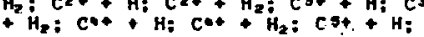

$\mathrm{Cs}_{3}+\mathrm{H}_{2} \mathrm{O} \mathrm{Cos}_{4}+\mathrm{H}_{\mathrm{i}} \mathrm{Cos}^{+}+\mathrm{H}_{2}$

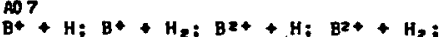

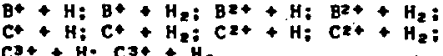

1954

E01

$\rightarrow H$

$+5$

1955

$\stackrel{\mathrm{CO} 2}{+}+\mathrm{HO}$

1956

ED 3

$\because+\mathrm{H}_{2} \mathrm{O}-+\mathrm{D}_{2}$

EO $+\mathrm{H}_{2} \mathrm{O} \mathrm{C}+\mathrm{D}_{2}$

$1957 \quad$ HO6

Unde?

$1958 \quad$ HOS

1959 An i

Under

1960 .

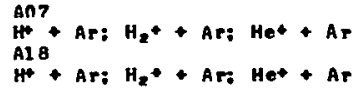

$1961 \stackrel{\mathrm{EOS}}{\mathrm{C}+\mathrm{He*}}$ $\begin{array}{cl}\text { EXP } & \text { ENERG } \\ \text { OR } & \text { RANGE } \\ \text { THEDR } & \end{array}$

REF ERENCE்

$\varepsilon \quad$ Under

$0.7-500 \mathrm{keV}$

$\varepsilon$

$0.7-500 \mathrm{keV}$

. Ande

, An dersen, T.: 01 sen, J. 0 .

Collsion-induced al Ignment In qus si-one-electron

polarisots tewatl cal trends in resonance-ilno

Denuark

$0.25-1 \times e V$

Ponce, V. H. Dptlinl sed transiation factors for lou energy proton-hydrogen-atom colitalons. J. Phys. B 12, 3731 (1979)

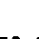

Matsuzawa, M. Theraal colllolong between hlgh-Rydberg,
atoms and rare-gas otoms. J. Phys. B 12, 3743 (1979) ators

$100-2530$

Gorle, T. V.: Shah, M. B.: Gtlbody, H. B. One-alectron capture and loss by rast multifly charged toron and carbon Ions In $\mathrm{H}$ and $\mathrm{H}_{2}$. J. Phys. B 12, 7763 (1979)

Mecarthy, I. E.: MCDowall, M. R. C. Total react Ion $30-200$ \&U cross sectlons in the atoule coupledtchannel optical cross. Soctions in the atomic coupled-ch
(1979)

Austral is

$100-1400$ ov

Dalbo, G.: Fornabinl P.; Lazzlezera, I.: Aanterl, G.: for Intermediate energy el eetren seattering. I. He. J. Phys, 8 12, 3787 (1979)

Italy

E $\quad 11-16 \circ V$

Bose, H.: Linder, F. Throshold excltation in $H_{2}$ and $D_{2}$ by electron tapact and prodissoclation of triplet otates wasured by electron photen colneldences. J. Phys. B 12, 3805 (1979)

Weat Germany

Dy, H.-B.: van Regeasorter. H. Photol onl eati on from low nrenigh axclted ot otep: J. Phys..B 12, L716 (1979) France

Arastrong, L., Jr. Bl stabll ity erfoets In cooperatt ve multiphoton lontoation. J. Phys. B. 12, L719 (1979) United states

Jakubassa, D. H.: Aaundsen, P. A. Equlvalonce of the adlabatlc approxifat ion and the Born opproximation for oxcltatlons in slouly colliding soyemetric aystess. Jo Phys. B 12, L726 (1979)

Ho ruay

E. 5-20 keV

Sotaka, M.: Urakawa, J.: Oda, N. Measurements of doubl- differentlat eross sectlong for electrone ejected by 5-20 $\mathrm{KeV}^{+}, \mathrm{H}_{2}+$ and Het Iepacts on argon. J. Phy e. B 12, L729 (1979) a pan

T 3.0-3.25 By
Morgan, L. A. Electron lapaet axeltation or the $n=2$ states of Het. J. Phy B. B 12, 1735 (1979)
(19) 


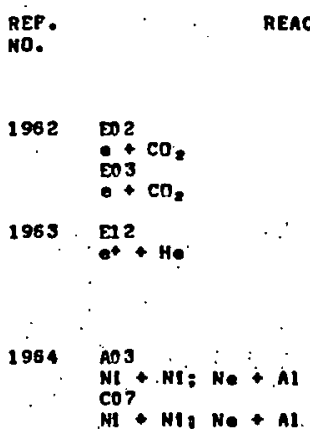

E'NERG

RANGE

REF ERENCE

$M+N H_{0}+A$

1968

202

$17 \times 0$

1966

EO 3
E1
+

$1967.50 \mathrm{~S}$

Eos
E2t Lt
$0+i 2$

1968 205

1959 E1 2

$\infty^{2}+H e$

1970

$\mathrm{Cl}_{r}^{2}+\mathrm{He}_{\mathrm{O}} \mathrm{Cr}+\mathrm{Ar} ; \mathrm{Cr}+\mathrm{N}_{2} ; \mathrm{Cr}+\mathrm{H}_{2}$

1971

503

1972

203

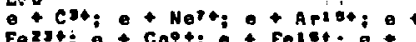

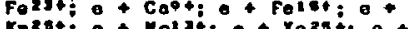

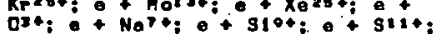

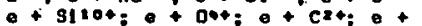

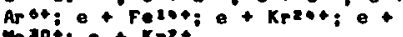

1973

$m \cdot+m$

loo No

$1974 \mathrm{HLO}^{4}$

Under

1925 A1?

Al? + $\mathrm{HCl}$; DCl + $\mathrm{HCl;} \mathrm{Ar}+\mathrm{HCl}$

1976

Go 2

Unde?
$573 R$

$20-503 \bullet V$

$0.3-1.2 \mathrm{MeV}$

$5=300 \bullet V$

$15-190:$ eV

$80-5000 \bullet V$

$109-300$ ov

$2-20$

threshold

$1-80$

throohold

A12

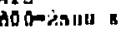

Ho2

$1090 \mathrm{~cm}^{-1}$

I Uil- 480 K

$309 \times$
Haddad, G. N.: El Ford, M. T. Low energy electron seottering crose sections in carbon dloxide. J. Phys. B 12, L.743 (1979)

Australl.

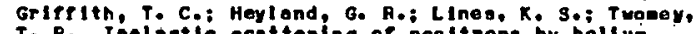
T. R. Inelestle scattering of pcaltrons by hollug atong at Interaedi ate energleo. J. Phys. B 12, 1747

UnIted KIngdon

Meyorhor, E. E.: Anholt, R, Recoll epfects on molecular-orbital x-ray spectra In solid targeta. J. Phys. B 12, 3919 (1979)

Klover, Mo: Deorlagu, H. J. Mo: van der Wiel, Mo Jo

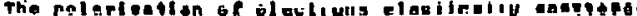
Prow monon at enerylos between s ana 300 ev. J. Phys. D 12, 3950 ( $137+$ )

Catalan, G.: Roberta, M. J. The rorm of electron-aton axcltation amplitudos at high sooentun trangfer in the Faddeev-Vatson approxteation. J. Phys. B 12,3947 (1979)

United KIngdoo

Shuttloworth, T.: Burgess, D. E.: Hender, M. A.; Solth, A, C. H. Inalagfie ocettering of olectrons by.littil um atoas. J. Phys. B 12, 3967 (1979) UnIted KIngdon

Kumar, A.: Roy, B. N. Electren ingact lonisation of alkali batal lons. J. Phys. B 12, 3979 (1979) Ind Ia

Gten. T. T. Tntal croes dootlong of posttivn-lieltua colliclons at intermedlate energles. J. Physe B 12 , 3987 (1979) Canado

Pujol, G.: Henlger, S. Broadenlng and ohl ft of neutral chroalua absorption lines by vartous perturbino gasas. J. uuant. Spectrósc. Radiat. Fransier 22. 145 (1979) Franco

Younger, S. M. Colllston strengths and Gaunt factors for hlghly lanlzed atons of the copper laoelectronle sequence. J. Quant. Spectrosc. Rodlat. Tranger 22 $155(1979)$ Untrea states

rouinguer, 5. H.: Uliese, U. L. An assessment of the offectlive Gaunt toctop npproxinotion. J. Quant Spectrosc. Radiat. Trangfer 22, 161 (1979) Unlted states

Sulzann, K. G. P. Frppragalone for the laral ereotpal ihopption cootílolont ful currelation aosorption ipectroscopy. J. Quent. 3 pectrose. Radat. Transfer $22,195(1979)$

Quack. M. Master equations Por photochenl stry with Quack, M. Master equations for photochenl atry with
Intenge infrared light. Ber. Bungenges, Phys. Ches. 9. 757 . ( 1 970)

Sohrans, D.; Leuchs, $U$. Second virlal coeffictents of HCl, DCl and Ar-HCl mixtures. Ber. Bungenges, Phys. Chem. 9, 847 (1979) West Germany

Relinhardt, D.: Lowe, A.: Dialer, $K$. Prediet lon of the diffuglonal bohaviaur of three component systeas at diffuslonal bohaviaur of three component systeas diagrass. Ber. Bunsenges, Phyo. Ches.9, 956 (1979) diagrans. Geriany 


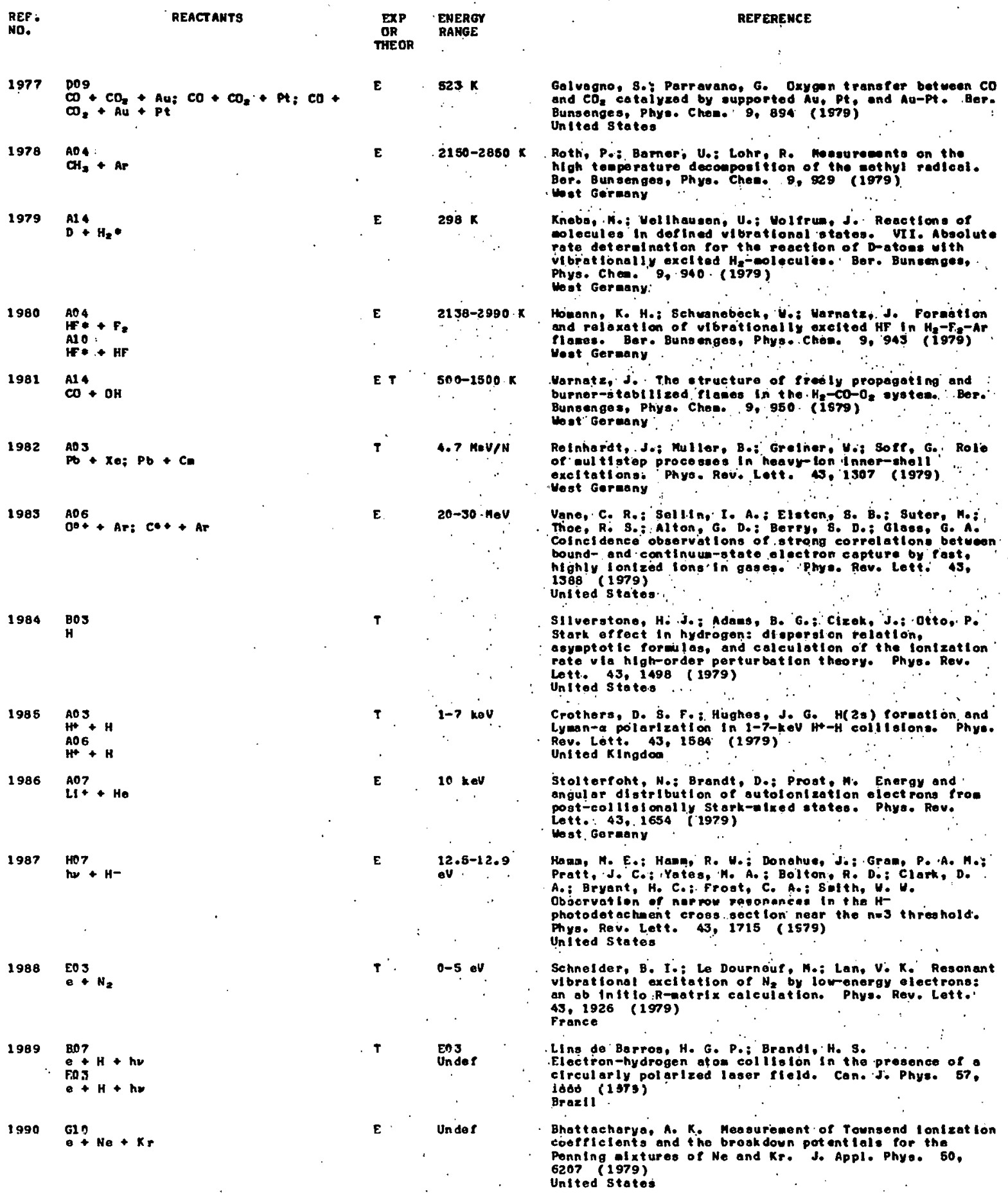




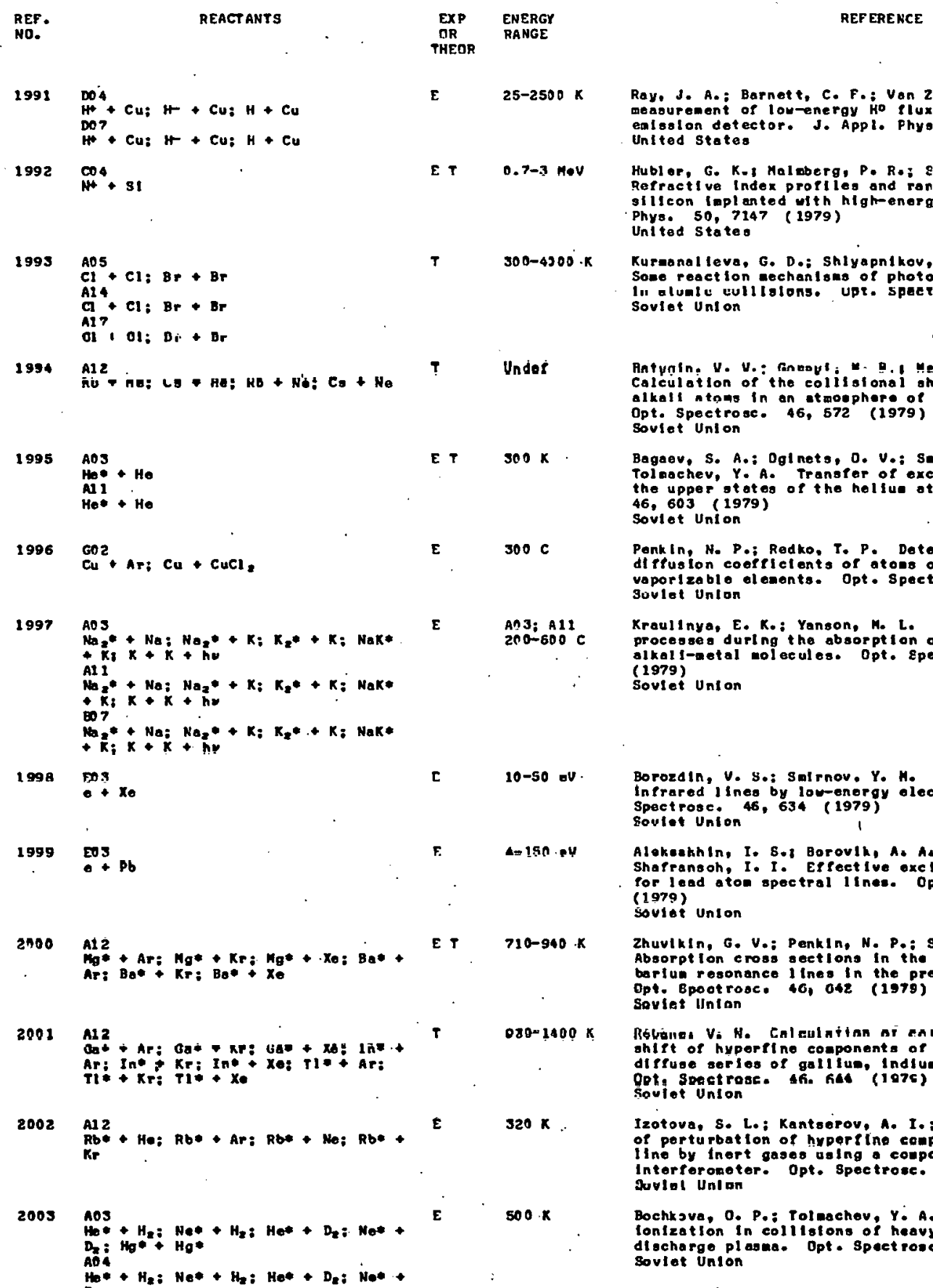




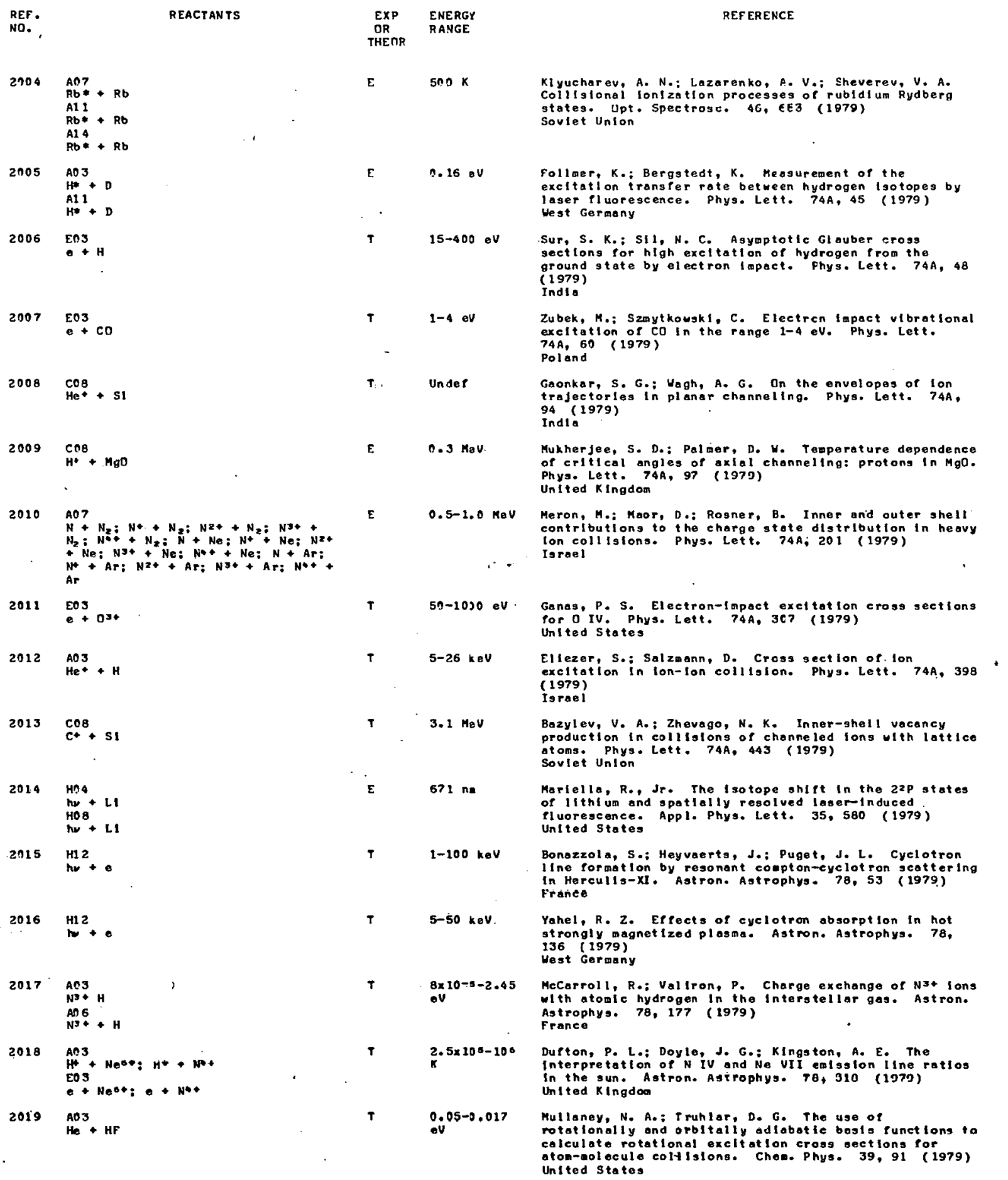




\begin{tabular}{|c|c|c|c|c|}
\hline $\begin{array}{l}\text { REF. } \\
\text { NO. }\end{array}$ & REACTANTS & $\begin{array}{l}\text { EXP } \\
\text { DR } \\
\text { THEOR }\end{array}$ & $\begin{array}{l}\text { ENERGY } \\
\text { RANGE }\end{array}$ & REFERENCE \\
\hline 2020 & $\mathrm{H}_{2}^{\mathrm{AO3}}+\mathrm{HD} ; \mathrm{HD}+\mathrm{HD}$ & T & $0.35-20 \mathrm{eV}$ & 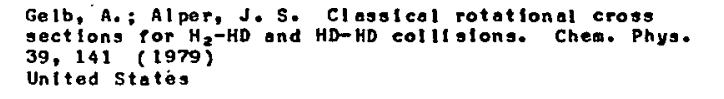 \\
\hline 3021 & $\stackrel{A 14}{F}+\mathrm{HBr}$ & E & $\operatorname{sen} K$ & 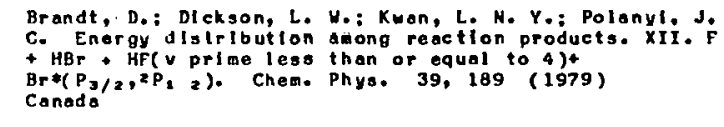 \\
\hline 2022 & $\stackrel{\text { Al }}{\mathrm{F}} \rightarrow \dot{\mathrm{DH}}$ & $T$ & $3 n 0 \mathrm{~K}$ & 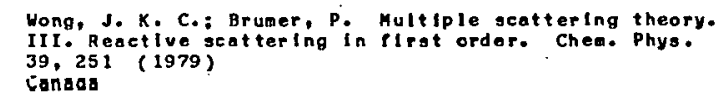 \\
\hline 2023 & ${ }_{M G}^{A n s}+r_{2} C_{a}-P_{2}: S P+H_{2} Z H_{a}+F_{2}$ & $F$ & $300 \mathrm{~K}$ & 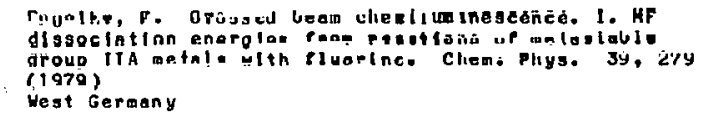 \\
\hline 2024 & $\stackrel{A 14}{F}+H_{2} ; H_{1}+F_{2}$ & $T$ & $0.1-2.8 \mathrm{eV}$ & 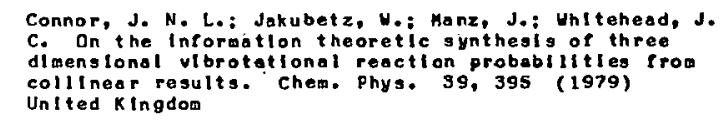 \\
\hline 2025 & $\begin{array}{l}\mathrm{AOZ} \\
\mathrm{LI}+\mathrm{CO} \\
\mathrm{AOS}+\mathrm{CO} \\
\mathrm{LI}+\mathrm{CO} \\
\mathrm{AlO}+\mathrm{CO} \\
\mathrm{LI}+\mathrm{CO}\end{array}$ & E & $4-7 \mathrm{eV}$ & 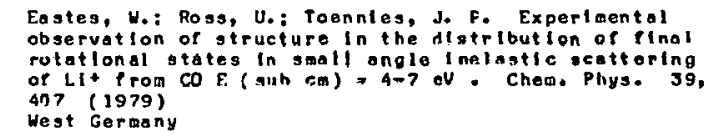 \\
\hline 2026 & $\mathrm{C}_{2}+\mathrm{O}_{2}$ & E & $298 \mathrm{~K}$ & 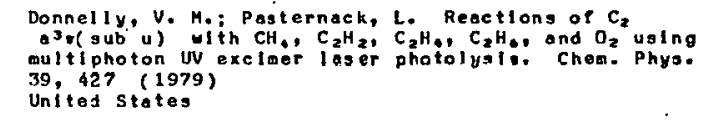 \\
\hline 2027 & $\begin{array}{l}\mathrm{A03} \\
\mathrm{H}_{2}+\mathrm{H}_{2} \mathrm{H}_{2}+\mathrm{He}: \mathrm{H}_{2}+\mathrm{CS} ; \mathrm{H}_{2}+\mathrm{OCS}: \\
\mathrm{He}+\mathrm{HCl} ; \mathrm{He}+\mathrm{CO} ; \mathrm{He}+\mathrm{HN}_{2}^{+} ; \mathrm{Ar}+ \\
\mathrm{HCl}\end{array}$ & $T$ & $\begin{array}{l}\mathrm{es}^{6-1}{ }^{60300} \\
\because\end{array}$ & 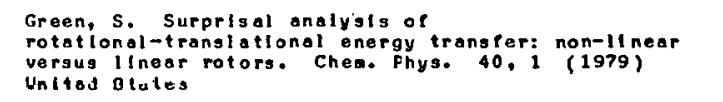 \\
\hline 2028 & 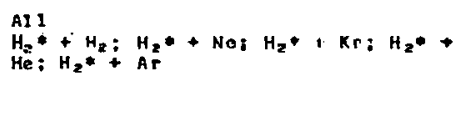 & $\varepsilon$ & $300-3300 \%$ & 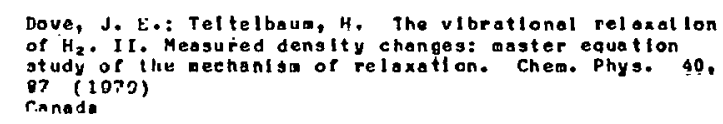 \\
\hline $20 \Sigma 9$ & $\begin{array}{l}\text { Al } 1 \\
\mathrm{~N}_{2} *+\text { Ar }\end{array}$ & $T$ & $600-12000$ & $\begin{array}{l}\text { Nyeland, C.: Bllling, G. D. Approxlmatlue treatments } \\
\text { of rotat anal relaxatlon. Cheo. Phys. 40, } 103 \text { (1979) } \\
\text { Denmark }\end{array}$ \\
\hline 2030 & 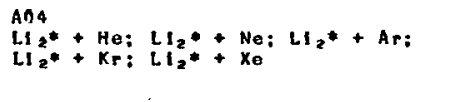 & $\varepsilon$ & $300 \mathrm{~K}$ & $\begin{array}{l}\text { Ennen, Get Dttinger, C. Collloion Induced digsoctatlon } \\
\text { of 1ager-exclted Li, Bin(sub u). Chem. Phys. } 40,127 \\
\text { (1979) } \\
\text { Hest Germany }\end{array}$ \\
\hline 2031 & 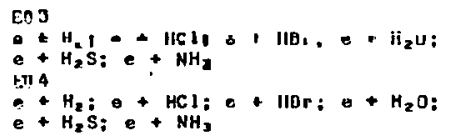 & $E$ & $3^{0-2009} \mathrm{eV}$ & 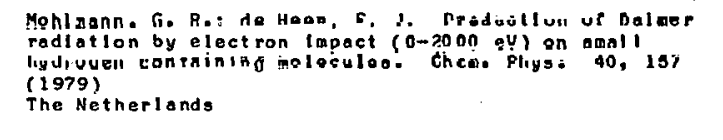 \\
\hline 2032 & $\begin{array}{ll}\text { Al } 14 \\
\text { CIO }\end{array}$ & T & $\begin{array}{l}: 220-1000 \mathrm{~K} \\
\vdots\end{array}$ & $\begin{array}{l}\text { Jarfe, R. L. Calculated rate congtants for the } \\
\text { reaction c10 }+0 \text { C C1 }+0_{2} \text { between } 220 \text { and } 1000 \mathrm{~K} \text {. } \\
\text { Chem. Phys. } 40 \text {. ios }(1979) \\
\text { Untited Stateg }\end{array}$ \\
\hline 2033 & 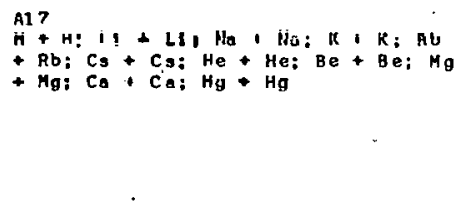 & $T$. & $\begin{array}{l}\because \operatorname{lndar} \\
\therefore \\
\therefore \\
\vdots \\
\vdots\end{array}$ & 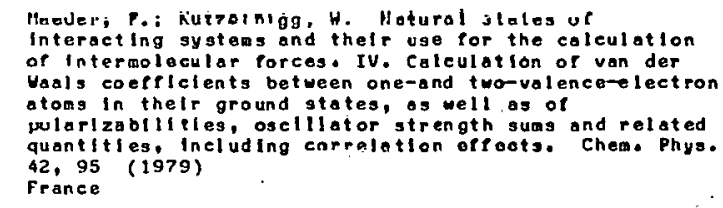 \\
\hline
\end{tabular}




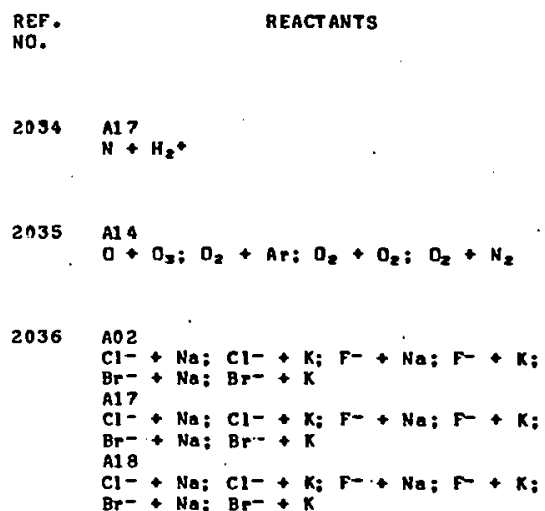

ENERGY

RANGE

THEOR

Under

$262-335 k$

E $5-1000 \bullet V$

Velweyer, H.: Pauly, H,: Duker, H. Comparlson of

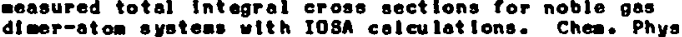
42, 389 (1979)

West Gernany

Under

Brunott1, B.; Cambi, Ro: PIronl, F.: VecehlocattIvi, f.: Touasint, $M$. The Interatcale potentlal of noon Prom ecattering, gaseous and solld atate data. Cheis. Phys. 42,397 (1970)

Boundo, D. G. Pal potentlals and pol arlzobilltles for. Lif and KF. Chen. Phys. 42, 408 (1979) Canodo

Herbst, E.; Payne, L. G.; Champion, R. L.; Doversplke, L. D. An experimental and traject ory study of the reactl on $D^{-}+D_{2} \cdot D^{-}+D_{0}$ Ches. Phys. 42,413 (1979) United statea

$730 k$

Rowe, M. D.: MeCaffery, A. J. Transfer of state

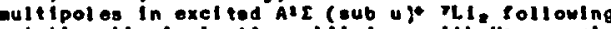
rotationally Inelastic collistiono ut th tho oxperiant and theory. Chem. Phys. 43, J6 (1979) United Kingdom

Unesoto, H.: Tsunashiea, S.: Sato, S. The obsolute croos sect tons for quenchlng of cadrium 5 गp, and $53 p$ at ons by eethane, nitrogen and l sotople hydrogens. Chem. Phys. 43, 93 (1979)

Viehl and, L. A.: LIn, S. L. Applicetion of the three-tenperature theory of gaseous Ion trangport. three-tenperat ure thoory of
Chem. Phys. 43, 135 (1979) Unitod states

Pastarnack, L.: Medonald, J. F. Reactions of C. $x_{1} \varepsilon(s u b g)$ produced by ult iphoton uv exciner laser phorolyolso chas: phye.

Rol Dastldar, T. K.; Ral Dastldor, K.: Bose, M. Use of

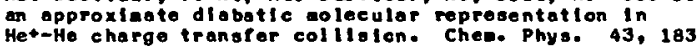
(1979) Indis

Kuntz, P. J.: Doran, M.: HIlller, I. H. Ab $1 n 1 t 10$ Caleulation of the potentiol energy surface and eross Ne.t Weat Garaany

Donnelly, V. M.; Oaronavsk 1, A. P.: MeDonald, J. Re Exclted state dynaeles and bicolecular quenching processes for Nlla(A ilide sAa). Chom. Phyo. 43, 283 (1979)

United stateo 
REF.

REACTANTS

2948

$\mathrm{e}+\mathrm{CO}_{2} ; \mathrm{e}+\mathrm{N}_{2} \mathrm{O}$

en $+\mathrm{CO}_{2} \mathrm{O} e+\mathrm{N}_{2} \mathrm{O}$

$2049 \quad{ }^{A 11}+D$

2050

$\mathrm{Al}_{2} \mathrm{O}+\mathrm{Cl}_{2} \mathrm{O}$

2951

2952

2053

2354

2055

$\mathrm{H}+\mathrm{F}_{2}$

2056

$\stackrel{\mathrm{A} 17}{\mathrm{H}+\mathrm{NO}}$

2057.

2950

$205 \theta$

$\mathrm{e}^{\mathrm{E}}+\mathrm{N}_{2} \mathrm{Z} \mathrm{e}+\mathrm{O}_{2}$

en $+\mathrm{N}_{2}: e+\mathrm{O}_{2}$

$e+\mathrm{N}_{2} ; e+\mathrm{O}_{2}$

2060

$\mathrm{NH}_{2}+\mathrm{NO}_{3}$

$3051 \quad 004$

$\mathrm{DO4}+\mathrm{Nb}: \mathrm{O}+\mathrm{Nb}_{2} \mathrm{O}_{3}$

$2062 \quad \mathrm{HAO}_{\mathrm{H}}^{\mathrm{An}}+\mathrm{HI}_{2}$

2053
203

ext $\mathrm{N}_{2}$
EXP

THEOP.

$0.1 \mathrm{eV}$.

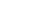

Under

60-100 ev

e

298-5J5 K

$E \quad n-3$ kev

T. $0.1-1.2$ ov

E

$30 \mathrm{~B}-500 \mathrm{eV}$
FNERGY
RANGE

REF ERENCE

Hitcheock, A. P.: Brlon, C. E.: van der Wtel, M. J. Ionic fraguentation of inner shell excltedistotes or $\mathrm{CO}_{2}$ and $\mathrm{N}_{2} \mathrm{O}$. Chem. Phys. Lett. $6 E, 213$ (1979) Canada

rernando, R. P.: Solth, I. H. M. Vibrattonal relaxation of No hy atomlo oxygen. Chet. Phys. Lett. $66,218$ ( 1979$)$
United Kingdom

NIkl, H.: Maker, P. D.: Savage, C. M.: Breltenbech, L. P. Fourler transform iR spectroseople deternination or the equillbrtum constant for $\mathrm{H}_{2} \mathrm{O}+\mathrm{Cl}_{2} \mathrm{O}$ golng to or coning from 2 HOCl. Ched. Phyg. Lett. 66, 325 (1979) United States

Rakshtt, A. B. The reaction ef $\mathrm{CO}_{2}$ dimer lons with sulfur dioxide. Chem. Phys. Lett. 66, 373 (1979) wegt Germany

Tlirush, B. A.: Wllkinson, J. F. T. Pressure dependence of the rate of reaction hatwoen $\mathrm{HO}_{2}$ rodicals. Chem. Phys. Lett. 66, 441 (1979) Unted Kingdom

Barroux, C.: Audibert, M. M. Experimental study or the vibrational relaxation nf $H D$ in collislun with HD and iHe in the range 80-400 K. Chem. Phys. Lett. 66, 403 (1979)

Mayne. H. R. Quastclassical trajectory calculations Por $11+H_{2}(y=1)$ on a neu potential energy surface. Chea. Phys. Lett. 66, 487 (1979) West Germany

Clary, D. C.: Connor, J. N. L. Application of the vibratlonally adlabatlc and statle distertad Hevo Born approxlmatiuns to the reaction $H+F_{2}(v=0, J=0)$. HF( v prIme, J prine) + F. Chen. Phys. Lett. 66, 493 (1979)

Nomura, 0.: Iwata, S, Potentlal energy curves of lou-lying atates nn Hun. Shoü, fliyst Let?. 66, 523 (1979)

Morrison, M. A.: Lane, N. F. Threshold gtructure in the vibrational excltation of $\mathrm{CO}_{2}$ by low=ehe electrons. Cham. Dhye. Lette 00,327 (197y)

Lee, E. P. F.; Pottg, A. W. Configuration Interaction effects in the He IId $5 p^{-1}$ phctoel ectrun spectirnm of i. =hom. Phys. Lett. 66, 553 (1979) United Kingdom

Locht, R.: Mosigny, J, DI ssoclative lonlzat ton by law energy electron impoct. Energy distribution and energy electron lapoct. Energy drstributlon and by lau appearance energy of doubly lonized rraginents Prow $\mathrm{N}_{2}$ Helolum

Kurasave, H.: Lesclaux, R, Kinetics of the reaction or $\mathrm{NH}_{2}$ ith $\mathrm{NO}_{2}$. Chem. Phys. Lett. 66,602 (1979) France

Lavarec, M.; Bocquet, P.; Septler, A. Vartation du Lavarec, M.: Bocquat, P.; Septler, A. Varlation du coerficient d en lssion electronlque secondalre eleotron laue urlanire. Compt Rendo 280,77 77 (1979)

van vijk, H. ; Razavy, M. Collinear colllision of an atom with a homoncleer di atomlc molecule. Int. J. Quantur Chem. 16, 1249 (1979) Canoda

IIdo, T. Measurements of energy-loss croso section for 300,400 , and 500 eV electrons in the continuun of $\mathrm{N}_{2}$. Jap. J. Appl. Phys. 18, 1667 (1979) 


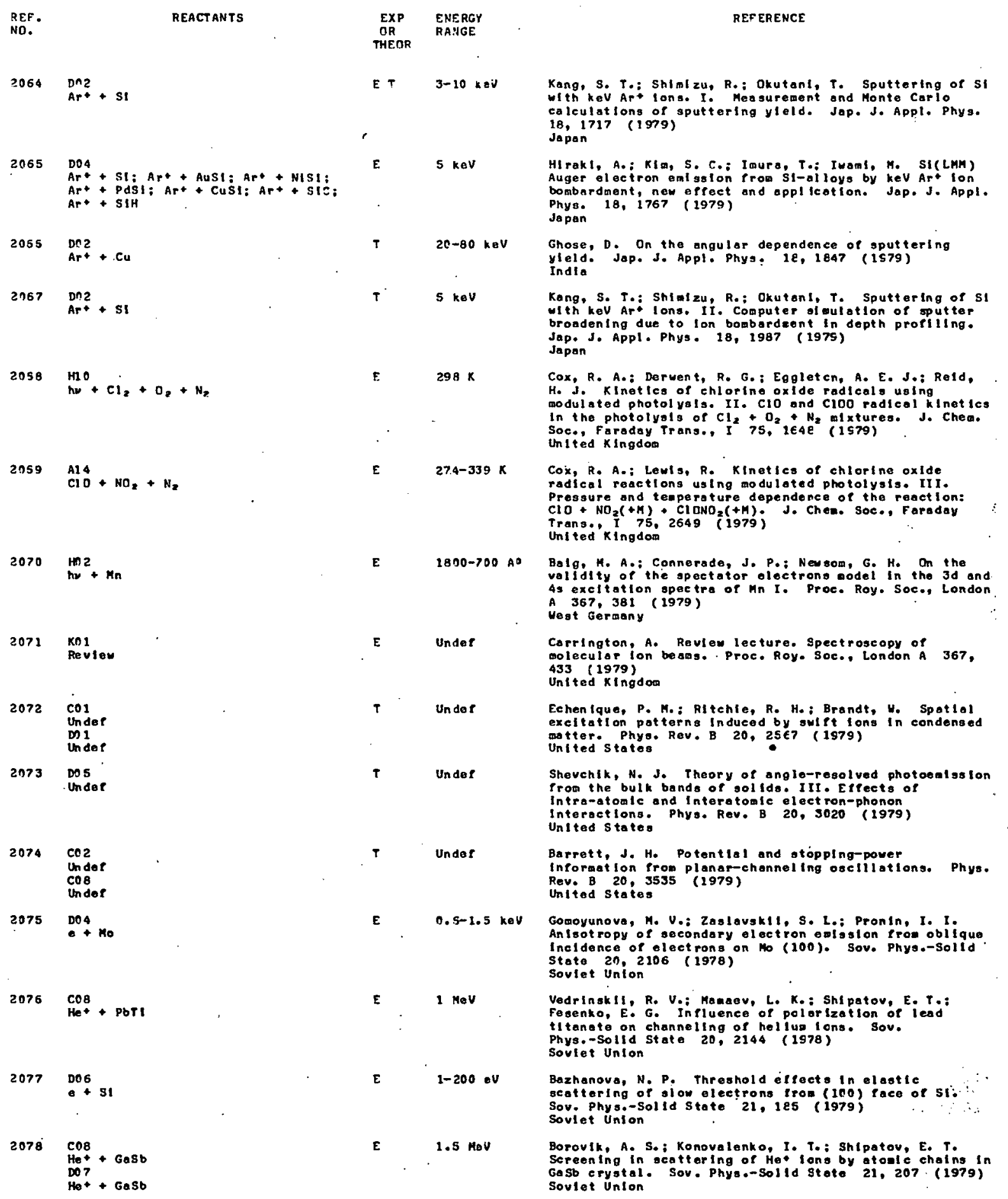




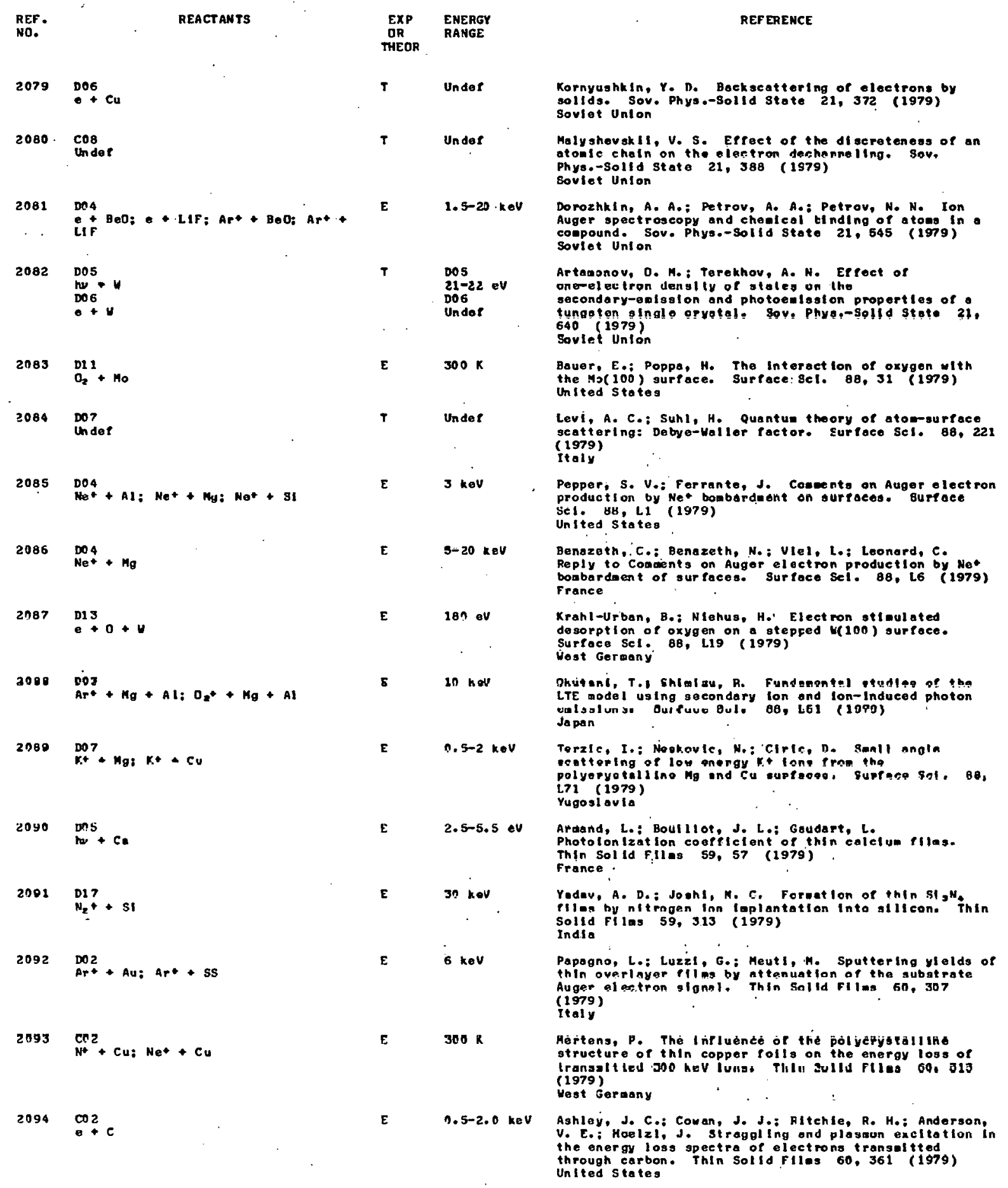




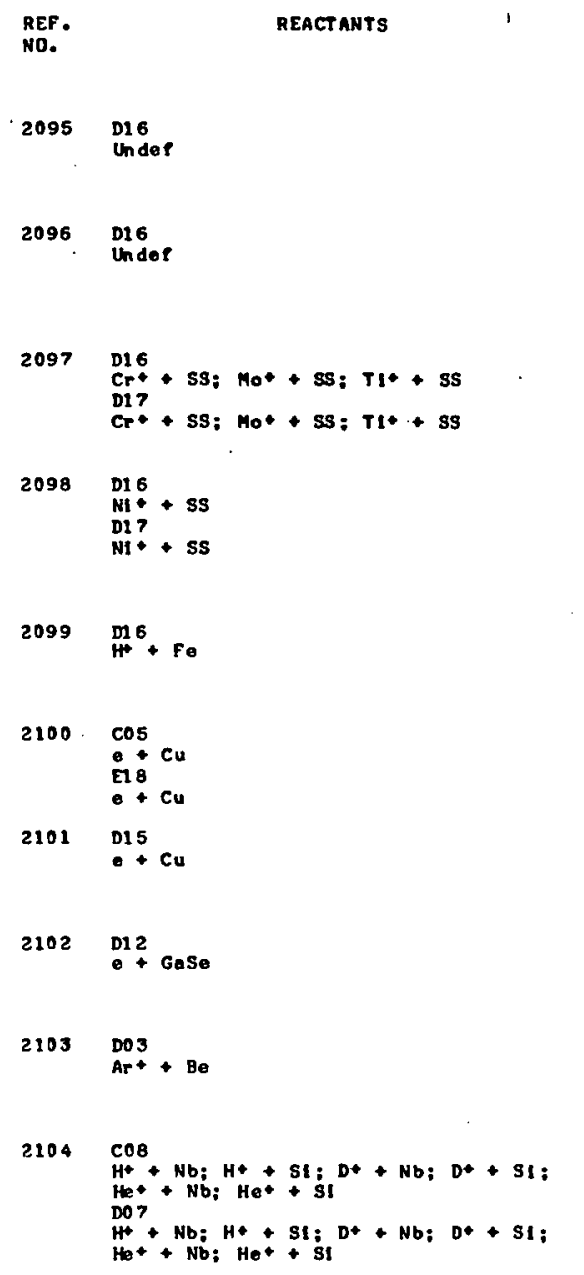

$\begin{array}{lll}\text { EXP } & \text { ENERGY } \\ \text { OR } & \text { RANGE } & \text { REFERENCE } \\ \text { THEOR } & & \end{array}$

Pleraux, S. T.: Myors, S. Mo: Foll otaodt, D. M.

E Undor Meroalioy layar foration by lon inplantation. Then Solld Filas 63, 1 (1979) United 8 tates

E Undat

HI rvonon, J. K.: Carosoll o, C. A.: Kent, R. A.: SInger, I.: Vardian, Ro: Rath, B. B. Improvacent of Eotal

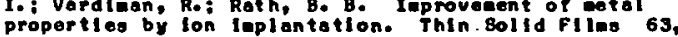
5 (1979) Unitod states

$75 \mathrm{kev}$

Unde:

$\mathrm{T} \quad 20 \mathrm{keV}$

T Under

$\mathbf{T}$

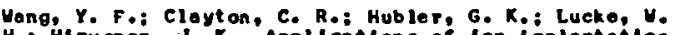

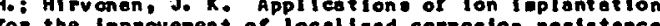
ror the improvenent of local ized corroston United States

Agarwal, S. B.: Uang, Y. F.: Clayt on, C. Ro: Merman, H.: Hirvonen, J. K. Interpretation of ol oct rochenical behavt or of nickel implanted type-430 stainless steol using $x$-ray photoelectron spectroscopy and transelssion using x-ray photoelectron opectroscopy and transelssion United states

Myers, S. M.: Pieraux, S. T.: Stoltz, R. E. Deuterlun troppling in iron alloys toreed by Ion Implantation. Thin solid films 63,37 (1979)

United states

Ralph, J. E. Phot oluel nescence of Cd8: Cu doped by diffusion. Phys. Stetus Solldi A 50,611 (1978) Finiand

Laffers, T.: SIngh, B. N. Loop growth and polnt-defect protil during hVE irradlation. Physe. Status Solldi A 54, 621 (1979)

RaIno, A.: Mancinl, A. M.; Kurrl, R.: Rizzo, A.; Vosanell 1, L. Cathodoluai nescence of Gase Ingle crystals, Phys. Status Solldi A 63, 46 ? (1979) Italy

Mladenov, G. M.: Braun, M. Ion bobardwent Induced Itne radtation erom sputtered beryll1us. Phys. Status Solidi A 53, 631 (1979) sueden

Chublsov, M. A.: Akkeraan, A. F. Conputer glnulation of lou energy hydrogen and hellue lon reflection fron aonocryatalline surfoces. Phys. Stotus Soltdi A 55, K63 ( 1979 ) 


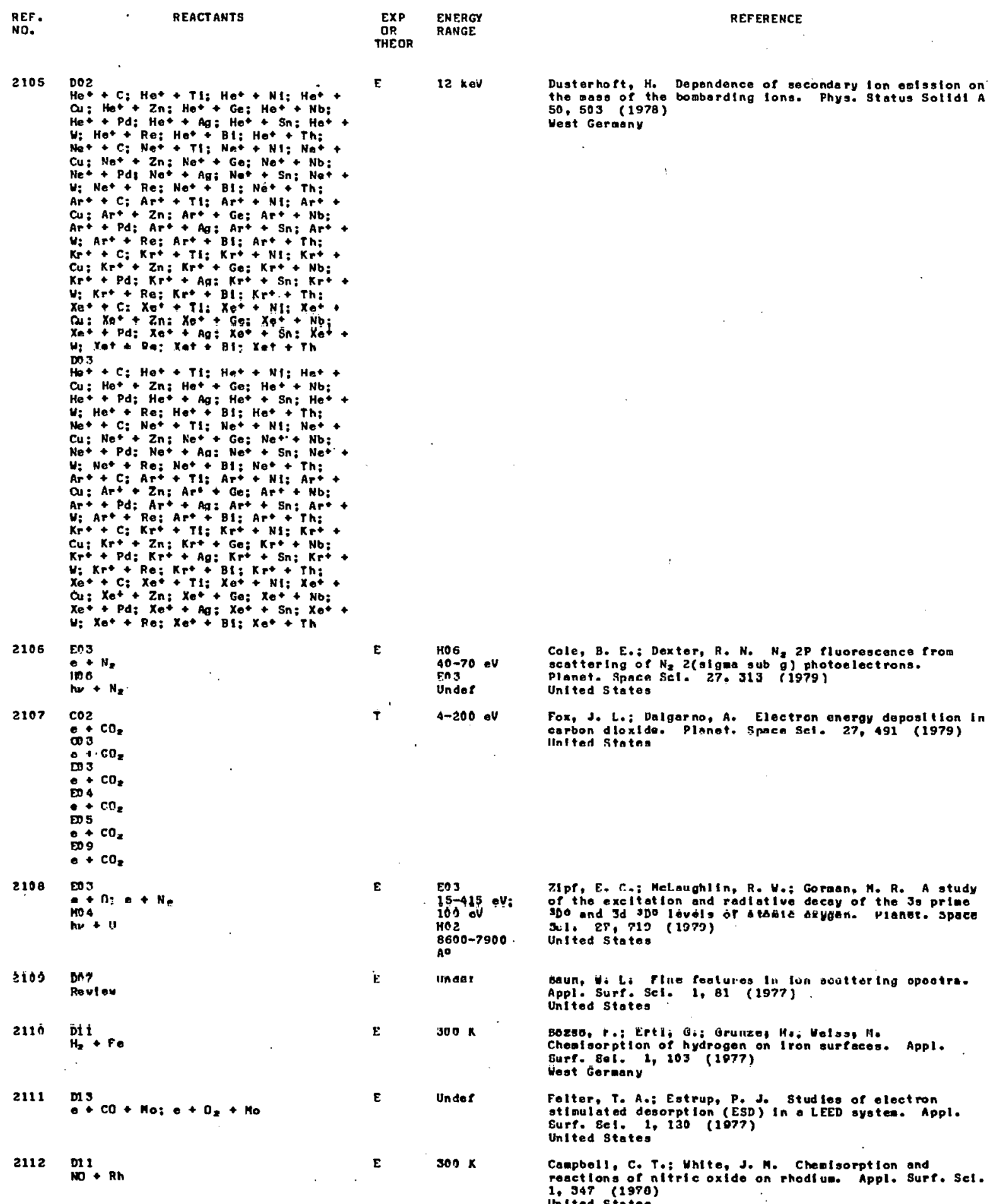

E03

105

$8600-7900$

Ao

E. Inaar

E $300 \times$

Unde ?

$\operatorname{son} \mathrm{K}$

Cole, B. E.: Dexter, R. N. $\mathrm{N}_{2} 2 \mathrm{P}$ rluoresence from scotterlng of $\mathrm{N}_{2} 2$ (olgas sub g) photoelectrons Plante Dustarhoft, H. Dependence of seciondary Ion emlssion on
the aas of the bombarding lons. Phys. Status Solldi A Heat Germany Fox, s. L.: Dalgarno, A. Electron energy doposilion in
carbon dloxide. Planet. Spece Sel. 27,491 (1979)
llnttert states

ZI pP, E. C.: Melaughiln, R. U.; Goraan, M. R. A study of the excilotlon and radi ot lue decay of the 33 prine

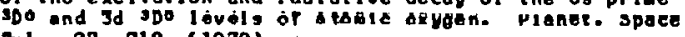
3il. 28,719 (1370)

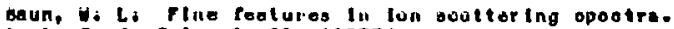

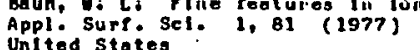

Bozso, P.: Ertli; Oa; Grulzes HA. Helss, HA Cheal sorption of hydrogen on iron surfeces. Appl. Eurf. Bal. 2, 103 (1077) West Germany

Folter, T. A.; Estrup, P. J. Stud fes of electron st mulated desorpt Ion (ESD) In L LEED systea. ADpl. Eurf. Bel. 1, 130 (1077) United states

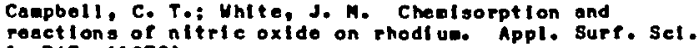
1.347 (1980) 


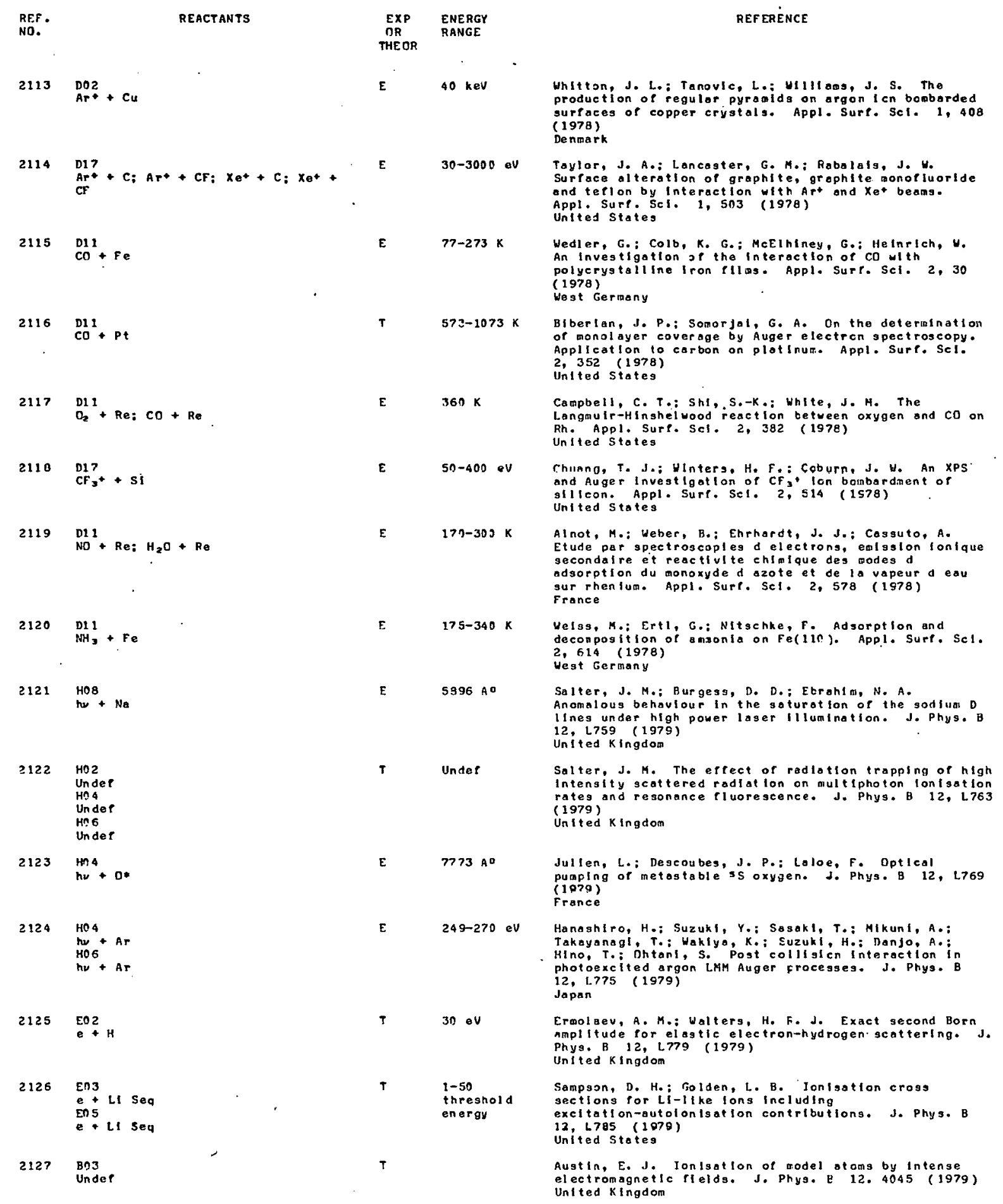




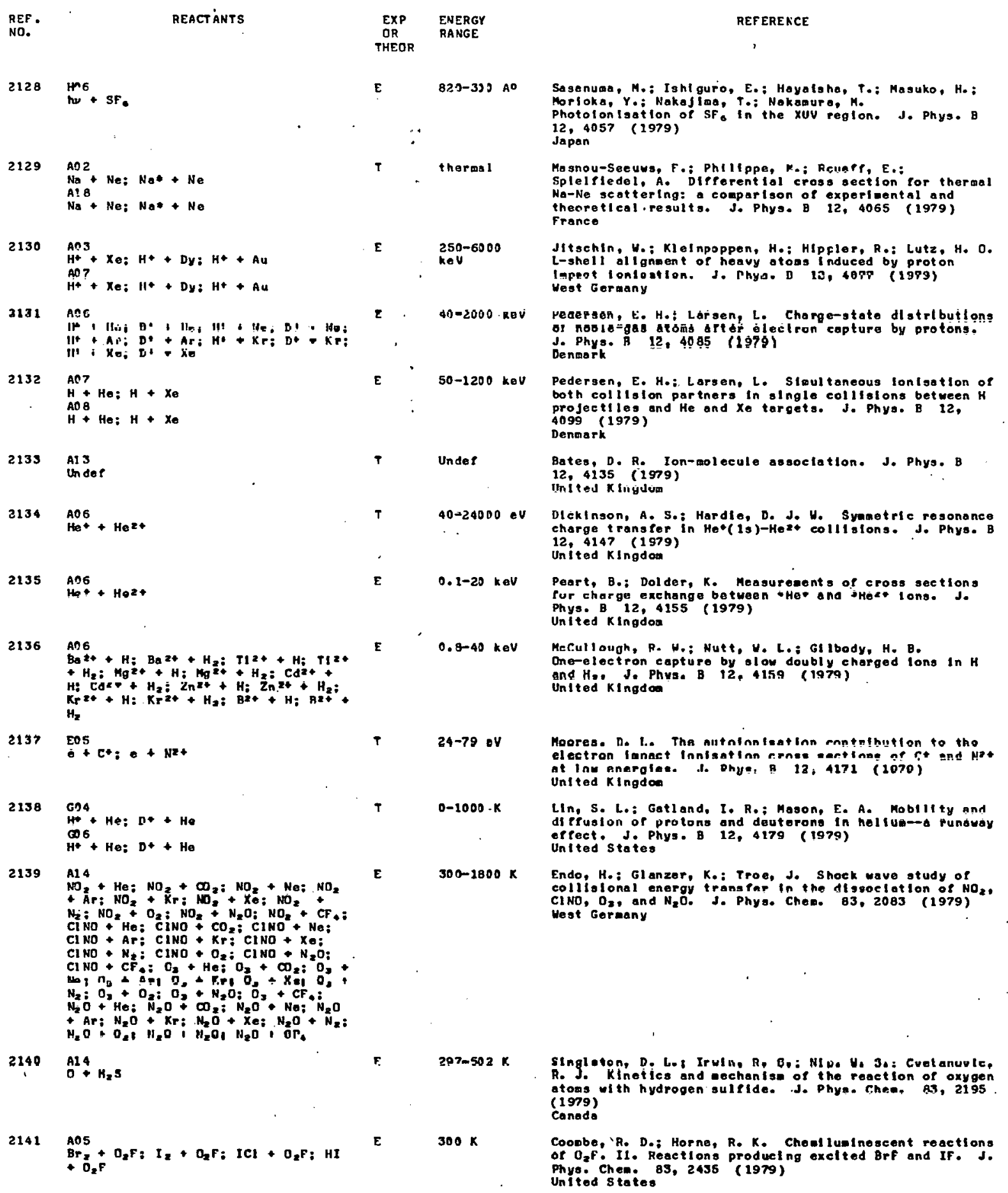




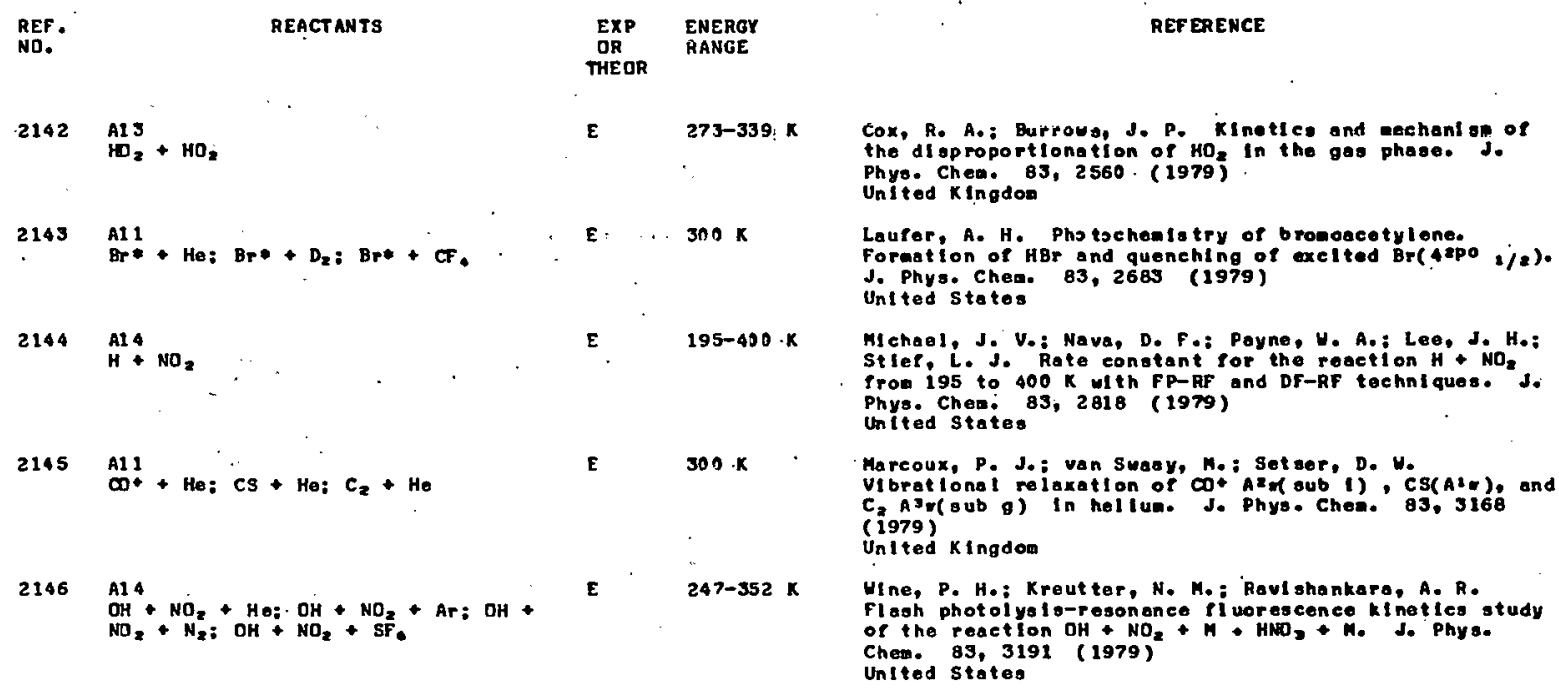


THIS PAGE

\section{WAS INTENTIONALLY \\ LEFT BLANK}


REACTANT INDEX 
THIS PAGE

\section{WAS INTENTIONALLY LEFT BLANK}


no1

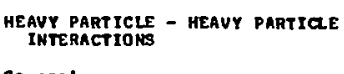

General

$$
\begin{aligned}
& \text { Ar }+ \text { Ar } \\
& \mathrm{Ar}+\mathrm{Kr} \\
& \text { Ar + } \begin{array}{c}
\mathrm{Ne} \\
2037
\end{array} \\
& A r=\underset{2037}{A+A r} \\
& A_{2}+K_{2037}
\end{aligned}
$$

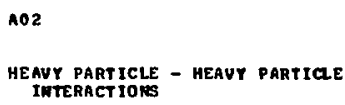

Elastle Scattering Colll at ons

$$
\begin{aligned}
& \mathrm{Ar}+\underset{232}{\mathrm{CO}_{2}} 1230 \\
& \mathrm{Ar}+\mathrm{N}_{2} \\
& \text { A5* + Cu } \\
& \text { Art }+ \text { CuO } \\
& \text { Art }+X e \\
& \text { Br- }+\underset{2 n 36}{+K} \\
& \mathrm{Br}-+\mathrm{Na} \\
& c+\underset{85}{c} \\
& c+\mathrm{ce}_{135} \\
& \begin{array}{r}
\mathrm{Cd}^{*}+\mathrm{xe}_{\mathrm{t}} \\
1629
\end{array} \\
& \mathrm{Cl}+\mathrm{xe}_{\mathrm{e}} \\
& \text { Cl- }+ \text { Ar } \\
& \text { CI- } \underset{2036}{+x} \\
& \text { C1- + }+ \text { No } \\
& \mathrm{Cl}-+\begin{array}{r}
+x_{0} \\
i \in 29
\end{array} \\
& \text { D* }+ \text { D } \\
& \text { D* + N1 } \\
& \mathrm{D}_{2}+\mathrm{D}_{2} \\
& r+A_{452}^{A r} \quad 1173 \\
& f+\pi_{452} \\
& \begin{array}{l}
\mathrm{Ne} \\
452
\end{array} \\
& F+x_{i 173} \\
& \text { F- K } \\
& \mathrm{PA}+\underset{2036}{\mathrm{Na}} \\
& \text { F- + } \begin{array}{c}
x_{e} \\
3 \text { fi2. }
\end{array} \\
& \text { H+ } \begin{array}{c}
\text { Ar } \\
\mathbf{2 0}
\end{array} 936 \\
& \mathrm{H}+\mathrm{C27}_{\mathrm{B}, \mathrm{H}} \mathrm{F}
\end{aligned}
$$

$\mathrm{H}+\mathrm{C}_{227}$

$H+80+6$

H + CO 1355

H + Ho

$\mathrm{Hen}$
+1351

$\mathrm{H}+\mathrm{Hg}_{\mathrm{gg}}$

$\mathrm{H}+\mathrm{KF}_{\mathrm{B} 27} \quad 936$

$\mathrm{H}+\mathrm{Ne}_{\mathrm{g}}$

$\mathrm{H}+\mathrm{O}_{\mathrm{B}}$

$H+x^{x_{e}}+936$

$\mathrm{H}+\mathrm{C}_{13 \mathrm{a}}$

$\mathrm{h+}+\mathrm{Cas}^{+3}$

$\mathrm{H}^{++}+\mathrm{Cs}^{\mathrm{ang}}$

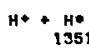

$\mathrm{H}+{ }^{\mathrm{H}} \mathrm{H}^{\mathrm{H}}$

$\mathrm{H}^{+}+\mathrm{HO}_{32.4}$

32.4940

$\mathrm{H}++\mathrm{He*}$

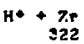

$\mathrm{H}_{2}+\underset{\mathrm{Ar}}{\mathrm{A2.7}} 936$

$\mathrm{H}_{2}+\mathrm{H}_{27} \quad 1909$

$H \neq+K r$

$327 \quad 939$

$H_{2}+\underset{827}{x 0} \quad 936$

HCl Ar
1807

$\mathrm{HO}+\mathrm{HD}$
$190 \mathrm{~g}$

HD $+\begin{array}{r}\text { Ho } \\ 335\end{array}$

He + Ar

$\mathrm{Ho}+\mathrm{CO}_{31} 934$

$\mathrm{He}_{\mathrm{e}}+\mathrm{CO}_{2}$
232

He + He

$\mathrm{He}+\mathbf{N}_{31}$

$\mathrm{Ha}+\mathrm{Na}_{1466}$

$$
\begin{aligned}
& \text { No }+\underset{203}{K R} \\
& \text { No }+ \text { Nes? } \\
& \text { No, }+ \text { Ar } \\
& \text { Ne. }+\mathrm{Kr}^{\mathrm{K}} \\
& \mathrm{Ne}=\underset{2037}{+\mathrm{Ne}} \\
& \text { Revlay } \\
& \text { Undef } \\
& \begin{array}{lll}
274 & 598 & 708 \\
717 & 718 & 740
\end{array} \\
& \begin{array}{lll}
717 & 718 & 740 \\
1308 & 1447 & 1619 \\
1689 & 1959 &
\end{array}
\end{aligned}
$$

$$
\begin{aligned}
& \text { Ho }+{ }_{31}^{\text {NO }} \\
& \mathrm{He}+\mathrm{O}_{31} \\
& \text { Ho* }+\begin{array}{r}
H e \\
1036
\end{array} \\
& \mathrm{He}+\underset{58 \mathrm{He}}{+} \\
& \mathrm{Hot}+\mathbf{K r} \\
& \mathrm{He}^{+}+\begin{array}{r}
\mathrm{xe}_{0} \\
162 \mathrm{O}
\end{array} \\
& \text { He=2 } 1863 \text { Ho } 1869 \\
& \text { Hoz. } 6 \mathrm{BO}^{4} \mathrm{LI} \\
& \mu F+\underset{928}{x} \\
& \text { I- }+\mathrm{Na}_{906} \\
& K+{ }_{9 j 6} \\
& \mathrm{Kr}+\stackrel{X_{0}}{1620} \\
& \text { LI } \begin{array}{r}
\mathrm{He} \\
\mathbf{6 3 9}
\end{array} \\
& \mathrm{LI}+\underset{\mathrm{CO}}{1446} 2025 \\
& 2 \mathrm{Lt+}+\mathrm{H}_{2} \\
& \text { Lt* } \mathrm{N}_{2} \\
& \text { LIF } \underset{525}{A+} \\
& \mathrm{Na}+\underset{770}{\mathrm{Ma}} \quad \mathrm{OSB} \quad 1117 \\
& \text { Na - } 115 \\
& \mathrm{Na}+\mathrm{Ha} \\
& \text { No* } 2129 \\
& \text { No+ }+x \in \\
& \begin{array}{r}
0+65 \\
135
\end{array} \\
& \text { D* } 131 \\
& 0+51 \\
& 51+c \\
& 81+0_{841}^{0} \\
& \begin{array}{r}
x x^{+}+x_{t} \\
1620
\end{array} \\
& 2 n+4 x \\
& \text { Unde? }
\end{aligned}
$$


103 MEAVY PARTICLE - HEAVY PARTICLE
INTERACTIONS

Exelsation

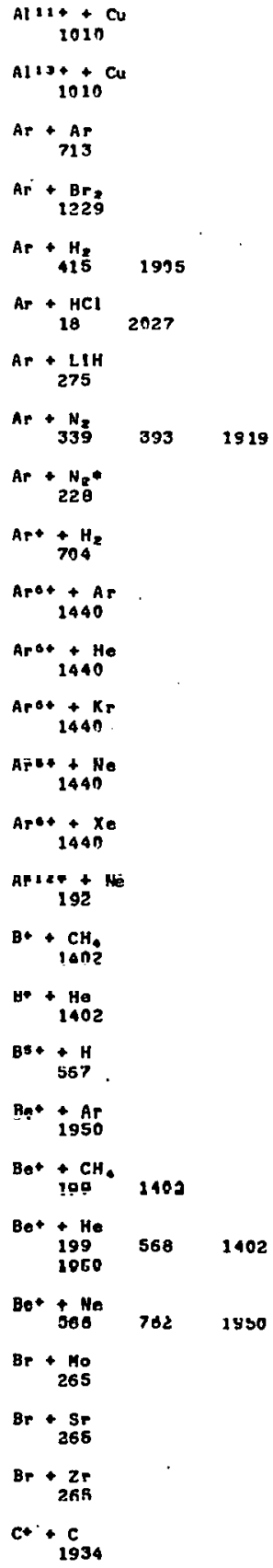

C. $+\underset{1432}{\mathrm{CH}}$

$C+\underset{14 n_{2}}{\mathrm{He}}$.

$C++\underset{29.35}{N}$

$\mathrm{C+}+\underset{14 \mathrm{Ne}}{\mathrm{Ne}}$

$c++0$

C24
+ Ar
146

Cos $+\mathrm{Cu}$

Co4 $+\underset{567}{+H}$

$\mathrm{Ca}_{1865}+\mathrm{H}$

$\underset{993}{\mathrm{Cll}}$

Cl10+ $+\mathrm{Ho}$

993

C1164+ Nd

993

$\mathrm{Cl}: \mathrm{Pt}+\mathrm{Cu}$

101n

- $\mathrm{Cl}^{+}+\mathrm{H}_{2}$

$\mathrm{CO}+\underset{342}{\mathrm{H}_{2}}$

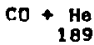

Ca* + cas

Caf + Ar

$r . F+60$

$\mathrm{CaF}_{1210}^{+\mathrm{CO}_{2}}$

$\mathrm{CaF}$ i He

Cor $+k r$

Cof $+H_{i 217}$

$\mathrm{CaF}+\mathrm{N}_{2} \mathrm{O}$

$1: 9+N+1210$

$c$ of $+\mathrm{sF}^{\circ}$

Cor $+8 a$

Cuot+Kr

Cuzst+ Kr

Cunot $+x_{r}$

$\mathrm{Cu} 21+\mathrm{Kr}_{1270}$

F+ + Ho
$\mathrm{F}^{2+}+\mathrm{He}$

Fo+ He
1017

Fo+ + He
1017

p.. 5

Fst $+\mathrm{He}$
1017

Fs+ +5

1024

Fot + Ce

Fot + Cr
1027

Pl: Fe
1027

Fo+ + He
1017

Fot $\begin{array}{r}51 \\ 1024\end{array}$

Fo+ + TI
1027

Fe+ $+v$

FP+ $+\mathrm{Co}^{2027}$

Frt + Cr

Fr+ + Cu

Fre + Fe

FP+ He
1017

foe + 81 1024

Fot +71

F'.

Fo+ + AL

p*4 1 e

Fot $+\mathrm{Cr}$

Fe+ + Cu

cat : Fo

POI He
101?

Fet $\underset{993}{+}$

Fa. $+\mathrm{Nd}^{\circ}$

Fe* 4 st

Fe+ + TI

fes + v 
161
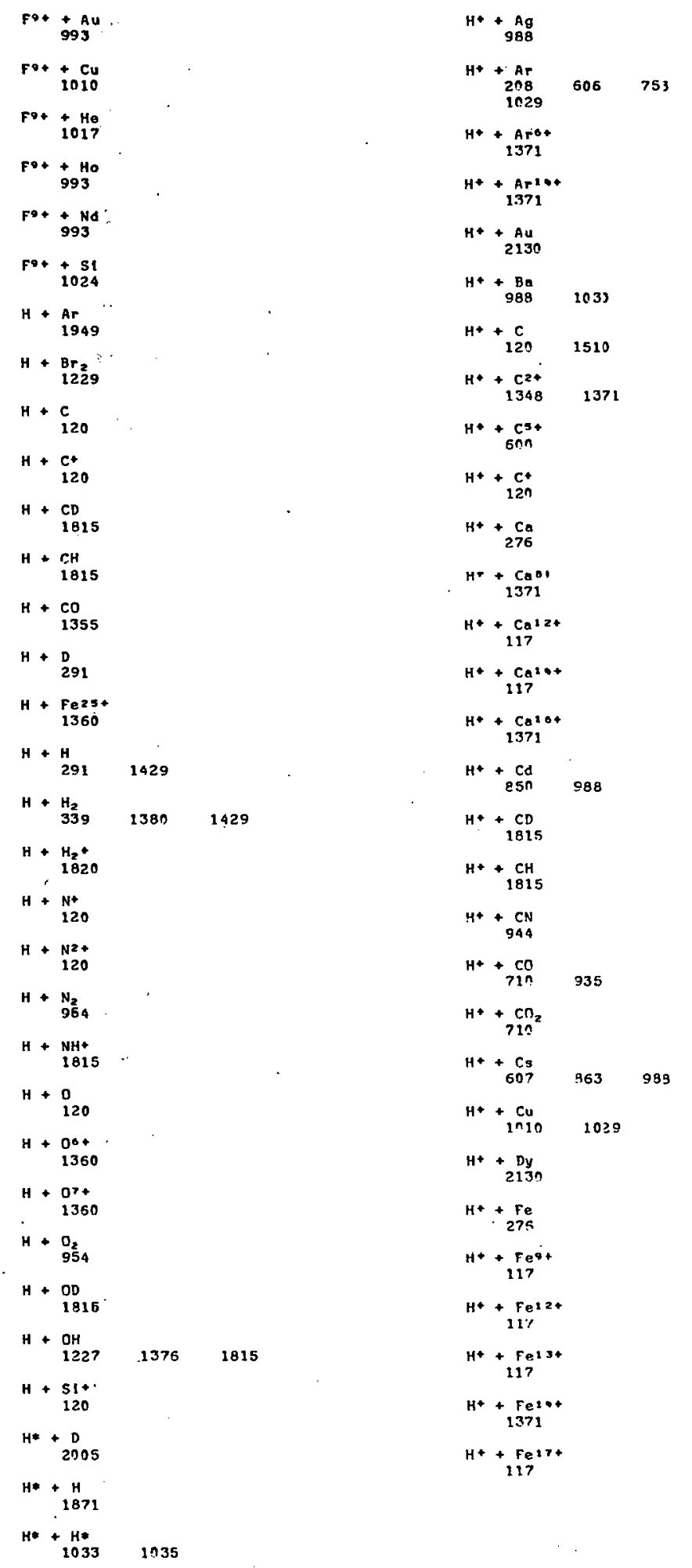
$\begin{array}{ccc}H *+H & & \\ 120 & 240 & 599 \\ 1255 & 1438 & 1449 \\ 1286 & 1933 & 194\end{array}$ $\begin{array}{lll}1255 & 1438 & 1449 \\ 1867 & 1933 & 1942 \\ 1961 & 1985 & \end{array}$




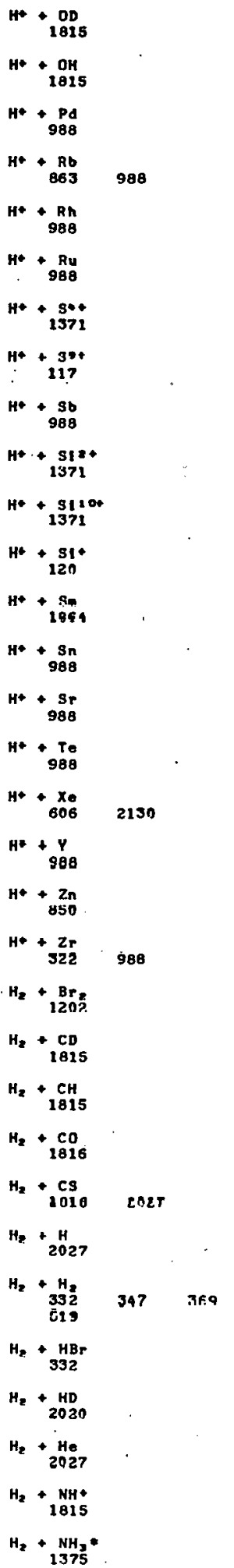

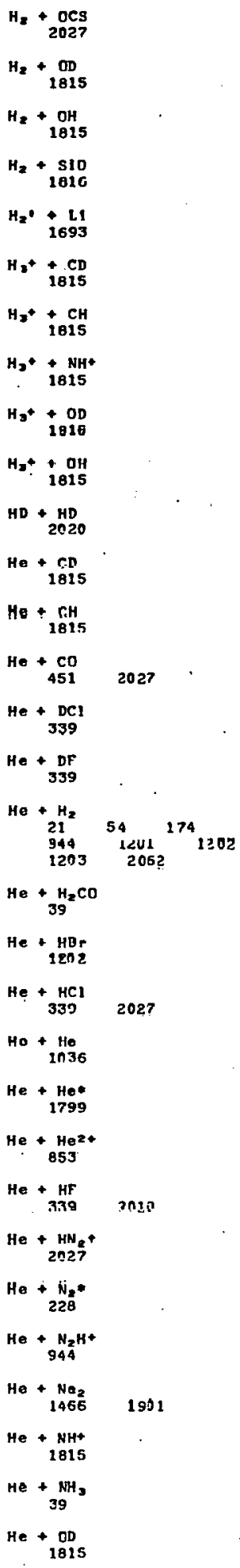

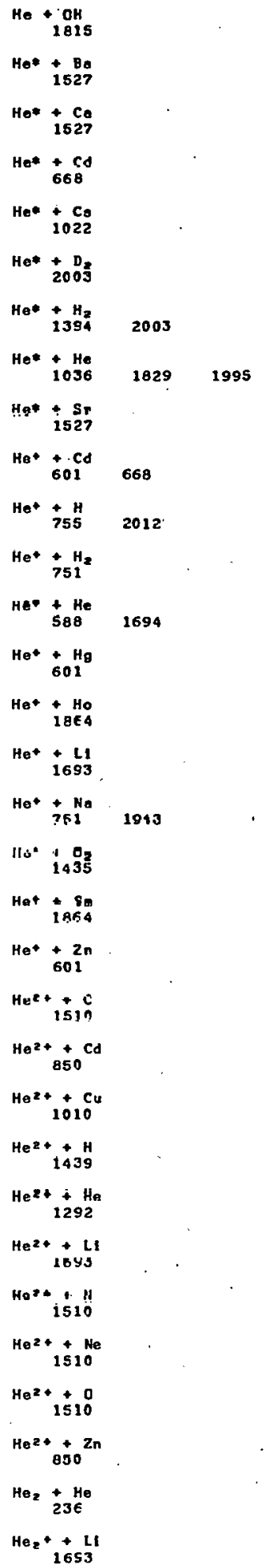




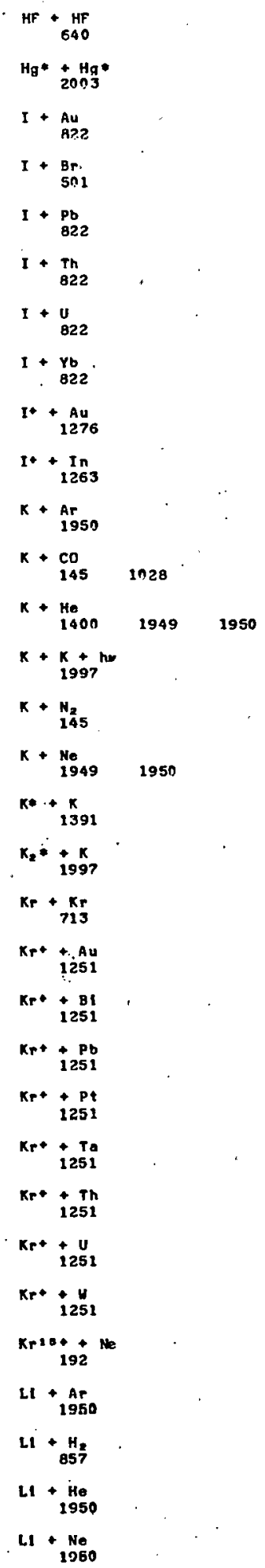

$$
\begin{aligned}
& \begin{array}{r}
\mathrm{N2}^{2+} \\
1510
\end{array} \\
& \text { N3. H } \\
& \text { NT+ + CO } \\
& \mathrm{N}_{2}+\underset{332}{\mathrm{CO}} \\
& \mathrm{N}_{2}+\mathrm{N}_{332} \quad 1202 \\
& \mathrm{~N}_{2}+\begin{array}{r}
\mathrm{O}_{2} \\
\mathbf{3 3 2}
\end{array} \\
& \mathrm{N}_{2} *+\mathrm{CO} \\
& \mathrm{N}_{2}+\underset{20 \mathrm{Br}}{+\mathrm{Ar}} \\
& \mathrm{N}_{2}+\underset{2 \mathrm{OB}}{+} \\
& \mathrm{N}^{+}+\underset{1855}{K} \\
& \mathrm{~N}_{2}++\mathrm{LI} \\
& \mathrm{N}_{2}+\underset{208}{+\mathrm{N}_{2}} \\
& \mathrm{~N}_{2}++\mathrm{Na} \\
& \text { Na + A } \\
& 1950 \\
& \begin{aligned}
\mathrm{Na}+\mathrm{He} \\
1950
\end{aligned} \\
& \text { Na }+ \text { Ne } \\
& \mathrm{Na*}+\mathrm{Ar} \quad 1952 \\
& \begin{array}{c}
\text { Na* He } \\
715
\end{array} \\
& \text { Na* + } \begin{array}{r}
\text { Na } \\
1391
\end{array} \\
& \text { Na* } 71 \mathrm{Ne} \\
& \begin{array}{r}
\mathrm{Nat}+\mathrm{Cd} \\
1487
\end{array} \\
& \mathrm{Na*}+\underset{1262}{+\mathrm{H}_{2} \mathrm{O}} \\
& \text { Not + HC.N } \\
& 1646 \\
& \mathrm{Hat}+\mathrm{N}_{2} \\
& \text { Nat + Ne } 1262 \\
& \begin{array}{r}
\mathrm{Nat}+\mathrm{O}_{2} \\
1262
\end{array} \\
& \mathrm{Na}_{2}+\mathrm{Xe}_{375} \\
& \mathrm{Na}_{2}+\frac{K}{1957} \\
& \text { Nne " } 1997 \\
& \mathrm{Na}_{2} *+{ }_{486} \mathrm{Xe}^{-} 1550 \\
& \text { Nax }+\ldots \\
& 1997 \\
& \mathrm{No}+\mathrm{Al}
\end{aligned}
$$




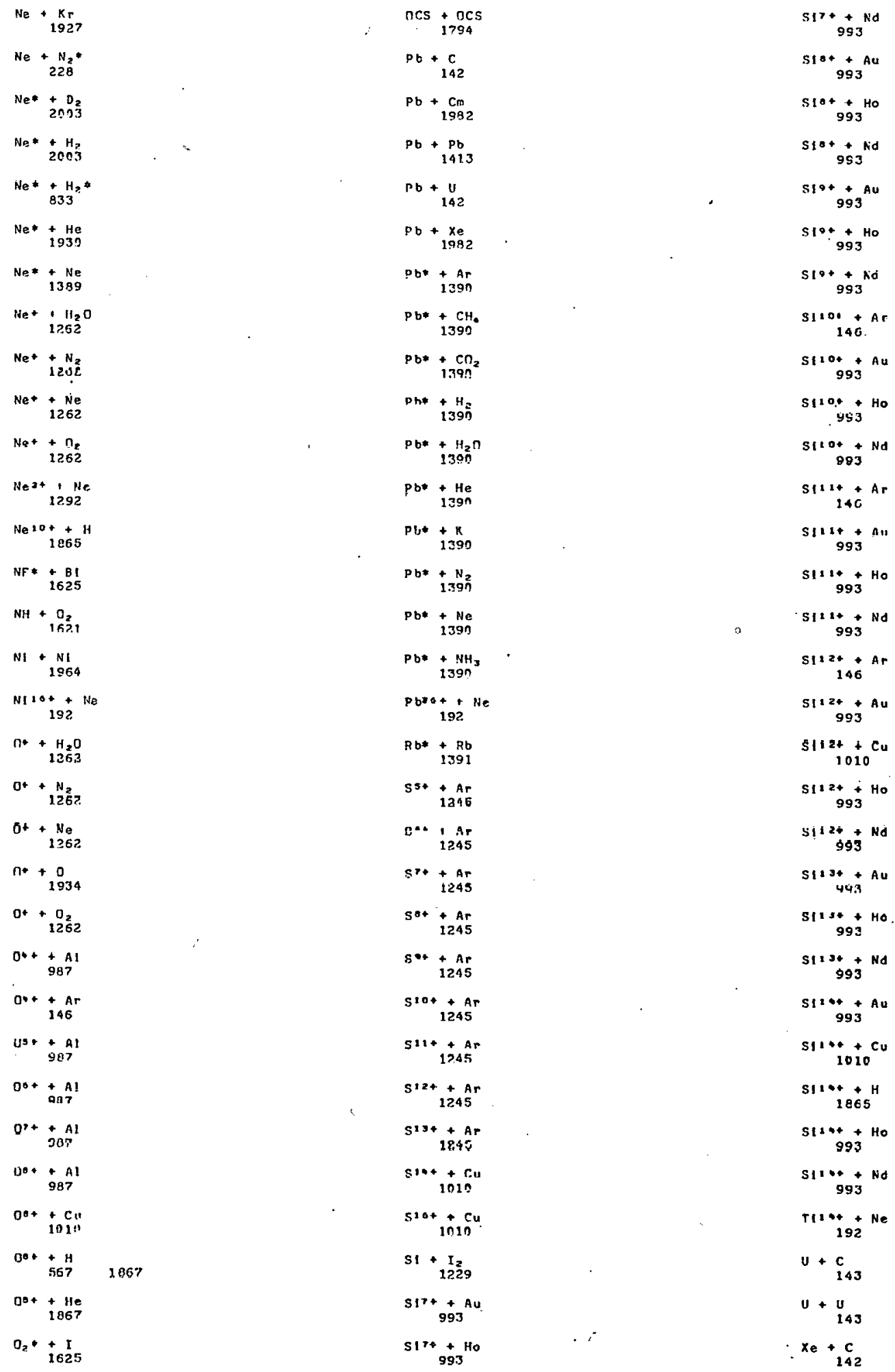




$$
\begin{aligned}
& \begin{array}{r}
\mathrm{Xe}+\mathrm{Pb} \\
\text { ABR }
\end{array} \\
& X_{0}+\text { U } \\
& \text { Ke* } \begin{array}{r}
+\mathrm{H}_{2} \mathrm{O} \\
1700^{\circ}
\end{array} \\
& \text { Xe* }+{ }^{+\mathrm{H}_{2} \mathrm{~S}}
\end{aligned}
$$

104

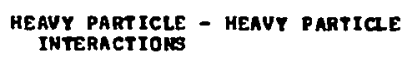

Ditasoctation

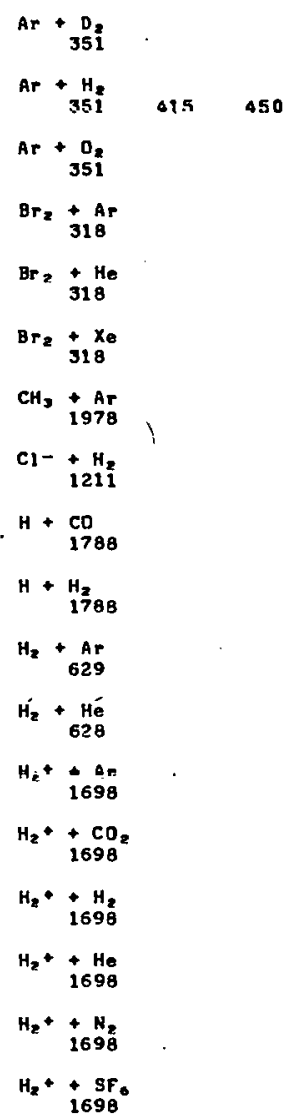

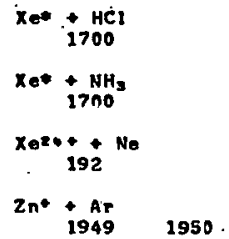

$\mathrm{H}_{2}+\underset{1698}{+}+\underset{ }{+X_{e}}$

$\mathrm{H}_{2}+\underset{684}{+} \mathrm{H}_{2}$

$\mathrm{Hg}^{*+}+\underset{1634}{\mathrm{Mg}}$

$\mathrm{HCl}+\mathrm{AP}$

He $+\mathrm{H}_{349}^{+*}$

He* $\begin{array}{r}+\mathrm{D}_{2} \\ 2 \mathrm{CO}_{3}\end{array}$

He* $+\begin{array}{r}+\mathrm{H}_{2} \\ 2003\end{array}$

$\mathrm{He}+\underset{1476}{+\mathrm{CO}_{2}}$

$\mathrm{He}^{+}+\mathrm{n}_{2}$
1435

HeHt $+\underset{2698}{+}$

$\mathrm{HeH+}+\underset{1698}{\mathrm{CO}_{2}}$

HeH* $\underset{1698}{+}$

$\mathrm{HeH}^{+}+\mathrm{He}$
1698

$\mathrm{HeH}_{169 \mathrm{~B}}^{+}$

HeH* $+\mathrm{SF}_{1698}$

HeH+ $+\mathrm{Xe}_{169}$

$H F *+F_{2}$
1980

$I_{2}+\underset{21 A}{\operatorname{Ar}}$

$I_{2}+\begin{aligned} \mathrm{He} \\ 318\end{aligned}$

$I_{2}+X_{3 e}$

LI $2+2+a^{A r}$

$41:+40$

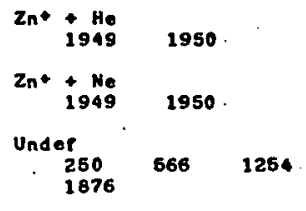

$L 1=* K_{2030}^{K r}$

$\mathrm{LI}_{2} * \underset{2030}{+}$

$L I_{2} * \underset{2030}{*} x_{e}$

$\mathrm{N}_{2}+\underset{20 \mathrm{Ar}}{+}$

$\mathrm{N}_{2}+\underset{208}{\mathrm{He}}$

$\mathrm{N}_{2}+\mathrm{H}_{20} \mathrm{~N}^{2}$

$\mathrm{Ne}+\mathrm{H}_{2}$

$\mathrm{Ne}+\underset{348}{\mathrm{He}_{2}}$ *

Ne* $+\mathrm{D}_{2}$

$\mathrm{Ne*} \underset{2003}{+} \mathrm{H}_{2}$

$\mathrm{Ne}+\underset{833}{\mathrm{H}_{2} *}$

$\mathrm{Ne}_{2}+\underset{20}{+\mathrm{He}}$

NO + NO

$\mathrm{NO}_{2}+\underset{62 \mathrm{OP}}{\mathrm{Ar}}$

$\mathrm{NO}_{2}+\underset{620}{\mathrm{He}}$

$\mathrm{NO}_{2}+\underset{62}{\mathrm{~N}}$

Nur $i$ 620 Ar

NoC $1+$ He

NoCl 620 N2

$\mathrm{O}_{2}+\underset{330}{\mathrm{Ar}}$

$\mathrm{SO}_{2}+\underset{163}{\mathrm{Ar}}$ 


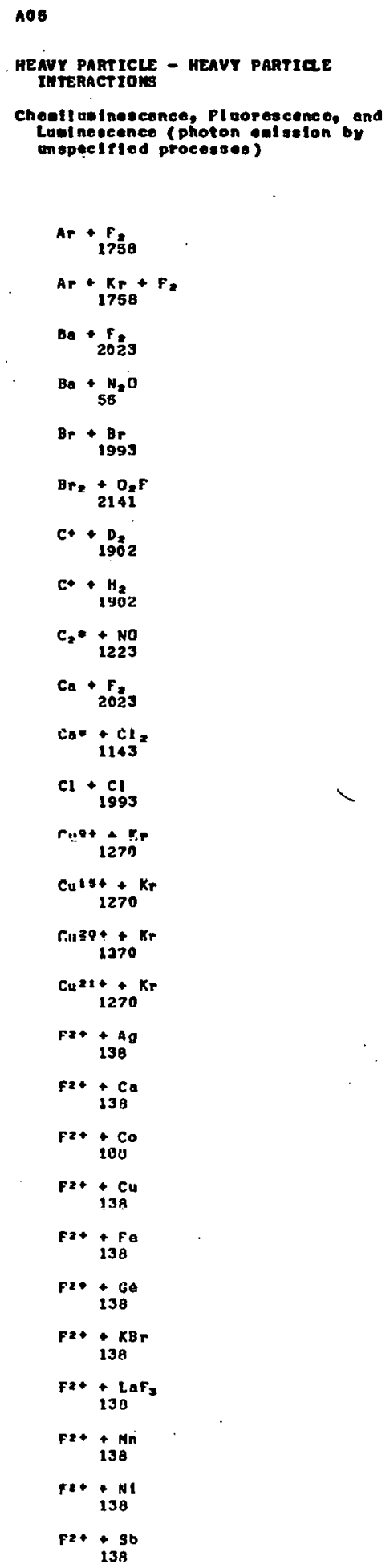

$\mathrm{F}=4$

F $2 * \underset{138}{\mathrm{ZnS}}$

F $+\underset{138}{+A g}$

Fot + Ca

$\begin{aligned} \mathrm{F}^{3+} & +\mathrm{Co}_{0} \\ & +38\end{aligned}$

$\mathrm{F}^{2+}+\mathrm{Cu}_{138}$

$\mathrm{Fa+}^{+\mathrm{Fe}}$

cat $\frac{138}{138}$

Fin $+\mathrm{KBr}$

138

F $24+\underset{138}{\text { LaF }}$

F3+ + Mn

138

F $94+N$

$\mathrm{F}^{3+}+\mathrm{3b}$

138

F $+\underset{158}{+T}$

F34 + V

$\mathrm{Fat}+7 \mathrm{~ns}$

Fo+ $+\mathrm{Ag}_{138}$

ra4
270

$\mathrm{F*+}+\mathrm{Co}_{138}$

$\mathrm{Fo+}+\mathrm{Cu}_{138}$

Fo* $+\mathrm{Fe}$

F*4. + +60

Fo. + KBr

138

Fit + taFs

For + $\mathrm{Mn}$

Fit +

Fot + Sb

138

$F+*+T$

138

Fo+ + v

Fo+ $+2 \mathrm{~ns}$

$\mathrm{FS}^{\mathrm{S}+\mathrm{Ag}}$
F** $+\mathrm{C}$

Fse + Co 138

Fs. + Cu

PEF + Pe
138

Fs+ + Ge 138

Fst ${ }^{3} \mathrm{KBr}^{\circ}$

Fs+ $\underset{138}{\operatorname{LaF}}$

Fst $\underset{138}{\mathrm{Kn}}$

P.. , 11 138

Fs+ + Sb 138

$\mathrm{Fs}^{\mathrm{st}}+\underset{138}{\mathrm{Tt}}$

F*t $v$ 138

pst+2ns 138

Fo+ + Ag

Fot $+\underset{138}{\mathrm{Cs}}$

Fot $+\mathrm{Co}$

Fot $+\mathrm{Cu}$

Fot $+\underset{138}{F e}$

Fot +60

Fot $+\mathrm{KBr}$

13

$\mathrm{FO}_{+} \mathrm{LaF}_{3}$

fot + in

Fot $+\mathrm{NL}$

Fo* + 8b

138

Fot $\frac{T 1}{138}$

fot $+v$

rat ing

$F_{2}+51$

$F_{2}+510$

$F=+51 H_{4}$

$\mathrm{B}$
$\mathrm{Br}$
161

$\mathrm{H}^{+}+\mathrm{Ar}+\mathrm{N}_{2}$ 


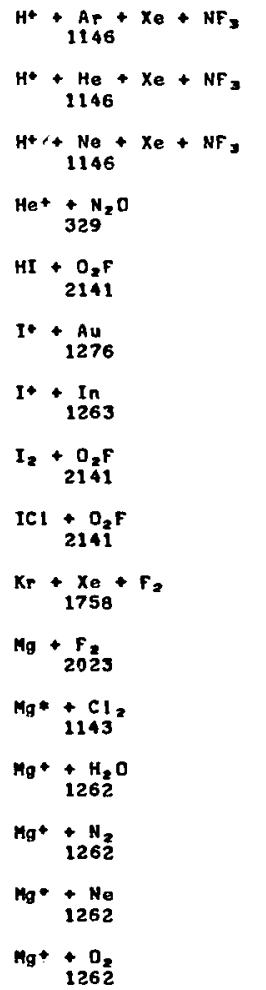

$\mathrm{N}+\mathrm{S}_{376}^{\mathrm{S}}$

$\mathrm{N}+\underset{376}{\mathrm{~S}_{2} \mathrm{Cl}_{2}}$

- $\mathrm{N*}+\mathrm{H}_{2} \mathrm{O}$

$\mathrm{N}++\frac{N_{2}}{1262}$

$\mathrm{N}+\mathrm{Ne}$

$\mathrm{N}+\mathrm{O}_{2}$

Nat $+\mathrm{H}_{2} \mathrm{O}$

$\mathrm{Na}+\mathrm{N}_{2}$
1262

$\mathrm{Nat}+\mathrm{Ne}$

$\mathrm{Net}+\mathrm{O}_{2}$
1262

Net $+\mathrm{H}_{2} \mathrm{O}$

$+\mathrm{N}_{2}$
1262

$\mathrm{Ne}++\mathrm{Ne}$

$\mathrm{Ne}+4 \mathrm{O}_{2}$

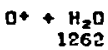

$\mathrm{O}+{ }^{+} \mathrm{N}_{2}$

O* + Ne

1262

$\mathrm{D}+\mathrm{O}_{12}$

$S+A r$

$s+H_{2}^{\prime}$

S+ $\begin{array}{ll}\mathrm{He} \\ 1126\end{array}$

$s+\frac{K r}{i .176}$

S+ $\mathrm{N}_{276}$

$s+x e$

Se $+\begin{array}{r}02 \\ 905\end{array}$

S1se $+\mathrm{C}^{\mathrm{C}}$

$\mathrm{Sr}+\underset{2023}{\mathrm{~F}}$

$\mathrm{Sr} *+\mathrm{Cl}_{1143}$

$x_{0}+\begin{array}{r}x_{e} \\ 397\end{array}$ 
AD6 HEAUY PART ICLE - HEAUY PARTICLE
INTERACTIONS

Electron Capture

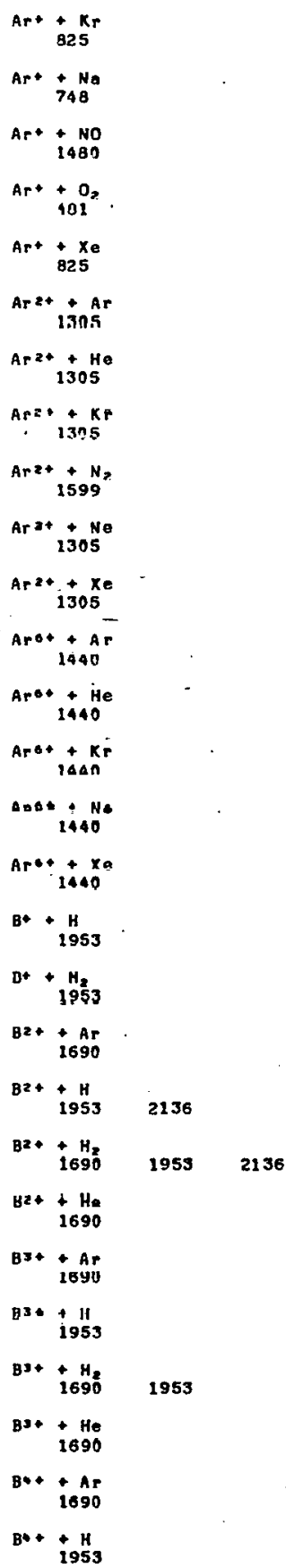

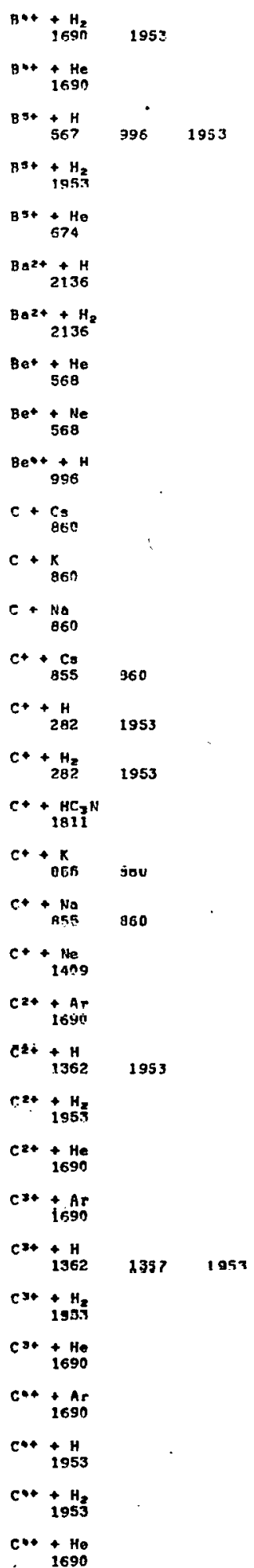

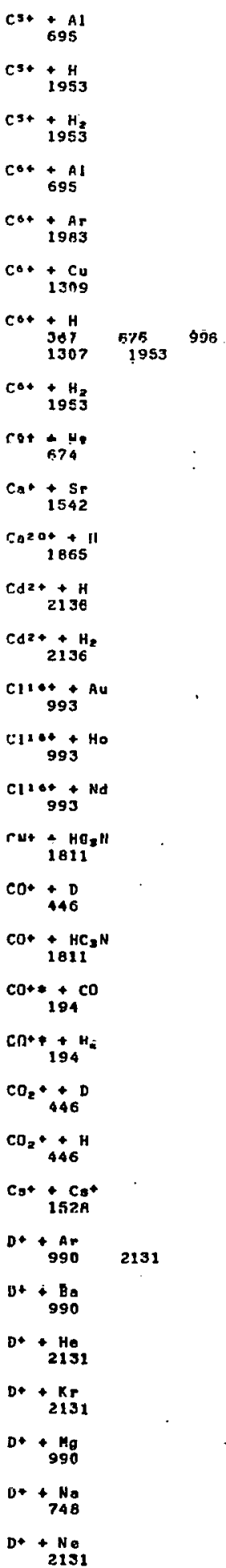

1953

1953

695

1983

$13 n 9$

$1307 \quad 1953 \quad 996$

1953

674

1542

1865

$\mathrm{Cd}^{2+}+\underset{\mathrm{H}}{\mathrm{H}}$

$\mathrm{Cd}=+\underset{2136}{+\mathrm{H}_{2}}$

$\underset{993}{C 11+4}$

C11.4+ Ho

Cl10* $+N$

$+4 \mathrm{H}^{2}$

1811

440

$\mathrm{CO}+\underset{1811}{\mathrm{HC}_{3}}$

$\mathrm{CO}+\underset{194}{\mathrm{CO}}$

$\mathrm{CO}+{ }_{194}^{+}$

$\mathrm{CO}_{2}+\underset{46}{D}$

$\mathrm{CO}_{2}+{ }_{446}^{+}$

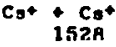

D+ $+\underset{9 P}{A+} 2131$

D+ + B

D* + He

$+K_{r}$
2131

D* + $\begin{array}{r}\mathrm{Mg} \\ 990\end{array}$

D+ $+\mathrm{No}$ 

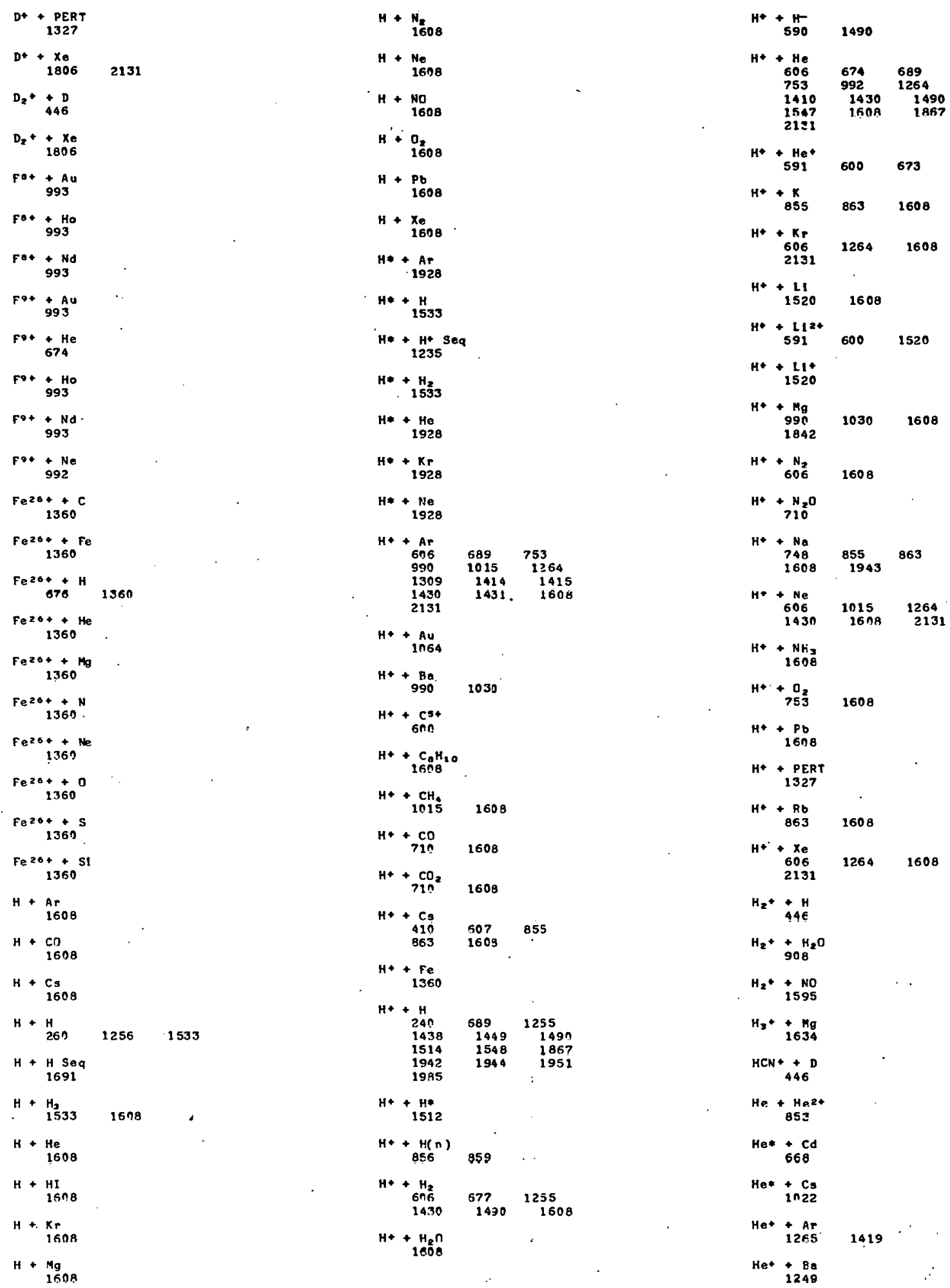


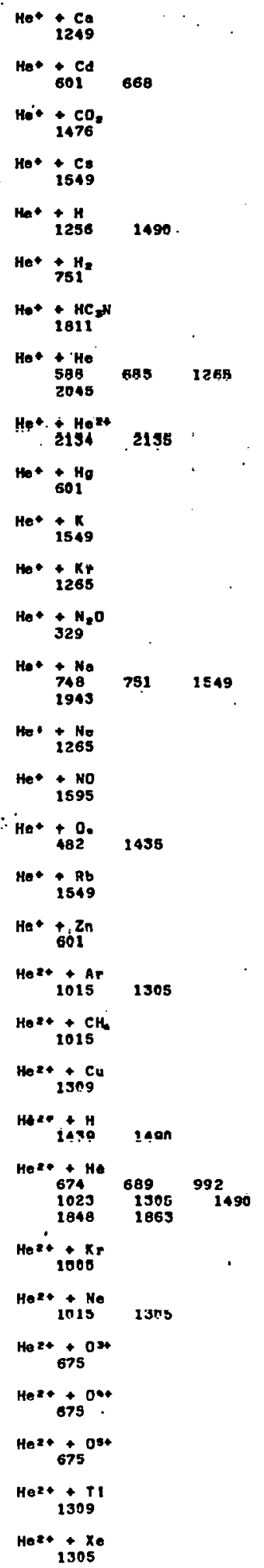

\begin{tabular}{|c|c|c|}
\hline $\begin{array}{l}\text { Net } \\
\text { Ner }\end{array}$ & $\begin{array}{l}+\mathrm{He} \\
283 \\
+\mathrm{Ne} \\
283\end{array}$ & 1690 \\
\hline$N=$ & $\stackrel{A r}{\text { Ar }}$ & \\
\hline$N=*$ & $\stackrel{+}{H}$ & 2017 \\
\hline Na* & $\stackrel{K_{0}}{+650}$ & \\
\hline Ne* & $\stackrel{A r}{A 6 s 0}$ & \\
\hline Not & $+H_{0}^{H 69}$ & . \\
\hline No * & $+\begin{array}{c}A r \\
1690\end{array}$ & \\
\hline Ho! & $\begin{array}{l}\div 1 \mathrm{lg} \\
1690\end{array}$ & \\
\hline $\mathrm{HP}$ & $+{ }^{4}$ & \\
\hline N>* & $\stackrel{+N_{0}}{992}$ & \\
\hline $\mathrm{N}_{\mathbf{2}}$ & 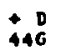 & \\
\hline $\mathrm{H}_{2}{ }^{+}$ & $\stackrel{k}{1855}$ & \\
\hline $\mathrm{N}_{3}{ }^{*}$ & $+L_{1895}^{\prime}$ & \\
\hline $\mathrm{N}_{2} *$ & $\stackrel{\mathrm{Na}}{1895}$ & \\
\hline $\mathrm{Na}$ & $\begin{array}{l}+ \text { Ne } \\
991\end{array}$ & \\
\hline Ne & $+\underset{198}{\mathrm{CH}}$ & \\
\hline Not & $\stackrel{+A=}{576}$ & 1419 \\
\hline $\mathrm{Ne}+$ & $\begin{array}{r}8 \mathrm{Ba} \\
1249\end{array}$ & \\
\hline Nö & $+{ }_{576}^{H e}$ & . \\
\hline Ne* & 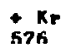 & ค2. \\
\hline Ne* & $+\mathrm{N}_{2} \mathrm{O}$ & \\
\hline $\mathrm{Ne}^{*}$ & $\dot{7}_{48}^{\mathrm{No}}$ & \\
\hline & sre & \\
\hline & $+\mathrm{O}_{2}$ & . \\
\hline $\mathrm{NoOS}$ & ${ }^{\prime}+{ }^{A r}$ & 1305 \\
\hline Neee & $+\stackrel{+H}{137 \mathrm{e}^{-}}$ & \\
\hline Meze & $+{ }^{+}+\mathrm{He}$ & $13 n 5$ \\
\hline $\mathrm{Ne}^{2}$ & ${ }_{576}{ }^{\mathrm{Kr}}$ & 1305 \\
\hline He $=0$ & $+{ }^{+}+{ }^{+N e}$ & 1305 \\
\hline Nexo & $\begin{array}{c}+x_{e} \\
1305\end{array}$ & \\
\hline Neor & $+t_{576}^{A r}$ & \\
\hline
\end{tabular}




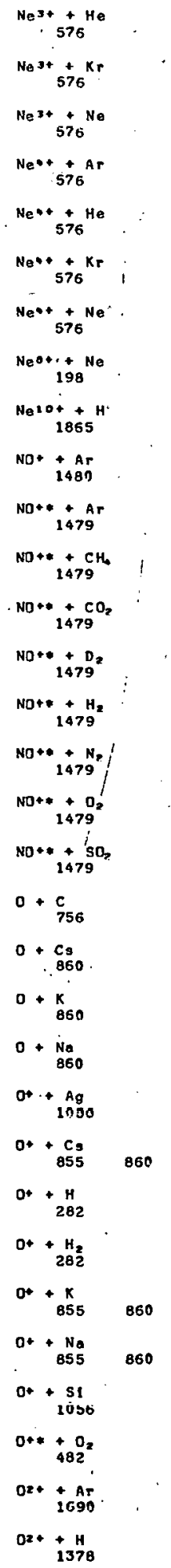

$\mathrm{Oz+}+\mathrm{He}$
1690

O3. $\begin{gathered}\text { Ar } \\ 169 \text { ? }\end{gathered}$

O $3+$ + $\mathrm{He}$

118

Do+ + Ar
1690

$\mathrm{O}+4+\mathrm{He}$

$\mathrm{Ost}+\mathrm{Ar}$

$\mathrm{O}^{5+}+\mathrm{He}$

$00++\mathrm{Ar}$

$00++\mathrm{He}$

$\mathrm{OPH}+\mathrm{He}$

Do+ + Ar

oot $\begin{array}{r}+\mathrm{Cu} \\ 13 n 9\end{array}$

$00++H$
567
185

676

$\mathrm{OOO}+\mathrm{He}$

674

$\mathrm{OAO}+\mathrm{Ne}$
$99 ?$

PERT ${ }_{746}$

PERT + H H
$13 \cap 7$

PERT + He
$13 n 7$

$s+{ }_{756}^{A 1}$

$s+c$

$s+c_{860}$

$s+k_{860}^{k}$

3. $\mathrm{Na}_{860}$

$\mathrm{St}+\mathrm{Cs}$

$s+x+\frac{260}{255}$

$\mathrm{St}+\mathrm{Na}$

So+ +Ar .. :

.201

Sot + $\mathbf{K r} \quad \because$,

$301+\mathrm{No}$

sot $+x_{e}$

201

Ge + AP

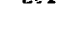

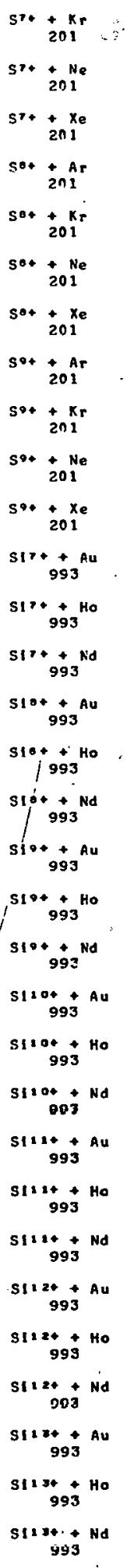

ST+
201
201

Sit 201

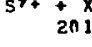

sot $\operatorname{tar}_{1}$

$\mathrm{Se}_{201}^{+K r}$

Sot 201

So+ $+x^{x e}$

Sot 201

So+ $+{ }_{2 n} 1$

So+ 201

So. 201

${ }_{993}{ }^{\mathrm{Au}}$

$\mathrm{SI}_{993}{ }^{\mathrm{HO}}$

993

${ }_{993}{ }^{\mathrm{Au}}$

Slot t Ho

$\mathrm{Siot}+\mathrm{Nd}$

siot. Au

$/ \mathrm{SLC}_{993}^{\mathrm{S10+}+\mathrm{No}}$

993

$\mathrm{S}^{210+} \mathrm{Ag3}$

$\mathrm{Sl}_{9 \mathrm{OO}}+\mathrm{HO}$

S1100+ Nd

007

993

$\mathrm{SI}^{12+}+\mathrm{HO}$

993

$\mathrm{SII}_{993}^{2+}+\mathrm{A}^{2}$

SII3+ + Ho

993

S1124.

S1125+ Au

S113t+ Ho

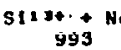




$$
\begin{aligned}
& \text { s!1 } 9+4+A 4 \\
& 5110+4+\mathrm{H} \\
& \text { S11.t+ } 674 \text { He } \\
& \text { S110. + Ho } \\
& \text { SII } 99+\text { Nd } \\
& \begin{array}{r}
\mathrm{TI}^{2+}+\mathrm{H} \\
2136
\end{array} \\
& \mathrm{Ti}^{2+}+\mathrm{H}_{2} \\
& 2136 \\
& \mathrm{UF}_{4}+\mathrm{Cs}_{424}
\end{aligned}
$$

$W F_{0}+\mathrm{Cs}_{424}$

$\mathrm{Xe}+\frac{\mathrm{Kr}}{825}$

$\mathrm{Xe}^{2+}+\mathrm{Ar}^{\mathrm{Ar}} 1305$

$\mathrm{Xe}_{295}^{2+}+\mathrm{CO}_{2}$

$\mathrm{Xe}^{2+}+\mathrm{H}_{2}$

$\mathrm{Xe}^{2+}+\mathrm{He}$

$\mathrm{Xe}^{2+}+\underset{13 n}{+} \mathrm{Kr}^{2}$

$$
\begin{aligned}
& \mathrm{Xe}^{2+}+\mathrm{N}_{2} \\
& \mathrm{Xe}^{2+}+\mathrm{Ne} \\
& \mathrm{Xe}^{2+}+\mathrm{CSS}^{\mathrm{O}} \\
& x^{2+}+x^{x e} 1305 \\
& \mathrm{Zn} 2+\frac{\mathrm{H}}{2136} \\
& \mathrm{Zn2*}+\mathrm{H}_{2} \\
& \text { Under }
\end{aligned}
$$$$
293 \quad 719
$$ 


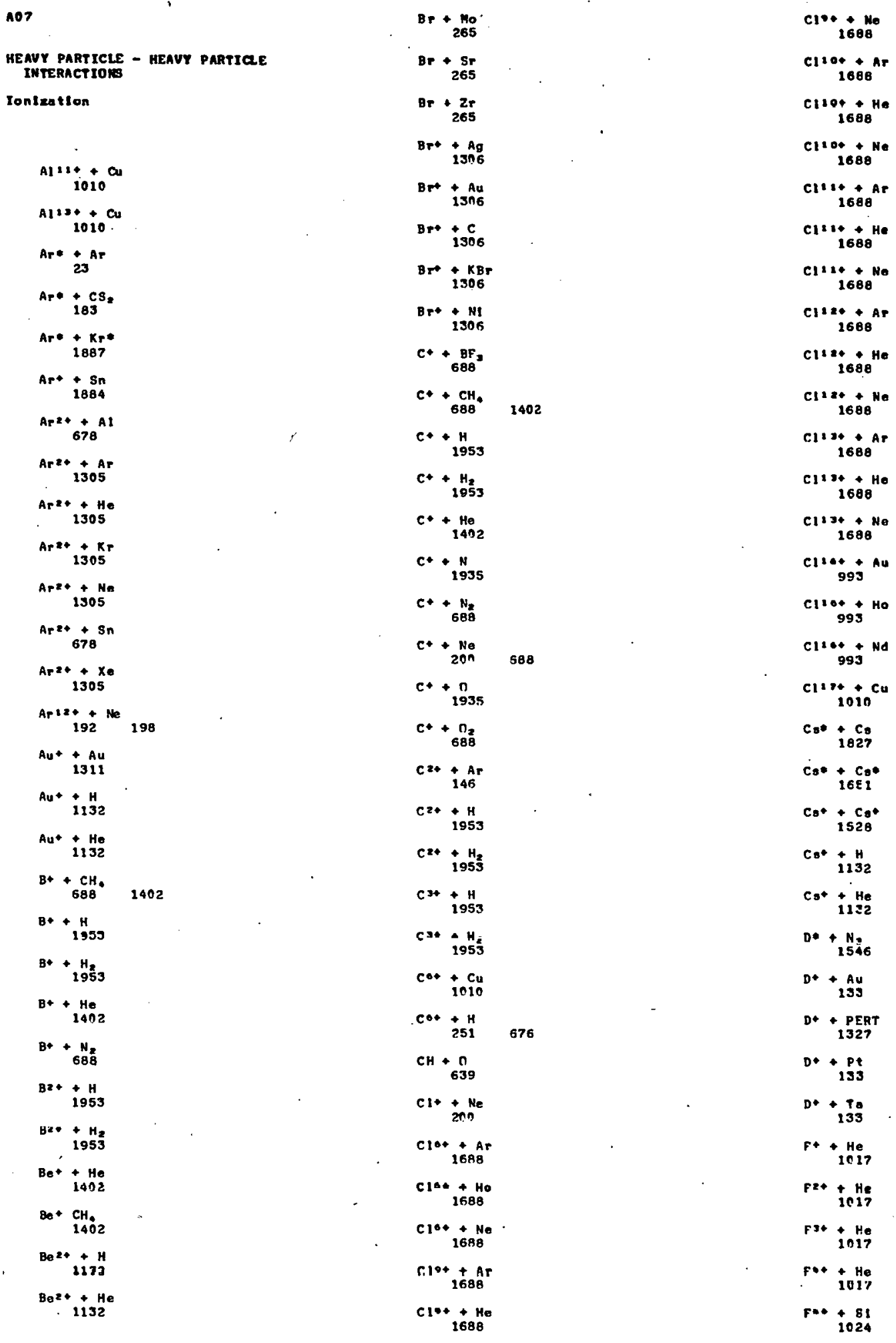


$\therefore 174$

\begin{tabular}{|c|c|}
\hline & $\begin{array}{l}+\mathrm{He} \\
101 ?\end{array}$ \\
\hline$F^{5+}$ & $\begin{array}{r}+51 \\
1024\end{array}$ \\
\hline Fo+ & $\begin{array}{l}+ \text { co } \\
1027\end{array}$ \\
\hline $\mathrm{r}+4$ & $\begin{array}{l}\text { C } \mathrm{cr} \\
1027\end{array}$ \\
\hline fot & $\begin{array}{l}+\mathrm{Fe} \\
\text { in27 }\end{array}$ \\
\hline $\mathrm{Fot}^{6+}$ & $\begin{array}{r}+\mathrm{He} \\
1017\end{array}$ \\
\hline Fo+ & $\begin{array}{l}+51 \\
1024\end{array}$ \\
\hline$r *+$ & $\begin{array}{l}1 \\
\text { in? }\end{array}$ \\
\hline rod & $\begin{array}{c}10 y \\
1027\end{array}$ \\
\hline$F^{\prime+}$ & $\begin{array}{l}+ \text { Co } \\
1027\end{array}$ \\
\hline F'* & $\begin{array}{l}+\mathrm{Cr}_{\mathrm{r}} \\
1027\end{array}$ \\
\hline$F^{\prime+}$ & $\begin{array}{l}+\mathrm{Cu} \\
1010\end{array}$ \\
\hline Fr* & $\begin{array}{l}+F e \\
1027\end{array}$ \\
\hline$F^{\prime+}$ & $\begin{array}{r}+\mathrm{He} \\
1017\end{array}$ \\
\hline$F^{\prime+}$ & $\begin{array}{l}+5 t \\
1024\end{array}$ \\
\hline Fit & $\begin{array}{l}+T 1 \\
1027\end{array}$ \\
\hline Fr* & 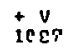 \\
\hline por & $\begin{array}{l}\text { Hu } \\
99.3\end{array}$ \\
\hline ral & $\begin{array}{l}\text { in } c u \\
\text { in }\end{array}$ \\
\hline $\mathrm{FO}^{\mathrm{O}}$ & $\begin{array}{l}+\mathrm{Cr} \\
\ln 27\end{array}$ \\
\hline $\mathrm{FB}^{\mathrm{B}+}$ & $\begin{array}{l}+\mathrm{cu} \\
1010\end{array}$ \\
\hline $\mathrm{F}^{\mathrm{B}+}$ & $\begin{array}{l}+\mathrm{Fe} \\
1027\end{array}$ \\
\hline $\mathrm{FAt}^{-}$ & $\begin{array}{r}+\mathrm{Ho} \\
1017\end{array}$ \\
\hline fo+ & $\begin{array}{l}+\mathrm{HO} \\
993\end{array}$ \\
\hline $\mathrm{Fat}$ & $\begin{array}{l}+\mathrm{Nd} d \\
\mathrm{ge} 3\end{array}$ \\
\hline Fo+ & $\begin{array}{l}+51 \\
\ln 24\end{array}$ \\
\hline For & $\begin{array}{l}+11 \\
1027\end{array}$ \\
\hline Fot & $\begin{array}{l}+v \\
\text { in } 2 \text { ? }\end{array}$ \\
\hline Fo+ & $\begin{array}{l}+\mathrm{Au} u \\
993\end{array}$ \\
\hline $\mathrm{Fo*}$ & $\begin{array}{l}+\mathrm{Cu} \\
1010\end{array}$ \\
\hline $\mathrm{Fat}$ & $\begin{array}{r}+\mathrm{He} \\
1017\end{array}$ \\
\hline Fa. & $\begin{array}{l}+\mathrm{HO} \\
993\end{array}$ \\
\hline
\end{tabular}

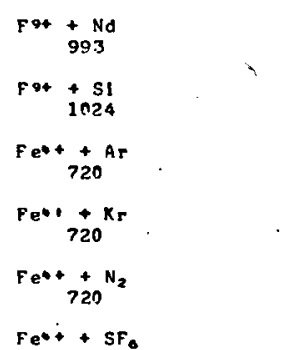

$72 \mathrm{n}$

$\mathrm{Fent}_{720} \times$

reas: 67 "

11 I $\mathrm{A}, \mathrm{CB}$

$H+{ }_{260}^{H} \quad 1255 \quad 1533$

$\mathrm{H}+\underset{1323}{\mathrm{H} \text { Seq }}$

$\mathrm{H}+\mathrm{H}_{2}$

$H+\frac{H \cdot}{2132}$

$\mathrm{H}+\underset{\mathrm{He}}{\mathrm{H} 23}$

$\mathrm{H}+\mathrm{Xe}_{2132}$

$H *+\underset{1533}{H}$

$\mathrm{H*}+\underset{1050}{\mathrm{H*}}$

$H=+\mathrm{He}^{2}$

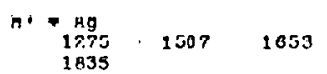

$\begin{array}{rll}\mathrm{H}^{+}+\underset{1415}{A r} & 1415 & 1451 \\ 1445 & 1697 & 1960\end{array}$

$\mathrm{H}^{+}+\underset{15 \mathrm{As}}{\mathrm{An} 7} \quad 1835$

$\mathrm{H}++{ }_{76}^{A 4} 1275 \quad 2130$

$\mathrm{H}+\underset{15 \mathrm{Kr}}{\mathrm{Kr}} \quad 1835$

$H+4 \underset{1510}{r}$

$\mathrm{H}^{+}+\mathrm{Ca}$

7 1

$\mathrm{H}^{+}+\underset{\mathrm{BES}}{\mathrm{Cd}} 2607 \quad 1938$

$\mathrm{H}^{+}+\underset{76}{\mathrm{Ce}}$

$\mathrm{H}+\underset{1015}{\mathrm{CH}_{4}}$

$\mathrm{H}++\mathrm{Co}$

1835

$\mathrm{H}^{+}+\underset{1507}{\mathrm{Cr}} \quad 1835$

$\mathrm{H}+\mathrm{Cs}$

$$
\begin{aligned}
& \begin{array}{ccc}
\mathrm{H}^{+}+\mathrm{Cu}_{76} & \\
2653 & 1010 & 1507 \\
1835 &
\end{array} \\
& \mathrm{H}^{+}+\underset{2130}{\mathrm{Dy}} \\
& \begin{array}{ccc}
H++F e & \\
+66 & 1507 & 1835 \\
1856 & &
\end{array}
\end{aligned}
$$

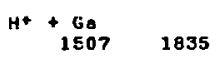

$$
\begin{aligned}
& \mathrm{H}^{+}+\underset{\mathrm{Gd}}{1833} \\
& \begin{array}{c}
\mathrm{H}+\mathrm{Ge} \\
1507 \quad 1835
\end{array} \\
& \mathrm{H}+11 \mathrm{IJ} \\
& \text { II' }+112 \\
& \mathrm{H}^{*}+\underset{1132}{\mathrm{He}} \quad 1410 \\
& H^{+}+\underset{76}{\mathrm{Hn}} \quad 1864 \\
& \mathrm{H}^{*}+\underset{1507}{\mathrm{In}} \quad 1835 \\
& H^{*+K r} \begin{array}{lll}
K 445 & 1507 \quad 1836
\end{array} \\
& \mathrm{H}+\underset{1842}{\mathrm{Mg}} \\
& H^{*}+\operatorname{Mnn}_{i \operatorname{sn} 7} \quad 1835
\end{aligned}
$$

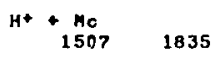

$$
\begin{aligned}
& \mathrm{H}+\underset{1510}{N} \\
& \mathrm{H}^{*}+\underset{16}{\mathrm{~N}} \mathrm{~g}_{2} \\
& \mathrm{H}^{+}+\underset{1943}{\mathrm{Na}} \\
& \mathrm{H}^{*}+\mathrm{Nb} \\
& 1507 \quad 1835
\end{aligned}
$$

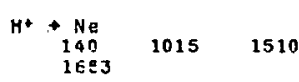

$$
\begin{aligned}
& \mathrm{H}^{+}+\begin{array}{c}
\mathrm{NI} \\
1507
\end{array} \\
& \begin{array}{c}
\mathrm{He}^{2}+ \\
+0 \\
1510
\end{array}
\end{aligned}
$$

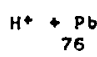

$$
\begin{aligned}
& \mathrm{H}^{+}+\underset{1007}{\mathrm{Pd}} \quad 1095
\end{aligned}
$$

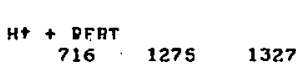

$$
\begin{aligned}
& \text { 11. } \begin{array}{l}
n e \\
15 n 7
\end{array} 1835
\end{aligned}
$$

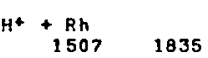

$$
\begin{aligned}
& \begin{aligned}
H++R_{4} \\
15 n 7 \quad 1835
\end{aligned} \\
& \mathrm{H}^{*}+\underset{\mathrm{Sc}}{1507} \quad 1835 \\
& \mathrm{H}^{+}+\underset{76}{\mathrm{Se}} \quad 1507 \quad 1835 \\
& \mathrm{H}^{+}+\underset{76}{\mathrm{~S} 5} \mathrm{IS64}
\end{aligned}
$$




\begin{tabular}{|c|c|c|}
\hline $\mathrm{H}^{+}+\underset{76}{5 \mathrm{Sn}}$ & $150 ?$ & 1235 \\
\hline $\mathrm{H}^{+}+\underset{1507}{\mathrm{Sr}}$ & 1835 & \\
\hline H' + Th & & \\
\hline $\begin{aligned} \mathrm{H}^{+}+ & \mathrm{TI} \\
& 76 \\
& 1856\end{aligned}$ & 1507 & 1835 \\
\hline $\mathrm{H}^{+}+\underset{1833}{\mathrm{II}}$ & & \\
\hline $\mathrm{H}^{+}+\underset{1507}{\mathrm{~V}}$ & 1835 & \\
\hline $\mathrm{H}^{+}+\underset{76}{\mathrm{H}}$. & 1833 & \\
\hline $\begin{array}{r}\mathrm{H}^{+}+\mathrm{Xe}_{\mathrm{e}} \\
2.130\end{array}$ & & \\
\hline $\begin{array}{r}\mathrm{H}+\underset{76}{\mathrm{Y}} \\
76\end{array}$ & 1507 & 1835 \\
\hline $\begin{aligned} H++ & \mathrm{Zn} \\
& 850 \\
& 1856\end{aligned}$ & 1507 & 1835 \\
\hline $\mathrm{H}^{+}+\underset{1507}{2 \mathrm{r}}$ & 1835 & \\
\hline $\mathrm{H}_{2} * \underset{1641}{\mathrm{Ar}}$ & & \\
\hline $\mathrm{H}_{2} *+\begin{array}{r}\mathrm{He} \\
1641\end{array}$ & & \\
\hline $\mathrm{H}_{2} * \begin{array}{c}+\mathrm{Xr}_{\mathrm{r}} \\
1641\end{array}$ & & \\
\hline $\begin{array}{r}\mathrm{H}_{2}^{*}+\underset{\mathrm{Ne}}{\mathrm{Na}} \\
1641\end{array}$ & & \\
\hline $\mathrm{H}_{2} * *+\mathrm{H}_{8.64}^{+\mathrm{H}_{2} \mathrm{O}}$ & & \\
\hline $\mathrm{H}_{2} * *+\mathrm{SF}_{864}$ & & \\
\hline $\mathrm{H}_{2}+\underset{1960}{+\mathrm{Ar}}$ & & \\
\hline $\mathrm{H}_{2}+\underset{1152}{N_{2}}$ & & \\
\hline $\begin{array}{c}\mathrm{He} *+\mathrm{Ar} \\
517\end{array}$ & 546 & \\
\hline $\begin{array}{r}\mathrm{He} * \mathrm{Ba} \\
1527\end{array}$ & & \\
\hline $\mathrm{He}+\underset{\mathrm{Ca}}{\mathrm{Ca}}$ & & \\
\hline $\mathrm{He}+\underset{668}{+\mathrm{Cd}}$ & $2 n 03$ & \\
\hline 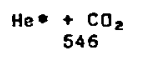 & & \\
\hline $\begin{array}{r}\mathrm{He} *+\mathrm{Cs} \\
1022\end{array}$ & & \\
\hline $\mathrm{He} *+\underset{1248}{H}$ & & \\
\hline 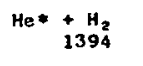 & & \\
\hline $\mathrm{He*}+\underset{2003}{+}$ & & . \\
\hline $\mathrm{He}+\underset{546}{+N_{2}}$ & & \\
\hline $\begin{array}{l}\mathrm{He} *+\mathrm{N}_{56} \mathrm{NO} \\
\end{array}$ & & \\
\hline $\mathrm{He} * \underset{546}{+\mathrm{NO}}$ & & \\
\hline
\end{tabular}

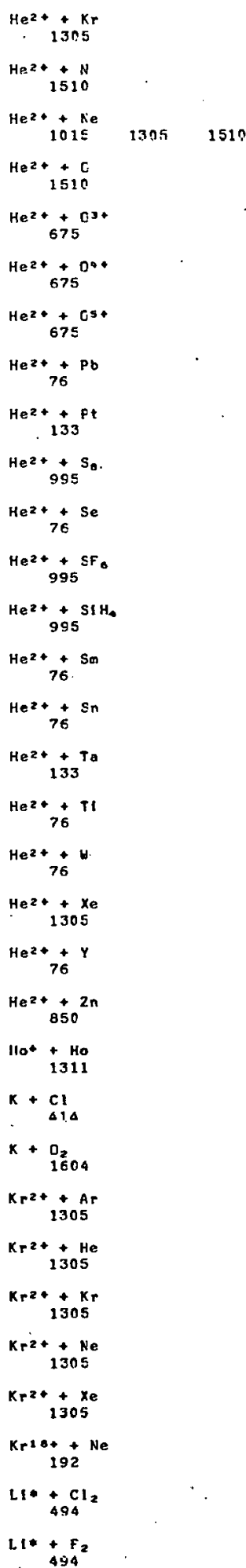




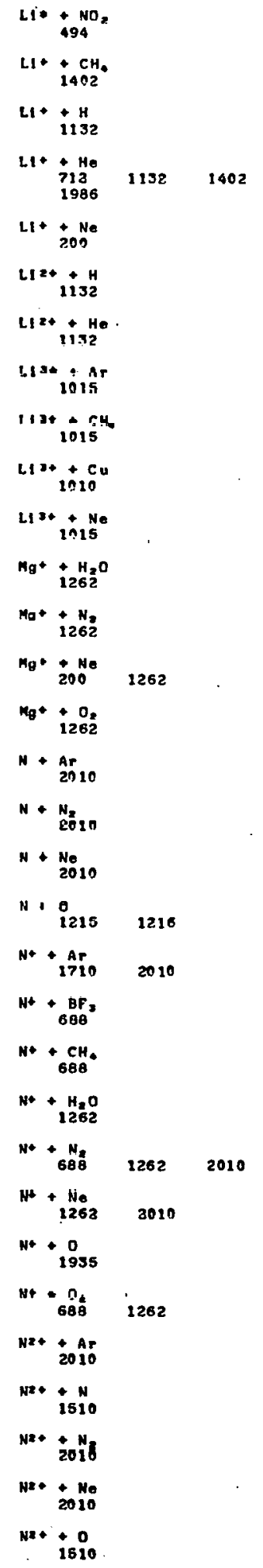

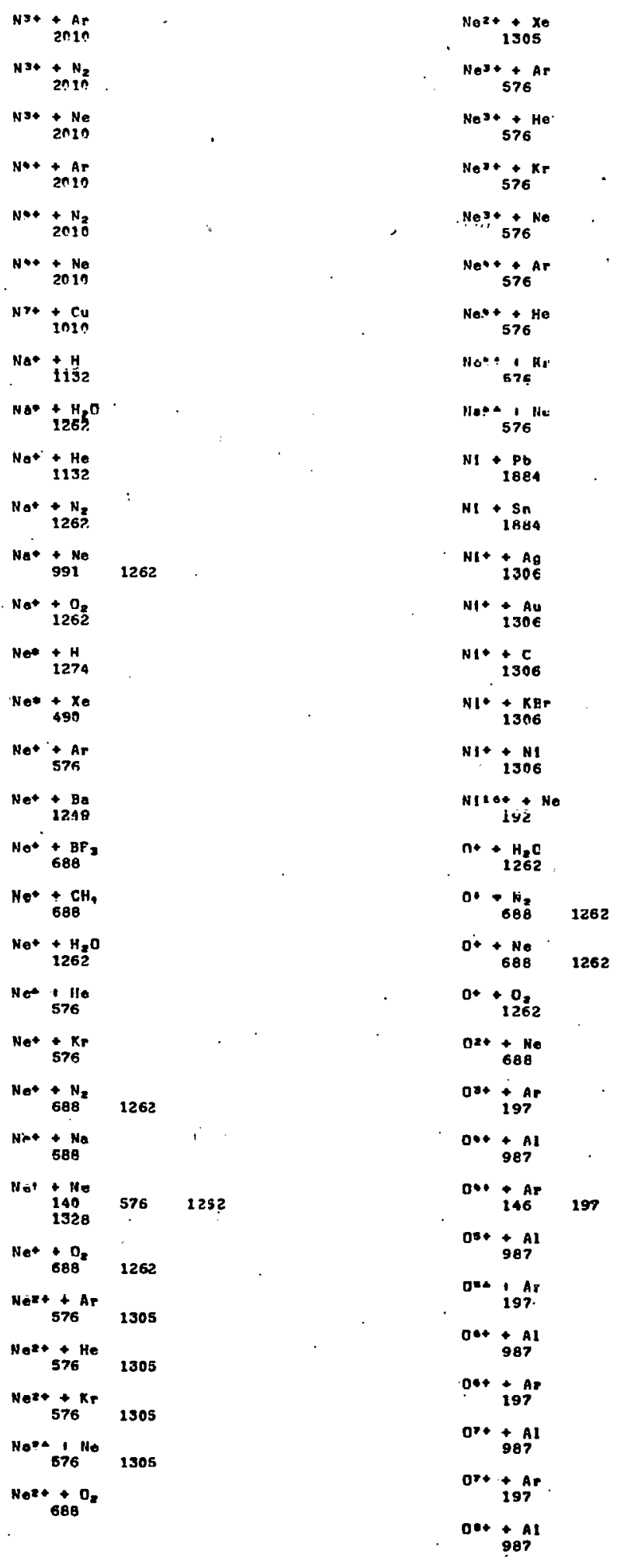




\begin{tabular}{|c|c|}
\hline $00++A r$ & \\
\hline${ }^{00+}+c_{0}$ & \\
\hline $00 * \underset{676}{111}$ & 1013 \\
\hline $\mathrm{PH}+\underset{2 \mathrm{No}}{\mathrm{No}}$ & \\
\hline $\mathrm{Pe+}+\underset{200}{\mathrm{Ne}}$ & \\
\hline $\mathrm{Pb}+\stackrel{1312}{A g}^{A}$ & \\
\hline $\mathrm{Pb}+\underset{142}{c}$ & \\
\hline $\mathrm{Pb}+\mathrm{Pb}$ & \\
\hline $\mathrm{Pb}+\operatorname{Sm}_{1312}$ & \\
\hline $\mathrm{Pb}+\underset{1312}{\mathrm{Sn}}$ & \\
\hline $\mathrm{Pb}+\mathrm{U}_{142}^{\mathrm{U}}$ & 1312 \\
\hline$P b++\frac{P b}{1311}$ & \\
\hline$\underset{192}{\mathrm{~Pb}}$ & \\
\hline $\begin{array}{r}\mathrm{Rb} * \begin{array}{r}\mathrm{Rb} \\
2004\end{array} \\
0\end{array}$ & \\
\hline sint $+\mathrm{cu}_{1010}$ & \\
\hline sios $+\underset{1010}{c c u}$ & \\
\hline $\mathrm{SIT+}_{993}^{+A \mathrm{u}}$ & \\
\hline $\mathrm{SITH}_{993}^{+\mathrm{HO}}$ & \\
\hline $\mathrm{SI}_{993}^{+\mathrm{Nd}}$ & \\
\hline SIOA $+\underset{993}{A U}$ & \\
\hline $\mathrm{SlOH}_{993}^{+\mathrm{HO}^{\circ}}$ & \\
\hline $\mathrm{SIOA}_{993}^{+\mathrm{Nd}}$ & \\
\hline $\mathrm{CI}_{993}^{\mathrm{Au}}$ & \\
\hline $\mathrm{Sl}^{\mathrm{O}+}+\mathrm{H93}^{\mathrm{HO}}$ & \\
\hline
\end{tabular}

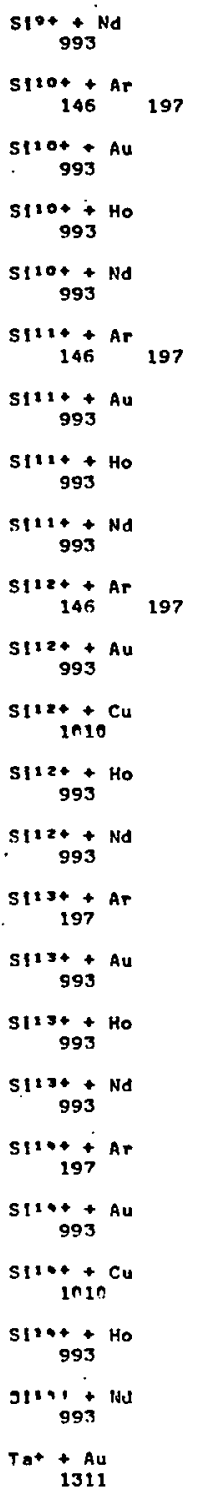

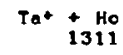

$\begin{aligned} &+P b^{\circ} \\ & 1311\end{aligned}$

Tot +59
1311

$+5 n$
1311

Tat $+T_{13 i}$

$\begin{aligned} & +\mathrm{Th} \\ 1311 & \end{aligned}$

Tilet+ Ne

$u+c$

$u+c e$

$u+u$

U*+Th

$\mathrm{UF}_{0}+\mathrm{Cs}_{424}$

$\mathrm{HF}_{\mathrm{s}}+\mathrm{Cs}_{424}$

$x e+142$

$\mathrm{Xe}+\mathrm{Pb}$
$868 \quad 1312$

$x_{e}+U_{142} \quad 1312$

$\mathrm{Xe}^{2+}+\underset{1305}{\mathrm{Ar}}$

$\mathrm{Xe}^{24}+\mathrm{He}$

$x e^{2+}+\operatorname{kins}$

$\mathrm{Xe}^{2+}+\mathrm{Ne}$

1305

$x^{2+}+x_{e}$
$130 E$

$\mathrm{Xe}^{204} 192 \mathrm{Ne} 198$

Under $\begin{array}{lll}250 & 292 & 1250\end{array}$ 
$A 08$

HEAVY PARTICLE - HEAVY PARTICLE
INTERACTIONS

stripping

$\mathrm{Be}++\mathrm{CH}$

$\mathrm{Be}+\begin{gathered}+\mathrm{He} \\ 199\end{gathered}$

$\mathrm{Br} *+\mathrm{N}_{2} \mathrm{O}$

$D++x e$

$2^{+}+x_{e}$

Fe $20+t+H$

Fol $+1+H$

Fez2t+ +11

$\mathrm{Fe}^{23+}+1360$

$\mathrm{Fe}^{2+*+\mathrm{H}}$

$\mathrm{Fe}^{25+}+\mathrm{H}$

1360

$H+{ }_{1608}$

$\mathrm{H}+\mathrm{CH}_{168}$

$H+\underset{1600}{C D}$

$\mathrm{H}+\mathrm{CO}_{168}$

$H+\mathrm{Cs}_{\mathrm{s}}$

$H+\underset{260}{H}$

1256

$\mathrm{H}+\mathrm{H}_{2}$

$\mathrm{H}+\mathrm{H}_{2} \mathrm{O}$

H+ He 1608 2132

H + HI

$H+K_{r}$

$\mathrm{H}+\mathrm{Mg}_{1000}$

$H+\underset{1608}{N_{2}}$

$H+\mathrm{Ne}$
1608

H+ NH $_{\text {IfกA }}$

$H+\underset{1608}{160}$

$H+\begin{aligned} & \mathrm{O}_{2} \\ & 1608\end{aligned}$
$\mathrm{H}+\mathrm{Pb}$

ox+ AT

$H+X_{e}$
1698

2132

$\mathrm{H}^{-}+\underset{1608}{\mathrm{Ar}}$

$H^{-}+\underset{1600}{\mathrm{CO}}$

$H-+\underset{1608}{C g}$

$H+{ }_{1608}^{\mathrm{H}_{2}}$

$\mathrm{H}-+\underset{1600}{\mathrm{He}}$

$\mathrm{H}-+\underset{1608}{\mathrm{Kr}}$

if $: \frac{H y}{1608}$

$+\frac{N_{2}}{1608}$

$\mathrm{H}-+\mathrm{Ne}$

$\mathrm{H}^{-}+\mathrm{NO}$

$H-+O_{2}$
1608

$\mathrm{H}^{-}+\underset{1608}{\mathrm{Xe}^{2}}$

Het $+\underset{1256}{H}$

$\mathrm{He}+\mathrm{He}$
$\mathrm{es} 4$

$\mathrm{He}^{2+}+\mathrm{LI}$

$\mathrm{i}++\mathrm{N}_{2}$

I2*+ $+\mathrm{N}_{2}$

I $34+\begin{array}{r}+\mathrm{N}_{2} \\ 1834\end{array}$

$+\cdots+\mathrm{N}_{2}$

$I^{3+}+\mathrm{N}_{2}$

I*. $\operatorname{ing}_{15 n}$

$I_{10+}^{+N_{2}}+\underset{1506}{2}$

$\frac{120+\underset{15 n}{+} \mathrm{N}_{2}}{2}$

$130+\underset{15 n 6}{*} \mathrm{~N}_{4}$

I th+ $\underset{1506}{+} \mathrm{N}_{2}$

$150+\underset{1506}{+N_{2}}$

$\mathrm{Is1+}+\mathrm{N}_{2}$
1506

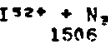

$\mathrm{LI}+\underset{191}{\mathrm{CH}}$

$\underset{191}{+}$
$03+\begin{gathered}H \\ 1360\end{gathered}$

O*+ $197^{\text {AT }}$

$04+\underset{1360}{H}$

0 + 197

Os+ $+\underset{1360}{H}$

$00++$ Ar
107

$00+\underset{1360}{+H}$

QT) 197

$0 \%+\underset{1360}{+}$

Oot + Ar

Sot
201

So* $+\underset{201}{K r}$

Sot $\underset{201}{+N e}$

S*+ $+x_{20}$

Srt + Ar

S?* $+\mathrm{Kr}$

$\mathrm{Set}+\mathrm{Ne}$

$37+x_{0}$

So+ $+{ }_{201}^{A r}$

Sot t Kr

Sot + Ne

sot tyo

Sot + Ar

$30+\mathrm{Kr}^{\mathrm{Kr}}$

So+ $+\mathrm{Ne}_{201}$

$51+10$

$51104+A$

197

S111. Ar

S1:24+Ar

S113* + A r

S11 +2. A 


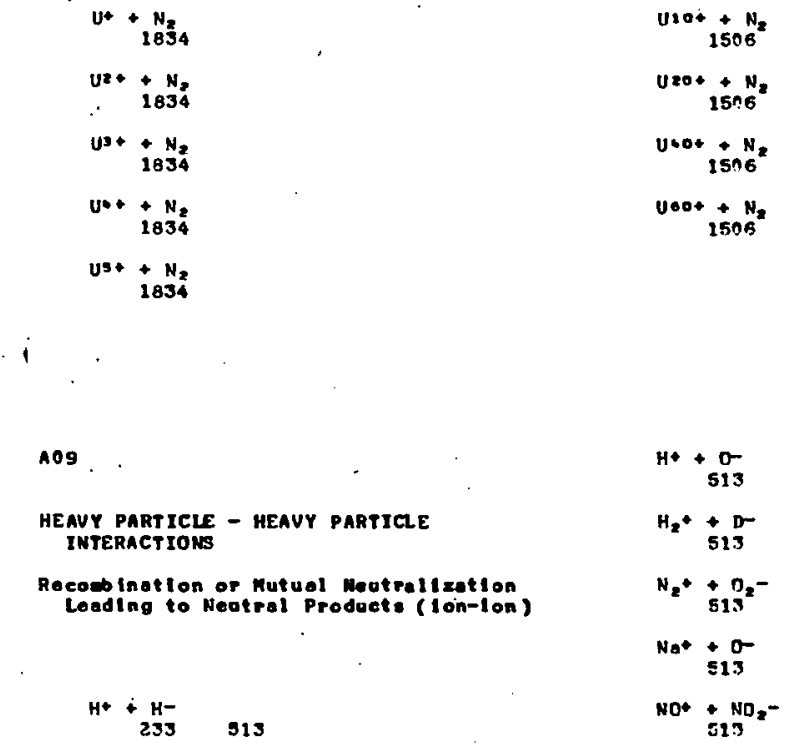

$\stackrel{.00++N_{2}}{1506}$

$1100++k^{k}$,
1506

$000++k_{2}$

$\frac{1501+\mathrm{N}_{2}}{1506}$

$\mathrm{Nn}+\ldots \mathrm{NO}_{3}-$

NO+ +0

D+ +513

$\mathrm{O}_{2}+\mathrm{C}^{2}$ 


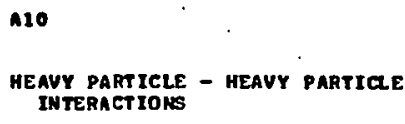

Electronle, Vibratlonal, and Rotatlonel

Energy Trons fer (process unknown)

Ar $+N_{393}$

$A r *+\underset{458}{+} \mathrm{N}_{2}$

$\mathrm{BaCl}+\mathrm{Ar}$

$\mathrm{BaCl} \underset{\mathrm{id}}{+} \mathrm{Ho}$

Bacl $+N_{2}$

$\mathrm{Br}+\stackrel{+\mathrm{D}_{2}}{521}$

Br* $+\mathrm{H}_{\mathbf{5}}$

$B r *+$ HD

$\mathrm{Br}=+\mathrm{N}_{2} \mathrm{O}$

$\mathrm{CO}+\underset{\mathrm{CO}}{1473}$

$\mathrm{CO}+{ }_{7}^{\mathrm{CO}} 9 \mathrm{n} 3$

$\mathrm{CO} *+\mathrm{CO}_{3}$

$\mathrm{CO}_{2}+\mathrm{Ar}$

$\mathrm{CO}_{7} *{ }_{184}^{+A \mathrm{r}}$

$\mathrm{CO}_{2} \cdot+\mathrm{H}_{2}$

$\mathrm{CU}_{2} \mathrm{O}+\mathrm{He}$

$\mathrm{CO},+\underset{184}{+K r}$

$\mathrm{CO}_{7} *+\mathrm{Ne}$

$\cos _{2}+x_{29}$

$\mathrm{HB}$
T5A

$D F+\frac{n F}{441}$

DF* $+\mathrm{CO}_{285}$

$\mathrm{DF} *+\underset{405}{+\mathrm{D}_{2}}$

$D F * \underset{485}{+} \mathrm{H}_{2}$

bF* + + HF

$\mathrm{DF} *+\mathrm{N}_{4}$

$F+H_{459}^{+}$
$F+H_{3}$

408

$F+\begin{aligned} & x e \\ & 459\end{aligned}$

$F *+\underset{1226}{H_{2}}$

$H+\underset{1068}{\mathrm{CN}}$

$H+F_{2}$

$H+\underset{1068}{N}$

$H *+\underset{1239}{\text { Ar }}$

H. 篻产

H* " Ilo

$H *+\underset{K r}{K}$

$\mathrm{H} *+\mathrm{Ne}$

$\mathrm{H}_{2}+\mathrm{H}_{2}$

$\begin{array}{lll}332 & 347 & 1473\end{array}$

$\mathrm{H}_{2}+\underset{332}{\mathrm{HBr}}$

$\mathrm{H}_{2}+\underset{1232}{\mathrm{HCl}}$

$\mathrm{H}_{\mathbf{2}}+\underset{628}{\mathrm{He}}$

$\mathrm{H}_{2}+\mathrm{LI}_{1}+$

$\mathrm{HBr}+\mathrm{CO}_{2}$

$\mathrm{Br}+\mathrm{N}_{2} \mathrm{O}$

411

HCl + HBP

$\mathrm{HCl}+\mathrm{HCl}$

$\mathrm{HCl}+\mathrm{HF}$

$\mathrm{HCI}+\mathrm{HI}$

IICI* 21

624

$\mathrm{HO}_{\mathrm{O}}+\underset{21}{\mathrm{H}}$

$\mathrm{He}+\underset{463}{\mathrm{H}_{2}}$

$\mathrm{He}+\mathrm{Ar}^{+}$

$\mathrm{He}+\underset{53}{+\mathrm{CO}}$

$\mathrm{He}+\mathrm{CO}_{2}$

$\mathrm{He*}+\mathrm{H}_{2}$

$\mathrm{He}+\mathrm{H}_{2} \mathrm{H}$

$\mathrm{He}_{53}^{+} \mathrm{HRT}$

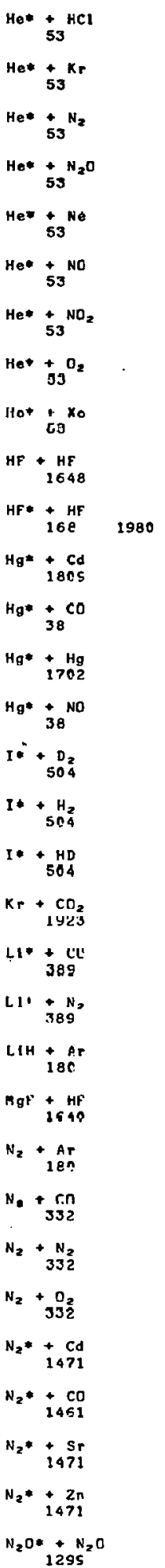

He* $+{ }_{53} \mathrm{HCl}$

He* $+\underset{53}{K}$

$\mathrm{He} * \leftarrow_{3} \mathrm{~N}_{2}$

$\mathrm{He*}+\mathrm{N}_{2} \mathrm{O}$

$\mathrm{He}+\mathrm{S3}_{3}$

$\mathrm{He}+{ }_{53}^{\mathrm{NO}}$

$\mathrm{He}+\mathrm{53}_{2}$

$\mathrm{He}+\mathrm{J3}_{3}$

Ifo+ : ${ }^{x 0}$

$\mathrm{HF}+\mathrm{HF}$

$\mathrm{HF} *+\mathrm{HF}$

I* $\mathrm{HO}_{\mathrm{SO}}^{\mathrm{H}}$

$I *+\quad H D$

$\mathrm{Kr}+\underset{1 \mathrm{YO}^{2} 3}{\mathrm{CO}_{2}}$

$410+\mathrm{C}$

L1. $\div$ N,

$\mathrm{LIH}+\mathrm{Ar}$

ingt + HF

$N_{2}+A T$
$18 ?$

N. $+\mathrm{rn}$

$N_{2}+N_{2}$

$\mathrm{N}_{2}+\mathrm{O}$

$\mathrm{N}_{2} *+\mathrm{Cd}$

$\mathrm{N}_{2} * \mathrm{CO}$

$\mathrm{N}_{2} *+\mathrm{Sr}$

147

$1295^{2}$ 
181

\begin{tabular}{|c|c|}
\hline & $+{ }_{2.39}^{A r}$ \\
\hline & $+\underset{2.39}{\mathrm{CO}_{2}}$ \\
\hline & 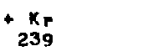 \\
\hline & $+{ }_{239}^{N}$ \\
\hline Na & $\begin{array}{l}+\mathrm{Ne} \\
239\end{array}$ \\
\hline & $\begin{array}{r}+x_{e} \\
239\end{array}$ \\
\hline & $\begin{array}{l}\text { Ar } \\
\text { in } 26\end{array}$ \\
\hline & ${ }_{902}^{+D_{2}}$ \\
\hline & $+H_{1218}^{H_{2}}$ \\
\hline & $+\mathrm{H}_{2} \mathrm{O}$ \\
\hline & ${ }_{151} \mathrm{H}_{2} \mathrm{O}+\mathrm{Ar}$ \\
\hline & $+\begin{array}{c}\mathrm{He} \\
\text { in } 26\end{array}$ \\
\hline & $\begin{array}{l}+\mathrm{N}_{2} \mathrm{O} \\
902\end{array}$ \\
\hline & $\begin{array}{l}+\mathrm{Na} \\
1703\end{array}$ \\
\hline
\end{tabular}

Na* $\begin{gathered}+X_{e} \\ \text { in } 26\end{gathered}$

$\mathrm{Na}_{2}+\mathrm{Xe}$

$\mathrm{NA}_{\mathrm{B}} *+{ }_{1219}^{+\mathrm{r} \cdot \mathrm{H}_{4}}$

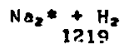

$\mathrm{Na}_{2}+\underset{12.19}{\mathrm{He}}$

$\mathrm{Na}_{2}{ }_{1219}^{+\mathrm{N}_{2}}$

NoF $+M F$

$\mathrm{NH}_{2}+\underset{1069}{\mathrm{H}}$

NO* + NO

$n+\begin{aligned} & \text { Ar } \\ & 1472\end{aligned}$

$0+\frac{K r}{1472}$

$0+\mathrm{Ne}$

$0+x_{e}$
OCS + Ar

ocs $+\mathrm{CO}_{2}$

ocs $+\mathrm{He}$
327

OCs $+\mathrm{N}^{2}$

oCs $+{ }_{327}^{\mathrm{NH}_{3}}$

ocs + ccs

$S_{2}+\begin{array}{r}A r \\ 941\end{array}$

$S_{2}+\begin{array}{r}H e \\ 941\end{array}$

$s_{2}+\begin{array}{r}x_{0} \\ 941\end{array}$

$\mathrm{SO}_{2}+\mathrm{SO}_{3}$

$\mathrm{Ca}$
+758

$\mathrm{Zn*}+\mathrm{NO}$

Under 1701 


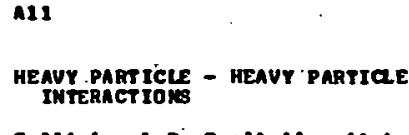

Collistonal bi-Exoltation (total)

$$
\begin{aligned}
& \text { Ar }+\underset{415}{H_{2}} \\
& \text { Ar }+\underset{1802}{N a *} 180 J \\
& \text { Ar* }+H_{2}^{H} \\
& \mathrm{Ar}_{2} * \underset{168}{ \pm} \mathrm{CH}_{6} \\
& \operatorname{Ar}_{2} * \underset{462}{+} \operatorname{Co} \\
& A F_{2}=\underset{462}{+C U_{2}} \\
& \mathrm{Ar}_{2} * \underset{462}{\leftarrow H_{2}} \\
& A r_{2}={ }_{462}^{+} \mathrm{Kr}_{r} \\
& \operatorname{Ar}_{2}+{ }_{187}^{+N_{2}} 462 \\
& \mathrm{Ar}_{2}+\underset{462}{+} \mathrm{H}_{2} \mathrm{O} \\
& \text { Ar }=\underset{462}{+} \text { NO } \\
& \mathrm{Ar}_{2} *{ }_{462}^{*} \mathrm{O} \\
& \because A r_{2} *+462 \\
& \operatorname{Ar} *{ }^{*}+X_{e} \\
& \mathrm{Ba} * \underset{922}{+\mathrm{CO}} \\
& \mathrm{Ba}+\underset{922}{+\mathrm{D}_{2}} \\
& \mathrm{Bo}+\underset{\mathrm{9} 22}{+\mathrm{H}_{2}} \\
& \mathrm{Ba} *+\mathrm{N}_{2} \\
& \mathrm{BaCl}_{32}^{+} \mathrm{Ar} \\
& \mathrm{BaCl}_{32}+\mathrm{He} \\
& \mathrm{BaCl}_{32}+\mathrm{N}_{7} \\
& \mathrm{Br}=+\underset{2143}{\mathrm{Cr}} \\
& \text { 무 }+2143 \\
& \mathrm{Br}+\mathrm{Het} \\
& 2143 \\
& \mathrm{C}_{2}+\mathrm{He}_{2145} \\
& c_{z} *+{ }^{4 r} \\
& \mathrm{C}_{2} * \text { + Nn } \\
& \mathrm{c}_{2} *+\mathrm{O}_{2}
\end{aligned}
$$

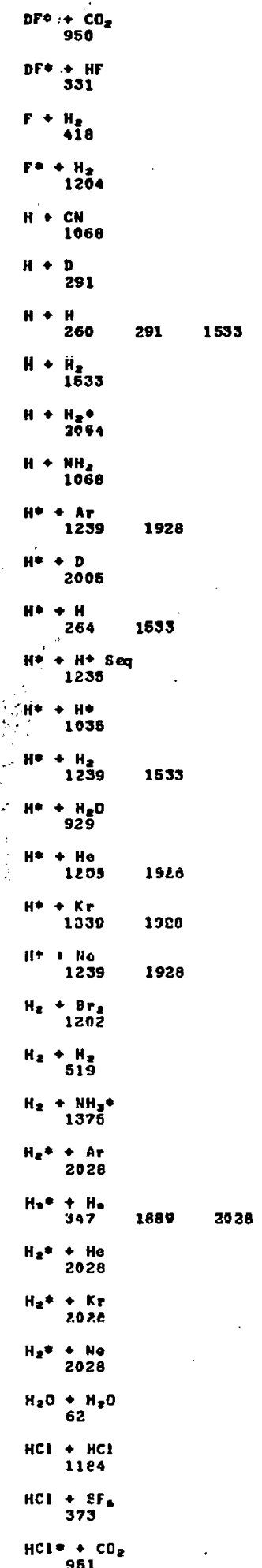

20

280




\begin{tabular}{|c|c|c|}
\hline HCN* $+{ }_{1482}^{A R}$ & & . \\
\hline $\mathrm{HCN} * \underset{1482}{+\mathrm{CO}}$ & & \\
\hline $\begin{array}{c}\mathrm{HCN} * \mathrm{CO}_{1482} \\
+\mathrm{CO}_{2}\end{array}$ & & \\
\hline$\underset{1482}{\mathrm{HCNO}}+\underset{\mathrm{HO}}{\mathrm{Ha}}$ & & \\
\hline $\begin{array}{c}\mathrm{HCNE} \\
1482\end{array}$ & & : \\
\hline $\mathrm{HD}+\underset{335}{\mathrm{He}}$ & . & . \\
\hline 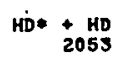 & & \\
\hline $\mathrm{HD}+\underset{2053}{+\mathrm{He}_{8}}$ & & \\
\hline $\mathrm{He}+\underset{333}{\cos }$ & & \\
\hline $\mathrm{He}+\underset{54}{\mathrm{H}_{2}}$ & 1202 & 1203 \\
\hline $\mathrm{Ho}+\underset{1202}{\mathrm{KBr}}$ & $\therefore$ & \\
\hline $\mathrm{He}+\underset{190}{\mathrm{HD}}$ & & \\
\hline He + ${ }^{H o *}$ & & $\because:$ \\
\hline $\mathrm{He}+\mathrm{HF}^{\mathrm{H33}}$ & . & $\because$ \\
\hline $\mathrm{Ho}+\mathrm{No*}^{\mathrm{NoO2}}$ & 1803 & ; \\
\hline $\begin{aligned} & H o *+A P \\
& 1428\end{aligned}$ & & $\because$ \\
\hline 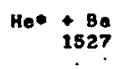 & $\cdot$ & · \\
\hline $\begin{array}{r}+\mathrm{Ca} \\
1527\end{array}$ & . & \\
\hline $\mathrm{He*}+\mathrm{Cd}_{2003}$ & & $\therefore$ \\
\hline 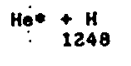 & . & \\
\hline $\mathrm{He}:>_{01}$ & 1995 & . \\
\hline $\begin{array}{c}\mathrm{He*}+\mathrm{Hg} \\
\quad 2003\end{array}$ & & \\
\hline 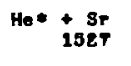 & & $\cdot$ \\
\hline $\begin{array}{r}H 0 *+2 n \\
2003\end{array}$ & & . \\
\hline $\mathrm{HF}+\mathrm{HF}_{1184}^{\mathrm{HF}}$ & $\cdot$ & \\
\hline $\mathrm{HF}+\underset{173}{\mathrm{CO}_{2}}$ & 950 & $:$ \\
\hline$\stackrel{\mathrm{FF}}{+\mathrm{D}^{2}}$ & & \\
\hline $\mathrm{HF} * \stackrel{\mathrm{DF}}{\mathrm{JSI}}$ & & . \\
\hline $\mathrm{HF} * \underset{i \times 3}{+\mathrm{Hz}_{2}}$ & & . \\
\hline $\mathrm{HF} *+\mathrm{HFF}$ & & \\
\hline 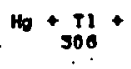 & $\mathrm{N}_{\mathbf{2}}$ & \\
\hline $\mathrm{Ha} *+\mathrm{Cl}_{398}$ & & \\
\hline
\end{tabular}

\begin{tabular}{|c|c|c|c|c|c|c|c|c|}
\hline $\mathrm{HgBr}_{112}^{+} \mathrm{Ar}$ & & & & & & 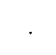 & $x+\begin{array}{l}x_{e} \\
870\end{array}$ & \\
\hline 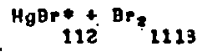 & 3 & . & & & & & $x *+\underset{1948}{A r}$ & \\
\hline $\mathrm{HgRr}_{112}+\mathrm{CCl}_{2} \mathrm{Br}_{2}$ & $=$ & $\because$ & & & & & $K *+\begin{array}{c}H_{0} \\
1948\end{array}$ & \\
\hline $\mathrm{HgBr}_{112} \mathrm{CH}_{2} \mathrm{\theta r}$ & & & & & & . & $x *+\sum_{1948}^{\text {Ne }}$ & \\
\hline $\mathrm{HgBr}_{111 \mathrm{~B}} \mathrm{CO}$ & & $\because$ & : & 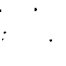 & & & $k *+x_{1948}$ & \\
\hline $\mathrm{HgBr}_{1118}+\mathrm{CO}_{2}$ & $\therefore$ & $\begin{array}{r}\quad: \\
\therefore\end{array}$ & & & $\therefore$ & & 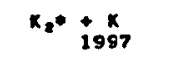 & \\
\hline $\mathrm{HgBr}_{1118}+\mathrm{H}_{2}$ & , & $\because$ & & & & & $\mathrm{Xr}_{\mathrm{r}}+\underset{1802}{\mathrm{Mat}}$ & 1803 \\
\hline$\stackrel{\text { HgBr: }}{\mathrm{HBr}}$ & . & . & & & & & Krete ${ }_{1308}^{\text {He }}$ & \\
\hline $\mathrm{HgBr}_{112}$ & . & $\cdot$ & . & & & '. & $21+\operatorname{Apr}_{870}$ & \\
\hline${ }_{112}^{\mathrm{HgBr}} \mathrm{Hg}$ & & $\vdots$ & : & & . & & $24+H_{070}$ & \\
\hline${ }_{112}^{\mathrm{HgBr}}+\mathrm{HgBr}_{118}$ & : & $\therefore \quad$. & & & , & & $L+\underset{870}{K r}$ & \\
\hline${ }_{112}^{H_{2}} \mathrm{~N}_{2}$ & 8 & . & & $\because$ & & & $4+N_{870}$ & \\
\hline $\mathrm{HgBro}_{112}+\mathrm{Ne}:$ & . & $\therefore$ & & & & $\therefore$ & $L+x_{870}$ & \\
\hline $\mathrm{Hg}^{\mathrm{BOH}}+\mathrm{O}_{\mathrm{Z}}$ & : & $\therefore$ & & $:$ & ' & & 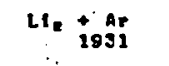 & \\
\hline${ }_{112}^{\mathrm{HgBr}^{2}} x_{0}$ & $\therefore$ & $\therefore$ & & $\therefore$ & : & & LI. He Hi & \\
\hline${ }_{1118}^{H g r^{*}}$ & & $:$ & . & & & & $41 .+\begin{array}{c}k_{F} \\
1921\end{array}$ & \\
\hline Hgro Ar: & $\therefore$ & $\therefore$ & & & & & L1. : 21031 & \\
\hline $\mathrm{HgT}_{112}^{+}+\mathrm{CFj \textrm {I }}$ & $\cdots$ & $\therefore$ & & . & & & $L=$ + Mo & \\
\hline${ }^{\mathrm{HgI}}+\mathbb{1 1 2}^{*} \mathrm{Hg}$. & $\therefore:$ & 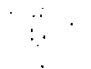 & & . & & $\vdots$ & L. & \\
\hline $\mathrm{Hgr*}+\mathrm{HgI}_{12}$ & : & $\because \vdots$ & & $\cdot$ & 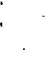 & & Lit $+\underset{1207}{\mathrm{DCl}}$ & 1208 \\
\hline $\mathrm{HgIO}_{112}^{+\mathrm{HI}}$ & . & $\therefore$ & & . & : & . & LIH + ${ }_{1207}^{\text {HCI }}$ & 1208 \\
\hline $\begin{array}{l}\mathrm{Hgr} *{ }^{+\mathrm{N}_{2}} \\
\because \quad 12\end{array}$ & & $\cdot$ & & 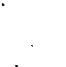 & & & $N=+N$ & \\
\hline$\underset{1 i e^{H}}{H g l *}$ & $\cdot$ & $\because$ & & $\therefore$ & & & $N o+\begin{array}{c}N_{2} \\
1889\end{array}$ & \\
\hline${ }^{\mathrm{HMO} *}+\mathrm{S77}^{\mathrm{N}_{2}}$ & & : & & $\cdot$ & & $\therefore$ & $N_{2}+N_{1202}$ & . \\
\hline $\mathrm{I}: \mathrm{He}^{\mathrm{He}}, 1195$ & & $\because$ & $\therefore$ & : & & . & $\mathrm{H}_{2} \cdot+\underset{2029}{\mathrm{Ar}}$ & \\
\hline $\mathrm{ICl}_{487}^{+} \mathrm{ICl}$ & & & & & & & $\mathrm{N}_{2} *+\mathrm{H}_{361} \mathrm{H}$ & \\
\hline $\begin{array}{l}\mathrm{ICl} \bullet+\mathrm{Ne} \\
: 487\end{array}$ & & : & & & $\therefore$ & & $\mathrm{N}_{2} \mathrm{O}+\mathrm{CO}_{95}$ & \\
\hline$X+\underset{870}{A r}$ & & & & & & & $\frac{\mathrm{M}=0 * \mathrm{Ar}}{1299}$ & \\
\hline $\mathrm{K}+\underset{\text { ห⿻?口𧘇 }}{\text { He }}$ & & $\therefore$ & & & & & $\begin{array}{c}\mathrm{M}_{2} \mathrm{O}+\mathrm{Ho}_{\mathrm{H}} \\
1299 .\end{array}$ & \\
\hline$K+\frac{K}{1997 .}$ & . & & & ، & & & $\mathrm{N}_{200}+\mathrm{Kr}_{\mathrm{r}}$ & \\
\hline$\dot{x}+x_{\mathbf{\theta r 0}}^{\mathbf{x}_{\mathbf{r}}}$ & & & $\therefore$ & ; & & & $\frac{\mathrm{N}, \mathrm{O} *+\mathrm{No}}{1299}$ & \\
\hline$x+\sum_{870}$ & & & & & & & $\mathrm{~N}_{2} \mathrm{Oe}$ & \\
\hline
\end{tabular}




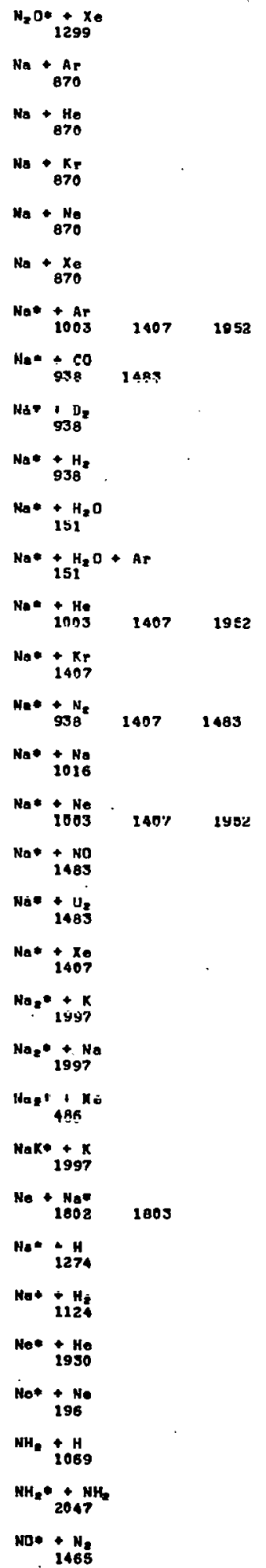

$0 *+\begin{array}{r}x e \\ 529\end{array}$

$\mathrm{O}_{2}+\mathrm{O}_{464}$

$\mathrm{O}_{2}+\begin{gathered}0_{3} \\ 464\end{gathered}$

$\mathrm{O}, *$ He

$\mathrm{O}_{2 *+\mathrm{N}_{2}}$

$\mathrm{O}_{2} *+\begin{array}{r}\mathrm{O}_{2} \\ 1157\end{array}$

$\mathrm{OH}^{\circ}+\mathrm{Al} \mathrm{r}$

OH* $+\mathrm{H}^{\circ} \mathrm{H}^{\circ}$

$\mathrm{OH}^{+}+\mathrm{N} \mathrm{N}$

$\mathrm{OH}+\mathrm{O}_{2}$

$\mathrm{PH} *+\mathrm{CO}$

$\mathrm{PH}=\mathrm{HO}_{4} \mathrm{H}_{2}$

$\mathrm{PH}+\mathrm{CO}_{4}^{\mathrm{Ke}}$

$\mathrm{PHO}+\mathrm{N}_{4}$

$\mathrm{PH} *+\mathrm{NO}$

$\mathrm{Rb}+\frac{\mathrm{Ar}}{\mathrm{OTO}}$

$\mathrm{Rb}+\mathrm{He}$

$\mathrm{Rb}+\begin{array}{r}\mathrm{K}_{\mathrm{r}} \\ 070\end{array}$

Pb $+\mathrm{Na}$

$R b+x_{e}$

No* A Ar

1418

$\mathrm{Rb}+\begin{array}{r}\mathrm{He} \\ 1407 \quad 1418\end{array}$

Rb* + $\mathrm{Kr}$

Rbe $+N=$

REF. + Na

Rbe + Rb 1418 , 2009

Rb* $\begin{array}{r}\text { Xe } \\ 1407\end{array}$

So* + ocse

Se, + ocso

S1*. $\mathrm{CH}^{\mathrm{CH}}$

$\mathrm{si*}+\underset{537}{+\mathrm{Cl}_{2}}$

S1* + $\mathrm{CO}_{53}$ 


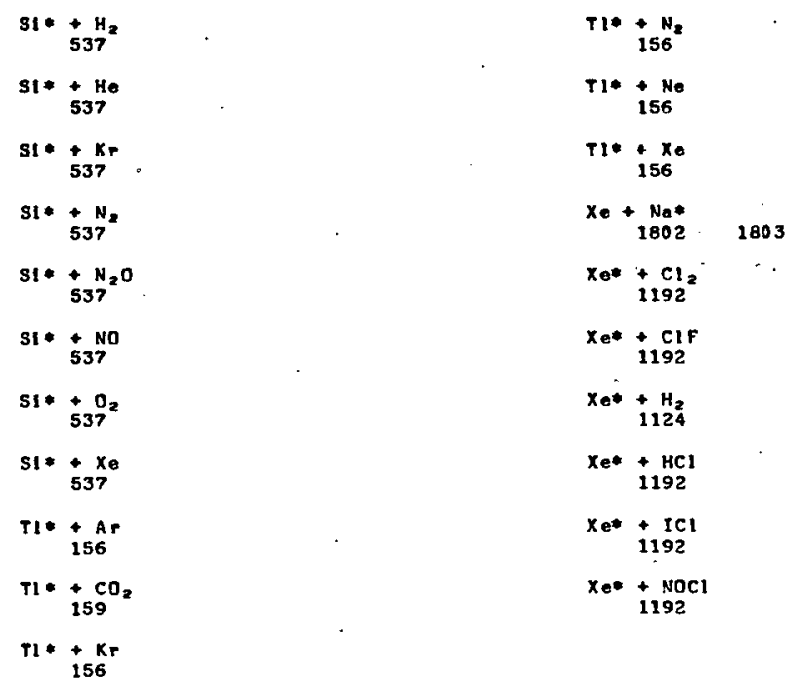

$\mathrm{Xe}^{+}+\mathrm{SCl}_{1}$

$\mathrm{Xe}^{2+*}+\mathrm{He}$ 1305

$\mathrm{Xez} *+\mathrm{Ne}$ 1305

$\mathrm{Zn*}+\mathrm{CH}_{63}$

$\mathrm{Zn}+\underset{637}{\mathrm{CO}}$

$\mathrm{Zn*}+\mathrm{D}_{2}$

$\mathrm{Zn} *+\mathrm{H}_{2}$

$2 n *+4 \mathrm{HD}$

$\mathrm{Zn}+\mathrm{N}^{637}$ 
12

HEAVY PARTICLE - HEAUY PARTICLE INTERACTIONS

Colltelonal Line Broadenting

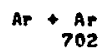

AP + ocs

$B a *+$ Ar

$\mathrm{Ba}+\underset{159}{\mathrm{Kr}} 2000$

Ba* $+\underset{2000}{+\times 6}$

$\mathrm{CO}+\mathrm{co}$

$\mathrm{CO}+\underset{1807}{\mathrm{CO}_{2}}$

$\mathrm{CO}+\begin{array}{r}\mathrm{HCl} \\ 1154\end{array}$

$\mathrm{CO}+\underset{1807}{\mathrm{~N}_{3}}$

CO* + Ar

co* + He

1113

$\operatorname{co*}+\mathrm{Kr}$

$\mathrm{CO} * \mathrm{Ne}$
1113

$\operatorname{co*}+x_{e}$

$\mathrm{CO}_{2} * \underset{1196}{\mathrm{CO}_{2}} 1111 \quad 1115$

$\mathrm{CO}_{0} *+\mathrm{N}_{106} \mathrm{~N}=1740$

$C r+A r$

$\mathrm{Cr}+\underset{1970}{\mathrm{H}_{2}}$

Cr $+\underset{i \dot{9} 70}{\mathrm{He}}$

$\mathrm{Cr}+\mathrm{N}_{2}$

Cs+Cs

$\mathrm{Cg}+\mathrm{He}$

$\mathrm{Cs}+\mathrm{Ne}$

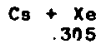

D+ Art

D* + Ar

Ga* + Ar

Ga* + $K_{r}$
Go* 200

H + Ar*

$H *+$ Ar

He + Ar

$H *+\underset{1735}{\mathrm{H}_{2}}$

$H *+\underset{130}{H e}$

H* + $\begin{array}{r}H * \\ 582\end{array}$

$\mathrm{H}_{2} \mathrm{O}+\mathrm{H}_{2} \mathrm{O}$

$\mathrm{H}_{2} \mathrm{O}+\mathrm{Iin}^{+} \mathrm{N}$

$170 p$

HCI * MCI

$\mathrm{HCl}+\mathrm{N}_{\mathbf{2}}$.

HCl $\underset{1113}{+\mathrm{Ar}}$

$\mathrm{HCl} *+\mathrm{He}$

HCl $* \begin{array}{rr}\mathrm{Kr} \\ 1113\end{array}$

HCl * + Ne

$\mathrm{HCl} * \pm \mathrm{Xe}^{+}$
1113

$\mathrm{Ho}+\underset{\substack{\mathrm{He} \\ \mathrm{Inn}}}{\mathrm{an}}$

He $+\underset{1792}{\text { ncs }}$

$\mathrm{He*}+\underset{1735}{+\mathrm{H}_{2}}$

Hé* He

HF* + Ar

1316

$\mathrm{HF} *+\mathrm{He}$

1113

$H F=+R P$

2316

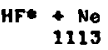

$\mathrm{HF}+\mathrm{YA}$
1113

$I n *+A r$
$2 n n !$

$I_{n} * \begin{array}{r}K_{r} \\ \text { ennI }\end{array}$

In* $+x e$

K. Ar

$K=+\frac{H e}{1948}$

$K *+\mathrm{Ne}$

1948

$x *+x_{e}$
$K++\frac{K r}{644}$

$L I+x_{0}$

$\mathrm{Mg} *+\frac{\mathrm{Ar}}{2000}$

Mg* $+\mathbf{K r}$

$\mathrm{Mg}_{\mathrm{g}}^{+} \mathrm{X}_{\mathrm{e}}$

$\mathrm{H}_{2}+\mathrm{HCl}$

$\mathrm{Na}+\mathrm{CO}$

1127

$\mathrm{Na}+\mathrm{He}$

$\mathrm{No}+\mathrm{N}_{2} \mathrm{O}$

$\mathrm{Na}+\mathrm{Na}$

No + Ne

$\mathrm{Na}+\mathrm{NO}$

Na $+x 6$

$\mathrm{Na*}+\mathrm{He}$
$185 ?$

$\mathrm{Na*}+\mathrm{Kr}$
$185 ?$

$\mathrm{Na*}+\mathrm{No}^{+185 ?}$

Ne + He

$1495 \quad 1513$

Ne + Ne

1495

Ne* $\begin{array}{r}\mathrm{He} \\ 114 \mathrm{C}\end{array}$

Ne*+ Ho

NH* $\underset{1125}{\mathrm{NH}_{3}}$

$\mathrm{NI}+\mathrm{NI}$

1926

No+ 552

No* $+\mathrm{N}_{2}$

No* + NO

$\mathrm{D}+\mathrm{HCl}$

$\mathrm{O}_{2}+0_{89}$

ocs + ocs

1794

$\mathrm{UH}=\underset{648}{+}$

$\mathrm{OH} * \underset{648}{+\underset{\mathrm{N}}{\mathrm{N}} \mathrm{H}}$

$\mathrm{OH} *+\mathrm{NO}_{2}$

648

$R b+A r$ 


\begin{tabular}{|c|c|c|}
\hline $\mathrm{Ab}+\mathrm{He}_{1994}^{\mathrm{He}}$ & & Rb* +Ne \\
\hline$R b+\underset{305}{K r}$ & & $S F_{0}{ }_{1753}^{+S F_{0}}$ \\
\hline $\mathrm{Rb}+\begin{array}{r}\mathrm{Na} \\
305\end{array}$ & 1994 & $3 r+\underset{3 n 4}{A r}$ \\
\hline Rb $+\begin{array}{r}x_{e} \\
3 n 5\end{array}$ & & $\begin{aligned} & +\mathrm{He} \\
& 1114\end{aligned}$ \\
\hline Rb* + $\begin{array}{c}A r \\
20 n 2\end{array}$ & & $\mathrm{Sr}^{*}+\begin{array}{r}+\mathrm{xe}_{\mathrm{e}} \\
1114\end{array}$ \\
\hline $\begin{array}{l}\mathrm{Rb} * \begin{array}{r}\mathrm{He} \\
2002\end{array} \\
200\end{array}$ & & $T 1+\underset{271}{A r}$ \\
\hline
\end{tabular}

TI* AR

$T I *+\underset{K r}{K}$

Tie + $\begin{array}{r}x_{e} \\ 2001\end{array}$

$x e+\begin{array}{r}x e \\ 644\end{array}$

$152 \quad 646 \quad 1142$

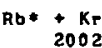


A 13

HEAUY PARTICLE - HEAVY PARTICLE
INTERACTIONS

Heavy Partiele Interchange.

Rearrangevent, and Association (one

or aore (onle roactants)

$\left(\mathrm{CO}_{2}\right)_{2 \mathrm{n}_{1}}^{+}+\mathrm{SO}_{2}$

$\mathrm{Ar}^{+}+\mathrm{CF}_{33}$

$A r+\mathrm{Cl}_{2}$

$\mathrm{Ar}^{+}+\mathrm{y}_{\mathbf{y} \mathrm{I}^{2}} \mathrm{H}^{2}$

$\mathrm{Ar}^{+}+\mathrm{I}_{3}$

$\mathrm{Ar}_{2}+\underset{924}{+\mathrm{CO}}$

$A r={ }_{924}^{+} H_{r}$

$\mathrm{ArC}^{*}+\mathrm{HBr}$

$A r=+{ }_{924}^{+} \mathrm{HCI}^{-}$

$\mathrm{Ar}_{2}+\underset{924}{+} \mathrm{Kr}$

$\mathrm{Ar},+\underset{924}{+} \mathrm{N}_{2}$

$\mathrm{Ar}_{2}+\underset{\mathrm{OZ}_{4}}{+} \mathrm{N}_{2} \mathrm{O}$

arat + NO

$\mathrm{Ar}_{2}+\mathrm{O}_{2}$

Arpt+ $x_{0}$

$\mathrm{Ar}_{2}^{+}+2 \mathrm{Ar}$

$\mathrm{B}+\mathrm{D}^{\mathrm{D}}$

$\mathrm{C}+\mathrm{CH}_{4}$

$\mathrm{C}^{2}+\mathrm{CD}$

$\mathrm{C*}+\mathrm{CO}$

911

$\mathrm{C}++\mathrm{D}_{2}$

$\begin{array}{lll}+\mathrm{H}_{2} & & \\ 911 & 1366 & 1902\end{array}$

$\mathrm{C}++\mathrm{H}_{2}+\mathrm{He}$

C* $+\underset{911}{\mathrm{H}_{2} \mathrm{O}}$

$\mathrm{C}++\underset{911}{\mathrm{NH}}$

C* + No

$C++a_{2}$
$\mathrm{C2*}+\mathrm{Ar}$
1469

C24+ $+\begin{array}{r}\mathrm{H}_{2} \\ 1469\end{array}$

C24 $+K r$

$\begin{aligned} & \mathrm{C} 2++\mathrm{N}_{2} \\ & 1459\end{aligned}$

$\mathrm{C} 2+$
$+\mathrm{Ne}$
1459

$\mathrm{C2}+\mathrm{O}_{2}$
1469

C2t $\begin{gathered}x e \\ 146 y\end{gathered}$

$r_{C} H_{C}+$ Hrit

$\mathrm{rH}_{2}+\mathrm{H}_{1366}$

$\mathrm{CH}_{2}+\underset{1811}{+\mathrm{HC}_{3} \mathrm{~N}}$

$\mathrm{CH}_{3}+\underset{1356}{+\mathrm{CO}}$

$\mathrm{CH}_{3}+\underset{1354}{+\mathrm{H}_{2}} \quad 1355$

$\mathrm{CH}_{3}+\underset{1366}{+\mathrm{H}_{2} \mathrm{O}}$

$\mathrm{CH}_{3}+\mathrm{ICO}_{3} \mathrm{~N}$

$1+D I$

$\mathrm{Cl}+\mathrm{HI}$

$\mathrm{Cl}+\mathrm{CO}$

400

$\mathrm{Cl}^{-}+\mathrm{SO}_{2} \mathrm{Cl}=$

CO* D

$\mathrm{CO}_{2}+\underset{446}{+\mathrm{D}}$

$\mathrm{CO}_{2}+\underset{146}{+\mathrm{H}}$

$D_{2}+\frac{D}{446}$

$F+n_{1900}$

$F+\mathrm{H}_{3}$

$F+$ HD $_{1900}$

$F_{7}-+\underset{82}{s} F_{2}$

H.

$\mathrm{H}+\mathrm{H}^{+}$

1212

$+\mathrm{H}+\frac{15}{124}$

$\mathrm{H}+\mathrm{He}$

$\mathrm{H}_{2}^{+}+\mathrm{Ar}$

$\mathrm{H}_{2}+\mathrm{Cn}$
$H_{2}+\underset{446}{H}$

$\mathrm{H}_{3}+\underset{1811}{\mathrm{HC}_{3} \mathrm{~N}}$

$\mathrm{H}_{3} \mathrm{O}+\underset{824}{+} \mathrm{CH}_{2} \mathrm{CO}$

$\mathrm{H}_{3} \mathrm{O}+\underset{82}{+} \mathrm{CH}_{2} \mathrm{O}$

$\mathrm{H}_{32} \mathrm{O}+\underset{824}{\mathrm{CHN}}$

$\mathrm{H}_{3} \mathrm{O}+\underset{824}{+\mathrm{H}_{2} \mathrm{~S}}$

$\mathrm{H}_{3} \mathrm{O}+\underset{1811}{+\mathrm{HC}_{3} \mathrm{~N}}$

$11,0 \cdot \frac{1}{238}$

$11,00{ }_{824}^{1} \mathrm{HIl}$

$\mathrm{H}^{-+\underset{356}{\mathrm{NH}} \mathrm{H}^{-}}$

$\mathrm{HCN}_{446}^{+}$

$\mathrm{HCO}+\mathrm{HC}_{3} \mathrm{~N}$

1811

$\mathrm{He}+\mathrm{H}^{\mathrm{H}} \mathrm{H}^{*}$

$\mathrm{He}^{*}+\mathrm{AF}$

1419

Hot $+\mathrm{N}$,

Het $+\mathrm{O}_{2}$

$\mathrm{HeH+}+\mathrm{H}$

$\mathrm{HO}_{2}+\underset{2142}{\mathrm{HC}_{2}}$

$\mathrm{Krl}=\mathrm{CP}$.

$\mathrm{Kr}+\underset{33}{\mathrm{Cl}_{2}}$

$\mathrm{Kr}+\mathrm{SF}_{\mathrm{S3}}$

Kr2* + Ar

$k=2 *+4.499$

$K r 2+\underset{146 S}{K r}$

$R_{r}=+\underset{1469}{*} N_{2}$

K+34 + Ne

Rrot $0_{2}$

$K r 2+\underset{1469}{+X_{e}}$

$\mathrm{N+}+\underset{35}{\mathrm{CH}} \cdot 36 \quad 911$

$\mathrm{N}+\mathrm{CO}$

$\mathrm{N}+\underset{911}{\mathrm{CO}_{2}}$ 


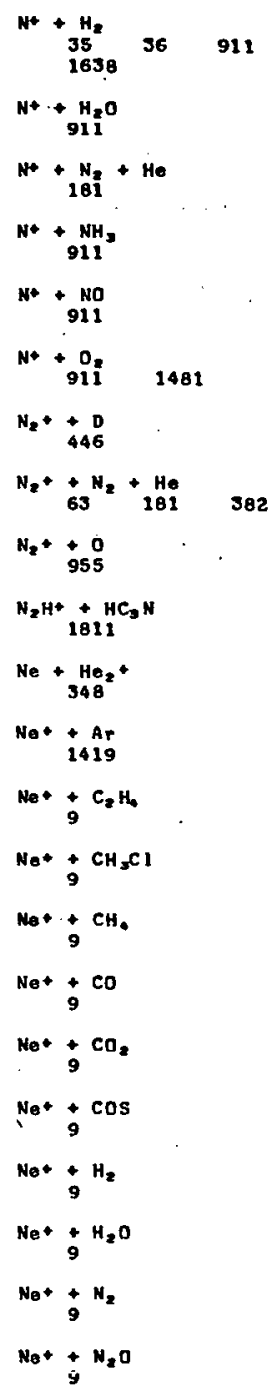

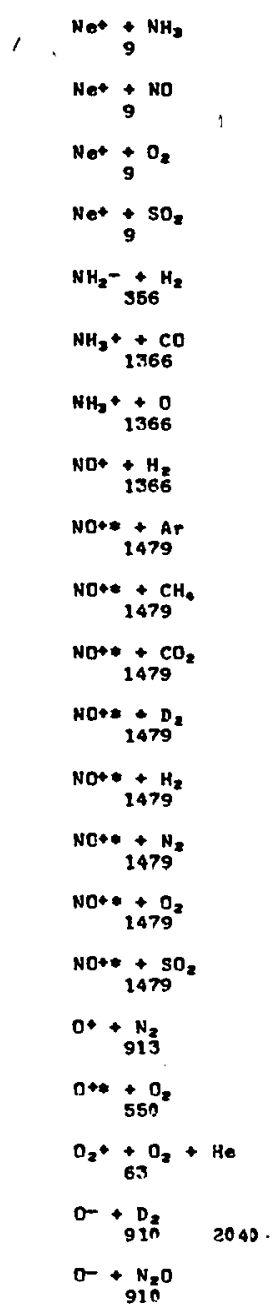

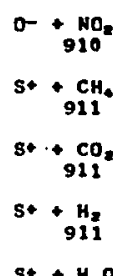

S* $+\underset{911}{\mathrm{H}_{2} \mathrm{O}}$

$3+\underset{911}{N H_{3}}$

$5 *+\underset{911}{N O} 1467$

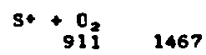

$\mathrm{SO}_{2}-\underset{82}{+\mathrm{SO}_{2} \mathrm{Cl}_{2}}$

$\mathrm{SO}_{2} \mathrm{Cl}_{2 \mathrm{e}}+\mathrm{SO}_{2} \mathrm{Cl}$

so- $\mathrm{SO}_{91 \mathrm{n}}$

$X_{e}=+\underset{1469}{A+A r}$

$\mathrm{Xe}=\underset{1469}{+\mathrm{H}_{2}}$

$X e^{2+}+K_{r}$
1469

$\mathrm{Xe}^{2+}+\underset{14 \in S}{\mathrm{~N}_{2}}$

$\mathrm{Xe}+* \mathrm{Ne}$
1469

$\mathrm{Xe}^{2+}+\mathrm{O}^{2}$

$x e^{2+}+x \in 9$

Review

1372

Undef $1190 \quad 2133$ 

A14
HEAUY PART TCLE - HEAUY PARTICLE
INTERACTIOHB

Houvy Papticle Intorchange,

Rearraggenent, and Aasoolotion (onir

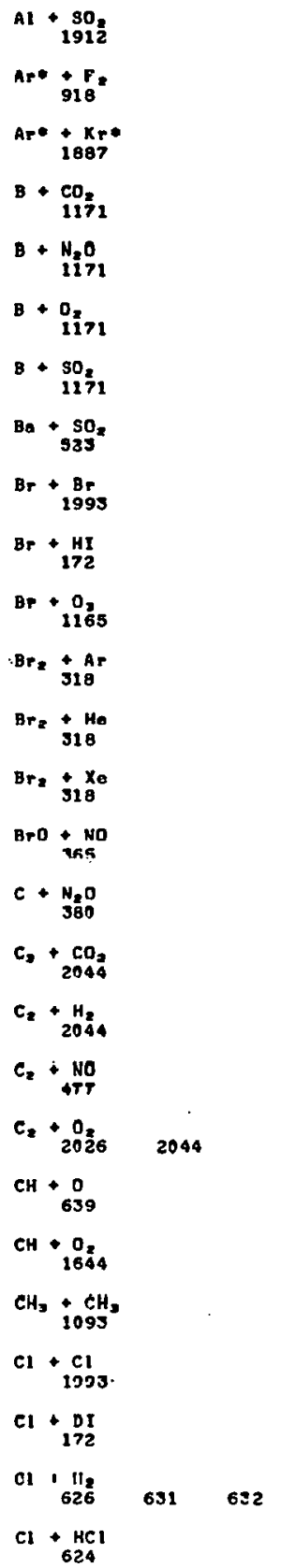

C1+ HD

Cl +

$\mathrm{C}+\mathrm{NO}+\mathrm{Ha}$

620

$\mathrm{Cl}+\mathrm{NO}+\mathrm{N}$

$\mathrm{Cl}+\mathrm{NO}_{2}+\mathrm{N}$

C1 + ${ }^{\top} \mathbf{T}^{\mathrm{Z}}$

$\mathrm{Cl}_{2} \mathrm{O}+\mathrm{H}_{\mathbf{2}} \mathrm{O}$

CINO + Ar

rikn + ris.

CINO $+\mathrm{CO}^{2}$

CINO + He
2139

2139
C1NO
$213 \%$

$\mathrm{CINO}+\mathrm{N}$,
2139

$\mathrm{CINO}+\mathrm{N}_{2} \mathrm{O}$

$\mathrm{C}$ Ino
2139

CINO $+\mathrm{O}_{2}$

CINO $+x_{0}$

C10 1163

010 ig18

C10 $+\mathrm{NO}_{222}+n$

$\mathrm{C} 10+\underset{3069}{\mathrm{NO}_{2}}+\mathrm{N}_{2}$

C10
2032

$\mathrm{CH}+\underset{1981}{\mathrm{nH}}$

$c+c 0 *$

D+ 6

D - ${ }^{\mathrm{H}}{ }^{*}$

$D_{0}+\underset{188}{r}$

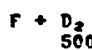

630

$F+\underset{2 n 22}{\mathrm{DH}}$

$\mathrm{F}+\mathrm{H}_{2}$

$\begin{array}{ll}\mathrm{H}_{2} & \\ 447 & 468 \\ 542 & 626 \\ 631 & 1173\end{array}$

503

630

$\mathrm{F}+\underset{47}{\mathrm{H}_{2}}+\mathrm{mo}$
$F+\underset{543}{\mathrm{HBr}}$

2021

$\mathrm{F}+\mathrm{HCl}_{545}$

$F+41$

$P=+\frac{D}{625}$

$F_{2}+\underset{626}{H}$

$F *+\frac{T}{625}$

$\mathrm{H}+\mathrm{Br}_{162}$

1635

H + $\mathrm{Cl}_{\mathrm{B}}$

$651 \quad 1170$

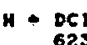

$H+\mathbf{F}_{20}$

2055

$\mathrm{H}+\mathrm{H}_{28} 527 \quad 630$ $631 \quad 632.641$ $\begin{array}{lll}642 & 1154 & 1164\end{array}$

$H+\mathrm{H}_{2} *$

$H+\mathrm{H}_{2} \mathrm{O}$

H + $\underset{1635}{\mathrm{MBr}}$

H + HI

10921186

H + ${ }_{315}$

H+ I8 102

$H+I_{1404}$

$\mathrm{H}+\mathrm{N}_{2} \mathrm{C}$
622

$\mathrm{H}+\underset{\mathrm{OSO}}{\mathrm{NO}}$

$\mathrm{H}+\mathrm{NO}_{2}$

2144

$4+\frac{n}{1356}$

H. $\mathrm{O}_{3}$

$11+\mathrm{OH}$

1356

$\mathrm{H}_{2}+\underset{623}{\mathrm{Cl}}$

$\mathrm{H}_{2} \div \frac{\mathrm{r}}{\mathrm{B}}$

$\mathrm{H}_{2}+\mathrm{I}_{1 \mathrm{leg}}$

$\mathrm{He}+\frac{n}{1356}$

$H_{*}+\underset{1956}{\mathrm{OH}}$

$\mathrm{H}_{2} *+\mathrm{Ar}$

$\mathrm{H}_{2} *$ He 


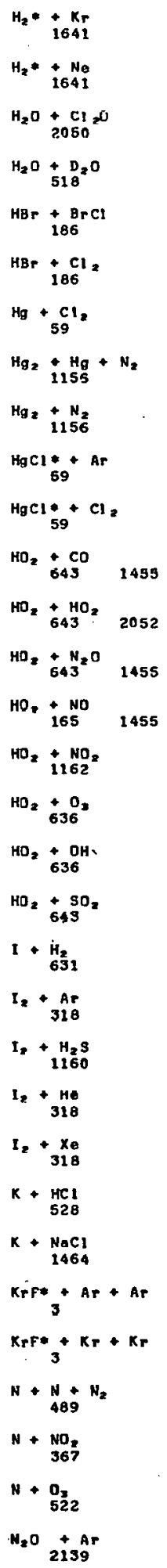

$\mathrm{N}_{2} \mathrm{O}+\mathrm{CF}_{2139}$

$\mathrm{N}_{2} \mathrm{O}+\mathrm{CO}_{2}$

$\mathrm{N}_{2} \mathrm{O}+\mathrm{Ho}_{2139}$

$\mathrm{N}_{2} \mathrm{O}+\mathrm{Kr}_{2139}$

$\mathrm{N}_{2} \mathrm{O}+\mathrm{N}_{2}$

$\mathrm{N}_{2} \mathrm{O}+\mathrm{N}_{2139} \mathrm{O}$

$\mathrm{N}_{2} \mathrm{O}+\mathrm{Ne}$
2139

$\mathrm{N}_{2} \mathrm{O}+\mathrm{O}_{2}$

$N_{2} \mathrm{O}+\mathrm{Xe}_{6}$
2139

$\mathrm{Na}+\mathrm{Na}_{1437}$

$\mathrm{Na}+\mathrm{Na}_{336}$

$\mathrm{Ne}_{2}{ }^{+}+\mathrm{He}$

$\mathrm{NH}_{2}+\mathrm{NO}_{2}$

NO. $+\mathrm{N}_{2}$

$\mathrm{NO}_{2}+\mathrm{Ar}$

$\mathrm{NO}+\mathrm{CF}$.

$\mathrm{NO}_{2}+\mathrm{CO}_{2}$

$\mathrm{NO}_{2}+\mathrm{He}$

NO. $+K_{r}$

$\mathrm{NO}_{2}+\mathrm{N}_{2} \mathrm{O}$

$\mathrm{NO}_{2}^{+} \mathrm{No}$

$\mathrm{NO}_{2}+\mathrm{O}_{2}$

No. + Y.

$0+\mathrm{CH}_{2}$
1354

$0+\underset{350}{\mathrm{CHF}}$

$0+\mathrm{Cl}_{2}$
984

$\mathrm{O}+\underset{930}{\mathrm{Cs},} 983$

$\mathrm{O}+\mathrm{H}_{214} 626$

1132

D. $\mathrm{H}_{2140}$

$0+\mathrm{N}_{2}$

$0+N_{2} O$
167

D+

$$
\begin{aligned}
& \mathrm{O}+\mathrm{NO}+\mathrm{He} \\
& 620 \\
& \mathrm{O}+\mathrm{NO}+\mathrm{N}_{2} \\
& 0+0_{2} \\
& 0+\frac{0}{2036} \\
& \text { D. }+\begin{array}{r}
\mathrm{H}_{2} \\
1630
\end{array} \\
& 0 *+\mathrm{N}_{2} \mathrm{O} \\
& \mathrm{O}_{2}+{ }_{635}^{A r} 2035 \\
& 0 .+N_{2} \\
& \begin{array}{l}
\mathrm{O}_{2}+\mathrm{O}_{2} \\
325 \\
0
\end{array} \\
& \text { 0, } 2139 \\
& 0,+ \text { Ar } \\
& 0,+\mathrm{CF} \\
& \mathrm{O},+\mathrm{CO}_{2} \\
& \text { O. } \begin{array}{c}
\mathrm{He} \\
2139
\end{array} \\
& 0,+\begin{array}{c}
K_{r} \\
2139
\end{array} \\
& \begin{array}{r}
\mathrm{O},+\mathrm{H}, \mathrm{C} \\
2139
\end{array} \\
& \mathrm{O},+\mathrm{Ne} \\
& 0,+02 \\
& 0,+x_{0} \\
& \mathrm{OH}+\mathrm{CO} \\
& \mathrm{OH}+\mathrm{H}_{621} \\
& \mathrm{OH}+\mathrm{HEr} \\
& \mathrm{OH}+\mathrm{NO}_{2}+\mathrm{Ar} \\
& \mathrm{OH}+\underset{2146}{\mathrm{NO}_{2}}+\mathrm{He} \\
& \mathrm{OH}+\underset{2146}{\mathrm{NO}_{2}}+\mathrm{N}_{2} \\
& \mathrm{OH}+\underset{2146}{\mathrm{NO}_{3}}+\mathrm{SF}_{*} \\
& \mathrm{OH}+\mathrm{O}_{46} \quad 509 \\
& \mathrm{OH}+\mathrm{OH} \\
& P+\underset{\mathbf{S}_{2}}{\mathrm{C}_{8} \mathrm{H}_{2}} \\
& P+\underset{538}{C_{2} H_{0}} \\
& \begin{array}{l}
P+c 1= \\
538
\end{array} \\
& \text { p. No }
\end{aligned}
$$


$P+\mathrm{O}_{\mathbf{2}}$.

$P+\underset{538}{P C 1,}$

$R b *+R b$

2004

$\mathrm{S}+$ OH s* + ocs

SO + OH

1159

$x e+F$

Xee $+\mathrm{Br}_{918}$

$H+H$

$\mathrm{Lt}_{2}+\mathrm{Ar}$

$\mathrm{Lt}_{2}+\mathrm{He}$

io'
$\mathrm{Xe}^{*}+\mathrm{Cl}_{218}$

$\mathrm{Xe} *+\mathrm{HC}_{918}$

$\underset{532 \quad 533}{\text { Rev Iew }}$
A15

HEAVY PART TCLE
IMTERACT TOKS

Spln Exehange

$$
\text { Cs }+\begin{gathered}
\text { Ar } \\
845
\end{gathered}
$$

A16

HEAUY PARTICLE - HEAUY PARTICLE INTERACTIONS

Electron Defochment Proe Negative Ione Into Continum

Br- + He
$\mathrm{H}-\mathrm{Ar}$
687
$\mathrm{H}$ 2269

$1269 \quad 1872$
$\mathrm{NH}_{2} \div \underset{10 \mathrm{O}}{\mathrm{H}}$

$\mathrm{Ab}+\mathrm{He}$

$\mathrm{I}-+\mathrm{Ne}$ 


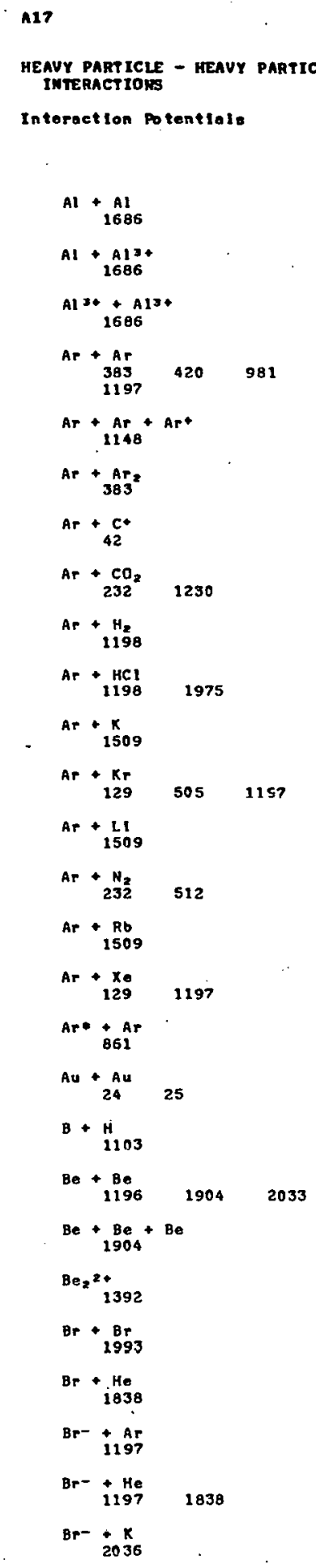

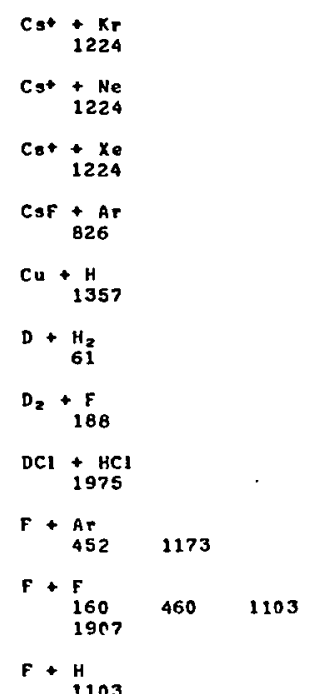$$
1103
$$$$
\begin{array}{r}
F+k_{r} \\
452
\end{array}
$$$$
F+\mathrm{Ne}_{452}
$$$$
F+x_{173}
$$$$
\text { F- }+\underset{2036}{K}
$$$$
\mathrm{F-}+\underset{203 E}{\mathrm{Na}}
$$$$
F-+x e
$$$$
\mathrm{Fe}+\mathrm{D}_{136 \mathrm{E}}
$$$$
\mathrm{Fe}+\mathrm{Fe}_{40}
$$$$
\text { Fe }+H_{1362}^{H}
$$$$
\mathrm{FH}_{2} 1475
$$$$
\text { Ge }+0
$$$$
\text { Ge* }+0
$$$$
H+{ }_{359}^{A r} \quad 936
$$$$
\begin{aligned}
H+\text { Br } \\
1798
\end{aligned}
$$$$
H+\mathrm{Cd}_{1758}
$$$$
\mathrm{H}+\mathrm{Cl}_{17 \mathrm{~s}}
$$$$
\begin{array}{r}
\mathrm{H}+\mathrm{Cl}_{2} \\
188
\end{array}
$$$$
\mathrm{H}+\mathrm{CO}_{44 \mathrm{~B}}^{\mathrm{C}} 947
$$$$
H+F \quad 1793 \quad 1798
$$$$
\mathrm{H}+\mathrm{H}
$$
$\begin{array}{lll}379 & 503 & 1103 \\ 1793 & 1790 & 2033\end{array}$ 


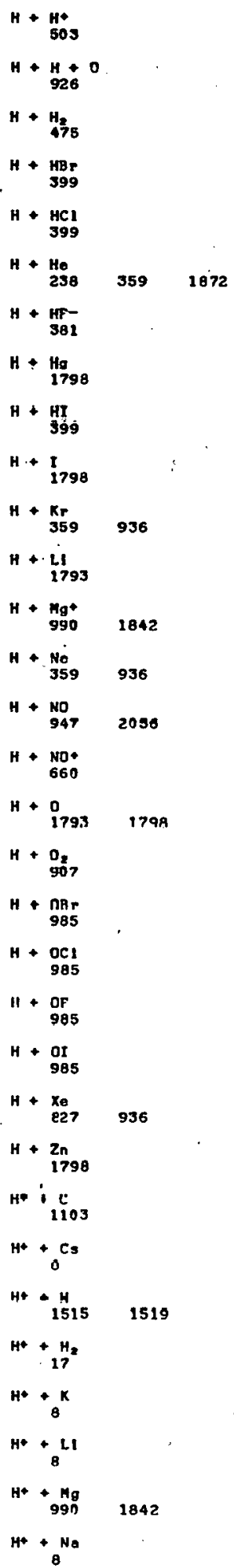

H* $+\mathrm{Rb}_{8}$

H* 834

$\mathrm{H}_{2}+{ }_{026}^{\mathrm{Ar}} 936$

$\mathrm{H}_{2}+\underset{\mathrm{O26}}{\mathrm{CH}}$

$\mathrm{H}_{2}+\mathrm{CO}$

$\cdot \mathrm{H}_{2}+\underset{188}{\mathrm{~F}}$

$\begin{array}{rll}\mathrm{H}_{2}+\mathrm{H}_{2} & \\ 707 & 826 & 991 \\ 982 & 1484 & 1925\end{array}$

$\mathrm{H}_{\mathrm{O}}+\underset{\mathrm{O} 44}{\mathrm{H}_{4}}+\mathrm{He}_{\mathrm{e}}$

$\mathrm{H}_{2}+\underset{836}{\mathrm{Ho}_{0}} \mathrm{O81}$

$\mathrm{H}_{2}+\underset{826}{\mathrm{Kr}} 936$.

$\mathrm{H}_{2}+\underset{\mathrm{B}}{\mathrm{N}_{2}}$

$\mathrm{H}_{2}+\mathrm{Ne}$

$\mathrm{H}_{2}+\mathrm{O}_{145 \mathrm{~B}}$

$\mathrm{Hz}_{2}+\underset{826}{\mathrm{Xe}_{\mathrm{e}}} \quad 936$

$\mathrm{H}_{2}+\underset{443}{+} \mathrm{He}^{-}$

$\mathrm{H}_{2} \mathrm{O}+\mathrm{H}_{2} \mathrm{O}$

$\mathrm{H}_{2} \mathrm{O}$ - $\mathrm{NO}$

$\mathrm{H}_{3}, 1475$

$\mathrm{H}-+\mathrm{H}_{2}$

$\mathrm{H}-+\mathrm{He}$

H- HF

$\mathrm{H}^{-}+\mathrm{Mg}^{2+}$

HCI HCI

HD $+\mathrm{He}$

335

$\mathrm{He}+\mathrm{Ar}$
29
1463

$129 \quad 1197$

$\mathrm{He}+\mathrm{Ar}+$

$\mathrm{He}+\underset{58}{\mathrm{CH}}$

$\mathrm{He}+\mathrm{CD}_{31} 934$

$\mathrm{He}+\underset{232}{\mathrm{CO}_{2}}$

$\mathrm{He}+\mathrm{Cs}_{149}$

$\mathrm{He}+\mathrm{H}_{2}$
1198

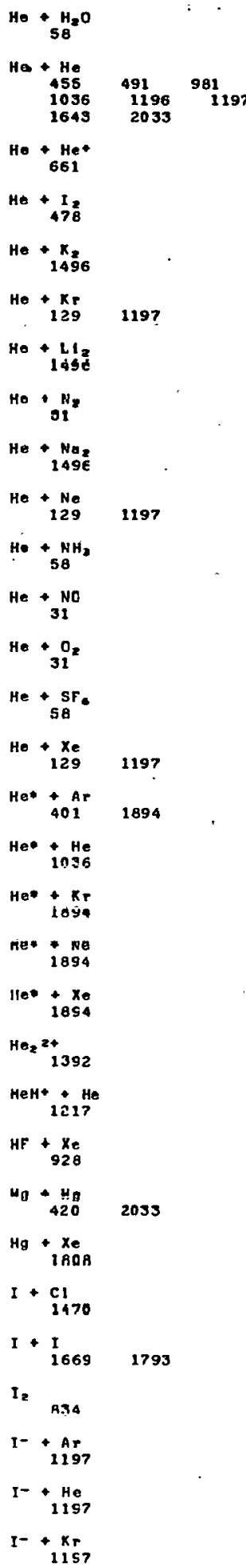




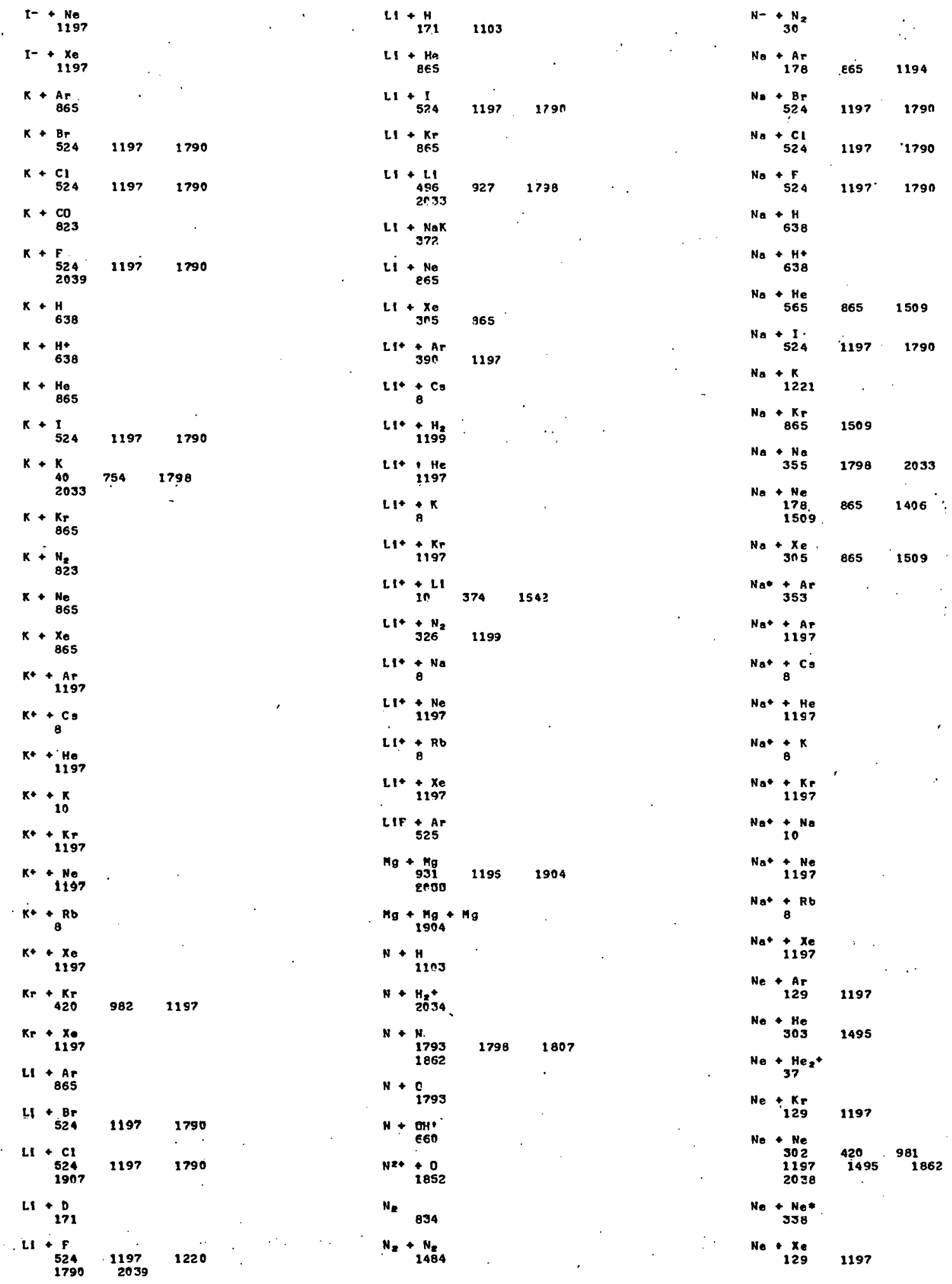




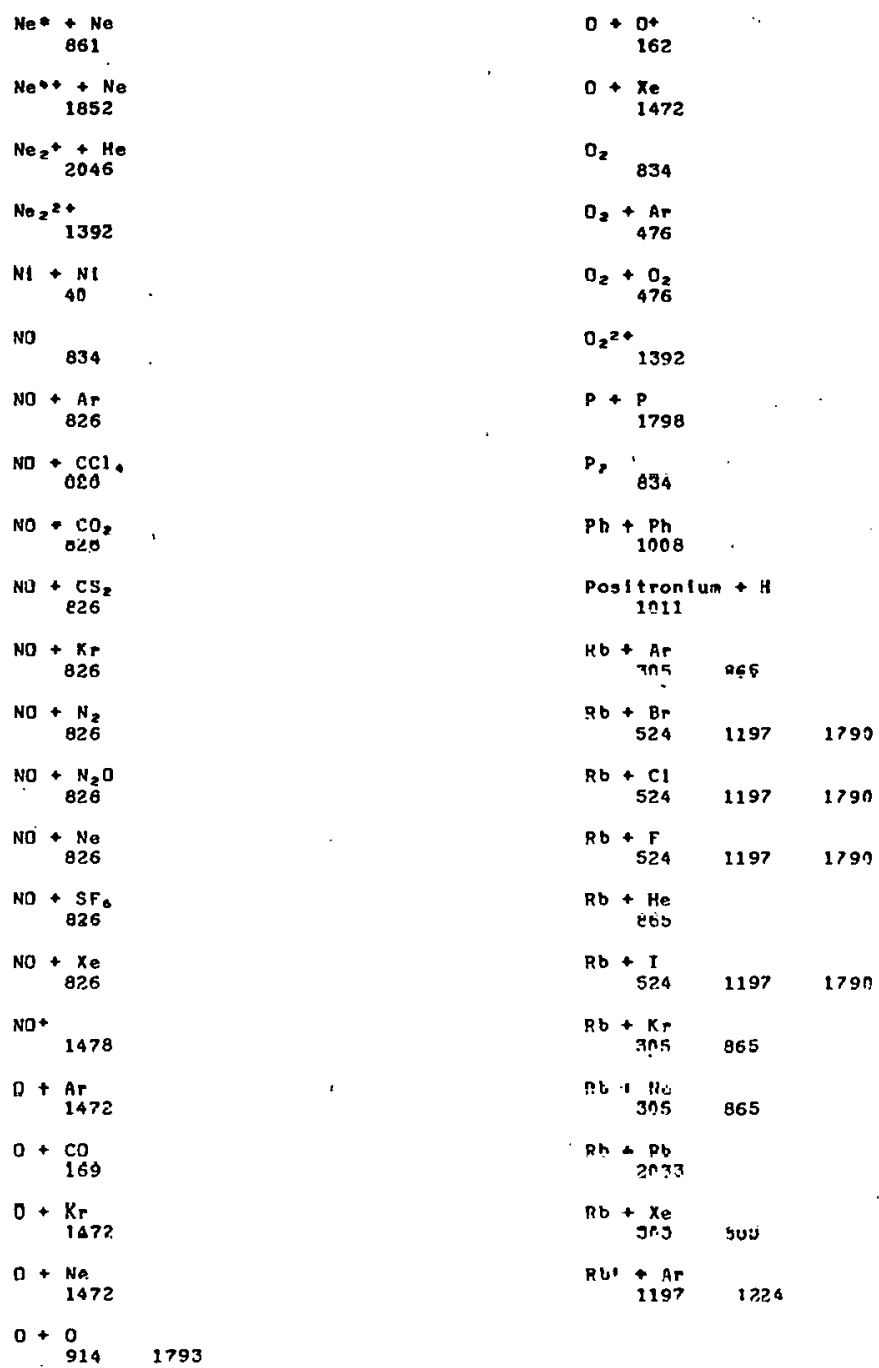


197
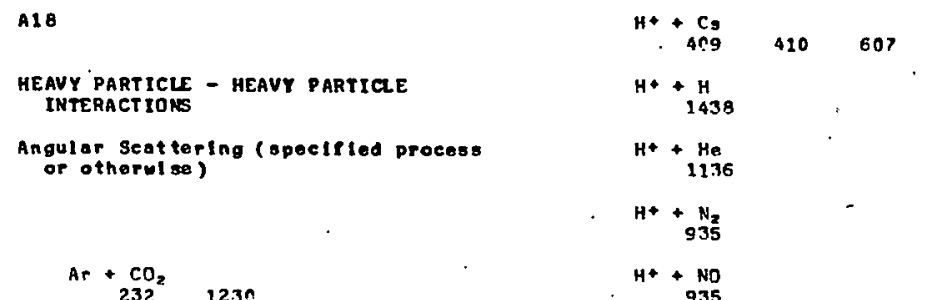

$\mathrm{Ar}+\underset{232}{\mathrm{CO}_{2}} \quad 123 n \quad+\mathrm{H}^{+}+\underset{935}{\mathrm{NO}}$

$H^{*}+\underset{322}{2 r}$

$\mathrm{H}_{2}+\underset{196 n}{\mathrm{Ar}}$

LI+ $+\mathrm{H}_{2}$

$\mathrm{He}+\mathrm{Ar}$

$11+\underset{1293}{11}$

$21++N_{2}$

LIF $\underset{525}{+ \text { AT }}$

$M g^{+}+A_{14 R}^{A R}$

$\mathrm{Hg}+\underset{1262}{+\mathrm{H}_{2} \mathrm{O}}$

Art $\begin{array}{r}\text { Xe } \\ 1620 \quad 1851\end{array}$

$\mathrm{Be}+\mathrm{He}$
568

29

1408

$\mathrm{Be}+\mathrm{Ne}$

$+\underset{58}{\mathrm{CH}_{4}}$

- $12 \epsilon 2$

Br- $+K$

$\mathrm{He}+\mathrm{CO}$

$+\mathrm{Ne}$
1262

1408

$\mathrm{Br}+\mathrm{Na}$

$\mathrm{He}+\underset{232}{\mathrm{CP}_{2}}$

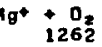

$c+\mathrm{Ca}_{135}$

$c++x_{e}$

$\mathrm{He}+\underset{58}{\mathrm{H}_{2} \mathrm{O}}$

$\mathrm{N*}+\begin{array}{r}\mathrm{H}_{2} \mathrm{C} \\ 1262\end{array}$

$N++\underset{605}{N}$

1262

$\mathrm{He}+\mathrm{He}_{1 \mathrm{n} 36}$

H* $+\begin{aligned} & \text { No } \\ & 1262\end{aligned}$

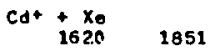

$\mathrm{He}+\underset{31}{\mathrm{~N}_{2}}$

$\mathrm{He}+\underset{+9 a_{2}}{\mathrm{Na}_{2}}$

$\mathrm{Cl}+\underset{\mathrm{Xe}}{352} \quad 1173$

$\mathrm{He}+\underset{58}{\mathrm{NH}_{3}}$

$\mathrm{Cl}-\stackrel{\mathrm{Ar}}{996}$

C1 - $+\frac{K}{2036}$

$\mathrm{Cl}-\stackrel{\mathrm{Na}}{2936}$

$D+\begin{array}{r}H_{2} \\ 61\end{array}$

$D++\underset{1869}{D}$

D* + NI

$F+\underset{452}{A r} 1173$

$F+\underset{452}{K r}$

$\mathrm{He}+\mathrm{NO}$

$\mathrm{He}+\underset{21}{\mathrm{n}_{2}}$

$\mathrm{He}+\underset{58}{\mathrm{SF}}$

He* $\begin{array}{r}+ \text { He } \\ 1036\end{array}$

He+ + Ar
$196 ?$ ?

$\mathrm{He}+\underset{588}{+}$

$\mathrm{He}+\mathrm{Kr}$
$162 ?$

$\mathrm{He}+\frac{\mathrm{Xe}}{1620}$

$\mathrm{N}+\mathrm{O}_{2}$

$\mathrm{Na}+\mathrm{Hg}$

$\mathrm{Hg}$
1155

$\mathrm{Na}+\mathrm{Ne}$

Na* + Ar

$\mathrm{Na} *+\mathrm{Ne}$

$\mathrm{Na}+\underset{12 \epsilon 2}{\mathrm{H}_{2} \mathrm{O}}$

$\mathrm{Na}+\begin{array}{r}+\mathrm{N}_{2} \\ 1262\end{array}$

Not + Ne 1262

$\mathrm{Na}+\mathrm{O}_{2}$
1262

$\mathrm{Ne}+\underset{1262}{+\mathrm{H}_{2} \mathrm{O}}$

Net+ $+\mathrm{N}_{2}$

$\mathrm{Ne}+\mathrm{Ne}$

Net $+\begin{array}{r}\mathrm{O}_{2} \\ 1262\end{array}$

$\mathrm{Ne*}+\mathrm{Xe}_{\mathrm{e}}$

$1620 \quad 1851$

$F^{-}+\underset{2036}{\mathrm{Na}}$

$\mathrm{He}_{1863}^{+\mathrm{He}}$

1859

HF $+\begin{array}{r}x_{e} \\ 928\end{array}$

$+\mathrm{Ne}$
$90 \mathrm{G}$

$0+$ Co $_{155}$

$0+\frac{N 1}{136}$

$0+\operatorname{si}_{681}$

$0+56$

$\mathrm{O}+\mathrm{H}_{2} \mathrm{O}$
1262 
$0++\frac{N 8}{1262}$

O+ + Ne

or $+0_{2}$
O- $D_{2}$

$U+\frac{k r}{175}$

$x_{0}++x_{0}$

$162 n$
$2 n+* x_{0}$

1851

Under 


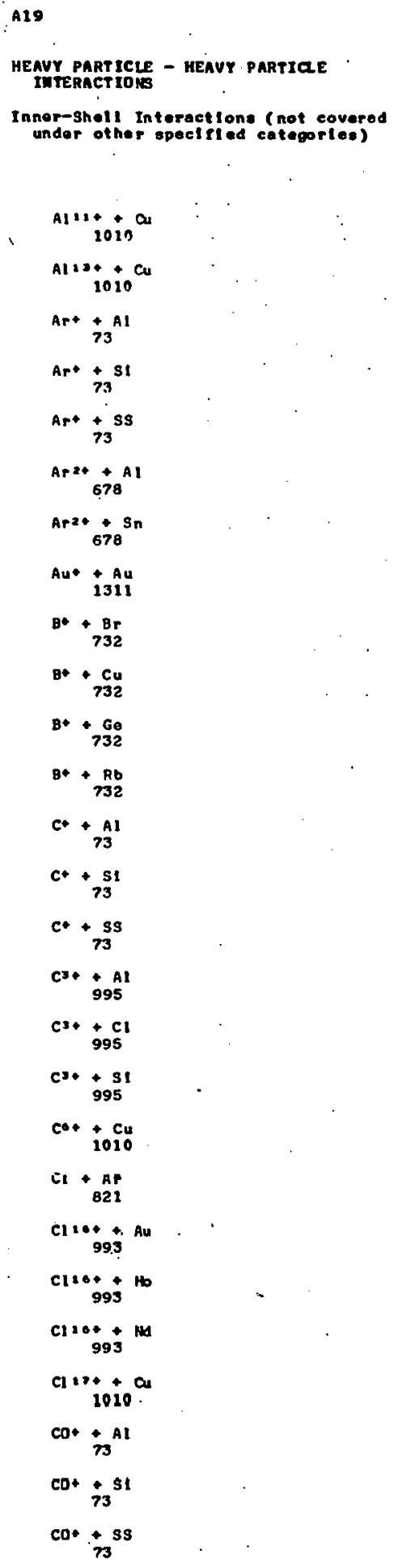

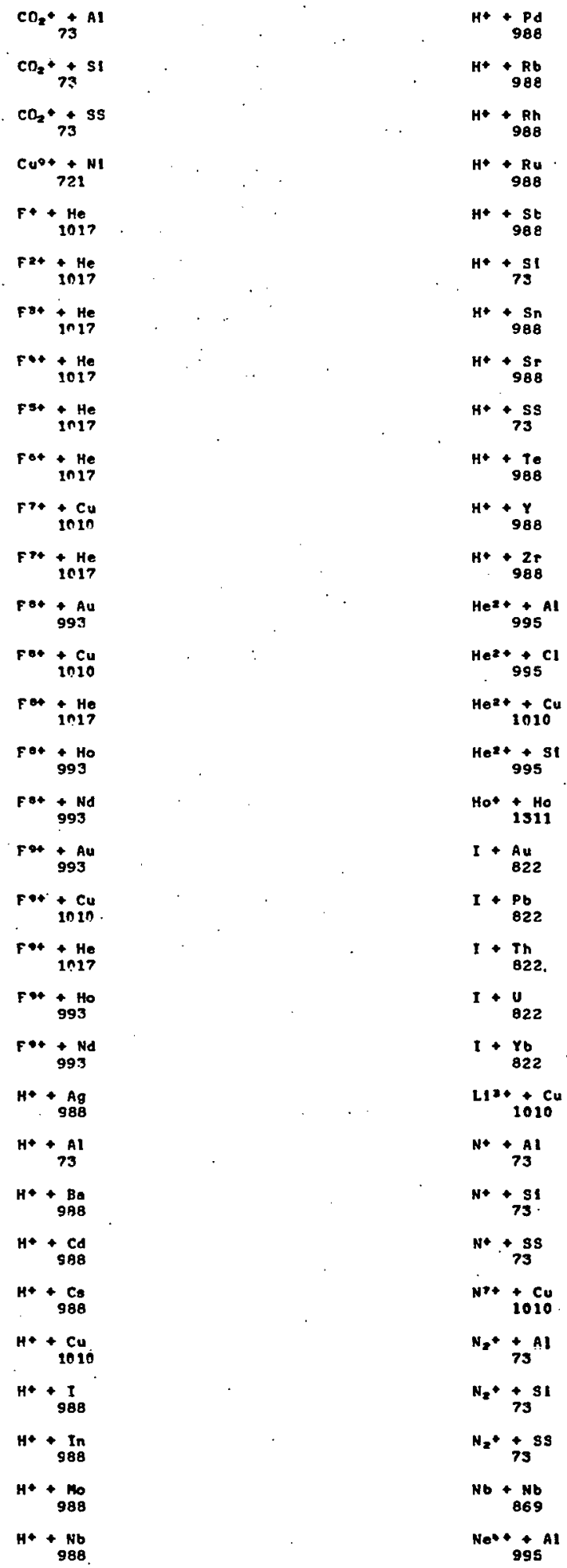




$$
\begin{aligned}
& \mathrm{He}+2+\mathrm{Cl} \\
& \text { Nat. } 995 \\
& \mathrm{NI}+\underset{691}{\mathrm{Mn}} \\
& \mathrm{NI}+\underset{691}{+\mathrm{Pb}} \\
& \mathrm{HI}+\underset{691}{\mathrm{Sn}} \\
& \text { O* + } 73 \\
& 0++\frac{51}{73} \\
& 0+\frac{55}{73} \\
& \text { nat }+41 \\
& \text { O3+ }+\underset{996}{C 1} \\
& \begin{array}{c}
03++51 \\
995
\end{array} \\
& \text { olt }+ \text { He } \\
& \text { oot }+ \text { Cu } \\
& \text { Oo+ }+ \text { He } \\
& \mathrm{Pb}++\mathrm{Pb} \\
& 1311 \\
& s+\text { Al } \\
& s+c_{821} \\
& 3+21 \\
& s+\mathrm{Mg}_{\mathrm{g}} \\
& 9+\begin{array}{c}
\text { Na } \\
\text { O21 }
\end{array}
\end{aligned}
$$

$3+$ se

$s+\begin{gathered}s ! \\ 821\end{gathered}$

$\mathrm{S}+\mathrm{T1}$

seat
1010

S200+ Cu

Sile+Au

S17. 4 HO $^{\mathrm{H}}$

sirt A 993

$5100+$ YU

$\mathrm{SIOH}+\mathrm{Ho}$

$\mathrm{Sl} 0+{ }_{993}^{+\mathrm{Nd}}$

$\mathrm{S10+} 993 \mathrm{Au}$

S10. + Ho

$\mathrm{SI}+4+\mathrm{Nd}$

S110+ Au

993

$31100+110$

993

$\mathrm{S} 210+09+\mathrm{Nd}$

S121+ + A4

$\mathrm{S}_{921+}+\mathrm{HO}$

$\mathrm{SI} 21+\mathrm{Nd}$
A20 HEAUY PARTICLE - HEAUY PARTICLE
INTERACTIUSS

Attenuation (unspeel fled process)

$$
\begin{gathered}
\mathrm{NB}+\underset{\mathrm{CS1}}{\mathrm{C}_{2} \mathrm{H}_{4}} \\
\mathrm{Na}+\underset{ }{\mathrm{CH}_{3} \mathrm{H}_{4}} \\
\mathrm{No}+\underset{\mathrm{CO}}{\mathrm{CO}}
\end{gathered}
$$

801

INTEHACI LUNS WIIH STATIC OR TIRE-VARYING ELECTRIC AND MAGETIC

General

$$
\begin{aligned}
& \text { 31128+ Au } \\
& \mathrm{SI}^{2+2+}+\mathrm{Cu} \\
& 1010 \\
& \text { SI:RA+Ho } \\
& \text { Slixt+Nd } \\
& \mathrm{Sl}_{13+}+\mathrm{Au} \\
& 993 \\
& 5120+\mathrm{Ho} \\
& \text { situo + Nd } \\
& 993 \\
& \text { SI } 10+\text { Au } \\
& 993 \\
& \text { siset in } \\
& \text { Blint Ho } \\
& 993 \\
& \text { SI } 14+\mathrm{NA} \\
& 993 \\
& \mathrm{TA}^{+} \mathrm{AL} \\
& \mathrm{Ta}+\mathrm{HO} \\
& \text { Tat }+\mathrm{Pb} \\
& \text { Ta* + 5n } \\
& T a^{\circ}+\mathrm{Sn} \\
& 1311 \\
& \text { Ta* Ta } \\
& \text { Tot Th } \\
& 11 *+1 \%
\end{aligned}
$$

$$
+\frac{8}{85}
$$

$$
1738
$$


Bos

INTERACT IONS UITH STATIC OR

TIME-UARYING ELECTRIC AND MAGETTC FIELDS

Ionteation

1715

B04

INTERACT IONS UITH STATIC OR TIME-VARYING ELECTRIC AHD MAGNETIC
FIELS

Detachaent

$\mathrm{H}^{-}$

323

Bo5

INTERACTIONS UITH STATIC OR

TIME-VARYING ELECTRIC AND MAGNETIC

FIELDS

Quench Ing

He*

1836

B06

INTERACT TONS UITH STATIC OR

TIME-UARYIMG ELECTRIC AND MAGETIC FIELDS

Exeltation

c*

206
$D_{2} \quad 1715$

H

$\begin{array}{lll}1416 & 1417 & 1715\end{array}$

H*

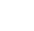

289

$H_{2} \quad 1715$

$h v+\frac{5-}{699}$ $h v+\begin{array}{r}P 6 \\ 767\end{array}$

Na* 602

Undef
2127 


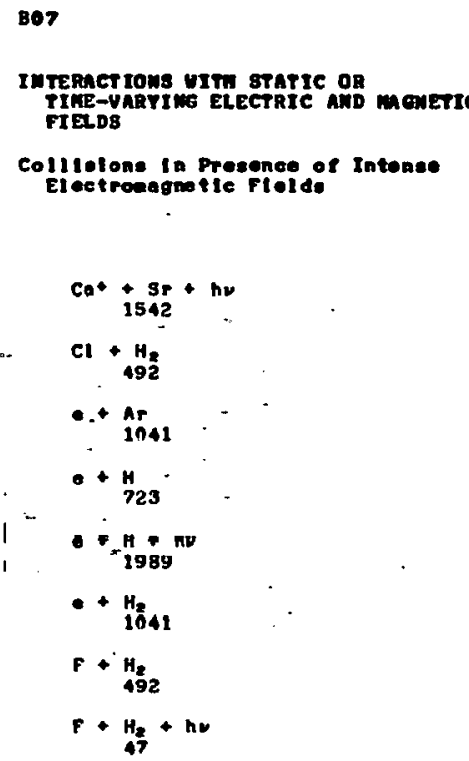

$\cos$

PANT YCLE PEHETRATION IH MACAOSCOPTC matten (JONS, NEUTRALs, AMD EL ECTRON8)

general
$\mathrm{F*}+\mathrm{H}_{12 \mathrm{a}}$

$H+\underset{1212}{40}$

H $+\mathrm{H}_{\mathbf{B}}$

492

$H+\operatorname{lif}_{484}$

nut $\mathrm{Ca}+\mathrm{SP}$

$n=+N_{a}+K_{r}$

643

hw Under

728

He 4

ne* ir

$110+\div 17 y$

$K+\frac{K}{1997}+h$

$K_{2} *+\frac{K}{1997}$

clt 81

$\mathrm{H}_{2}+\underset{169 \mathrm{C}}{\mathrm{C}}$

HeH+ $+\mathrm{C}$
1698

$$
\begin{gathered}
\text { Nat+Na } 1703 \\
\mathrm{Ha}_{2} *+\mathrm{K} \\
1997 \\
\mathrm{Ha}=+\mathrm{Na} \\
1997
\end{gathered}
$$

Nake + $\mathrm{x}$

1957

$85+\underset{758}{6=}$

spe ca $*$ ho

1123

$x_{0}+\underset{657}{F}+$ tho

3hv $\rightarrow H$

1590

Undef

$\begin{array}{lll}277 & 693 & 698 \\ 771 & 772 & 11+1\end{array}$

$\begin{array}{lll}1246 & 1287 & 179\end{array}$

$\begin{array}{lll}1317 & 1326 & 1349 \\ 1353 & 1701 & 1712\end{array}$

$\begin{array}{lll}1353 & 1701 & 1712 \\ 1713 & 1749 & 1840 \\ 1878 & 1883 & \end{array}$
100
800

Under 2072

Ar. 481 


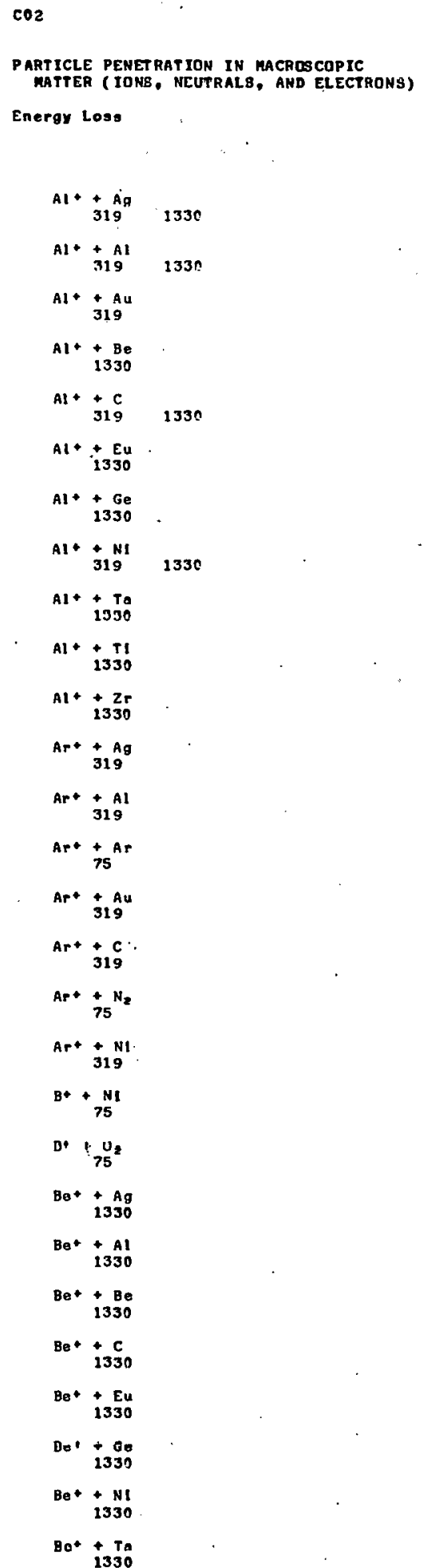

e+ Au

$-+6$

$\mathrm{CO}$
$21 \mathrm{C} ?$

e+ Cu

N1
105

e + PERT
1616

e+ 51

$\mathrm{F}+\mathrm{Ag}$
319

$+\quad 41$
319

F* + Au
319

$F++\frac{C}{319}$

$\mathrm{Ft+}+\mathrm{NL}^{\mathrm{N}} 319$

$\mathrm{f+}+\mathrm{O}_{75}$

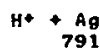

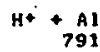

$\mathrm{H}+\mathrm{AU}$
791

$\mathrm{H}^{+}+\underset{1675}{\mathrm{c}}$

H* + Cu
791

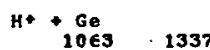

$\mathrm{H}+\mathrm{MO}$
$\mathbf{7 9 1}$

$\mathrm{H}^{+}+\mathrm{NI}$

$\mathrm{H}^{*}+\mathrm{SI}^{2}$

$+891063$

H* $+T^{701}$

$\mathrm{H*}+\mathrm{TI}$

H* +

$+791 \quad 1337$

$M++2 n$
791

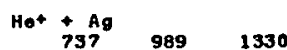

$118 *+\stackrel{A 1}{989} \quad 1330$

$\mathrm{He}_{737}+\mathrm{Au}$

$\mathrm{He}^{+}+\mathrm{Bg}^{\circ}$

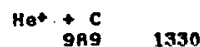

$\mathrm{He}_{989}$ 


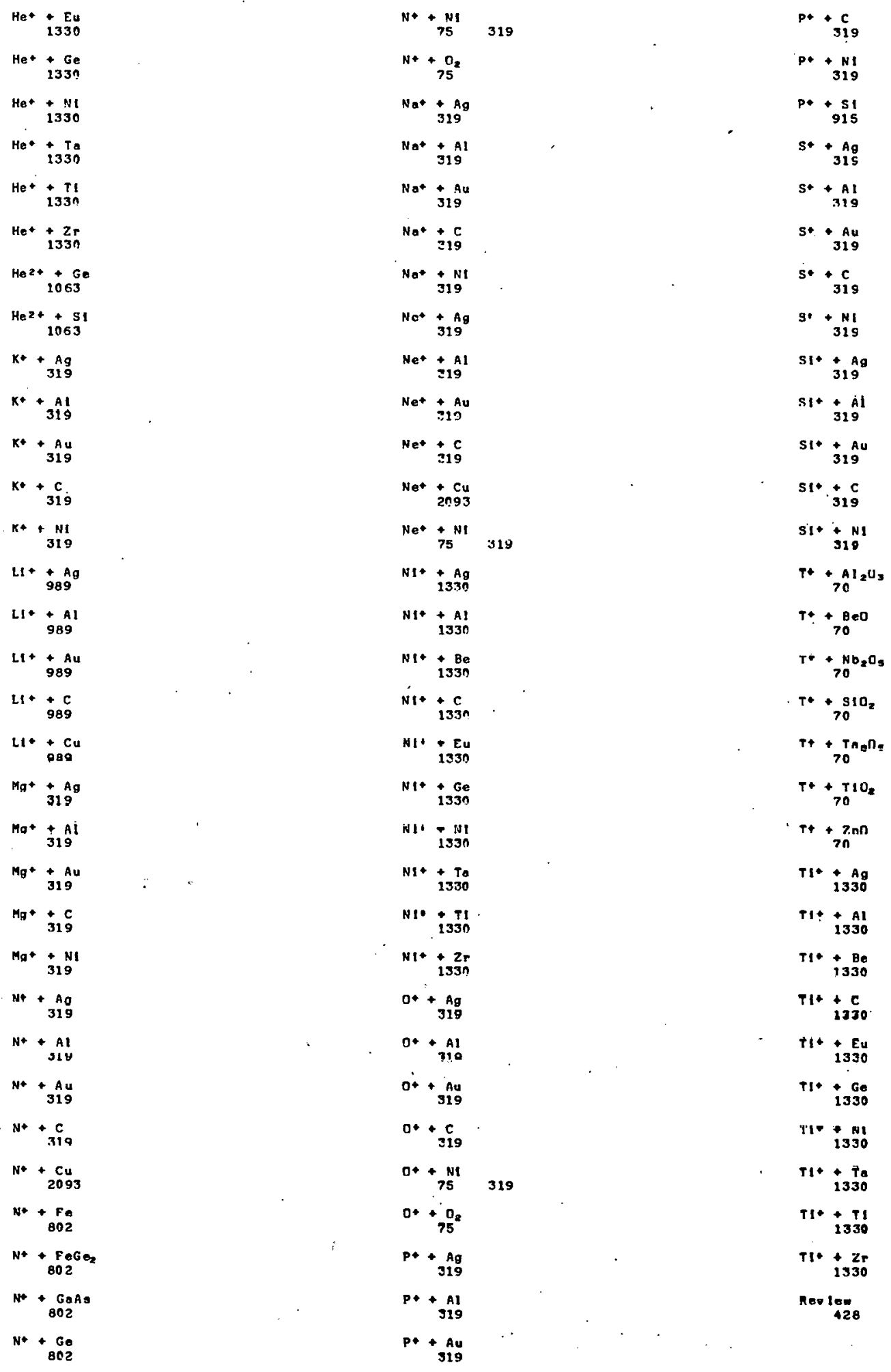




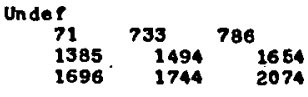

$\cos$

PARTICLE PENGT RATION IN MACROBCOPIC MATTER ( IONS, MEUTRALS, AMD ELECTRONS)

Enorgy to Croate an Ion Palr

$+\mathrm{CO}_{2}$

$\cos$

PARTICLE PENETRATION IN MACROSCOPIC.

MATTER (IORS, NEUTRALS, AND ELECTRONS)

Particle Range

$C+\underset{75}{A g g}$

$D++\underset{1662}{C}$

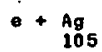

+ Al $_{104}$

$104 \quad 105 \quad 1736$

Cos

Patricle penetration in macros copic

MATTER ( IOWS, MEUTRALs, AND ELECTRONB)

Multiplo scattering

C3+ $+\underset{695}{+ \text { A1 }}$
$-\mathrm{H}_{2}$

$-\stackrel{\mathrm{He}}{1841}$

$e+\mathrm{Ne}_{1841}$

$0+$ Au

$-+\operatorname{lu}_{1 \mathrm{~s}}$

1735

$0+\mathrm{NaCl}$

- + Nt

$+\mathrm{Pb}_{173}$

- 31

H* + PERT

Cot + Al

$D++\underset{1662}{C}$

- + cu

$H++\underset{1675}{C}$
H. + He

$\mathrm{H}^{\circ}+\mathrm{Ne}$

$x+0_{8}$

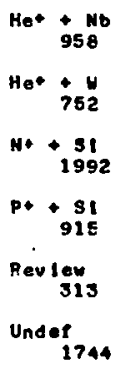

H* + $\operatorname{sio}_{\text {Ines }}$

$\mathrm{He}^{+} \underset{1066}{\mathrm{SIC}_{2}}$

$N++\operatorname{SiO}_{2}$

Under 
co6

PARTICle penetration IN machoscopic MATTER (IONS, NEUTRALS, AHD ELECTRONS)

Charge State Populations.

Art $+\underset{1043 \quad 1044}{C}$

Bo+ +714

Bo+ + Au

Br + PERT

$c++\mathrm{Cs}_{855}$

C+ $+\underset{\text { BSS }}{\text { K }}$

$C++$ No

Cs+ $+\mathbb{7 1 9}^{\mathrm{Ag}}$

Cs+ + Au

Cl * PERT

Cuot + C C

D+ $+\begin{gathered}\text { Ba } \\ 1302\end{gathered}$

D+ $+\mathrm{Ca}_{1302}$

$\mathrm{D}+\stackrel{\mathrm{Mg}}{\mathbf{M g}}$

$D++\underset{1352}{\mathbf{s r}}$

$n_{2}+$ An
1508

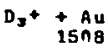

Fi+ $+{ }_{714}^{A g}$

Fr+ + Au

$\mathrm{F}^{\mathrm{O}+}+\mathrm{Al}_{4}^{\mathrm{Ag}}$

$\mathrm{Fa}+\underset{714}{\mathrm{Au}}$

Feot 720 Ar

$\mathrm{Fe}_{720}^{+}+\mathrm{Rr}$

Fe+. $+{ }_{720} \mathrm{~N}_{2}$

$\mathrm{Fe}_{72 n}^{+} \mathrm{SF}_{6}$

Feot +720 Xe
Felot $+{ }^{H}$

Fetr*t 4

Felat $+H$

$F e^{20 t+H}$

$\mathrm{Fe}^{20 t+H}$

Feal + + H

$F e^{2 z+}+H^{+H}$

Fen $3+$ *

$F e^{2+t+h}$

Feest $+H$

Fees * * H

$H+\underset{1078}{A u}$

H+ C

$\mathrm{H}+\underset{1675}{\mathrm{C}}$

$\mathrm{H}++\begin{array}{r}\mathrm{Cg} \\ 855\end{array}$

H* + $\underset{855}{K}$

$\mathrm{H}+\underset{855}{\mathrm{Na}}$

$\mathrm{H}_{2}+\underset{1 \in \mathrm{CO}}{\mathrm{C}}$

$\mathrm{H}_{3}+\underset{\mathrm{B} 36}{\mathrm{C}}$

$\mathrm{HeF}+\underset{1936}{+\mathrm{Ag}}$

Hot + Al

$\mathrm{He}_{72}^{+\mathrm{Au}} 1936$

$\mathrm{He}^{+}+\underset{1936}{\mathrm{C}}$

$\mathrm{Hn}+\underset{1936}{\mathrm{NI}}$

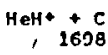

$I+\begin{array}{r}\text { PERT } \\ 1532\end{array}$

$\mathrm{N}+\mathrm{Au}$

Not $+\underset{714}{+\mathrm{Ag}^{-}}$

Not + Au
714
$\mathrm{Ne}+\underset{867}{\mathrm{C}}$

$\mathrm{Ne}^{2+}+\mathrm{C}$

$\mathrm{NaO}+\mathrm{C}^{\mathrm{C}}$

$\mathrm{NeOt+C}$

$\mathrm{Ne}^{\mathrm{s}+}+\mathrm{C}$

141

$\mathrm{NeO}+{ }_{141}^{+} \mathrm{C}$

Nort $+\mathrm{C}$

$0++\underset{100}{1056}$

$0++\underset{895}{c 9}$

$0++\frac{K}{666}$

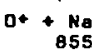

$0++\begin{gathered}51 \\ 2056\end{gathered}$

$00+{ }_{987}^{A 1}$

$0 *+\begin{gathered}H \\ 1360\end{gathered}$

Ds+ ${ }^{A 1}$

ose $+\underset{1360}{H}$

Oo+ + A1

$00+\underset{1360}{H}$

Or+ $+\mathrm{ABg}^{\mathrm{A}}$

Q"4 A1

Or+ + Au

$00++11$

00+
+ A1
987

oot $+\underset{1000}{H}$

$\mathrm{s}+\underset{856}{\mathrm{Cg}}$

S* + K

$s++\mathrm{Na}$

$\mathrm{Xe} * \mathrm{Cu}$
1075 
607

PARTICLE PENET RATION IN MACBOSCOPIC MATTER (IONS, NEUTRALS, AND ELECTRDNS)

Exclted State Populationg

$$
\begin{aligned}
& \mathrm{B}^{4+4}+\mathrm{Ag}^{\mathrm{Ag}} \\
& \text { But }+\mathrm{Au} \\
& \mathrm{C}+\mathrm{c} \\
& 1297 \\
& \mathrm{Cs}+\underset{714}{\mathrm{Ag}} \\
& \begin{array}{c}
\mathrm{Cs}^{+}+\mathrm{Au} \\
714
\end{array} \\
& \text { Fit }+\mathrm{Ag}^{\mathrm{Ag}} \\
& \begin{array}{c}
+14 \\
714
\end{array} \\
& \mathrm{Fot}^{+}+\mathrm{Ag}^{\mathrm{Ag}}
\end{aligned}
$$

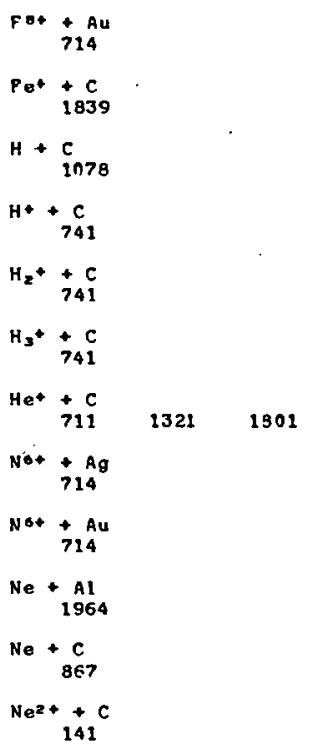

$\mathrm{Ne}^{\mathrm{J}}+\mathrm{C}_{1}^{\mathrm{C}}$

$\mathrm{Nen++C}$

Ner+ + C

$\mathrm{NeO}+\underset{141}{+C}$

$\mathrm{No}+\underset{141}{+\mathrm{C}}$

$\mathrm{NI}+\mathrm{NI}$

1964

$0 *+\frac{C}{741}$

Ore + Ag

Ore + Au

$\mathrm{O}_{2}+\underset{741}{\mathrm{C}}$

$\mathrm{Xe}^{+}+\underset{\mathrm{Cu}}{1075}$ 
$c 0$

papricle penetrarton in machoscopte

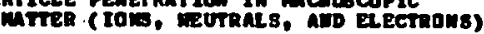

encommiting

1681

Art $\begin{array}{r}\text { NI } \\ 1074\end{array}$

2683

Bot +119

on. : A14

C. $+\mathrm{NI}$

c. + 81

C+ + 89

Cs. $714^{99}$

Co+ 314 Au 1800

CI + aud

Cl $+\begin{array}{r}60 \\ 642\end{array}$

C1 + 81

$c 1+\frac{v}{842}$

$D++60$

D* + No

De. Whn 795

$00+51 \quad 2104$

$-\stackrel{\text { A1N }}{160 ?}$

$-+c$
Art c cu
81
1067

Fre $+714^{A g}$

Fit 114

Fer $+714^{A g}$

Fot 714 Au 1863

$H+\underset{842}{\text { Bu }}$

$H+6 e$
842

H + S1

H +

H+ $+\underset{1064}{\text { Au }}$

$\mathrm{H}+\underset{60}{1063}$

$\mathrm{H}+\underset{2 n g \mathrm{Mg}}{\mathrm{M}}$

$\mathrm{H*}+\underset{1747}{\mathrm{NaCl}}$

$\mathrm{H} *+$ Nb

$\mathrm{H}++\mathrm{Nt}$

$\mathrm{H}++\mathrm{St}$

84
2104
21 $1063 \quad 1070$

$H++\frac{y}{69}$

$\mathrm{He}+{ }_{842}^{\mathrm{Au}}$

$\mathrm{He}+\mathrm{Oo}$

$\mathrm{He}+\mathrm{SI}$

He + 842

$\mathrm{He}_{72}^{\mathrm{AU}} \mathrm{C92}$
Ho+ + Gosb

$\mathrm{Hec}_{82}^{+} \mathrm{HoO}^{\circ}$

Hot 692

Hot+ $+\begin{array}{r}\mathrm{Nb} \\ 2104\end{array}$

$\mathrm{Hot}+\mathrm{NI}$

Ho+ $\begin{gathered}+ \text { PbTI } \\ 2076\end{gathered}$

Het $+\underset{892}{\mathrm{Pt}}$

$\mathrm{Hat}+\mathrm{SI}$

$892 \quad 2008: 2104$

Ho+ $+\mathrm{UC}_{5} 896$

$\mathrm{HO}^{+}+{ }_{790} \mathrm{~V}_{3} \mathrm{SI}$

Hot + 892

$\mathrm{He}+\mathrm{Ge}$
1068

Ho2+ + sI

1063

$\mathrm{N+}+\mathrm{AP}^{\mathrm{Au}}$

$\mathrm{Nat}+\mathrm{AB}^{\mathrm{Ag}}$

Not + Au

De. Ag

$0++51$

0 D" $+A_{0}$

Ort + AU 1500

$\mathrm{Xos}_{+\mathrm{Cu}}$

Und of

$\begin{array}{lll}878 & 1057 & 1336\end{array}$ $1387 \quad 1494.2074$

Do1

$\mathrm{Kr}+\underset{\mathbf{E}}{\mathbf{W}}$

$x_{0}+\frac{y}{871}$

Ne $+\mathrm{y}$

PATTICUE INTERACTIDW UIT SOL IDS

Goveral 
Do2

PARTICLE INTERACTIONS VITH SOLIDS

Sputtering by Electrons, Meutrons, and Heavy Part leles (totel resovel Heavy Part felos

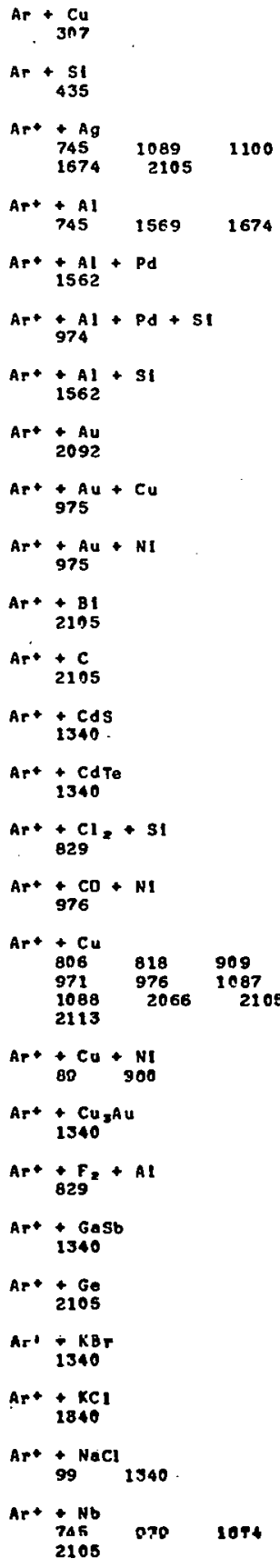

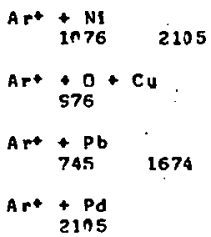




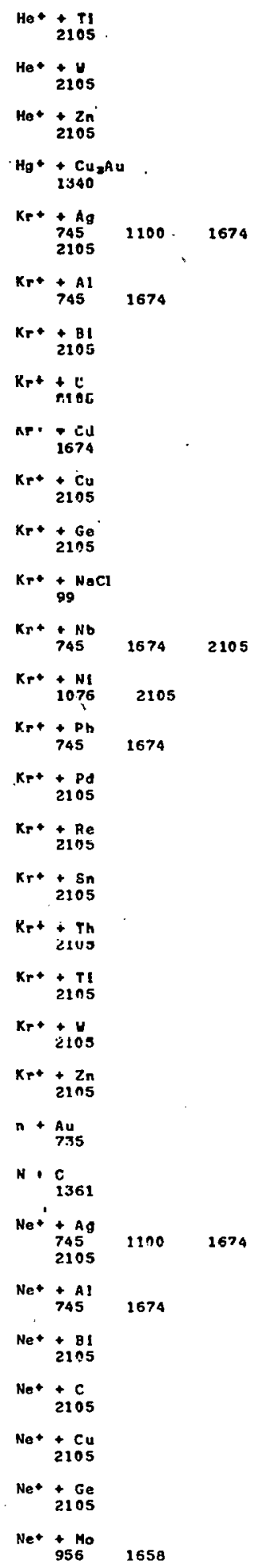

$$
\begin{aligned}
& \mathrm{O}_{2}+\mathrm{Pa}^{\mathrm{P}} \\
& 0 .+ \text { Pd } \\
& \mathrm{D}_{2+}+\mathrm{Ru} \\
& 0 .+56 \\
& 0_{2}+\mathrm{SI}^{+11} \\
& 0 .+2+5 n \\
& 0_{2}+T_{11} \\
& 0 .+11 \\
& \text { ח.+.+ TI } \\
& \begin{array}{r}
0_{2}+U \\
821
\end{array} \\
& \mathrm{O}_{2}+\stackrel{\mathrm{V}}{\mathrm{B} 11} \\
& \mathrm{O}_{2}+{ }_{811} \quad 922 \\
& 0_{2}++2 n \\
& \mathrm{O}_{2}+\mathrm{Zr}^{21} \\
& \begin{array}{l}
\mathrm{P}+\mathrm{Ag} \\
11 \mathrm{~g}
\end{array} \\
& \mathrm{P}_{2}+{ }_{113}^{\mathrm{Ag}} \\
& \text { PERT + }{ }_{307} \\
& \text { Iut } 11 \mathrm{AO}^{\mathrm{A}} \\
& \text { So+ }+ \text { Au } \\
& \text { cot } \\
& \mathrm{Sb}_{2}+{ }_{11.3}^{\mathrm{Ag}} \\
& \text { 86.: } 113 \\
& \mathrm{Sb}_{2}++_{113}^{\mathrm{Pt}} \\
& \mathrm{Sb}_{3}+113^{\mathrm{Ag}} \\
& \mathrm{Sb}_{3}+\ldots \mathrm{Au} \\
& \mathrm{Sb}_{2}+\ldots \mathrm{Pt}^{\mathrm{Pt}} \\
& \mathrm{Se}^{+}+\mathrm{Ag}^{\mathrm{Ag}} \\
& \mathrm{SO}_{2}+{ }_{113}^{+{ }^{A B}} \\
& \begin{array}{c}
\mathrm{T}+\mathrm{Fe} \\
30 \mathrm{E}
\end{array} \\
& T+\begin{array}{l}
\text { Mo } \\
308
\end{array} \\
& \begin{array}{c}
\mathrm{Te}+\mathrm{Ag} \\
113
\end{array} \\
& \mathrm{Te}_{2}+{ }_{113}^{\text {Ag }}
\end{aligned}
$$


211

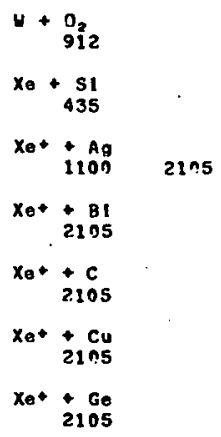

Xo* + In

$x+T 1$
2108

$\begin{aligned} x_{0}+ & +4 \\ 2108 & \end{aligned}$

$\times 0+\begin{array}{r}2 n \\ 2108\end{array}$

Undef

$880 \quad 973^{\circ} \quad 1082$ 
Dos

PARTICLE INTERACTIOHS UITH BOLIDS

Sputtered Particle Charge and Quantue (Exelted) state Dietribution

$A \mathbf{r}+\mathbf{S}$

Ar* + Ag
1089

Ar* $+\underset{230}{A 1}$

$\mathrm{Ar}++\mathrm{Al}^{2} \mathrm{OS}$

Art $+A s$

Art $+A$

$\mathrm{Ar+}+\mathrm{Be}$

2193

Ar* + BI

Art $+C$

Art $+\mathrm{Cu} 004 \quad 1772$

Art + CuO

Ar* +60

$A r+$ Ge

Art $+\mathrm{KCICaCl}$

$A r+t \mathrm{Mg}_{20 \mathrm{AI}}$

A.' $\div$ Nu

Ar*

$\mathrm{Ar}+\underset{1544}{\mathrm{O}}+\mathrm{Cu}$

Ar * + Pd

APV + Ke

Ar* ${ }_{80}^{51} 804$

Ar* +51 H

Art $+S_{3108}$

Art + SS

Ar* + Th

Art $+\mathrm{TI}$
2105

$\mathrm{Ar}++\mathrm{Y}$

2105
Art $\begin{array}{r}+7 n \\ 2105\end{array}$

Kr+t Th

Cot + Ta
ins

$\mathrm{Kr}^{+}+\mathrm{TI}$

$\mathrm{Kr}^{+}+\underset{\mathrm{H}}{21 \mathrm{~ns}}$

$K r+\frac{Z_{n}}{2105}$

$\mathrm{Ne}+\underset{80}{\mathrm{SI}}$

$\mathrm{Ne}^{+}+\mathrm{Ag}$

$\mathrm{Ne}^{+}+\mathrm{BI}$

$\mathrm{Ne}+\mathrm{C}$

Ned iu

$\mathrm{Ne}+\mathrm{Ge}$

$\mathrm{Net}+\mathrm{Nb}$

Net $+\mathrm{NI}$

2205

Net+ Pd

$\mathrm{Ne}^{+}+\mathrm{Re}$

Hot + EIII

$\mathrm{He}^{+}+\mathrm{Re}$

Het + Sn

Het t Th

$118 * 17$

$\mathrm{Hot}+\mathrm{V}$

$\mathrm{He}+\mathrm{Zn}$

$K r+S_{\text {gn }}$

$\mathrm{Kr} * \mathrm{Ag}$

$\mathrm{Kr}++$ BI

Kr+ + C

+ Sn

Net 3105

$\mathrm{Ne}+\mathrm{TI}$

Net+

Ne+ $+2 n$

$\mathrm{O}_{2}+\mathrm{Mg}_{\mathrm{g}}+\mathrm{Al}$

$X e+5 i$

Xe* + A9

$x_{0} \cdot+81$

$\mathrm{Xet}^{+} \mathrm{C}$

$\mathrm{Xo*}+\mathrm{Cu}$

$16 \mathrm{O}+\mathrm{Ge}$

2105

$\mathrm{Xet+Nb}$

$X=1+N I$

Yat $\begin{array}{r}\text { Pd } \\ 2105\end{array}$

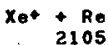

$\mathrm{Xe}^{+}+\mathrm{SI}^{+}$ 
$x_{0+}+5114$

$x_{0}++\begin{array}{r}5 n \\ 2105\end{array}$

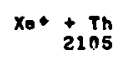

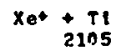

$x_{0}+4$

2105

$x 0^{+}+2 n$ $\begin{array}{ccc}\begin{array}{c}\text { Reviey } \\ 781\end{array} & 782 & \\ \text { Undea } & \\ 90 & 893 & 1066\end{array}$ 


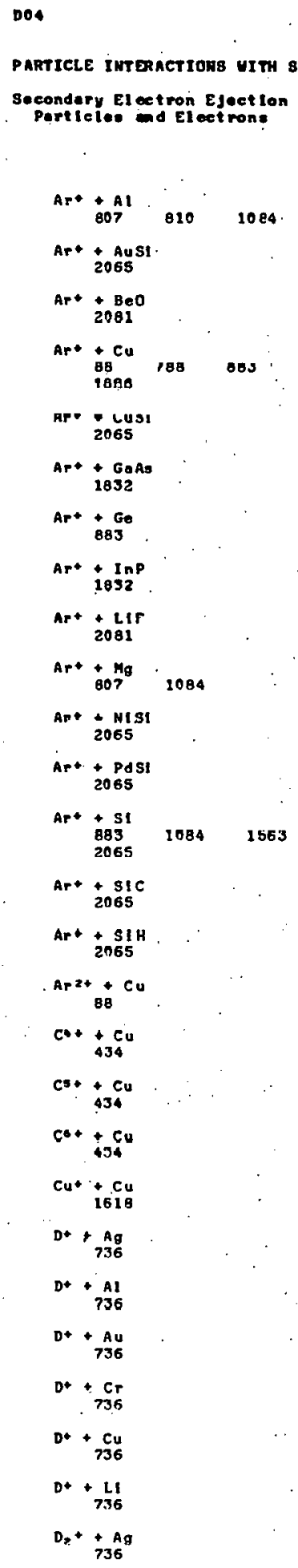

$$
\begin{aligned}
& \text { e+ }{ }_{831}^{\mathrm{P}_{2} \mathrm{O}_{3}} \\
& \therefore+\text { PbI } \\
& \text { c + PoS } \\
& \text { - RbBr } \\
& \text { - + RbI } \\
& 1053 \\
& \text { - + Se }
\end{aligned}
$$$$
\text { e }+ \text { si }
$$$$
\because T
$$$$
\because \text { is } 3 \text { inn }
$$$$
\text { - } \mathrm{THH}^{2}
$$$$
\text { - } \mathrm{v}_{8} \mathrm{O}_{2}
$$$$
\text { H + Cu }
$$$$
\mathrm{H*}+\underset{739}{\mathrm{Ag}}
$$$$
\text { H* + Al } 736
$$$$
\text { H4: + Art } 1618
$$$$
\text { H. } \because \text { AU }
$$$$
6650^{\circ} 736.1064
$$$$
\mathrm{H}+\underset{736}{\mathrm{Cr}}
$$$$
\mathrm{H}+\mathrm{Cu}_{4}
$$

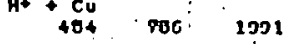$$
\text { H* + } \begin{array}{ll}
736 \\
736
\end{array}
$$$$
\mathrm{H}+\mathrm{Mgg}_{007}^{\mathrm{Mg}}
$$$$
\mathrm{H}_{2}^{+}+\stackrel{\mathrm{Ag}_{\mathrm{g}}}{736}
$$$$
\mathrm{H}_{2}+736
$$$$
\mathrm{H}_{2}+{ }_{665}+{ }_{736}
$$$$
H_{2} *+{ }_{736} \mathrm{Cr}^{2}
$$$$
\mathrm{Hz}^{+}+\underset{736}{+\mathrm{Cu}}
$$$$
\mathrm{H}_{2}+\underset{736}{+\mathrm{Lt}}
$$$$
\begin{array}{r}
\mathrm{H}^{-}+\mathrm{Cu}^{-} \\
+1991
\end{array}
$$$$
\text { Mọ" + cut + ve }
$$$$
760
$$$$
\mathrm{HoO}+\mathrm{CO}^{\circ} \mathrm{Y}
$$$$
\text { He* }+\mathrm{N}
$$$$
\mathrm{He*}+\mathrm{Pd}^{\circ}
$$$$
\text { He* }+409
$$$$
\mathrm{He}+\stackrel{\mathrm{Ag}}{736}
$$ 


\begin{tabular}{|c|c|c|}
\hline $\mathrm{He}$ & ${ }_{736}^{A 1}$ & 807 \\
\hline He* & ${ }_{1618}^{A r^{*}}$ & \\
\hline $\mathrm{Ha}$ & $\begin{array}{l}+\mathrm{Au} \\
665\end{array}$ & 736 \\
\hline $\mathrm{He} \cdot$ & $+c$. & \\
\hline $\mathrm{He}+$ & $+{ }_{736} \mathrm{Cr}$ & \\
\hline $\mathrm{Hat}$ & $\begin{array}{l}+\mathrm{Cu} \\
665\end{array}$ & 736 \\
\hline $\mathrm{He} *$ & $\begin{array}{l}+k^{+}+ \\
1559\end{array}$ & NI \\
\hline $\mathrm{He}+$ & $+L_{736}^{\prime \prime}$ & \\
\hline $\mathrm{He}+$ & $+{ }^{M g}$ & \\
\hline
\end{tabular}

D08

PARTICl.E TMTERACTIONS UITH BCQ IDS

Photoelectric Ejection of Elcotrons (coefficlonts)

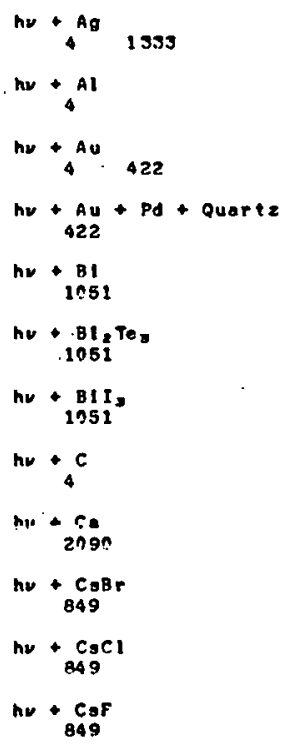
(coesficlents)

$$
\begin{aligned}
& \begin{array}{r}
\text { - Al } \\
\text { a17 }
\end{array} \\
& \text { - C } \mathrm{Cr}, \mathrm{O}
\end{aligned}
$$

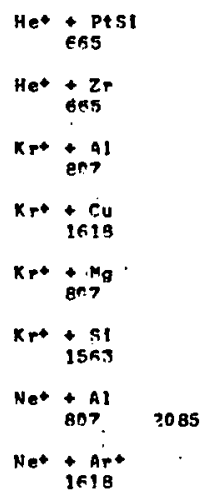

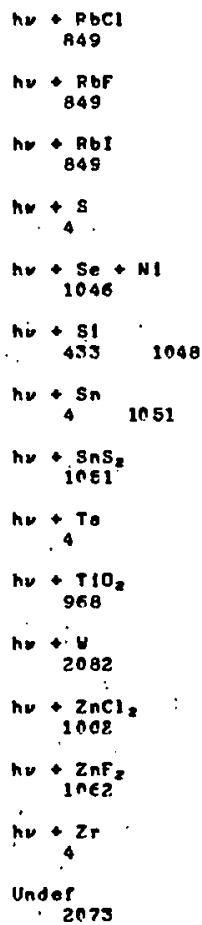

$$
\begin{aligned}
& \text { e+ S1 } 801 \text { 2iny } \\
& \text { e+ Sn } \\
& \text { - + Y66 } 2003 \\
& \text { Review } 428 \\
& \text { Under }
\end{aligned}
$$


Do7

PARTICLE INTERACTIONS UITH SOL IDS

Reflectlon of Heavy Partieles Prow Surfaces ( total rafiection coefeletents)

\begin{tabular}{|c|c|c|c|}
\hline & $+\operatorname{Lif}_{102}$ & & \\
\hline Ar & +516 & & \\
\hline Ar & $\begin{array}{r}+ \text { WC } \\
516\end{array}$ & 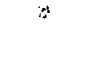 & \\
\hline & $+\begin{array}{c}+A U \\
796\end{array}$ & & - \\
\hline & $\begin{array}{l}+\mathrm{Cu} \\
97 \\
895\end{array}$ & $\begin{array}{l}111 \\
1676\end{array}$ & 2ดธ่ $168 \mathrm{i}$ \\
\hline & $+\begin{array}{l}+N 1 \\
\text { In?4 }\end{array}$ & 1339 & I7ES \\
\hline c+ & $\begin{array}{r}\text { NI } \\
892\end{array}$ & & \\
\hline c* & $+\underset{892}{W}$ & & \\
\hline D + & $\begin{array}{r}\ln f f \\
107\end{array}$ & 1568 & \\
\hline & $\begin{array}{r}\text { NaF } \\
107\end{array}$ & 1568 & \\
\hline$D+$ & $+\underset{1661}{c}$ & 1662 & \\
\hline D* & $+\underset{2104}{\mathrm{Nb}}$ & & , \\
\hline $\mathbf{D}^{+}$ & $+\underset{T P 4}{N 6 O}$ & 79.5 & \\
\hline$D+$ & $\begin{array}{c}5 t \\
1661\end{array}$ & 2104 & \\
\hline II ' & $\begin{array}{l}\text { Cu } \\
1801\end{array}$ & & $\cdot$ \\
\hline H + & $+\underset{1568}{\mathrm{H}_{2} \mathrm{O}}+$ & $\mathrm{KCl}$ & \\
\hline H + & $+\operatorname{l1f}_{1568}$ & & \\
\hline H + & $+\begin{array}{l}\text { NaF } \\
1568\end{array}$ & & \\
\hline $\mathrm{H}^{+}$ & $+\underset{199}{\mathrm{Cu}}$ & & \\
\hline $\mathrm{H}^{*}$ & $+\underset{1337}{G e}$ & & \\
\hline H* & $\begin{array}{r}\text { Mo } \\
919\end{array}$ & & \\
\hline
\end{tabular}

$\mathrm{H}^{+}+\mathrm{Nb}$

2104

$\mathrm{H}++\mathrm{NI}$

H* + Pq

$\mathrm{H}++\mathrm{Si}$

2104

$\mathrm{H*}+\underset{225}{\mathrm{U}} \quad 992$

1337

$\mathrm{H}^{+}+\underset{325}{\mathrm{WO}}$

$\mathrm{H}_{2}+\underset{\mathrm{Ag}}{\mathrm{An}}$

$\mathrm{H}_{2}+\underset{1.1 \mathrm{~F}}{1.55}$

$H_{2} * N_{101}^{N 1}$

$\mathrm{H}^{-}+\mathrm{Cu}_{1991}$

$\mathrm{He}+\underset{\mathrm{Ag}}{\mathrm{Ang}}$

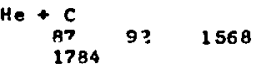

$\mathrm{He}+\underset{\mathrm{Cu}}{\mathrm{Cu}}$

$H e+\underset{474}{L 1 F}$

1047

$+\mathrm{NaF}$

ก9 1

$\mathrm{He}+\underset{73 B}{\mathrm{~N} I \mathrm{O}}$

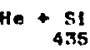

He. 51

B日

He + 516

$\mathrm{He}_{\mathrm{B}}^{+\mathrm{Au}}$

$\mathrm{He}^{+}+\mathrm{Cu}$

Het $\underset{2 n 78}{\text { GaSb }}$

He* * Mo

$\mathrm{He}+\mathrm{Nb}$

2104

IIol 1. Hall

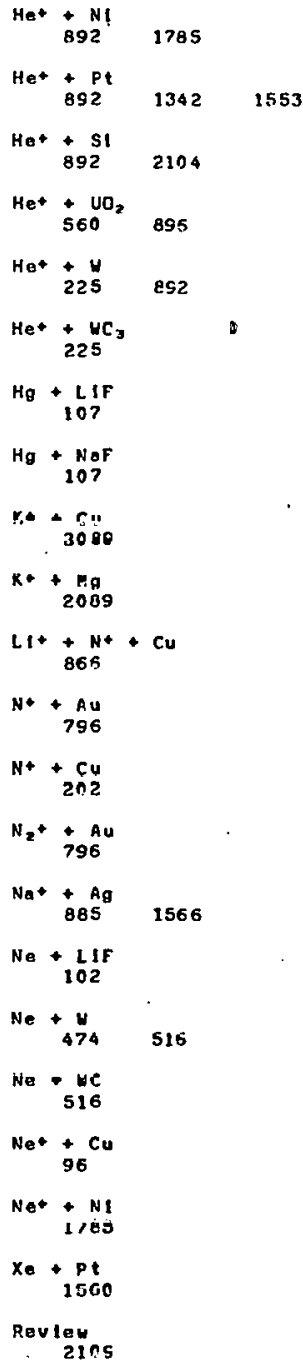


Dos

PARTICLE INTERACTIONS UTTH SOLIDS

Charge and quantua state Diotributione or Reflacted Heavy Particles at Macroscople DIstances fron Surfeces

\begin{tabular}{|c|c|c|}
\hline & $+{ }_{202}^{+C u}$ & 783 \\
\hline B* & $+\underset{204}{c}$ & \\
\hline $8+$ & $+\begin{array}{r}s 1 \\
2 n 7\end{array}$ & . \\
\hline & $*_{207}^{+} \mathrm{S1}$ & \\
\hline & $+\underset{1828}{\mathrm{Cu}}$ & \\
\hline H + & $+{ }_{+}^{+} \mathrm{Cg}_{1395}$ & \\
\hline
\end{tabular}

D09

PARTICLE INTERACTIONS WITH SOL.IDS

De-axcit at lon, Neutrallzation.

Ionlaction, or Dissoclation or

Particles interacting with surfaces

$\mathrm{CO}+\mathrm{CO}_{7}+\mathrm{Au}$

1977

$\mathrm{CO}+\mathrm{CO}_{2}+\mathrm{Au}+\mathrm{Pt}$

$\mathrm{CO}+\mathrm{CO}_{107}+\mathrm{Pt}$

D10

PARTICL.F. INTERACTIONS UITH SOLIDS

Interactlon Potentlals Between Surfaces and Free Particles Located External to the Surface (olectrons and heavy

particles)

$\mathrm{CO}+\underset{\mathrm{Ru}}{\mathrm{TfB}}$

$D y+W$

40+

$\mathrm{H}+\mathrm{AL}$

$1 n 78$

$\mathrm{H}+\mathrm{r}$

Ho $+w$
$\mathrm{He}^{+}+\underset{204}{\mathrm{C}}$

$\mathrm{Hol}+\mathrm{N3}^{\mathrm{N}}$

$\mathrm{He}^{+}+\mathrm{Pb}$

$\mathrm{He}+$ + Under

1399

$\mathrm{N}+\mathrm{AL}^{\mathrm{AL}}$

$N++\underset{2}{C}$

$\mathrm{Ne}+\underset{96}{+\mathrm{Cu}}$

Reviey

205

Li* $+\mathrm{Ph}$

$\mathrm{LI}+\mathrm{T}^{\circ}$

$\mathrm{BH}+\mathrm{C}$

$T b+W$

Undef

1081
$H+\mathrm{C}_{1778}$
$K r+\underset{493}{C}$
$\mathrm{H}_{2}+\underset{1598}{\mathrm{CC}}$
Ne $+\underset{4 y 5}{C}$
$x_{e}+\underset{493}{C}$

$A r+\underset{493}{C}$ 
D11

PARTICLE INT ERACTIONS UTT BOCTDS

st Ieking Coofficlente (therael energleo)

\begin{tabular}{|c|c|}
\hline Ar + $\begin{array}{c}y \\
871\end{array}$ & \\
\hline $\mathrm{Br}_{2} \stackrel{+446}{\mathrm{Y}}$ & \\
\hline $\mathrm{CH}^{+} \mathrm{NI}^{\mathrm{N}}$ & \\
\hline $\mathrm{Cl}+\mathrm{Fe}_{1766}$ & \\
\hline $\mathrm{Cl}_{2}+\mathrm{H}$ & \\
\hline$c u+F e$ & \\
\hline $\mathrm{CO}+\underset{1762}{\mathrm{FO}^{2}}+\mathrm{K}$ & \\
\hline $\mathrm{CD}+\underset{970}{\text { In }}$ & \\
\hline $\begin{aligned} \text { Co }+ \text { pt } & 2116\end{aligned}$ & \\
\hline $\mathrm{Co}+\mathrm{He}$ & \\
\hline $\mathrm{CO}+\underset{\mathrm{Ru}}{\mathrm{Ru}}$ & \\
\hline $\mathrm{Co}+\underset{1763}{u}$ & \\
\hline $\mathrm{CO}_{2}+{ }_{970}{ }^{\mathrm{In}}$ & \\
\hline
\end{tabular}

D12

PARTICLE INTERACTIONS UITM SOLIDS

El ect romagnotie Radf at ion Induced by Electron or Heary Particle Inpoct on Surfaoes

$$
\begin{aligned}
& \mathrm{APt}+\mathrm{BaSU}, \\
& 725 \\
& \text { Art + Mo } \\
& \text { Art? Ss } \\
& \text { C+ + Al } \\
& \text { C+ }+\mathrm{c} \\
& \text { c. +N1 } \\
& \text { e+ AlN } \\
& \text { e + Co } \\
& + \text { Cr }
\end{aligned}
$$

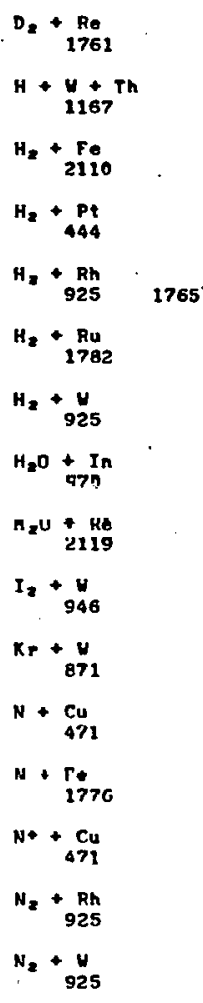

$\mathrm{N}_{2} \mathrm{O}+\mathrm{Cu}$

Ne + 47

$\mathrm{NH},+\mathrm{Fe}$

NO $+\mathrm{Re}$

No + Fin

$0 .+1768$

$\mathrm{O}_{2}+\mathrm{Cu}$

$\mathrm{O}_{2}+$ In $_{\text {ITO }}$

$0 .+70$

O. Re 2117

$0 .+81$

$02+1763$

$\mathrm{xe}+\mathrm{Cu}$

$x e+P d$

$x_{0}+471 \cdot 1763$

Under

1335

$\mathrm{H}^{+}+\underset{1000}{\mathrm{Sic}}$

$\mathrm{H}_{2}{ }^{+}{ }_{780} \mathrm{NACl} 1682$

$11 e^{+}+\mathrm{NaCl}$

$40++510$

1680

$\mathrm{N}+\mathrm{NaCl}$

$+\mathrm{NACl}$
780

$\mathrm{H}^{+}+\underset{1680^{2}}{ }$

$\Psi_{*}+\underset{780}{\mathrm{HaCl}} 1682$

$0++A_{9}$

$0++51$

$0++310^{2}$

$\mathrm{Pb}+\mathrm{Pb}$

Review 
D13

PARTİCLE INTERACTIONS UITH SOLIDS

Dosopption of Bados fron Surfaces

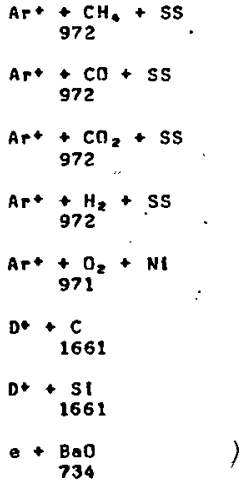

D14

PARTICLE INTERACTIONS WITH SOLIDS

Bllatering, Wolds, and Surface strain In Metals

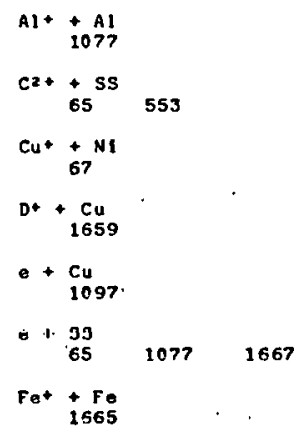

$e+\underset{\mathrm{SP}_{2}}{\mathrm{CH}_{2}}+\mathrm{SS}$

$+\mathrm{CO}+\mathrm{Mo}$

$\mathrm{e}+\mathrm{CO}+\mathrm{NI}$

$++\mathrm{CO}_{972}^{+} \mathrm{ss}$

$+\mathrm{Cn}_{177 n}+\mathrm{H}$

$e+\mathrm{CO}_{2}+\mathrm{si}$

$+\underset{B B 7}{H}+M_{0}$

$\mathrm{e}+\underset{972}{\mathrm{H}_{2}}+\mathrm{SS}$

$\mathrm{H}+\mathrm{H}+\mathrm{H}$
1567

$M_{930}$
734

$e+\frac{0+C d s}{1086}$

\begin{tabular}{|c|c|c|c|}
\hline $\mathrm{Fe}^{+}$ & $\begin{array}{l}+\mathbf{S 5} \\
\mathbf{1 6 6 5}\end{array}$ & & \\
\hline $\mathrm{He}^{+}$ & $\stackrel{A 1}{A 44}$ & & \\
\hline $\mathrm{He}^{+}$ & $\begin{array}{l}+c \\
203\end{array}$ & & \\
\hline $\mathrm{He}^{+}$ & ${ }_{784} \mathrm{C}_{5}+$ & NI & \\
\hline $\mathrm{He}^{+}$ & ${ }_{\mathrm{e}}^{+\mathrm{Nb}}$ & 958 & \\
\hline $\mathrm{He}^{+}$ & $\begin{array}{l}\text { SS } \\
22.6\end{array}$ & 784 & \\
\hline $\mathrm{He}^{+}$ & $\stackrel{2 r r}{226}$ & & \\
\hline$n+$ & $\begin{array}{l}\text { Al } \\
\text { In>? }\end{array}$ & & \\
\hline$n+$ & $\begin{array}{l}\text { fe } \\
1665\end{array}$ & 1655 & \\
\hline$n+$ & $\begin{array}{l}\text { sS } \\
959 \\
1666\end{array}$ & 960 & 1655 \\
\hline
\end{tabular}

$$
\begin{aligned}
& \begin{array}{ccc}
0+734 \\
1332 & 087 & \\
1767 & &
\end{array} \\
& \begin{array}{l}
0+4177 \quad 2087 \\
91
\end{array} \\
& \text { - }+\mathrm{O}_{2111}+\mathrm{Mo}^{2} \\
& \begin{array}{l}
0_{2} * \text {, } \\
15 \in 5
\end{array} \\
& \begin{array}{r}
5 r 0 \\
754
\end{array} \\
& \text { e+ No, } \\
& \text { - } \begin{array}{l}
\text { Zno } \\
734
\end{array} \\
& \mathrm{H}_{2}+\mathrm{Cu}_{\mathrm{O} 46} \\
& \mathrm{H}_{2}+\underset{846}{\mathrm{NI}} \\
& h v+\underset{879}{\mathrm{H}_{2} \mathrm{O}}+\mathrm{Glase}
\end{aligned}
$$

$$
\begin{aligned}
& n+\mathrm{Zr}_{566}+\mathrm{Nb} \\
& n+\underset{555}{\operatorname{zr}}+g_{n} \\
& N++\begin{array}{c}
N 1 \\
959
\end{array} \\
& \mathrm{NL}+\mathrm{Fe} \\
& \mathrm{N1}+\underset{559}{+\mathrm{Nb}} \\
& \text { NI } \underset{559}{+} \text { Nb }+\mathrm{TI} \\
& N 1+\underset{66}{+N 1} 67 \quad 1077 \\
& \text { NI+ }+\begin{array}{r}
\text { SS } \\
1665
\end{array} \\
& \mathrm{NI}+{ }_{65}^{+} \mathrm{SS} \text { 553 } \\
& \text { Und ef } \\
& \begin{array}{lll}
789 & 1071^{\circ} 1665
\end{array}
\end{aligned}
$$


D15

PARTICLE INTERACTIONS UITH SOLIDS

Rodiat Ion Dosage in Metals

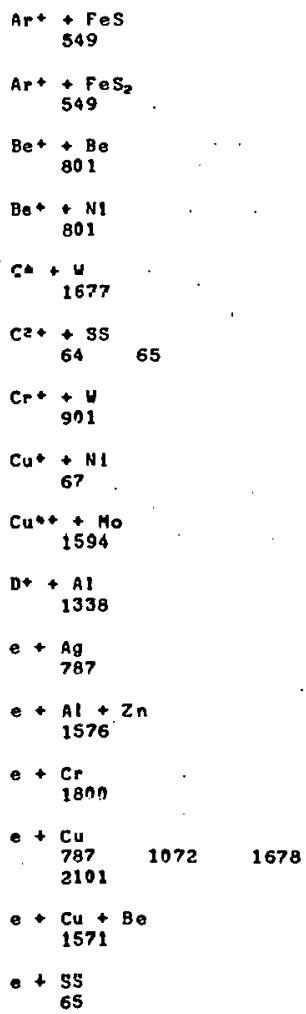

D16

PARTICLE INTERACTIOHS UITH SOLIDS

Partielo Ioplantation In Matal

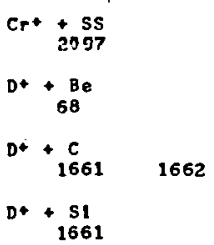

$\mathrm{H}+\underset{\mathrm{Bn}}{\mathrm{Bn}}$

$\mathrm{H}+\mathrm{Ni}$

ho $+\underset{109 g}{P d}+S$

$h 2+p t+s t$

$\mathrm{He}^{+}+\mathrm{Al}$

$+\mathrm{Mg}$
+168

$\mathrm{He}^{+}+\mathrm{NI}$

558

Mel + NI

In*: Ma

Mot +4

$n+$ Al

+ Be

$n+i$

$n+\mathrm{Fe}_{3}$
243

$n+M_{0}+\mathrm{TI}$

$n+N b$

$n+N \mathrm{I}$

$n+N I+r r+N+A I+T I$

$n+51 c$

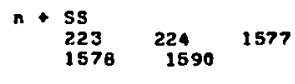

$n+\begin{aligned} & n \\ & 1584\end{aligned}$

$n+\begin{array}{ll}2 r \\ 222 & 1660\end{array}$

$n+\mathrm{Zr}_{\mathbf{1 G G}}+\mathrm{Nb}$

$N++\underset{1079}{1079}$

$\mathrm{NI}+\mathrm{Al}_{+}+\mathrm{Mn}+\mathrm{TI}+\mathrm{Cr}_{r}$

Nit + Ban
Ro?

NIt GK $^{\text {NI }} 67$ An!

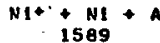

$\mathrm{NI}+\mathrm{NI}+\mathrm{Al}+\mathrm{Mg}+\mathrm{SI}$

$\mathrm{NI}+\underset{1572}{+}+\mathrm{NI}$ I589

$\mathrm{NI}+5$

NI 64 SS 65

$\mathrm{O}_{2} * \underset{549}{+ \text { Fes }}$

$\mathrm{O}_{2}+\mathrm{FeS}_{549}$

บ* +

Reulew

158

Und er

$\begin{array}{lll}793 & 797 \quad 1073\end{array}$

$1579 \quad 1580$ 1581

1507 10T5

$[n++$ He

Mo* + SS

$N++70$

$\mathrm{NI}+\mathrm{ss}$

$T 1++5 s$

Berten

Uituof

$6612095 \quad 2096$ 


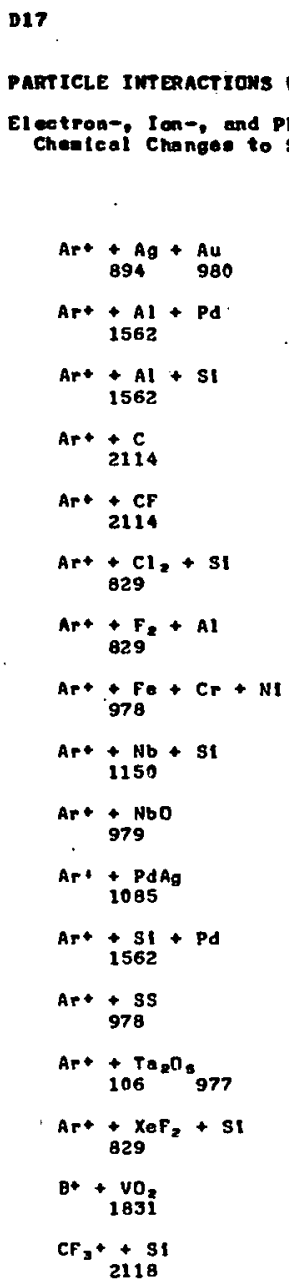

EO1

ELECTROH-PARTICLE INTERACT YOWS Ganera 1
Crt + ss

- $+\mathrm{CO}+\mathrm{NI}$ 108

$-+\underset{108}{\mathrm{CO}_{2}}+\mathrm{NI}$

$+G a P+2 n$ 1729

- + NaCl

$+\mathrm{s10}_{2}$

$+\mathrm{Ta}_{432} \mathrm{O}_{3}$

$\cdots+y_{432}$

e+ $x_{829}+3 I_{3} V_{4}$

$-\underset{829}{\mathrm{XeF}_{2}}+\mathrm{s1O}_{2}$

$\mathrm{H}_{3}++\mathrm{SS}$

557

hv $+\operatorname{BaF}_{2}$

$\mathrm{h}=+\mathrm{CaF}_{3}$

1122

hv + LIF

1122

$h v+\mathrm{MgF}_{2}$

he + PrF

ho $+\mathrm{SiO}_{2}$

1122

$h v+T_{1825}$

$h v+\underset{1545}{102}$

nv + Znse

hv $+\mathrm{ZrO}_{1122}$

$-\mathrm{N}^{2} 13$ 2921

Revt ew
$\mathrm{Kr}+\underset{108}{\mathrm{Ta}}=$

Hot $+5 S$

$\mathrm{N}_{2}+\underset{633}{+\mathrm{CO}_{0}}$

$\mathrm{N}_{2}+\underset{633}{\mathrm{Cr}}$

$\mathrm{N}_{2}+\underset{633}{+} \mathrm{Cu}$

$\mathrm{N}_{2}+\underset{633}{+\mathrm{Fe}}$

$\mathrm{N}_{2}+\underset{633}{+\mathrm{Mn}_{n}}$

$\mathrm{N}_{2}+\underset{633}{+\mathrm{NI}}$

$\mathrm{N}_{2}+\mathrm{PdAg}$

$\mathrm{N}_{2}+2 \mathrm{SI}$

$\mathrm{N}^{2}++21$

$\mathrm{N}_{*}+\mathrm{V}$

633

$\mathrm{N}_{2}+2 \mathrm{2n}$

$\mathrm{Ni}+$ SS

2058

$\mathrm{O}_{2}+\mathrm{Te}_{108} \mathrm{Te}_{3}$

$T 1++5 S$

$\mathrm{Xot}+\mathrm{C}$

$\mathrm{Xot}+\mathrm{CF}$

$\mathrm{Xet}+\mathrm{Ta}_{206} \mathrm{O}_{6}$

Revien 


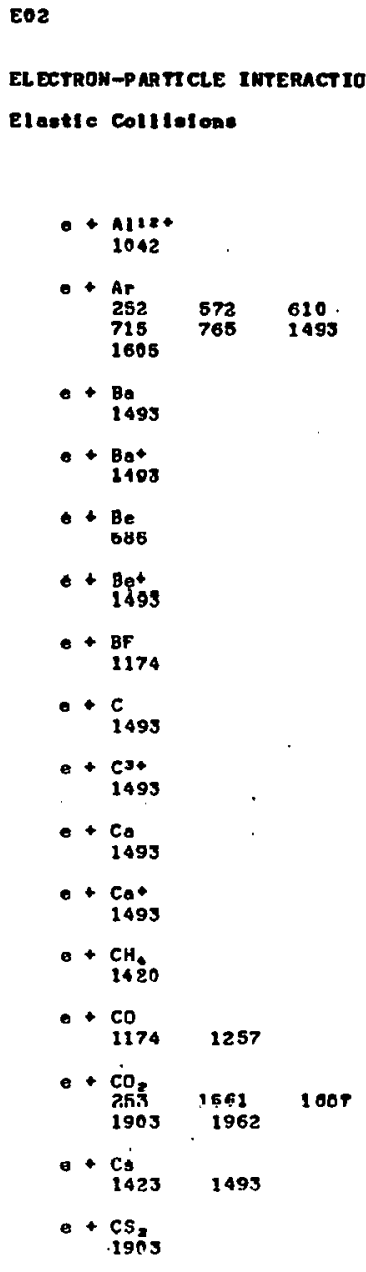

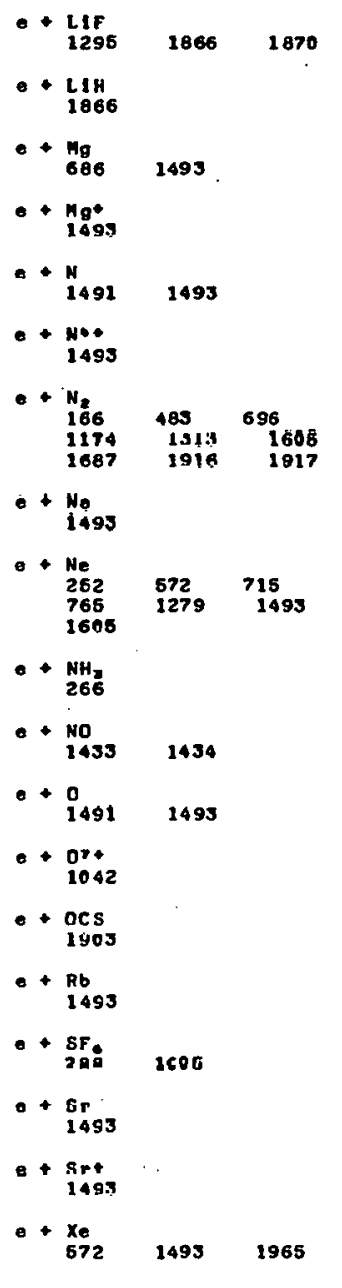




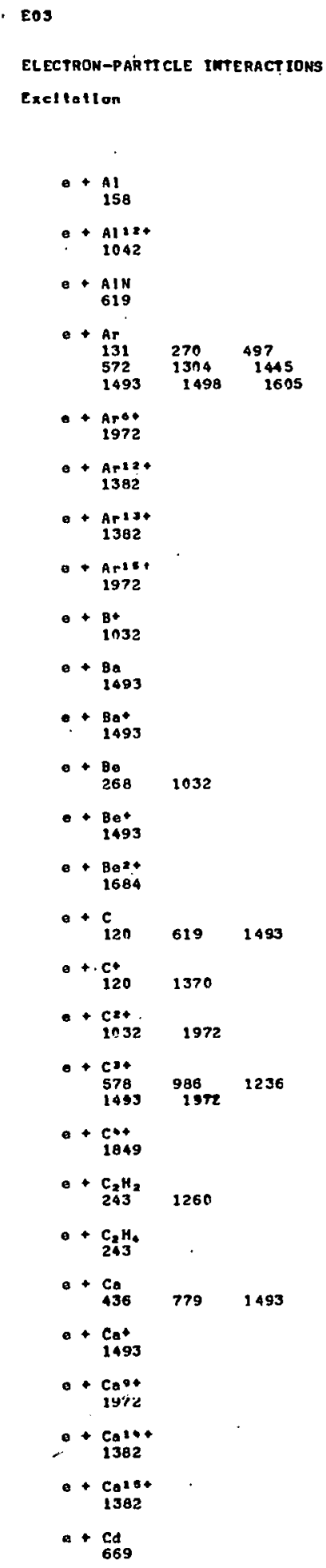

$e_{i}+c_{243}$

e+ $\mathrm{CH}_{1344}$

e+ 243

$0+$ en

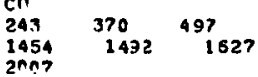

$\begin{array}{ccc}0+\mathrm{CO}_{2} & & \\ 185 & 243 & 253 \\ 497 & 1561 & 1962 \\ 3057 & 2137 & \end{array}$

$\cos$
243

$0+c_{9}$

1493

$2.0 F$
1492

- $+\underset{584}{\mathrm{Cs}}$

$-\mathrm{Cu}$
1727

- Cu Seq

D
497
1956

1956

$0+D_{20}$

o+ F* 1914

$0+\mathrm{FeO}^{\circ}$

$0+$ Fete
1972

- Fe1s+

$7020+$
1343

- fezie

9861972

- fe204
1814

69
158

- Ge2t
1971

$\dot{D}+H$

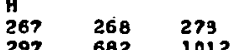

$\begin{array}{lll}297 & 682 & 1012\end{array}$

$\begin{array}{ll}1491 & 1493 \\ 1966 & 2006\end{array}$

C+ He $264 \quad 1379$

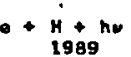

- 1 1809

- $+\mathrm{H}_{2}$

$\begin{array}{lll}\mathrm{H}_{2} & & \\ 285 & 246 & 255 \\ 280 & 339 & 497\end{array}$

$\begin{array}{lll}280 & 339 & 497 \\ 573 & 1031 & 1329\end{array}$

$\begin{array}{lll}1539 & 1847 & 1889 \\ 1956 & 2031 & \end{array}$

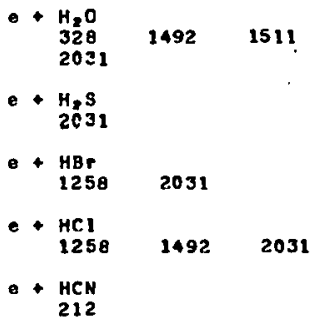




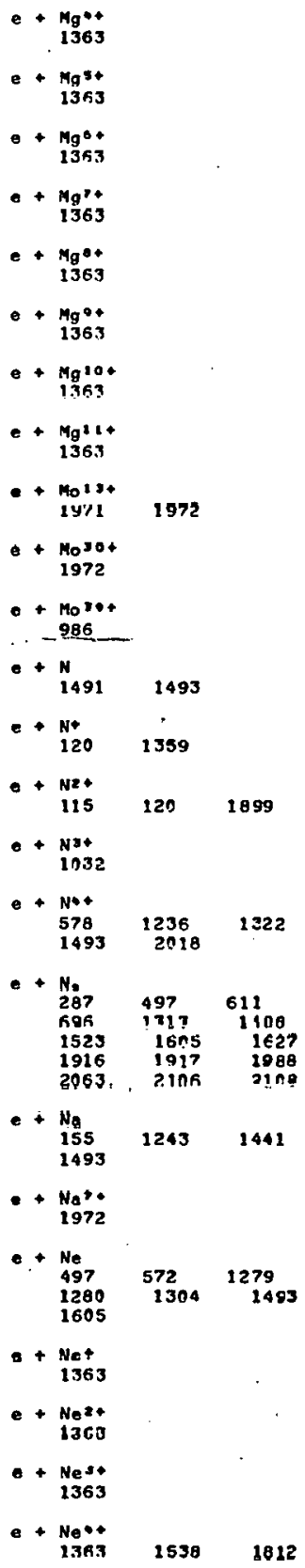

e
Nest
1363

e+ Neo+

2018

$-+\mathrm{Ne}^{\mathrm{TH}}$

1972

e+ NeO+

e+ $\mathrm{Ne94}$

$0+\underset{2 n 31}{\mathrm{NH}_{3}}$

-+ No
1627

$c+0$

$1493 \quad 1284,1491$

$02+$
1552

e+ 03*

197? 2021

0.4
594

0.0
678

$1932 \quad 1972$

004
690

1230

e+ $0 \times+4$

0
02
1284

e + Pb
1999

$\mathrm{kb}$
155

25़ 149

c+ st.

- F

e+ $\begin{gathered}534 \\ 1303\end{gathered}$

- 1 1363

1363

- + sst

e+ sot 1563

- + s7.

$0+504$
$+504$

e sia.

\begin{tabular}{r} 
e \\
\hline 124 \\
$13 \in 3$
\end{tabular}

e+ 5324

- $513+$

e+ 514.

$6+513 *$

1363

e+ $5 b$.

Se
1523

$-+\mathrm{SF}_{1 \in \mathrm{S}}$

e+ $51+$

$e+s_{190+}$

$5110+$
1972

$5112+$
1849

e+ $\begin{array}{ll}S 5 \\ 255 & 1493\end{array}$

$0+3 r^{+}$

- 7 Tes

+11
150

$-+4 F_{49}$

471
986

- $4 \mathrm{ur}^{\mathrm{u}}$

e+xe

$497 \quad 572 \quad 1304$

xezse
1971
1972

Undor 1565 


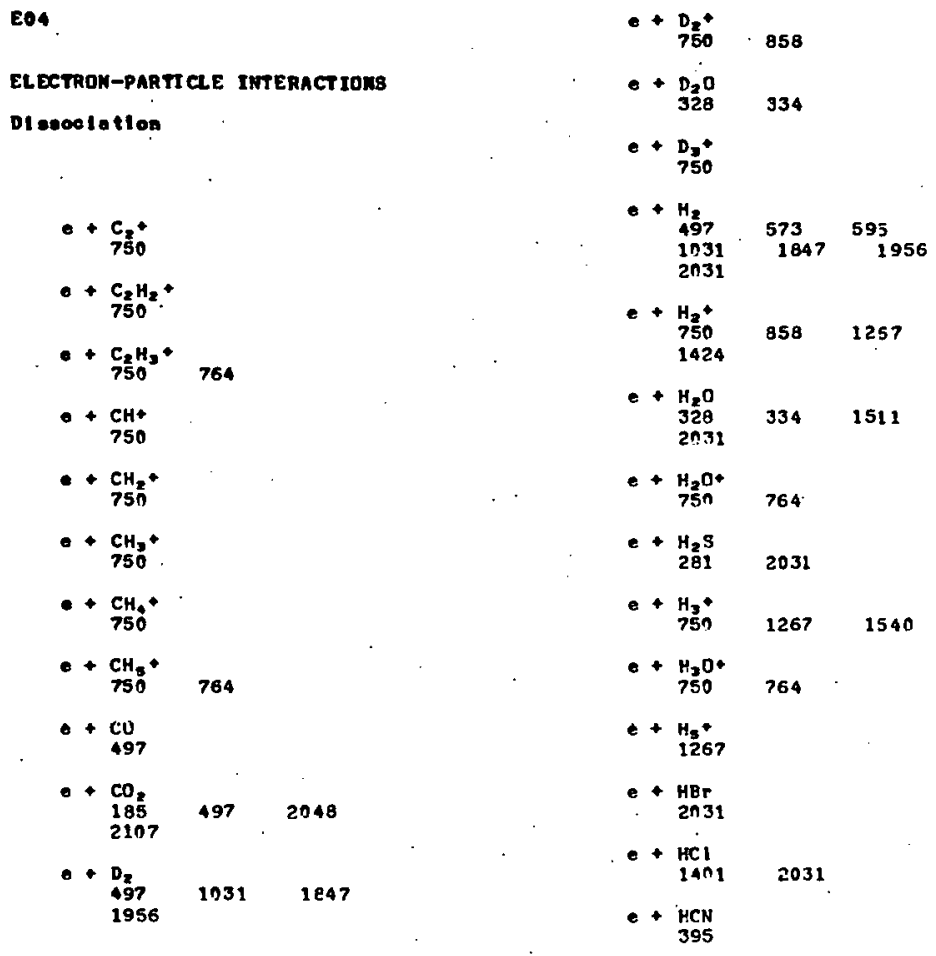

$$
\begin{aligned}
& \text { C + HD } \\
& 1031 \\
& \text { - + HDP } 750 \\
& \text { e+ }{ }^{\mathrm{HD}} \mathrm{HSO}^{\circ} \\
& \begin{array}{lll}
N_{2} & \\
287 & 497 \quad 2059
\end{array} \\
& \begin{array}{c}
-+N_{2}+ \\
612
\end{array} .750 \\
& \text { e+ } \underset{750}{\mathrm{~N}_{2} \mathrm{H}^{+}} \quad 764 \\
& e+\mathrm{N}_{2} \mathrm{O} \\
& \text { e + } \mathrm{NH}^{+} \\
& 750 \\
& \text { e }+\underset{2031}{\mathrm{NH}_{3}} \\
& \text { e+ } \begin{array}{c}
\text { NOA } \\
612
\end{array} 250 \\
& \begin{array}{l}
0_{2} \\
2059
\end{array} \\
& \begin{array}{c}
0_{2} \\
612
\end{array}>0 \\
& \begin{array}{r}
\text { C+ } \\
750
\end{array} \\
& \text { Under } \\
& 1259 \quad 1296
\end{aligned}
$$




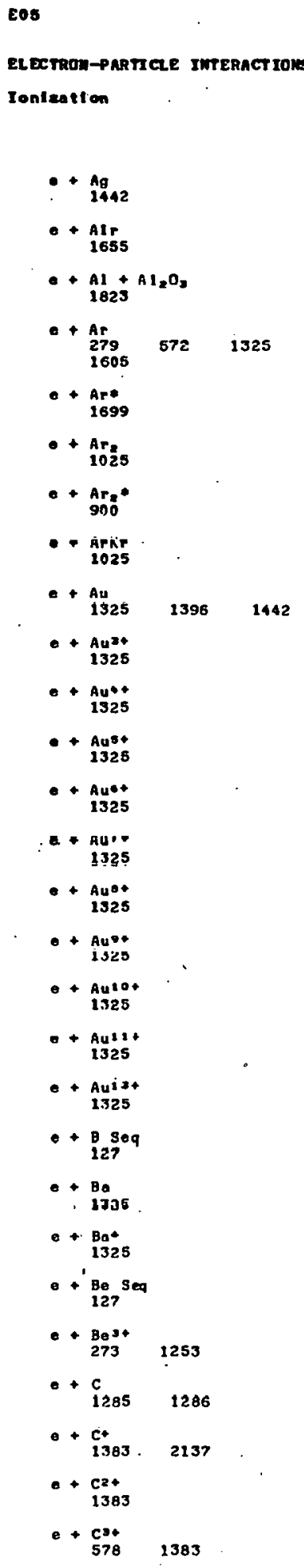

- + $\begin{gathered}\mathrm{Hg} \\ 1659\end{gathered}$

- $+\frac{k}{779}$

$-K *$
1968

- $\begin{gathered}k_{r} \\ 572\end{gathered}$

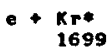

Kro
1025

krxe
1025

- L1 1102

- + t19.

$0+L_{127}^{11}$ Seq $1521 \quad 2126$

4128
273
0 253

- - Mg 229

- + $\mathrm{Mg}^{+}$

C+ $\mathrm{Mg}^{24}$

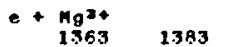

$79 *+$
$1363 \quad 1383$

$M_{954}$
1363
0.983

- $\mathrm{MaOA}_{13 \in 3} 1383$

$e+M_{1363}^{M 9 *}$

e+ $\mathrm{Hg}^{\circ+}$

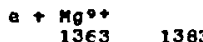

$\mathrm{Mg}^{104}$
1363
1383

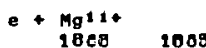

$=+$ Mo324

1374

- 11128

1286

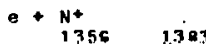

$\begin{array}{lll}1359 & 1383 \quad 2139 \\ 1359 & 0\end{array}$

e N3+
1383

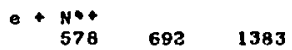

1383
1393

e+ No+ 


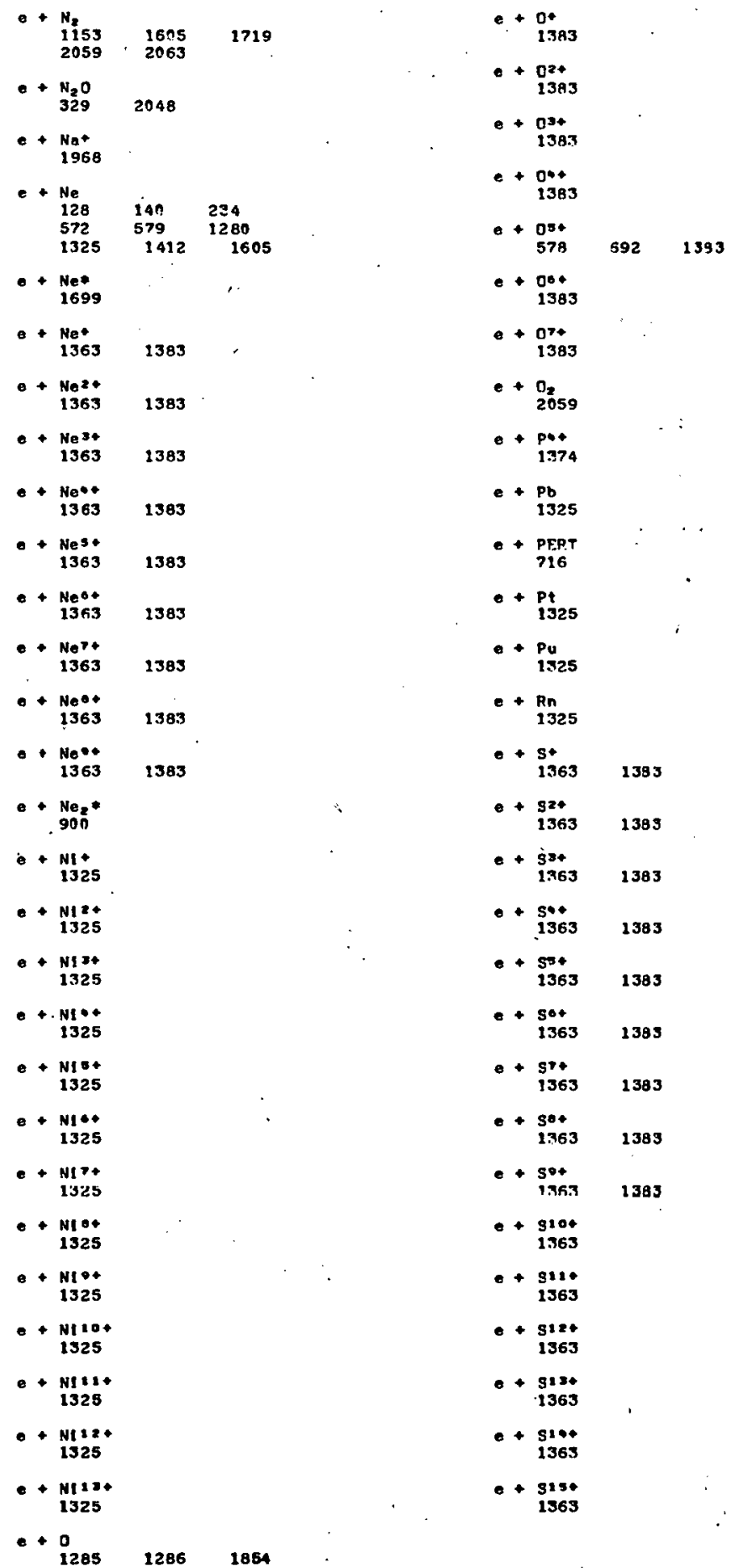

$$
\begin{aligned}
& \text { e+ } 56 \\
& \begin{array}{l}
\text { e } \\
1522
\end{array} \\
& \text { e+ SF. } \\
& \text { e+51+ } \\
& \text { C }+5120 \\
& \text { e+ } 5130 \\
& 0+5100 \\
& \text { c + } 5150 \\
& \text { e+ } 5104 \\
& \text { e+ } 517 . \\
& \text { e+ } 5104 \\
& \text { c }+5104 \\
& \text { o+ } 3110 \text {. } \\
& \text { e+ } 5111 . \\
& \text { e+ } \begin{array}{c}
51224 \\
1383
\end{array} \\
& 0+\underset{1383}{1214} \\
& \text { e+ Sin } \\
& \text { e+ } \begin{array}{c}
3 r \\
255
\end{array} \\
& \begin{array}{r}
\text { e Tez } \\
54 \mathrm{~B}
\end{array} \\
& \text { e+ Th } \\
& e+11 \\
& \begin{array}{r}
\text { e T1* } \\
1325
\end{array} \\
& \text { - + } 1014 \\
& \begin{array}{c}
x+x \\
572
\end{array} \\
& \begin{array}{r}
-169 \\
1699
\end{array} \\
& \begin{array}{r}
x e^{-2} \\
1025
\end{array} \\
& \begin{array}{l}
0+Y b \\
1325
\end{array} \\
& \text { - }+2 n \\
& \underset{603}{\text { Under }} 1654
\end{aligned}
$$


E06

EL ECTRON-PARTI CLE INTERACT IONS

Recosbination (electron-tion)

$$
1497
$$

A111.
1358

arto
1358

e $+\mathrm{C}^{+}$
1383

- + c24

e + ist

e t cast

$1358 \quad 2383$

- cst

1383

cos
1383

$c_{2}+$
$c_{50}$

$-+\underset{750}{\mathrm{C}_{25} \mathrm{H}_{2}}$

- + $\mathrm{C}_{250} \mathrm{H}_{3}+{ }_{764}$

$-400+$
$149 ?$

- $+\mathrm{CH}+$

- $+\underset{750}{\mathrm{CH}_{2}+}$

$-+\mathrm{CH}_{750}+$

o+ $\mathrm{CH}_{4}$ *

$\mathrm{CH}_{35}+{ }_{754}$
$\mathrm{C}^{2}$

-+6160
1497

$\mathrm{CO}_{2}$
$\mathrm{CO}^{2}$

- + $\begin{gathered}\mathrm{D}_{2}^{+} \\ \mathrm{P}_{\mathrm{KO}}\end{gathered}$

$D_{3}$
750

$-\mathrm{Fe}^{3+}$
1383

$0 .+\mathrm{Fe}^{\circ+}$
1383

$0+b^{\prime \prime t}$
1383

e + Feot
138.3

- $\begin{array}{r}\mathrm{FeP} \\ 1383\end{array}$

a $+\mathrm{Fen}^{10}$

1383 e+. $\mathrm{Fe}^{12}$

1383

e+ Fel 24

+ $\mathrm{Fe}^{13+}$

1383

1383
+ Fe10.
1383

Q + Fe15+

1383

e Felot
1383

C+Fez3.

195663

1814

$H^{+}$Seq
1381

$=+45$

e $+\underset{48}{\mathrm{H}_{2} \mathrm{O}}$

$\mathrm{H}_{2} \mathrm{O}^{+}$
$75 \mathrm{O}$

e + $\mathrm{H}_{\mathbf{3}}$ *

$++\mathrm{H}_{3} \mathrm{O}^{+}$

764

$\mathrm{HD+}$
$750 \quad 058$

$-\mathrm{HD}_{2}+$
750

- He-

$+x+$

$-\mathrm{K}$
1615

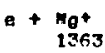

$1393 \quad 1497$

e+ Mg2+

e+ $\mathrm{Mg}^{34}$

1383

1309

- $+\mathrm{Mg*4}$

$+\mathrm{Mg}$

1383

$\mathrm{Mg}^{5+}$
1353

1383

e+ $\mathrm{Mg}_{1363}$

e+ $\mathrm{Mg}^{\mathrm{P}}$

1383

$+4000$

1983

$+\mathrm{Tg}_{1363}^{0.4}$

1383

e + $\mathrm{Mg} 9+$

$+M^{10+}$

1363

e+ $\mathrm{Hg}_{\mathrm{g}} \mathrm{il}$

$1363 \quad 1383$

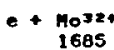

14000
1358

- N N

1359

1324
1359

$1359^{\circ} 138$ e+ N3.

e+ Not

1383

$\mathrm{e}+\mathrm{N3}$
1383

e+ no.

1383

C. N70

$e+N_{2}+$
612

$\mathrm{N}+\mathrm{N}_{2} \mathrm{D}$
126

e+ $+\mathrm{N}_{2 \mathrm{H}}+\mathrm{H}^{136}$

C+ Not

1700

- + Ne 24

$1363 \quad 1383$

$0+\mathrm{Ne}^{\mathrm{J}}$
1363

1383

C+ Neve

$13 \in 3 \quad 1383$

e+ Nese

$1363 \quad 1383$

e+ Neos

$13 \in 3 \quad 1383$

e+ Nert

$1363 \quad 1393$

e+ NeOP

$1363 \quad 1383$

e+ Neot

1383

C+ Ne10.

e+ $\mathrm{NHE}^{\circ}$

- . NII,

40.

612

e+ ot

1383

$0+024$

E

$+0.4$

1303

e. 034

1383

e +00.

$+07$

1000

13831649

$a+00 \%$

$0+0_{2+}^{*}$
612

OH*
750

$S+$
1363 


$$
\begin{aligned}
& \begin{array}{r}
0+\underset{52+}{1363} \\
1383
\end{array} \\
& \begin{array}{ll}
\text { s34 } \\
1363 \quad 1385
\end{array} \\
& \text { - S.* } 1363 \quad 1383 \\
& \begin{array}{l}
\text { S5+ } \\
1363 \quad 1383
\end{array} \\
& \begin{array}{c}
\text { Sot } \\
1363 . \\
0.583
\end{array} \\
& \text { + }+ \text { S74. } 1363 \\
& \text { C+ sot } 1363.1383 \\
& \text { - + } \begin{array}{c}
\text { S.4 } \\
1363
\end{array} \\
& \begin{array}{r}
\text { + } \\
1363
\end{array} \\
& \begin{array}{r}
\$ 124 \\
1363
\end{array}
\end{aligned}
$$

- +522

1565

e + s23.

1365

- $\$ 104$
1963

e+ siot

1365

- +51.

- +5124

e $+\begin{array}{r}5134 \\ 1383\end{array}$

- + $\begin{array}{r}510 . \\ 1383\end{array}$

- + sts.

1383

-
$138+$
1383

$$
\begin{aligned}
& \begin{array}{c}
\text { Ar } 134 \\
1382
\end{array} \\
& \begin{array}{r}
e+c^{2+} \\
1362
\end{array} \\
& \begin{array}{r}
+c 34 \\
1362
\end{array} \\
& \begin{array}{c}
-\mathrm{Ca}+\mathrm{H} \\
1382
\end{array} \\
& \begin{array}{c}
\text { C.15* } \\
1382
\end{array}
\end{aligned}
$$

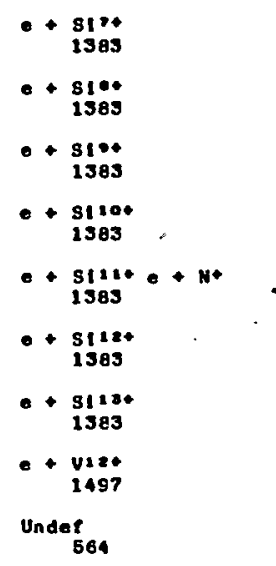

- He*
127 1799

KrFo
1610

- + N3.

1362

- $+N_{2}$

e+ XeF*
E0 8

ELECTRON-PARTICLE INTERACT IONS Collistonal Lne Broadening

$$
\begin{aligned}
& \begin{array}{r}
\text { e } \\
116
\end{array} \\
& \text { - } \begin{array}{c}
\text { A112 } \\
1042
\end{array} \\
& \begin{array}{r}
\text { e Art } \\
1345
\end{array}
\end{aligned}
$$

E09

EL ECTRON-PARTI CLE IWTERACT IOIS Negative Ion formation

$$
\begin{aligned}
e+\mathrm{Ar}_{1605} \\
\mathrm{e}+\mathrm{Cl}_{2} \\
\mathrm{C} \\
\mathrm{C}+\mathrm{Cl}_{2}+\mathrm{Ar} \\
1 \\
=+\begin{array}{l}
\mathrm{CO}_{2} \\
2107
\end{array}
\end{aligned}
$$

$$
\begin{aligned}
& \begin{array}{l}
\text { - } D_{2} \\
1847 \\
-5
\end{array} \\
& \begin{array}{r}
\mathrm{D}_{2} \mathrm{O} \\
\mathbf{3 3 4}
\end{array} \\
& \begin{array}{ll}
H_{2} \\
595
\end{array} \\
& \text { e+ }+\mathrm{H}_{334} \mathrm{O} \\
& \begin{array}{r}
e+\mathrm{H}_{2} \mathrm{~S} \\
281
\end{array} \\
& \begin{array}{r}
+4 \\
1540
\end{array} \\
& -\underset{1401}{H C 1}
\end{aligned}
$$

$$
\begin{aligned}
& \text { - }+\mathrm{Mg}^{12} \\
& \text { C+NeP } \\
& \text { c+ } 010 \\
& -+5112+0 \\
& \text { - S11a* } \\
& \text { Under } \\
& 123
\end{aligned}
$$

$$
\begin{aligned}
& \begin{array}{r}
-+N_{2} \\
1605
\end{array} \\
& e+\underset{51}{N_{2} \mathrm{O}} \quad 1474 \\
& \text { e+ } \mathrm{N}_{2} \mathrm{O}+\mathrm{CO}_{2} \\
& \begin{array}{l}
\text { e+ Ne } \\
1605
\end{array} \\
& \text { e+ } 0_{2}+\infty_{2} \\
& \begin{array}{l}
\$ F 5 \\
1605 \quad 1893
\end{array} \\
& \text { Undef } \\
& 1259 \quad 1296
\end{aligned}
$$


E10

ELETRON-PARTICLE IMTERACTIONS

spin Exehonge

$-+\frac{n}{724}$

e11

RLECTRON-PARTICLE IITERACT TOE

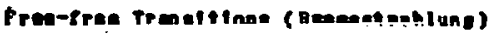

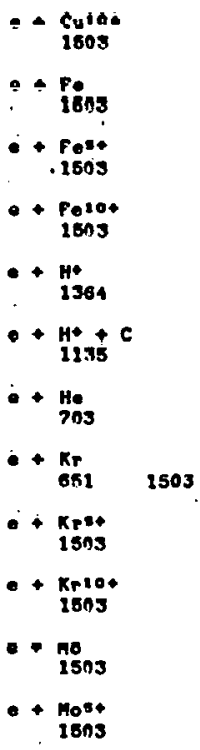

- +

- 1502

0
-+903

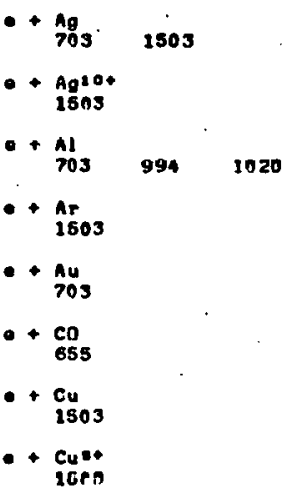

E12

$$
\begin{aligned}
& \text { at He tar } \\
& 1530 \\
& 0++\mathrm{He}_{\mathrm{OSO}}+\mathrm{C}^{\circ} \mathrm{H}_{2 \mathrm{O}} \\
& \therefore+\mathrm{He}_{150}+\mathrm{CCl} \mathrm{F}_{\mathrm{P}} \\
& \because+\underset{153 n}{\mathrm{He}_{3}}+\mathrm{Co} \\
& 0++\mathrm{He}_{1530}+\mathrm{CO}_{2} \\
& \text { e.+ } 1530+\mathrm{H}_{2} \\
& \text { - + }+\mathrm{He}_{1530}+\mathrm{Kr} \\
& \bullet+\mathrm{He}+\mathrm{N} \text {, } \\
& 1530 \\
& \circ+\mathrm{He}+\mathrm{Ne} \\
& 153 n \\
& 153 \text { ? }
\end{aligned}
$$$$
\text { - Pb }
$$$$
-+ \text { posor }
$$$$
1803
$$$$
\text { - + y }
$$$$
994
$$$$
\text { - }+ \text { Yos }
$$$$
0+\text { usot }
$$$$
1503
$$$$
\text { - } x_{0}
$$$$
\begin{array}{r}
x+100 \\
1503
\end{array}
$$$$
\text { Und ef }
$$
1138

$$
\begin{aligned}
& \because+\underset{1530}{\mathrm{He}}+x_{0} \\
& \text { e+ +het sea } \\
& 0+1 \\
& 0++\begin{array}{c}
k r \\
1704
\end{array} \\
& \begin{array}{c}
\text { +. } 11 \\
1277
\end{array} \\
& \text { o* + Nat? } \\
& \text { ot+ }+ \text { No } 1310 \\
& \text { o+ } \begin{array}{c}
51 \\
765
\end{array} \\
& \therefore+\begin{array}{c}
x e \\
1704
\end{array}
\end{aligned}
$$$$
\text { i3y } 301587
$$$$
613999.1131
$$ 
E15

ELECTRON-PARTTCLE INTERACTIONS

Electron Dotecheont fron Megative Jone

$+\underset{300}{c-} 1421$

E14

ELECTRON-PARTI CLE INTERACT IONS

BInary Electron-Elect ron Coll1 sl one

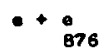

E15

EL DCTRON-PARTICLE INTERACT IONS

Inner-shell In teract Ions

- Au

E16

ELECTROH-PARTI CLE IMTERACT IOHS

fl coreseenee and Lual neacance

$$
\text { o Ar } 19
$$

E17

ELECTRON-PARTI CLE INTERACT IOW

Anguler seattering (apecteled process)

$$
\begin{aligned}
& \text { - }+A_{1442} \\
& \text { - + Ar } 252 \quad 610 \\
& \text { - Au } 1442 \\
& \text { - Bo74 } \\
& \text { - + } \mathrm{C}_{12} \mathrm{H}_{2} \\
& -+ \text { CF } \\
& \text { - }{ }_{1420}^{C H_{9}} \quad 1921 \quad 1922 \\
& \begin{array}{ll}
\text { co } \\
\text { cos }
\end{array} \\
& -+\mathrm{CO}_{2} \\
& 1921 \\
& 1922
\end{aligned}
$$

$$
\cdot+261
$$

$=+\underset{300}{0-} 1421$ 
E18

EL ECTRON-PARTI CLE INTERACTIONB

Attenuation (unapecifled process)

$-+C_{2100}$

G0?

THANSPORT PHENONENA AYN AU FBAFF PANPFATTEA PN GASFR

Diffusion of Neutrale

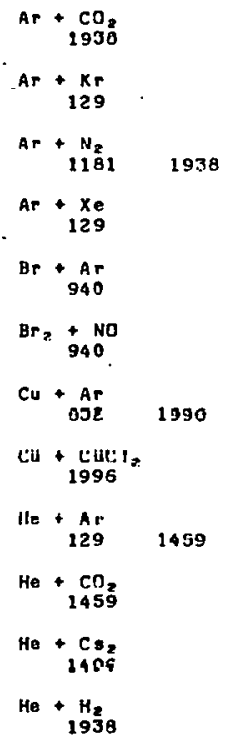

603

TRANSPOAT PHENORENA AND AVERAGE PROPERTIES IN GASES

Diffusion of Electrons
- + \$5\% 
604

TRANGPORT PHENOLENA AND AVERAGE PROPERTIES II OABES

diffuston of Ione

$$
\text { Ar+ }+ \text { He }
$$$$
\text { C* + He }
$$$$
1598
$$

605

TRANGPORT PHENOHEMA AND AVERAGE PROPEATIES IN GASES

Deift Volocity of Electrone

$$
\begin{aligned}
& \mathrm{Ar}_{74} \\
& \therefore+\mathrm{Pr}_{\mathrm{7}}+\mathrm{C}_{2} \mathrm{H}_{4}
\end{aligned}
$$

606

TRANSPORT PHDNOKENA AND AV ERAGE PROPERTIES IH GRSES

Drift velocity of Ione

$$
\begin{aligned}
& \mathrm{Ar}+\underset{1315}{\mathrm{He}} \quad 1598 \\
& \text { Aret }+ \text { He } \\
& A u+\underset{1603}{S F} \text {. } \\
& \begin{array}{c}
\text { C+ Ho } \\
1598 \quad 1910
\end{array} \\
& \mathrm{CH}+\stackrel{\mathrm{He}}{+1598} \\
& \mathrm{CH}^{\circ}+\underset{\mathrm{is} 9 \mathrm{be}}{+\mathrm{He}} \\
& \mathrm{Cl}-\underset{480}{+\mathrm{Ar}}
\end{aligned}
$$

\section{Go?}

TRANSPORT PHENONENA AND AVERAGE PROPERTIES IN GASES

Seatterling and Energy Losa Parametere of Electrons. Neutrals end Ions in Gasos

Ar $+\mathrm{N}_{2}$

$$
\begin{aligned}
& \mathrm{CH}+\mathrm{He} \\
& \mathrm{CH}_{2}+\text { He } \\
& \text { C1- }+ \text { Ar } \\
& \mathrm{Cl}+\mathrm{Kr} \\
& \mathrm{Cl}^{-}+\mathrm{Ne}
\end{aligned}
$$

$$
\begin{aligned}
& \mathrm{Cl}+\mathrm{Xe}_{\mathrm{e}} \\
& \text { Cs* Ar } \\
& \text { D+ + He } \\
& \mathrm{H}^{+}+\mathrm{He}
\end{aligned}
$$

Under

$294345 \quad 1602$

$$
\begin{aligned}
& -+\mathrm{Ar}_{74}+\mathrm{CH}_{77} \\
& \text { - }+\underset{77}{\mathrm{Ar}}+\mathrm{CO}^{2} \\
& \text { - }+\mathrm{C}_{24} \mathrm{H}_{4} \\
& +{ }_{74}^{\mathrm{CH}_{4}} \quad 1716 \\
& \begin{array}{l}
-\mathrm{CO} \\
132
\end{array}
\end{aligned}
$$

$-+\mathrm{CO}^{2}$

e+ca

e+ $\mathrm{H}_{2}$

1095

1453

o+ He

Neg
1096

1453

$C_{480}+K_{r}$

Not+ He

$\mathrm{Ne}+2+\mathrm{He}$

1310

No+ $+N^{118 ?}$

$\mathrm{O}_{2}+\underset{1187}{\mathrm{~N}_{2}}$

st + $\begin{gathered}\mathrm{He} \\ 1910\end{gathered}$

$x e^{+}+\begin{array}{r}\text { He } \\ 1315\end{array}$

$\mathrm{Xe}^{+}+\mathrm{Ne}$

1315

$\mathrm{Xe}^{2+}+\mathrm{He}$

$\mathrm{Xez}^{+}+\mathrm{Ne}$

1315

Undef

$294 \quad 345$

$$
\begin{aligned}
& \mathrm{Hg}+\mathrm{Ar} \\
& \mathrm{Hg}+\underset{43}{\mathrm{Kr}} \\
& \mathrm{Hg}+\mathrm{Xe}_{\mathbf{4 3 1}}
\end{aligned}
$$

$\begin{array}{ll}N_{8} \\ 679 & 1096\end{array}$ 
008

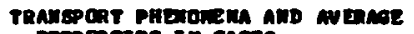

PROPERTIEs In anges

Enery Dietptbetion ( oneror detribution of Ione and atrone Ith eppiled electife and menctio fielde)

Ar * Are oss

609

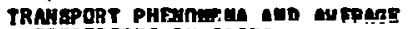
PROPEATIES IN OABE:

Hocontue Trenser

Ar + Net

Au* + 4

Au+ + He

Bo:+ $+\mathrm{H}$

Bese+ He 1132

Co+ +1152

$\mathrm{Cs}+\mathrm{He}$ 1132

919

TRAMgPORT PHENONEMA AND AVERAOE PROPERTIES IA GASES

FIrst and seeond Pouneend Conffietents

$$
-+\underset{616}{\mathrm{CO}_{2}}+\mathrm{N}_{2}+\mathrm{He}_{0}
$$

611

TANISTUR PHEUTER AND AVERAE PROPERTIES IA GABES

Electron Attecheont Coesflolente

$$
-\frac{18}{1213}+N_{*}
$$

HO1

PHOTOH COLLIOION8 UITH HEAVT PARTICLES, G ECTROHS MP PHOTONB In OASES CHO $100 \mathrm{keV})$

General
$A r+$ Fe

$n=+x_{r}$

A. $+\frac{x}{144}$

- Ar
468

- $+\mathrm{CO}_{2}$
$-+\mathrm{CO}_{616}+\mathrm{H}_{2}+\mathrm{H}_{6}$

- + H

- $\frac{\text { Hef }}{314} 1601$

$H, 0+\hat{A r}_{60}$

$\mathrm{H}=\stackrel{\mathrm{O}}{\mathrm{He}} \stackrel{\mathrm{He}}{\mathrm{a}}$

H* * He

1132

Ho* + 4

He* + He

LI* + $\frac{1}{1132}$

Lt* $+\mathrm{He}$

$412+\frac{1}{1132}$

LIR+ He

$\mathrm{Nat}+\mathrm{H}$

Hat + He

1132

$v+K_{175}$

H1 Th

ct $+\mathrm{He}_{0}+\mathrm{Rr}$

- + HI $_{34} 2008$

1990 


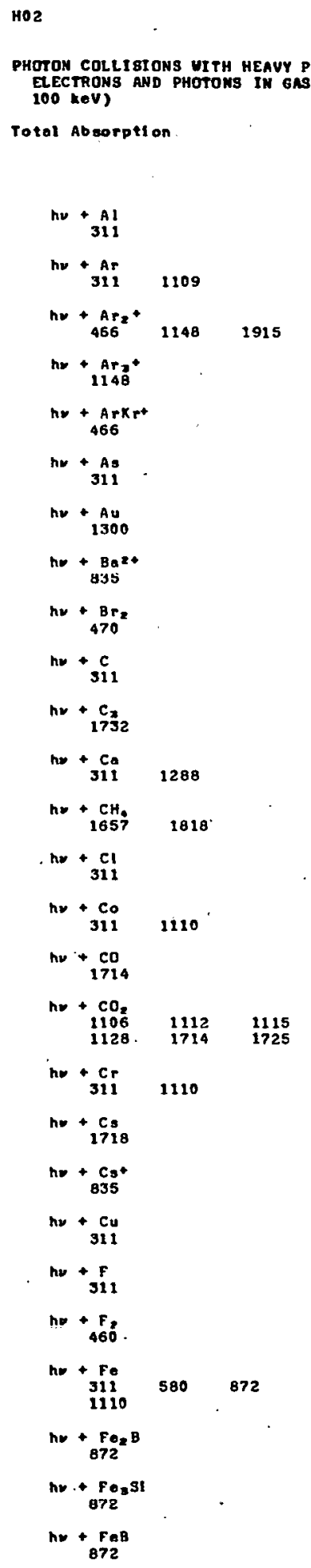

$$
\begin{aligned}
& \mathrm{HW}+\mathrm{Ne}+\mathrm{NF} \\
& \mathrm{How}+\mathrm{Ne}+\mathrm{Xe}_{e} \\
& \text { a } 1612 \\
& \mathrm{nu}+\mathrm{Ne}+\mathrm{x}+\mathrm{x}+\mathrm{NF} \\
& \text { ho }{ }^{+} \mathrm{Ne}^{+*} \\
& \mathrm{hu}+\underset{1112}{\mathrm{NH}} \\
& \text { ho. } \begin{array}{c}
N I \\
311
\end{array} 872 \quad 1110 \\
& \mathrm{Hu}+\mathrm{Ni}_{272} \mathrm{~B}
\end{aligned}
$$

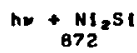

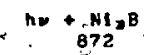

$$
\begin{aligned}
& \text { hu }++_{872}^{N 1351} \\
& \text { ho }+\mathrm{KIT}^{\mathrm{N}} \mathrm{B} \\
& \text { no + Nis! } \\
& \text { ho }+\underset{872}{\mathrm{NISI}}= \\
& \text { no }+ \text { NO } \\
& \text { ho }+311 \\
& \text { ho }+\mathrm{Og}^{\circ} \mathrm{Bg}^{\circ} 1105 \quad 1714 \\
& \text { hy }+\begin{array}{l}
05 \\
898
\end{array} 943 \\
& \text { ho ocse } \\
& \text { no }+ \text { OH } \\
& \text { ho }+ \text { p } \\
& \text { ho + PERT } \\
& \text { he + Rb } \\
& \text { nu }+5 \\
& \text { ho }+\begin{array}{r}
\text { Sc } \\
311
\end{array} 1110 \\
& \text { ho + }+ \text { SF }_{1273} \cdot \\
& \text { ho }+\frac{51}{311} \\
& \text { ho }+514 \\
& h=+810 \\
& \text { h. } \begin{array}{c}
\text { Sn } \\
1726
\end{array} \\
& \text { ho + TI } \\
& \text { no }+\operatorname{TIN}_{467} \\
& \text { ho. } 72
\end{aligned}
$$



ho + vy
hv $+\begin{array}{r}x_{e} \\ 835\end{array} \quad 1612$
$\mathrm{hu}+\mathrm{Xez}_{1915}^{+}$
ho + $\begin{gathered}\text { Xesr } \\ 416\end{gathered}$
he $+\underset{416}{x e C l}$
ho $+\begin{gathered}x_{e 1} \\ 416\end{gathered}$
no $+\frac{z n}{311}$

$$
\begin{aligned}
& \text { he }+ \text { ZRT }_{467} \\
& \text { nhe + RC1, } \\
& \begin{array}{r}
\text { nhe }+ \text { co } \\
1427
\end{array} \\
& \text { nhe + SF. } \\
& \text { nhe } 1120 \text { UF } \\
& \text { 2he }+ \text { In } \\
& \begin{array}{c}
2 h y+N^{2} \\
259
\end{array}
\end{aligned}
$$

$\mathrm{He}+\underset{1 \mathrm{CH}}{\mathrm{CH}}$

he + co 1037

no. + $\mathrm{rn}_{\substack{4 \\ \text { ints }}}$

hs: : 04

he $+\begin{array}{r}\text { Dy } \\ 586\end{array}$

hu+ é

ho + FH

he $+\mathrm{H}_{2}$

$\mathrm{ne}+\begin{gathered}\mathrm{He} \\ 13 \mathrm{C}^{2}\end{gathered}$

$\mathrm{hv}+\mathrm{Hg}$

$n=+\begin{gathered}k r \\ 1 n 37\end{gathered}$

ho + to

$\mathrm{hu}+\mathrm{N}$

ho $+\begin{gathered}\mathrm{H}_{2} \\ 1037\end{gathered}$

$\mathrm{H}=\mathrm{C}_{6} \mathrm{H}_{2} \mathrm{O}$

1174

HO4

MOTON COLLISIONS UTTH HEAVY PARTICLES, E.ECTRONS AND PHOTOHS IN OABEB (hD is $100 \cdot k e V)$

Excitation

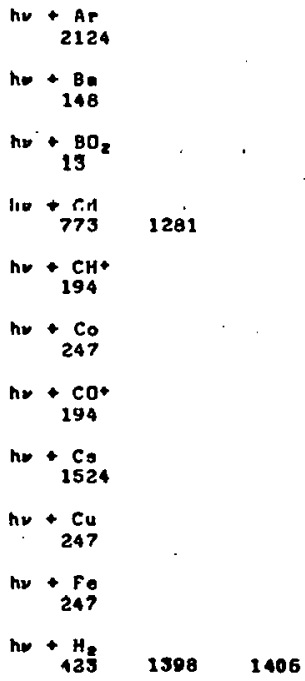

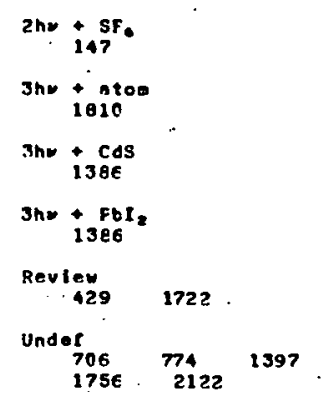

$\mathrm{Nu}+\begin{array}{r}\mathrm{N} 2 \mathrm{O} \\ 1037\end{array}$

ho + NA

ht i Nt

110 $+\frac{1}{100}$

$\mathrm{he}+\mathrm{O}_{2}$

no + pb 320
1796 $586 \quad 1707$

$\mathrm{h}=+$ PERT
962

nv $+\underset{1037}{S F}$

hv + Sn

$586 \quad 1796$

$h_{2}+T_{320} T^{\circ} \quad 1796$

ho + Th

ho +2 rr

Und ef

438

h. $+\begin{array}{r}\text { PbF } \\ 1622\end{array}$

ho. $\begin{array}{r}\text { Ro } \\ 775\end{array}$

nhe + Atou

1805

uhe it: co

$2 \mathrm{hr}: \mathrm{Ar}$

2 ino +12

2he 1867

$2 \mathrm{ho}+\mathrm{Ne}$

the + Be

the + $\begin{array}{r}\text { II } \\ 1750\end{array}$

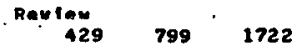

Under

$771 \quad 772 \quad 774$.

10041006106 


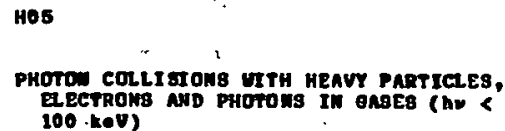
$(00 \cdot(\mathbf{k})$ V)

Di ascelation $h=+{ }_{194}^{H}{ }^{\circ}$

$h v+\underset{932}{\mathrm{H}_{2} \mathrm{O}}+\mathrm{H}^{2} \mathrm{O}$

ho $4 \mathrm{HBr} *$

hv $+\underset{1896}{\mathrm{HCO}}=$

$\mathrm{H}=+\underset{1896}{\mathrm{HCO}}-+\mathrm{H}_{2} \mathrm{O}$

hv $+\underset{1751}{\mathrm{Heq}}$

hy + HeHe

119

hw HeHe+

$\mathrm{hv}+\underset{35 \mathrm{gg}}{\mathrm{Hg}}+\mathrm{Hg}$

hv + HNCO

$+1 . H$

ho ${ }_{1195}^{\mathrm{I}_{2} \mathrm{He}}$

hy $+\mathrm{I}_{2 \mathrm{He}}$

hv $+I_{12}^{I_{2}} \mathrm{He}_{3}$

hv $+\underset{473}{\text { ICN }}$

he $+{ }_{5} \mathrm{KI} \quad 1477$

$h \mathrm{~h}+\underset{193}{\mathrm{Kr}_{2}}+1039$

hv $+t_{1626}^{42^{\circ}}$

$h=+\underset{1479}{147 ?}$

hv $+\underset{1369}{\mathrm{MgH}}$

$\mathrm{hu}+\mathrm{Na}^{\mathrm{N}} \mathrm{2}^{\circ}$

hv $+\underset{1477}{\mathrm{NaI}}$

$\mathrm{hr}+\underset{1 \mathrm{Na} \mathrm{N}^{*}}{\mathrm{Nat}}$

he $+\underset{1733}{\text { NH }}$

$\mathrm{he}+\mathrm{NO}^{2}$

$\mathrm{hy}+\underset{1908}{\mathrm{NO}_{2}-}+\mathrm{H}_{2} \mathrm{~J}$

$\mathrm{H} 2+\mathrm{NO}_{1998}-$

hv $\underset{1908}{\mathrm{NO}_{8}-}+\mathrm{H}_{2} \mathrm{~J}$

$h v+\mathrm{O}_{2}$

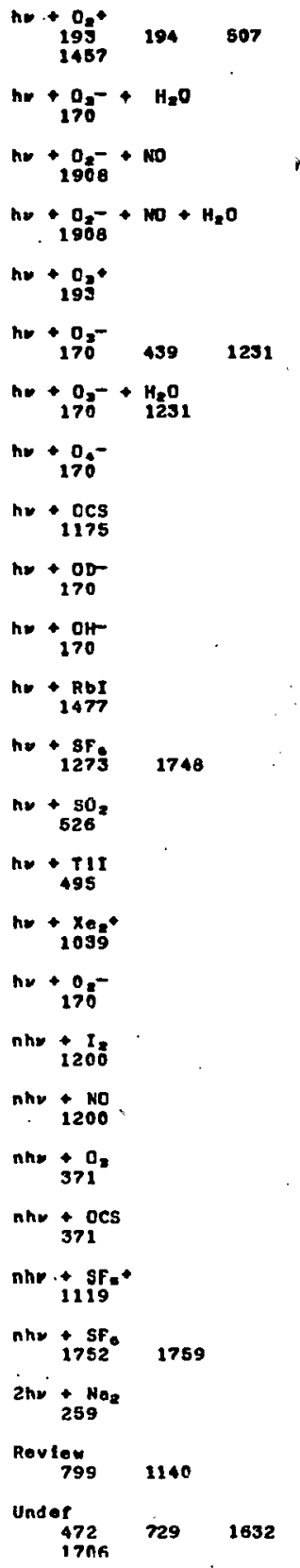

$+195$

$\mathrm{nn}+{ }_{170^{-}}+\mathrm{H}_{2} \mathrm{O}$

no $+{ }_{1968}^{0}+$ No

he +193

he $+\mathrm{D}^{2}$

$+\mathrm{O}_{3}-+\mathrm{H}^{2} \mathrm{O}$

hv $+\frac{0}{170}$

he ocs

HD + OD

ho + OHe

ho 147

ho 1273

526

495

1099

170

1200

. 1200

371

371

1119

1752

259

7

$472 \quad 729 \quad 1632$ hr $+\mathrm{O}_{208}^{-}+\mathrm{MO}+\mathrm{H}_{2} \mathrm{O}$

1337 

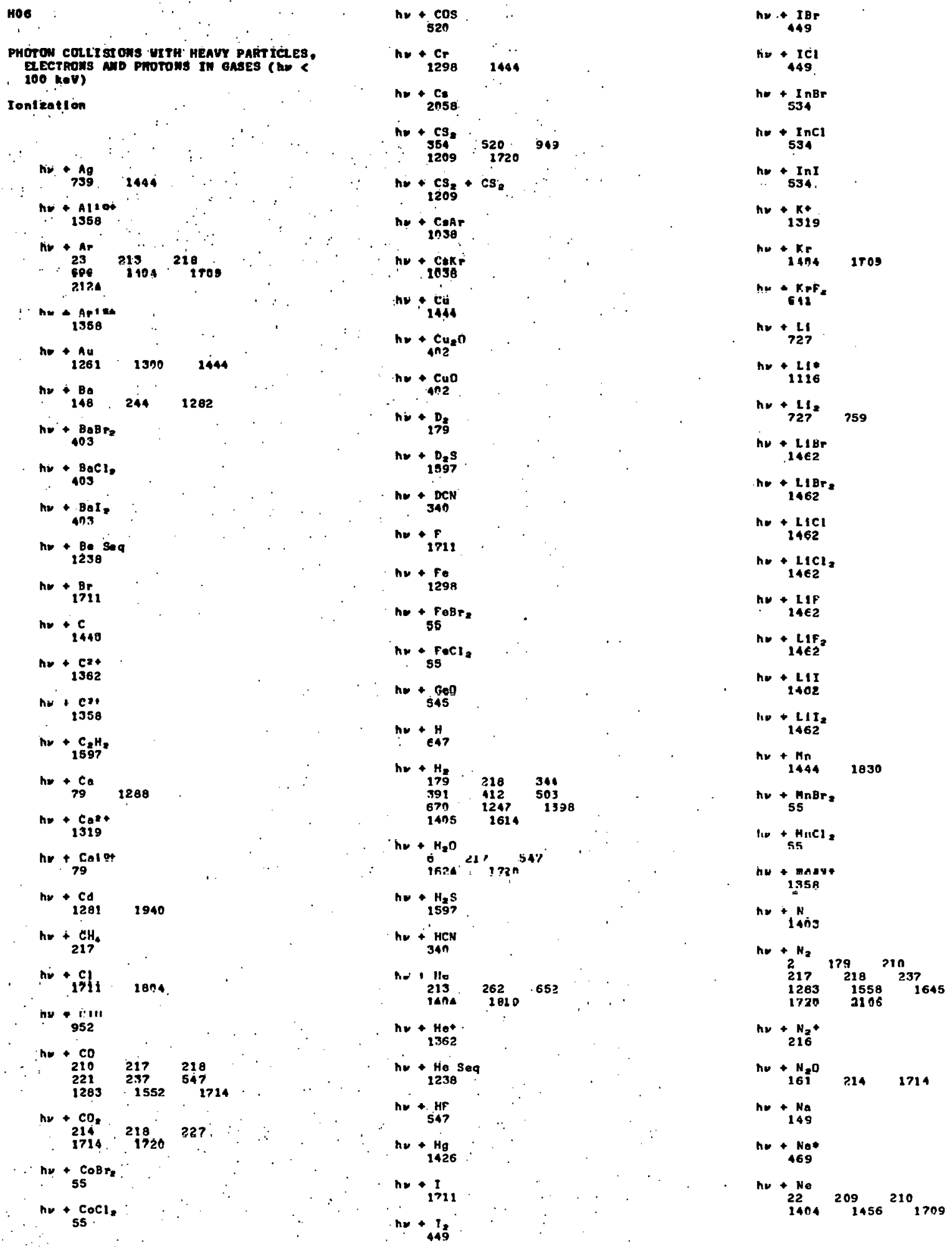


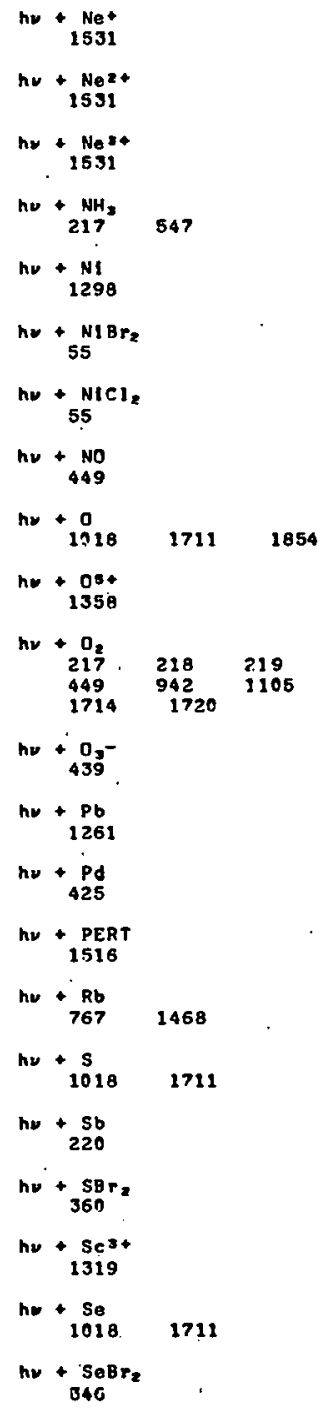

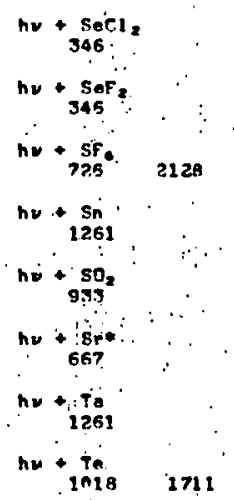

niv $+\underset{79}{+7 n} 1261$

ho + Th>2+

he +. Thoot

ho. 1298

he + Ti1 1319

he +.118r

ne + TIC!

$\because$ E\$?

ne' TII $^{\circ}$

he $\cdots x_{e} \because$

$\begin{array}{ll}213 & 212 \\ 272 & 309 \\ 596 & 769\end{array}$

596: 769

He* $\div$ Ar

nhe +4

nhe $+i_{12}$ nhe +1200

nhe $+85_{1}$

nho ${ }_{666}^{\mathrm{UT}}$

$2 \mathrm{hp}+\mathrm{Co}$

$\begin{array}{lll}258 & 645 & 1000 \\ 1233 & 1268 & \end{array}$

2hvice ${ }_{645}$

ahv :

2ho i. He

2ho + In

inv + iNes

2no iunder

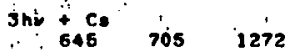

Sinv + Co* 1947

3he $\mathrm{H}$

She $1: x$

sho 2

1499

$4 \mathrm{hw}+\mathrm{Ca}$

$\begin{array}{lll}648 & 706 & 1272 \\ 1939 & 1948\end{array}$

$4 h v+C s=$

Revi leiv

1140

Und of

211 '290' 730 '

$\begin{array}{lll}1289 & 1397 . & 1517\end{array}$

$1626 \quad 1671 \quad 1708$

$1861 \quad 1874$ 


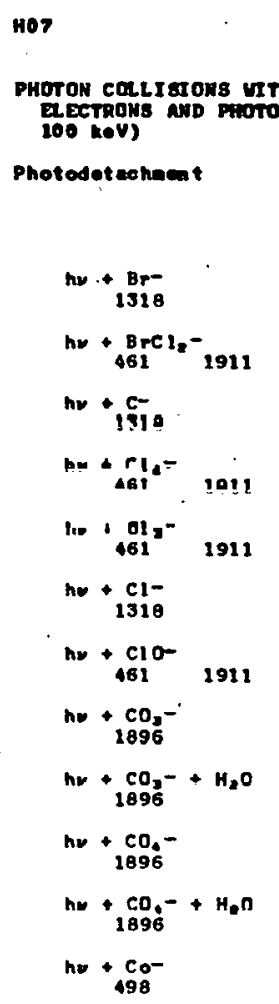

ตั่

PHOTON COLLISTONS UITH HEAVY PARTICLES ECETRONB AND PHOTOWS IN OASES The 6 LQ⿱日一⿻口卄日 nevi

Fl uoreseence and Lusinasence yidide

\begin{tabular}{|c|c|}
\hline$A B \cdot{ }_{2 A}^{A r} \cdot$ & Ar \\
\hline $\mathrm{hv}+\mathrm{Ba}$ & 1001 \\
\hline$n v+\underset{647}{B r}=$ & \\
\hline he + & 531 \\
\hline$h=\frac{01 i_{6}}{1108}$ & \\
\hline$h v+\underset{539}{c 1}=$ & 948 \\
\hline$h e+\frac{50 *}{387}$ & \\
\hline he $+\underset{\text { es }}{\text { ens }}$ & \\
\hline $\mathrm{h}=+\mathrm{C}_{457}+$ & $x e$ \\
\hline $\mathrm{hv}+\underset{421}{\mathrm{CS}_{2}}$ & 536 \\
\hline$h v+\underset{1205}{c_{s}}$ & 1225 \\
\hline
\end{tabular}

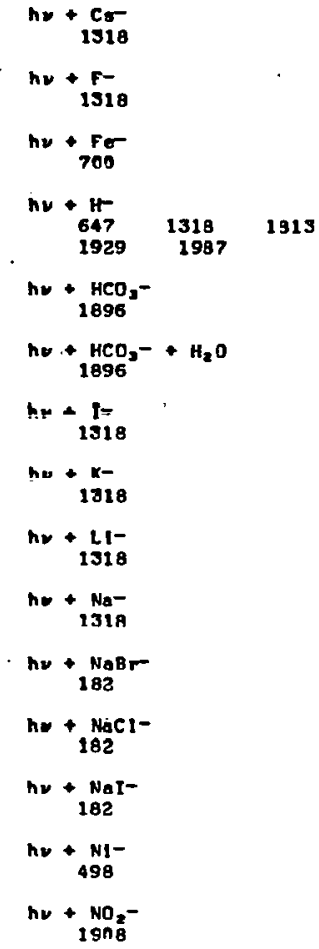

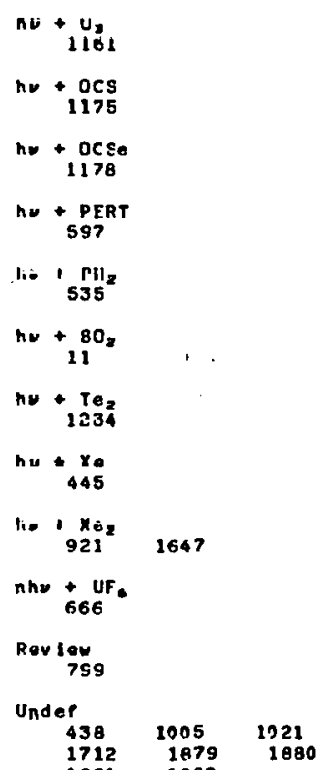


H10

PHOTON COLLI SIONS UTTH HEAUY PARTICLES ELECTRONS AND PHOTONS IN GASES (HD < $100 \mathrm{keV}$

photochedist ry

$$
\begin{aligned}
& h \nu+\frac{A r}{2 B}+A r \\
& h_{v}+\frac{B r z}{416}+X_{e} \\
& \mathrm{hv}+\underset{492}{\mathrm{Cl}}+\mathrm{H}_{2} \\
& \mathrm{hv}+\underset{416}{\mathrm{Cl}} \mathrm{z}+\mathrm{Kr}
\end{aligned}
$$

$$
\begin{aligned}
& \text { hy }+\underset{316}{\mathrm{NO}_{2}} \\
& \text { he+ } \begin{array}{r}
03 \\
1161
\end{array} \\
& \mathrm{hu}+\mathrm{SO}_{2} \\
& h_{v}+x_{445} \\
& \text { nhe }+\mathrm{O}_{371} \\
& \begin{array}{c}
\text { nhe }+ \text { ocs } \\
321
\end{array} \\
& \text { Undef } \\
& 1721
\end{aligned}
$$$$
1974
$$

111

PHOTON COLLI gI ONS MTH hEAUY PARTICLEg, EIECTRONS AND PHOTONS IN GASES (he? $100 \mathrm{kev}$ )

Free-frec Absorption or Inverse Bremestrahlung

$$
\text { hy }+ \text { C: } 1739
$$

H12

PHOTON COLLISIONS UITH HEAYY PARTICLES, ELETRONS AND PHOTOWS IN GASES (ho < $100 \mathrm{kev}$ )

Photon-Electron scattorting

$\begin{array}{ccc}\text { hu }+ \text { e } & & \\ 122 & 1320 & 1365 \\ 1373 & 1745 & 1746 \\ 1786 & 1787 & 1755 \\ 1817 & 1821 & 2015 \\ 2016 & & \end{array}$

H14

$$
\begin{aligned}
& h_{2}+\frac{e}{874} 1875 \\
& h_{2}+\underset{1041}{e+A r} \\
& h_{2}+\underset{655}{e}+\mathrm{CD}
\end{aligned}
$$

$$
\begin{aligned}
& 1041 \\
& \text { hy }+\frac{H-}{1347} \text {. } \\
& \text { Under }
\end{aligned}
$$

hi $+\mathrm{N}_{2}=$

PHOTON COLLISTONS YITH HEAUY PAATICLES, EIECTRONB AND PHOTONS IN GABES (ho < $100 \mathrm{keV}$ )

Photo-De-Exeltation, Quenching, and

st leulated Ealadion ( trobs ceetions) 
242

so1

DATA COMPILATION

Heavy PertIele

Unde?
312

Jns

DATA COHPILATION

Dentaop?

$h v+\underset{963}{\text { PERT }} .964$

KO1

REviEUS AND BODKS

Heavy Particlo

Revi on 1488

$1489 \quad 2071$

K92

REVIEUS AND BOOKS

Electrons

$\begin{array}{rrr}\text { Ravion } & \\ \text {.. } & 1488 & 1499\end{array}$

KOS

REVTEUS AND BODRS

Phutuns

$$
\begin{array}{ccc}
\text { Revien } & & \\
430 & 683 & 1488 \\
1489 & 1501 & 1722 \\
1755 & &
\end{array}
$$


243

Ko4

REVIEUS AND books

Portleles on surfaces and sollde

$$
\begin{aligned}
& \text { Revi on } \\
& 1489
\end{aligned}
$$

$x 06$

REVIEIS AND BOOKS

Strueture

$$
\begin{array}{rrr}
\text { Revley } & & \\
427 & 428 & 429 \\
683 & 799 & 1489
\end{array}
$$

$\operatorname{Los}$

BI BLIOCRAPHIES

Photcas

$$
\text { Unde ? } 310
$$


THIS PAGE

\section{WAS INTENTIONALLY LEFT BLANK}


AUTHOR INDEX 
THIS PAGE

\section{WAS INTENTIONALLY LEFT BLANK}


247

A01:

HEAVY PARTICLE - HEAVY PARTIClE INTERACTIONS

General

$\bullet$

$$
\begin{aligned}
& \text { Anundoen, P. A. } \\
& 1959 \\
& \text { Daunens, } M \text {. }
\end{aligned}
$$

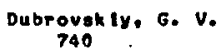

$$
\begin{aligned}
& \text { Duker, m. } \\
& \text { Fano, U. } \\
& \text { Fischer-HJ thars, I. }
\end{aligned}
$$

Ford, A. L.

Franco, $v$.

Fueno, 717 . 718

Inokut1, M. 1447

I tt kava, $Y$.

Jakubassa, D. H. 1959

Mats uzaua,
1447

Mlt suoke,
1447

Pouly, ${ }_{\text {żก37 }}$

Perroud, M.
P.eoding, s. F.

Russek, ${ }_{15 \text {. }}$

Saho, H. $_{4}$ P.

S11, ${ }_{274} \mathrm{C}$

Takatauka, K.

Thomes, H. K.

Turner. J:E.

Vohaoyer, $H$.
2037

$\underset{1619}{\underset{y}{\text { Unternt } z,} \text {. }}$ 


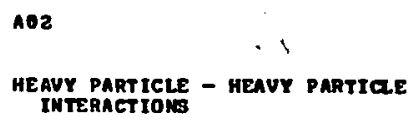

El wetic seattering colltelons

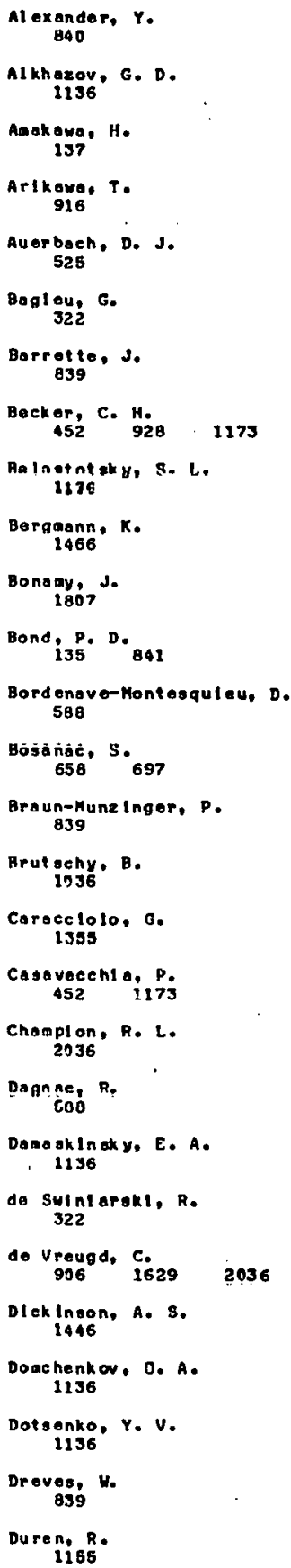

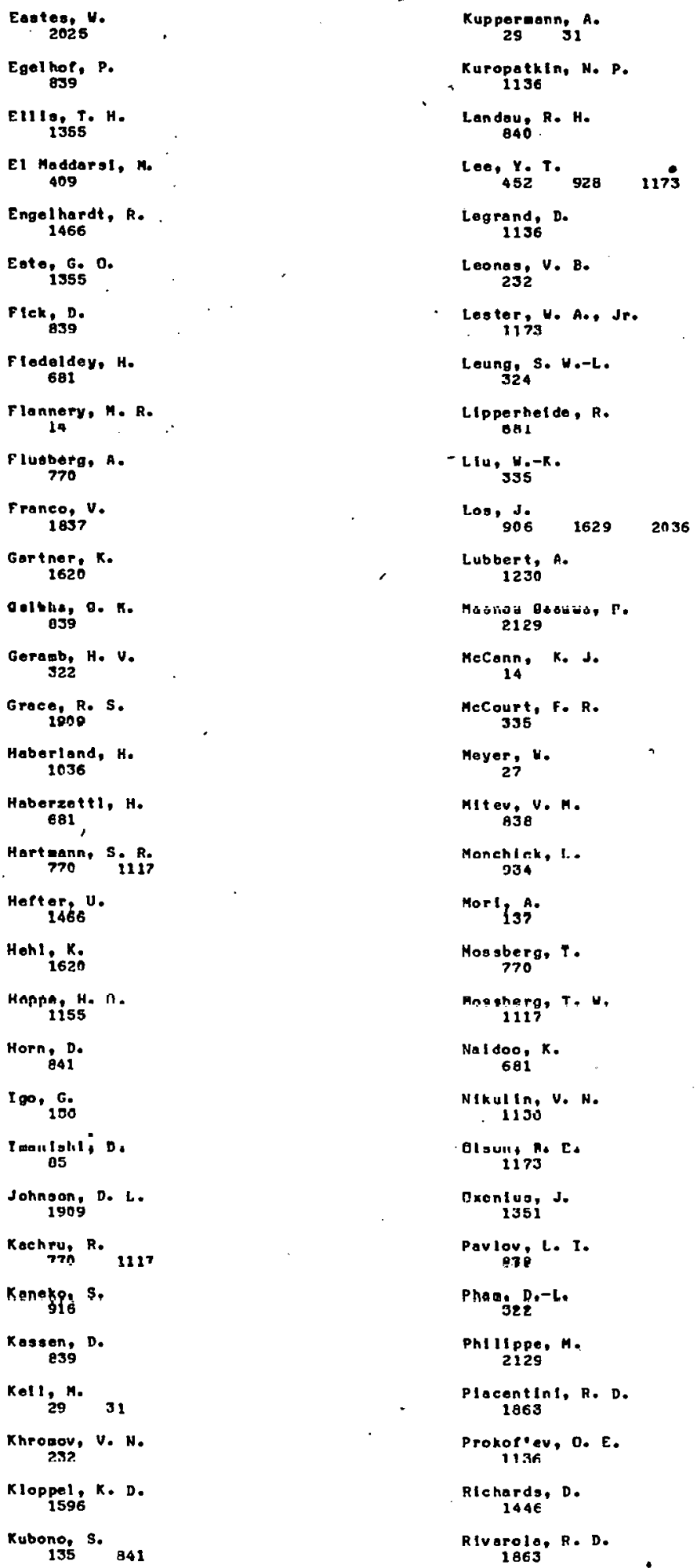


$249 !$

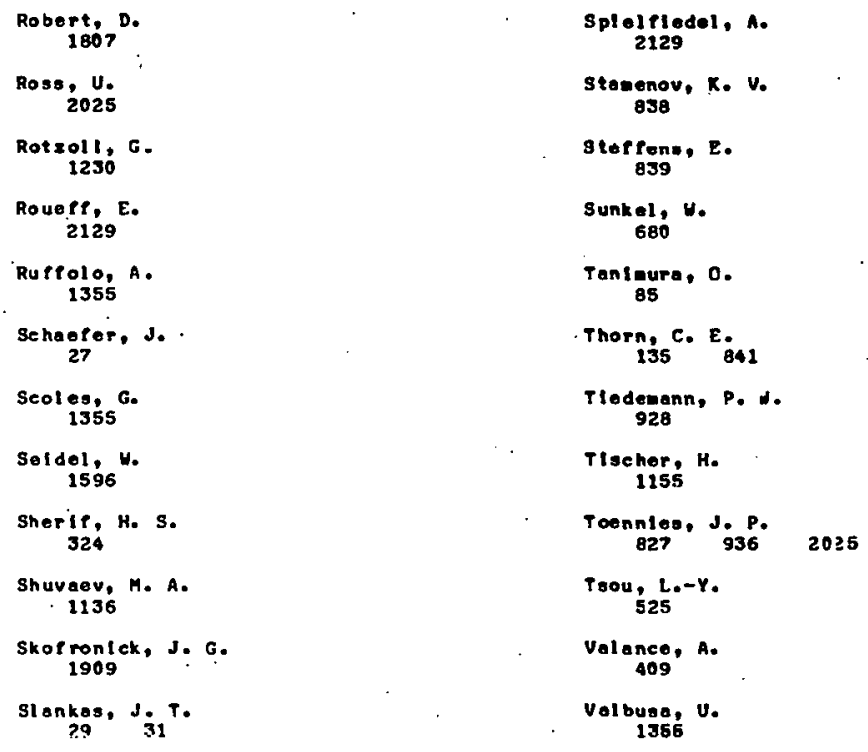

Valentini, d. S.

$$
928
$$

Volkov, s, 8.

1136

vadehro,.d. T.

Malker, R. B.

Uet e. " No

Wo1 ב,

$\underset{\text { Uharton, }}{525}$.

Wt Jnaendt s von Resondt, R. U.

vitt, Jo

Yol P. 6.

Yamejt, 5 .

Yozakf, $K$.

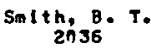




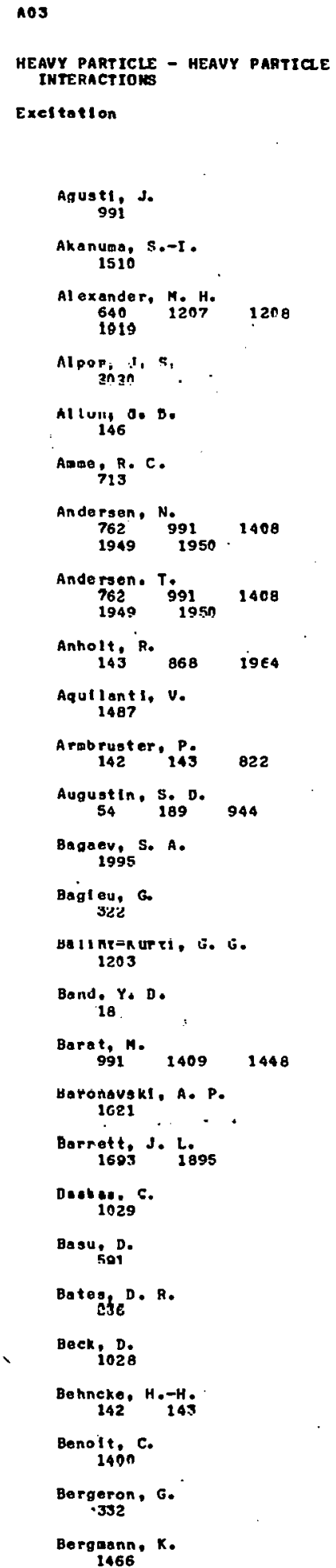

103

HEAVY PARTICLE - HEAVY PARTICLE

INTERACTIONS

Exeltation

${ }_{991}^{\text {Agusti, }}$ J.

Akanuma, S.-I .

Al exander, $M$. H. 640 1207 1208

Alpopi, ${ }_{2 n}^{1,} s_{1}$

AluII, 0.5 .

ADed, R. C.

Andersen, N. 762 991 1408

Andersen. T. $\begin{array}{ll}762 & 991 \\ 1949 & 1408\end{array}$

Anholt, $R$ 143868 ige4

Aqut lant I, $v$.

Armbruster, ${ }_{142}$. 022

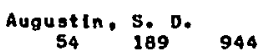

Bagaev, S. A.
1995

Bagleu, $G$

Ba 1 I KT=R UP 21, G. 5. 1203

Band. Y. D. is.

Barat,
991

Baronavskl, A. P. 1621 .

Barrett, J. L. 16931895

Dester, 5 .

Basu, D.

Bategfe $D$.

Beck, D.

Behneke, H.-H.

Benolt. $C$. $140 n$

Bergeron, $G$. -352

Bergaann, $K$. 1466

Bergstedt, $K$. 2005

Bert ande, J. lann

Beyer, H. F.

Bnotla, A. $\mathrm{K}$.

Bhat tocharyya, S. S.

B 111 ing, G. D.

BIrk Inshaw, $K$.$$
1435
$$

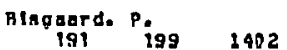

Plalg, "I. ".

Bloenen, E.

Bochkova, O. P:
83003

Bolshow, M. A.

Bordenave-Monte squil au, D. 580

Borkenhagen, U. 1210

Botteher, $C$.

Boul wer, J.

Bouman, J. Y.

$\underset{704}{\text { Brandt. } D .}$

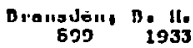

$\underset{\substack{\text { Brennan, J. G. } \\ 1029}}{\text { J }}$

Breuckmann, B. 1043

B rou ckmann, E. 1943

Bnidoux, M. 1628

Briggs, J. S.

Broun, $T$
1371

Bruch: Re log 1432

n Punner, T. A. 4861550 Brut achy, B. .

Burfa, 6

Caracelolo, G. 1755

Carl son, T. 'A. 228

Casavechla, $P$. 1487
Chan, F. T

Chang, A. M.

Chapulsat, $x$. 332

Cheung, J. T. 1211

Clary, D. C.

Cleaens, E.

Cocke. C. L. 1120

Courb In-Gauesorguee, $C$

rirnss. Fi. de. Jr.

$\underset{1438}{\text { Crothers, D. }}$ S. F. $_{1449} 1985$

Cuvellter, J.

Dagdlglan, P. I. 1207

Dagnac,

Dahl, P. 568

Dahler, J. S.

Dortus, E.

Dasheugkayo, E. I.
1391

Datz, 3

Desagr, H. P.

de Heer, F. J.

Del peeh, J.-P.
1759

de mesnoy, J.

DePrloto, A.E. ${ }_{375}, 944$

Dessaux, 0 . 1628

de $\underset{322}{\text { Sulnlarskl, R. }}$

Devos, F.
IVyy

do Vrloo, c. r. 1022

Dewangan, D. P.

Dlekinson, A. S. 275

DIIllnghas, $A$ 1024

Dixon, F. N. $181 E$

Douek, $D$ 


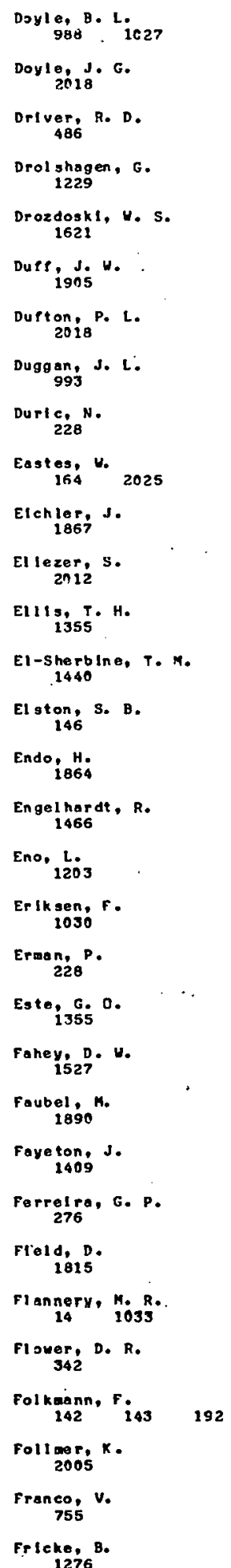

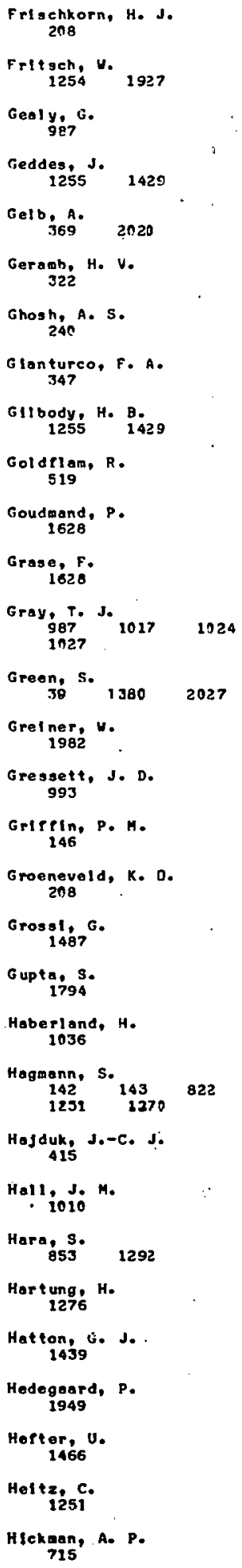

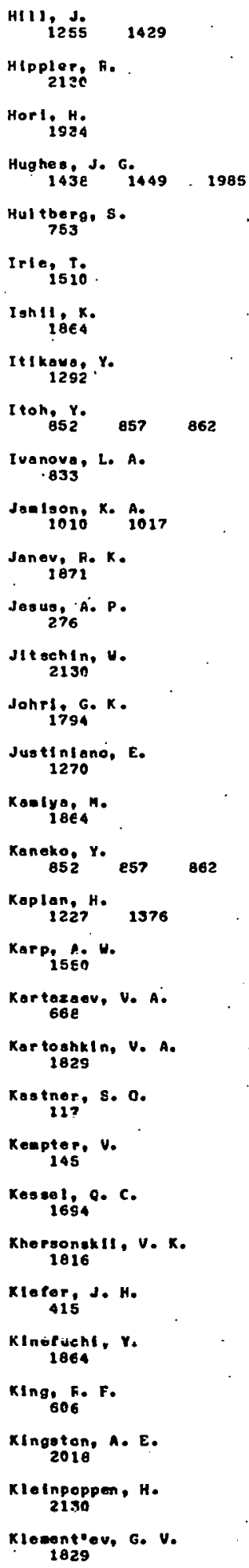



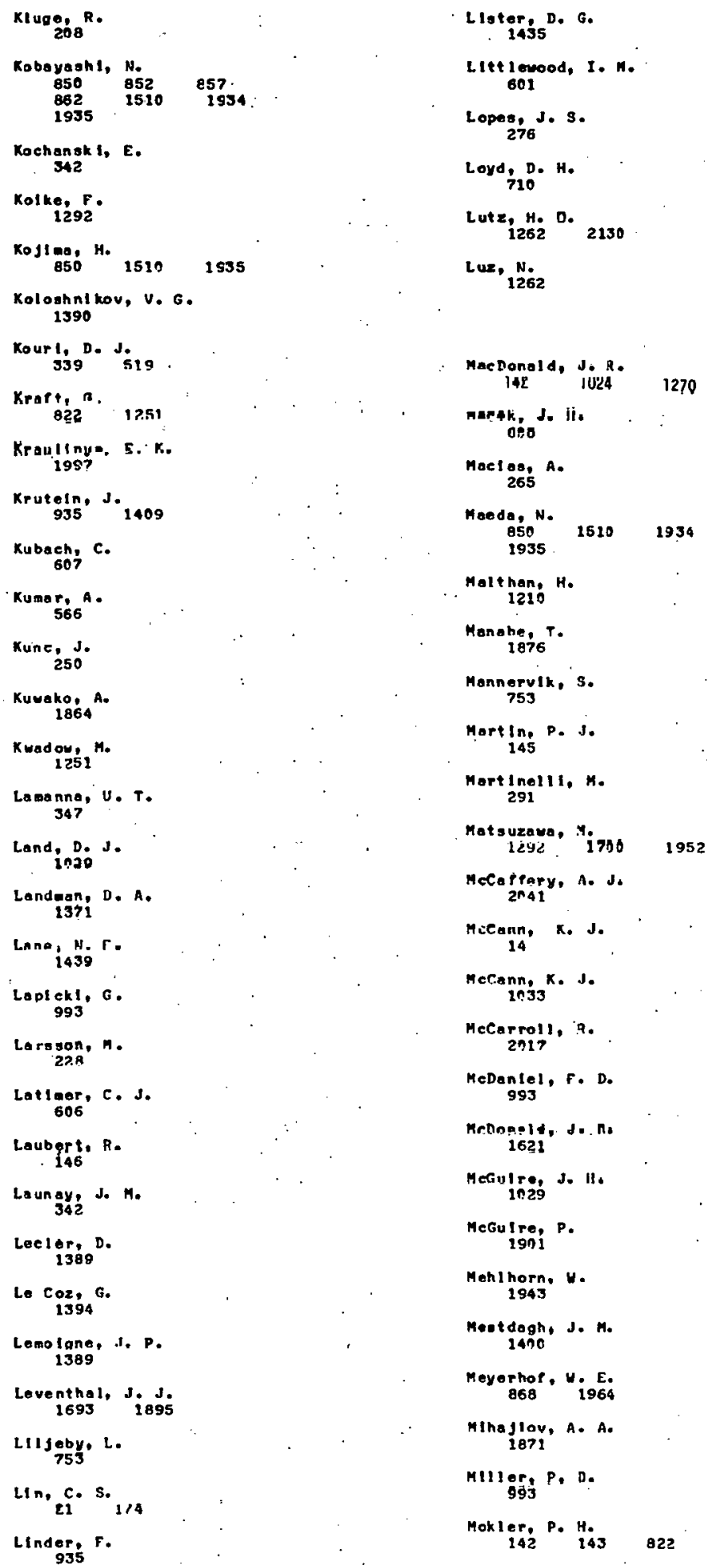


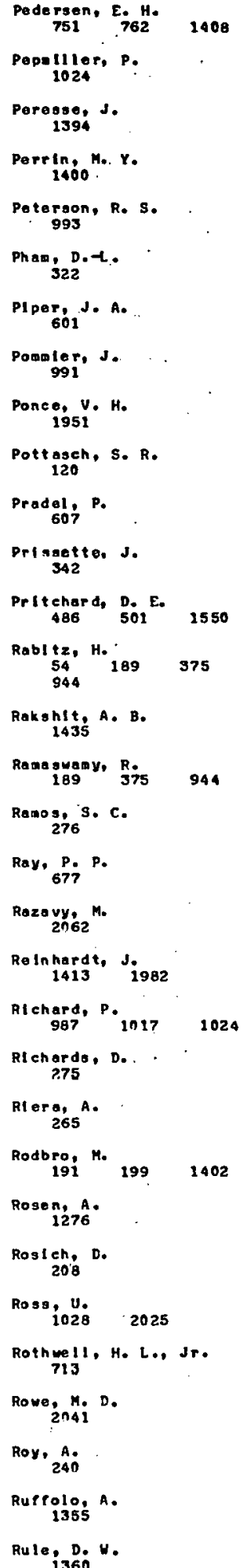

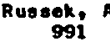

Shav, Mo do

she hok I nou, Y. A. 1820

sheridon, $R$ 984

Shi aonura, It 1292

shi ral, T.

S14tag ve 691

s11. S9i C. $_{600} 673$

$\underset{600}{\operatorname{sinhog} c .} 673$

Snoding, A. G.

$$
120
$$

Sut rnov, V. $\theta_{0}$

Sitth, N. 1550

sott, G.

Sou za, M. D.

Splose, 6.

Ste in, H.-J.

Steinfold, 'J. I.

$$
1626
$$

$\underset{868}{\text { stoller, }}$

Storey, P. J.

Stott, Y. R. 1269

Suenkov, A. A.
1020

Sur, s. $\mathrm{K}$.

Suter, ifen

Sut ton, D. G. . 1628

Tarrinl, 291

Taulbjerg, K. 605

${ }^{\text {Tawara, H. }} 1024$

The is: J J

Thoodostou, C. E. 1943

Thielmann, U.

$$
1405
$$

Thoe, R. S.

Thomas, L. D.

TIehy, H. 
254

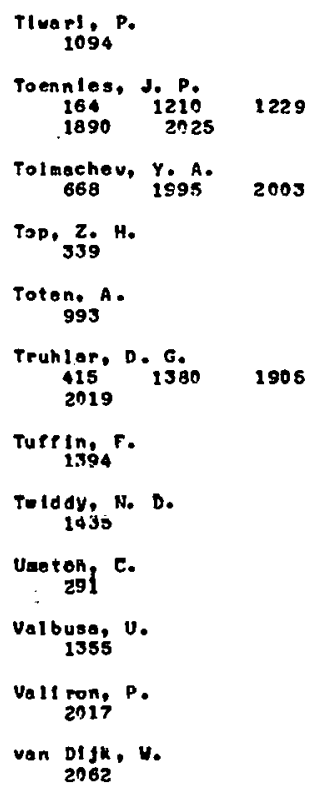

Vane, C. R.

van zy1, 8.

Vorshal ovich, D. A.

Vede1, $K$.

$\underset{\text { S54 }}{\text { VIdnar, } R . ~}$

Vol Pp, J,

$\underset{\text { Uadalngton, J. C. }}{1263}$

$\underset{1448}{\text { Uahnon, }}$

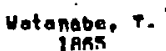

Uebb, $c$. E.

Webster, $M . J$.
19jo

UIleomb, B. E.

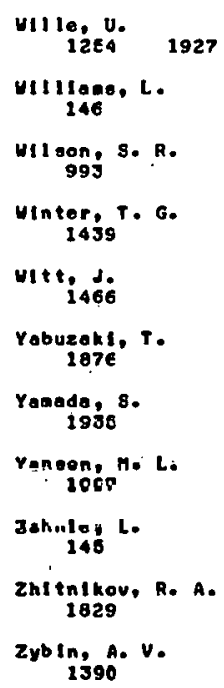




\section{A04}

HEAVY PARTICLE - HEAUY PARTICLE

DI osociation

Asaba. T'

Babu, s. v.

Barner, 1978

BIrk Inghaw, $R$.

Birks, J. W.

Bl al ${ }_{415}$ N. C. $_{450}$

Bochkovs, O.P.

Choung, s. T.

$\underset{1698}{\text { Cooney. P. J. }}$

Coplan. m. A

1476

Curt19, P. H.

Dal garno, A.

Datz, S.:

Doran, 1 .

Dove, J. E

Ennen, $G$.

Frischkom, H. S. 208

Gemall, D. S.

1698

Grillo, $A$

Groeneveld, K. O. 208

Groeneveld, K. -0 .
HaJ duk, J.-C. J.

$351 \quad 415$

Hoveanan, $U$.

$\underset{349}{H e r m a n,} Z$.

H111 Ier, I. H.

Homenn, K. H.

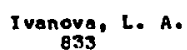

Kanter, E: P.

$\underset{348}{\text { Kendrlek, J. }}$

Rlerer: J. H.

Kluge, ${ }_{208}^{R .}$

Koont; 1158

Kunt x, ${ }_{348}^{P .} J_{2045}$

Lee, $\underset{\text { d.2n }}{H}$.

Lohr, T.

Leter, 0.6.

Lohr. R.

Memt Ilan, M. R. 1476

Michoel, 3. V. 620

net $\operatorname{lnger}_{20} 30$ C.

Pletsch, U.J. 1698

Porter, P. F.

Prit ehard, H. J. 629

Nakohlt, h. B: 1435

Renakitlehne, M.

Ratkougk1, A. J.
Raynor, 9.

Poe d, 163 .

Roberge, w. $G$.

Ros Ich, D.

Roth, P.

Sehader. J.
2ne

sehneider,
349

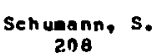

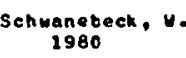

slack, H. W.

Snider, N.

Tilenyius.

Tol machov, Y. A. 2003

Trunlar. C. 45.

Tulday. N. D.

vager. 2.

$\underset{684}{\text { Vogler, }}$

1980
Wornatz, J.

unitton, w. N. 348

UIIIIans, B. W. 1634

Yau, A. Y.

zabranaky, B. J. 1658

Zunrt, c.

Zulleke, L. 
nos

GEAUY PARTICLE - GEAUY PARTICE IWTERACTIONS

Cheoll win Inesconce, FI uorescence, and Luel nescence (photon enl saton by

unapec (fited procesees)

Arustrong, R. A.

1460

Basovi N. G.

$\underset{905}{\operatorname{senard,} D .}$.

Black, G.

Buek, U.

Buttlar, $H, V$.

Coske, c. $\mathrm{t}$

Coosber R. D.

Dant Iychev, V. A.
1758

Davt s, 5. J.

Bolgikh, v. A.

den, J46 6 .

Fannel, ro.

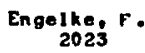

Fricke. 1 .

$\underset{329}{\operatorname{Gerard,} M}$

Golden, J.

Govers, T. R.

Heakg, D.

Hagmann, $S$.
Henmer, D. A.

I151

Hort ung, $H$.
1276

Heckmainn, P. H ezo

Horne, R. K.

Just intano, $E$.

$127 n$

Ker Injov, O. M.

Knar, B.

Kowa 1 sk1, A. 1143

Kurmanalieva, G. D. 15\$0

KusunokI, I 1902

Lebedev, v. S.

Lute, H. $O$.

ᄂ หะด, พุ.

MacDonald, J. R.

Mahapfey, R. A.

Manglr, M.

Morx, $R$.

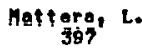

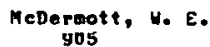

Mefórlañe, H. A. 1151

MIIEr; $0 . J$

$\underset{1758}{\text { Mol e hanov, A. }} \mathbf{5}$

Moroule, T.

18TE

Mura i, H.

กtI, $\underset{376}{k .}$ ottinger, $c$. 1902

Pasour, J. A.

Presser, $G$.

Pruett, J. G.

Put t, D.

Relsler, $\mathrm{H}$.

Rosen, A.

Sackmann, 3 .

1262

Scheldt-Becking, H.

12.70

Sepp, $D$.

Shackleford, W. L. 1611

Sharpleas, R. L.

1176

Shl yaphinov, G. v.

Shmatov, 1. P.

Stahler, J.

Stott, H. R.

Tanaka, I.

Torres-Fitho, A.

Trabert, E.

azn

Heddingtop, i, $C$.

1263

Uaynant, P. W.
1146

Ulley, L. G.

utetig, $\mathrm{c}$ 1223

Yog $1, T .5$ 
no6

HEAYY PARTICLE - HEAVY PARTICE
IWTERACTIONS

Eleetron Capture

Adaeg.' N. Go

Ague 1 , J.
991

$\underset{1479}{\text { Albe Ittion, D. Lo }}$

Al1, 8. P.

Al ton, G. D 1983

Andersen, F. D.

Anderson, $N$

Anderson, $T$.
991

Ange $1,6 . c$ 1528

Anteleh, $v$. 446

Appleton, B. R. 1056

Awad, 6. $L$

Banyard, $K$. E。

1520

Barot, $\mathrm{H}$.

Bards1 ey, J. N.

arrett. J. L.

$$
1895
$$

Basu, D

Bayeteld, J. E.$$
1690
$$

Becker, R. Le $_{135}$

14.71

Be11, 756

Bender, C. F.

Berry, S. D.

Betz, H. D.

DI yubrtaff, J. A.

Blond1, M. A.

B1 rk!nshar, $\mathrm{K}$.

Bt lean, S.

Bl oemen, $F$.
Bordenave-Montesqul es, D. 588

Bose, M.

Brandt, 132 .

Brouckeann, B.
1943

Breuckanan, $E$.
1943

Briggs, J. S.

Brou 111 ard, $F$.

Burgdorfer, J.

1547

But 1 er, s. E.

arrington. A. 194

Chan, f. T. $\begin{array}{lll}746 & 1235 \quad 1307\end{array}$$$
18
$$
1307

chinieny, to. To 1650

Christengen, R. B. 1367

Claeys,

Cocke, C. L.

Cohon, d. 5 .

Compton, R. N.

424

Coplan, M. A.

Courbin-Gaussorgues, $c$. 991

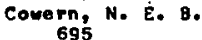

Crandel1, D. H.

Crothers, D. S. 293 i438 1449

19.

Curt 19, P. M.

Dagnac, $R$.
Seg

Dah1, P.

$\underset{118}{\text { Dalgarno, }}$ A.

Dota, s.

Dowaon, H. 9.

de $\underset{576}{ }, F \cdot \begin{aligned} & \text { J:er: } \\ & 1440\end{aligned}$

Deral, R. 482

de Urles, C. P.
Dicklnson, A. S. 2134

Dol dere Ke:

Dotan, I. 1475

Dousan,
1806

Douek, D.
1409

Doyleg B. 1.

Duggang \&. L.

Duan, E. L.

Dunn, $K_{-} F_{\text {. }}$

Dutte, $N$.

Elenler. $746 \quad 1295 \quad 1307$

Ell suorth. L. $D$. 709

El Maddaral, M. 410

El-sherbine, T. M. 1440

EI Shorbint, T. n 576

Elston, S. B. 1983

Ertksen, F

Eriksen, F. J.

Fayeton, $J$.

Fohsentel d, F. $C$.

Fonlstein, 8 .

Fit chard, $A$. 1305

fol knonn, F.

198

Ford, ${ }_{1309}^{\text {A. L. }} 1415$

1431

Freeman, C. G.

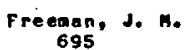

Frohileh, D.
PAP

$\underset{1690}{\underset{10}{\text { Gardner, }} \text { L. D. }}$

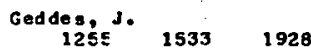

Gels, M. W.

Geller, R.
180e 


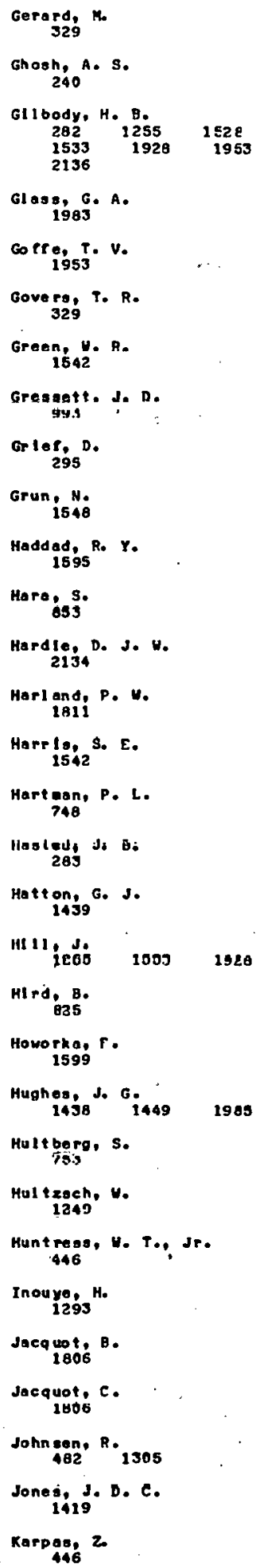

Kortazaev, V. A.

Khan, M. N.

KIng, R. $F$.

Ktta, s.

xleyn, $A . W$.

Koch, P. M.

Kronast, $W$.

Krutein. J.

Rubach, $\mathrm{C}$

Kumar, A.

Lane, N. F. 1439

$\underset{993}{\text { Laplekl, } 6 .} 1327$

Larsen, L.

Latlmer, C. J.

Leventhal, s.

LIgne, J. C. 1179

LIl leby, $t$

LIn, C. D. 1416

Lindinger,

$$
\text { I } 5 \text { yy }
$$

i ister, 1. G. $1419 \quad 1435$

Litl lewood. I. A.

LIu, B.

1842

Los, J.

Loyd, D. H.

Lucas. M. W.

MacAlptne, G. H.
Mas?

Macdonald, J. R.

MacDonald, J.R. 1015

Mecketlar, $A . D$.
1256

mahr, ${ }_{748}$

Mallory, M. L.

Mann, $R$.

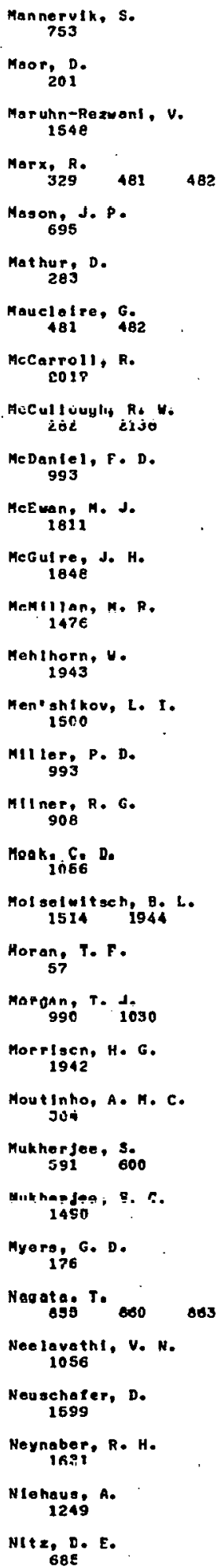




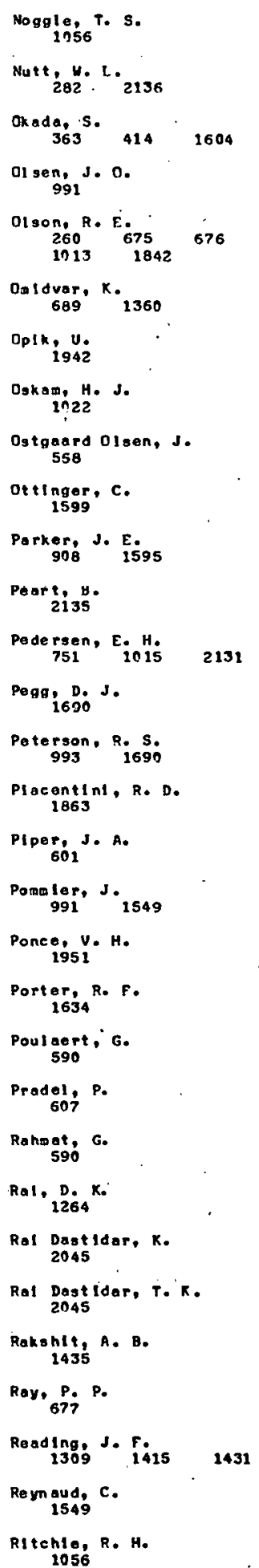


260

$$
\begin{aligned}
& \underset{1056}{\text { verbeek, } H .} \\
& \underset{1023}{\text { Wodehra, J. }} \\
& \text { Warnack, R. j. } \\
& \text { Watanabe, } T \\
& 996 \quad 1865 \\
& \underset{1367}{\text { Wotson, }} \text {. D. } \\
& \text { Nobb, } c, E_{601} \text {. }
\end{aligned}
$$

Wal ohelt, J. $C$

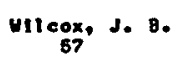

U111 16as, B. U.

w11 son,
993

uinter, T. 6.
Voorlee, P. H.

$\underset{1542}{\text { Urtght, }}$ D. D.

Young; J. F.

Zimeriann, s.
ISSS 
107

\section{HEAUY PARTICLE - HEAVY PARTICLE IUTERACTIONS}

Ionizati on

Agust 1,
991

Akenuma, $S .-I$.

1510

Al bat, $70^{R}$.

Alton, 6. D:

Andersen, N.

991

Andersen, $T$.

Andrews, M. C., III$$
1856
$$

Ange 1, G. C.

${ }_{143}{ }^{\text {Anholt, }} 716 \quad 8 \in \theta$

Arobruater.
142

Anay a, $Y$.

Barat, $M$.

Becker, R. $L$.

1256 . $1415 \quad 1431$

Behncke,
142

Bell um, J. C.

Berezhko, E. G. 1445

$\underset{23}{\operatorname{Berkow}}$. J.

Bet 2 , 131 .

Deyer " ${ }^{\prime} H, F$,

Blondl, Mo A.

Bi sgaard, $P$

1402

Btat t, S. L.

Bochkova, D. P.

2003

Boving, E. G.

Braidt, D.

Brandt, $U$.

Rroueknann, B. 1943

Breuckeann, E. 1943
BrIdwell, L. B.

720

Brlggs, J. 3.

Bruch, $R_{0}$

Celler, $^{A-}$

Coeke, C. L.

Compton, R. $N$

424

Courbin-Gauss or gues, $c$.

991

Dagdi glan, P. J. 1152

Danler, J. S.

de Heer, F. J.

Dewarest, J. A.

Devdarialn, A. $z$.$$
1827
$$

de Urleg, C. P.$$
11 \text { inghas, } R \text {. }
$$$$
1024
$$

Doering, J. P.

Doyle, B. L.

Duggan, J. L.

Duncan, $M$. M.

Dunn, $K_{15} F$.

Edl und, $K$.

Etehiar, J.

El sherbini, T. M.

fiston, s. $\frac{8 .}{146}$

Endo, ${ }_{1864}$

Engel gann, C.

Fohey, D. W.

Fint $z_{678} P$.

Flannery, M. R.

Falkgann, $F_{\text {i4s }}$ i9?

142
198

Ford, A. L. 2431

f uk u yase, T i641
Gea ${ }_{987} 6$.

Geddes, $d$

1803

Gentry, H. R.

George, T. F.

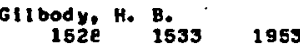

Gillesple, G. H.

1132

Gotte, T. V.

Gray, T. J. $967 \quad 1017 \quad 1024$ 1027

Grelner, W. 1312

Grosett, J. D.

Gritein, P. H.

Gulllause, 6.

678

Hageann, S. 143

Hall, J. M.

HII I J

${ }_{140}{ }^{H i p p l e r, ~} R \cdot 2130$

Hiratshl, H.

Hor Pann, v.

Hot op, ${ }_{546}^{H .}$

Hul teseh, H. 1249

Irte, ${ }^{T}$.

I saacson, A. D.

Isnit, $k$.

Jam toon, K. A
1010
1017

Jesue, A. P. 1835

Jit schin, $u$.

Johnsen, fo $130 E$

Johnson, E. M. 678

Jones, C. $M$.

Jones, K..

Jundt, $F$. C. 


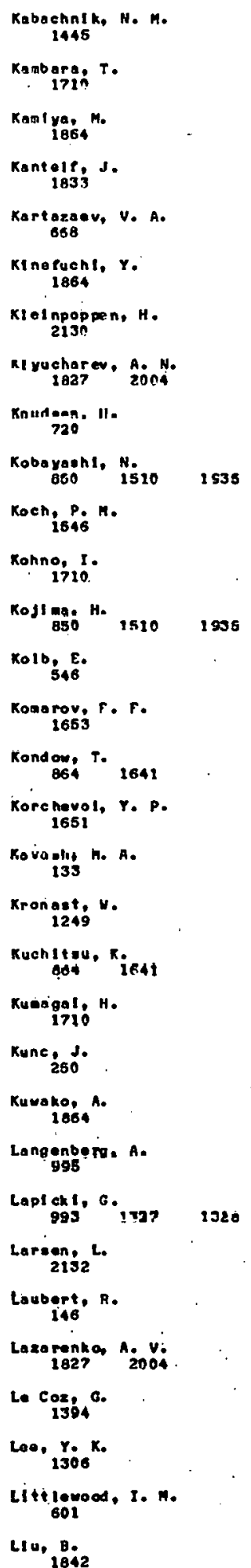

Lopog, J. S. S. 1035

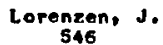

Losonsky,
1328.

Loontana, M.

Lutz, ${ }_{1262}^{H_{0}}$ O. 2139

L.u2, N.

macdonal a, J. A. 140

MacDonal d, ti $R$. in15 ioa

matiag: A.

Maekellar, A. D. 1256

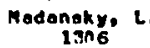

Modt son, D. H. 1697

moede, No $_{950} 1510 \quad 2935$

Mann, R.

Nenson, S. T.

Maor, D.

Marfollo, R. P., Jr. 494

Mart le, n. Me

Mecann, $x, J$. L93

Meclintoek, J. A. I3no

Modanie1, F. D. 993

Mehinorn, 1943

Monandez. H. Go 687

Meron, N-

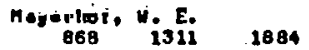

matler, P. Dg.

moth 320 o.

Mokl arp P. H.

Morgan, J. P.

Morgnor, Ho

1274.1428

Morita, $s$.

Muller, B. 1312

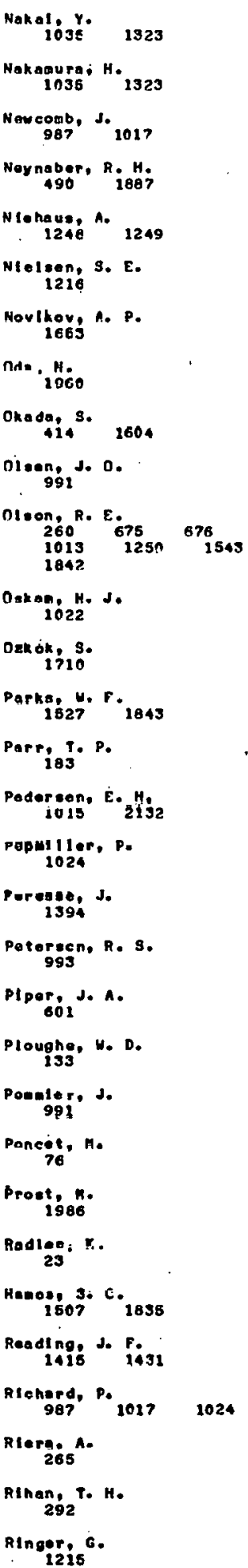




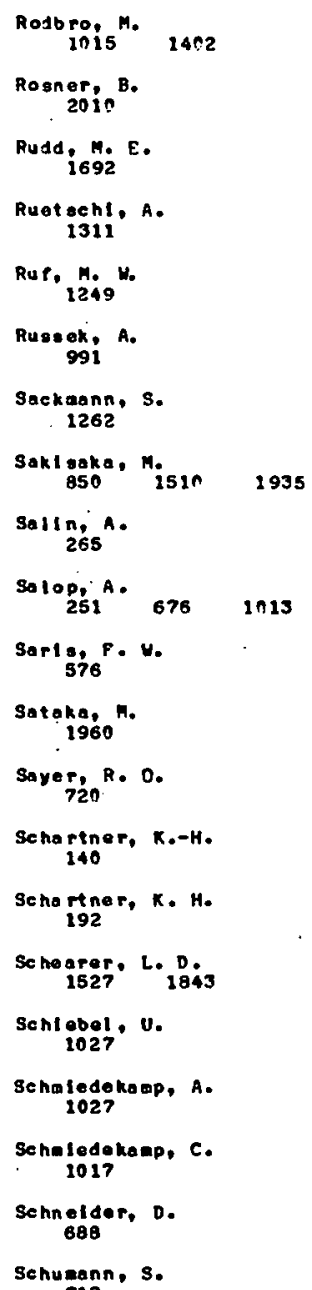

Schaledekamp, C. 1017

Sehneldar, $D$. 688

Schusann, S.

sellin, I. A.

Shah. M. M. .

Sheverev, $V_{0} A_{4}$
2004

Shirat, $1035{ }^{T} 1323$

siaig, $v$.

Sopt ${ }^{G}$.

Sonobe, B. I.
995

$\underset{200}{\text { Soransen, }} G$.

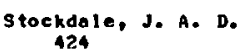

Stock11,

$\underset{8 \times 8}{\text { stoller, c. }} 1311$

Stol tersoht, N. N.

Suter, ${ }_{146}^{M .}$

Smapford, G. L. 1415

Tang, S. Y. ${ }_{1887}$

Taulbjerg, $k$.

1410

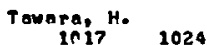

Theodostou, C. E. 1943

Thoe, R. S.

TIwory, S. N.
Toburen, L. H.

Tolmechev, Y. A.

Tonuma, $17{ }^{\top}$.

Toton, A.

Turpin, F.

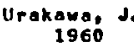

vane, C. F. 712

$\underset{639}{\text { Vinekler, }} \mathrm{C}$.

Waragek, R. J.

Hatgon, R. L.

Weob, $c$. E.

WIIItoes, 1.

146

wileon, S. R.

WII son, W. E. 1034

$\underset{57 \epsilon}{\text { Wórlee, }}$ P. $H$.

Nolrit, $W$. 1311

Yamoda, 1935 .

Zander, A. F.

zoran, U.

Zyilez, Jo 
nOB HEAUY PARTICLE - GEAUY PARTICE
INTERACTIONS

stripping

Arlkava, $T$.

854

$\underset{1256}{\text { Beck er. }}$ L.

BI sgoard, P:
191

ค1 $10 \times n$; 9 .

Bruen, ${ }_{1 y_{1}}$. 199

uahl ig.

DmItrtev, I. 5.
1506

Dousson, s.
264
A09

$\underset{513}{H \text { lekman, A. P. }}$

HEAVY PARTICLE - HEAVY PARTTCE INTERACTIONS

Recosb Ination or Mutual Neutratization leading to Noutral Products (Ion-Ion)
Gell er, $R$.

${ }_{\text {I noue, }}$ M.

Jaequot, B.

Jacquot, $C$.

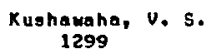

Larsen, L.

Mackellar, A. D.
Labe

Maar, D. द्रo10

maron, the

Narughtma, $K$.

01 son, R. E.
Dal duar, $K$.

Pedersen, E: H.

Rodbro, $^{\text {M. }} 199$

$\underset{201}{\text { Rosner, }} 2010$

Rule, D. $_{1360}$

Sellin, T. A.

Toohoobs Y. A.

Tawara ${ }_{106}$.

Tseker is, J.
1806

Zalkov, V. P.

Peart, ${ }_{233}$

Forregt, R. A. 


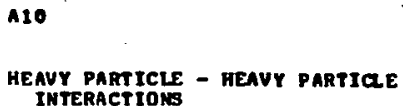
IMTERACTIONS

Electronte, Vibrat lowal, and Rotational Energy Tranter (procese unkaom)

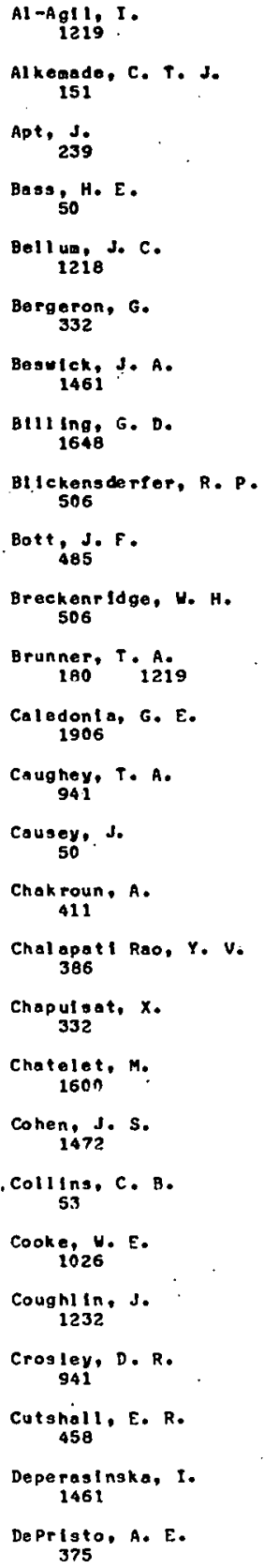

DeVrles, P. L. 408

DIxon, R. N. 1069

Dove, J. E.

Doyennette, L. 411

Driver, R. $D$.

Edalsteln, S. A.

Engelke, F. 1640

Evans, W. D.

F ahey, D. W.

Fleld, $D$. 1n68 1069

Fltepatrlek, d.

collaghor, $t$. F. 1026

Gay, J. C. $_{1701} 1702$ 1703

Gedd og, J.

$\underset{408}{\text { George, } T . P .}$

Gtantureo, F. A.

GIIbody, H. B.

Gough, W.

$\underset{\text { Green, B. D. }}{\text { I9n6 }}$

Green, Y. R.

Grimley, A. J.

Harr 1s, 3. E.

Hay, P. J

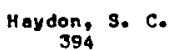

Henry, $l$.

Hamann, K. H. $15 B 0$

Hor I guehl, H.

Horwltz, A. B.

$E 15$

Houston, P. L.

Huest Is, D. L.

Joll lard, $G$.

454

Karp, A.
Knebe,

Korseh, H. J. 413

$\underset{327}{\text { Kukol I Ch, S. G }}$

Kus haveha, v. s. 129

Kuok, H. $_{168}$

Lamanne, $U . T$. 347

Lee, F. W.

Leone, S. R.

Lepoutre, $F$ :

184

Lin. c. S.

Louls, $G$.

Lukap Ik. W.

Marcus, $A$.

cus A. A

Mergottin-maclou, $M$.

Mecurdy, c. w.

MeGuire. $P$.

$\underset{394}{\text { MeIntosh, A. I. }}$

MeKee, J. D. A.

Meyer, H. D.

Moyer, $12,-D$.

Micha, D. A.

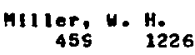

Mulloney, $t$. 1923

Murphy, $R \cdot$ E. $_{6}$

Musch!ltz, E. E., Jr. 468

Nutt, G. F.

Oba, D.

Oksengorn, $\mathrm{E}$. 1600

Parkgitu. $F$.

Poulaen, L. L. 1648

$\underset{160}{\text { Prttcherd, }} \underset{239}{\text { E. }} 1219$

Rabitz, H. 
266

$$
\begin{aligned}
& \text { Rama guamy, R. i } \\
& \underset{628}{\text { Raynor, }} \\
& \text { Rosen, D. }
\end{aligned}
$$

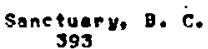

$$
\begin{aligned}
& \text { Schate, G. C. }
\end{aligned}
$$

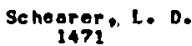

$$
\begin{aligned}
& \begin{array}{c}
\text { Sehnelder, } \\
1702
\end{array} \\
& \text { gshnelder. M. M. } \\
& \begin{array}{c}
\text { Sahuanehert, } u . \\
\text { I980 }
\end{array} \\
& \begin{array}{c}
\text { Secrest, D. } \\
21
\end{array} \\
& \text { Seoud! } 8 . \\
& \text { shin, }{ }_{903} K .{ }_{1473} \\
& \underset{+180}{\text { Setth, } N .} 1219 \\
& \text { Talne, J. }_{184} \\
& \text { Taylor, H. S. } \\
& \begin{array}{c}
\text { Taylor: R. L. } \\
122^{2}
\end{array} \\
& \text { Toennles, J.P. } \\
& \operatorname{Tramer}_{1461} A . \\
& \underset{38}{\text { Tsuchtya, }} \text { s. } \\
& \text { Turfag }{ }_{163}^{A . F .} \\
& \text { van olfh, } i . A \text {. }
\end{aligned}
$$

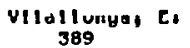

$$
\begin{aligned}
& \text { Uadt, } U_{1472} R \text {. } \\
& \text { Wainger, } \\
& \text { Walker, R. B. } \\
& \begin{array}{c}
\text { Uarngts, J. } \\
198 \text {. }
\end{array}
\end{aligned}
$$

$$
\begin{aligned}
& \text { Was oam, H. A., Jr. } \\
& \text { Hest: } 50 \\
& \text { Uidenlocher, } G \text {. } \\
& 1600 \\
& \text { UIIkIng, R. L. } \\
& \text { U1111ans, J. R. } \\
& \begin{array}{c}
\text { MIIson, J.R. } \\
758
\end{array} \\
& \text { UnI frum, J. }
\end{aligned}
$$

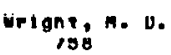

$$
\begin{aligned}
& \underset{60}{\text { Uyatt, }} \text {. E. } \\
& \text { Hys s, } J \\
& \text { Young, d. F. }
\end{aligned}
$$

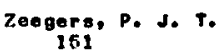




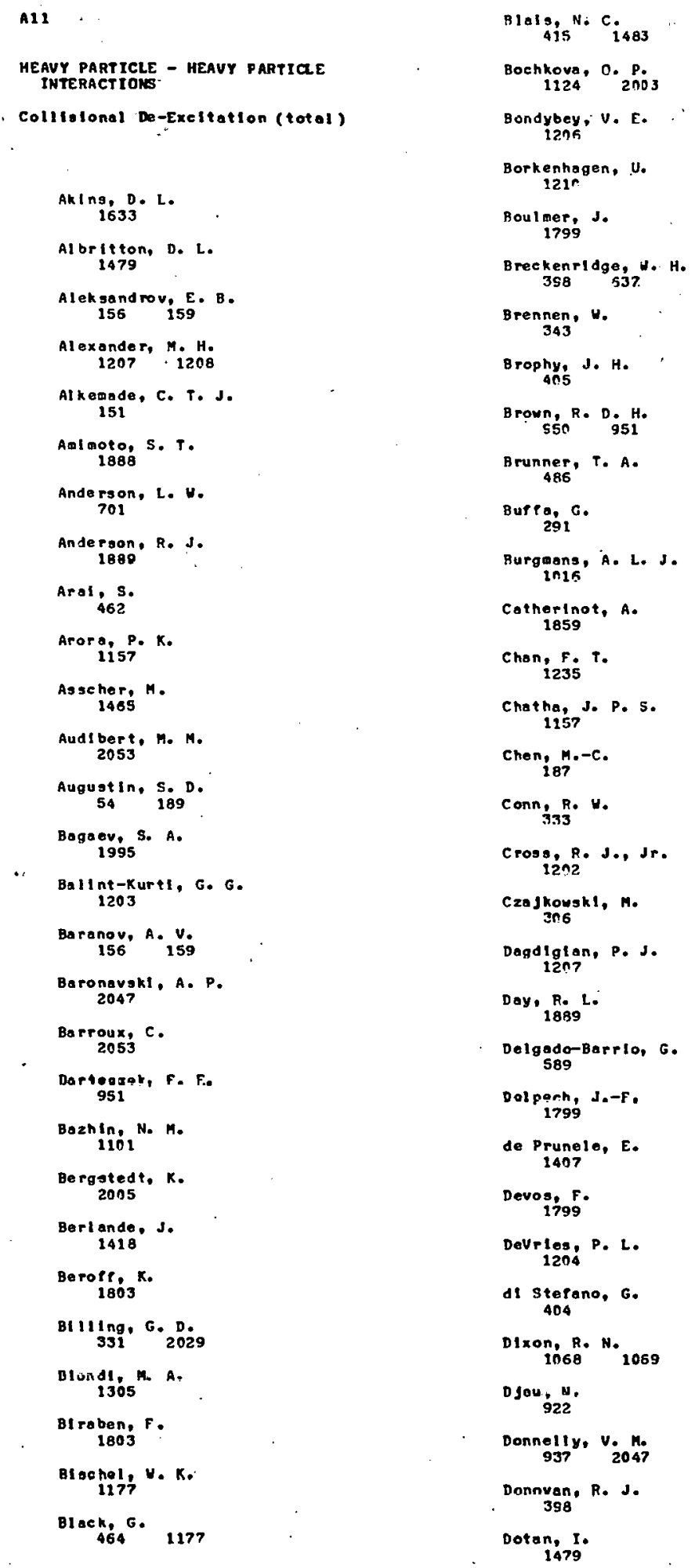



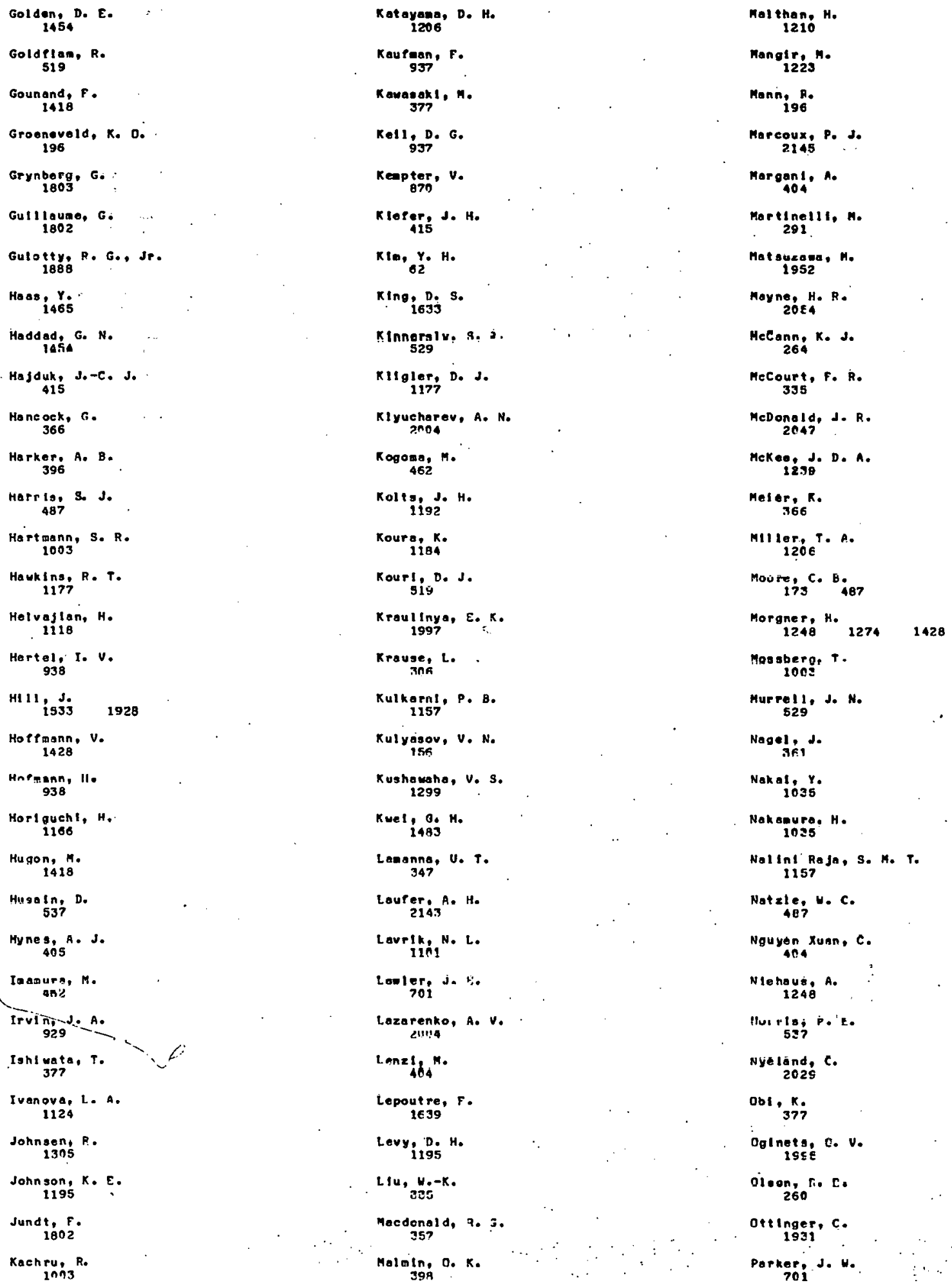


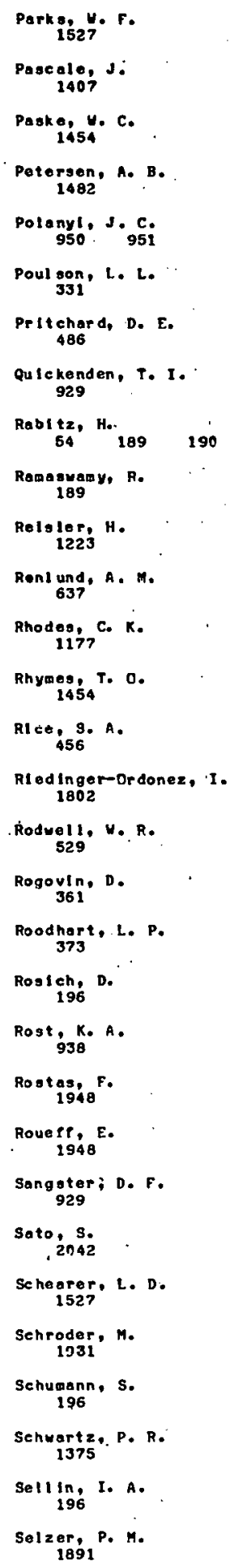

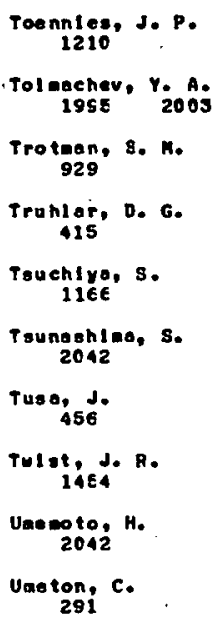


la12

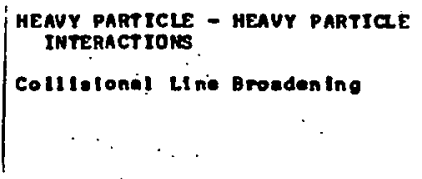

Bal ucan 1. U.

202

Bastard, D

648

Batyoin, $v, v$

199

Belgang, $R$

1316

Berang: $\mu$. $H$.

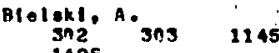

1495

Bterea, S.

Btrnbaus, 6

1112

Bjorkhiol m, J. E.

1857

Boese, R. U.

Bonaty 1 .

Bretonoux, A.

Bulanin, M. D.

t12s

Bulychev, V. P.

1125

Butayso

Bystrá, $K$.

Calvert, s. 6 .

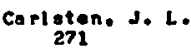

Chembed, 240

Charru, A.

Chobotain, V: P.

1753

Chen i s. Y.

Chong.s. $Y$.

Damany, $\mathrm{K}$

Darowlek1, $H$.

1145

de Priato, A. E.

1113

Dokumo, 1495
Ehrleh, H.
Blg

Eng, R. So 210 ?

Frish, H. So

$20 \times 2$

rusteoto. $T$ :

Fukuda; $K$.

Golking V. D.

1129.

asllagner, A.

Gentry, 8 .

11 bert, $D$ :
1948

Iver, $L$. P.

Goldenlth, J. E. M.

$13 n$

Gomyl, N. B.

Greenberg, $A . S$.

1926

Gude enko, $\mathrm{L}$ : I.

Gupt o, 5

Henry, ${ }_{\text {ss. }}^{A .}$

Henry, 2 .

Hull l cek, J.

646

Iminun, S. I..

Johr1, G. K.

Kant serov, A. I.

Kelloher, D: $E$.

Niensovic, $\mathrm{No}$

Korfe D.

Lodviahchenko, Y. M.

1.125

Lakoba, 1.9 .

Laporte, P.

LeDuts, $Y$.

Lee, ${ }_{\text {8. }}$.

Levy; ${ }_{248}$

L100, P. F.

LIn. C. $L$
Ltstekl, E.

LIStsa, V. S.

liting, ${ }^{\text {late }}$

Monaba, T. 1513

Mentz, A. W. 110 ?

Mat Isov, B. G.

199

Mehrotra, s. c.

1752

nlllte, $P$ 248

Mitev. v. M.

Nesterov, $M$.

Ntemax, $K$

Niantulat, 0 . 162

Ogawa, 1.

Pateh, F. W.
1735

Povlov, L. I.

PenkIn, N. P.

PIcherlt, F.

Ptenler, $G$.

271

D1.moticus.

Popov. K. G.

1127

Pujol. 19.

Rabita, H.

1113

Roging H, Oo

Rebane, V. N.

RIdard, J.

Robert, D.

Rostas, $F$.

Rouert. E.

Rub tsove, N. N.

Ruzov, V. P.

Sal asky, E. T.

Solganik, Y. A. 
Sehnelder, R.

$$
1316
$$

sehuller, $F$.

Schuemaer, $G$.

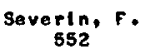

$\underset{\substack{\text { Shabanova, L. N. } \\ \text { 2nog }}}{ }$

Shoug J. H.

sivkov, v. N.
liz?

Skvortsov, M. N.

Sptel rtedel, A.
194 B

Srivastava, S. L.

1792

Stamonov, $K$. $V$.

838

Buoroz, C. D.
1115
Sul zaann, K. G. P.

1573

sỵtsiko, Y. I.

i7s:

szudy, J.

Tettemor, G. L.

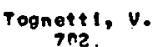

Tskhal, N. S.

Uedo, $x$.
is?

Valero, F. P. J.

Vallourt, $R$.

$7 n 2$

vesilienko, L. S.

Vincent, $P$.

1926

$\therefore \because$
Hang, S. Y.

Ueber. E. U.

Neber, $K \cdot-H$

Wen iger. 5.

Ulese, U. L

uIlkersen. T. D.

$\underset{69}{\operatorname{Hoerdabn}}$ J. P.

Wol nikguskt joj.

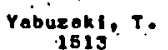

Yakovleniko, S. $I$.
17eI

zhuvikin, G. V.

zono 
A13

\section{hEWY PATITCLE - hEAUY PARTICLE Internctions \\ Heavy Partiele Interchange, \\ Rearrangonent, and Asecoletion (one \\ or more Iont e reactant $b$ )}

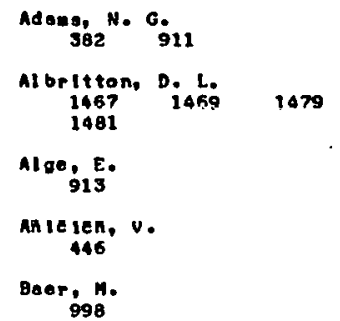

Bates, D. R.

Beoraan, G. H.

I lek, J.A.

998

Blontek, R. J.

BI lotes, R. M.

1898

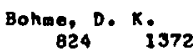

Burrousi J. P.

$\underset{356}{\text { Carsky. }}$

changl on, R. $L$.

2040

Coll ins, C. B.

924

Convay, D. C.

Cox, ${ }_{2142}$.

îa iy raus ha

$$
124
$$

Davidson, J. A. 1469

Donahue, $K$.

Deten; I

$910 \quad 1467 \quad 1465$

$1479 \quad 1481$

Doveraplke, L. D.

Farrar. J. M.

Fehsenfeld, $F, C$.

$\begin{array}{lll}63 & 1467 & 1469\end{array}$

Fergusan, E. E.

63

fischer, s. F.
Flower,

Franklin, J. L.

Freecan, C. G.

rees, $L . C$.

Futrell, J. H.

$$
1469
$$

Gentry. ${ }_{1190}$. R.

GIteIns, H. A.

Green, 5 .

Hanson, W. 9.
5s0

Harland, P. $H$.

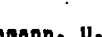

$$
340
$$

Hel क: ${ }_{913}^{H}$

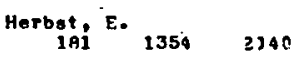

Herman, $Z$.

HI rst, D. M.

Horegan, J. H.

Molzasnn, $\mu$.

913

Hopk Inson. A. C.

howorka, P: ijss

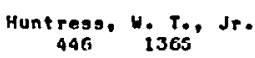

Jones, $A . C$.
1228

Joneg, J. D. C.

1419

Karpas, $z$.

Koufaan, J, J.

$$
\text { 17: }
$$

Kayser, D.

Kello, v.

Kendrick, J.

340

KleInglo $F$.

Xook1, U. S. $_{4228}$

Kuen, 1 .

Kuntz, P. J.

Kusunok?, I.
Lee, F,

Lindinger, H. 912

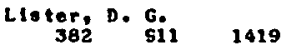

Mackay, G. I.

MeEwan, H. J.

1811

Mitehe11, G.F. 1366

NIer. A. U.

ottinger, c.

1902

Pah1,

Payne, L. G.

Poor1, P. L.

Poek, J. M.

Proun Inger, F. N.

Rakshit, A. B。 ${ }_{2051} 382911$

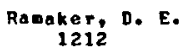

$\underset{\text { Robb }}{\text { Ront, R. }}$

Rouerf, E.

Rusoh, D. W.

Douoro, I

Senter, H. I.

Sehnelder, $\dot{F}$.
349

Cot th; D. 911

sonderuaard. H. A*
I22e

stort, 4 .

$\underset{824}{\operatorname{Tanner,} S . D .}$

TIehy, $n$.

Torr, D. G.

Tor r, $N$. $\mathrm{A}$

Turner, D. L 1214

Tulddy, NE D. 911 $9_{1419}$

Urban. 
273

Varney, R. $N_{\text {. }}$

Vene 1, 6.

Vigoteno. A. A. 1481
Uhit ton, $U$. $N$.
Sis

$\underset{366}{\text { Zahradnith, }}$ zunet $^{c}$.

Zul icke, $L$.
S49 
114

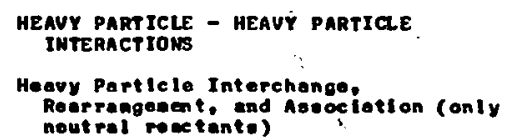

Adang, J. E.

Addison, M. C.

Albertl, H.

Anastasl, $C$.

Arnold, G. S.

Arnold, I.

Atkingon, P.

Boer isg;

Bane 517 .

Bolduin, A. C.

Basco, N.

Bass, H. E.

Beadle, P. 043

Behrens, R., Jr.

Bensuls 50

B1y, s. $_{1635}$ H. P.

Bott, Soo $^{2 .} \mathrm{F}$.

$\underset{\text { Bas }}{\text { Bowang }}$ C. T.

Bowangin, J. H.

Arandt,
1636

Brel tenbech, L. P.$$
2050
$$

Brooks, P. R.

$\underset{2183}{\text { Brophy, J. H. }}$

${ }_{367}$ Brown, R.

Brown, J. $c$.

Bruser, $P$.
1464

Butl ar, J. E.
Byrne, C. D.

Calloar, A. B.

1156

Chang, J. 5 .

ChI u, $Y_{3}-\mathrm{N}$.

Chovdhury, M. A.

533

chrtstov, S. G.

1164

Clary, D. C.

clausen, H.

Combourteu, j.

1159

Comes, F. J.

Connor, J. N. L.

2055

Costes, $x$.

Cox, R. A. 2069

$\underset{2140}{\text { Cvet anoulc, R. J. }}$

avidovits, P."

Davldaon, J. A.

167

A. $\operatorname{linng}_{1437} 0$,

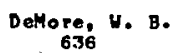

negtelinu, $M$

DeVrles, P. L.

DIckson; L. $Y$.

DIGluaeppe, T. G. 1171

b Impli, H. L.

Dlapert, H. H.

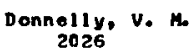

vorovan, H. J.

Doran, H.

2046

Dorthe, G.

Durf, J. J.

Dunn, M. R.

Elltzur. M.

1356

Emanuel, G.
Endo, ${ }_{213}$

Falst, M. B.

Fehsenteld, F. C. 167

Felder,

Fil seth, s. v. 1644

Finlaysen-pitts, B. J.

Fontisn, A.

Freednan, A.

Fuk uyass, 1.

1641

Gardner, C. E.

Garrett, 8. C.

Gels, N. W.

George, T. F

1897

Glanzer, $K$.

Claos, C. P.

Golden, D. M.

Gorry. ${ }^{\text {P. }}{ }^{\text {A }}$

$\underset{643}{\operatorname{Grahan,} R \cdot A *}$

Gray, J. C.

Groon, $S$.
1354

${ }_{983}^{\text {Geice. }} 984$

Grosser, A. E.

unniigard, Ha lle $11 \mathrm{aa}$

Hack, H.

Hanson, R. R.

635

Iluldusio, n. F., I3I 50

Herogty 1354 .

Herm, R. R.

$$
523
$$

Hersehboeh, D. R 642

HIIIIOF, I. H

HIralshl, H 1641

Hovard, C. J. 167 liss 1918 


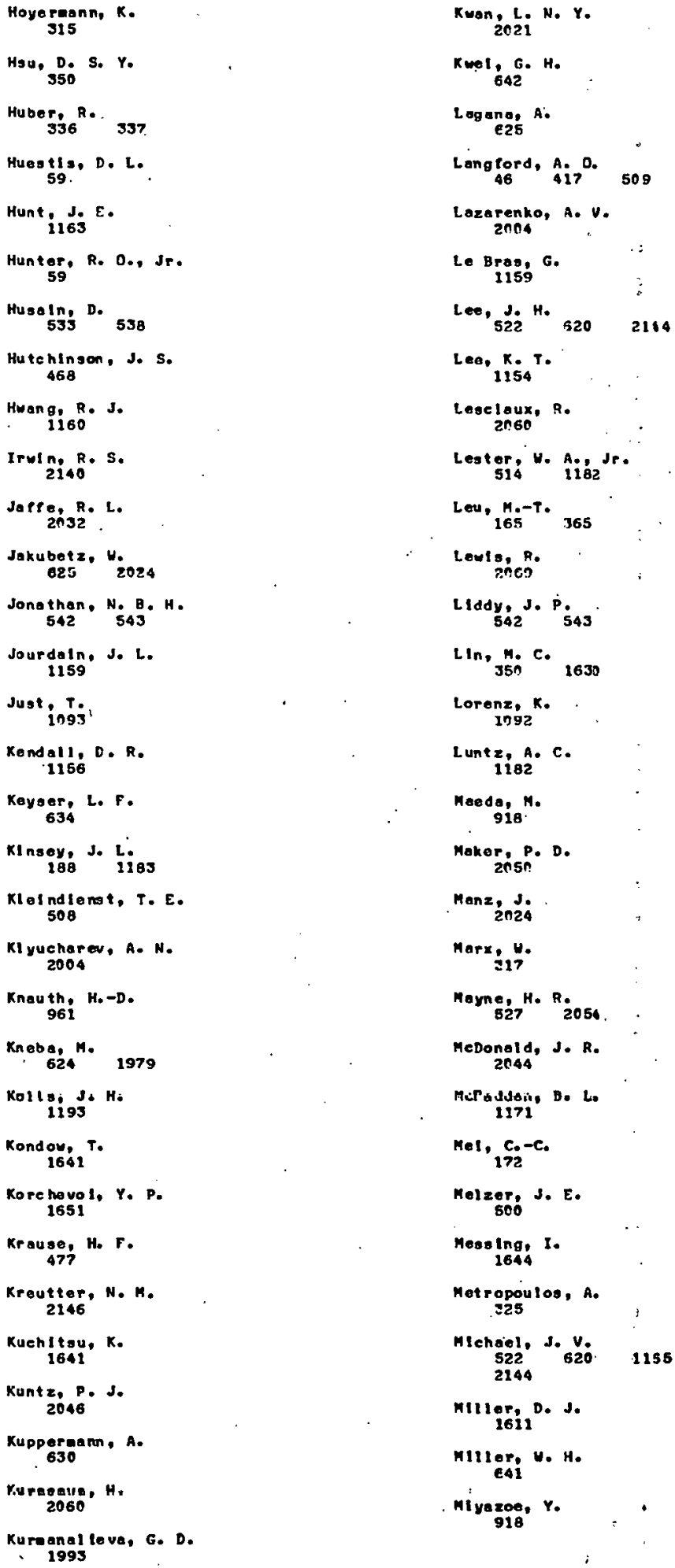

Kuan, L. N. Y.

Kmel, G. H.

Logana, A.

Langford, ${ }_{41}^{A}{ }^{O}{ }^{0} \quad 509$

Lazarenko, A. V.

Le Bras, $G$.
I159

Lee, J.. H. $520 \quad 2114$

Lee, K. T.

Lesclaux, R.

25.60

Lestor, W. A., Jr.

Leu, M.-T. $165^{365}$

Lew s, R.

Liddy, J. P. $_{543}$

LIn, H. $_{350}$ C. 1630

Lorenz, $k$.

Luntz, A. C.

Maedo, $M$

918 .

Mokor, P. D.

Manz, J. J.

Marx, 117

Mayme, H. R.

Medonald, J. R. 2044

Miroldsiin, B. L. 1171

Mel, c. c.-e.

Mel zerp s. E.

Mesolng, I. 1644

Metropou los, A.

Hichaiel, J. V. $522 \quad 620 . \quad 1155$

IIlep, D. J.

millar, W. H.

Miyazoe, $Y$.

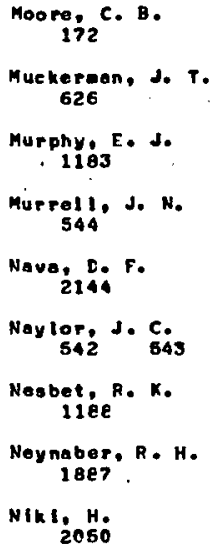




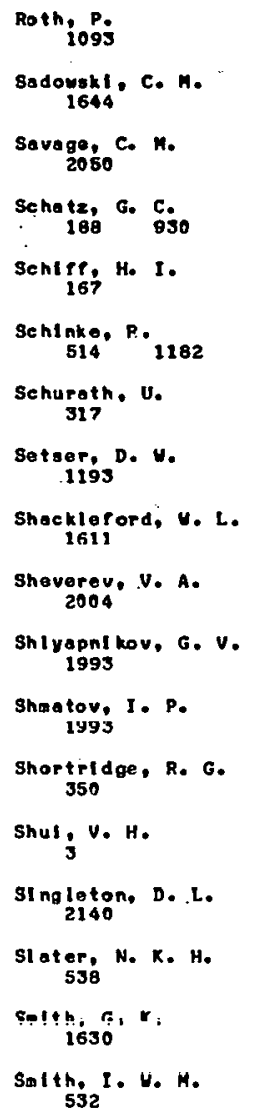

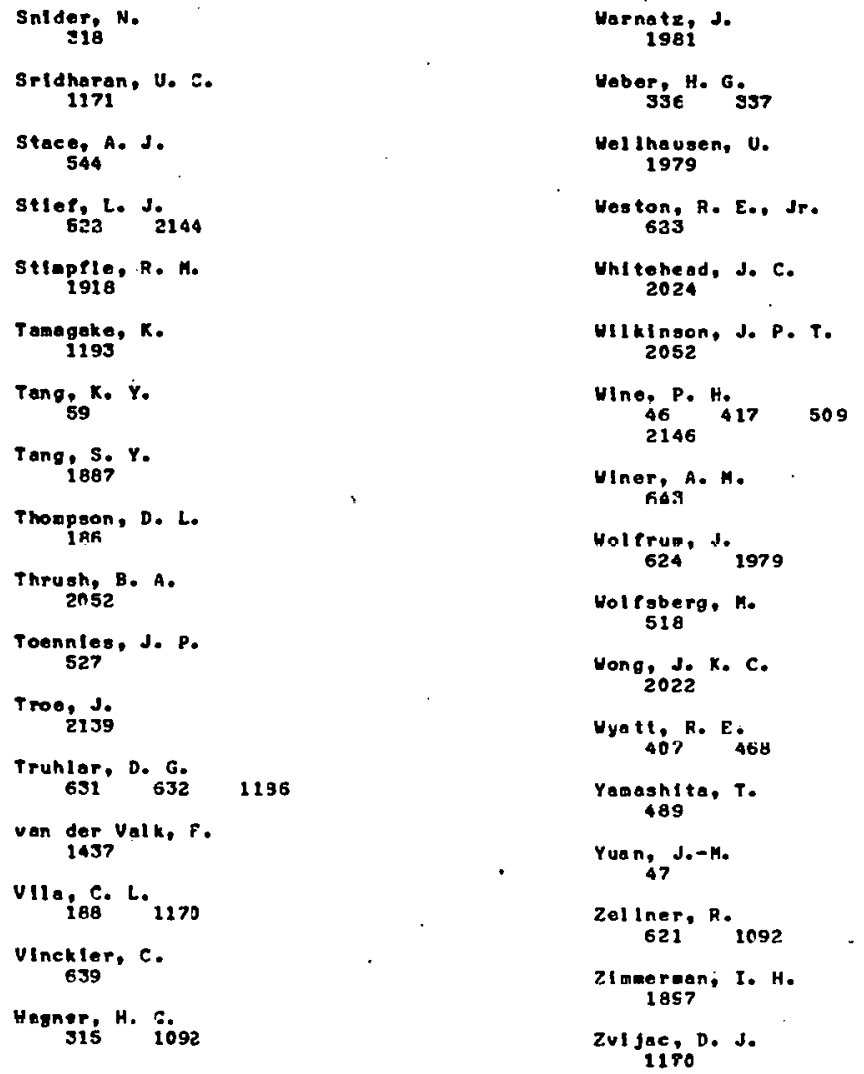


A15

HEAVY PART ICLE - HEAUY PARTICE INTERACTIONS

Soin Exehange

D1 xon, $R \cdot N$.

A16

HEAVY PARTICLE - HEAVY PARTICLE INTERACTIONS

Electron Detachent from Negat lue Iono into ContI nuus

de $\underset{906}{\operatorname{reugd}}, \mathrm{c}$.
Fleld, 0.
Ins9

$\underset{66 ?}{\text { Jeyes, S. F. }}$

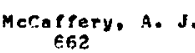

Posingkl, K.

e45
Rove, M. D.

Shl zgal, P.

sleradzan, A.

845

sleradzon, $H$

845 nunean, $M . M$.

Gauyaca, J. $P$ 1269

Herzenberg, A 18 ?

LIU, B.

Los, I.
Menendez, M. G.$$
687
$$

njho, P.

01 gon, F. E.

Wijnoendts van Resandt, R. W. onf

, 


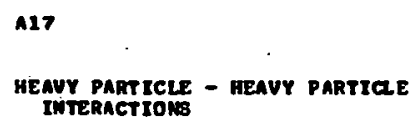

Interaction Potentiale

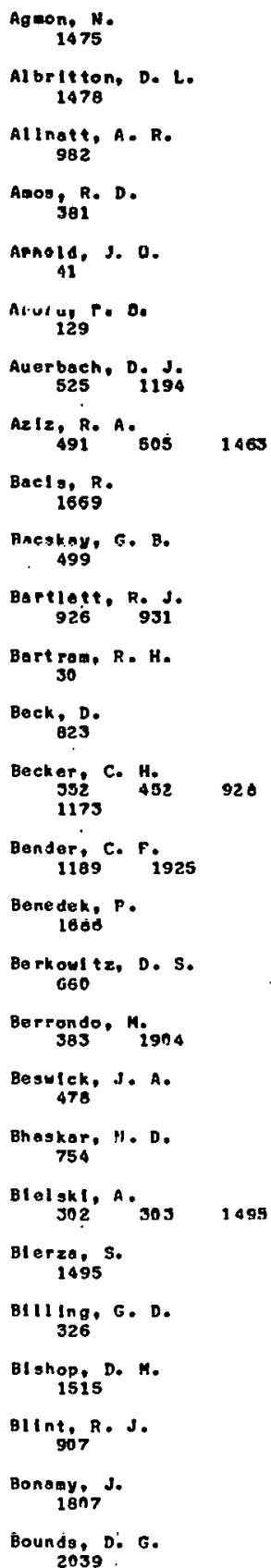

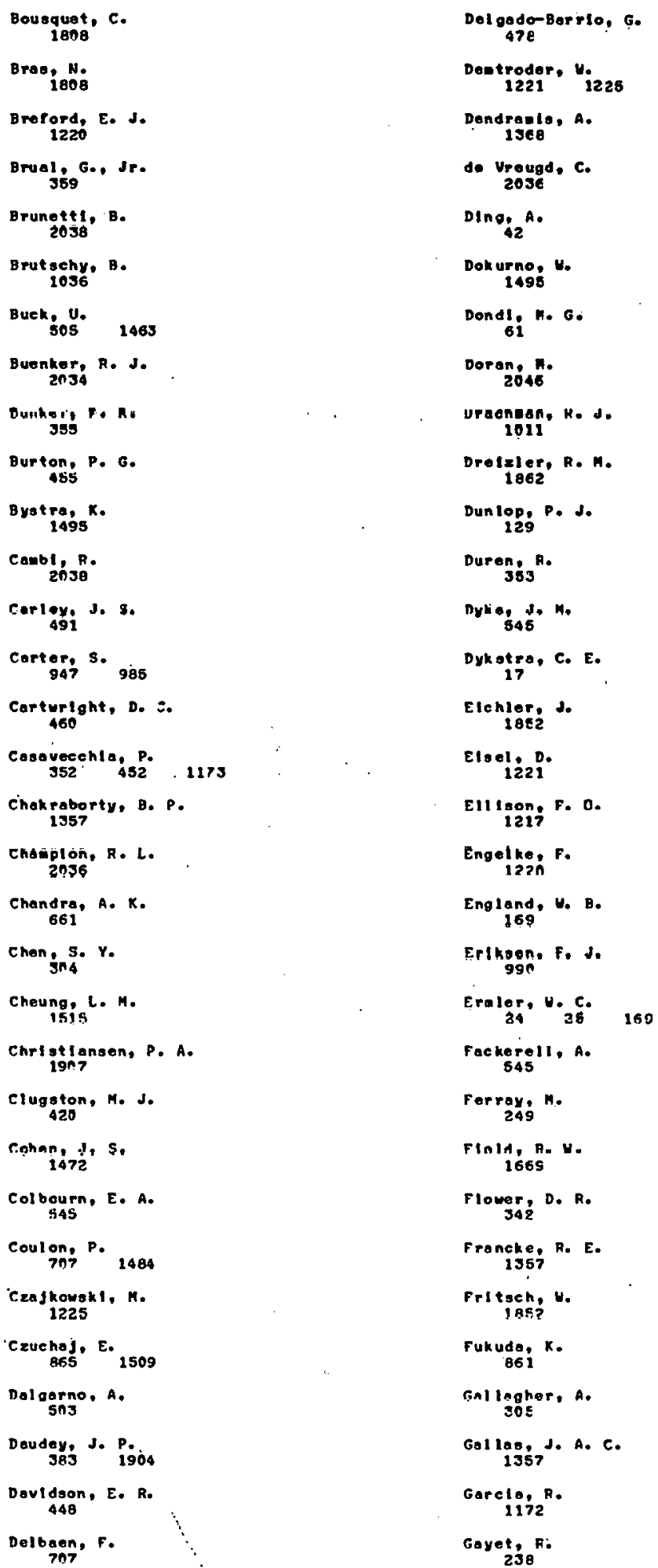

Del godo-Borilo, G.

Destroder, $y$ 1221 i228

$\underset{\substack{\text { Dendraglo, } \\ \text { IJee }}}{ }$.

d- Uraugd, $C$.

$\operatorname{ling}_{42} A_{1}$

Dokurnog,

Dond1. H. 6 .

Doran, 2046

vraenasen. K. d. 1011

Drolzler, R. M. 1862

Dun lop: P. J.

Duren, Fe

Dylieg 5. 14

Dyk atro, C. E.

Elehler. $d$.

Esel. 0.

Ellison, F. O.

Engetke, $F$

England, $W$. B. 169

Erlkoen, f. J.

Eraler, $Y . C$. 


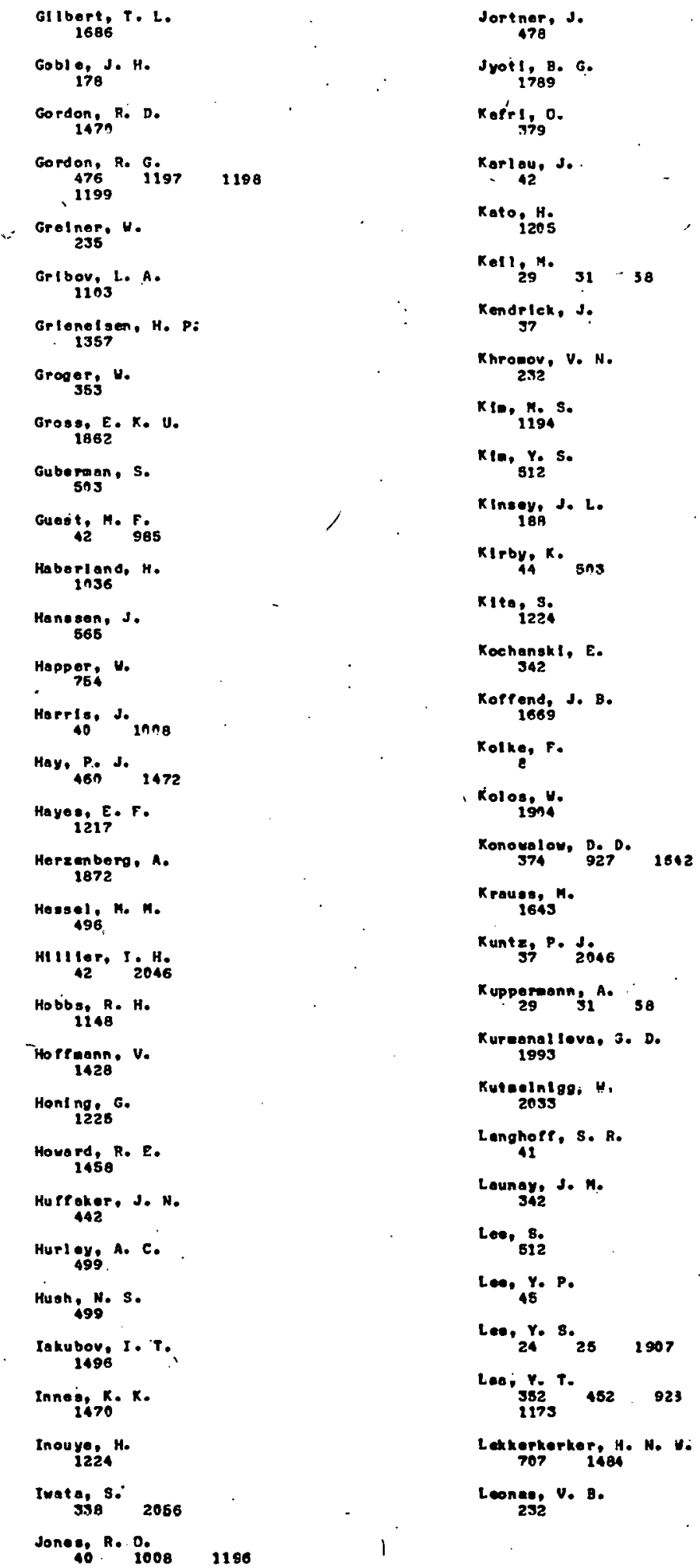

Jortner, J. 478

Jrot1, 8. 6.

Kof́r 1, 0 .

Karlau, J.

Kato, $\mathrm{H}$.

Koll ${ }_{29}{ }^{M \cdot} 31-38$

Kendrlek, d:

Khromov, V. N.

Kin, n. s.

KIn, sid s.

Kinsey, J. L.

KIrby, R.

Kita, $s$ s.

Kochangkt, E.

Koffend, J. B.
1669

Kolke, $F$.

Kol os ign:

$\underset{374}{\text { Konowal D. D. }}{ }_{927}^{\text {D. }} 1842$

Krause, 1643

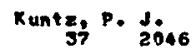

$\mathrm{Kuppermonn}_{29}$ i. $_{30}$

Kureanalleva, 3. D. 1993

Kuteeln1gg; $u$, 2035

Langhope, s. R. 41

Lounay, J. N.

Loe, 812

Loo. Y. P.

Lee, Y. S. $25 \quad 2907$

Leo; $Y . T$

382482.923

Lekkerkerker, H. N. V. $707 \quad 1484$

Leonee, V. 8.

Las Bâd

Losterpy. Apistr.

Leuehs, U.

Lev Ine, A. D.

Levy is: H.

LI, K. C.

LIoickl, E.

Liu, 8. $390 \quad 1030$

1

LIU, Y..

Loev6. G. H.

Loo, 20.

Loz Inget, J.

Lubbert, $_{1250}$.

Luyehx; A. 1484

Lyvre,

Meclage ${ }_{15}^{A}$.

-Macder, ${ }_{2035}$.

Mattlend, G. C.

Manake, 6 .

Masnou-soeuma, ${ }_{250}{ }_{1406}$

$\underset{689}{\text { Mason. }} \mathbf{A}$.

Matsuurs, $y$ 861

Metorroll. Re

Necielland, T.

784

MeConville, 6. T. 491

Mecoupt.

338

Moleughiln, D. R. 443

Mel ean, A. D. $24,{ }_{660} 1480$

Meeth, W. J.

Nol or: P. P.

Mol luv, C. P. 
Mlehal s, H. H.

1118.7 I . M.

Mt ngel grin, U.

Mohanaad, S. N.

1790

Monchtek, L.

934

Morgan; T. J.

$\underset{1428}{\operatorname{Morgnar,}} \mathrm{H}$

Morrls; A.

Mulfar, F.

Mull er, $B$.

Muon lgatel, V. M.

Murrel1, J. N,

NaIn. V. P. s.

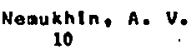

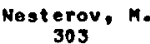

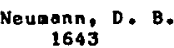

$\mathrm{Ng}, \underset{982}{K .-C .}$

Noda, K.
LeC4

Nomura, 0 .

Noor Moll dim rad, 3.

Novare, D. 1904

Numrich, R. U.

Obara, $s$.

Ohno, $\mathrm{n}$.

Qtha. P.

01 son, M. L.

Olgon, R. E. ग440 $\quad 11 \% 3 \quad 2838$

Orel, A. E.

Pathak. A. P.

Penkina, N. N.

Perevozehlkov, V. I. 1103

Peyerlahof, S. D.
PhIl 1ppe,
1406

PhIllippe, M.

$\underset{2038}{\text { PIranl, }}$

$\underset{24}{\text { Pitzer, K. } 35} 1907$

Polt shehuk, A, Y.

1496

Presley, J.

Prissette, J.

342

Puru19, G. D.

${ }_{42}{ }^{2}{ }^{N}, C_{2}$

Radzlo, E.

Reghumansh!, S. S.

Ragone, A.

Robane, $T$. $K$.
1392

Ree, F. H.

Rescigno, T. N.

1189

Reuss, J.

Rlera, A.

BNay n. W.

$\underset{52}{\text { Roberae. R. }}$

Robert, D.

Robjohns, HI. L.

129

Rosenkrantz, $_{374}^{\text {M. }}{ }_{1642}$ :

Ross , J.

Ross, U.

Ros 1, A. H. Ki 112

Rothgteln, S. M.

Pantenl1, 9

123 .

Russek, A.

Sabell1, N. H.

1086

Sal ahub, D. R.

63

$\underset{914}{\text { Sasakl, }} F$.

$\underset{249}{\text { Sayer. }}$
Schaofler, H. F., III

Sehatz, G. C.

enepper, 4.

823

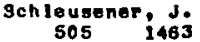

Sehooltekop?, A. L.

1478

Sehraup, 8.

Schwaln, U.

50019864.

Oobũllail, Ko L。

662

Shapt ro, M.

Sharag, L. K.

Shavstt, I.

Shl yapnlkov, G. V.

Shatov, 1. P.
160

Snollrain, 5. E.

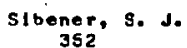

Sienklewlez, J. oes

Slsko. P. E.

$\underset{29}{\operatorname{sinks}, ~ J . ~}{ }^{\text {Tl }} 58$

Smalley, R. E.

smlth, e. $r$.

Sort, 6 .

stoponou, N. P.

Stevons: W. J.

Stock, M.

Stolte. $s$ s.

Stwalley, U. C.

Swope, t. $c_{17}$

Tanak o, $k$.

$162^{\circ} 448914$

Tat evakl, H.

Taylor, P. Re

Tavlor, W. L. 


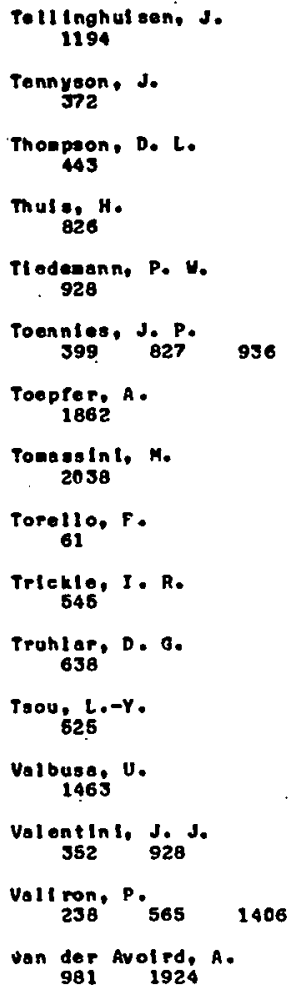

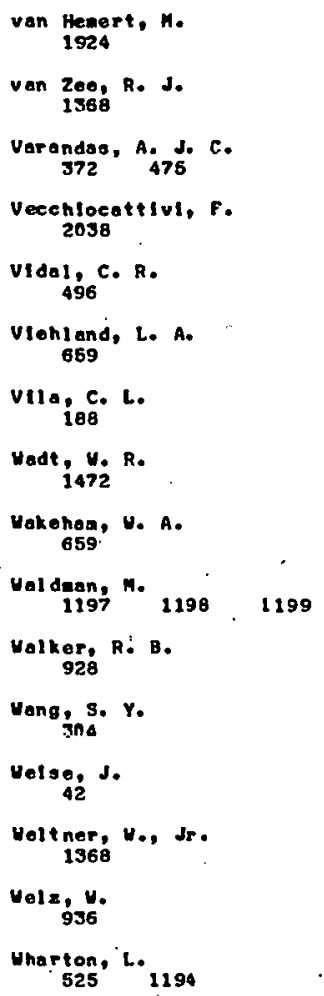

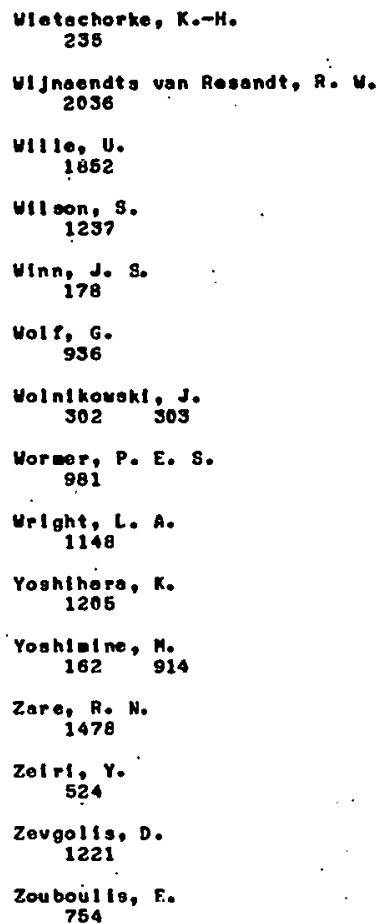




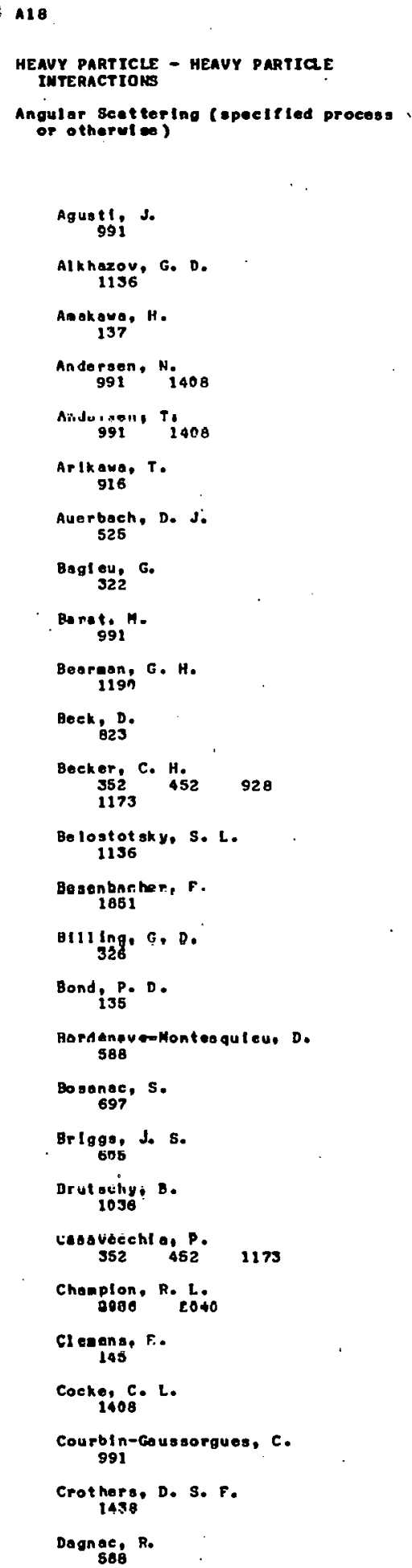

Dahl, P68

DasaskInsky, E. A 1136

de SwIntarskl, R.

de Vreugd, C.

DIckInson, A. S. 1446

Dosehenkov, O. A. 1136

Dond I, M. G

Dotgenko, $Y . V$. 11.85

Deverep!ke, b. D.

Dureng

353.1155

Eastes.

El Maddaral, M.

Fledeldè, $H$. 6B1

Gortnor, K.

Gentry, U. R.

Geramb, H. V.

Groger, 4 .

Haberland, $H$.

Hoberzettl, $M$.

$\theta 0$

Hohl, K.

Horbat, E.

Hoppe, H. O.

Nuylies Ji is

ton, $x=$

Inouyo, II"

Jenden, 0.5 .

Kaneko, 9

Kell, ${ }_{29}^{M .} 3158$

Rester, $v$.

Khrowov, V. N.

KID, $8 .-T$.

K1ta, s.

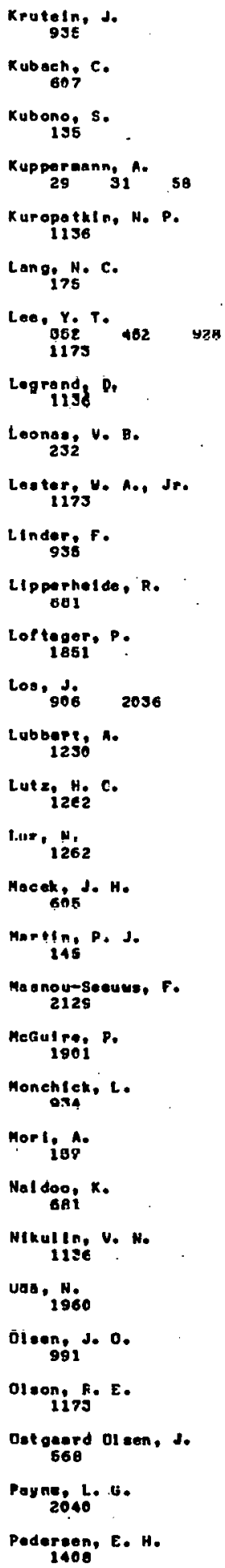




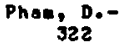

PhII Ippe, M.

Placentinf . R. D.

1863

Ponaler, J.

Prodel, P.

Prokof'ev, O. E.

$$
1136
$$

Richards, D.

$$
1446
$$

Rivarole, R. D.

Rose, U.

8232025

Rotzol1 123 . 6 .

Roueff, E.

Rues tk: A.

Sackmann, s.

Satoka. 1960 .

Sehepper.

$$
823
$$

Sentinke, R
Shuvaev, M. A.

1136

Slbener, S. J.

stals, $v$.

$\underset{29}{\operatorname{slankas,} \mathrm{J}_{31}^{\mathrm{T}}} \mathbf{5 8}$

Smsth, B. T$$
2 n 36
$$

Sorensein, V. S.

Splelifledel, A.

$$
2129
$$

Splegs, 6.

n, $R \cdot C$

$$
{ }_{175}{ }^{R . C .}
$$

Sunkel,

Taulbjerg, K.

Thorn, ${ }_{135} c . E$.

$\underset{928}{\underset{9}{\text { Tledemann }}}$ P. W.

$\underset{\text { TIseher, H. }}{\text { IISS }}$

$\underset{2 \text { Toes }}{\text { Tountes, J. P. }}$
Torello, F.

61

. Tsou, $1,-$

Urakawa, J.

Valance, A.

Val entinl, J. J.

Vedel, $k$.

volkov, 5 . S.

Hadehro, J. M.
leES

Walker, R. B.

Wharton, $L$.

Wijaendts van Resandt, R. Y.
gne

Yamaj!, 5 .

Yazak1, $K$

Zehnle, $L$.

I901 
ค19

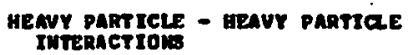

IMUEnact Iols.

Canop-shell Intereotione ( wot covered uader other epeefified eatepories)

Anholt:
869

Annett, C. H.

$\underset{622}{\operatorname{Arab} r u s t}$ er, P.

Aranti,

ceirne, 1. A.

Cocke, C. L.

Curnutte. B.

$\underset{995}{\underset{90 a}{\text { Deat }}}$

Doyle, B. L.

unga an, J. L.

EII: $\underset{709}{\operatorname{mog} \text {. L. D. }}$

Fint 2,9 .

Goukler, $G$.
oe1

Gray T. J.

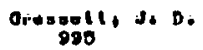

180

hEAVY PAMTICLE - hEAVY PARTICLE

InTERACTIOKS

Attenuation (anspecl fled proeses)

H+ ติ

801

INTERACTIONS WITH STATIC OA TIKE-VARYIUS ELEctric and magnetic FIELos

Gemerol
Gull liane,
678

Hogaenn, $S$.

822

Hat 1 J. J. $M$.

Hol I oway, D. F.

Janison, K. A.
1026

Johnaon, B. M.

Joneg. K. H.

dundt, $F \cdot C$

Krac i jo

Kruseg, T. H.

Langenbero, A.

995

Laptek1, 6.

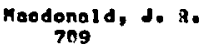

$\underset{732}{\operatorname{Mebant}} \underset{993}{ }$

Morteg 752 .

Meyerhop, W. E.
1311

MIIler: P. D.

$\underset{822}{\text { Mokl or, }}$ P.

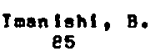

Rey. S.

Finken, ${ }_{1743} . H$.

rondevio, $\mathrm{N}$.

Plallsa, M.

1738
Newconb, J. $_{1017}$

Potorzon, R. S.

Rlehard, P.

Rustech!, A.

1312

Schlebel, U.

seholdt-Bocking, Ho

$$
821
$$

sohertedektiap, $C$.

$$
1017 \text {. }
$$

senuen, $\mathrm{K}$.

Shs froth, S. H.$$
988
$$

Sonobe, B. I.$$
\text { Stolng } \mathrm{In}_{0}-\mathrm{J} \text {. }
$$

stocklt, $m$. stoller,

Tawara, H.

Tol en, A.

Uat son, R. L.

Wt son. S. R.

Molf11, N.

Tan imuro, 0.

DI altrijoule, M.

$1+15 j 00$
1758 
Bo3 IWTERACT IONS YITH STATIC OD MA TESTC PIELos

Ionisetion

Adans, B. G.

Austin, E. J.

Barnott, $C$. F.

1715

Brodsky, A. M.

Cisek, J.

Bo4

INTERACT IONS UITH STATIC OR

TINE-VARYING ELECTRIC AND MANETIC PIE⿺3

Detachuent

Bl uaberg, W. A. H.

699

B.o5

I WTERACTIONS UITH STATIC OR TIKE-VARYING ELECTRIC AND WAGETIC FIELDS

Quenehing

Drake, G. H. F.

Bo6

INTERACTIONS UTTH STATIC OR TIME-UARYI ELECTRIC AND WAGETIC TIME-U
FIELDS

Excitation

Beiting, $5 . \mathrm{J}$ 469

Crossuhl te, $H$. 757

Dunn Ing. $F$. B. 409
Damburg, R. J. 1416

Duong, H. T.

Feneullie, S. 767

Kol osov, v. v. 1416

Leopold, J. G.

Liberean, S.

Morgan, T. $J$.

1715

otto, P.

Itano, H. M.

Larson. D. J.

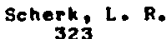

Perelual, I. C.

289

PInard, J

Ray, J. A.

Rus rok, A.

SII verstone, H. $\mathrm{H}$. 1984

Tal eb, A.

VIalle, J.-L. van wijngaarden, A.

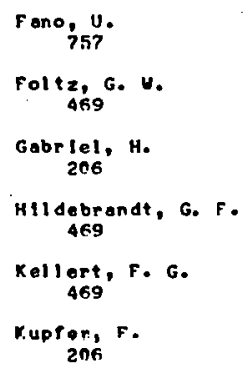

Loopold, J. $G$ 28

Lu, K. ${ }_{757}{ }^{\top}$

Perclual, I . C 289

Rau, A. R. P.

Solth, K. A

$4 \in S$

stebbings, R. F, 469 
807

INTERACT HONS UITH STATIC OR

TIME-UAYIMG ELECTAIC AND MONETIC TIme-varios

Collistone in Presence of Intenae El catronagnetle field

Altenberger-siczok, A. 484

Ande roon, $V . E$.

1713

Apensecevloh, P. A. 1749

Reche! !er, 4 ,

Bell ung, J. C.

Berang, P. $R$.

Bornor, 6.

Brondt, H. S.

Clarke, E. M.

Cauchoj. $E$.

Deguehi, $K$.

Devrles, P. L.

Durrant, c. J.

Forrante, G.

Qay. J.: ¿. $1702 \quad 1705$

$\begin{array}{ccc}\text { George, T. } & F . \\ 67 & 488 \\ 657 & 617\end{array}$

Green, W. R.

758 ilas 1642

Harpis: S. É:

$\cos$

PARTICle penetration IN machoscopic

WATER ( TONS, WEUTALL, AND ELECTRONS)

Gampram

$\underset{83}{\text { Ah }} \underset{\text { d, N. A. G. }}{ }$

Brandt, 2072

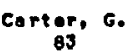

Chrl stodoulides, C. E.
Holmes, J. K.

Jaforpour, Mo

1883

Jung, $C$;

Kazantsov, A. P. 1141

Kolller, 8.

Kraulinyo, E, $K$.

‥, $4 ., 4$

Leone, $\mathrm{C}$.

Light, J. C.

lins da Barros, H. G. P.

Lo caselo, L.

$$
1291
$$

Lue-Koen1g, E.

$$
1556
$$

L ukasik, J. 758 J. 1123

Meszoros, P.

$$
1353
$$

MII 11 , W. 11.

Mitt lesan, M. H. 6936981216

$$
1878
$$

Nizovtsev, A. P.

1740

Nuxzo, 5 .

Õrei . A. E.

Parne, 17. 6.

Peek, s. $M$.

Ragaker: D. E.

$\cosh 6 y, P$.
1698

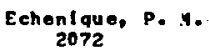

G E.M.11, J. 5 .

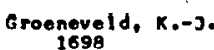

I nokut!, $\mathrm{H}$.

ItIkava, $Y$.

$\underset{1698}{\text { Kantorg. P. }}$
Rasarao, 1

1803

Robingon, E. J 604

Rosenberg, $1317^{1326} 1849$

Sehnelder, W. $B$ 1712 i7ns

sehnelder, W. $M$ 170

Silth, C. R. 1883

Iml llig n: A:

sokelpy, V. P.

Taylor, H. S.

Turner, J. E.

Helngarts tho fer, A. 1041

Wililsen, J. R.

Uright, H. D.

758 . 11251542

Yan oon, H. L.

1997

Yarts, $R$

Yeh, S.

Young, J. F.

Yuan, J. $-H_{4}$

zonene;

Zhukova, N. I.
IISi

$20110 \mathrm{Fi}^{\mathrm{P}}$

Plotsch, U.

$\underset{\substack{1698 \\ \text { Rathougt }}}{\text { A. J. J. }}$

$\underset{2072}{\text { Ritehte R. H. }}$

Turner. J. E.

vager, 2.
1698

Zabranaky. E. J. 
$\mathrm{CO} 2$

PART ICle PENEt Ration In macroscopic MATTER (IONS, REUTRALS, AND ELECTRONS)

Energy Loss

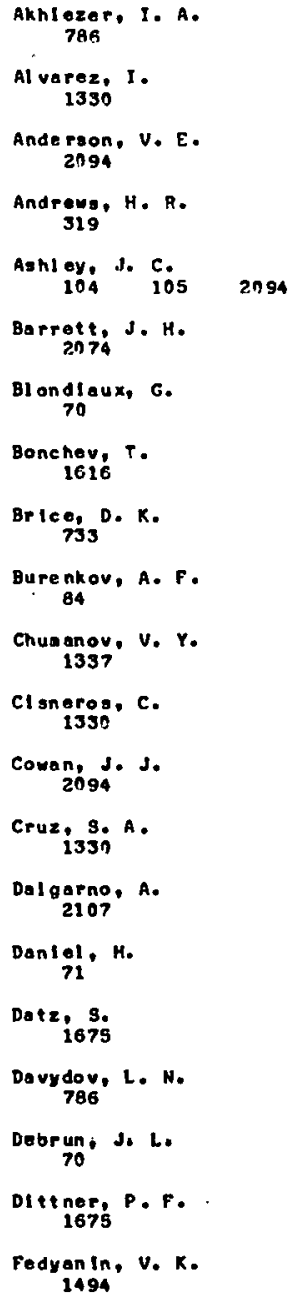

Fontell, A.

Fox, J., th.

Gourl lanko, G. . 1494

Grozdanov, $M$.

Hrao, T.

Hoolzl, J.

I Afrata, G. J.

Inoue, ${ }^{K}$.

Ishit, $k$.

I zaa 1lov, s. z.

Jarulg, O. N.

Khoknlov, V. D.

Kema rov, $F$. $F$.

Lennard, U. N.

Leon, ${ }^{M}$.

L11kov, v.

Lueas, M. W.

Luomajarul, ${ }_{791}^{M}$

Medvedev, $Y$. 1654

Mertens, P.
S89

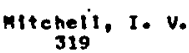

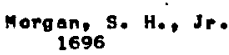

$\underset{7 S}{\text { Mukhergl, } s .}$

Nayak, A. K.

Neshov, F. G.

Douthat, D. A.

$$
28 n
$$

Fox, J. L. L.
Ovorbury, S. H.

Pokntl, G. P.

Puzanov, A. A.

Ritenle, R. ${ }_{105}$

2094

Ros soun, C. J.,

Rud, N:

sherucod, A. C.

Strotinin, E. I. 1337

Sung, ${ }_{1696}$ c.

Takayanagl, S.

915

Thoe, R.:

Tuiinov, A. F.

Tung, c. J. 105

Vehalkin, v. v. 1744

$\underset{802}{\text { Urmanov, A. R. }}$

valladen, ${ }_{70}$.

Ved'anov, G. D.

802

Walker, R. B.
J19.

Ward, D.

Uholan, M. J. 619 1607

unt teheod, $C$.
lo63,

WIIIans, R. H.

Yeoyashl, $Y$. 915

zlogler, J. F. 1385 
$\cos$

PAmTCLE PEnetration In maceoscopic MATTER ( IONS. MEUTRALS, AMD ELECTRONS)

Partielo Range

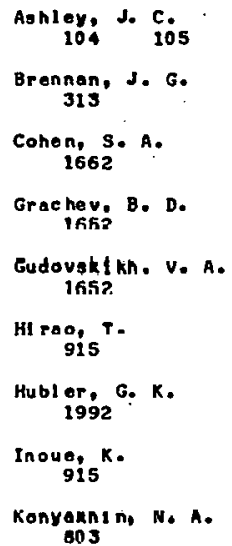

Korobochko, Y. S. 1652

Kozlovakif, 3. S 1652

Krasnov, N. N 803

Land, 0.5

Molnuerg, P. R。 1992

McCracken, G. M.

Aneev. v. $T$. 1652

ironov, v. N.

Huklier 1 l, 5.

75 ,

Noyak, A. $K$.

Pet rochenko, A. F 1652

$\underset{\text { SSR }}{\text { RIseh, R. }}$

Cowern, N. E. B.

natx, 5. Della Mea, G.
1065

$\underset{1675}{\text { DIttnerg }}$ P.

brigo, A \& $v$.

Freeman, J. M.

L.Holr, A.

Lo Russo, $s$.
In65
Riterise, A. ${ }_{104}^{H .}$

Rot h, J..

Scherzer, B. M. U.

Set dian, D. N.

Sotth, T. P., III

Takoyonog 1, s.

Tuny ${ }_{104}$. J. 105

Uchatgln, $V, V$.

Wogner, $A$.

Wienke, B. R.

Yaegash1, Y.

915

$$
\begin{aligned}
& \text { Mason, J. P. } \\
& \text { Mecracken, G. M. } \\
& \text { l6ez } \\
& \begin{array}{c}
\text { Dverbury. S. H. } \\
1675
\end{array} \\
& \text { Rolph, de E. } \\
& \begin{array}{c}
\text { Schaugs, } D . \\
\text { IC65 }
\end{array} \\
& \begin{array}{c}
\text { Sopiold, c. J. } \\
698
\end{array} \\
& \text { Sug Iyama, } H \text {. } \\
& \text { Thoe, R. } 8 \text {. }
\end{aligned}
$$



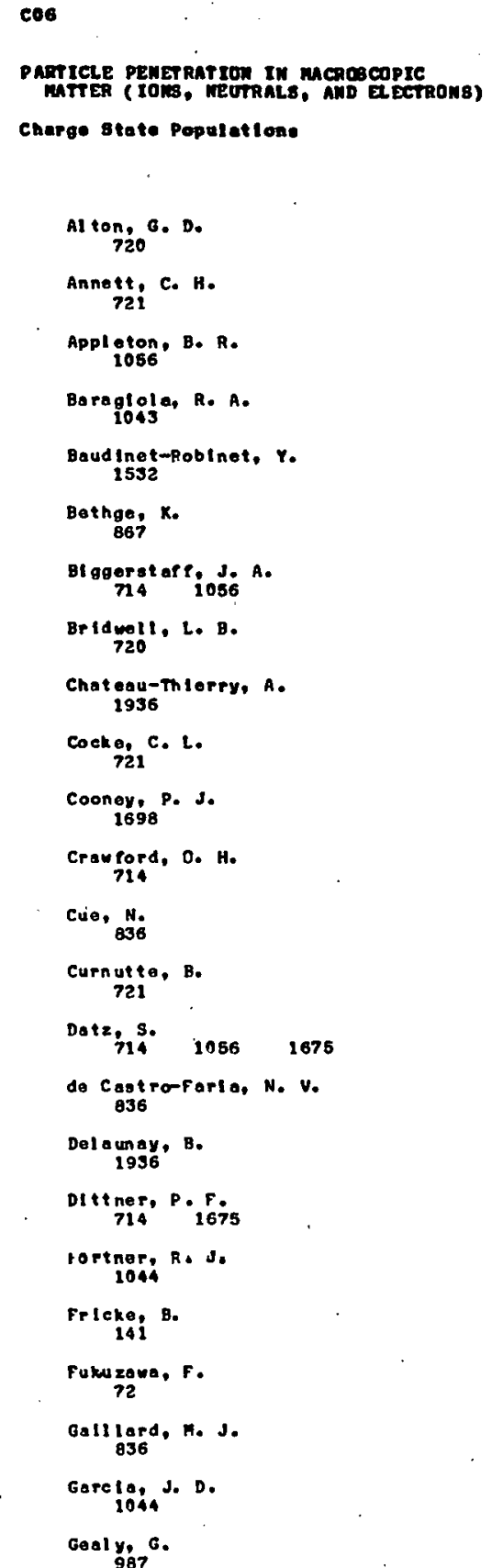

Geanal1, D. S.

Gledieux, A.

1936

Geare del Caupo, d. 214

Gray, T. J.

Groeneveld, K. 0. 141

Groeneveld, $K_{0}-0$. 2698

Hvel plund, $p$

$$
714
$$

Iton, A.

Joneg, C. M.

Kantor, E. P.

Kerkow, 1075 .

K Ido, $Y$ Y

KIoura; $K$.

Knadsen, H.

Krause, H. P.

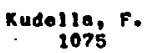

Kuroge, J. J.

Kyoshlea, $A$.

$\underset{1532}{\text { Lanotte, } D .}$

$\underset{1508}{\text { Liran, }} 5$.

Mann isô:

Mannaal, $M$.

107

mayo, $m$.

M11 Ier; P. D.

Mosk; C. D. $720 . \quad 1056$

Morgan, T. J.

Nagata, T.

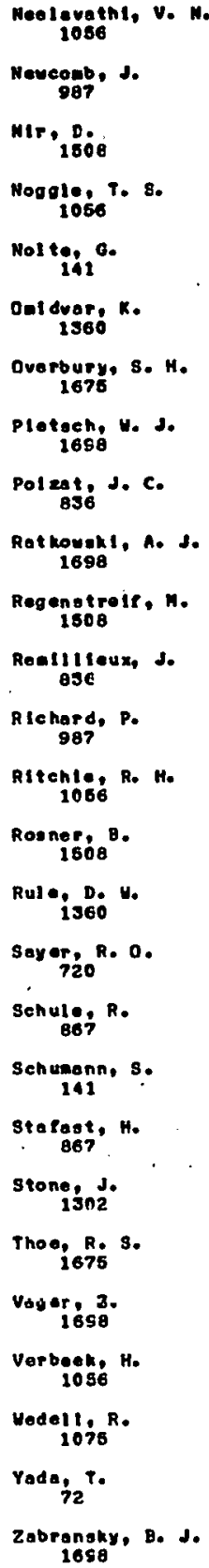


$\cos$

PARTICLE PENETRATION In MACROS COPIC

MATTER ( TONS, NEUTRALS, AND ELECTRONS)

Exclted state Populations

Andresen, B.

Anholt, ${ }_{1964}$

Baudinot tioblnet, $Y$.
Igsp

Berry, H. G.

Betnge, $x$.

Blemont, $E$.
1839

Bl ggerstaf, J. A.

Burns, D. J

Crawford, O. H.

Datz, 5 .

Dent 9,0 A.

Desesquell es, J.

1801

ot tiner, P. F.

714
Dumont, P. D.

Frlcke, B.

Gorntr, H. P.

Gay, ${ }_{71}$ j.

Gomez del Canps, J.

714

$\underset{1321}{\operatorname{Greene}}$ C. $H$.

Frovesco, N.
1839

1839

Groenevel 1, R. 3.

HIght, R. D.

$\underset{741}{\text { Hult berg, }} \mathrm{s}$.

Hvel plund, $P$.

Itoh in A. $^{-}$

Jelenk ovle, B.
741

Jengen, S. B.

1297

Kerkow, $H$.

Klaure, $K$.

Knudgen, $H$.
Krouee, H. F.

Kudello, $F$.

Kyoohina, $A$.

1.11 Jeby, L.

Mannaul, M.

2078

$\underset{741}{\text { Mannerutk, }}$.

Meyerhop, $U$. E.

1964

Atller, P. D.

Noak, C. D.

Nol te, G.

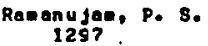

sohule,
867

$\underset{142}{\text { Sehumann, S. }}$

stalast, H.
867

Vese, E. 1297

Vedell, 1075 . 
608

PARTICle PENETRATION In machoscopic MATTER (IONS. NEUTRALs, AND ELECTRONS)

Chenruling

Akkeraan, A. $\mathrm{f}$. $21 n 4$

Apploton, B. R. 1056

Araour, D. G.

Barratt, J.H.

2074

Bazylev, V. A.

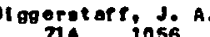

Borovik, A. S.

Burenkov, A. F.

Chublsov, M. A. 2104

Chul , s. ${ }^{\mathrm{T}}$.

Cravford, O. ${ }_{\text {P14 }}$

ceerionlak, J.

842

Datz, S. 1058

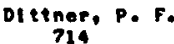

Dol. ${ }_{794}$. 795

E1 119, . W. P.

Evdok100\%, I, N.

Fodyentn, U. K.

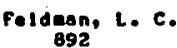

$\underset{2076}{\text { Foenko, E. } 0 .}$

Fujeno, Y. 795

Fukuzewe, F.

$\underset{2000}{\text { Gaonkar, } 5.0 .}$

Gurr! Ienko, O. M. 1494

Coese del Ceppo, d.

HI rabayash 794 .

trel pl und, P.

Dอ1

PAortcte ImERactrows utm sCLIDS

eomer
Jarvis, 0.

KallnIn, B. N. 1138

Konurin, $Y$. M. 174

Kapl $\operatorname{lin}_{1138} v . v$.

Karpuzov, Dis. 1683

Kaufaonn, R.

Kerkow, $H$.

Khan, M. N.

1064

Khel feta, $\mathrm{s}$.

KIdo, ${ }_{72}$

Knlght, ${ }_{1387}$.

Rmudeen, $H$. 714

Kol wo, $794 \%$

Kemarow, F. $F$.

Konovaleniko, $I$. I. 2n.78

Kravse, H. F.

Kude Ila, F.

Xunrt, E.

Kunakhov, M. A.

Kurasowa, T.

560

Lueas, M. M.

Mal eckl, $H$.

Mal yshevakll, v. S.

2080

Moasev, L. $K$.

Noyer, 0 .

miller, P. D.

Moak, C. D. 1055

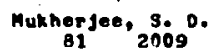

Hasu, 5 ,

'Heel avathi, V. N.

Bren ig, $u$.

Echenlque, P. : .
Noggle T. S.

Ozona, $^{K}$. 795

Palaer, D. W.
2009

Pathak, A. P.

Potyltesy, A. P 1138

Rltente, R. H. $1056 \quad 1868$

Ros sour, C. J. 607

Sherwood, A. C. 1062

Shlozava, $k$. 560

Shlpatov, E. T.

Silvernon, P. J. 892

Stensgaard, I. 892

Takahash1, J.

Takeuchl,

1681

Taylor, T. N

van den Berg, J. A.

Vedringkit, R. $v$.

$$
2076
$$

Vorboek, $H$.

Vorob"ev, S.A.

Wogh, $A_{200 B} G$.

Yebb, F.

Yedell, $1067^{R .} 1070 \quad 2075$

Whelan, $160 ?$.

Whl teheid, $c$.

- 1063

Yods, $T$.

Yakovlev, V. Y.
1747

$\underset{794}{\text { Yasaguehl, }}$.8.

Yan amura, $Y$

Zhovago: H. K.

$210 \ln _{\text {A78 }} N . I$.

Mul ler, $H$.

$\underset{207 a}{\text { Ritchle, R. H. }}$ 
Do2

PARTICLE IITERACTYONB UTTH SOLIDS

Spottering by El octrons, Mautrone, and Havy Partieles ( Rotal removel have Partiel

Abatsno, C. 1087

Ak I Eune, $H$

Al totetter, $c, J$. 959

Aildo rsen; H. H.

Due 1, 1088

Bay, H. $_{847}^{\text {L. }} 1076$

Behr tsch, R.

Bi ank, $P$.

Bohdanaky, J.jo $1646 \quad 1661$

Br uckaul ler, R.

99

Chu, ${ }_{1562}$.

$\underset{\text { Cobg }}{\text { Cobur, J. }}$.

Conner, R. A.

Dawe on, J.

$\underset{\text { Degreg }}{ } F$.

$\underset{971}{\text { Delgass, U. N. }}$

Dral ne, B. $T$.

1961

Dusternoft, H.

210

Eckateln, U.

1661

Ennoth, B.

1089

Erents, S. $K$.

1661

Flelsch. T.

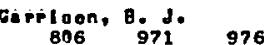

Gose, D.

Coto, s. $^{K}$.

Greenwood, L. R.

Gruen, D. M.

Uinters, H. F. 829

UIt mack, $K$.
Harrison, D. E., Jr. $\underset{806}{971} 973$

Heentl, E.

Holnrieh, R. R.

Helng, C.. R.

HIl derbrandt, $D$

Ho, P. S. 1562

Holl I gugy, P. H.

Hou, H.

Hua I naky, H.$$
.99
$$

Jimenez-Rodriguez, J. J.

$$
1340
$$

Johar, $S$. $S$.

${ }_{\text {Kang, S. T. }}^{T \text { T. }}{ }_{205 ?}$

KIrk, M. A.

Koshtkava, T.

$$
89
$$

Loty, P.

Lewlag, J. E.

Lusn Ikgy; $A$. V.

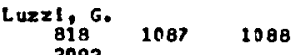

$$
2092
$$

$\underset{\text { Hacg }}{\operatorname{Mnal}}$ d. R. J.

Maderlechner, 6.

$\underset{735}{\operatorname{moleulekl}}$ R. L.

${ }_{745}{ }^{\text {Manns, }} 1674$

Matteson, S. 1661

$\underset{65}{\operatorname{mattax}}$ D. RP

Neut $t, M$.

$\underset{965}{\text { Meyer, } C, H ., J r . ~}$

Meyer, $H$. K.

Morrlman, G.H

Nelson, G. C.

Nlohus, $H$.

Bos

Ontsuka, $H$.

Worntak, D. G.
Okudo, 8

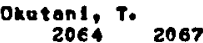

Papegno, $818^{1087} \quad 1088$ 2002

Plotnev, vo vo 1082

Rob Inson, Het.

Rodrlguez-VIdal, $n$. 1340

Roth, Jepi

Rudat, H. A ait

Saokl, N.

Saldoh, M. 1658

Seherzer, B. H. U.

308

Gehwarz, \&. A.

$$
1100
$$

Soe thanen, D. 1589

Sharp. D. J

ShImIzu, F. 89, $2064 \quad 2067$

Solth, J. N., Jre 965

snowdon, K. J.

Sone, $K$. 1658

sta ib, $r$.

Staudanmeler, $G$. 1661

$\underset{\substack{966 \\ \text { Ste inbruche } 1, C .}}{c .}$

Sugata, E.
ค9

Tanovlc, $L$ L.
2113

Thnapenn, n. A 113

Tompk ins, H. G.

Tor torel11, P. F 979

Valleg-Aborca, J. A. 1340

Wahner, G. K.

unttton, J. L. 2113

Uillians, J. S (119

Winograd, N. $\underset{806}{971} 976$

Yameda, R. 
D03

PARTICLE ImTERACTIOWS UITH SOLIDS

spattered Pertlele Charge and Quantue (Exclted) State Disiribetion

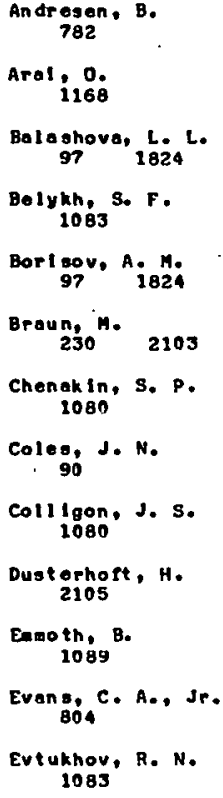

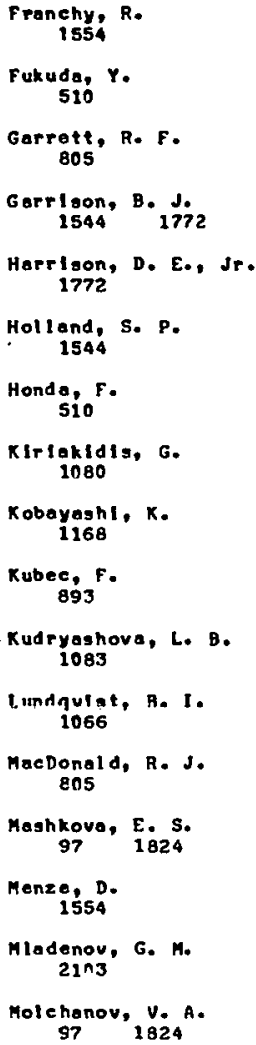

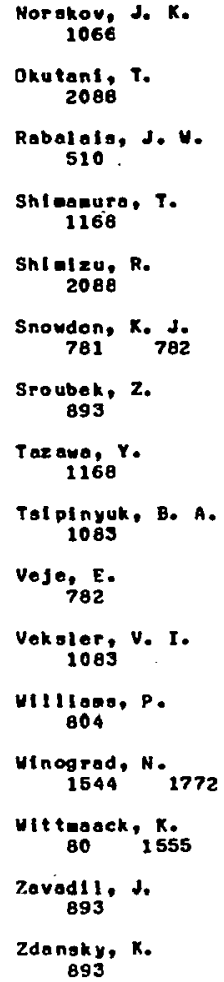


Do4

PANTTEe ImTEACtrons WIT SQR TOS

secondery El cotron Ejection by Heavy Partloles und Electione

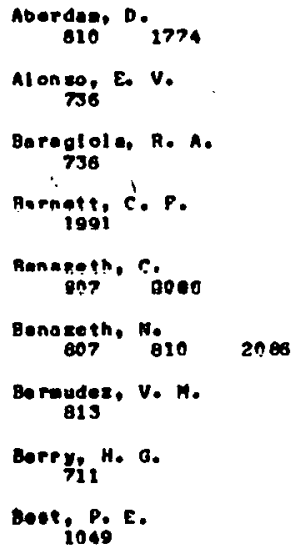

Geropd, $K$.

comoyunova, N. V.

2375

Heberland, $H$.

Hagstrua, H. D. 1559

Henke, B. L.

Hennequin, J.t.

Hirakly a.

Irol anani 0 :

1 mora, $r$.

I want, M.

Johnyon, P. D.

Josnt, A.

Khan, m. $^{2} \mathrm{~N}$.

Kta, s. c.

Kroeg, 1084

Kuk 10 if C.

$\underset{760}{\text { Kuppers, }} \mathrm{J}$

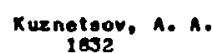

Lentert H.

Lovaree,
2081

Leongrdy 20.

Lleoggang, 1 .

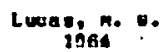

Maltezon, s.

nisenlor, J.$$
010
$$

Holehanor, Y. A.

Negra, $n$.

Nleolet, M. -A.

665

Novttakit, M. 3. 881 olive Florle, A.

736

Padasaee, $\mathrm{H}$

16

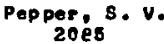

Pet rov, A. A. A. $_{2081}$

Pot rov, N: ${ }_{\text {Bes }}^{\text {Nog1 }}$

Place, J. D

Pruintnd Io to 18222075

Protopger, O. D.

Prutton, M.

Ray, ${ }_{1992}{ }^{A}$.

RIPIn, B. H .

RItx, Y. H.

Rostolng. P.

solent, $n$.

Senou, Jo

${ }_{200 i^{A}}^{\text {Septterp }}$

sloknsug, E. N.

$\underset{1618}{\text { Stgeund, } p \text {. }}$

Sol the g. D.

$\underset{\text { Spanjagerd, D. }}{\text { D. }}$

Svengaon. 16.

Von zyy 1001 :

vierig de Leeegno, p.

Viel,

vrakking, J. J. 1084

Uang, s. H.

vit tanecek, $x$. ises

$\underset{\text { Zesievekil, s. L. }}{\text { Lez2 }} 2078$ 
PART ICLE INTERATIONS UTth soLIDs Photoolectric Ejection of Elcotrone
(coerrieleme)

Allen, $F .6$.

$\underset{2090}{\text { Armend, } L .}$

Artanonov, O. K.

2082

Becker, H.

Boul1104, J. L. $2090^{\circ}$

Coapagne, M.

1557

Cartor, 1.6 .

Celotta, R. J.

$$
1149
$$

Chtang, G. C.

Chlang, 3

Chrl gtaan, 8. B.

1051

Chrlatophorou, L. 6.

Dahl back. No

1331

Den 1 is, D.

Dietze.

Eastann; D. E.
iose

Goudare, L.

006

PARTICLE INTERACTIONS UITH 80. IDS

Reflection of Electrone froe surfaces (coutriclent s)

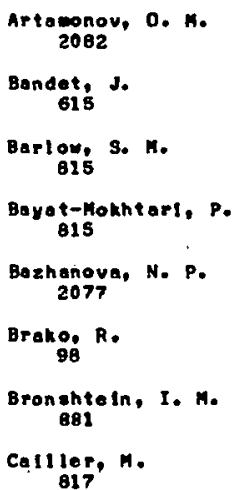

Gerhardt, $U$.

‥ 1048

coidd, $\mathrm{A}$

Gudat, $y$.
1557

H Iopse1, F. J.

1059

Hops ter; H.
155?

Jepren, D: W.

Keivan, s. D.

kisker: 5

Knapp Iñse J. A. 1059

Kuhlieann, $E$. 1557

Lasoerifor.

I. $\mathrm{C}$

$$
1046
$$

Margariltondo, 3 .

$\because 2 n 51$

Moore, I. D

Nilison, P, 0 .

1331

Okada, S. $K$.

Pesein; 1 .

plerce, D. i.

: 1149

Podt oucky", R. 1060

Pong. $y$.

Pulteswany, K. $s$.

4

$\therefore$

Colotita, Nid.

disterano, T: H.

2960

Duni ap, B. I.

Foldkanp, L: A.

$$
1617
$$

Gallon; T. E.

Ganichérad, J. P.

$\because \quad 617$

Qasorunger; H. $v$.

Jach, $T$.

Rornyushk1n, Y. D.

2079

tence, $z$.

Maliavồiอud, A.

618

novitekt1; M. 3.
Powe, Jugi $E$.

San jeeval oh, B.

Gasak1, T.
049

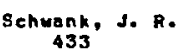

$\underset{433}{\text { Shahrlary, I. }}$

Shevehlk, N. J.
2073

Snirley, D. A.

$510008, K$.

Sitin, N. V.

$\underset{849}{\text { Sugovara, }}$ H.

Sund, A.

Talt, 8: H.

Tar oknov, A. N. 2082

Tong, 5.

Wang, G. C.

1149

Uehner: $P$. S.

Holnberger, Po

$$
1060
$$

Uni to, J

HIIIAas, R. S. 1333

WIm aer. E.

MSoree, D. T.

Pronin, I. 1.

1822 .

Quomener, $Y$.

Shinozokl, s. S.

$$
1617
$$

Sokeevle, $D$.

Stearna, M. B.

Bunjle, $M$.

Torekhoy, A. N.

2082

Vano, ${ }_{766}=-C_{*}$

WIIILAs, R. H.

428 .

Zog loysk11, 8. t. 

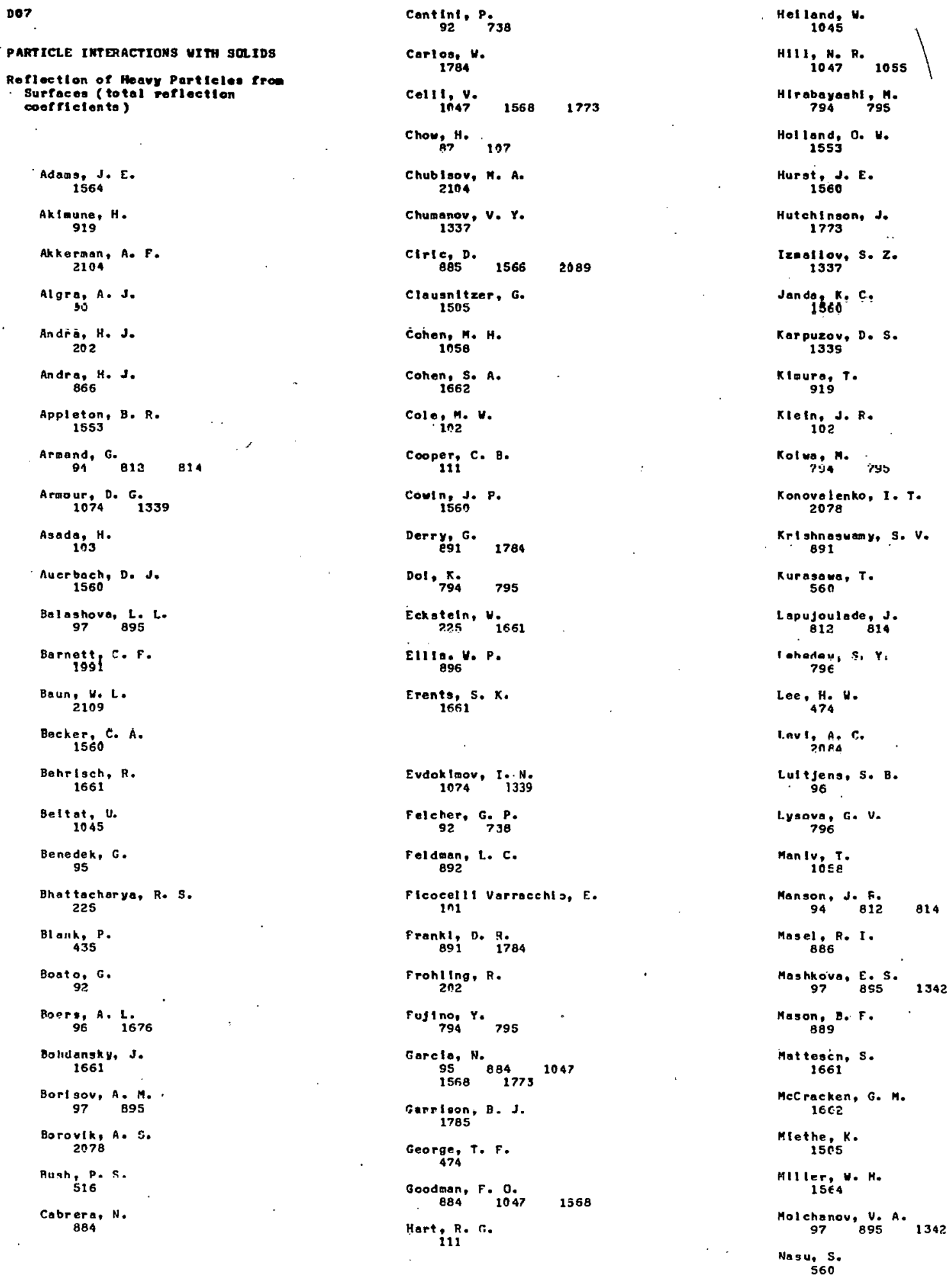


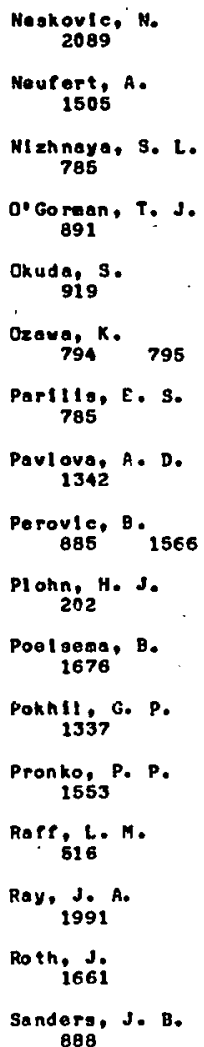

Sorts, $_{\theta \theta_{B}} F \cdot W$.

Sehlebel, $U$. 1 ebel,
1505

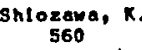

Shipatov, E. T 2078

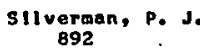

Irot $\ln \ln$, E. I. 1337

Snlear, V. A.

1342

stalo, $P$.
1661

staudenmaler, 5 . 1662

sten sgaar $d, 1$

Suhl, $H$.

Tagl ouer, $E$ $1 n 45$

Takahasht, J.

Takeuch!, $W$$$
1681
$$

Tat a rek, R.

Tayl or, T. N.

$\underset{285}{\text { Terzle, I. }}$ 1565 2089
Thoopson, E. D.

Tul Inov, A. F 1337

Turaev, N. Y. 785

van den Berg, J. A. 107

van der Veen, $J$. F. 888

Van $2 y 1, B$

Verbeek, H. 225

Mesner, D. 1784

Wharton, L. 1560

WI11 1008, B. R. (189

Wtl son, S, R. $15 \mathrm{E}$

${ }_{202}{ }^{H 66}$

Hit tmoack, $K$. 435

Uittmann, $H$. 202

Yamagueht, s.

Yamamura, $Y$. 
DO8

PARTICLE INTERACTIONS UITH SOLIDS Charge and Quentus State Dlatr lbutions
of Reflected. Heavy Particles at Macroseople diatances iron Surfaces

Al gra, A. J.

Anme, R: $C$

Aludiast ${ }_{202} 11 . J$.

Boers ${ }_{9 b}$ :

Carl son, L. W.

$\underset{2 n 4}{\text { Chureh, D. A. }}$ 2n?

Frohllng, R.

Fukuzawe, $F$.

Goltz, $k$.

009

PARTICLE INTERACTIONS UITH SOLIDS

De-oxcltotion, Neutrel IzotIon,

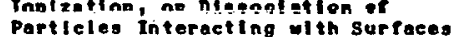

Breskln, A.

Dresser, M. J.

Falolg, A.

Floyd, $R$. L.

Galvagno, S.

vagno,

D10

PARILLE INIEHATIUNS UITH SUR IDS

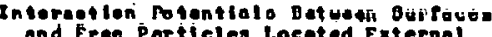
and Froe Particles Loceted External to the Surface (electrons and heavy part(eles)

Carlos, 1784
Granam,
1395

Hell and, W. 1425

Holag, $Y_{1}-Y$. R.

KIdo, $\dot{72}$

Kraug, s. 5 .

Kurniaev, V. A.
1828

L Bna, ixg.

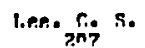

Lut t Jeno, S. B.

Wac Donald, R. J. 1425

NetP, S. H.

Nizhnaya, S. L. 785

Part119, E. S.

foldrlng, $G$.

Hoss: 749

Toh, As.

Rolm, $R$

KImura, In>8 $_{8}$

Kyoshina, A,

Lundqulot, 3. I.

Mannamt:

Marlella, P. P., Jr.

Carlos, 1551 . 5.

colo, H. is i.

Derry, 6.

Frank1, D. R.

$\underset{493}{\text { Plerott1, R. A. }}$

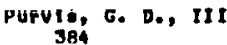

Plonn, H. J.

$\underset{205}{\text { Sehroder, H. }}$

Stz $\operatorname{man}_{93}$, .

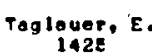

Tolk, N. Ho 1425

Tully, J. C.

Vorol oo, C.
93

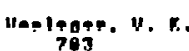

vinter. $H$

$$
\ln 2
$$

witemann, $v$

202

Yoda, ${ }^{\text {T. }}$

7habrev. 6. I
Rybol t, T. R.

Schuartz, $c$.

videll, Go.

Wener: D.

Wotken, G., Jr. 
D1 1

PARTICLE INTERACTIONS UITH SOLIDS stlektny coefrfteiento (theraol energles)

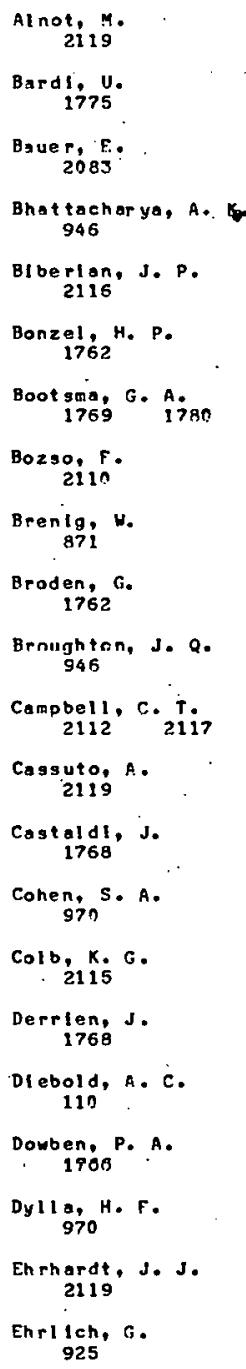

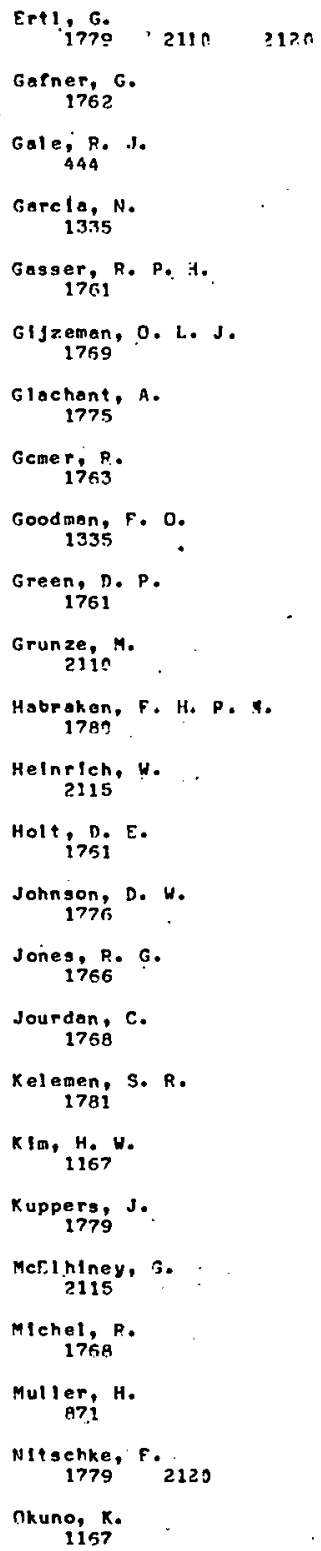

Perry, D. L.

Pollzzott1, R. S. 925

Poppa, t.

Roberts, M. $U$. 1776

Rossnogel, S. M. 970

Sal mercn, M. 444

Schouten, $F, C$. $17 E S$

Schuarz, J. A.
1781

Sh1, $\sin _{211}^{-K}$

Somorjal, G, A $444 \quad 2115$

Sparnaay, M.J.

ThI P., P. A.

TIbbetts, G. G.

$$
421
$$

van silthout, A. 1771

Wandelt, $K$.

Wang, C.

Neber, 8.

vedler, $\mathrm{G}$

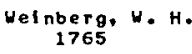

Wetsa, M. $212 n$ White, J. M. 2117 Uleringa, P. E. Wolken, G., Jr. Yates, J. T., Jr. 


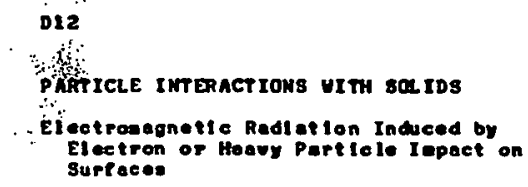

Agullar, $M$.

Ahlen, $S$. P.

$\therefore$ Appiozon, B. H.

$\therefore$ A Arobrugter. P.

in

Hartomonov, O. M.

is 792

Bozhin, A. I.

Bhattacharya, R. s.

$$
617
$$
BI ggerstät, J. A.

Curne, E. J. T. 1147

chandler, P.J. 1682

Clark, D. L

Datz, 5 .

Dosehek, C. A.

1147

Duley, W. W.

Ferpgtrorth, A, V., JP. $\underset{1147}{ }$ fol dman,

F111 ppov, E. 1 . 1656

Folkaann, $F$. 134

Gonde, s.

Hassel 1 kanp, D.
617

Joque, $F$.
If80

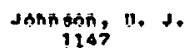

Knzal te. V. V.

Kuswa, G.

. 1147

Lobein, v. G. 1656

Mancint, A. M. 2102

Meyerhor, $v, E$ 134

Mlosue, s ingo

Mook, $C$. $D$.

Mokler, P. H.

Morenzon!, E. 134

Murrl, n.

$\sin 2$

Neelavathl, v. N. Ins6
$\operatorname{Noggleg}_{1056} T .8$

Nuroh, $k$.

Prince, P. H.

Ralno, A.

$\underset{1056}{\text { RItchle, R. H. }}$

RIz20, A.

Sal agon, H. H.

sementn, s. $M$

702

Soy or, R. A.

$\underset{617}{\text { Schartner, }}, K_{*}-H_{0}$

stoller, $C$ 134

Toungend, P.
IGAA

Tounsend, P. D.

780

Vasanelli, L. 2102

Verbeek, $H$ $105 E$

Vincent, $P$. 134

Nolf11;

134

Yoshida, s. 
D1s

Particle interactions UIT sa Ios

Decoppti on of Gases froe surfeces

Bohrlseh, Ro

$$
1661
$$

Bollard, s. W.

Bohdansky, J.

$$
1661
$$

Cralg, J. H. Jr. 1770

Del gess, U. N.

971

Eekstein, $y$

1661

Edwards, D., Jr.

Erents, S. K.

Estrup, P. J.

Felterp T. A.

flel seh, $T$.
D14

PARTICLE INTEACTIONS UITH SOLIDg

siletering: Volds, and surtoce serain
In Wotel:

Arke 11, D. R.

Arnstrong, T. R.

1659

Berber, S. B.
559

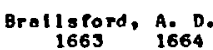

Bukou, H. H.

Buttlar, H. $v$

203

Chél nokov, O. I.

784

Das, S. Ko

Debras, G.

Fonske, 6.
830

foreaan, A. J. E.

$$
65
$$

Ghonlea, N: M.

GI11 es, J. M.
Garpleon, B. J.

Harel son, D. E., Jr 。 971

Hoek, J. 1 B87 $1565 \quad 1567$

Keoge1', N. I.

KIrsehner, J. 1777

Krahl-Urban, B. 2087

Kuks InokI I, N. I. 879

Lagousk1, J 1086

Lientenetolger, $n$. 1086

L Ichtman, D. $687 \quad 1565 \quad 1567$

Los, s.

Matteson, S.

1661

Menzel, $D$.

177 .

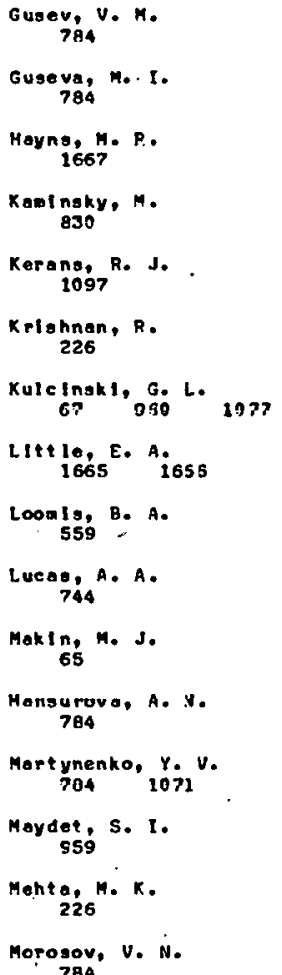

N to hus, $H$ BOB 208 ?

PhIIIIpe, J.C.

Roth, J

1661

Shubalova, N. M. 079

seatb, $p$ 1661 1777

staudenaler, 6 . 1661

Steinbruete $1, c$. 846

Taglrov, R. B. 879

Verhoeven, J. 108 $\therefore$ utlllasis, $\varepsilon$. H.

HIIHang, P. M. 1086 971

Yu, M. L. ${ }_{967}$ 1332 $\because 1767$

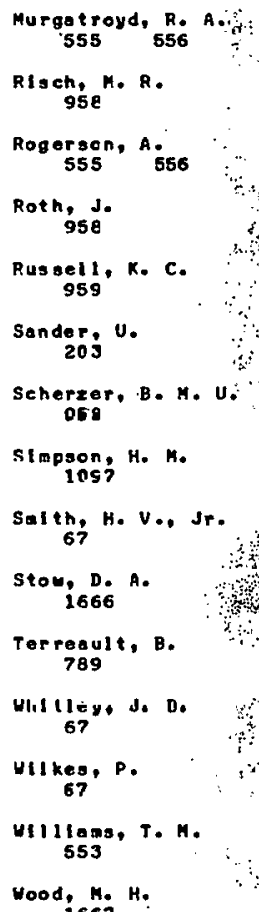


D15

PARTICLE INTERACTIONS WITH $80 Z$ IDS

Radiation Damage In Metals

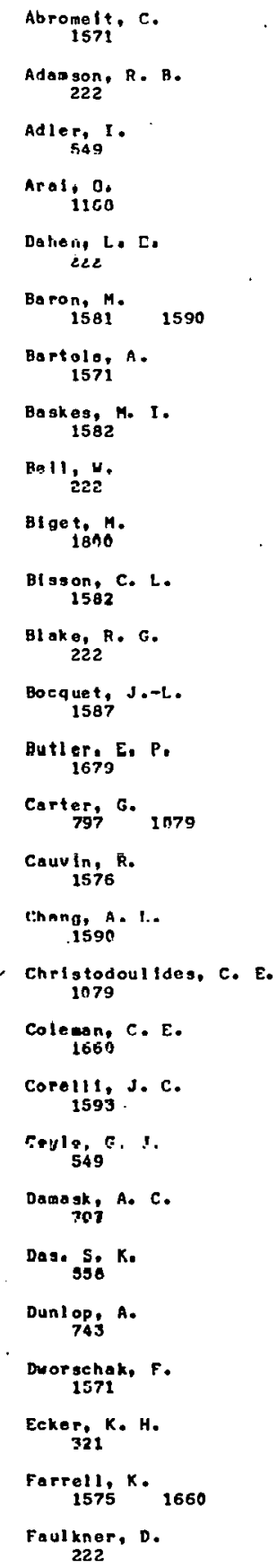

Fenske,
558

Fisher, s. 8:

Foreman, A. J. E.

Foster, R. E.

Gelles, D. "s.

Gllbert, R. ${ }_{222}$.

GIrard, P.

An1...u, 11. 4.

Houston, J. T.
IS75

Howe, L. M.

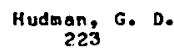

I got e. N.

Johi.son, R. A.

Jostsons, A. 222

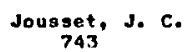

Kaul naky, M.
558

Relly, P. $M$.

Kentk, E. A.

KInney, J. H.

Kobayashl, $k$. 1158

Kozina, G. 801

Kuleingk1, G. L

Lem. N. O 1579

Lauzler, $j$.

Lee, $\dot{E}, H$.

Leffers, $T$

frers, $T$
21ni

Lou, K,-Y.

1594

t.nrensenliti, $\mathrm{N}$ 743

Lucasson, A. 1800

Lucasson, $P$. 1800

L'vov, A. H.

Madden, P. K.
Makin, M. J

Manaur, L. K. 1580

Martin, 6

$\underset{1572}{\operatorname{Marwlek},}$ A. D.

Matheny, R. A.

Houry, Fore

MeVay, G. 'L.

Hougent II,-p.

M Igalenya, V. Y.

miler, K. M.

$\underset{787}{\text { Minter, }}$.

Nobee in 1.

$\underset{222}{\text { Northuood, D. } 0 .}$

offersann, P. 321

Okacoto, P. R.
1579

$\underset{1572}{\text { Pliler, }}$ C. $_{1573}$

Porter, D. L. 2.8. 157 A

Pot ters D. I.

Rocknagel, E. 1133

Rowel Iffe, A. F. 1577

Russell. K. C. 1586

Sato, s.

Sahulnen, Fi. M. 1692

Sel dean, D. No,

snibata, R. 1677

Shl lyoev, B. A. Rot

Shi easura, $T$.

1168

singh, B. N 2101

stuell, P. M. 1572

Slabospltakll, R. P. 801

Safth, H. V., Jr

Sunson, M. L. 
D17

PARTICLE INTERACTIONS UITH SQL IDB

Electron-, Ion-, and Photon-Induced

Chenteal Changes to Surfaces

Agarval, 5. B.

2098

Altstetter, C. J.

Antoghin, A. A.

1729

Bett!s, J. T.

Bonch-Bruevich, A. M.

Cliu, ${ }_{1562}$ K.

Chuang, T. J.

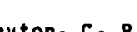

$\underset{2097}{C l a y t o n, ~ C . ~ R . ~}$

Coburn, J. U.

Frledenberg. A.
1783

$\underset{\substack{\text { Gatalisk } 11, G, V . \\ 1729}}{\text {. }}$

Guenther, A. H.
1122

Herman, ${ }_{2098}$

Hirvonen, J. K.

Ho, P. S.

Holl OWgy, P. H.

House, R. A., II

Hubler, $G$. $K$.

2097

Ieni wura, 5 .

1388

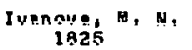

Fol

Is

ELECTMON-PARTI CLE IMTEANCT IOHS

General

$$
\begin{aligned}
& \text { Aronson, I } \\
& 999 \\
& \text { Blau, } \bar{R} \text {. } \\
& \text { Dube, L. } \\
& \text { Fink, M. }
\end{aligned}
$$

Jones, $V$.
1545

Josh 1, M. C.

2091

Konayama, $T$.
1150

Kol1 y. $R$.

Knotak, M. L.

KochengIna, $K$. K.$$
1825
$$

Korshunov, $\varepsilon_{0} P$.$$
27 z y
$$

Lancoster, G. M.

1. au! o; 1. r.

Lau, Z. L.

Libe, ${ }^{M .}$ N.

Libenson, H. N. 1825

Lehtman. D. 432

LIn, T. T.

Los J J

Lueke, W. H.

Mok In, v. S.

$\underset{\text { meintyre, N. }}{\text { Sy }}$
.

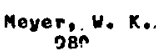

Murt1, D. $K$.

Nelson, G. C.

Okamoto, P. R. 157

Pootag J. $M$. Pudkov, S. D.

Fueng $14^{T}, 718$

mogery, ".

Herzenberg, A.

$$
1313
$$

Inokuti. M.

$8 n n$

Itikawa, $Y$,

Raldor, $U$.

694

Kloinman, c.J.

Mecarthy, I. E.
Rabalats, J. No

Rehn, L. E.

Rehn, v.

Shaplra, Y.

Shl ol zu, $R$.

Sluoser, G. J.

Sal th, J. N., Jre

stanchell, F. U.

976

Suvorny. A. Y.

Tanoue, $\mathrm{H}$

Taylor, J. A.

Terukov, E. I. $\underset{979}{\text { Tortorell, }}$ P. F.

Tau ruaniea, T. 1150

Verhoeven, J.

108

Uang, $_{2097} F_{2098}$

Vatanabe, $K$.

Re4

$\underset{980}{\text { Uehner, }}$. $K$.

Winograd, $N$.

Unterg, H. F.
829

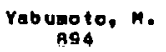

Yadav, A. D.

Yamashino. T. YS4

\footnotetext{
MeDove 1I, M, R. C 1954

Huv log 19200

Rosenberg, Lo 999

Sprueh, L.

999

Taketsuke, K.

Turner, J. E.

Yates, A. C.
} 
EO2

ELECTRON-PARTI CLE INTERACT IOLS

Eleatle collitotono

Abde 1-Raour, M. A.

$$
1534
$$

Band, Y2. B.

Barratt, $R$, $F$.

Beer lage, $H_{1423} \mathrm{~J}_{1965} \mathrm{H}^{\circ}$

$\underset{266}{\operatorname{Bennan1} \text {. A. L. }}$

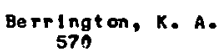

Berry, 609 . S.

Bhad ra, $K$.

Bl alia, $M$.

Bronsden, B. $H$.

Buckaan, S. J.

$\underset{570}{\text { Burke, } P \text {. }}$

Coll away. J.
1009

Chol, B. H.

Chrl st ophorou, L. $G$. 1605

Clark, $C \cdot$.

Collins, L. A. ${ }_{574} \cdot{ }_{1866}^{A}$

corcoran, C. 1695

Dalba, G.

Das, J. N.

do Heer, F.J.

572

Dehmer, J. Liges

Dhal, s. S. 1420

DA II, D. 1903

Di xon, D. A.

1422

Drachnan, R. J. 1945

Dube il.

Duguet, A.

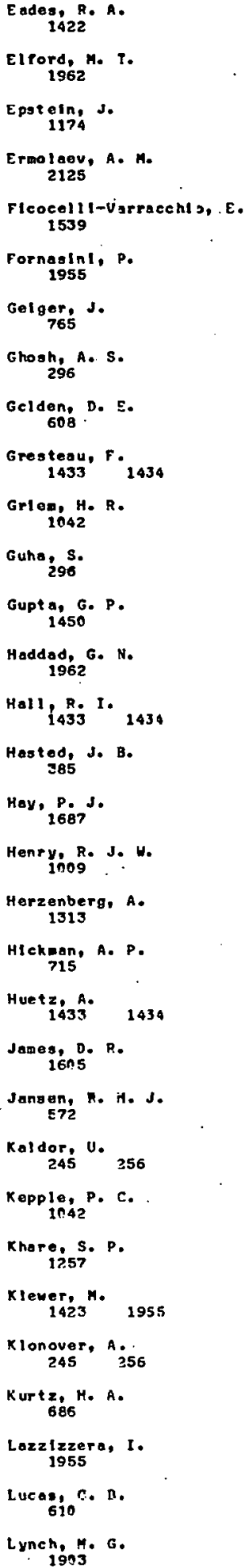

Mathls, R. A.

Mat hur, $D$.

Mat hur, $K . C$.

Mazeau, J. 1433

MeDonald, L.

MeDowell, M. R. C.

MeKoy, v.

Mol seyev, $N$
1695

$\underset{\substack{\text { Moron-teon, D. } \\ 765}}{ }$

Morrison, M. A. l6e?

Mukher jee, A.
1277

Nesbet, R. K.
1007 1305

Noble, C. J.

$\underset{574}{\operatorname{Norerog9}, 0}$ iB66

Noro, 1.

Ohrn, ${ }_{686}$

OMalley, T. F. $k$.
166
1917

Peach, G.

Poe, R. T.

65.6

Ras, D.

Ranieri,
195S

Reltan, A.

Ritente, $B$.

Robb, H. 5 .

Pobson, B. A.

241

Rohr, K

Rumble, J. R.

Sogokt, $F$.

Sehold, G. B.

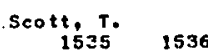


306

$$
\begin{aligned}
& \text { Shan, } Y .{ }^{598} \\
& \text { Shingel, }{ }_{1278}{ }_{1420} \\
& \text { slegel, J. } \\
& \text { Sinfollam. A. L. } \\
& \text { Silvastava, B. B. } \\
& \underset{284}{\text { SrIvastava, M. K. }} \\
& \operatorname{stepn,~}_{608} \text {. L. } \\
& \text { stevart, } \overline{\text { P. }} \overline{\text { F. }} \text {. } \\
& \text { Sun, J. C. } \\
& \text { Sural, D. P. }
\end{aligned}
$$

Tatevikt, $H$.

Toyal. 5.5.

Taylor: $H, S_{4}$
1535

Teubner, P. J. ?.

Trlpethl, A. N.

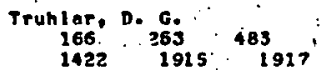

Valley, $\mathrm{H}$.

van der Risy, $\mathrm{U}$ van der utel, M. $_{1423} J$.

Viehon, D.

Wolterg, H. R. J.
2125

Wateon, D. K.

$4 e 1 \mathrm{ch}, J$.

Uellenste In, H. F.
266

HIJISen, J.F.

zeces, $n$. 
Eos

EL ECTROH-PAR TICLE INTERACT JONS Exeltation

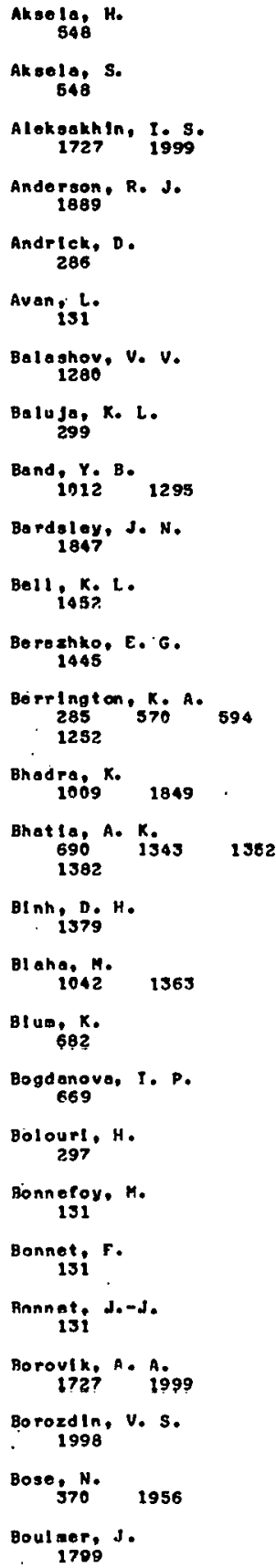

Brandt, H. S.

Brangden, B. H. 1493

Breuckiann, B. 1825

Brton, C. E.

Brongerene, H. H.

Buekman, S. J. 1245 S. J. 1441 1451

Burgess, D. E. 1967

Burke, $P . G$. $570 \quad 594 \quad 1232$

Burrow, P. D.

Callavay, Jogeg 1191

Cardinot, $J$.

$$
1498 \text {. }
$$

Carturight, D. C. 1914

Catolan, $G$.

Cedarbouw, L. 3 . 1258

Chot, B. H.

Chrlotophorou, L. G.
lens

Ciark, R. E.

Cole, B. E.

2106

Col.1Ins, i. A.

comer. 19.1260

Crandall; $D_{\text {. }} \mathrm{H}_{\text {. }}$

, 578 . 1322

Crove, ${ }^{A}$.

Dalgarno, A.

Das, A. K.

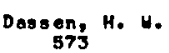

Dovl s, J.

Day, R. L.

de Henr, F. J.

Deherer, J. L.

Delpech, J. $-F$. 1999

Dere, K, P

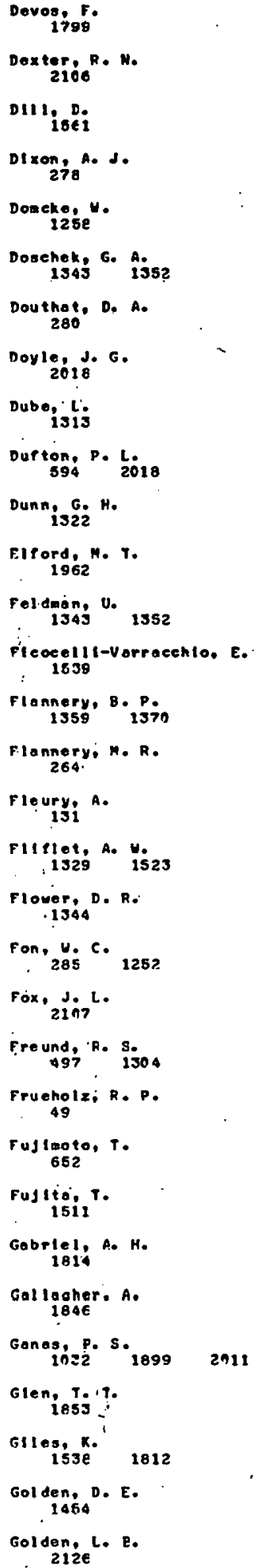




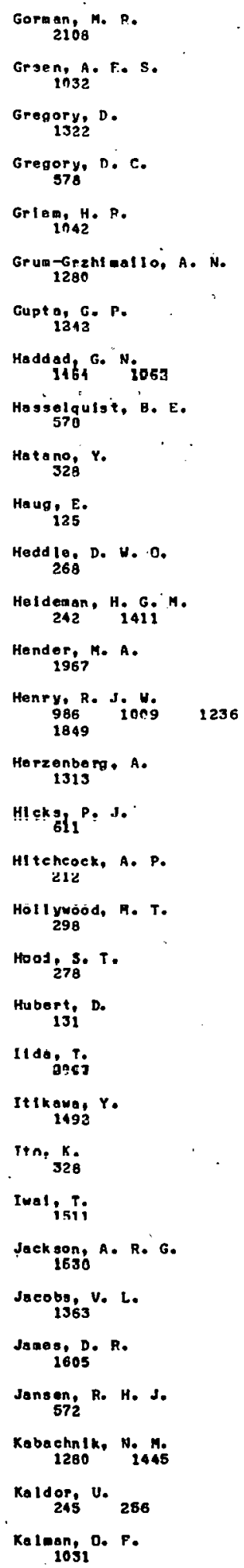

Kospar, $F$

Kaul $\underset{5 \in 2}{R .} D$.

Kepple, P. C.

Kats, F; B:

Xhakoo, M. A.

$X$ Ing, ${ }_{2.43} c$.

KIngaton, A. E. 285 S84 1252

riolnpoppón, H. BOD

KIonover, $A$.
2456

Roref, D. F.

$\underset{32.8}{\text { Noueh, }}$.

Kour1, D. J.

Kunar, 5 .

Kuppermann, A.

Lamb, Wo3i E., Jr.

Lon. ${ }_{1988}^{K}$.

${ }_{\text {Lane }} \frac{N}{2 n} 5 ;$ F.

Ledourneuf,.

$$
12 \dot{y}
$$

Lo voupheur, h. 1988

Leo. E. $_{1850}^{\text {T. P. }}$

Levin, D. A.

LIoplinsh, A, K. 256

Lin, c. $c$.

i. Intar. r.

1956

Lins de Barros, H. G. P. 1980

Madison, D. H.

1637

Magunou, A. $I$.

Matti, N.

malcolu, I. C. 240 S7J

Monafleld, M. d. D.

Morehand, P. 1498

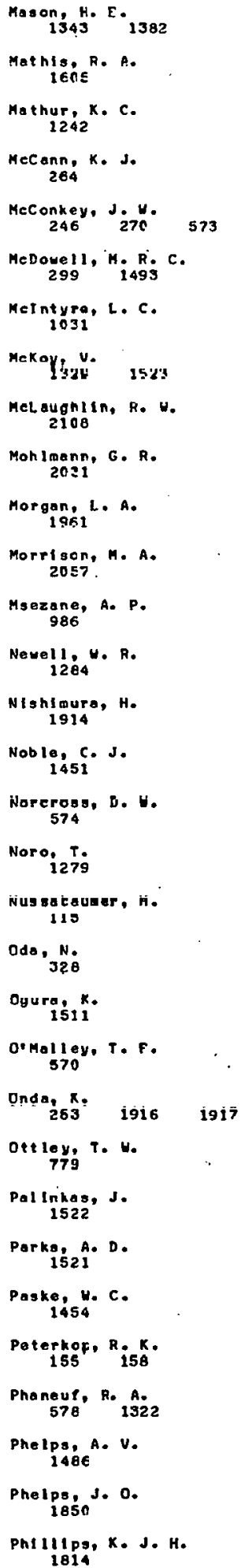




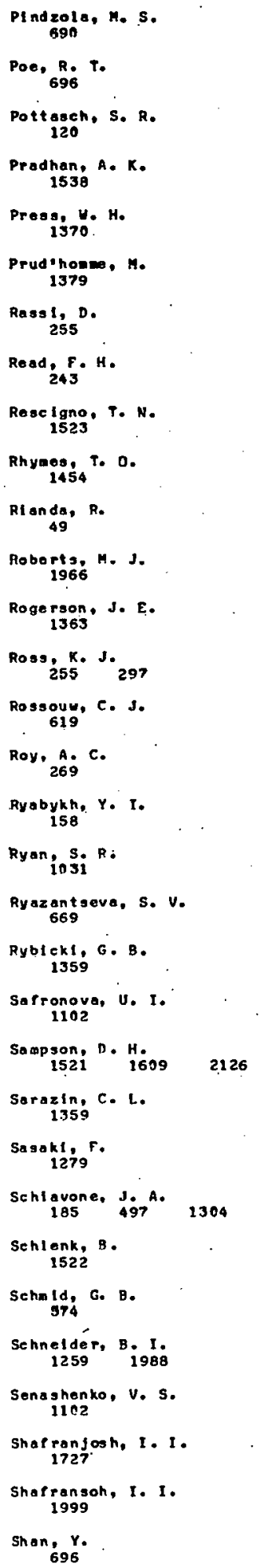

Shorpton, F. A.

1889

shuttleworth, I. 1967

slegel, J.

sil, N. C $269.1240-1584$

Sinfallas, A. L.

SIngh, s: N.

sinhe, $C$

swoding, A. G.

I rnov, Y. M.

1998

Salth, A. C. H.

Solomon; J. E.$$
1850
$$

spence, 0

Spereak 1, J. J.

Srlvastava, M. $x$.

1294

Starodub. V. P.

storey, P. J.

strakhova, S.. I

1280

$\underset{1377}{\text { Sumers, }}$ H. P.

sun, $\mathrm{J}_{696} \mathrm{C}$.

Sur, S. $\mathrm{K}$.

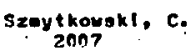

Tachlbane, K.
TARG

Tarr, S. H. 1304

Totemakt. $H$.

1279

Taylor, $T$. R.

Temklng: $A$.

Toubrioe, P. J.J. 1451

Top, Z, H.

Trojest, S. 1992

Trone, ${ }_{24}{ }^{M .}$

Truhl ar, D. G. 1917
Teubol, $T$

Tully, J. A

Tul st, d. R.

1454

$U_{g r ! n_{4}} 8 . Y$.

Vol ok. $A$.

wan der kaey, $y$.

ander poo rt on, $R$. 267

van Eck, Jo

uan Regenorter, H.

1379

ven Sprang, H. A.

van Uyngsorden, $U$. L.

$\underset{548}{\underset{5 a y r y n e n,}{d}}$

Vuskovie, L.

1892

vadehra, J. $m$.

Matanabe, $S$. 1511

Watanabe, $Y$.

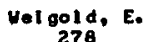

Uel ch, j.

vasterveld, H. $^{\text {B. }}$

Whelan, N. J.

Unl te, M. D. 297

viding. $k$. $G$.

viese, $L$.

197

viden, D. $G$.

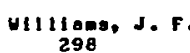

uing, H. H.

vinterg, K. $H$.
267

Yakhontova, V. E. 665

Younger, S. M.

Zejone, A

ZIpR. E. C

Zubok, K. 
E04

ELECTRON-PARTICLE INTERACTIOHS

DI seceletton

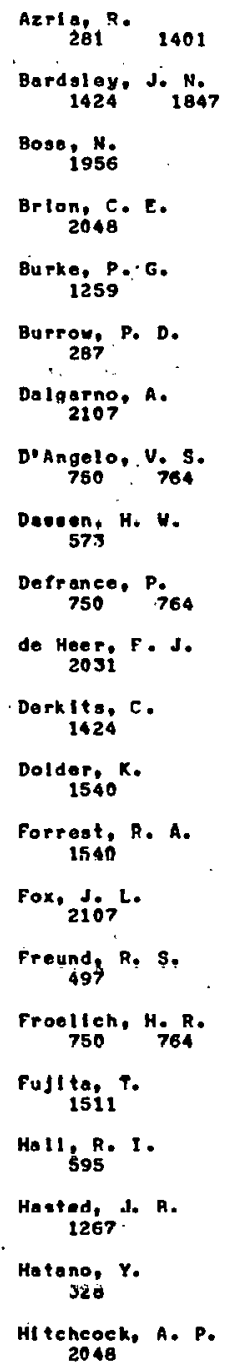

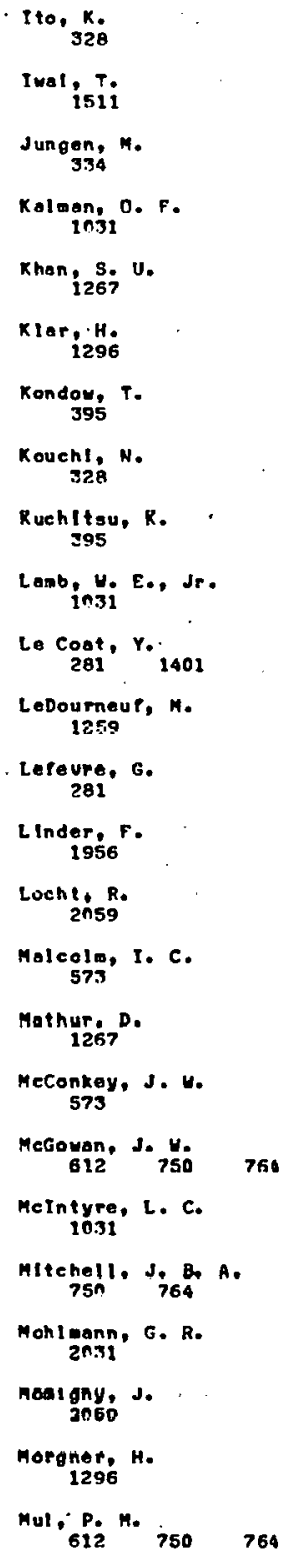

Nisht yone, I.
39s

Oda, N2B

Ogura, $k$.

Peart, $E$.

1540 .

Ral Destldar, $K$ B58

Ral Dastldar, T. K. B58

Ryan, 34 R.

Sehermann, C

Śchiovone, J. A 185497

Sehnelder, E. I. 1259

Staon, D. $281 \quad 1401$

Spence, D. 287

Spezesk1.J.J.

Sto easoler, $v$ 334

Tarres. H.

Tavlor, H. S.

Trnnes to

Tsubol, T

328

von der Wtol, M. J.

Vog t, J.

Vadehra, J. M..

Uat anabe, 5 . 1511

Uat anabe, $Y$. 1011

Ving, H. H. 
E05

ELECTRON-PARTI CLE INTERACT IONS

Ionleatl on

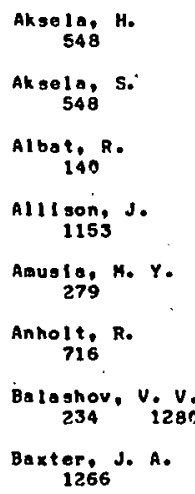

Dimftrijevic, M. S.

$$
1253
$$

Douthat, D. A.$$
\text { 2Bn }
$$

Ebding,
579

Fantan1, R.

Flannery, B. P.$$
1350^{\circ}
$$

Flonnery, $M$. R.

Fox, J. L.

2107

Fuss, I. I. ' 250

Gel tman, S:

Genz, 1396

Gerard, $M$.

Glardin! Guldonl. A. 1412

Glardint-Guldonl, A.

Gotden, L. B.$$
2126
$$

Govers, T. Q.

$\underset{578}{G \text { regory, D.C. }}$

Grujte, P. V.

Grum-GrzhIm:110, A. V.

- Holpern, A. M.

Horrison, M. F. A.$$
1285
$$

Hosh tzune; A.

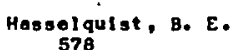

Hazl, A

Hel a, H.

Hicks, P. J.
1266

HInk, 57.

HIppler. R.

HIt cheoek, 4. P.

$$
2 n 48
$$

Hof Paann, D. H. H. 1396

Holder, c. H., Jr.

Hol zer, T. E.

${ }_{215}^{\text {Hood, S. T. }}$
Hynon, H. A.

1655

I1da, $T_{20}$

sncobs, v. $t$.

se

James, D. $P$

1605

Sansen: R. H. J.

Joachain, C. J. 529

Joselying J. A.

Jung, $x$.

KaboehnIk, N. N. $234 \div 1280$

Keskl-Rahkonen, 0 . 229

Khokhloy, V. D.

Kianang J. T.

Kingoton, $A, E$. $14 E 2$

Komna, 1442

Kon don, $T$.

Krasllenthova, N. A. 1823

Kuatar, A.

-

Ruoppala, R.

Lal, $\begin{gathered}\mathrm{Me} \\ 569\end{gathered}$

Locht, R.

Low, 1396

Magunov, A. ${ }_{234}$

Mann, J3. 15.

Mansisield; M. N. D.

Mark, To2. D. 1285

Morx, ${ }^{\text {P. }} 9$

Mathls, R. A.

Mecann, K. J. 900

Mecarthy, 1 . E.

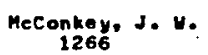

MeDovell, M. R. C. $1954^{\circ}$

Megulre, E. J. 


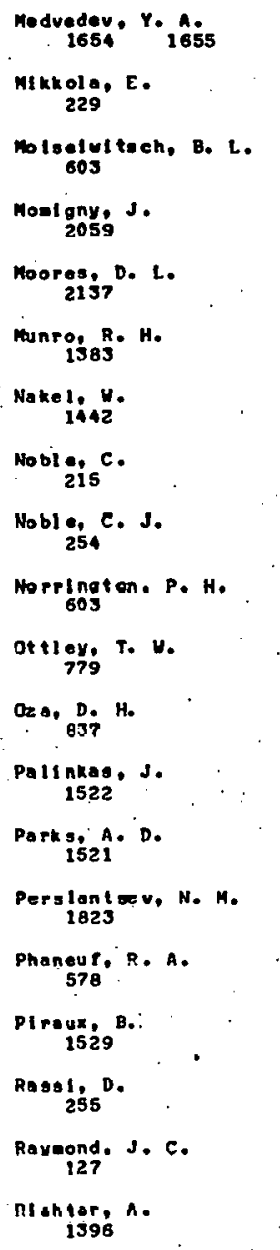

Robort, J., R.

Rogerson, J. E.

Ross, K. J.

$\underset{\text { RAK4 }}{\text { Roun troe, S. P. }}$

Rowan, U. L.

Roy, ${ }_{1968}^{\text {B. }}$ N.

Ryblekt, G. B.

Sofronova, U. I. 1102

Sampaon, B. H.

sarazin, c. l.

Sehortner, $X_{0}-H$. 140

Sehlenk, B.

Sehmllt, H. P. 579

Schubert, E.

Schuek, A.

Sehuesslar. H. A. 1099

Senashonko, V. S. 11 ! 2

sheinerman, S. A.

Sulth. A. C. H.

Oalth, d. di $\underset{569}{\text { Splvastaus, M. X. }}$

Stefant, ${ }_{1423} 1710$

Stephan, $k$.
1025

Strakhove, S. I.

Taylor, It R.

Thonas, 15 . K.

$\underset{569}{\text { Tripothi, A. N. }}$

Valok, 1522 .

van der Kooy, i.

van der ylol, M. J.

van T11burg, $n$. 1529

van yingerden, B. 1520

$\underset{5 a \theta}{\text { Vayrynen, } J . ~}$

Vernazzo, J. E. 127

Uasoda, N.

Volgold, E. $215 \quad 254 \quad 1529$

Uhl to, H. D.

Winters, K. H.
H.
1241

Zare, R. N. 
E06

ELECTROW $\rightarrow$ ARTICLE INTERACTIONB

Rocomb ination (olectron lon)

Aleksakhin, I. S.

$\underset{48}{\operatorname{Arnstrong}}$, D. A.

Bardoley, J. N.

Bartleld, W. D. 1358

Boly-Dubau, F.

Blaha, M.

Brocklehurgt, M. 1381

DoAngelo, ve 5 .

Dạvi sij J.

$\underset{750}{\text { Defrance, }} \mathrm{P}_{764}$

Derk1ts, ${ }_{1424}^{\circ}$.

Dubou, J.

Foueher, $P$.

E0?

ELECTRON-PARTICLE INTERACT IOUS

Colllolonal Do-Exeltation

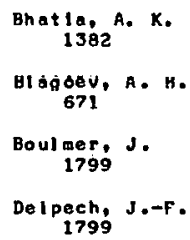

EOa

ELETTRON-PARTI CLE INTERACT IUNS Collislonal Line Broaden Ing

$$
\begin{aligned}
& \text { Blahg, M. } \\
& 1042 \\
& \text { Ocupurg J. } \\
& 123 \\
& \text { Enrteh, H. } \\
& 584
\end{aligned}
$$

Flannery, B. P. 1359

Froelleh, H. R. 750 . 764

Gabrtel, A. ${ }_{195}$.

1814

Hol zer, $T$. E.

Ime, A. $I$.

Jecobs, V. L.

Joselyn, J. A.

Loul orgue, '
195

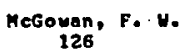

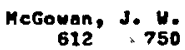

Mendas, 1

564

mital, H.P.

1497

$\underset{750}{\operatorname{Altehel} 1, J_{764} \text { B. A. }}$

Mul, P. M

$126 \quad 612 \quad 759$

Hunro, R. H.

Narain, $u$

Dere, K. P.

Devo a, F.

Dube, $\mathrm{L}$.

Grujle, P. $v$.

Hazt, A. U.

Herzenberg, A.

1313

$\underset{1271}{\text { Koledin, }}$

Grlem, H. R.

irujic, r.

Heiblg, $v$.

Kepple, $P$ In42 $C$.

Koledin, D. 1.27i

Kuseh, ${ }_{116}^{H \cdot J}$ is45

Lee, ${ }_{581} W^{\circ}{ }_{582} 583$
PhtIIIps, K. J. H. 1814

Rat Deatldar, K. 850 :

Ral Deatlder, T. K. 858

Rogergon, J. E.

Ros zaan, L. J. 1685

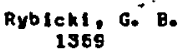

sal on,

Sarazin, C. L. 1359

Sennhauser, E. S. 48

Steonman-clark, $L$. 195

Vol onte, s. 195 65

Wadehra, J. M. 1424

varaan, J. N.

Zapesochnyl, A.. I. 1615

Zndanov, v. P. 1645

MacalpIne, G. M. 1362

Mason, H. E。 1382

Ore 1, A. E.

Popov, T. K.

671

Reselgno, T. $N$.

1610

shuder.J: $\mathrm{K}$.

viding, K. G.

Ptel, A.

Puedingahov, L. A.
1346

Richter, $H$.

Pondigs 116 G. 1345

Sobol, I. M.

Sunyoev, F. A. 


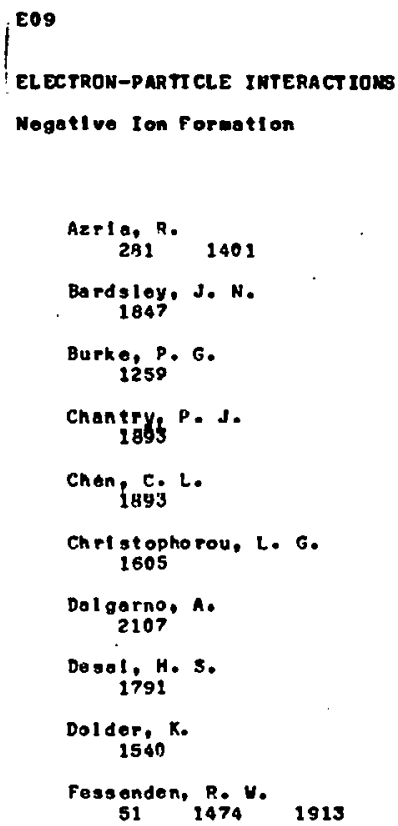

E11

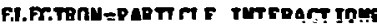

Prec-free Transtelons (Brousst rohiung)

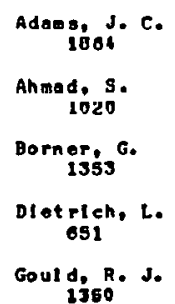

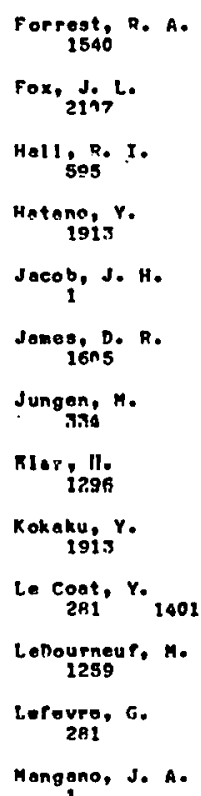

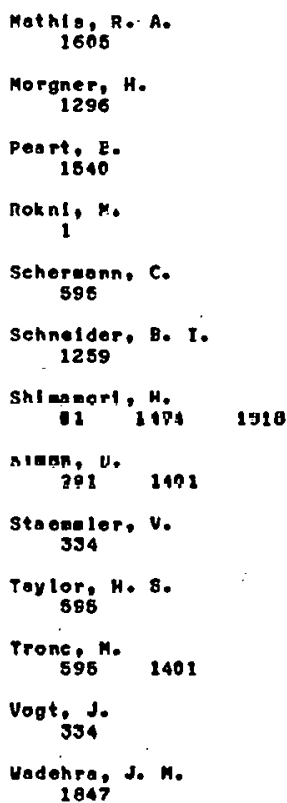

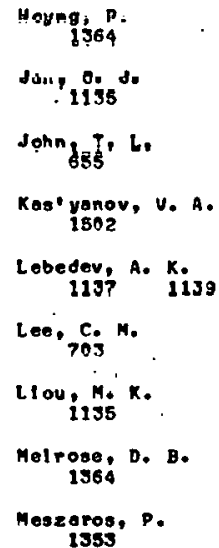

Porngtiog c.

Pausp a. $x$ Pentt, g. U. $703 \quad 994$ Reese, 7 . singh ing. starostin, A. N. 1502

Tsong, H. R. 994 Zhdanov, V. P. 


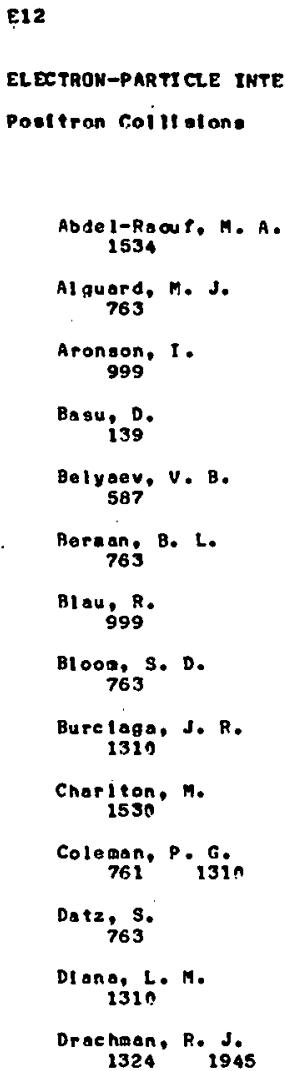

Gion T969 T.

Grlpelth, T. C. 1963

Guha, 5.3 . 1432

Gupt A. G. P. 145 ?

Hay, ${ }_{1697}^{\text {P. J. }}$

Heyl and, G. R.

1963

Klelnman, c: J.

999

Kuasknov, M. A. 1rG?

Lal, Me.

$\operatorname{Ling9,~}_{868}$. S

1963

Mandal, $P$. 1432

mothur, ${ }^{K}$. c.

Mef.aehran, R. P. 575

MeNutt, J. D.

Morrison, M. A.

muknersee, $\mathrm{A}$. 1277

Pantell, R. H.

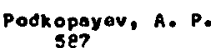

$$
\begin{gathered}
\underset{261}{\text { Dolder, K. T. }} \\
\text { Forrest, R. } \\
261
\end{gathered}
$$

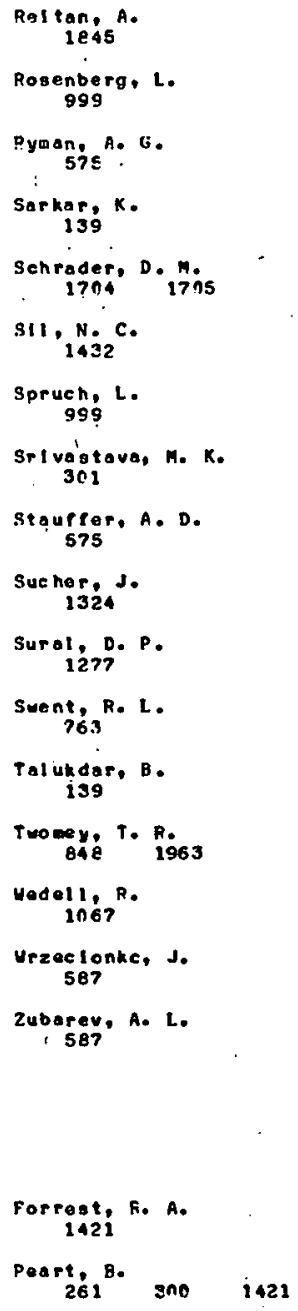

Forrest, F. A.

Rocanov, Y. I. 
E15

ELETRON-PARTCLE IATERACT IOHS

Inner-shell Interactions

Brton, C.E

341

E16

EL ECTRON-PARTT CLE TNTERACT TONB

Fluorescence and Lun Inescence

Avan, $\mathrm{L}$.

Bonnefoy, M.

131

Bonnat, $F$
Genz, $\begin{gathered}H . \\ 1396\end{gathered}$

Hitcheock, A. P

341

Hof Pann, D. H. H. 1396

Bonnet, J.-J.

Firestone, $q . F$.

19

Fioury, A.

Gerard, $M$.

Govers, T. M.

329

Hubert, D.
Low , ${ }_{1396}$

Alchter, A 1396
Maede, M.

$\operatorname{Mar} x_{329} R$.

Higave, I.

Neshitarual zu, T.

918

Oka, 19

Takao, s. 
E17

EL DCTRON-PARTT CLE IWTERACT IONB

Angolar seat toring (opecitied procese)

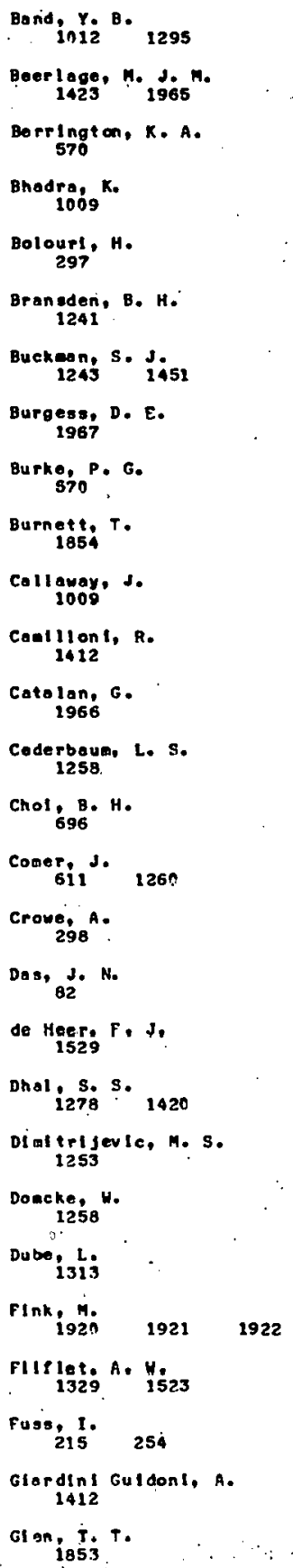

Golden, D. E。

Gregory, D. 1921

$\underset{1253}{\underset{\text { Grugle, }}{\text { P. }} \text {. }}$

Hay, P. J.

Hender, M. A. 1967

Henry, R. J. W.

Herzenberg, A.

$$
1313
$$

Hieks, P. J

611

Holl ywood. .1. T.

Hood, S. T.

Jooehain, C. J. 1529

Kaspar, F.

Khakoo, M. A.

Khare, S. P.

KImonen, J. T.

Ktar, ${ }_{1296}^{H .}$

Klever, M. 14231965

Konag, M.

Lovin, D. A.

Lucas, c. B.

Mad t gon, D. H 153

MeDoneld, L. $6 n 8$

MeKoy, V. 1523

Moore, P. G.

Morgner, $H$ ${ }_{1296} H$

Marrison, $M$ : A. 168

Nake !1: We

Neabet, R. K. $577 \quad 1303$

Newe II, U. R. 128

Noble, $C$.

Noble, C. J.

maliey, $T$ s7n

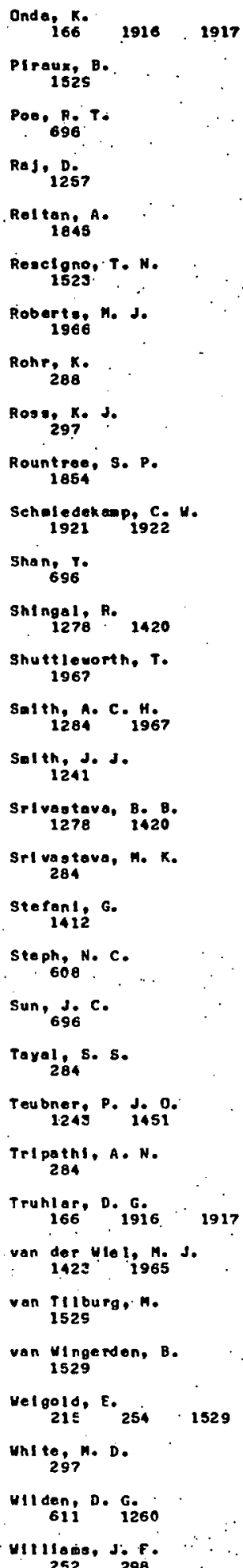


608 .

TRANBPOAT PHENONEMA AMD AUERMGE
PROPERTIES IN GASES

Energy Dietelbution Ceneryy distribution of fone and electrons uth applica electrie and en gnetie (I) das)

Azharonok, $v, v$. 1724

Bogneux.: J. M.

Blouin, H. A.

Copltol11, H. 1001

Chubrik, N. I. 1724

Des'yaneruk, A. S. 1723

Dillonardo, M. 1601

609

TRANSPORT PHFNONFWA AHD AVERAGE PROPERTIES IN GASES

Momentue Tromstor

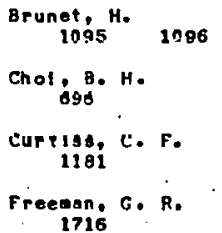

610 PRAMSPORT PHENONENA AND AVERAGE
PROPERTIES IN GASES

first and second Fownend coeptielente

Bhat tacharya, A. $K$

$$
1990
$$

BIevIn, H. A.
Eroshenko, L. E.

1723

Fletcher, 3 .

Glel zes, A.

Gubkeylch, V. A.

Hul, $\underset{650}{\text { A. }} \mathrm{K}$.

Hunter, s. 9.

Kofrount, $H$.$$
653
$$

Kaneko, s. 619

Hekeover. M. R. 65 ? Meehov, V. S.

Rull inusen, $p$. 144
Sakal, ${ }_{616}$

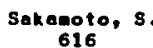

Schumacher, $M$.

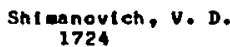

Saend, $F$.

Tagaghtra, Ho Tollinghutsen, J.

Tapeler. $M$.
466

Vacqule, s. 693

varulsch, P. 465

zlegeler, $L$.

Saelee, $H . T$ T.

Gee, $N_{1716}$

Giliesple, H. H.

ItIkama, Y.

l.ang, N. $r$

Lueas, J.

Neobet, R. 4.

Ond a, K. $1915 \quad 1317$

Poe, $R_{6.5} T$.

Shan, Y.

Stern, R. C.

sun, $\underset{6 \mathrm{~S}}{\mathrm{~J}} \mathrm{C}$.

Truhlar, De G.

Vincent, H. 1096

Hood, R. R.

i18i
Kaneko, $S$.
Kif

Sakal, Y.

Sakamoto, S.

Tagashira. H.

$\underset{1055}{\operatorname{vincent}, F .} 1096$

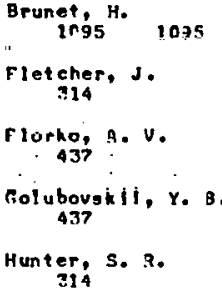


611

TRANSPORT PHDNONENA AND AUERAGE PROPEATIES IN GASES

Electron Attechment Coeprictents

$\underset{1213}{\text { Brooks, H. L. }}$

HO1

PHOTON COLLISIONS MITH HEAUY PARTICLES, ELECTRONS AND PHOTONS IN OASES (AV $100 \mathrm{keV}$ )

General

$\underset{1757}{\text { Andreev, N. F. }}$

Bespal ov., V. I.
1757
Hunter, s. s.

Nygaurd, K. J.

inas J. Wh. Jr.

Cook i877. J.

KIselev, A. H.

Lam, J. Fo

1673

Let ikhov, v. s

563

Morse, 1742

PasmenIK, G. A.

1757

shepeleulch, V. V.

shore. B. 4 .

Steel, T. G.

Minogln, V. G. 
$\mathrm{HO}_{2}$

PHOTON COLLISIONS UITH HEAVY PARTICLES. EECTRONS AND PHOTONS IH GASES ThE $100 \mathrm{keV}$ )

Total Abcorptlon

AJ1ka, T. $G$

Ak Inoto, $Y$.

$165 ?$

Al bert I, H.

Antonov, $E$. N.

1112

Bala. M. A. izon 2070

Bakhlrós I.. P.

Bolduln, K. G. H. 1244

Bnptista, G. B.

$$
311
$$

Barrus, D. M.

1714

Bastard, D.

Battaglis, A.

IHIB

Boordall, J. S.

$$
1129
$$

Bo Igang. P.

Doukt e.t i.

oneohor, $N$. D.

754

Btsehel, W. K.

BI ake, $R$. L.

2714

Boege, Q. W.

Bakar, J.

Boudourla, $G$.
IBIs

Bower, L. P.

$\underset{648}{\operatorname{Brotenoux} A}$.

Betant : :

Brown, R. J.

Burek. A. J.

Conn, M. U. P.

Cant ${ }_{1300} A . M$.

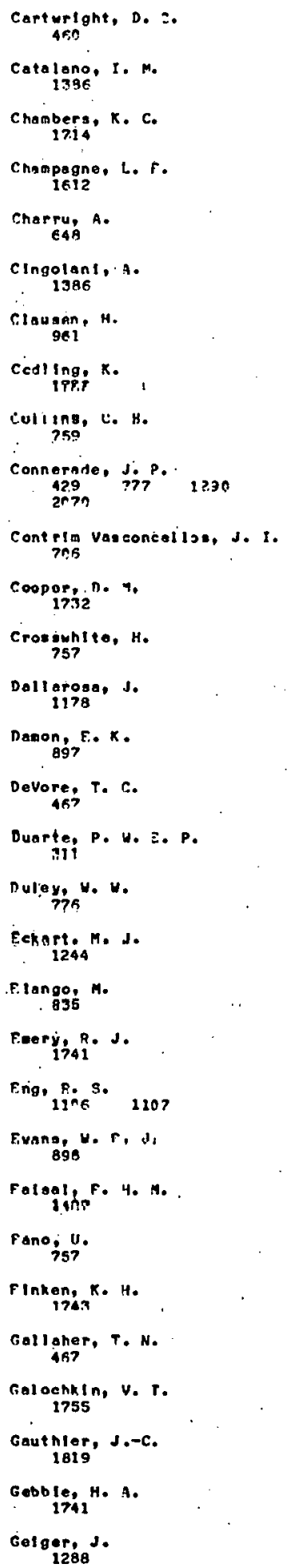

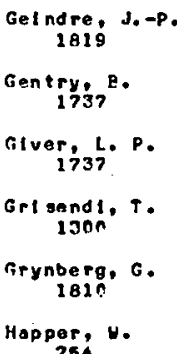


Long, H. H... Jr.

Lu, K. T.

Lucstorto, T. B.

14

Lucht, R.' A.

malste, $A$

Mal ste, A. A.

Mansfiel d, M. W. D.

Manson, S. T.$$
\text { Mintz, A. W. }
$$

Markano, A. n.

Meclelland, T.

nell rath, T. J.

149

Metz, F.

Miehels, H. H.

MIrza, H. Y.

mefat, P. H.

$\underset{\substack{\text { Moloney, } \\ \text { I4. }}}{ }$

Montenegro, F. C.
311

Mulder, T. F. A.

Muradov, V. G.
l726

Neusser, H. J.

$\underset{2 \mathrm{Cig}}{\text { Nouson, }}$. H.

Nienolls, R. W. 898

$N 1$ t2, D. F. .

Norerosa, D. W.

Nordatrom, R. $s$.

Nuron, $K$.

On1. H.

1657 Oraevsk11, A. N.
1755 nverchenko, $Y$. $V$. 1725

Park, r.

Parkinson, d. H.

Parsons, M. L.

Penner, s. 3 .

Peterson, J. C.

Pettini, M.

Pleherlt, $F$.

Platonenko, $v . T$. 1756

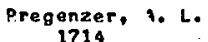

Prostatsov, G. P.

Qulgley, G. P.

Radtke, E.-?.
257

Rau, A. R. P.

Reoves, E. " 1.

Rellman, R. F.

Rhodes, C. K.

Rink, J. P.

Puus, R.

Ruus, R. E.

solter, J. .

Senearer,
259

$\operatorname{sen} \underset{774}{\operatorname{ag}, F .} u$.

Schnetder,

1316

Schwemmer, G.

senaahenko, v. S 593

shootak, S. L. 418

Selth, S. J.

259

Sojka, B. Z

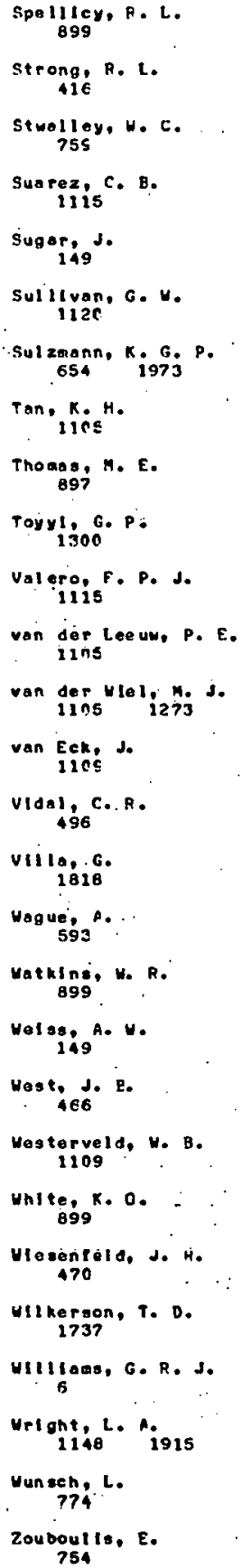


H03

PHOTON COLLISIONB UTTH HEAUY PARTICLES, ELECTRONS AND PHOTONS IN GASES (hV < $100 \mathrm{keV}$ )

Elostle scattering

$$
\text { Alay 11, Y. }
$$

Aparasevleh, P. A. 438

Balueant,, ,

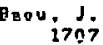

Dusl lkuv; Pa $u$.

Chaudhurt, N.

Epstain, J.
Hubhell, J. H.$$
962
$$

Kenneth, T. J. $32 n$

$x+11$, s. $y$

Kopytor, G. 875

Kusa Paju, s.

Lakshar Inarayans, V. 1796

Lobure, Y.

Naras I ina Hurty, R.

1'ינ丶万

Narasimha Murty, V. A. 1796

Qverbo, I.
PaU1, N. C:

Prestulch, W. V.

Romanathan, N. 320

Roy, S, C

Satyaendra Prasad, A. 1756

Sen Gufta, S. K.

skourones. M. 1ก37

Stevart, R. F.

1178

Tognett1, $v$.

Vallourt, R. 


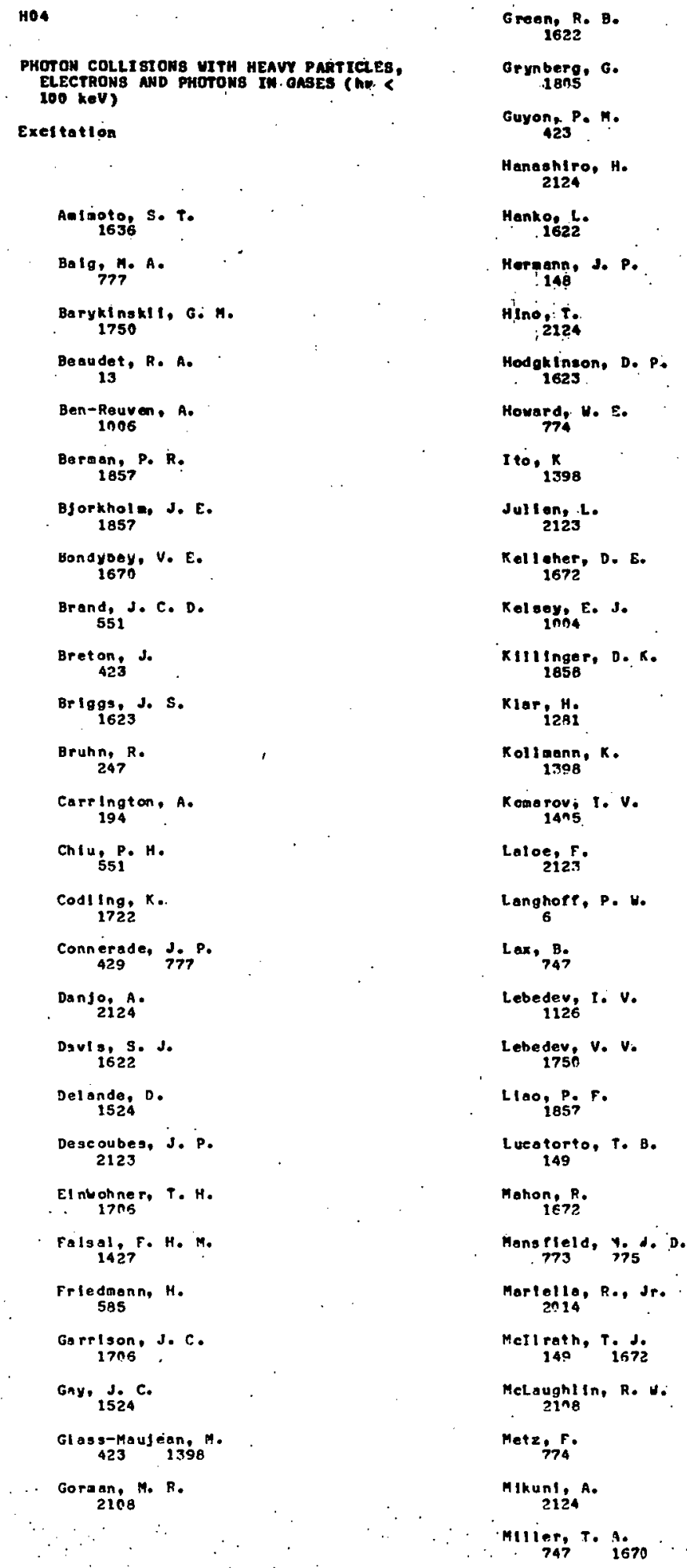

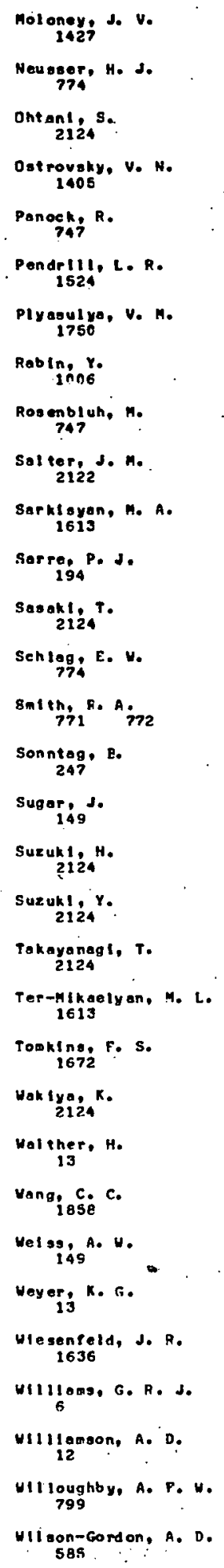


Holte, H. W.

Uong, Je

Wungeh, $L$.
Wynne, J.'J.

Yakovlevo, V. S. 1126
Young, R. H,

1636

$2 t p t, F \cdot c$.

774 


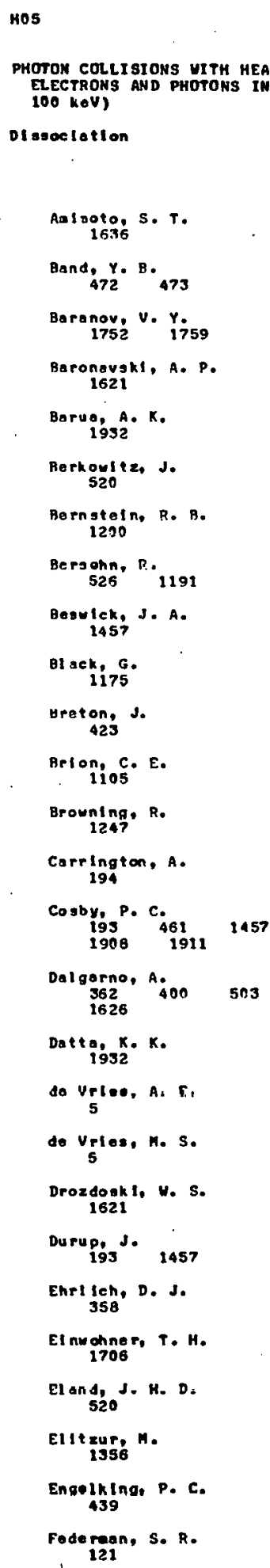

HOS 100 neV)

DIssoclation

Anlooto, S. T.

163

${ }_{472}{ }_{473}$

$\underset{1752}{\text { Baranov, V. Y广59 }}$

Baronavakl, A. $P$. 1621

Baruo, A. K.

Re rk oulte, J.

Bernsteln, R. B. 1290

Bergohn, P.

Beguick, J. A.

1457

Bl ack, G.

ureton, J.
423

Belon, C. E.

Browning, R.

Carrington, A.

194

Cosby, P. C. $461 \quad 1457$

Dalgorno, A. $\begin{array}{lll}362 & 400 \quad 583\end{array}$

Datta, $x$. K.

do vrlae, A, $E$.

verias, M. S.

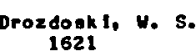

Durup, J. 193

Ehrl leh, D. J.

EI num ohner, T. H.

El and, J. H. D:

Elltzur, 1356

Engolk ing, P. C. Fode raan, s. R.

PHOTON COLLISTONS UITH HEAUY PARTICLES, ELECTRONS AND PHOTONS IM GASES ( $\mathrm{NL}^{\circ}<$
Fel doenn, $D$
1119

Flover, D. R.

Fournler, A. P.

${ }_{472}^{\text {reed, }}{ }^{2} F_{i 73}$

$\underset{526}{\underset{5}{*} \text { reedman, }}$.

Futrell, J. H.

Garrison, J. C.

Glogsgold, A. E.

Glase-mauge in, 423 ".

$\underset{\sin 3}{\operatorname{Guberman}}, \mathrm{s}$.

Gudzenko, L. I. 1751

Guest, J. A.

Guyon, P. M:

Hltcheock, 4. P. 1273 .

Hogan, P, R.

su, n. ${ }^{\mathrm{K}}$

Huang, K. -N.

1to, $\mathrm{K}$

Joekson, K. H.

Janson, M. L.

419

Johnson, K. E.

1195

Joneg, $P . L$.

Judge, D. L.

$\underset{\text { En? }}{\text { Kot oyama }}$ D. H.

Kat 0 , $\mathrm{H}$.

Kavasak!, No

$\underset{1752}{\text { Kazakov, S. A. }}$

Kirby, ${ }_{\text {Shy }}{ }^{2}$

Koll aann, $R$.

Kolow118k11, $Y_{1752}{ }_{1759}^{R .}$

Kome rov, $1 . v$.

Kraulinya, E. K. 419

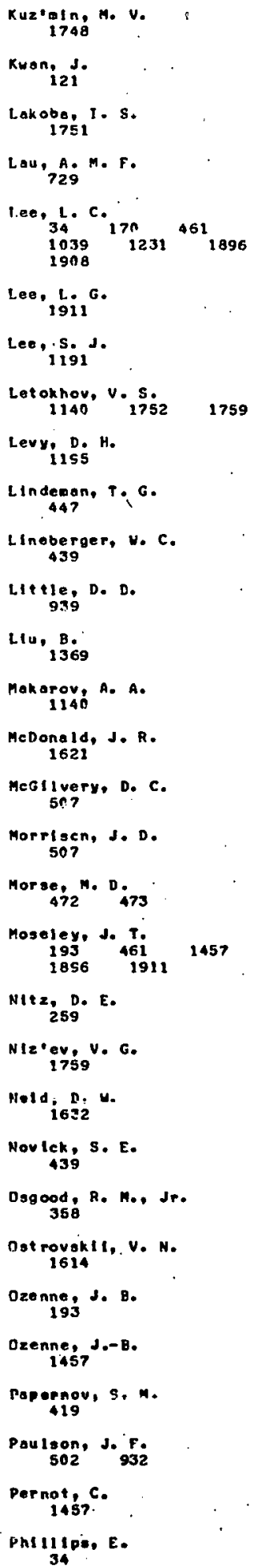




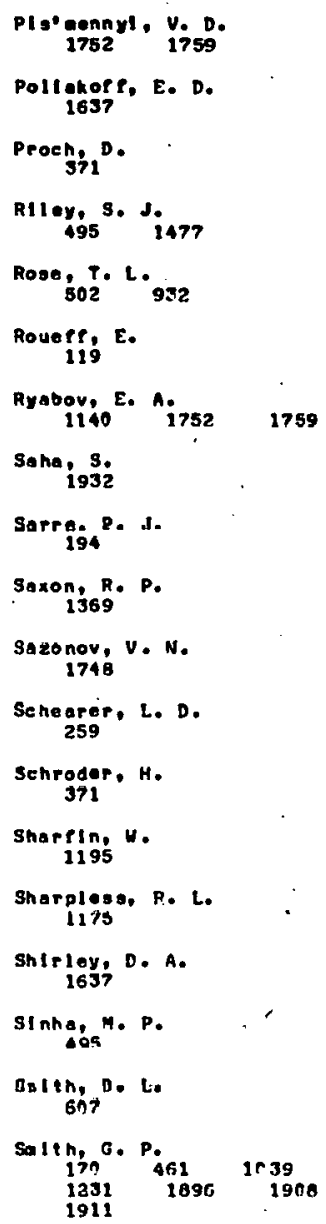

Salth, S. J.

Solth, H. H.

Southuorth, S. H.

Starace, A. F.

Starodubtsev, A. I.

1752

Stine, J. R.

stratindee, 9.

1247

Su, R. ${ }^{T}$

Ju, Ti-ii: $n$.

Syts pko, $Y .1$.

Tan, Kins $^{H .}$

Thomos, T. F.

Uasp, T6? $400 \quad 1626$

van der Laeuw, P. E.

van ter W101, i27j J.

von veen, N. J. A.

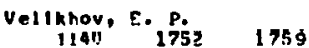

VIt osov, F. I.

Vinogradov, I. P.

von Hellfeld, A.

$$
1119
$$

Vel ge, K. H.

Vol sh. J. A.

Wharton,
ils

Wiesenfeld, J. ${ }_{447}^{R .}$

WIIloughby, A. F. U.

Wong, JP.

Wu, C. Y. R.

$\underset{\substack{\text { Yakoul lonko, s. } \\ \text { I7SI }}}{1 .}$

Yang; S. S. -C.

Yoghlhora, $k$.

120

Young, R. H.

zandee, L.

Zare, A. H.

$\underset{935}{21++01, P . F .}$ 
Hn 6

PHOTON COLLISIONS VITH HEAVY PARTICLES ELECTRONS. AND PHOTONS IN GASES (ho C 100 keV)

Ionization

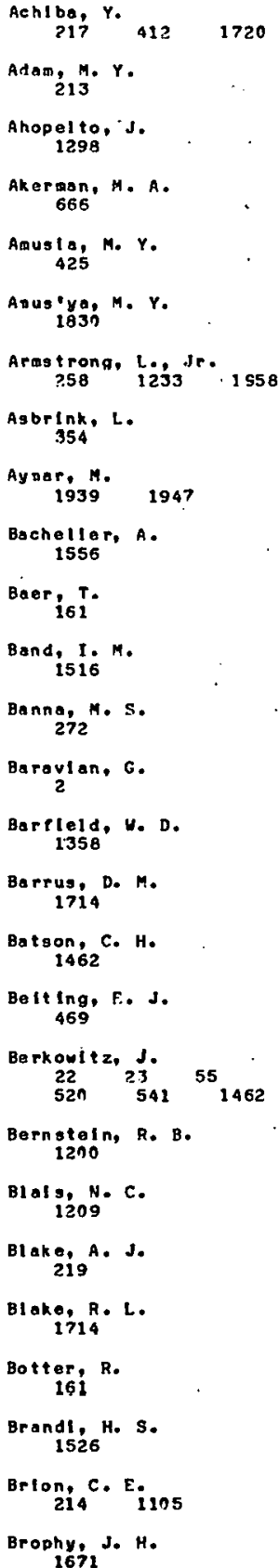

Brown Ing, $R$.
1247

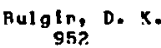

Burek, A. J.

Burke, P. G.

Burnett, $T$.

Cont u, A. M. ${ }_{7300}$

Carver, I. H.

Cederboun, L. S.

Chambers, $K . C$.

Chang, T. N.

Chellehmalzadeh, M. A. 1n38

Cheng, $K$. $T$. Cherepkov, N. A.
596

Codling, $\mathrm{K}$.

$\underset{\text { Sal }}{\text { Colbourn, E. A. }}$

Cole, B. E. 2136

Col1 Ins, C. 8 .

Combet farnoux, $f$.
1804

$\underset{244}{\text { Connerade, J. P. }}$

Cooke, W. E.

Coppens, P.

Crance, $M$.

$1939 \quad 1946 \quad 1947$

Doosoh, H. R.

algarno, A.

$$
5 n 3
$$

Danso, ${ }_{2124}^{A}$

Dovidovieh; $L$.

$$
1526
$$

Davidson, E. R.

1456

Dehmer, J. L.

1558

de $\operatorname{Lang}_{346}^{\circ}, C_{360} A^{\circ}$

de Leeuv, D. M.

Dexter, R. N.

$0111,0$.

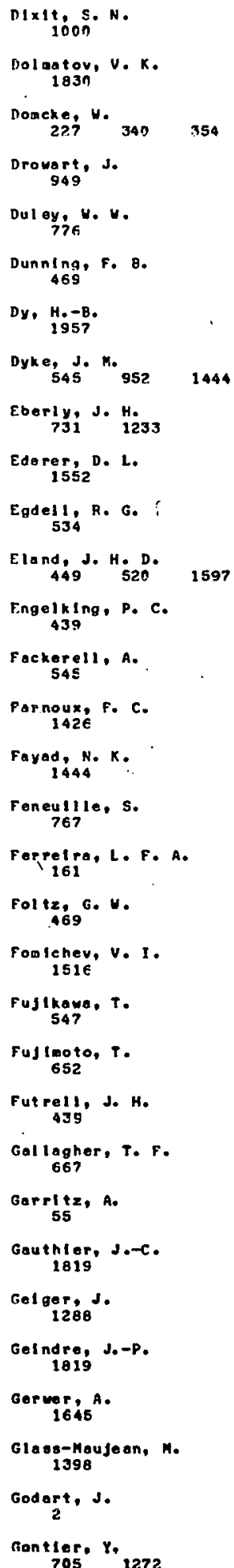




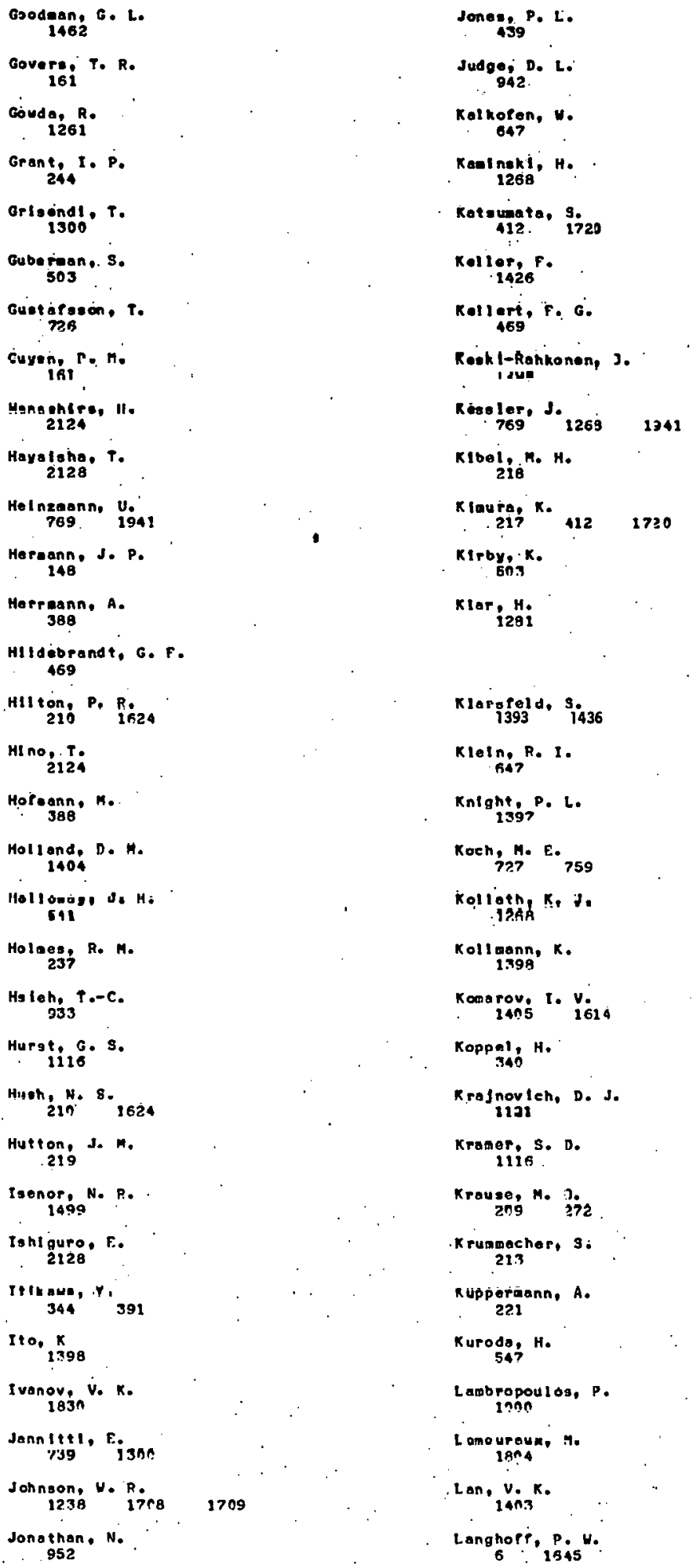

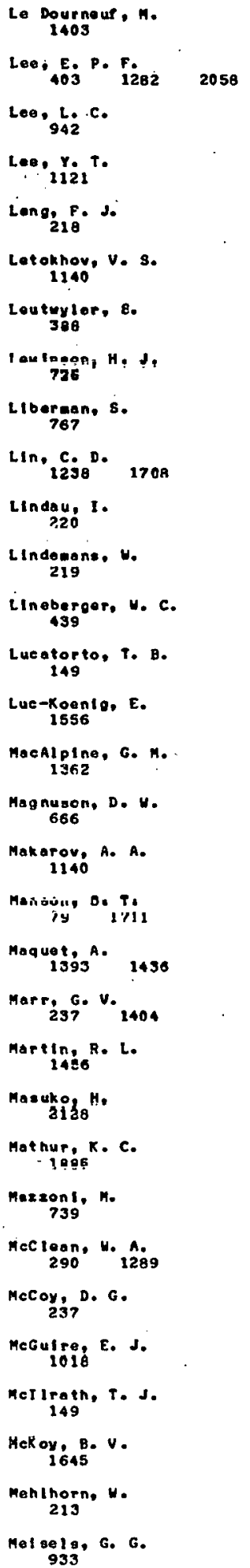




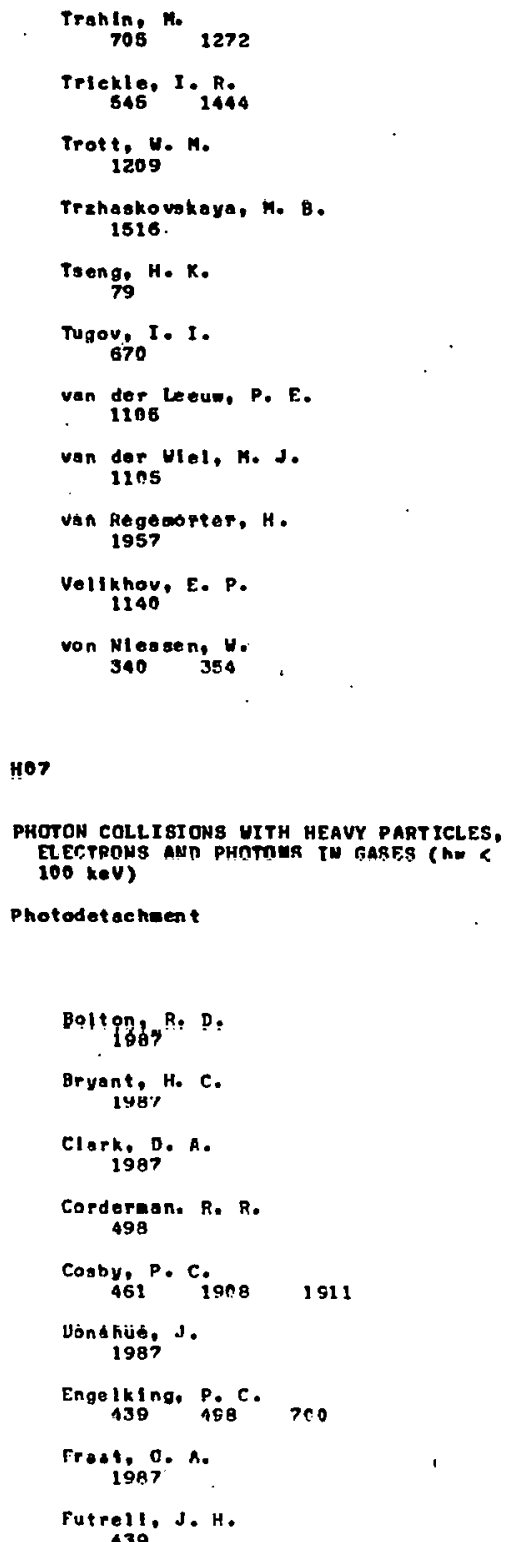


HO8

PHOTON COLLISIONS YITH HEAVY PARTICLES IIECTRONS AND PHOTOUS IU GASES (hE, IOn keV)

Fl uoresence and Lun inescence Yields

Agarual, G. S

Akeragn, 19 . A

665

Anderson. $H$. R.

Apsnasevich, P. A.

438

Auerbach, n. J.

1194

Baronavgki, A. P.

378

Barrous. R. F.

Ranediet. R. P.

$$
457
$$

Bhaskar. N. D

Blalkowskl, s. E.

BIsohel. Y. K.$$
1178
$$

Block, G
1175

Bl gekburn, M. B.

$$
114
$$

Bokoris J.

Brophy, J. H.

Brown, R. D.

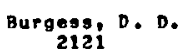

$\underset{316}{\text { Canosa, } C .}$

ChI Ids, J. J. 1873

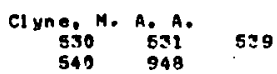

Crofts, J. $G$.

Crosley. D. S.

ovotonavie, n. $s$ 1161 CaujkougkI. $M$.

Dall arosa, J.

$$
1178
$$

Dang-Nhu, 1108

Dent roder, W.

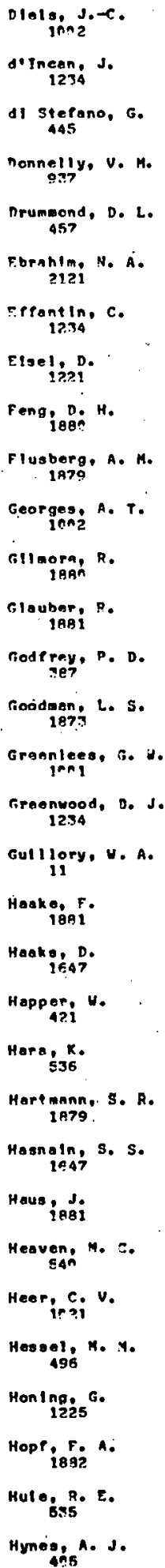




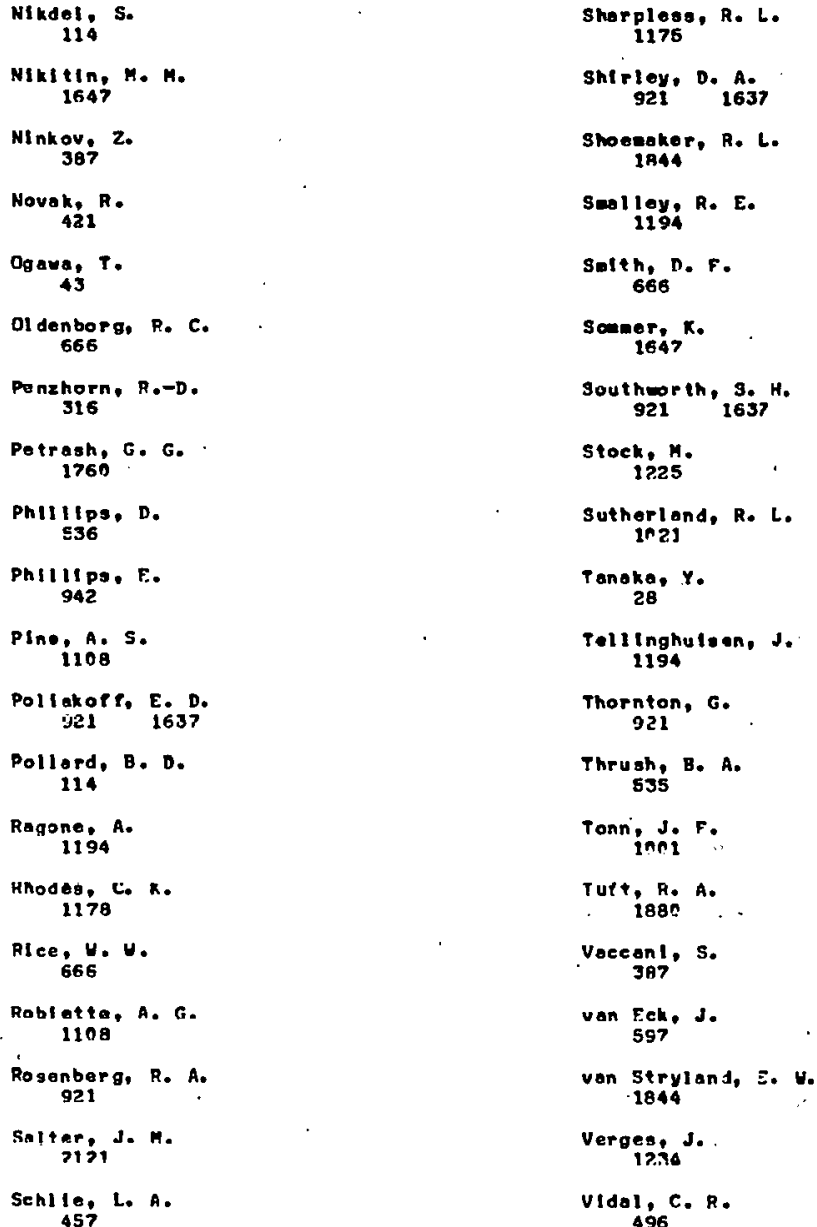

Nikdel: S.

$\underset{1647}{\text { Nikltin, } M . M .}$

$\underset{387}{\text { Ninkov. }}$

Novak, $R$.

Ogava, $T$.

O1 denborg, R. C.

Penzhorn, R.-D.
316

Petrash, G. G.

176

Phillips, D

Phlllipa. E.

942

PIno, A. S.

1108

Poll lakote, E. D.

j21 1637

Pollerd, B. D.

114

Ragone: $A$.

Hnodes, $c . x$.

1178

Alce, U. U.

Roblatta, A. G

$$
1108
$$

Rosenberg, R. A.

Saltar, J. H.

Senlle, L. A.

Sharpless, R. L.

$$
1176
$$

shirley, O. A.

Shoenaker, R. L.

Salley, R. E.

$\underset{668}{\operatorname{senteh}, D . F .}$

somer, $_{1647}$

Southoorth, ${ }_{921}^{3 .}$ H.

stock, 1 .

Sutherland, R. L.
Inzl

Tanake, $Y$.
28

Tellinghuteen, J 1194

Thornton, $G$.

921

Thrush, B. A.

Tonn inni $F$.

ute, $R, A$.

i88

Voeesanl, $S$.

3 A7

van Eek. J.

van seryland, $\Xi$.

Vergest J.

VIdaI, C. R.

schroder $G$.
VII e*ov, F. I

$$
187
$$

Vinogradov, I. P.

$$
167
$$

von Sonntag, $C$.

$$
316
$$

Walker, H. C.

yampler, F. B.

Uenci, H. D.$$
1647
$$

$\underset{666}{\text { Verner, } G .}$ K.

tharton. $t$. 1194

unlto, in. $\hat{G}$. $\underset{\substack{\text { ulloghby, A. F. W. } \\ 799}}{\text {. }}$

Utuarordnar, I. 0 . 114

Wu, C. Y. R.

Yakushev, O. F. 1760

Yoshihara, K. 1205

Voshino, $x$. 28

Zore, P. $_{0}$

Zovgol19. D. 1221

zlomorer, G. 1647

Zol ter:P.

Zouboulls, E. 421 
H10

PHOTON COLLISTONS UTTH HEAVY PART ICLES,
ELECTRONS AND PHOTONS IN GASES ( 100 kev)

\section{Photochenlat ry}

Benediet, R. P.

Blalkowskl, S. E.
11

Canssa, $C$.

$\operatorname{Cox}, \operatorname{Rin}_{2 \sin }^{A \cdot}$

Cvetanoule, R. J.

1161

$\underset{2968}{\operatorname{Daruent}}$. $G$.

di Sterano, $G$. Drusmond, D. L.

Hะ1

PHOTON COLLISIONS UTTH HEAVY PARTICLES, ELECTRONS AND PHOTONS IN GASES (hD < 100 neV)

Free-free abeorption or Inverse

Bremsetrahlung

$\underset{\text { Clarke, E. M. }}{\text { Ind }}$

H12

PHOTON COLLI SIONS UITH HEAUY PARTICLES, ELECTRONS AND PHOTONS IH GASES (hN < loo nev')

Photon-Electron seattaring

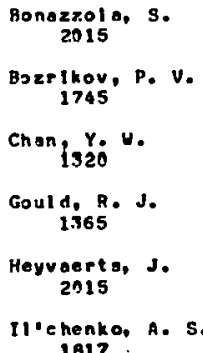

Eggleton, A.: E. J J.$$
\text { 2n68 }
$$

F.hr I I ch, D. J.

Gutl tory, 4 . A.

$$
11
$$

Kasimoto, 0 .

Lenz I, 445

Margan $1 . A$

$\underset{402}{\operatorname{Mller}}$ H. H. .

$\underset{445}{\text { Nguyen }}$ Xuan, $c$.

Orel, A.

492

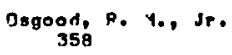

Penzhorn, $R,-D$.
II6
Proch, $D$.

Quock, M.

Peld, H. J.

Schlle, L. A.

Sehroder. $H$.

Shostok, S. L.

416

Strong, . L.

Tanake, $Y$.
28

von Sornteg, $C$.

Walker, W. C.
28

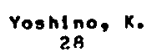

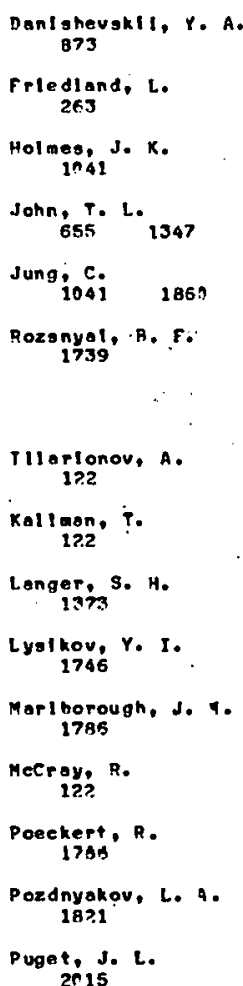


H14

PHOTON COLLISHONS UTTH hEAUY PARTICLES, EECTRONS AND PHOTOWS IN OASES (no < $200 \mathrm{keV}$ )

Phot orde-txeitetlon, quanehing, and

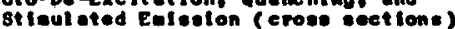

HI ne hen, J. J.

15

Jo1

Data COMPILATION

Heovy Particle

Benko, ${ }^{0 .}$

sos

DATA COMPILATION

Photons

Krauge, M.

xo1

REvirus avp gnoks

Ho avy Poiptlele

Botegi D. R.

KO2

REUIEUS AND BOOKS

Electrons

Bates, D. R.
Hobbe, R. H.

Mal on kova, L. D.
877
Kropt, Aiz:

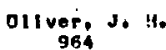

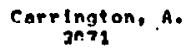

$\underset{240^{4}}{\operatorname{Lorenz}}$

Laranz, A. 
337

$\mathrm{KOJ}$

REVIEUS AND BOOKS

Photans

$$
\text { Bates, D. P. }
$$

KO4

REVIEUS AND BOOKS

Particles on Surfaces ond Sollds

Lorenz, A.

Kө6

REUIEUS AND BDOKS

structure

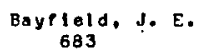

L.03

BI BLIOGRAPHIES

Photons

$$
\text { Way, Ka: }
$$

\# U.S. GOVERNMENT PRINTING OFFICE: 1981--310.912:1062
Boyfleld, f. E.
683

$\operatorname{cod} \operatorname{lng}_{1722} x$.

Galochkin, v. T.
loss

tetokhov, V. S. Isn 1
Lorenz,
1469

nraevok11, A. N.

stenholm, $s$.

$43 n$
Cisinerade, J.P.

Lorenz, $A$.

Stern, F. A.

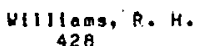

Willoughby, A. f. U.
7s5 
United tes

Departi.uout of Energy

Washington, DC 20545

Otficial Business

Penatty for Private Use, $\mathbf{8 0 0}$
Postage and Fees Paid

U.S. Desartment of Energy DOE-350 